\title{
W. AHRENS
}

\section{SCHERZ UND ERNST}

\section{IN DER MATHEMATIK}

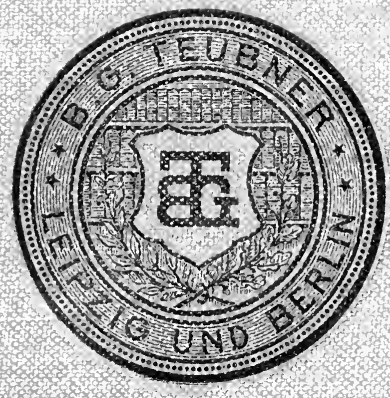




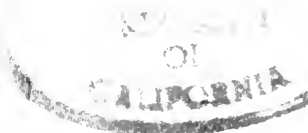

"4. 1. TTAT

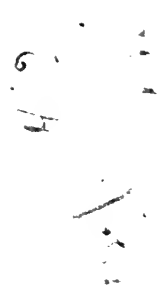

$\therefore$ 






\section{SCHERZ UND ERNST IN DER MATHEMATIK}

GEFLÜGELTE UND UNGEFLÜGELTE WORTE

GESAMMELT UND HERAUSGEGEBEN

VON

DR. W. AHRENS

IN MAGDEBURG

冤

LEIPZIG

VERLAG VON B. G. TEUBNER

1904 
Alle Rechte, einschlieblich des Übersetzungsrechts, vorbehalten. 


\section{Vorwort.}

Bücher $\left.{ }^{1}\right)$ sind, einem bekannten Wort Beaumarchais' zufolge, wie die „Kinder der Frauen“: „conçus avec volupté, menés à terme avec fatigue, enfantés avec douleur". Der Autor des vorliegenden Buches, der der bequemen und ungefährlichen Kunst gehuldigt hat, ein Buch zu "schreiben“, das von anderen „verfaßt", ja der sogar bei der Wahl des Titels diesem Grundsatze treu geblieben ist, ist begreiflicherweise über jene erste der drei Entwicklungsphasen Beaumarchais' kaum hinausgekommen. Doch vielleicht ist gerade dies der schlagendste Beweis für die Unfertigkeit dessen, das nunmehr der Öffentlichkeit übergeben wird. Wie dem immer auch sei - ich hoffe das bescheidene Ziel, das ich mir gesteckt, zu erreichen; würde ich doch meine Mühe hinreichend belohnt finden, wenn das zunächst zu meiner eigenen Unterhaltung und Erholung gesammelte Material auch anderen Fachgenossen Unterhaltung und Erheiterung in Mußestunden gewähren würde.

Bei dieser Bestimmung des Buches für die Unterhaltungslektüre habe ich, wenn auch die Anordnung der Citate keine ganz willkürliche ist, von einer strengen Systematik, - die, wie ich befürchten mußte, nur ermüdend wirken, übrigens auch kaum durchführbar sein würde, - ebenso wie von einer Einteilung des Ganzen in Abschnitte, von vorneherein absehen zu sollen

1) In einem Buch, dessen vornehmste und einzige Aufgabe darin besteht, richtig zu citieren, darf hier nicht die Bemerkung unterlassen werden, daB der Urvater des „Figaro" an der betreffenden Stelle („Lettre modérée sur la chute et la critique du barbier de Séville"; Oeuvres complètes, t. 1 (1809), p. 372) nur von Dramen spricht. 
greglaubt. Durch Berïcksichtigung auch derjenigen Literatur, die dem Fachmathematiker ferner liegt, hoffe ich auch diesem noch einiges zu bieten, auf das seine Aufmerksamkeit bisher vielleicht nicht gerade gelenkt wurde und das ihn doch interessieren möchte. Insbesondere dürften aber angehende Nathematiker hier eine bequeme Möglichkeit finden, sich auch bibliographisch über die, wenn ich so sagen darf, „schöngeistige“ mathematische Literatur zu unterrichten, auf die hingewiesen zu werden, sie in ihrem offiziellen Bildungsgang naturgemäß wenig Gelegenheit haben, und möchten somit aus diesem Buch die Anregung zu weiterer Lektüre schöpfen. Dieser Umstand bestimmte mich auch, die Titel von Vorträgen usw. möglichst üherall genau anzugeben, auch dort, wo das eigentliche Thema der betreffenden Schrift mit dem daraus entnommenen Citat nur in ganz losem Zusammenhang stand und wo ich daher in meinen ursprünglichen Aufzeichnungen den Titel bisweilen ganz fortgelassen hatte. Schließlich habe ich mir noch die Aufgabe gestellt, solche Worte, die für die Fach- oder selbst Laienwelt als geflügelte gelten dürfen und ihrem Inhalte nach hierher gehören, aufzunehmen, jedoch ohne daß diesen etwa eine gesonderte oder gar hervorragende Stelle zugewiesen wäre; ihre Einreihung unter andere Citate war oft nicht ohne gewissen Zwang möglich, so daß einige von ihnen, wenn eben nicht

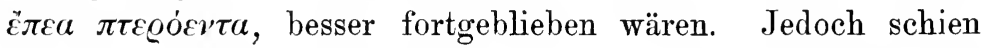
mir die Aufnahme solcher Worte um so wünschenswerter, als die Untugend, falsch zu citieren, bekanntlich nicht bloß eine Spezialität der Tagespresse ist, vielmehr auch aus der wissenschaftlichen Literatur mit Leichtigkeit vielfache Belege dafür beigebracht werden könnten, daB nur zu oft nach dem Xenienwort verfahren wird:

Allegire der Erste nur falsch, da schreiben ihm zwanzig

Immer den Irrthum nach, ohne den Text zu besehn.

Für diejenigen Leser zumal, welche das Buch auch als Citatenschatz für gelegentliche literarische oder sonstige Zwecke zu benutzen gedenken, habe ich ein umfangreiches Register beigegeben, das jedoch, ohne ungebührlich lang zu werden, nicht 
so ausführlich gestaltet werden konnte, wie dies bei dem heterogenen Inhalt des Buches an sich möglich und manchem vielleicht erwünscht wäre. - Der Begriff Vollständigkeit entbehrt schon bezüglich der sogenannten "geflügelten Worte“ — von den nicht geflügelten natürlich gar nicht zu reden - jeder Bedeutung, und ich hoffe daher, man werde mir das Fehlen solcher Worte, die man vielleicht mit Bedauern vermißt, insbesondere auch aus der nichtfachlichen Literatur, umsoweniger zum Vorwurf machen, als ich eben, wie schon oben gesagt, nur die Gelegenheitsfrüchte einer in der Hauptsache ganz unsystematisch betriebenen Lektüre zu bieten mir vorgenommen. ${ }^{1}$ ) Es war auch nicht einmal mein Bestreben, jeden Autor unbedingt gerade mit solchen Aussprüchen auftreten zu lassen, die für seine wissenschaftliche Stellung, seine Denkweise, ïberhaupt seine ganze Individualität besonders charakteristisch sind; auch wird man in der Zahl der Citate nicht einen Maßstab für die dem betreffenden Autor beigelegte Bedeutung erblicken dürfen, vielmehr waren hier neben persönlichen Liebhabereien vorwiegend mehr zufällige Momente ausschlaggebend und mußten es aus naheliegenden Gründen sein. So knüpft z. B. eine recht große Zahl von Citaten an die Namen Gauss und Jacobi an. Geben nun allerdings auch diese beiden glänzenden Namen an sich hierfür schon eine hinreichende Rechtfertigung, so war dabei für mich doch weiter noch bebestimmend, daß über den ersteren ein größeres biographisches Werk - fast 50 Jahre nach seinem Tode - immer noch nicht existiert und bezüglich des letzteren, daß wenn auch hier ein derartiges Werk von hervorragendster Seite ganz nahe bevorsteht, infolge der diesjährigen Centenarfeier noch ein besonderes erhöhtes Interesse in Fachkreisen vorausgesetzt werden darf. Prinzipiell ausgeschlossen sind natürlich solche Aussprïche, für die Belege aus der Literatur nicht beigebracht werden können, auch wenn sie an sich hinreichend verbürgt erscheinen.

1) Natürlich würde ich etwaige Wünsche und Winke aus dem Leserkreise bereitwilligst und mit Dank berücksichtigen, falls eine neue Auflage sich vernotwendigen sollte. 
loh habe mir ferner nicht verhehlt, dals eine solche Simmlung ron zum betrïchtlichen Teil der modernen Literatur entnommenen Citaten leicht die Gefahr bietet, Anstoß zu erregen, was unter Enständen rielleicht schon durch die Gruppierung ron an sich harmlosen Worten geschehen könnte. Mehrere Citate meiner Simmlung sind daher mit Rïcksicht auf noch lebende Personen fortgelassen worden. Die Selbst-Kompromittierung - zweifellos eins der elementarsten Menschenrechte - habe ich dagegen niemindem, weder Toten noch Lebenden, verwehren zu diurfen greglaubt; der Leser mag entscheiden, welche Worte er als hierher gehörig betrachten will.

Das eimzige ältere Werk, das man mit dem vorliegenden seinem (harakter nach vergleichen könnte, ist, so viel mir bekamnt, das Buch Rebières "Mathématiques et Mathématiciens"

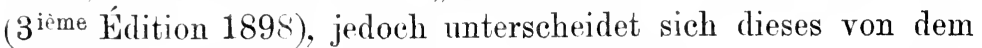
meinigen so wesentlich, ${ }^{1}$ ) dab es mir für meine Zwecke keinerlei irgendwie nennenswerte Dienste leisten konnte: Zunächst wird dort der Stoff nach gewissen, allerdings mehr äußerlichen Grundsïtzen angeordnet, indem z. B. in einen ersten Teil ,morceaux "hoisis et pensées", in einen zweiten "variétés et anecdotes", in einen dritten „paradoxes et singularités“ usw. verwiesen werden. Sodann fehlt dort jede Angabe der Quelle; dies erschwert eine Kontrolle nicht nur sehr, sondern schließt sie oft geradezu aus und hat den weiteren Nachteil, daß es einem Interessenten recht unbequem gemacht wird, über ein aus dem Zusammenhang gerissenes und daher leicht mehr oder minder beeinträchtigtes Wort an der Originalstelle im Zusammenhang genauere Orientierung zu suchen. Für manche der Rebièreschen Citate dürften Belegstellen aus der Literatur überhaupt nicht beizubringen sein, und ich habe daher auch nur ein einziges Citat von dort ïbernommen (s. S. 437), dessen Aufnahme ich möglicherweise

1) Noch weit mehr gilt dies von Maupin, „Opinions et curiosités touchant la Mathématique", über dessen Anlage ich allerdings nur nach seinem ersten Teil urteile, während ein - wofern ich nicht irre - kürzlich ersehienener zweiter Teil mir noch unbekannt ist, ebenso wie auch das Buch Rebière's "Pages choisies des Savants modernes". 
auch schon gezwungen sein werde, mit dem „se non è vero, è ben trovato" zu motivieren. Im Gegensatz zu dem Rebièreschen Buch ist bei mir überall die betreffende Belegstelle möglichst genau angegeben, bei mehrfach abgedruckten Sachen oft sogar mehrere Stellen, obwohl ich eine Verpflichtung in letzterer Hinsicht allerdings nicht anerkannt habe. Alle von mir angegebenen Stellen sind auch von mir selbst eingesehen, Ausnahmen hiervon sind extra bemerkt. - Für einige Aussprüche, für welche mehrere oder vielfache Belege aus der Literatur gegeben werden könnten, eignete sich aus äußeren Gründen die älteste der mir bekannten Stellen für meine Zwecke weniger und mußte daher einer neueren weichen; jedoch ist dann tunlichst auf solche ältere Stellen verwiesen (s. z. B. S. 228). Im Gegensatz zu Rebière, der alle Citate nur in französischer Sprache wiedergibt, habe ich die Originalsprache stets ${ }^{1}$ ) beibehalten und auf hinzugefügte Übersetzungen, so weit mir dies irgend angebracht erschien, verzichtet. Dies letztere gilt natürlich zunächst von den französischen Citaten, die fast so zahlreich wie die deutschen sein dürften, sodann aber auch von den weniger häufigen englischen, italienischen und lateinischen. Den vereinzelten Citaten aus einer anderen Sprache habe ich eine deutsche Übersetzung beigegeben, falls nicht meine Quelle mir die Reproduktion einer Übersetzung in eine andere der oben genannten Sprachen gestattete. In allen Citaten sind Wortlaut und Schreibweise der Belegstellen getreu beibehalten, und zwar normierte bei solchen Sachen, die an mehreren Stellen erschienen sind und dort fast regelmäßig in Kleinigkeiten divergieren, eine der angegebenen Belegstellen, jedoch nicht immer gerade die an erster Stelle angeführte. Die buchstabengetreue Reproduktion eines Citats, z. B. einer Briefstelle, scheint mir an sich das

1) Eine Ausnahme bilden allerdings begreiflicherweise ein paar russische und ein hebräisches Citat, bei denen ich - auch ohne meine völlige Unkenntnis der betreffenden Sprachen - auf eine Reproduktion der Originale verzichtet haben würde, da diese für die Mehrzahl meiner Leser doch wahrscheinlich wertlos gewesen wären, zumal die betreffenden Citate nicht für sonderlich wichtig angesehen werden dürften. 
einzig richtige $\mathrm{zu}$ sein, wobei ich mir gestatte, auf die Worte zu verweisen, mit welchen die Herren Stäckel und Schmidt dieses bei Herausgabe des Briefwechsels Gauss-W. Bolyai von ihnen beobachtete Verfahren rechtfertigen, wo l. c. p. 172 mit Recht bemerkt wird, daß anderenfalls „das Charakteristische zerstört und dem Biographen wie dem Sprachforscher wichtiges Material entzogen wird, ganz abgesehen davon, daß auch die neue Rechtschreibung der Gefahr des Veraltens ausgesetzt ist", welch' letzteres Moment mir um so berücksichtigenswerter zu sein scheint, als in Deutschland schon seit geraumer Zeit bei dem beständigen FluB der sogenannten „Rechtschreibung“ und dem dadurch bedingten Antagonismus zwischen den jeweiligen amtlichen Vorschriften und den selbst in Amtsstuben gebräuchlichen Spezial-,Orthographien“ von einer "Rechtschreibung" überhaupt kaum noch die Rede sein kann. Allerdings hat die Befolgung dieses Prinzips der getreuen Wiedergabe in meinem Falle einige UngleichmäBigkeiten zur Folge, hierfür nur ein Beispiel: Während ich also einen Brief Gauss' an Bolyai dem oben angegebenen Grundsatze der Herausgeber zufolge in der Original-Schreibweise zu reproduzieren in der Lage bin, ebenso übrigens auch Briefe Gauss' an H. C. Schumacher, bin ich gezwungen, einen Brief des "Princeps mathematicorum" an Bessel in einer der vor 25 Jahren üblichen Rechtschreibung einigermaßen assimilierten und einen solchen an Olbers in einer noch neueren, natïrlich auch „Orthographie“ genannten Schreibweise wiederzugeben. ${ }^{1}$ ) Einige offenbare Versehen oder Druckfehler

1) Zu welchen Ungereimtheiten dies führt, dafür nur einige Beispiele: Während Gauss in seinen Briefen an Bessel noch "Coefficient" schreiben darf, darf er in seinem Verkehr mit Olbers nur mit "Koefficienten" operieren; wird eine andere Gauss'sche Korrespondenz, etwa die bisher uur auszugsweise publizierte mit Encke, einen Herausgeber gleich modernisierender Tendenzen finden, so werden dort sicherlich nur noch „Koeffizienten“ gestattet sein — und da möglicherweise die wandelbaren Neigungen unserer amtlichen Rechtschreibung auch von dem Gesetz beherrscht werden: "On revient toujours à son premier amour", so dürften eventuell spätere Editionen Gauss'scher Briefe wieder die umgekehrte Entwickelung zu der Original-Schreibweise zurück nehmen. 巳̈brigens 
in den Citaten habe ich allerdings verbessert; späterhin, seitdem ich den Plan einer Publikation hatte, sind auch solche Änderungen, wie unten angegeben, gekennzeichnet, jedoch schien mir die große Mühe, alle Citate auf diese und ähnliche Kleinigkeiten vor dem Druck nochmals zu prüfen, in gar keinem Verhältnis zu dem Wert der Sache zu stehen, zumal ja die Worte an den angegebenen Originalstellen von Interessenten in besonderen Fällen doch jedenfalls nachgeprüft werden würden. Bei einigen wenigen Citaten habe ich auch wohl zwei Absätze umgestellt, wenn hierdurch das aus dem Zusammenhang gerissene Citat leichter verständlich wurde, auch sonstige unwesentliche Änderungen vorgenommen, z. B. ein durch Aufhebung des Zusammenhangs überflüssig gewordenes Verbindungswort fortgelassen oder dgl., ohne daß es mir - aus dem angegebenen Grunde - möglich war, dies immer anzugeben resp. anzudeuten. Einige wenige Citate werden erst durch die vorhergehenden verständlich, andere haben erläuternde Zusätze erhalten, von denen einige („Noten“) aus technischen Gründen an das Ende des Buches gesetzt werden mußten. Jedoch glaubte ich hinsichtlich solcher Erläuterungen nicht allzu weit gehen zu dürfen, da allzuviele Anmerkungen den Leser nur belästigen; bei einzelnen vielleicht überflüssig erscheinenden Zusätzen wolle der deutsche Leser berücksichtigen, daß das Buch sich vielleicht auch außerhalb Deutschlands einige Freunde erwerben möge.

kann man hierfür leicht schon jetzt Belege beibringen: ich greife drei Briefe Gauss' heraus, welche alle dem Jahre 1814 angehören und auf einen Zeitraum von nur 2 Monaten (13. Sept. - 13. Nov.) entfallen; in dem 1860 erschienenen Band I des Briefwechsels Gauss-Schumacher heiBt es p. 108: "diesmal" (Original-Schreibweise), in dem 1880 publizierten Briefwechsel Gauss-Bessel dagegen (p. 203) modernisiert: „diess Mal", schlieBlich nach abermals 20 Jahren in dem 1900 ausgegebenen Briefwechsel Gauss-Olbers (p. 561) wieder: „diesmal". - Zudem wird mit der Modernisierung in keinem der beiden erwähnten Werke wirklich radikal vorgegangen, so daß man selbst in dem neueren Werke Schreibweisen findet, die schon jetzt als veraltet anzusehen sind und daher möglicherweise von einzelnen Lesern störend empfunden werden, womit dann natürlich das Hauptargument für das beobachtete Verfahren ziemlich binfällig wird. 
Ließen sich erläuternde /usätze zwanglos in den Text des Citats selbst einfïgen, so ist dies geschehen, zumal immer dann, wenn hierfür auch eigene, vorher oder nachher gebrauchte Worte des citierten Autors benutzt werden konnten; solche Zusätze im Text sind in eckige Klammern [] eingeschlossen, während runde Klammern () original sind, also von dem eitierten Autor herrühren. Ebenso bedeuten in den Stellenangaben und bei Übersetzungen eckige Klammern Zusätze von mir, während eine dem betreffenden citierten Werk entnommene Übersetzung oder die \%usïtze von Herausgebern - z. B. in einer Briefstelle das vom Herausgeber zugesetzte Datum oder dgl. - in runde Klammern eingeschlossen sind. Die mit Ziffern 1) etc. eingeleiteten Fußnoten rühren, wofern nicht anders bemerkt, von mir her, während die dem Citat als solchem angehörenden Anmerkungen unmittelbar auf das Citat folgen und durch Asterisken (*) eingeführt sind (z. B. S. 50). Bei den Hinweisen, welche die Fußnoten geben, bezieht sich, wenn die Seitenzahl durch „S." bezeichnet ist, der Hinweis auf dieses Buch selbst, während bei einem Hinweis auf eine andere Stelle des citierten Werkes diese durch ein "p." charakterisiert ist. Sind in den Stellenangaben Verlagsort und Verlagsjahr vermerkt, so sind diese zur Unterscheidung von anderen Orts- und Zeitangaben in runde Ǩlammern geschlossen, so daB eine Angabe, wie z. B. „Verhandl. des 1. intern. Mathem.-Congr. Zürich 1897 (Leipzig 1898)" ohne weiteres verständlich sein dürfte. Bei Zeitschriften, bei denen mehrere Serien zu unterscheiden sind, folgt die wie ïblich in runde Klammer geschlossene Serienzahl auf den Namen der Zeitschrift, worauf dann die Zahl des Bandes kommt. Bei den sehr häufigen Stellen aus Briefen sind, abgesehen von einigen besonderen Fällen, Ort und Datum, unbeschadet der Schreibweise des Originals, der Einfachheit und Übersichtlichkeit halber, in gleicher Weise angegeben und zwar nach dem folgenden Muster:

Magdeburg, 18. V. 1904.

W. Ahrens. 
Der Zweck der Wissenschaft ist, die Wirklichkeit zu begreifen und das Vergängliche aufzufassen als die Erscheinungsform des Unvergänglichen, des Gesetzes.

H. von Helmholtz.

s. „Anspr. u. Red. geh. bei der HelmholtzFeier 2. XI. 1891" (Berlin 1892), p. 13.

Omnis philosophiae difficultas in eo versari videtur, ut a phaenomenis motuum investigemus vires naturae, deinde ab his viribus demonstremus phaenomena reliqua.

IsaAC Newton.

„Principia mathem. philosophiae naturalis“, Auctoris praefatio ad lectorem.

Unter Erklären versteht der Naturforscher nichts anderes, als das Zurückführen auf möglichst wenige und möglichst einfache Grundgesetze, über die er nicht weiter hinaus kann, sondern sie schlechthin fordern muss, aus ihnen aber die Erscheinungen erschöpfend rollständig als nothwendig ableitet.

\section{Gauiss.}

"Erdmagnetismus und Magnetometer." s. Werke, Bd. 5 (1877), p. 315316.

Das endliche Ziel der theoretischen Naturwissenschaften ist, die letzten unveränderlichen Ursachen der Vorgänge in der Natur aufzufinden. Ob nun wirklich alle Vorgänge auf solche zurückzuführen seien, ob also die Natur vollständig begreiflich sein müsse, oder ob es Veränderungen in ihr gebe, die sich dem Gesetze einer nothwendigen Causalität entziehen, die also in das Gebiet einer Spontaneität, Freiheit, fallen, ist hier nicht der Ort zu entscheiden; jedenfalls ist es klar, dass die Wissen-

A hrens, Scherz und Ernst i. d. Mathem. 
schaft, deren Zweck es ist, die Natur zu begreifen, von der Voraussetzung ihrer Begreiflichkeit ausgehen mïsse, und dieser Voraussetzung gemäss schliessen und untersuchen, bis sie vielleicht durch unwiderlegliche Facta zur Anerkenntniss ihrer schranken genöthigt sein sollte.

\section{HELMHOLTZ.}

t"ber die Erhaltung der Kraft", Vortr. phys. Ges. Berlin 23. VII. 1847.

s. Ostwald's Klassiker d. exakt. Wiss. No. 1, p. 4.

Ein Gewitter, welches die Sonne verdunkelt, heisst Zufall: eine, durch den Mond rerursachte Sonnenfinsterniss heisst nicht Zufall: von dem einen Ereignisse wissen wir nicht die Ursachen, von dem anderen sind sie uns sehr bekannt; - es hat aber eine Zeit gegeben, wo eine Finsterniss auch Zufall hiess viele Dinge, welche jetzt Zufall heissen, werden in der Folge diesen Namen verlieren und es ist überhaupt klar, dass der ganze Begriff relativ ist. Als Newton anfing Licht in der Welt zu verbreiten, wurde Vieles aus dem dunkelen Reiche des Zufalls hervorgezogen; ein anderer Newton würde die Ursachen anderer Dinge enthüllen, und es ist ein Verstand denkbar, für welchen wenig Zufälliges übrig bleiben würde.

F. W. Bessel.

"ther Wahrscheinlichkeitsrechnung."

6. Populäre Vorlesungen, herausg. v. Schumacher (Hamburg 1848), p. 391.

Tous les événemens, ceux même qui par leur petitesse, semblent ne pas tenir aux grandes lois de la nature, en sont une suite aussi nécessaire que les révolutions du soleil. Dans l'ignorance des liens qui les unissent au système entier de l'unirers, on les a fait dépendre des causes finales, ou du hasard, suivant qu'ils arrivaient et se succédaient arec régularité ou sans ordre apparent; mais ces causes imaginaires ont été successivement reculées avec les bornes de nos connaissances, et disparaissent entièrement devant la saine philosophie qui ne 
voit en elles, que l'expression de l'ignorance où nous sommes des véritables causes.

Nous devons . . envisager l'état présent de l'univers, comme l'effet de son état antérieur, et comme la cause de celui qui va suivre. Une intelligence qui pour un instant donné, connaîtrait toutes les forces dont la nature est animée, et la situation respective des êtres qui la composent, si d'ailleurs elle était assez vaste pour soumettre ces données à l'analyse, embrasserait dans la même formule, les mouvemens des plus grands corps de l'univers et ceux du plus léger atome: rien ne serait incertain pour elle, et l'avenir comme le passé, serait présent à ses yeux. L'esprit humain offre dans la perfection qu'il a su donner à l'astronomie, une faible esquisse de cette intelligence. Ses découvertes en mécanique et en géométrie, jointes à celle de la pesanteur universelle, l'ont mis à portée de comprendre dans les mêmes expressions analytiques, les états passés et futurs du système du monde. En appliquant la même méthode à quelques autres objets de ses connaissances, il est parvenu à ramener à des lois générales, les phénomènes observés, et à prévoir ceux que des circonstances données doivent faire éclore. Tous ses efforts dans la recherche de la vérité, tendent à le rapprocher sans cesse de l'intelligence que nous venons de concevoir, mais dont il restera toujours infiniment éloigné. Cette tendance propre à l'espèce humaine, est ce qui la rend supérieure aux animaux; et ses progrès en ce genre, distinguent les nations et les siècles, et fondent leur véritable gloire.

LAPLACE.

"Essai philosophique sur les probabilités", 3ième éd. (Paris 1816), p. 2-4.

Kennte man alle Kräfte der Natur und wüßte man, welches der Zustand der Materie in einem Zeitpunkte ist, so würde man ihren Zustand für jeden späteren Zeitpunkt durch die Mechanik ermitteln, und ableiten können, wie die mannigfaltigen Naturerscheinungen einander folgen und begleiten. Das 
höchste Ziel, welches die Naturwissenschaften zu erstreben haben, ist die Verwirklichung der eben gemachten Voraussetzung, also die Ermittelung der Kräfte, welche in der Natur rorhanden sind, und des Zustandes, in dem die Materie in einem Augenblicke sich befindet, mit einem Worte, die Zurückführung aller Naturerscheinungen auf die Mechanik.

G. KirchinofF.

„Cber das Ziel der Naturwissenschaften“, Akad. Festrede Heidelberg 22. XI. 1865, p. 9.

Naturerkennen - genauer gesagt naturwissenschaftliches Erkennen oder Erkennen der Körperwelt mit Hülfe und im Sinne der theoretischen Naturwissenschaft - ist Zurückführen der Veründerungen in der Körperwelt auf Bewegungen von Atomen, die durch deren ron der Zeit unabhängige Centralkräfte bewirkt werden, oder Auflösen der Naturvorgänge in Mechanik der Atome. Es ist psychologische Erfahrungsthatsache, dass, wo solche Auflösung gelingt, unser Causalitätsbedürfniss vorläufig sich befriedigt fühlt. Die Sätze der Mechanik sind mathematisch darstellbar, und tragen in sich dieselbe apodiktische Gewissheit, wie die Sätze der Mathematik. Indem die Veränderungen in der Körperwelt auf eine constante Summe ron Spannkräften und lebendigen Kräften, oder von potentieller und kinetischer Energie zurückgeführt werden, welche einer constanten Menge von Materie anhaftet, bleibt in diesen Veränderungen selber nichts zu erklären übrig.

Emil du Bois-Reymond. "Über die Grenzen des Naturerkennens", Vortr. Naturf.-Vers. Leipzig 1872 s. Reden, Bd. 1 (1886), p. 105/106.

Im ganzen möchte das Laplacesche Ideal der überwiegenden Mehrzahl der heutigen Naturforscher kaum fremd sein.

E. MaCH.

„Die ökonomische Natur der physikalischen Forschung“, Vortr. Wien Akad. d. Wiss. 1882.

s. „Populär-wissensch. Vorles.“, 3. Aufl. (Leipzig 1903), p. 217. 
Nur vom Boden der exakten Wissenschaften her, für welche wieder die Mathematik der Lebensnerv ist, entspringt für uns eine einwandfreie Erkenntnis; sie sind nach meiner Auffassung berufen, das letzte Wort in allen Fragen nach dem Wesen der Dinge zu sprechen. Dass .. hier voreilige Verallgemeinerungen auftreten können, die uns verwirren und deprimieren, muss zugegeben werden. Die Welt nach dem Bilde Dubois-Reymond's, aufgelöst in ein Wirrsal reinen Centralkräften unterworfener Atome und Moleküle, deren Bewegungsgleichungen auch schon durch einen überlegenen Geist integriert gedacht werden können, ist eine trostlos öde Grundlage für eine ethische Weltanschauung.

\section{A. Stodola.}

„Über die Beziehungen der Technik zur Mathematik." s. Verhandl. d. 1. intern. Nathem.-Congr. Zürich 1897 (Leipzig 1898), p. 270.

$=$ Zeitschr. d. Ver. deutscher Ingenieure, Bd. 41, No. 44 (1897), p. 1260.

Ausser der Zusammenfassung möglichst vieler Thatsachen in eine übersichtliche Form hat die Naturwissenschaft noch eine andere Aufgabe, die ebenfalls ökonomischer Natur ist. Sie hat die complicirteren Thatsachen in möglichst wenige und möglichst einfache zu zerlegen. Dies nennen wir erklären. Diese einfachsten Thatsachen, auf die wir die complicirteren zurückführen, sind an sich immer unverständlich, d. h. nicht weiter zerlegbar, z. B. die, dass eine Masse der andern eine Acceleration ertheilt.

Es ist nun wieder nur eine ökonomische Frage einerseits und eine Frage des Geschmackes anderseits, bei welchen Unverständlichkeiten man stehn bleiben will. Man täuscht sich gewöhnlich darin, dass man meint, Unverständliches auf Verständliches zurückzuführen. Allein das Verstehn besteht eben im Zerlegen. Man führt ungewöhnliche Unverständlichkeiten auf gewöhnliche Unverständlichkeiten zurück. Man gelangt schliesslich immer zu Sätzen von der Form, wenn A ist, ist 
B, also Sützen, die aus der Anschauung folgen müssen, die also nicht weiter verständlich sind.

Die Newton'sche Gravitationstheorie beunruhigte bei ihrem Auftreten fast alle Naturforscher, weil sie sich auf eine ungewöhnliche Unverständlichkeit gründete. Man trachtete die Gravitation auf Druck und Stoss zurückzuführen. Heute beunruhigt die Gravitation keinen Menschen mehr. Sie ist eine gewöhnliche Unverständlichkeit geworden.

\section{E. МАС̆.}

„Die Geschichte und die Wurzel des Satzes von der Erhaltung der Arbeit", Vortr. Böhm. Ges. d.

Wiss. 15. XI. 1871 (Prag 1872), p. 31/32.

Warum fragt niemand nach dem Wesen des Goldes oder nach dem Wesen der Geschwindigkeit? . . . . Mit den Zeichen „Geschwindigkeit“ und „Gold“ verbinden wir eine grosse Zahl ron Beziehungen zu anderen Zeichen, und zwischen allen diesen Beziehungen finden sich keine uns verletzenden Widersprüche.

Auf die Zeichen ,Kraft" und „Elektricität" aber hat man mehr Beziehungen gehäuft, als sich völlig mit einander vertragen; dies fühlen wir dunkel, verlangen nach Aufklärung und äussern unsern unklaren Wunsch in der unklaren Frage nach dem Wesen von Kraft und Elektricität.

Heinrich Hertz.

„Die Prinzipien der Mechanik" (1894), p. 9.

Es ist äusserst wahrscheinlich, dass es mit der Fernkraft eine ähnliche Bewandtnis wie mit den angeführten Beispielen [algebr. Aufl. der Gleichungen des $5^{\text {ten }}$ und höherer Grade; Quadratur des Kreises; Perpetuum mobile] hat, um so mehr, als ein Kennzeichnendes für die Unlösbarkeit eines Problems die schliesslich ganz verzweifelten Anstrengungen der Forschung, die geradezu extravaganten Hilfsmittel sind, zu denen sie ihre Zuflucht nimmt, wohin Lesage's Kastenatome und Zöllner's durch Lust und Unlust bewegte Atome gehören. Steht es erst so um ein Problem, dann liegt ausreichender Grund zu der 
Vermutung vor, dass es mit Hilfsmitteln, die unserem gemeinen Verstande angemessen sind, sich nicht werde lösen lassen. So glaube ich denn, dass man die Schwerkraft als etwas menschlich Unfassbares, etwas mechanisch Unbegreifliches ansehen muss, und ich will versuchen, dies zu beweisen. - - -

Fernkraft und Materie sind Eins.

Paul du Bois-Reymond.

„Über die Grundlagen der Erkenntnis in den exacten Wissenschaften" (Tübingen 1890), p. 35/36 u. 103; s. a. Naturwiss. Rundschau 3 (1888), p. 171.

Gegenüber den Räthseln der Körperwelt ist der Naturforscher längst gewöhnt, mit männlicher Entsagung sein „Ignoramus" auszusprechen. Im Rückblick auf die durchlaufene siegreiche Bahn trägt ihn dabei das stille Bewusstsein, dass, wo er jetzt nicht weiss, er wenigstens unter Umständen wissen könnte, und dereinst vielleicht wissen wird. Gegenüber dem Räthsel aber, was Materie und Kraft seien, und wie sie zu denken vermögen, muss er ein für allemal zu dem viel schwerer abzugebenden Wahrspruch sich entschliessen:

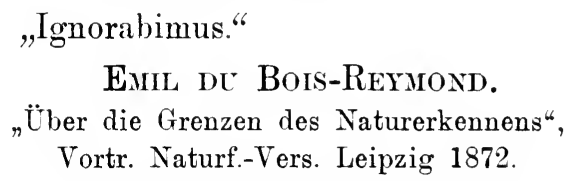

EMIL DE Bors-Reymond. „Über die Grenzen des Naturerkennens", Vortr. Naturf--Vers. Leipzig 1872.

s. E. du Bois-Reymond, Reden, Bd. 1 (1886), p. 130.

Geheimnissvoll am lichten Tag

Lässt sich Natur des Schleiers nicht berauben, Und was sie deinem Geist nicht offenbaren mag, Das zwingst du ihr nicht ab mit Hebeln und mit Schrauben.

GoEThe.

„Faust", Teil I, 672-675.

s. Werke, Grosse Weimarische Ausg., Abth. I, Bd. 14 (1887), p. 39. 
Eine falsche Hrpothese ist besser als gar keine.

Goethe.

"Analyse und Synthese."

s. Werke, Grosse Weimarische Ausg., Abth. II, Bd. 11 (1893), p. 70. $=$ Goedeke sche 10-bändige Ausg., Bd. 9, p. 715.

Hspotheses non fingo.

\section{Newton.}

„Principia mathematica philosophiae naturalis“ (Amsterdam 1714), liber III, scholium generale, p. 484.

Wir beobachten eine stetige Thätigkeit unserer Seele. Jedem Act derselben liegt etwas Bleibendes zu Grunde, welches sich bei besonderen Anlässen (durch die Erinnerung) als solches kundgiebt, ohne einen dauernden Einfluss auf die Erscheinungen auszuüben. . . . . .

Von dieser Thatsache geleitet, mache ich die Hypothese, dass der Weltraum mit einem Stoff erfüllt ist, welcher fortwährend in die ponderablen Atome strömt und dort aus der Erscheinungswelt (Körperwelt) rerschwindet.

Beide Hypothesen lassen sich durch die Eine ersetzen, dass in allen ponderablen Atomen beständig Stoff aus der Körperwelt in die Geisteswelt eintritt. Die Ursache, weshalb der Stoff dort verschwindet, ist zu suchen in der unmittelbar vorher dort gebildeten Geistessubstanz, und die ponderablen Körper sind hiernach der Ort, wo die Geisteswelt in die Körperwelt eingreift.:

* In jedes ponderable Atom tritt in jedem Augenblick eine bestimmte, der Gravitationskraft proportionale Stoffmenge ein und verschwindet dort.

Es ist die Consequenz der auf Herbart'schem Boden stehenden Psychologie, dass nicht der Seele, sondern jeder einzelnen in uns gebildeten Vorstellung Substantialität zukomme.

\section{B. Riemanx.}

Fragment über "Neue mathem. Principien d. Naturphilosophie“. s. Werke, herausg. r. H. Weber, 2. Aufl. (1892), p. 528/529. 
Hypotheses . ., in Philosophia quae circa experimenta versatur, pro nihilo sunt habendae.

I. Newton.

„Optice“, latine redd. S. Clarke (1740), Quest. XXXI, p. 329.

Witzige Einfälle verschaffen ihrem Autor bald den Namen - eines geistreichen Mannes. Unter einer grossen Zahl solcher Einfälle werden ja auch wohl einige sein müssen, die sich schliesslich als halb oder ganz richtig erweisen; es wäre ja geradezu ein Kunststück, immer falsch zu rathen. In solchem Glücksfalle kann man seine Priorität auf die Entdeckung laut geltend machen; wenn nicht, so bedeckt glückliche Vergessenheit die gemachten Fehlschlüsse. Andere Anhänger desselben Verfahrens belfen gern dazu, den Werth eines "ersten Gedankens“ zu sichern. Die gewissenhaften Arbeiter, welche ihre Gedanken zu Markte zu bringen sich scheuen, ehe sie sie nicht nach allen Seiten geprüft, alle Bedenken erledigt und den Beweis vollkommen gefestigt haben, kommen dabei in unverkennbaren Nachtheil. Die jetzige Art, Prioritätsfragen nur nach dem Datum der ersten Veröffentlichung zu entscheiden, ohne dabei die Reife der Arbeit zu beachten, hat dieses Unwesen sehr begünstigt.

In den Letterkästen eines Buchdruckers liegt alle Weisheit der Welt zusammen, die schon gefunden ist und noch gefunden werden kann; man müsste nur wissen, wie man die Lettern zusammenzuordnen hat ${ }^{1}$ ). So sind auch in den Hunderten von Schriften und Schriftchen, die alljährlich erscheinen über Aether, Beschaffenheit der Atome, Theorie der Wahrnehmung, ebenso wie über das Wesen der asthenischen Fieber und der Carcinome, gewiss schon längst alle zartesten Nüancirungen der möglichen Hypothesen erschöpft und unter diesen müssen noth-

1) Dies Bild vom umgestürzten Schriftkasten kommt nach E. du Bois-Reymond, „Culturgeschichte und Naturwissenschaft", Vortr. Köln 24. III. 1877, seltsamerweise schon bei den Alten vor (s. das Nähere in du Bois' „Reden“, Bd. 1 (1886), p. 254). 
wendig viele Bruchstücke der richtigen Theorie sein. Wer sie nur zu finden wüsste!

\section{Helmholtz.}

„Das Denken in der Medizin“, Festrede Berlin militairärztl. Bildungs-Anst. 2. VIII. 1877. s. Vorträge u. Reden, Bd. 2 (1884), p. 185.

De simples vues, quelque grandes, quelque heureuses qu'elles soient, ne peuvent, ni être mises sur la même ligne qu'une découverte précise et bien prononcée, ni diminuer le mérite de celle dont elles ont été le germe.

CONDORCET.

Oeurres, Édition d'Arago, t. 2 (1847), p. 6/7.

Im Allgemeinen würde ich gegen . . . . Phantasiespiele nachsichtig sein, und ihnen nur die Aufnahme in die wissenschaftliche Astronomie, die einen ganz andern Character haben muss, nicht einräumen. Gehören doch auch Lapla ce's Cosmogenische Hypothesen in jene Classe. Ja, ich leugne nicht, dass ich selbst mich zuweilen auf ähnliche Art amusire, nur würde ich dergleichen nie publiciren. Es gehören dahin z. B. meine Gedanken über die Bewohner der Himmelskörper. Ich meinerseits bin (gegen die gewöhnliche Meinung) überzeugt (was man in solchen Dingen Überzeugung nennt), dass, je grösser die Weltkörper, desto kleiner die Bewohner und sonstige Producte. Z. B. auf der Sonne würden Bäume, die in demselben Verhältniss grösser wären als die unsrigen, wie die Sonne die Erde an Grösse übertrifft, gar nicht existiren können, denn wegen der so viel grössern Schwere auf der Oberfläche der Sonne würden alle Zweige von selbst abbrechen, insofern die Stoffe nicht ganz heterogener Art mit irdischen sind. - - -

Gauss an Schumacher

Göttingen, 7. XI. 1847.

s. Briefw. Gauss-Schumacher, Bd. 5 (1863), p. 394. 
G auss hielt es für möglich, mit Hülfe von Heliotropen eine telegraphische Correspondenz zwischen Mond und Erde zu errichten und hatte in Bezug auf diese Frage sogar die Grösse der erforderlichen Spiegel berechnet, woraus sich ergab, dass eine solche Correspondenz eventuel ohne grosse Kosten sich würde einrichten lassen. Das wäre eine Entdeckung, pflegte er zu sagen, noch grösser als die von Amerika, wenn wir uns mit unseren Mondnachbarn in Verbindung setzen könnten hielt es jedoch nicht eben für wahrscheinlich, dass der Mond eine mit höherer Intelligenz ausgestattete Berölkerung besitze.

\section{F. A. T. WINNEKCE.}

„Gauss" (Braunschweig 1877), p. 25/26.

Un savant doit rejeter, sans hésiter, toute hypothèse qui serait en contradiction avec les vérités révélées. Ce point est capital, je ne dirai pas dans l'intérêt de la religion, mais dans l'intérêt des sciences, puisque jamais la vérité ne saurait se contredire elle-même. C'est pour avoir négligé cette règle, que quelques savants ont eu le malheur de consumer en vains efforts un temps précieux qui aurait pu être heureusement employé à faire d'utiles découvertes. Et en effet, que de travaux remarquables eussent pu être ajoutés aux importants mémoires compris dans nos recueils scientifiques, si la religion eût toujours guidé la plume de ces auteurs qui ont cru pendant quelque temps avoir découvert que les zodiaques de Denderah et d'Esneh avaient douze mille ans de date, que l'homme descendait du polype, qu'il avait existé sur la terre de toute éternité, que le déluge était une fable, que la création de l'homme et des animaux était un effet du hasard, et que de nos jours encore on les voyait sortir de terre dans les îles du grand Océan, que les Américains formaient une espèce d'hommes distincte de la nôtre, etc.

\section{Augustin Cauchy.}

„Sept leçons de physique générale“, Turin 1833, avec appendices par l'abbé Moigno (Paris 1868), p. 16. 
E Scriptura quidem via ad salutem, non verò ad Mathematica, discenda.

\section{O. v. Guericke.}

„Experimenta nova de vacuo spatio“ (Amsterdam 1672), liber VI, caput XVI, p. 218.

Die Wissenschaft in den einzelnen Erscheinungsgebieten verfïhlrt mit äusserster Rücksichtslosigkeit gegen die übrigen Erscheinungsgebiete. Es ist ein durchgehender Zug, dass sie mit jedem ihrer Elementarmechanismen nur ein bestimmtes Erscheinungsgebiet zu construiren trachtet, völlig unbekümmert darum, ob dieser Mechanismus anderweit noch verwertbar ist. Dies geht soweit, dass die Erscheinungen eines Gebietes in ihrem verschiedenartigen Auftreten zuweilen mit verschiedenen Ausgangsvorstellungen in Verbindung gebracht werden, wie denn z.B. der Wärmestoff noch in der Lehre von der Wärmeleitung in Gebrauch ist, während die kinetische Gastheorie ihn in die lebendige Kraft der bewegten Gasteilchen verlegt und die Wärmestrahlung in die des schwingenden Aethers.

Wenn auch directer Widerspruch (wie z. B. continuirliche Raumausfüllung und chemische Molekel) als Monstrosität anzusehen ist, so ist doch im allgemeinen dies Vorgehen der $W$ issenschaft ein durchaus richtiges.

\section{Paul du Bois-Reyiond.}

„C̈ber die Grundlagen der Erkenntnis in den exacten

Wissenschaften" (Tübingen 1890), p. 17/18.

Für die Unterwerfung eines neuen Gebietes unter die Theorie ist in vielen Fällen das Ausgehen von ganz bestimmten Vorstellungen über den Mechanismus des Vorganges nützlich gewesen. Ein Beispiel hierfür liefert die zur Erklärung der Cohäsionserscheinungen gemachte Annahme, dass die Körper aus discreten Molekiilen bestehen, welche fernwirkende Kräfte auf einander ausüben; ein anderes die für die Electricitätstheorie so fruchtbare Hypothese, dass es Fluida von sehr ge- 
ringer Dichte giebt, die sich durch ausgeübte Kräfte innerhalb der ponderablen Körper bewegen lassen.

Es ist offenbar tief in der Organisation unseres Geistes begründet, dass die Benutzung solcher anschaulicher Bilder der uns ihrem Wesen nach unbekannten Vorgänge das Auffinden ihrer Gesetze erleichtert.

\section{W. VoIgT.}

Göttinger Nachr. 1895, Math.-phys. Kl., p. 259.

Meine Begriffe über das Theoretisiren in den Erfahungswissenschaften sind die folgenden: Wer eine Theorie aufstellen will, muss sie auf alle damit in Bezug stehenden Thatsachen prüfen, ohne Vorurtheil zu Gunsten dieser Theorie, muss gleich offen ihre schwachen, wie ihre guten Seiten hervorheben. Er muss nie versuchen, Überzeugung hervorzubringen, wo nur Wahrscheinlichkeit ist; denn wer Probabilitäten als Wahrheit giebt, der wird, mit oder ohne Willen, ein Irreführer. Jeder Theoretiker muss Newton's Grundsätzen in dieser Hinsicht folgen, durch welche dieser unübertroffene Naturforscher noch die höchste Stelle behauptet, obgleich nach ihm ein Jahrhundert gefolgt hat, in welchem mehr für die Naturwissenschaften gemacht worden ist, als in der ganzen Zeit vor ihm.

JAC. Berzelies an J. Liebig.

Stockholm, 14. XI. 1843.

s. "Berzelius und Liebig, ihre Briefe von 1831-1845", herausg. von J. Carrière (München u. Leipzig 1893), p. 251.

Hatte einst Napoleon in der Kapuzinergruft zu Wien gerufen: „Alles ist eitel mit Ausnahme der Kraft“, so strich jetzt Kirchhoff auf einer Druckseite die Kraft aus der Natur, jenen deutschen Professor beschämend, von dem Karl Moor ${ }^{1}$ ) erzählt, dass er sich vermass, trotz seiner Schwäche auf seinem Katheder das Wesen der Kraft zu behandeln, aber doch nicht diese zu vernichten.

1) Śchiller, „Räuber“, 1. Akt, 2. Scene. 


\section{$-14-$}

Kirchhoff hat selbst das Wort Kraft später wieder eingeführt, aber nicht als metaphysischen Begriff, sondern bloss als abgekürzte Bezeichnung für gewisse algebraische Ausdrücke, welche bei der Beschreibung der Bewegung beständig vorkommen. - - - -

Kirchhoff hatte an der alten classischen Mechanik keine materielle Änderung vorgenommen; seine Reformation war eine rein formale. Viel weiter ging Hertz, und während fast alle späteren Autoren die Darstellungsweise Kirchhoff's nachahmten, hie und da freilich oft mehr gewisse, bei Kirchhoff stehende Ausdrucksweisen als dessen Geist, so habe ich Hertz's Mechanik zwar sehr oft preisen gehört, aber noch niemanden sah ich auf dem von Hertz gewiesenen Weg weiterwandeln.

\section{Boltzinann.}

„"̈ber die Entwicklung der Methoden der theoretischen Physik in neuerer Zeit", Vortr. Naturf.-Vers. München 1899. s. Deutsche Mathem.-Verein. Jahresber. 8, 1899, p. 82/83.

Die Mechanik ist die Wissenschaft von der Bewegung; als ihre Aufgabe bezeichnen wir: die in der Natur vor sich gehenden Bewegungen vollständig und auf die einfachste Weise $\mathrm{zu}$ beschreiben. ${ }^{1}$ )

\section{G. KirchiofF.}

„Vorlesungen über Mathem. Physik", Bd. 1 (Mechanik), (1876), p. 1.

Das Kirchhoff'sche Wort, dass die Mechanik bloss beschreibt, ist im Übermass nachgesprochen worden. In gewissem Sinn ist freilich jedes Erklären ein Beschreiben, aber doch zugleich von dem rein äusserlichen Beschreiben grundverschieden, und es dürfte sich wohl lohnen, dafür das unterscheidende Wort beizubehalten.

\section{O. HöLdER.}

„Anschauung und Denken in der Geometrie"(Leipzig 1900), p. 71.

l) Z̈ber verwandte Anschauungen und Aussprüche früherer Forscher, vor allem Adam Smith's und J. R. Mayer's, s. E. Mach, „Populär-wissenschaftliche Vorlesungen", 3. Aufl. (Leipzig 1903), p. 263 Anm. 
Dass Kirchhoff selbst nichts anderes beabsichtigte, als dem Ausdruck „Erklärung“ entgegenzutreten, schliesst der Verfasser aus einer Unterhaltung mit ihm aus der Mitte der $70 \mathrm{er}$ Jahre. Es handelte sich um das Weber'sche Gesetz, und der Verfasser bemerkte: es sei schade, dass man sich dabei nichts denken könne, worauf Kirchhoff erwiderte: das sei ja ganz gleichgültig, wenn es nur gelinge, damit die Erscheinungen „darzustellen“. Dies war sein wörtlicher Ausdruck. — - -

\section{Paul du Bois-Reymond.}

„Über die Grundlagen der Erkenntnis in den exacten

Wissenschaften" (Tübingen 1890), p. 15.

In order to obtain physical ideas without adopting a physical theory we must make ourselves familiar with the existence of physical analogies. By a physical analogy I mean that partial similarity between the laws of one science and those of another which makes each of them illustrate the other. Thus all the mathematical sciences are founded on relations between physical laws and laws of numbers, so that the aim of exact science is to reduce the problems of nature to the determination of quantities by operations with numbers.

\section{James Clerk Maxwell.}

"On Faraday's Lines of Force". cf. Scientific Papers, vol. 1 (1890), p. 156.

Dem Ausdruck Hrn. v. Helmholtz', dass die Erscheinungen Zeichen des Wirklichen seien ${ }^{1}$ ), möchte ich den inhaltloseren Ausdruck Zuordnungen vorziehen, da man in der Darstellung dieser zarten Verhältnisse nicht wählerisch genug in den Bezeichnungen sein kann.

\section{Paul du Bois-Reymond.}

„Über die Grundlagen der Erkenntnis in den exacten

Wissenschaften" (Tübingen 1890), p. 121/122.

1) s. die Rede über "die Thatsachen in der Wahrnehmung“, Berlin Univ. 3. VIII. 1878 in Helmholtz, „Vorträge u. Reden“, Bd. 2 (1884), p. 226. 
Wann ist unsere Auffassung der Welt wahr?

„Wenn der Zusammenhang unserer Vorstellungen dem Zusammenhange der Dinge entspricht."

Die Elemente unseres Bildes von der Welt sind von den entsprechenden Elementen des abgebildeten Realen gänzlich verschieden. Sie sind etwas in uns; die Elemente des Realen etwas ausser uns. Aber die Verbindungen zwischen den Elementen im Bilde und im Abgebildeten müssen übereinstimmen, wenn das Bild wahr sein soll.

B. Riemann.

Fragment über „Neue mathem. Principien der Naturphilosophie." s. Werke, herausg. г. H. Weber, 2. Aufl. (1892), p. 523.

Wir machen uns innere Scheinbilder oder Symbole der äusseren Gegenstände, und zwar machen wir sie von solcher Art, dass die denknotwendigen Folgen der Bilder stets wieder die Bilder seien ron den naturnotwendigen Folgen der abgebildeten Gegenstände.

Heinrich Hertz.

„Die Principien der Mechanik“ (1894), p. 1.

Zahlreiche Fragen, die früher unergründlich erschienen, entfallen hiermit von selbst. Wie kann, sagte man früher, von einem materiellen Punkte, der ein blosses Gedankending ist, eine Kraft ausgehen, wie können Punkte zusammen Ausgedehntes liefern etc.? Jetzt weiss man, dass sowohl die materiellen Punkte, als auch die Kräfte blosse geistige Bilder sind. Erstere können nicht Ausgedehntem gleich sein, aber es mit beliebiger Annäherung abbilden. Die Frage, ob die Materie atomistisch zusammengesetzt oder ein Continuum ist, reducirt sich auf die viel klarere, ob die Vorstellung enorm vieler Einzel- 
wesen oder die eines Continuums ein besseres Bild der Erscheinungen zu liefern vermöge.

\section{Boltzmans.}

„Über die Entwicklung der Methoden der theoretischen Physik in neuerer Zeit", Vortrag Naturf.-Vers. München 1899. s. Deutsche Mathem.-Terein. Jahresber. 8, 1899, p. 85.

Hertz hat versucht, in den Principien der Mechanik eine consequent durchgeführte Darstellung eines vollständig in sich zusammenhängenden Systems der Mechanik zu geben und alle einzelnen besonderen Gesetze dieser Wissenschaft aus einem einzigen Grundgesetz abzuleiten, welches logisch genommen natürlich nur als eine plausible Annahme betrachtet werden kann.

Freilich werden noch grosse Schwierigkeiten zu überwinden sein bei dem Bestreben, aus den von Hertz entwickelten Grundlagen Erklärungen für die einzelnen Abschnitte der Physik zu geben. Im ganzen Zusammenhange aber ist die Darstellung der Grundgesetze der Mechanik von Hertz ein Buch, welches im höchsten Grade jeden Leser interessieren muss, der an einem folgerichtigen System der Dynamik, dargelegt in höchst vollendeter und geistreicher mathematischer Fassung, Freude hat. Möglicherweise wird dieses Buch in der Zukunft noch von hohem heuristischen Wert sein als Leitfaden zur Entdeckung neuer allgemeiner Charaktere der Naturkräfte.

\section{H. von HeLmholtz.}

Vorrede zu Heinrich Hertz, Ges. Werke, Bd. 3 (1894), p. XIX u. XXII $=$ Zeitschr. phys. chem. Unterr. 8, 1894/95, p. 28 u. 29.

Nachdem durch Routh und v. Helmholtz die verborgenen Massen in die physikalische Mechanik eingeführt wurden, hat Hertz den kühnen Versuch unternommen die mechanischen Principien im Sinne dieser modernen Anschauungen mit Zugrundelegung des Princips der Erhaltung der Energie unter der Annahme „vollständiger" Systeme in ein abgeschlossenes System zu bringen.

Ahrens, Scherz und Ernst i. d. Mathem. 
Für die technische Mechanik ist eine nutzbringende Anwendung dieser - an und für sich ganz berechtigten - Anschauungen vorläufig aussichtslos, obwohl sie in gewissem Sinne das Ideal eines Systems der phrsikalischen Mechanik verwirklicht haben.

K. Heun.

„Die kinetischen Probleme der wissenschaftlichen Technik", Deutsche Vathem.-Verein. Jahresber. 9, 1900, p. 3.

Mit Recht weist Hertz darauf hin, dass in der Mechanik nicht die wenigen Experimente, aus denen gewöhnlich deren Grundgleichungen gewonnen werden, dass in der Elektrodynamik nicht die fünf oder sechs Fundamentalversuche Ampère's es sind, was uns von der Richtigkeit aller dieser Gleichungen so fest überzeugt, sondern vielmehr ihre nachherige Übereinstimmung mit allen bisher bekannten Thatsachen. Er fällt daher das salomonische Urteil, es sei das beste, nachdem man diese Gleichungen einmal habe, sie ohne jede Ableitung hinzuschreiben, dann mit den Erscheinungen zu vergleichen und in ihrer steten Übereinstimmung mit denselben den besten Beweis ihrer Richtigkeit zu erblicken.

Die Ansicht, deren Extrem hiermit ausgesprochen ist, fand die verschiedenste Aufnahme. Während die einen fast geneigt waren, sie für einen schlechten Witz zu halten, schien es anderen von nun an als einziges Ziel der Physik, ohne jede Hypothese, ohne jede Veranschaulichung oder mechanische Erläuterung für jede Reihe von Vorgängen Gleichungen aufzuschreiben, aus denen ihr Verlauf quantitativ berechnet werden kann, so dass die alleinige Aufgabe der Physik darin bestünde, durch Probiren möglichst einfache Gleichungen zu finden, welche gewisse notwendige formale Bedingungen der Isotropie etc. erfüllen, und sie dann mit der Erfahrung zu vergleichen. Dies ist die extremste Richtung der Phaenomenologie, welche ich die mathematische nennen möchte, während die allgemeine Phaenomenologie jede Thatsachengruppe durch Aufzählung und naturgeschichtliche Schilderung aller dahin gehörigen Erscheinungen zu beschreiben sucht ohne Beschränkung der dazu dienlichen Mittel, aber unter Verzicht auf jede einheitliche Naturauffassung, auf jede mechanische Erläuterung oder sonstige 
Begründung. Letztere ist charakterisirt durch den von Mach citirten Ausspruch, dass die Elektricität nichts anderes ist, als die Summe aller Erfahrungen, welche wir auf diesem Gebiete schon gemacht haben und noch zu machen hoffen $\left.{ }^{1}\right)$. Beide stellen sich die Aufgabe, die Erscheinungen darzustellen, ohne über die Erfahrung hinauszugehen.

\section{Boltzimann.}

„Über die Entwicklung der Methoden der theoretischen Physik in neuerer Zeit", Vortrag Naturf.-Vers. München 1899.

s. Deutsche Mathem.-Verein. Jahresber. 8, 1899, p. 89/90.

Das Studium von Analogieen, wie sie zwischen durchaus getrennten Gebieten der Physik auftreten, ist besonders seit den Untersuchungen von Maxwell zu einem wichtigen Instrument der Forschung geworden und hat, gerade in der abstracten Form, in welcher die Übereinstimmung in der analytischen Darstellung als Ausgangspunkt genommen ist, wesentlich dazu beigetragen, unsere heutige Auffassung der Beziehung physikalischer Vorgänge zu den correspondirenden mathematischen Formulirungen zu entwickeln. Sie steht im Gegensatz zu dem Glauben an die Möglichkeit einer uns zugänglichen absoluten Erklärung der Geschehnisse in der Natur, auf Grund pbilosophischer, wie rein mechanischer Vorstellungen und Hypothesen; einem Glauben, wie er uns zum Teil in mystischem Gewande bei den Gelehrten des vorigen Jahrhunderts, oder in rationalistischer Form bei den Encyklopädisten entgegentritt. - Bei Maxwell, später bei Kirchhoff und Hertz finden wir klar und schlicht die Anschauung vertreten, dass unsere Einsicht in physikalische Vorgänge nur eine relative, und wesentlich in der Aufstellung von Analogieen begründete ist, und dass insbesondere auch die mathematische Formulirung nur die Bedeutung einer zusammenfassenden Beschreibung besitzt.

W. Dyck.

„ய̈ber die wechselseitigen Beziehungen zwischen der reinen und der angewandten Mathematik", Akadem. Festrede München 14. XI. 1896, p. 12/13.

1) Vgl.z.B. E.Mach, „Die Analyse d. Empfindungen“, 3.Aufl.(1902), p. 251. 
Ce n'est pas que je prétende. avec un célèbre savant allemand, ${ }^{1}$ ) que la nature s'écrie toujours non! non! quand on veut soulever quelque coin de voile qui la recouvre.

Arago.

Oeurres, t. 1 (1854) p. $401=$ Werke, Bd. 1 (1854) p. 321

In der neueren Mathematik spielt die Frage nach der Unmöglichkeit gewisser Lösungen eine herrorragende Rolle und wir nehmen so gewahr, dass alte schwierige Probleme wie der Beweis des Parallelenaxioms, die Quadratur des Kreises oder die Auflösung der Gleichungen $5^{\text {ten }}$ Grades durch Wurzelziehen, wenn auch in anderem als dem ursprünglich gemeinten Sinne, dennoch eine völlig befriedigende und strenge Lösung gefunden haben.

Diese merkwürdige Thatsache neben anderen philosophischen Gründen ist es wohl, welche in uns eine Überzeugung entstehen lässt, die jeder Mathematiker gewiss teilt, die aber bis jetzt wenigstens niemand durch Beweise gestützt hat ich meine die Überzeugung, dass ein jedes bestimmte mathematische Problem einer strengen Erledigung notwendig fähig sein müsse, sei es, dass es gelingt, die Beantwortung der gestellten Frage zu geben, sei es, dass die Unmöglichkeit einer Lösung und damit die Notwendigkeit des Misslingens aller Versuche dargethan wird. - - - - -

Diese Überzeugung von der Lösbarkeit eines jeden mathematischen Problems ist uns ein kräftiger Ansporn während der Arbeit; wir hören in uns den steten Zuruf: Da ist das Problem, suche die Lösung. Du kannst sie durch reines Denken finden; denn in der Mathematik giebt es kein Ignorabimus ${ }^{2}$ )

\section{Hilbert.}

„Mathematische Probleme", Vortrag Mathem.-Congr. Paris 1900.

s. Göttinger Nachr., Math.-phys. Kl. 1900, p. 261/262 $=$ Arch. Nath. Phys. (3) 1 (1901), p. 51/52.

1) Chladni; s. Arago, Werke, Bd. 3, p. 26.

2) s. S. 7 (Emil du Bois-Reymond). 
On doit donner au problème une telle forme qu'il soit toujours possible de le résoudre, ce qu'on peut toujours faire d'un problème quelconque.

\section{N. H. ABel.}

„Sur la résolution algébrique des équations", (Mémoire posthume). voir Oeurres complètes, réd. par Holmboe (Christiania 1839),

t. 2, p. $185=$ Oeurres compl., édition de Sylow et Lie (Christiania 1881), t. 2, p. 217.

Il est beau de voir ainsi la théorie. . . . s'élever, par un enchaînement de vérités mathématiques, à tous les résultats habilement manifestés par le physicien qui fait parler les phénomènes. Telle doit être la véritable physique; telle, peut-être, par les efforts successifs des plus habiles géomètres, la verrons-nous quelque jour perfectionée dans toutes ses branches principales.

\section{Dupis.}

„Déreloppements de Géométrie“ (Paris 1813), p. 120.

Die Behandlungsweise der mechanischen Probleme ist zu einem Vorbild für die Kunst geworden, an die Natur Fragen zu richten.

\section{R. Lipschitz.}

„Bedeutung der theoretischen Mechanik“, Heft 244 der VirchowHoltzendorff'schen Sammlung gemeinverst. wiss. Vortr.(1876), p. 3

On a déjà plusieurs Traités de Mécanique, mais le plan de celui-ci est entièrement neuf. Je me suis proposé de réduire la théorie de cette Science, et l'art de résoudre les problèmes qui s'y rapportent, à des formules générales, dont le simple développement donne toutes les équations nécessaires pour la solution de chaque problème. $--\ldots$

On ne trouvera point de Figures dans cet Ourrage. Les méthodes que j'y expose ne demandent ni constructions, ni raisonnemens géométriques ou mécaniques, mais seulement des 
opérations algébriques, assujéties à une marche régulière et uniforme. Ceux qui aiment l'Analyse, verront avec plaisir la Iécanique en devenir une nouvelle branche, et me sauront gré d’en aroir étendu ainsi le domaine.

LAGRANGE.

„Mécanique analytique" (1811), Arertissement. $=$ Oeurres de Lagrange, t. 11 (1888), p. XI/XII.

Le trait distinctif du génie de Lagrange consiste dans l'unité et la grandeur des vues. Il s'attachait en tout à une pensée simple, juste et très-élevée. Son principal ourrage, la Hécanique analytique, pourrait être nommée la Mécanique philosophique; car il ramène toutes les lois de l'équilibre et du mouvement à un seul principe; et ce qui n'est pas moins admirable, il les soumet à une seule méthode de calcul dont il est lui-même l'inventeur. Toutes ses compositions mathématiques sont remarquables par une élégance singulière, par la symétrie des formes et la généralité des méthodes, et, si l'on peut parler ainsi, par la perfection du style analytique.

Fourier.

„Éloge historique de M. le Marquis de Laplace."

voir Mém. de l'Acad. des Sc. de Paris 10, 1831, Histoire, p. LXXXV.

L'illustre auteur [Lagrange] qui a voulu transformer la mécanique en une question de calcul, a sans doute rempli son objet avec toute la clarté et toute l'élégance qu'on en pouvait attendre. Mais si la véritable analyse brille quelque part dans la Mécanique analytique, j'oserai dire que e'est bien moins dans ces calculs que l'auteur range arec tant d'ordre et de symétrie, que dans ces lumineux rapprochements qu'il indique entre les méthodes, et surtout dans ces admirables préfaces qu'il a placées à la tête des différents livres de son ourrage, où il examine et discute les principes fondamentaux de la science, et fait l'histoire instructive du mouvement de l'esprit humain dans cette suite délicate d'idées fines et de solutions ingénieuses 
qui ont peu à peu formé la science de la Mécanique. C'est par là que ce bel ourrage pourra servir aux progrès ultérieurs de l'esprit, en lui montrant la route qu'il a suivie, et qui est encore la route où il doit continuer de marcher. Car ... gardons-nous de croire qu'une science soit faite quand on l'a réduite à des formules analytiques. Rien ne nous dispense d'étudier les choses en elles-mêmes, et de nous bien rendre compte des idées qui font l'objet de nos spéculations. N'oublions point que les résultats de nos calculs ont presque toujours besoin d'être vérifiés, d'un autre côté, par quelque raisonnement simple, ou par l'expérience. Que si le calcul seul peut quelquefois nous offrir une vérité nouvelle, il ne faut pas croire que, sur ce point même, l'esprit n'ait plus rien à faire: mais, au contraire, il faut songer que, cette vérité étant indépendante des méthodes ou des artifices qui ont pu nous y conduire, il existe certainement quelque démonstration simple qui pourrait la porter à l'évidence; ce qui doit être le grand objet et le dernier résultat de la science mathématique.

\section{Poinsot.}

„Théorie nouvelle de la rotation des corps", Journal de mathém. pures et appliquées 16 (1851), p. 88.

Bekanntlich verwandelt das Princip der virtuellen Geschwindigkeiten die ganze Statik in eine mathematische Aufgabe, und durch Dalemberts Princip für die Dynamik ist diese wiederum auf die Statik zurückgeführt. - - _ -

So sehr es in der Ordnung ist, dass bei der allmäligen Ausbildung der Wissenschaft und bei der Belehrung des Individuum das Leichtere dem Schwerern, das Einfachere dem Verwickeltern, das Besondere dem Allgemeinen vorangeht, so fordert doch der Geist, einmal auf dem höhern Standpunkte angelangt, den umgekehrten Gang, wobei die ganze Statik nur als ein ganz specieller Fall der Mechanik erscheine.

Gauss.

"C̈ber ein neues allgemeines Grundgesetz der Mechanik." s. Werke, Bd. 5 (1877), p. 25 u. 26. 
Es gibt eine Wissenschaft, die Mechanik, deren Aufgabe es ist, die Bewegung ron Körpern zu bestimmen, wenn die Ursachen, die diese bedingen, bekannt sind. - - - -

Die Mechanik ist mit der Geometrie nahe verwandt; beide Wissenschaften sind Anwendungen der reinen Mathematik; die Saitze beider stehen in Bezug auf ihre Sicherheit genau auf gleicher Stufe; mit demselben Rechte wie den geometrischen sützen ist auch den mechanischen absolute Gewissheit zuzusprechen.

\section{G. KirChinofF.}

„"̈ber das Ziel der Naturwissenschaften",

Akad. Festrede Heidelberg 22. XI. 1865, p. 4/5.

Mechanica ommis a geometria ita distinguitur, ut quicquid accuratum sit ad geometriam referatur, quicquid minus accuratum ad mechanicam. Attamen errores non sunt artis, sed artificum.

\section{IsaAc Newton.}

"Principia mathem. philosophiae naturalis", Auctoris praefatio ad lectorem.

Durch die Untersuchungen über die Grundlagen der Geometrie wird uns die Aufgabe nahe gelegt, nach diesem Vorbilde diejenigen physikalischen Disciplinen axiomatisch zu behandeln, in denen schon heute die Mathematik eine hervorragende Rolle spielt; dies sind in erster Linie die Wahrscheinlichkeitsrechnung und die Mechanik.

\section{Hilbert.}

„Mathematische Probleme“, Vortrag Mathem.-Congr. Paris 1900.

s. Göttinger Nachr., Math.-phys. Kl. 1900, p. 272 $=$ Arch. Nath.-Phys. (3) 1 (1901), p. 62.

Vielleicht lässt sich in der Mechanik die Deduction nicht so rein darstellen wie in der Geometrie. Vielleicht benutzen wir in der Mechanik, die mehr stofflichen Inhalt hat, noch 
ausser den Axiomen nebenher und unbewusster Weise gewisse Erfahrungsanalogien.

O. HöLDER. "Anschauung und Denken in der Geometrie", Antrittsvorles. Leipzig Univ. 1899 (Leipzig 1900), p. 22.

In England und Holland wird die Mechanik kurzweg als ein Teil der Physik angesehen. - $-\ldots-\ldots$ Auf dem Kontinent (mit Ausnahme Hollands) ist dagegen viel eher die Ansicht herrschend, dass die Mechanik als mécanique ratio[n]nelle ein Teil der Mathematik ist; denken Sie etwa an die grossen Meister Laplace und Lagrange! Auch haben niemals die Versuche gefehlt, die Grundsätze der Mechanik nach dem Muster der geometrischen Axiome als a priori gegeben festzustellen.

\section{Felix Kleis.}

„ढ̈ber die Encyklopaedie der mathematischen Wissenschaften, mit besonderer Rücksicht auf Band 4 derselben (Mechanik)",

Vortr. Naturf.-Vers. Aachen 1900.

s. Physikalische Zeitschr. 2, 1900/1901, p. 93.

Ich komme immer mehr zu der Überzeugung, dass die Nothwendigkeit unserer Geometrie nicht bewiesen werden kann, wenigstens nicht rom menschlichen Verstande noch für den menschlichen Verstand. Vielleicht kommen wir in einem andern Leben zu andern Einsichten in das Wesen des Raums, die uns jetzt unerreichbar sind. Bis dahin müsste man die Geometrie nicht mit der Arithmetik, die rein a priori steht, sondern etwa mit der Mechanik in gleichen Rang setzen. - -

Gauss an Olbers, Göttingen, 28. IV. 1817.

s. Gauss, Werke, Bd. 8 (1900), p. 177

$=$ „Wilhelm Olbers, Sein Leben und seine Werke", herausg. จ. C. Schilling, Bd. 2 (1900), p. 651/652. 
Der Mann, der noch einmal den elften Grundsatz des Euclides demonstrirt, verdient allenfalls den Namen eines sinnreichen Hannes; aber zur Erweiterung der Wissenschaften wird er nichts beitragen, was er nicht ohne diese Erfindung auch hätte thun können. „Aber, sagen sie, es geschieht, den Zweifler zu widerlegen." Den widerlegt ihr wahrhaftig nicht; denn welches Argument in der Welt wird den Mann überzeugen können, der eimmal Absurditäten glauben kann?

LichteNBerg.

Ausgew. Schriften, Reclam-Bibl. Nr. 1286-1289, p. 52.

Es thut mir sehr leid, dass ich unsere ehemalige grössere Nähe nicht benutzt habe um mehr von Deinen Arbeiten über die ersten Gründe der Geometrie zu erfahren; ich würde mir gewiss dadurch manche vergebliche Nühe erspart haben $u$. ruhiger geworden sein, als jemand wie ich es sein kann so lange bei einem solchen Gegenstande noch so viel zu desideriren ist. Ich selbst bin in meinen Arbeiten darüber weit vorgerückt (wie wol mir meine andern ganz heterogenen Geschäfte wenig Zeit dazu lassen); allein der Weg den ich eingeschlagen habe, führt nicht so wol zu dem Ziele das man wünssht und welches $\mathrm{Du}$ erreicht zu haben versicherst, als vielmehr dahin, die Wahrheit der Geometrie zweifelhaft zu machen. Zwar bin ich auf manches gekommen, was bei den meisten schon für einen Beweis. geltend würde, aber was in meinen Augen sogut wie NICHTS beweiset. z. B. wenn man beweisen könnte dass ein geradlinigtes Dreieck möglich sei, dessen Inhalt grösser wäre als eine jede gegebne Fläche so bin ich im Stande die ganze Geometrie völlig streng zu beweisen. Die meisten würden nun wol jenes als ein Axiom gelten lassen; ich nicht; es wäre ja wol möglich, dass so entfernt man auch die drei Endpunkte des $\triangle$ im Raume von einander annähme, doch der Inhalt immer unter (infra) einer gegebenen Grenze wäre. Dergleichen Sütze habe ich mehrere aber in keinem finde ich etwas Befriedigendes. Mach doch ja Deine Arbeit bald bekannt; gewiss 
wirst Du dafür den Dank nicht zwar des grossen Publikums (worunter auch mancher gehört der für einen geschickten Math. gehalten wird) einerndten, denn ich überzeuge mich immer mehr, dass die Zahl wahrer Geometer äusserst gering ist und die meisten die Schwierigkeiten bei solchen Arbeiten weder beurtheilen noch selbst einmal sie verstehen können - aber gewiss den Dank aller derer deren Urtheil Dir allein wirklich schätzbar sein kann.

\section{Gauss an W. Bolyai.}

Helmstedt, 16. XII. 1799.

s. „Briefw. zw. C. F. Gauss u. W. Bolyai“, herausg. v. Schmidt u. Stäckel (1899), p. 36/37.

In der Theorie der Parallellinien sind wir jetzt noch nicht weiter als Euklides war. Das ist die partie honteuse der Mathematik, die früher oder später eine ganz andere Gestalt bekommen muss.

C. F. Gauss.

"Ideen" (Nachlass), 27. IV. 1813.

s. Gauss, Werke, Bd. 8 (1900), p. 166.

Es wird wenige Gegenstände im Gebiete der Mathematik geben, über welche so viel geschrieben wäre, wie über die Lücke im Anfange der Geometrie bei Begründung der Theorie der Parallel-Linien. Selten vergeht ein Jahr, wo nicht irgend ein neuer Versuch zum Vorschein käme, diese Lücke auszufüllen, ohne dass wir doch, wenn wir ehrlich und offen reden wollen, sagen könnten, dass wir im Wesentlichen irgend weiter gekommen wären, als Euklides vor 2000 Jahren war. Ein solches aufrichtiges und unumwundenes Geständniss scheint uns der Würde der Wissenschaft angemessener, als das eitele Bemühen, die Lücke, die man nicht ausfüllen kann, durch ein unhaltbares Gewebe von Scheinbeweisen zu verbergen.

\section{F. Gauss.}

Göttinger Gelehrte Anz. 1816, April 20

$=$ Werke, Bd. 4 (1880), p. 364/365

$=$ Werke, Bd. 8 (1900), p. 170/171;

vgl. a. a. letzterem Orte, p. 183. 
Ich freue mich, dass Sie den Mut haben, Sich so auszudrücken, als wemn Sie die Möglichkeit, dass unsere Parallelentheorie, mithin unsere ganze Geometrie, falsch wäre, anerkennten. Aber die Wespen, deren Nest Sie aufstören, werden Ihnen um den Kopf fliegen.

Guess an Gerling. Göttingen, 25. VIII. 1818.

s. Gauss, Werke, Bd. 8 (1900), p. 179.

Meine Überzeugung, dass wir die Geometrie nicht vollständig a priori begrïnden können, ist womöglich noch fester geworden. Inzwischen werde ich wohl noch lange nicht dazu kommen, meine sehr ausgedehnten Untersuchungen darüber zur öffentlichen Bekanntmachung auszuarbeiten, und vielleicht wird diess auch bei meinen Lebzeiten nie geschehen, da ich das Geschrei der Boeoter scheue, wenn ich meine Ansicht ganz aussprechen wollte.

Gauss an Bessel.

Göttingen, 27. I. 1829.

Ich würde sehr beklagen, wenn Sie Sich „durch das Geschrei der Boeoter" abhalten liessen, Ihre geometrischen Ansichten aus einander zu setzen.

Bessel an Gauss.

Königsberg, 10. II. 1829.

s. Gauss, Werke, Bd. 8 (1900), p. 201

$=$ Briefw. Gauss-Bessel (1880), p. 490 u. 493.

aus Göttingen schrieb [nach Empfang der „Appendix“ von Joh. Bolyai] der Nathematische Riese, welcher aus erhabenen Thürmen, von den Sternen bis auf die tiefe Gründe mit gleichem Auge sieht; dass er überrascht war, gethan zu sehen, was er begonnen hat, um es unter seinen Papieren zu hinterlassen.

\section{W. Bolyai.}

„Kurzer Grundriss eines Versuchs". . (Maros Vásárhely 1851), p. 44. 
Wenn die Autorität von Gauss dahin gewirkt hat, die letzten logischen Grundlagen der Mathematik in der angegebenen Weise [nichteuklidische Geometrie] und auch bezüglich des Imaginären in einem mystischen Licht erscheinen zu lassen, so ist diese Thatsache aus der mit dem religiösen Aberglauben der Person gegatteten Beschränktheit ihres allgemeineren Denkens zu erklären.

\section{Eugen Dühring.}

„Kritische Gesch. der allgem. Principien der Mechanik,“ 2. umgearb. Aufl. (1877), p. 461.

Helmholtz fing an die a priorische Existenz der [geometrischen] Axiome in Zweifel zu ziehen und zwar nicht auf Grund abstracter mathematischer Betrachtungen, wie es zum Theil von Gauss und Riemann geschehen, sondern physiologisch-optische Untersuchungen hatten ihn veranlasst, über den Ursprung der allgemeinen Raumanschauung überhaupt nachzudenken, und sehr bald zur Überzeugung geführt, dass nur die Anschaulichkeit der Raumverhältnisse uns das als selbstverständlich voraussetzen lässt, was in Wahrheit eine besondere Eigenthümlichkeit unserer Aussenwelt ist, und wir dadurch die Axiome der Geometrie für durch transcendentale Anschauung gegebene Sätze halten.

\section{LEO KöNigsBerger.}

„Hermann von Helmboltz's Untersuchungen über die Grundlagen der Mathematik und Mechanik" (Leipzig 1896), p. 4.

Die Quelle der vollkommen irrigen, in der Bolyaischen Theorie zum Ausdruck gebrachten geometrischen Anschauung scheint mir der unglückselige Satz zu sein, „dass zwei parallele Linien sich im Unendlichen schneiden". Aus diesem Satze, den wohl auch diejenigen Mathematiker, denen er später in Fleisch und Blut ïbergegangen ist, bei seinem ersten Entgegentreten eben nur hinuntergewürgt haben, fliesst der Begriff 
„des unendlich fernen Punktes einer Geraden.“ Dieser Begriff ist in sich widersprechend, denn die Existenz einzelner unendlich ferner Punkte ist mit dem Begriff der Unendlichkeit nicht verträglich. - - - - -

Nun ist aber der ganze eben erwähnte Satz einfach unwahr. Zwei parallele Linien schneiden sich nie und nirgends, sie haben einen constanten Abstand, der, wie weit man auch geht, sich nicht vermindert und folglich auch „im Unendlichen" nicht gleich Null ist.

\section{F. Pietziker.}

Besprechung von Frischauf, „Absolute Geometrie“ (1876), Zeitschr. math. naturw. Unterr. 7 (1876), p. 470/471.

Eine missverständliche Auffassung der älteren Untersuchungen hat eine Polemik gegen dieselben hervorgerufen, die gegen Windmühlenflügel zu kämpfen scheint, umsomehr, wenn sich die Diskussion, freilich nicht ohne Schuld hervorragender Forscher, in das philosophische Gebiet verliert, das von jeher der Tummelplatz verschiedener Meinungen gewesen ist und immer bleiben wird.

\section{F. Schur.}

„Die Parallelenfrage im Lichte der modernen Geometrie", Paedag. Archiv 34 (1892), p. 546.

1. Es giebt nur einen einzigen Raum, in welchem alle Menschen leben und denken, und in welchem eine Klasse von Mathematikern nach eigenem Geschmack und eigener Willkür ihre besonderen Räume construirt hat und construirt. - - - -

2. Diejenigen Mathematiker, welche einen Raum durch reine Zahlen construiren, gleichen denjenigen Menschen, welche ihr Millionenvermögen im Traume construiren. - - - -

3. Diejenigen Geometer, welche die Definition der Parallelengeraden, das fünfte Postulat und den daraus folgenden Satz der Winkelsumme im geradlinigen Dreiecke fallen 
lassen und synthetisch eine ebene Geometrie construiren wollen, die entsprechen genau, kann man sagen, denjenigen Arithmetikern, welche das Axiom der Gleichheit fallen lassen und ihre algebraischen Probleme nicht durch Gleichungen, sondern durch Ungleichungew auflösen wollen.

\section{A. Karagiannides.}

„Die Nichteuklidische Geometrie vom Alterthum bis zur Gegenwart" (Berlin 1893), p. 43/44.

Von Seite des k. k. Unterrichtsministeriums wurden mir die Vorlesungen des Wintersemesters 1871/2 „Pangeometrie und Projectivität" als zu schwierig beanstandet - trotz der an unseren Universitäten doch herrschenden Lehr- und Lernfreiheit. Selbstverständlich hatte ich diese auf einer schon unglaublichen Ignoranz beruhende Beanständigung in gebührender Weise zurückgewiesen. Der betreffende Herr Referent kann sich nun hinsichtlich der in dieser Schrift gegebenen Pangeometrie von der Richtigkeit meiner damaligen Entgegnung überzeugen. - -

\section{J. Frischauf.}

"Absolute Geometrie nach Johann Bolyai" (Leipzig 1872), p. VII, Anm.

Für speculative Betrachtungen war Schröter nicht geschaffen. Wie er unklare Empfindungen im Leben von sich wies, so verhielt er in der Wissenschaft sich ablehnend gegen Theorien, die sich der Anschauung entziehen [nichteuklidische und mehrdimensionale Geometrie].

R. Sturi.

„Heinrich Schröter", Deutsche Mathem.-Verein. Jahresber. 2, 1891/1892, p. 37.

Wenn die gelehrte, unfruchtbare Theorie sich zu kühnem Fluge erhebt, da fliegt sie der wirklichen Welt aus den Augen, hinauf über die Wolken zu Abel und Riemann, wo die Theta-Funktionen verschwinden, wo der "spezielle" Begriff 
„Dimension“ durch den allgemeinen Begriff „Mannigfaltigkeit“ ersetzt wird und dann in einer. Welt von 4 und mehr Mannigfaltigkeiten geturnt werden kann.

A. RIEDLER.

.,Zur Frage der Ingenieur-Erziehung",

Heft 126 der "Wolkswirtsch. Zeitfragen" (Berlin 1895), p. 22/23.

Obgleich es uns nicht möglich ist, eine vollständig anschauliche Vorstellung von Räumen zu gewinnen, die mehr als drei Dimensionen besitzen, sind wir doch im Stande, uns im Einzelnen diejenigen Erfahrungen auszumalen, welche wir machen würden, wenn wir in einem Raume von mehr als drei Dimensionen lebten und uns nach sämtlichen Dimensionen willkürlich bewegen könnten.

Lidwig ScheEFFer. Doctorthese Berlin 1880.

Wir denken nicht Alles klingend und stellen uns die Molecularvorgänge nicht musikalisch, nicht in Tonhöhenverhältnissen vor, obgleich wir dazu gerade so berechtigt sind, wie dazu, uns dieselben räumlich zu denken.

.... Es liegt keine Nothwendigkeit ror, sich das bloss Gedachte räumlich d. h. mit den Beziehungen des Sichtbaren und Tastbaren zu denken, ebensowenig als es nöthig ist, dasselbe in einer bestimmten Tonhöhe zu denken. - - -

Zwischen n Punkten sind, wenn wir sie zu zweien combiniren $\frac{n(n-1)}{1 \cdot 2}$ Entfernungen denkbar, also im Allgemeinen mehr, als ein Raum von gegebener Dimensionszahl zu erfüllen vermag.

Je grösser nun die Zahl der Atome in einem Molecüle ist, einer desto höheren Dimensionszahl des Raumes bedürfen wir dann, um alle denkbaren Möglichkeiten solcher Verbindungen auch zu verwirklichen. Dies ist nur ein Beispiel, welches zeigt, wie beschränkt wir verfahren, wenn wir uns die chemischen Elemente räumlich (nach 3 Dimensionen) nebeneinander ge- 
lagert denken und wie eine Menge der Beziehungen der Elemente uns dadurch entgehen können, indem wir sie in einer Formel darstellen wollen, welche sie eben nicht zu fassen vermag.

E. МАCH.

„Die Geschichte und die Wurzel des Satzes von der

Erhaltung der Arbeit", Vortr. Böhm. Gesch. d. Wiss. 15. XI. 1871

(Prag 1872), p. 27-29.

Die Geometrie der Alten ... steht als ein scharf ausgeprägtes Gebilde vor unseren Augen. Jeder bewundert die Klarheit und Bestimmtheit ihrer Begriffe, die strenge Consequenz in deren Verbindung, die Einfachheit der Darstellung. . . . . . Wer aber die griechische Mathematik aus ihren Quellen selbst studirt hat, dem werden auch ihre Mängel nicht verborgen geblieben sein: die Alten kennen nur den synthetisch fortschreitenden Entwickelungsgang vom Einzelnen zum Einzelnen, vom Einfachen zum Zusammengesetzten; - es fehlen ihnen allgemeine Principien und Methoden. . . . . . . So opfert die antike Geometrie zu Gunsten einer scheinbaren Einfachheit die wahre Einfachheit auf, welche in der Einheit der Principien beruht, und erreicht eine triviale sinnliche Anschaulichkeit auf Kosten der Erkenntnis vom Zusammenhang geometrischer Gestalten in allem Wechsel und in aller Veränderlichkeit ihrer sinnlich vorstellbaren Lage.

\section{Hermann Hankel.}

„Projektivische Geometrie“ (Leipzig 1875), p. 1/2.

Wir verlangen die Zurückführung jeder logischen Begründung auf eine anschauliche; die Mathematik hingegen, wie sie vom Eukleides als Wissenschaft aufgestellt und bis auf den heutigen Tag im Ganzen geblieben ist, ist mit grosser Mühe bestrebt, die ihr eigenthümliche, überall nahe, anschauliche Evidenz muthwillig zu verwerfen, um ihr eine logische zu substituiren. Wir müssen finden, dass dies ist, wie wenn Jemand sich die Beine abschnitte, um mit Krücken zu gehen, 
oder wie wenn der Prinz, im „Triumph der Empfindsamkeit“, ${ }^{1}$ ) aus der wirklichen schönen Natur flieht, um sich an einer Theaterdekoration, die sie nachahmt, zu erfreuen. - - - -

Dass, was Eukleides demonstrirt, alles so sei, muss man, durch den Satz rom Widerspruch gezwungen, zugeben: warum es aber so ist, erfährt man nicht. Man hat daher fast die unbehagliche Empfindung, wie nach einem Taschenspielerstreich, und in der That sind einem solchen die meisten Eukleidischen Beweise auffallend ähnlich. Fast immer kommt die Wahrheit durch die Hinterthür herein, indem sie sich per accidens aus irgend einem Nebenumstand ergiebt. Oft schliesst ein apagogischer Beweis alle Thüren, eine nach der andern, zu, und lässt nur die eine offen, in die man nun bloss deswegen hinein muss. Oft werden, wie im Pythagorischen Lehrsatze, Linien gezogen, ohne dass man weiss warum: hinterher zeigt sich, dass es Schlingen waren, die sich unerwartet zuziehn ..... Indessen verdient übrigens die Art, wie rom Eukleides dieses durchgesetzt ist, alle Bewunderung, die ihm so viele Jahrhunderte hindurch geworden ist. $-\ldots$

\section{Sciopenhauer.}

„Die Welt als Wille und Vorstellung", Bd. 1, Buch I, § 15 . s. Werke, herausg. v. J. Frauenstädt, 2. Aufl., Bd. $2(1888)$, p. $82-84$.

Der Euklidische Mausefallenbeweis des Pythagorischen Lehrsatzes.

\section{Schopenhauer.}

„Über die vierf. Wurzel des Satzes vom zureichenden Grunde", Kap. VI, \& 39 .

s. Werke, herausg. v. J. Frauenstädt, 2. Aufl., Bd. I (1888), p. 139.

Nichts scheint mir zu beweisen, dass eine solche strenge, Schritt für Schritt fortschreitende Begründung, wie wir sie bei

1) Goethe, Werke, Grosse Weimarische Ausg., Bd. 17 (1894), p. 1-73. 
den Griechen in so hohem Grade bewundern, in Indien ein gleiches Bedürfniss gewesen sei. Es kann vielmehr dem phantasiereichen Geiste des Orients angemessener erscheinen, dass Wahrheiten, welche der unmittelbaren Anschauung zugänglich sind, nicht weiter zergliedert und auf einfachere zurückgeführt werden. Während z. B. bei den Griechen die Proportionalität der Seiten in gleichwinkligen Dreiecken als das Resultat einer langen Reihe von Schlüssen sich darstellt, kann dieselbe Wahrheit im Orient als durch Anschauung gegeben und mithin keiner ferneren Zurückführung bedürftig betrachtet worden sein. Jedem Menschen, und hätte er sich auch nie mit mathematischen Reflexionen beschäftigt, leuchtet in der That ein, dass jede Figur in beliebigem Grade vergrössert oder verkleinert werden kann, ohne dass die daran vorkommenden Dimensionen ihre Verhältnisse verändern.

\section{Dirichlet „nach Chasles". $\left.{ }^{1}\right)$}

s. Werke, herausg. v. Kronecker, Bd. 2, p. 345.

Es ist eine tief eingewurzelte Gewohnheit vieler Geometer, Sätze zu formulieren, die „im allgemeinen“ gelten sollen, $d . h$. einen klaren Sinn überhaupt nicht haben, zudem noch häufig als allgemein gültig hingestellt, oder mangelhaft begründet werden. Dies Verfahren wird trotz etwanigen Verweisungen auf Träger berühmter Namen späteren Geschlechtern sicher als ganz unzulässig erscheinen, scheint aber in unserem kritischen Zeitalter von vielen als eine berechtigte Eigentümlichkeit der Geometrie betrachtet zu werden.

\section{E. STudy.}

„Ein neuer Zweig der Geometrie,“

Deutsche Mathem.-Verein. Jahresber. 11 (1902), p. 100, Anm.

Allgemeinheit der Gesichtspunkte und Methoden, Präzision und Eleganz der Darstellung, sind seit Lagrange Gemeingut

1) Dirichlet hatte sich von Chasles einmal ein autographiertes Vorlesungsheft geliehen (s. G. Darboux, ,Notice sur les travaux de M. Chasles", Bull. des sc. mathém. et astron. (2) 4 (1880), p. 438). 
aller derer geworden, die auf den Rang wissenschaftlicher Mathematiker Anspruch machen dürfen. Und, wenn auch jene Allgemeinheit, zuweilen auf Kosten der Anschaulichkeit und Brauchbarkeit ïbertrieben, zum Abstrusen führt, so dass allgemeine Sätze aufgestellt werden, die in keinem speziellen Falle gelten; wenn ferner jene Präzision zuweilen in eine gesuchte Kürze ausartet, welche eine Abhandlung schwieriger zu lesen macht, als sie zu schreiben war; wenn endlich auch jene Eleganz der Form in unseren Tagen fast zum Kriterium über den Wert oder Unwert eines Satzes geworden ist, - so sind doch alle diese Bedingungen für die gedeihliche Entwickelung dadurch von höchster Bedeutung, dass sie das wissenschaftliche Material in den Grenzen halten, die innerlich und äusserlich notwendig sind, wenn die Mathematik sich nicht in's Kleinliche zersplittern oder am Überfluss ersticken soll.

Hermann HaNkel.

„Die Entwickelung der Mathematik in den letzten Jahrhunderten," Akad. Vortrag Tübingen 29. IV. 1869, 2. Aufl. (Tübingen 1884), p. 1415.

Il semble que dans l'état actuel des sciences mathématiques le seul moyen d'empêcher que leur domaine ne devienne trop vaste pour notre intelligence, c'est de généraliser de plus en plus les théories que ces sciences embrassent, afin qu'un petit nombre de rérités générales et fécondes soit, dans la tête des hommes, l'expression abrégée de la plus grande variété de faits particuliers.

\section{Dupis.}

"Déreloppements de Géométrie" (Paris 1813), IV me mémoire, § III, p. 265.

Durch gehörige Aneignung der wenigen [geometrischen] Grundbeziehungen macht man sich zum Herrn des ganzen Gegenstandes; es tritt Ordnung in das Chaos [aller Sätze, womit uns die ältere und neuere Zeit so freigebig beschenkt hat] ein, 
und man sieht, wie alle Theile naturgemäss in einander greifen, in schönster Ordnung sich in Reihen stellen, und verwandte zu wohlbegrenzten Gruppen sich vereinigen. Man gelangt auf diese Weise gleichsam in den Besitz der Elemente, von welchen die Natur ausgeht, um mit möglichster Sparsamkeit und auf die einfachste Weise den Figuren unzählig viele Eigenschaften verleihen zu können.

\section{J. Steiner.}

„Systemat. Entw. d. Abhäng. geometrischer Gestalten von einander", Vorrede.

s. Werke, herausg. v. Weierstrass, Bd. 1 (1881), p. 233.

Die Ansicht, dass es bei der Wissenschaft hauptsächlich auf Bequemlichkeit und Ersparniss in Denken ankommt, vertrete ich seit Beginn meiner Lehrthätigkeit. Die Physik mit ihren Formeln, mit ihrer Potentialfunction, ist besonders geeignet diese Ansicht klar zu stellen. Das Trägheitsmoment, das Centralellipsoid u. s. w. sind z. B. nichts wie Surrogate, durch die man mit Bequemlichkeit die Betrachtung der einzelnen Massenpunkte erspart. Besonders klar fand ich diese Ansicht auch bei meinem Freunde, dem Nationalökonomen E. Herrmann. Von ihm habe ich den mir sehr passend scheinenden Ausdruck angenommen: „Die Wissenschaft hat eine ökonomische oder wirthschaftliche Aufgabe."

E. MACH.

"Die Geschichte und die Wurzel des Satzes von der Erhaltung der Arbeit", Vortr. Böhm. Ges. d. Wiss. 15. XI. 1871 (Prag 1872), p. 55/56, Note 5.

Man könnte sich ja ein mathematisches - wenigstens ein geometrisches - Lehrgebäude denken, das auf den Begriff der absoluten oder unbegrenzten Genauigkeit verzichtete und seinen Sätzen nur eine angenäherte, also empirisch verifizierbare Form gäbe; etwa so: wenn in einem Dreieck zwei Seiten um nicht mehr als den 100. Teil ihrer Länge verschieden sind, unterscheiden sich die gegenüberliegenden Winkel höchstens um $3 \mathrm{Grad}$ - vorausgesetzt, dass der eingeschlossene Winkel nicht 
kleiner als 10 Grad ist. Aber das Beispiel zeigt schon, woran eine solche Einrichtung der Mathematik scheitert: an der Notwendigkeit von Zusätzen, wie der hier mit „vorausgesetzt, dass“ eingeführte, von denen die entsprechenden Sätze der idealen, genauen Mathematik frei sind. Wenn solche Zusätze schon bei so einfachen Sätzen, wie der eben genannte, erforderlich sind, so brauche ich nicht weiter auseinanderzusetzen, wie sie sich bei fortschreitendem Aufbau des Lehrgebäudes immer mehr häufen müssten, bis schliesslich die Sätze so lang würden, dass niemand mehr imstande wäre, den Anfang bis zum Ende im Gedächtnis zu behalten. Daher wird selbst der strengste Empirist, der die Gewinnung solcher der empirischen Verification zugänglichen Sätze als das eigentliche praktische Ergebnis der Mathematik ansehen möchte, dem Zugeständnis nicht ausweichen können: der ökonomischste Weg zu diesem Ziel führt über die Aufstellung absolut genauer Sätze, von denen aus man dann durch eine sogenannte Fehlerrechnung feststellt, welche Abweichungen man in den Voraussetzungen zulassen darf, wenn die Ungenauigkeit des Resultats eine vorgeschriebene Grenze nicht überschreiten soll.

\section{H. BuRkhaRdT.}

„Mathematisches und naturwissenschaftliches Denken," Antrittsvorl. Zürich Univ. 1897.

s. Münchener Allgem. Zeitg. Beilage 22. XI. 1897, Nr. 264, p. 2/3 $=$ Deutsche Mathem.-Verein. Jahresber. 11 (1902), p. 53/54.

Die Araber verwendeten in der Mathematik ihr Nachdenken darauf, das Nachdenken entbehrlich zu machen. Das consequent durchgeführte dekadische Zahlensystem [,] die praktischen Regeln des Rechnens in den vier Species sind von ihnen hauptsächlich so gut schematisirt worden, dass sie jetzt mit dem besten Erfolge auf Dorfschulen gelehrt werden können.

KuMMer.

(„Über die Bedingungen, unter denen die Wissenschaften, insbesondere die mathematischen, gedeihen und sich zur Blüthe entfalten"), Festrede i. d. Berliner Akademie 22. III. 1866.

s. Berl. Monatsber. 1866, p. 182. 
Am meisten ausgebildet ist die Gedankenökonomie in jener Wissenschaft, welche die höchste formelle Entwicklung erlangt hat, welche auch die Naturwissenschaft so häufig zur Hilfe heranzieht, in der Mathematik. So sonderbar es klingen mag, die Stärke der Mathematik beruht auf der Vermeidung aller unnötigen Gedanken, auf der grössten Sparsamkeit der Denkoperationen. Schon die Ordnungszeichen, welche wir Zahlen nennen, bilden ein System von wunderbarer Einfachheit und Sparsamkeit. Wenn wir beim Multiplizieren einer mehrstelligen Zahl durch Benützung des Einmaleins die Resultate schon ausgeführter Zähloperationen verwenden, statt sie jedesmal zu wiederholen, wenn wir bei Gebrauch von Logarithmentafeln neu auszuführende Zähloperationen durch längst ausgef ührte ersetzen und ersparen, wenn wir Determinanten verwenden, statt die Lösung eines Gleichungssystems immer von neuem zu beginnen, wenn wir neue Integralausdrücke in altbekannte zerlegen, so sehen wir hierin nur ein schwaches Abbild der geistigen Tätigkeit eines Lagrange oder Cauchy, der mit dem Scharfblick eines Feldherrn für neu auszuführende Operationen ganze Scharen schon ausgeführter eintreten lässt. Man wird keinen Widerspruch erheben, wenn wir sagen, die elementarste wie die höchste Mathematik sei ökonomisch geordnete, für den Gebrauch bereit liegende Zählerfahrung.

.. Im einzelnen vermag die Wissenschaft uns nichts zu bieten, was nicht jeder in genügend langer Zeit auch ohne alle Methode finden könnte. Jede mathematische Aufgabe könnte durch direktes Zählen gelöst werden

Wer Mathematik treibt, den kann zuweilen das unbehagliche Gefühl überkommen, als ob seine Wissenschaft, ja sein Schreibstift, ihn selbst an Klugheit überträfe, ein Eindruck, dessen selbst der grosse Euler nach seinem Geständnisse sich nicht immer erwehren konnte. Eine gewisse Berechtigung hat dieses Gefühl, wenn wir bedenken, mit wie viel fremden oft vor Jahrhunderten gefassten Gedanken wir in geläufigster Weise operieren. Es ist teilweise wirklich eine fremde Intelligenz, 
die uns in der Wissenschaft gegenübersteht. Mit der Erkenntnis dieses Sachverhaltes erlischt aber wieder das Mystische und Magische des Eindruckes, zumal wir jeden der fremden Gedanken, sobald wir nur wollen, nachzudenken vermögen.

\section{E. MACH.}

„Die ökonomische Natur der physikalischen Forschung“, Vortrag Wien Akad. d. Wiss. 1882.

s. Mach, „Populär-wissensch. Vorles.", 3. Aufl. (1903), p. $224 / 225,226,225 / 226$.

Durch die fortgesetzte Beschäftigung mit dem Unterrichte erweiterte sich, ohne dass ich es wusste und wollte, mein Streben nach wissenschaftlicher Einheit und Zusammenhang. Wie die in besonderen Abtheilungen verbundenen Sätze einer einzelnen mathematischen Disciplin, so, glaubte ich, müssten auch alle besonderen mathematischen Disciplinen auseinander hervorgehen; es schwebte mir die Idee der organischen Einheit

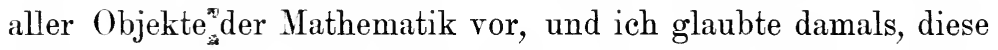
Einheit auf irgend einer Hochschule, wenn auch nicht als einen objektiv zu Stande gebrachten Lehrgegenstand, doch in der Form bestimmter Andeutungen zu finden.

\section{JaCOB StenNer}

in einer Eingabe an das preuss. Cultusministerium 16. XII. 1826. s. J. Lange, „Jacob Steiners Lebensjahre in Berlin“

(Berlin 1899), p. 19.

Es kann keine allumfassende geometrische Symbolik geben, wie sie Grassmann und Hamilton sich dachten.

Alles in Quaternionen zwängen zu wollen, ist zwecklos.

\section{H. BURKhaRdT.}

„Über Vectoranalysis“, Deutsche Mathem.-Verein. Jahresber. 5,1896, p. 52. 
Der wesentliche Vortheil, welcher durch Grassmann's Auffassung [der Geometrie] erreicht wurde, war der !Form nach der, dass nun alle Grundsätze, welche Raumesanschauungen ausdrückten, gänzlich wegfielen, und somit der Anfang ein ebenso unmittelbarer wurde, wie der der Arithmetik; dem Inhalte nach aber der, dass die Beschränkung anf drei Dimensionen wegfiel. Erst hierdurch traten die Gesetze in ihrer Unmittelbarkeit und Allgemeinheit ans Licht und stellten sich in ihrem wesentlichen Zusammenhange dar, und manche Gesetzmässigkeit, die bei drei Dimensionen entweder noch gar nicht oder nur verdeckt vorhanden war, entfaltete sich nun bei dieser Verallgemeinerung in ihrer ganzen Klarheit.

\section{F. Junghans.}

„Hermann Grassmann“, Zeitschr. Math. Phys. 23 (1878), Hist.-literar. Abth., p. 70

$=$ Zeitschr. math. naturw. Unterr. 9 (1878), p. 168.

In future times Tait will be best known for his work in the quaternion analysis. Had it not been for his expositions, developments and applications, Hamilton's invention would be today, in all probability, a mathematical curiosity; and there are those who think that, now Tait is gone, such will ere long be its fate. But I venture to think that Hamilton himself will prove the better prophet: for he wrote to Tait: „Could anything be simpler or more satisfactory? Don't you feel, as well as think, that we are on the right track, and shall be thanked hereafter? Never mind when."

Alexander Macfarlane. „Peter Guthrie Tait, his life and works", Bibl. math. (3) 4 (1903), p. 189.

In fact, with all my very high admiration ..... for Gauss, I have some private reasons for believing, I might say knowing, that he did not anticipate the quaternions. In fact, if I don't forget the year, I met a 
particular friend, and (as I was told) pupil of Gauss, Baron von Walter[s]hausen,.... at the second Cambridge Mieeting of the British Association in 1845, just after Herschel had spoken of my quaternions and your triple algebra, in his speech from the throne. The said Baron soon afterwards called on me here,........ he informed me that his friend and (in one sense) master, Gauss, had long wished to frame a sort of triple algebra; but that his notion had been, that the third dimension of space was to be symbolically denoted by some new transcendental, as imaginary, with respect to $\sqrt{-1}$, as that was with respect to 1 . Now you see, as I saw then, that this was in fundamental contradiction to my plan of treating all dimensions of space with absolute impartiality, no one more real than another.

W. R. Hamiltox to A. De Morgan.

Observatory (of Trinity College, Dublin), 6. I. 1852. ef. Graves, „Life of Sir William Rowan Hamilton", Vol. III (1889), p. $311 / 312=$ Vol. II (1885), p. 490.

Mit allen . . neuen Calculs verhält es sich so, dass man durch sie nichts leisten kann, was nicht auch ohne sie zu leisten wäre; der Vortheil ist aber der, dass wenn ein solcher Calcul dem innersten Wesen vielfach vorkommender Bedürfnisse correspondirt, jeder der sich ihn ganz angeeignet hat, auch ohne die gleichsam unbewussten Inspirationen des Genies, die niemand erzwingen kann, die dahin gehörigen Aufgaben lösen, ja selbst in so verwickelten Fällen gleichsam mechanisch lösen kann, wo ohne eine solche Hülfe auch das Genie ohnmächtig wird. So ist es mit der Erfindung der Buchstabenrechnung überhaupt; so mit der Differentialrechnung gewesen, so ist es auch (wenn auch in partielleren Sphaeren) mit Lagranges Variationsrechnung, mit meiner Congruenzenrechnung und mit Möbius [barycentrischem] Calcul. Es werden durch solche Conceptionen unzählige Aufgaben, die sonst vereinzelt stehen, und jedesmahl neue Efforts (kleinere oder grössere) des Er- 
findungsgeistes erfordern, gleichsam zu einem organischen Reiche.

Gauss an Schumacher.

Göttingen, 15. V. 1843.

s. Briefw. Gauss-Schumacher, Bd. 4 (1862), p. 148.

So wie niemand physisch bestehen könnte, wenn er die Blutbewegung, die Athmung, die Verdauung seines Körpers durch willkürliche, vorbedachte Handlungen einleiten und im Stande halten müsste, so könnte auch niemand intellectuell bestehen, wenn er genöthigt wäre, alles was ihm vorkommt zu beurtheilen, anstatt sich vielfach durch sein Vorurtheil leiten zu lassen. Das Vorurtheil ist eine Art Reflexbewegung im Gebiete der Intelligenz.

Auf Vorurtheilen, d. h. auf nicht jedesmal auf ihre Anwendbarkeit geprüften Gewohnheitsurtheilen, beruht ein guter Theil der Überlegungen und Handgriffe des Naturforschers, auf Vorurtheilen beruht die Mehrzahl der Handlungen der Gesellschaft. Mit dem plötzlichen Erlöschen aller Vorurtheile würde sie selbst sich rathlos auflösen. Und eine tiefe Kenntniss der Macht der intellectuellen Gewohnheit hat jener Fürst verrathen, der seine den rückständigen Sold ungestüm fordernde Leibgarde durch das übliche Commandowort zum Abzuge zwang, wohl wissend, dass sie diesem nicht widerstehen würde.

E. MaCH.

„Über Umbildung und Anpassung im naturw. Denken,“

Rectoratsrede Prag Univ. 1883 (Wien 1884), p. 14/15

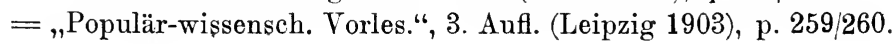

Es ist der Character der Mathematik der neueren Zeit (im Gegensatz gegen das Alterthum) dass durch unsere Zeichensprache und Namengebungen wir einen Hebel besitzen, wodurch die verwickeltsten Argumentationen auf einen gewissen Mechanismus reducirt werden. An Reichthum hat dadurch die Wissenschaft unendlich gewonnen, an Schönheit und Solidität aber 
wie das Geschäft gewöhnlich betrieben wird, eben so sehr verloren. Wie oft wird jener Hebel eben nur mechanisch angewandt, obgleich die Befugniss dazu in den meisten Fällen gewisse stillschweigende Voraussetzungen implicirt. ${ }^{1}$ ) Ich fordere, man soll bei allem Gebrauch des Calculs, bei allen Begriffsverwendungen sich immer der ursprünglichen Bedingungen bewusst bleiben, und alle Producte des Mechanismus niemals über die klare Befugniss hinaus als Eigenthum betrachten. Der gewöhnliche Gang ist aber der, dass man für die Analysis einen Character der Allgemeinheit in Anspruch nimmt, und dem Andern der so herausgebrachte Resultate noch nicht für bewiesen anerkennt zumuthet, er solle das Gegentheil nachweisen. Diese Zumuthung darf man aber nur an den stellen, der seinerseits behauptet ein Resultat sei falsch, nicht aber dem, der ein Resultat nicht 'für bewiesen anerkennt, welches auf einem Mechanismus beruht, dessen ursprüngliche wesentliche Bedingungen in' dem vorliegenden Fall gar nicht zutreffen. So ist es sehr oft mit Divergirenden Reihen. Reihen haben eine klare Bedeutung, wenn sie convergiren; diese Klarheit der Bedeutung fällt weg mit dieser Bedingung, und es ändert im Wesentlichen Nichts, ob man sich des Worts Summe oder Werth bedient. ... Nehmen Sie meinetwegen statt obigen Gleichnisses einer Maschine das von Papiergeld. Es kann dies zu grossen Arbeiten vortheilhaft benutzt werden, aber solide ist der Gebrauch nur, wenn ich gewiss bin, es jeden Augenblick in klingende Münze umsetzen zu können.

\section{Gauss an Schumacher.}

Göttingen, 1. IX. 1850.

s. Briefw. Gauss-Schumacher, Bd. 6 (1865), p. 107/108.

Eine Reihe ist convergirend, wenn ihre Glieder in ihrer Folge nach einander immerfort kleiner werden. Die Summe der Glieder nähert sich alsdann immer mehr dem Werthe der

1) s. Note 1 am Ende des Buches. 
Grösse, welche die Summe der ganzen ins Unendliche fortgesetzten Reihe ist.

\section{KLÜGEL.}

„Mathem. Wörterbuch“ (1803),

Abtheil. I, 1, Artikel „Convergirend“.

Zum Begriff des Unendlichen gelangt man durch das Studium der Mathematik - und der menschlichen Dummheit.

J. V.

Fliegende Blätter 1903, p. 20.

Sobald eine Reihe aufhört, convergent zu sein, hat ihre Summe als Summe keinen Sinn.

Gauss an Bessel.

Göttingen, 5. V. 1812.

s. Briefw. Gauss-Bessel (1880), p. 173.

Divergente Rækker ere i det Hele noget Fandensskab, og det er en Skam at man vover at grunde nogen Demonstration derpaa. Man kan faae frem hvad man vil naar man bruger dem, og det er dem som har gjort saa megen Ulykke og saa mange Paradoxer. Kan der tænkes noget skrækkeligere end at sige at

$$
0=1-2^{n}+3^{n}-4^{n}+\text { etc. }
$$

hror $n$ er et heelt positivt Tal. Risum teneatis amici. Jeg har i det hele faaet Øjnene op paa en meget forbausende Maneer; thi naar man undtager de allersimpleste Tilfælde for Ex: de geometriske Rækker, saa gives der i hele Mathematiken næsten ikke en eneste unendelig Række, hris Sum er bestemt paa en stræng Maade: med andre Ord det vigtigste af Mathematiken staaer uden Begrundelse. Det meeste er rigtigt; det er sandt, og det er overordentlig forunderligt. Jeg bestræber mig for at søge Grunden dertil. En overmaade interessant Opgave. 
(Les séries divergentes sont en bloc une invention du diable, et e'est une honte que l'on ose fonder sur elles la moindre démonstration. On peut en tirer tout ce qu'on veut quand on les emploie, et ce sont elles qui ont produit tant d'échecs et tant de paradoxes. Peut-on penser quelque chose de plus affreux que de dire que

$$
0=1-2^{n}+3^{n}-4^{n}+\text { etc. }
$$

où $n$ est un nombre entier positif. Risum teneatis amici. Je suis devenu prodigieusement attentif à tout cela; car si l'on excepte les cas de la plus extrême simplicité, par exemple: les séries géométriques, il n'y a presque pas, dans toutes les mathématiques, une seule série infinie dont la somme est déterminée d'une manière rigoureuse: en d'autres termes, ce qu'il y a de plus important dans les mathématiques est sans fondement. La plupart des choses sont exactes: cela est vrai; et c'est extraordinairement surprenant. Je m'efforce d'en chercher la raison. Sujet excessivement intéressant.)

ABEL à Holmboe. [Berlin], 16. I. 1826.

Der gives yderst faae Sætninger $\mathrm{i}$ den hoiere Analyse som ere bevisede med overbevisende Strænghed. Overalt finder man den ulykkelige Maade at slutte fra det Specielle til det Almindelige, og yderst mærkværdigt er det at der efter en saadan Fremgangsmaade dog kuns findes faae af de saakaldte Paradoxer. . . Efter mine Tanker ligger den deri at de Functioner som Analysen hidentil har beskjæftiget sig med mestendels lade sig udtrykke ved Potentser. - Saasnart der komme andre imellem hvilket rigtig nok ikke ofte er Tilfældet saa gaaer det gjerne ikke godt og af falske Slutninger opstaae da en Mængde med hinanden forbundne urigtige Sætninger.

(Il n'y a que très peu de propositions, dans l'analyse supérieure, qui soient démontrées avec une rigueur décisive. Partout on trouve la malheureuse manière de conclure du particulier au général, et il est très singulier qu'avec une pareille méthode, il ne se trouve malgré tout que peu de ce qu'on appelle paradoxes. . . A mon avis cela provient de ce 
que les fonctions dont l'analyse s'est occupée jusqu'ici peuvent, la plupart, être exprimées au moyen de puissances. Aussitôt que d'autres interviennent, ce qui, il est vrai, n'arrive pas souvent, alors ça ne va plus, et de conclusions fausses découlent une foule de propositions incorrectes qui s'enchaînent.)

ABEL à Hansteen.

Dresde, 29. III. 1826.

voir „Niels Henrik Abel, Mémorial publié à l'occasion du centenaire de sa naissance": "Texte original des lettres", p. 16 et 21/22 ou "Correspondance d'Abel" (traduction française), p. $16 / 17$ et 23 .

Der Gebrauch einer unendlichen Grösse als einer Vollendeten ist in der Mathematik niemals erlaubt. Das Unendliche ist nur eine Façon de parler, indem man eigentlich von Grenzen spricht, denen gewisse Verhältnisse so nahe kommen als man will, während anderen ohne Einschränkung zu wachsen verstattet ist.

Gauss an Schumacher.

Göttingen, 12. VII. 1831.

s. Briefw. Gauss-Schumacher, Bd. 2 (1860), p. 269.

Trotz wesentlicher Verschiedenheit der Begriffe des potenzialen und actualen Unendlichen, indem ersteres eine veränderliche endliche, über alle endliche Grenzen hinaus wachsende Grösse, letzteres ein in sich festes, constantes, jedoch jenseits aller endlichen Grössen liegendes Quantum bedeutet, tritt doch leider nur zu oft der Fall ein, dass das eine mit dem andern verwechselt wird. . . . Wenn aber aus einer berechtigten Abneigung gegen solches illegitime A. U. sich in breiten Schichten der Wissenschaft, unter dem Einflusse der modernen epikureisch-materialistischen Zeitrichtung, ein gewisser Horror Infiniti ausgebildet hat, der in dem erwähnten Schreiben von Gauss [Brief an Schumacher von 12. VII. 1831, s. Briefw. Gauss-Schumacher, Bd. 2, p. 269-271] ${ }^{1}$ ) seinen

1) s. das vorhergehende Citat. 
klassischen Ausdruck und Rückhalt gefunden, so scheint mir die damit verbundene unkritische Ablehnung des legitimen A. U. kein geringeres Vergehen wider die Natur der Dinge zu sein, die man zu nehmen hat, wie sie sind, und es lässt sich dieses Verhalten auch als eine Art Kurzsichtigkeit auffassen, welche die Möglichkeit raubt, das A. U. zu sehen, obwohl es in seinem höchsten, absoluten Träger uns geschaffen hat und erhält und in seinen secundären, transfiniten Formen uns allüberall umgiebt und sogar unserm Geiste selbst innewohnt.

\section{G. Cantor.}

„Zum Problem des actualen Unendlichen,“ Natur und Offenbarung 32 (1886), p. 226.

Dernièrement Leibnitz m'a fait une dissertation sur les infiniment petits: qui mieux que moi, ma chère, est au fait de ces êtres? ${ }^{1}$ )

\section{Sophie Charlotte Reine de Prusse \\ à son amie Mlle de Pöllnitz. \\ Wusterhausen, 7. VIII. 1702.}

roir Erman, „Mémoires pour servir à l'histoire de Sophie Charlotte" (Berlin 1801), p. 198.

Was in der Physik die Verbannung der Fernwirkungen, die Erklärung der Erscheinungen durch die inneren Kräfte eines raumerfüllenden Aethers ist, das ist in der Mathematik das Verständnis der Functionen aus ihrem Verhalten im Unendlich-Kleinen, insbesondere also aus den Differentialgleichungen, denen sie genügen.

F. KLleis.

„Riemann“, Vortrag Naturf.-Vers. Wien 1894.

s. Deutsche Mathem.-Verein. Jahresber. 4, 1894/95, p. 73.

1) Anspielung auf ihren Gemahl Friedrich I. und dessen Hof; s. Carlyle, „Gesehichte Friedrichs II. von Preussen", deutsch von J. Neuberg, Bd. 1 (Berlin 1858), p. 53, 54, 68, 384. 
Zwischen dem Wirken der natürlichen Zuchtwahl während einer Generation und dem Ergebnisse nach hunderttausend Generationen besteht etwa die Beziehung, wie zwischen Differential und Integral. Wieselten vermögen wir letztere Beziehung zu durchschauen, obschon wir sie der Rechnung unterwerfen. Bezweifeln wir deshalb die Richtigkeit unserer Integration?

Emil DU BoIs-Reymond.

„Darwin versus Galiani“, Rede Berliner Akad. 6. VII. 1876.

s. Reden, Bd. 1 (1886), p. 228.

Es ist überraschend, dass man in den Naturwissenschaften so oft das „Kleine“ gern in den Kauf nimmt, wenn man sich das „Grosse“ damit erklären zu können glaubt. Das erinnert an das Goethe'sche Wort:

\section{„Du kannst im Grossen nichts verrichten \\ „Und fängst es nun im Kleinen an." 1 )}

So meint man, die Massenattraction begreiflicher zu machen, wenn man einen Attractionsäther annimmt und die Kraft nun von Theilchen zu Theilchen wirken lässt; so „erklärt" die Darwin'sche Theorie die grossen Abweichungen, welche bei den Individuen einer Gattung organischer Wesen auftreten, indem sie lehrt, wie dieselben aus kleinen Änderungen hervorgehen.

L. KRONECKER.

Vorlesungen I (Integrale), herausg. v. Netto (1894), p. 3/4.

Bis auf die neueste Zeit hat man allgemein angenommen, dass eine eindeutige und continuirliche Function einer reellen Veränderlichen auch stets eine erste Ableitung habe, deren

1) Faust, I, 1360-1361 (Werke, Grosse Weimarische Ausg., Bd. 14 (1887), p. 68), Erste Szene zwischen Faust und Mephistopheles, wo es allerdings bekanntlich ,vernichten“ statt „,verrichten" heißt.

Ahrens, Scherz und Ernst i. d. Mathem. 
Werth nur an einzelnen stellen unbestimmt oder unendlich gross werden könne. Selbst in den Schriften von Gauss, Cauchy, Dirichlet findet sich meines Wissens keine Äusserung, aus der unzweifelhaft hervorginge, dass diese Nathematiker, welche in ihrer Wissenschaft die strengste Kritik überall zu üben gewohnt waren, anderer Ansicht gewesen seien.

Weierstrass.

„Cber continuirliche Functionen eines reellen Arguments, die tür keinen Werth des letzteren einen bestimmten Differentialquotienten besitzen", gelesen i. d. Berl. Akad. 18. VII. 1872. s. Werke, Bd. 2 (1895), p. 71.

Seit dem Versuche Ampère's, die Frage nach der Existenz eines Differentialquotienten zu beantworten, ist für dieses höchst interessante Problem weiter nichts geschehen, als dass die Nothwendigkeit, die Existenz des Differentialquotienten im Allgemeinen für stetige Functionen zu erweisen, oder die für seine Existenz wesentlichen Stetigkeitsbedingungen zu ermitteln, allgemeiner anerkannt ist, namentlich seit Dirichlet's* strenger Ableitung der Fourier'schen Reihen. Auch C. G. J. Jacobi hat, wie ich aus mündlicher Nittheilung weiss, in seinen Vorlesungen zuweilen von "Curven mit unendlich vielen Spitzen“ gesprochen, und Gauss: nimmt ausdrücklich auf solche Functionen Rücksicht.

* Crelle's Journ. t. IV. 1829. p. 169 [= Dirichlet, Werke, heransg. v. Kronecker, Bd. 1 (1889), p. 131/132].

** Allgem. Lehrsätze in Bez. auf die . . Kräfte. 1840. art. 16 $[=$ Gauss, Werke, Bd. 5 (1877), p. 218].

Hermanx Hankel.

Artikel „Grenze" in Ersch u. Gruber's Encyklopädie I, 90 (1871), p. 202.

Il y a cent ans, une pareille fonction eut été regardée comme un outrage au sens commun. Une fonction continue, aurait-on dit, est par essence susceptible d'être représentée par une courbe et une courbe a évidemment toujours une tangente..... 
On voit à quelles erreurs nous expose une folle confiance dans ce qu'on prend pour l'intuition. Par la découverte de cet exemple frappant, Weierstrass nous a donc donné un utile avertissement et nous a appris à mieux apprécier les méthodes impeccables et purement arithmétiques dont il a, plus que personne, contribué à doter la Science.

\section{H. Poincaré.}

"L'oeuvre mathématique de Weierstrass", Acta mathem. 22 (1899), p. 5/6.

In den letzten Jahrzehnten wandte die wissenschaftliche Forschung auch den metamathematischen Problemen sich zu, welche bisher nur Gegenstand laienhafter Anläufe gewesen waren. Solche Untersuchungen galten zuerst den geometrischen Grundbegriffen, Abziehungen [= Abstractionen] ganz besonderer Art, deren Zurückführung auf die notwendigen erzeugenden Vorstellungen schwierig war, später dem Grenzbegriff und damit Zusammenhängendem, - im Vergleich mit anderen Begriffen philosophisches Wild zu nennen, das aber im ersten Heft meiner Allgemeinen Functionentheorie, wie ich glaube, glücklich zur Strecke gebracht ist.

Paul du Bois-Reymond.

„Über die Grundlagen der Erkenntnis in den exacten Wissenschaften" (Tübingen 1890), p.22.

Der Begriff der Grenze ist, so zu sagen, die organische Kraft der Analysis, die aus dem niederen Material der Arithmetik ein Neues, qualitativ verschiedenes, schafft. Sie tritt überall da mit Nothwendigkeit ein, wo es sich um die Erzeugung eines wesentlich Neuen, um die Vergleichung von qualitiv Verschiedenem handelt; in der Geometrie also namentlich, wenn Krummes durch Gerades gemessen werden soll. Sie ist das Instrument, durch das die Mathematik mit ihrem Zahlenmaterial, das seiner Entstehung nach ein Discretes ist, das 
Stetige bezwingt. Nie wird man bei den Untersuchungen der Veränderungen des Stetigen den Begriff der Grenze entbehren können.

Hermanx Hankel.

Artikel „Grenze" in Ersch u. Gruber's

Encyklopädie I, 90 (1871), p. 201.

In unserem Jahrhundert treten die Begriffe Substitution und Substitutionsgruppe, Transformation und Transformationsgruppe, Operation und Operationsgruppe, Invariante, Differentialinvariante und Differentialparameter immer deutlicher als die wichtigsten Begriffe der Mathematik hervor.

Sophus LIE.

Leipziger Ber. 47 (1895), Math.-phys. Cl., p. 261.

Die Begriffe Invariante und continuierliche Gruppe sind so alt wie die Mathematik selbst,' wenn sie auch erst am Schlusse des vorigen Jahrhunderts in speciellen Fällen einigermassen deutlich hervortreten.

Sophus Lie.

Leipziger Ber. 45 (1893), Math.-phys. Cl., p. 370.

Der Gruppenbegriff, durch Gauss und Galois in die Mathematik eingeführt, hat in neuerer Zeit in allen Zweigen unserer Wissenschaft eine fundamentale Bedeutung erlangt.

G. Frobenius.

Antrittsrede in d. Berl. Akademie.

s. Berl. Sitzungsber. 1893, p. 627.

As all roads are said to lead to Rome, so I find, in my own case at least, that all algebraical inquiries sooner or 
later end at that Capitol of Modern Algebra over whose shining portal is inscribed "Theory of Invariants".

\section{J. J. Sylvester.}

(,Trilogy"), Philosoph. Transactions of the Roy. Soc. of London, Vol. 154, Part. III (1864), p. 579.

Fuchs: .... Fast möcht' ich nun moderne Algebra studieren. Meph.: Ich wünschte nicht euch irre zu führen.

Was diese Wissenschaft betrifft,

Es ist so schwer, die leere Form zu meiden,

Und wenn ihr es nicht recht begrifft,

Vermögt die Indices ihr kaum zu unterscheiden.

Am Besten ist's, wenn ihr nur Einem traut

Und auf des Meisters Formeln baut.

Im Ganzen - haltet euch an die Symbole.

Dann geht ihr zu der Forschung Wohle

Ins sichre Reich der Formeln ein.

Euchs: Ein Resultat muss beim Symbole sein?

Meph.: Schon gut! Nur muss man sich nicht allzu ängstlich quälen.

Denn eben, wo die Resultate fehlen,

Stellt ein Symbol zur rechten Zeit sich ein.

Symbolisch lässt sich alles schreiben,

Müsst nur im Allgemeinen bleiben.

Wenn man der Gleichung Lösung nicht erkannte,

Schreibt man sie als Determinante.

Schreib was du willst, nur rechne nie was aus.

Symbole lassen trefflich sich traktieren,

Mit einem Strich ist alles auszuführen.

Und mit Symbolen kommt man immer aus. ${ }^{1}$ )

Kurd Lasswitz.

Aus „Prost. Der Faust-Tragödie $(-\mathrm{n})$ ter Teil."

s. Zeitschr. math. naturw. Unterr. 14 (1883), p. 317.

1) nach Goethe, Faust I, "Schülerscene". 
Die Behandlung algebraischer Fragen übte von Anfang an einen besondern Reiz auf mich aus, und zu ihnen bin ich mit Vorliebe immer wieder zurückgekehrt, wenn ich nach anstrengenden analytischen Arbeiten einer Ruhepause bedurfte.

G. Frobenits.

Antrittsrede in d. Berl. Akademie.

s. Berl. Sitzungsber. 1893, p. 627.

Eine Wissenschaft kann niemals etwas anders sein, als die durchgeführte Entwickelung des ihr zu Grunde liegenden Begriffs. Dieser Begriff ist für die Algebra die Zahl. . . . Die Algebra' mag immerhin das ausführen, was der Zahl überhaupt und allgemein eigen ist, aber sie darf auch das nicht vergessen, was ausser der Grösse selbst, die Zahl characterisirt und weitere Unterscheidungen in derselben begründet. Unstreitig ist es daher auch nicht sehr wissenschaftlich, die Zahlenlehre, . . . . bei dem Studium der Mathematik, als einen isolirten und entbehrlichen Theil dieser Wissenschaft bei Seite zu setzen ..... Der Grund, aus welchem die häufig Statt findende Zurücksetzung der reinen Zahlenlehre im Unterrichte herrorgeht, liegt unstreitig weniger in dem Verkennen der oben geäusserten, gar nicht neuen Bemerkungen, als in der sehr vorherrschenden Richtung auf die angewandte Mathematik, für welche die Arithmetik unmittelbar keine Hülfsmittel darbietet. Aber die Mathematik nimmt eine selbstständige Würde in Anspruch und es muss der wesentliche Zweck des Unterrichts sein, das Bewusstsein dieser Würde zu erwecken, und ihre Anerkennung durch die Wissenschaft selbst zu begründen.

\section{Ferd. Minding.}

Voranzeige seiner „Höheren Arithmetik" (Berlin 1832) in J. f. Math. 7 (1831), p. 414.

Il est à croire . . qu'Euler avait un goût particulier pour ce genre de recherches [de la science des nombres], et qu'il 
s'y livrait avec une sorte de passion, comme il arrive à presque tous ceux qui s'en occupent.

\section{LEGENDRE.}

„Théorie des nombres", Préface.

In alio forte labore . occupatus, casu incidi in eximiam quandam veritatem arithmeticam (fuit autem in fallor theorema art. 108 [Omnium numerorum primorum formae $4 n+1,-1$ est residuum quadraticum, omnium vero numerorum primorum formae $4 \mathrm{n}+3$ non-residuum]), quam quum et per se pulcherrimam aestimarem et cum majoribus connexam esse suspicarer, summa qua potui contentione in id incubui, ut principia quibus inniteretur perspicerem, demonstrationemque rigorosam nanciscerer. Quod postquam tandem ex roto successisset, illecebris harum quaestionum ita fui implicatus, ut eas deserere non potuerim.

\section{F. Gauss.}

„Disquisitiones arithm.", Praefatio. s. Werke, Bd. 1 (1870), p. 6.

Nerkwürdig ist es immer dass alle diejenigen die diese Wissenschaft [die höhere Arithmetik] ernstlich studiren eine Art Leidenschaft dafür fassen.

$$
\begin{gathered}
\text { Gauss an W. Bolyai. } \\
\text { Göttingen, 2. IX. } 1808 .
\end{gathered}
$$

s. Briefw. Gauss-Bolyai, herausg. v. Schmidt u. Stäckel (1899), p. 94.

Göpel wurde, wie viele von denen, welche zur rein mathematischen Speculation berufen sind, zunächst von der höhern Zahlenlehre angezogen.

$$
\text { C. G. J. JACOBI. }
$$

\section{J. f. Math. 35 (1847), p. 313/314}

$=$ Ostwald's Klassiker der exakt. Wissensch., Nr. 67, p. 52

$=$ Jacobi, Werke, herausg. v. Weierstrass, Bd. 2 (1882), p. 148. 
Das Hauptinteresse der Mathematiker richtete sich auf die multiplicative Zusammensetzung der Zahlen. Und doch hätte unsere Wissenschaft [die Zahlentheorie] bei systematischem Vorgehen zuerst die Zerlegung der Zahlen in ihre Summanden erledigen müssen.

L. KroNecher.

Vorles. II, Abschn. 1 (Zahlentheorie), herausg. v. Hensel, Teil I (1901), p. 56.

Ich habe die Unart, ein lebhaftes Interesse bei mathematischen Gegenständen nur da zu nehmen, wo ich sinnreiche Ideenverbindungen und durch Eleganz oder Allgemeinheit sich empfehlende Resultate ahnen darf.

Gauss an Schumacher.

Götttingen, 17. IX. 1808.

s. Briefw. Gauss-Schumacher, Bd. 1 (1860), p. 2.

Ich gestehe, dass das Fermat'sche Theorem als isolirter Satz für mich wenig Interesse hat, denn es lassen sich eine Menge solcher Sätze leicht aufstellen, die man weder beweisen, noch widerlegen kann. Allein ich bin doch dadurch veranlasst, einige alte Ideen zu einer grossen Erweiterung der höheren Arithmetik wieder vorzunehmen. Freilich gehört diese Theorie zu den Dingen, wo man nicht voraussetzen kann, inwiefern es gelingen wird, dunkel vorschwebende entfernte Ziele zu erreichen. Ein glückliches Gestirn muss mit obwalten, und meine Lage und so vielfache abziehende Geschäfte erlauben mir freilich nicht, solchen Meditationen so nachzuhängen, wie in den glücklichen Jahren 1796-1798, wo ich die Hauptsachen meiner Disquisitiones Arithmeticae bildete. Allein ich bin überzeugt, wenn das Glück mehr thun sollte, als ich erwarten darf, und mir einige Hauptschritte in jener Theorie glücken, auch der Fermat'sche Satz nur als eines der am wenigsten interessanten Corollarien dabei erscheinen wird.

Gauss an Olbers.

Göttingen, 21. III. 1816.

s. "Wilhelm Olbers, Sein Leben und seine Werke", herausg. v. C. Schilling, Bd. 2 (1900), p. 629. 
Fermatius theorema suum inclytum non demonstravit.

\section{Kronecker.}

Doctorthese Berlin 1845.

s. Werke, Bd. 1, p. 73; vgl. hiermit a.

Kronecker, Vorles. üb. Zahlentheorie, I, p. $14 \mathrm{f}$.

u. Anm. des Herausgebers K. Hensel l. c., p. 497.

C'est en quelque sorte une tache pour la Géométrie moderne que l'on n'ait pu retrouver encore les démonstrations des théorèmes que Fermat nous a laissés, et qu'il nous assure avoir démontrés. . . . Le grand géomètre avait certainement une méthode toute particulière et peut-être fort simple qui l'a conduit aux différents théorèmes qu'il nous a laissés, et dont la démonstration ne nous paraît aussi difficile que parce que nous n'avons point encore retrouvé le fil de ses idées.

LAPLACE à Lagrange.

3. Il. 1778.

roir Oeurres de Lagrange, t. 14 (1892), p. 74.

Sie sagten einmal von Euklides, dass er Begriffe und Sätze der höheren Arithmetik besessen haben müsse, von denen sich in den Elementen keine directe Spur nachweisen lasse. - -

Bessel an Gauss.

Königsberg, 20. I. 18:20

s. Briefw. Gauss-Bessel (1880), p. 311.

Es erscheint geraten, die durch Euklid in die Zahlenlehre hineingetragene geometrische Tendenz allmählich abzustreifen.

L. KRONECKER.

Vorles. II, Abschn. 1 (Zahlentheorie), herausg. v. Hensel, Teil I (1901), p. 64.

Da man in der Wirklichkeit alle Brüche durch Einführung hinreichend kleiner Untereinheiten auf ganze Zahlen zurückführen könnte, so ist klar, was es mit dem nebelhaften Begriff einer Zahl, die das Stetige decke, und mit dem zugehörigen 
Unbegriff einer stetig interpolirten Zahlemreihe für eine Bewandtniss habe. Es ist hier einfach eine jener voreiligen Erdichtungen und Verdinglichungen im Spiele, zu welcher die Modernen, und zwar . nicht nach dem Vorgang der Alten, sondern im Gegensatz zu den strengeren antiken Vorstellungsarten auf Veranlassung oberflächlich aufgefasster Übereinstimmungen des Rechnungsschematismus gelangt sind.

Etgex Dühring.

„Kritische Gesch. der allgem. Principien der Mechanik," 2. umgearb. Aufl. (1877), p. 501.

Bei der geometrischen Anschauung gehen wir durch jede unendlich kleine Grösse, die in der gegebenen enthalten ist, und können eben deswegen diese nicht als Theile ansehn durch deren Zusammensezung das Ganze entstanden wäre; bei der Zusammensezung der Zahl hingegen gehen wir nicht durch alle unendlich kleine Theile woraus jede Einheit besteht, sonst würden wir uns die Zahl als Linie, als stetige Grösse denken, sondern wir nehmen die Einheit selbst, und durch diese bekommen wir natürliche Abschnitte, d. h. Theile.

Ich glaube, dass dies den wesentlichen Unterschied der Arithmetik von der Geometrie ausmacht. - _ -

\section{SChleiermacher}

als Erzieher im gräfl. Dohnaschen Hause an den damaligen Referendar, späteren preuss. Staatsminister Burggrafen Friedr. Ferd. Alex. zu Dohna.

Schlobitten, 16. XII. 1791.

s. H. Borkowski, ,Schleiermacher als Mathematiker", Arch. Math. Phys. (2) 16 (1898), p. 339/340.

L'algèbre n'est qu'une géométrie écrite, la géométrie n'est qu'une algèbre figurée.

\section{Sophie Germain.}

„Pensées diverses."

voir Oeurres philosophiques, édition de Stupuy (1896), p. 223. 
Die arithmetischen Zeichen sind geschriebene Figuren und die geometrischen Figuren sind gezeichnete Formeln.

D. Hilbert. „Mathematische Probleme“, Vortr. Mathem.-Congr.Paris 1900. s. Göttinger Nachr. 1900, Math.-phys. Kl., p. 259 $=$ Arch. Math. Phys. (3) 1 (1901), p. 50.

Ich fordere, dass die Arithmetik sich aus sich selbst heraus entwickeln soll... Sowie die negativen und gebrochenen rationalen Zahlen durch eine freie Schöpfung hergestellt, und wie die Gesetze der Rechnungen mit diesen Zahlen auf die Gesetze der Rechnungen mit ganzen positiven Zahlen zurückgeführt werden müssen und können, ebenso hat man dahin zu streben, dass auch die irrationalen Zahlen durch die rationalen Zahlen allein vollständig definirt werden.

R. Dedekind.

„Stetigkeit und irrationale Zahlen“ (1872), p. 17.

Die allgemeine Theorie der ganzen ganzzahligen Functionen gestattet alle der eigentlichen Arithmetik fremden Begriffe, den der negativen, der gebrochenen, der reellen und der imaginären algebraischen Zahlen, auszuscheiden.

\section{KroNeCKer. ${ }^{1}$ )}

„,̈ber den Zahlbegriff“, J. f. Math. 101 (1887), p. 345.

Ich glaube, dass es dereinst gelingen wird, den gesammten Inhalt aller mathematischen Disciplinen mit Ausnahme der Geometrie und Mechanik zu „arithmetisiren“, d. h. einzig und allein auf den im engsten Sinne genommenen Zahlbegriff zu gründen, also die Modificationen und Erweiterungen dieses

1) s. Note 2 am Ende des Buches. 
Begriffs * wieder abzustreifen, welche zumeist durch die Anwendungen auf die Geometrie und Mechanik veranlasst worden sind.

* Ich meine hier namentlich die Hinzunahme der irrationalen sowie der continuirlichen Größen.

\section{KRONECKER.}

„Über den Zahlbegriff“", Philos. Aufs., Eduard Zeller z. s. 50-jähr.

Doctor-Jubil. gewidmet (Berlin 1887), p. 265

= J. f. Math. 101 (1887), p. 338/339

$=$ Kronecker, Werke, herausg. v. Hensel, Bd. 3, 1 (1899), p. 253.

Ein rein formalistisch-literales Gerippe der Analysis, worauf die Trennung der Zahl nebst den analytischen Zeichen von der Grösse hinausliefe, würde diese Wissenschaft, welche in Wahrheit eine Naturwissenschaft ist, wenn sie auch nur die allgemeinsten Eigenschaften des Wahrgenommenen in den Bereich ihrer Forschungen zieht, schliesslich . . zum blossen Zeichenspiel hinabwürdigen, wo den Schriftzeichen willkürliche Bedeutungen beigelegt werden, wie den Schachfiguren und Spielkarten. - -

Ohne Frage wird man mit Hülfe von sogenannten Axiomen, von Conventionen, ad hoc erdachten Philosophemen, unfassbaren Erweiterungen ursprünglich deutlicher Begriffe nachträglich ein System der Arithmetik construieren können, welches dem aus dem Grössenbegriff hervorgegangenen in allen Punkten gleicht .... Allein man würde auf dieselbe Weise auch andere arithmetische Systeme sich ausdenken können, wie dies ja geschehen ist. Die gewöhnliche Arithmetik ist eben die einzige dem lineären ${ }^{1}$ ) Grössenbegriff entsprechende, ist gleichsam seine erste Registrirung, während die Analysis, mit dem Grenzbegriff an der Spitze, seine höchste Entwickelung bildet.

\section{Paul du Bois-Reymond.} "Allgemeine Functionentheorie" (1882), p. 53/54.

1) „lineär" bedeutet hier „wie Längen in der Richtung des Kileinsten und Grössten ausgedehnt und wie diese vergleichbar, messbar" (s. l. c. p. 23). 
Wenn ich die Strenge in den Beweisen als Erforderniss für eine vollkommene Lösung eines Problems hinstelle, so möchte ich andererseits zugleich die Meinung widerlegen, als seien etwa nur die Begriffe der Analysis oder gar nur diejenigen der Arithmetik der völlig strengen Behandlung fähig. Eine solche bisweilen von hervorragenden Seiten vertretene Meinung halte ich für durchaus irrig; eine so einseitige Auslegung der Forderung der Strenge führt bald zu einer Ignorirung aller aus der Geometrie, Mechanik und Physik stammenden Begriffe, zu einer Unterbindung des Zuflusses von neuem Material aus der Aussenwelt und schliesslich sogar in letzter Consequenz zu einer Verwerfung der Begriffe des Continuums und der Irrationalzahl. Welch' wichtiger Lebensnerv aber würde der Mathematik abgeschnitten durch eine Exstirpation der Geometrie und der mathematischen Physik? [!] Ich meine im Gegenteil, wo immer von erkenntnistheoretischer Seite oder in der Geometrie oder aus den Theorien der Naturwissenschaft mathematische Begriffe auftauchen, erwächst der Mathematik die Aufgabe, die diesen Begriffen zu Grunde liegenden Principien zu erforschen und dieselben durch ein einfaches und vollständiges System von Axiomen derart festzulegen, dass die Schärfe der neuen Begriffe und ihre Verwendbarkeit zur Deduktion den alten arithmetischen Begriffen in keiner Hinsicht nachsteht.

D. Hilbert.

"Mathematische Probleme", Vortr. Mathem.-Congr. Paris 1900.

s. Göttinger Nachr. 1900, Math.-phys. Kl., p. 258/259

$=$ Arch. Math. Phys. (3) 1 (1901), p. 49.

Die Arithmetik, die bei Diophant und Fermat den Character einer unterhaltenden Denkübung, eines geistreichen Spieles trug, war nach den Vorarbeiten von Euler, Lagrange und Legendre durch Gauss zu dem Range einer Wissenschaft erhoben worden. Die Königin der Mathematik nannte sie der Fürst der Mathematiker, und sie verdient diesen Titel nicht nur durch ihren hohen Rang, sondern auch durch die stolze 
Abgeschiedenheit, in der sie, fern von allen anderen Wissensgebieten, fern auch von den übrigen mathematischen Disciplinen thronte. Ihr machte das Genie von Gauss in seiner Lehre von der Kreistheilung die Algebra tributpflichtig, ihr legte Jacobi's siegreiche Kraft den unermesslichen Formelschatz der Theorie der elliptischen Functionen zu Füssen, in ihren Dienst zwang Dirichlet's Scharfsinn die feinsten Grenzmethoden der Analysis. Diese sich selbst genügende Wissenschaft zur ersten Dienerin der Algebra und Functionentheorie erhoben zu haben, ist Kronecker's unsterbliches Verdienst, dessen hohe Bedeutung erst jetzt allmählich anfängt, weiteren Kreisen zum Bewusstsein zu kommen, und dessen volle Würdigung nur von künftigen Geschlechtern zu erwarten ist.

\section{G. Frobenius.}

„Gedächnissrede auf L. Kronecker", Derl. Akad. 29. VI. 1893. s. Berliner Abh. 1893, p. 4.

Ähnlich wie bei den Beziehungen verschiedener Wissenschaften zu einander wird da, wo verschiedene Disciplinen einer Wissenschaft in einander greifen, die eine durch die andre gefördert und die Forschung in naturgemässe Bahnen geleitet.

L. KRoNeCKer.

Antrittsrede i. d. Berl. Akademie. s. Berl. Monatsber. 1861, p. 639.

Während ich sage, dass eine sog. irrationale Zahl eine so reelle Existenz habe wie irgend etwas anderes in der Gedankenwelt, ist es bei Kronecker jetzt ein Axiom, dass es nur Gleichungen zwischen ganzen Zahlen giebt. - - - -

Schlimmer ist es aber, wenn Kronecker seine Autorität dafür einsetzt, dass alle, die bis jetzt an der Begründung der Functionentheorie gearbeitet haben, Sünder vor dem Herrn sind. Wenn ein wunderlicher Kauz wie Christoffel sagt, in 20-30 Jahren wird die jetzige Functionentheorie zu Grabe ge- 
tragen und die ganze Analysis in die Theorie der Formen aufgegangen sein, so beantwortet man das mit einem Achselzucken. Wenn aber Kronecker den Ausspruch thut, den ich wörtlich wiederhole: „Wenn mir noch Jahre und Kräfte genug bleiben, werde ich selber der mathematischen Welt zeigen, dass nicht nur die Geometrie, sondern auch die Arithmetik der Analysis die Wege weisen kann, und sicher die strengeren. Kann ich es nicht mehr thun, so werden's die thun, die nach mir kommen.... und sie werden auch die Unrichtigkeit aller jener Schlüsse erkennen, mit denen jetzt die sogenannte Analysis arbeitet"; so ist ein solcher Ausspruch von einem Manne, dessen hohe Begabung für mathematische Forschung und eminente Leistungen von mir sicher ebenso aufrichtig und freudig bewundert werden wie von allen seinen Fachgenossen, nicht nur beschämend für diejenigen, denen zugemuthet wird, dass sie als Irrthum anerkennen und abschwören sollen, was den Inhalt ihres unablässigen Denkens und Strebens ausgemacht hat, sondern ist es auch ein directer Appell an die jüngere Generation, ihre bisherigen Führer zu verlassen und um ihn als Jünger einer neuen Lehre, die freilich erst begründet werden soll, sich zu scharen. Wirklich, es ist traurig und erfüllt mich mit bitterm Schmerz, dass das wohlberechtigte Selbstgefühl eine Mannes, dessen Ruhm unbestritten ist, ihn zu Äusserungen zu treiben vermag, bei denen er nicht einmal zu empfinden scheint, wie verletzend sie für andere sind. Aber genug von /diesen Dingen, die ich nur berührt habe, um Dir zu erklären, aus welchen Gründen ich an meiner Lehrthätigkeit .... künftighin nicht mehr dieselbe Freude haben kann wie bisher. Du wirst aber darüber nicht reden; ich möchte nicht, dass Andere, die mich nicht so genau kennen wie $\mathrm{Du}$, in dem Gesagten den Ausdruck einer Empfindlichkeit sähen, die mir in der That fremd ist. Niemand weiss besser als ich selbst, wie weit ich von dem Ziele entfernt geblieben bin, das ich in der Begeisterung der Jugend mir gesteckt hatte, niemand soll mir aber auch das Bewusstsein rauben, dass mein Streben und Wirken nicht ganz umsonst gewesen ist und der Weg, auf dem 
ich der Wahrheit nachgegangen bin, nicht als ein Irrweg sich erweisen wird.

Weierstrass an S. Kowalevski.

24. III. 1885.

s. Mittag-Leffler, „Une page de la rie de Weierstrass",

C. R. du deuxième congrès intern. des mathém. Paris 1900

(Paris 1902), p. 150-152.

Une certaine méfiance à l'égard de la géométrie est le caractère propre de l'Ecole de Berlin; pour ainsi dire elle ne cherche pas à roir, mais à comprendre.

Tout dérive done du nombre entier et participe par conséquent de la certitude de l'arithmétique; le continu lui-même se ramène à cette origine et toutes les égalités qui font l'objet de l'Analyse et où figurent des grandeurs continues ne sont plus que des șrmboles, remplaçant une multitude infinie d'inégalités entre nombres entiers.

Les notions analytiques sont donc pour Weierstrass. comme pour Kronecker, des constructions faites avec les mêmes matériaux, les nombres entiers. Mais il y a une différence entre les deux conceptions; Kronecker est surtout préoccupé de mettre en évidence le sens philosophique des vérités mathématiques; le nombre entier étant le fond de tout, il veut qu'il reste partout apparent; pour lui, les seules opérations licites sont l'addition et la multiplication; ce n'est que par une concession aux préjugés contemporains, qu'il consent quelquefois à admettre la division.

Tel n'est pas le point de vue de Weierstrass. Dès qu'il a élevé une construction, il oublie de quels matériaux elle est faite et n'y reut plus voir qu'une unité nouvelle dont il fera l'un des éléments d'une construction plus grandiose. Il peut le faire sans crainte, car il en a, une fois pour toutes, éprouvé la solidité.

Ces unités intermédiaires ne sont sans doute que des auxiliaires; mais notre esprit est si faible qu'il ne peut s'en passer; car il ne peut percevoir à la fois tous les détails d'un 
grand ensemble. Ces artifices sont donc nécessaires si l'on veut marcher toujours en avant et c'est là justement ce que veut Wei er strass. Kronecker, lui aussi, a fait bien des découvertes; mais s'il y est arrivé, c'est en oubliant qu'il était philosophe et en délaissant lui-même ses principes qui étaient condamnés d'avance à la stérilité.

H. Polncaré.

"L'oeurre mathématique de Weierstrass", Acta mathem. 22 (1899), p. 16/17.

Auf einem .. Spaziergange sagte Kronecker zum Verfasser dieser Zeilen: „Ich betrachte die Mathematik nur als eine Abstraction der arithmetischen Wirklichkeit." . . . . . Wie sehr ihm diese Anschauung Herzenssache war, und wie fest er auf ein Durchdringen seiner Auffassung des Zahlbegriffs vertraute, hat Verfasser oft Gelegenheit gehabt zu bemerken. So that er auch bei einem Besuche des Verfassers am 17. October 1890, als sich das Gespräch auf die Geometrie wandte, mit Bezug auf seine arithmetischen Grundanschauungen ganz siegesgewiss und triumphirend den Ausspruch: 'Mir gehört die Zukunft! Mir gehört die Zukunft!“

\author{
A. Gutzmer. \\ "Leopold Kronecker", \\ Naturwiss. Wochenschr. 8 (1893), p. 592.
}

Wenn ich nicht irre, geschieht die moderne Entwickelung der reinen Mathematik vornehmlich unter dem Zeichen der Zahl: Dedekind's und Weierstrass's Definitionen der arithmetischen Grundbegriffe und Cantor's allgemeine Zahlgebilde führen zu einer Arithmetisirung der Functionentheorie und dienen zur Durchführung des Princips, dass auch in der Functionentheorie eine Thatsache erst dann als bewiesen gilt, wenn sie in letzter Instanz auf Beziehungen für ganze rationale Zahlen zurückgeführt worden ist. Die Arithmeti- 
sirung der Geometrie vollzieht sich durch die modernen Untersuchungen über Nicht-Euklidische Geometrie, in denen es sich um einen streng logischen Aufbau derselben und um die möglichst directe und völlig einwandsfreie Einführung der Zahl in die Geometrie handelt.

D. Hilibert.

„Die Theorie der algebr. Zahlkörper", Vorwort, p. V in Deutsche Mathem.-Verein. Jahresber. 4, 1894/1895.

Je mehr ich über die Principien der Functionentheorie nachdenke - und ich thue dies unablässig - um so fester wird meine Überzeugung, dass diese auf dem Fundamente algebraischer Wahrheiten aufgebaut werden muss, und dass es deshalb nicht der richtige Weg ist, wenn umgekehrt zur Begründung einfacher und fundamentaler algebraischer Sätze das .,Transcendente“, um mich kurz auszudrücken, in Anspruch genommen wird - so bestechend auch auf den ersten Anblick z. B. die Betrachtungen sein mögen, durch welche Riemann so viele der wichtigsten Eigenschaften algebraischer Functionen entdeckt hat. (Dass dem Forscher, so lange er sucht, jeder Weg gestattet sein muss, versteht sich von selbst; es handelt sich nur um die systematische Begründung).

Weierstrass an H. A. Schwarz. 3. X. 1875 .

s. Werke, Bd. 2 (1895), p. 235.

Parmi les Sciences mathématiques, la première est la Science du Calcul, qui repose sur la seule notion de nombre et à laquelle on s'efforce de ramener toutes les autres.

APPELL.

„Mécanique rationnelle", 1 (1893), p. 1. 
Unter allen Disciplinen der Mathematik ist die Theorie der Differentialgleichungen die wichtigste. . . Sie giebt den Weg zur Erklärung aller elementaren Naturphänomene, die Zeit brauchen, ... . und hat auf der anderen Seite darin eine entsprechende theoretische Wichtigkeit, dass sie in rationeller Weise zum Studium neuer wichtiger Functionen, bez. Functionsclassen leitet.

Sophus Lik.

Leipziger Ber. 47 (1895), Math.-phys. Cl., p. 262; s. a. l. c. p. 53.

Die Mathematik hielt Gauss um seine eigenen Worte zu gebrauchen, für die Königin der Wissenschaften und die Arithmetik für die Königin der Mathematik. Diese lasse sich dann öfter herab der Astronomie und andern Naturwissenschaften einen Dienst zu erweisen, doch gebühre ihr unter allen Verhältnissen der erste Rang.

Sartorius v. Waltershausen. „Gauss zum Gedächtniss" (1856), p. 79.

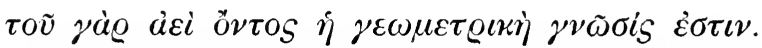
[denn die Geometrie ist die Erkenntnis des ewig Seienden].

Plato.

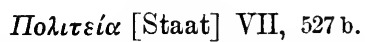

v. Opera II, Bibl. Didotiana 46 (Paris 1883), p. 132, 53-54.

Als eine vielleicht noch nicht allgemein geteilte persönliche Ansicht möchte ich . . aussprechen, dass mir die reine Mathematik bloss als ein Zweig der allgemeinen Logik erscheint: der Zweig, welcher auf die Schöpfung der Zahlen sich gründet, deren wirtschaftlichen Vorzügen die ungeheuere Entwickelung zu verdanken ist, die diesem besondern $\mathrm{Zweig}$ zuteil geworden im Vergleich mit den übrigen Zweigen der 
Logik, die bis in die jüngste Zeit fast stationär geblieben waren.

\author{
E. SChröder. \\ "Über Pasigraphie" etc. \\ s. Verhandl. d. 1. intern. Mathem.-Congr. Zürich 1897
}

(Leipzig 1898), p. 149.

Sehen wir von den Arbeiten der Gebrüder Grassmann in Stettin und des Professors Schroeder in Karlsruhe ....... ab, so bieten sämmtliche andere Darstellungen der Zahlenlehre in ihren grundlegenden Abschnitten bei ihren sogenannten Beweisen die bedenklichsten Zirkelschlüsse und Trugschlüsse, welche nichts beweisen und nur geeignet sind, die Leser an unwissenschaftliches Denken zu gewöhnen und sie zu verwirren.

Es mag diese Behauptung vielen Lesern übertrieben und anmasend erscheinen; aber sie ist durchaus wahr und darf im Interesse der Wissenschaft nicht verschwiegen werden. Fast alle Darstellungen der Zahlenlehre, auch die zuletzt erschienenen gründen (und dies ist ein Fehler, der zuerst gerügt werden muss, da er bereits die Möglichkeit strenger Wissenschaftlichkeit ausschliesst) ihre Beweise auf logische Schlüsse, obwohl ihre Schüler noch gar keine Logik studirt haben, und obwohl es bis in die neueste Zeit noch gar keine wissenschaftliche Darstellung der Logik gegeben hat.

R. Grassmann, [Bruder v. Hermann Grassmann d. Älteren]. „Das Gebäude des Wissens",

Bd. 23: „Die Formenlehre oder Mathematik“ (Stettin 1895), Erster Zweig: „Die Zahlenlehre oder Arithmetik“, Vorwort, p. IV.

Wir sehen in der Mathematik die bewusste logische Thätigkeit unseres Geistes in ihrer reinsten und rollendetsten Form; wir können hier die ganze Mühe derselben kennen lernen, die grosse Vorsicht, mit der sie vorschreiten muss, die Genauigkeit, welche nöthig ist, um den Umfang der gewonnenen 
allgemeinen Sätze genau zu bestimmen, die Schwierigkeit, abstracte Begriffe $\mathrm{zu}$ bilden und $\mathrm{zu}$ verstehen; aber ebenso auch Vertrauen fassen lernen in die Sicherheit, Tragweite und Fruchtbarkeit solcher Gedankenarbeit.

\section{HeLaiholtz.}

„Über das Verhältniss der Naturwissenschaften zur Gesammtheit der Wissenschaft," Prorectoratsrede Heidelberg 22. XI. 1862.

s. Vorträge und Reden, 4. Aufl., Bd. 1 (1896), p. 176.

Die Mathematik wird ja seit alten Zeiten als die unentbehrlichste Schule des philosophischen Denkens angesehen und die Forschung des Mathematikers in ihren höchsten Sphären ist ja der reinen Speculation am nächsten verwandt; sie ist die vollendetste Vereinigung zwischen exaktem Wissen und theoretischem Denken.

\section{E. Curtius.}

Festrede i. d. Berl. Akademie 3. VII. 1873.

s. Berl. Monatsber. 1873, p. 517.

Die Mathematik hat sich thatsächlich als eins der stärksten, wenn nicht als das stärkste Gährungsmittel im Denken vor Allem des siebenzehnten und achtzehnten Jahrhunderts erwiesen.

Karl LaMprecht. Zukunft, 12. IV. 1902, p. 62.

Uns fehlt eine Selbstkritik der Wissenschaft; Urtheile der Kunst, der Religion, des Gefühls über die Wissenschaft sind so zahlreich wie unnütz. Vielleicht ist dies die letzte Bestimmung der Mathematik!

Paul Movgré [Felix Hausdorfa] ${ }^{1}$ ). „Sant' Ilario, Gedanken aus der Landschaft Zarathustras" (1897), p. 342.

1) s. Kürschner's deutschen Litteratur-Kalender 1903. 
In the „Fortnightly Review" [1869] we are told that „Mathematics is that study which knows nothing of observation, nothing of experiment, nothing of induction, nothing of causation".1) I think no statement could have been made more opposite to the undoubted facts of the case, that mathematical analysis is constantly invoking the aid of new principles, new ideas, and new methods, not capable of being defined by any form of words, but springing direct from the inherent powers and activity of the human mind, and from continually renewed introspection of that inner world of thought of which the phenomena ar as varied and require as close attention to discern as those of the outer physical world (to which the inner one in each individual man may, I think, be conceived to stand in somewhat the same general relation of correspondence as a shadow to the object from which it is projected, or as the hollow palm of one hand to the closed fist wich it grasps of the other), that it is unceasingly calling forth the faculties of observation and comparison, that one of its principal weapons is induction, that it has frequent recourse to experimental trial and verification, and that it affords a boundless scope for the exercise of the highest efforts of imagination and invention.

\section{Sylvester.}

Address Meeting of the British Association Exeter 1869. cf. Report, Notices and Abstracts, p. 4.

... in the ... paper, there is a statement concerning the method of the mathematical sciences, which .... brought upon me, during the meeting of the British Association at Exeter, the artillery of our eminent friend Professor Sylvester.

No one knows better than you do, how readily I should defer to the opinion of so great a mathematician if the question at issue were really, as he seems to think it is, a

1) s. Huxley, „Lay sermons, addresses and reviews" (London 1870), p. 185 . 
mathematical one. But I submit, that the dictum of a mathematical athlete upon a difficult problem which mathematics offers to philosophy, has no more special weight, than the verdict of that great pedestrian Captain Barclay would have had, in settling a disputed point in the physiology of locomotion.

\section{Thomas Henry Huxley.}

"Lay sermons, addresses and reviews"

(London 1870), Prefatory letter to Tyndall, p. VI.

Auch die mathematische Untersuchung, welche mehr, als man zu glauben pflegt, inductiv rerfährt, besitzt, was dem metaphysischen Denken fehlt, das sichere Mittel zu entscheiden, ob sie richtig vermuthete oder nicht. Aber der Mathematiker schöpft die Entscheidung aus sich selber, und darum ist seine Beschäftigung minder als der Versuch geeignet, das Vertrauen auf die Speculation zu erschüttern. Daher konnte die Menschheit zwei Jahrtausende Mathematik treiben, ohne dass dies ihren speculativen Hang zügelte; und daher waren zwei der grössten Mathematiker des siebzehnten Jahrhunderts, Descartes und Leibniz, auch noch dessen kühnste Methaphysiker.

Enil dU BoIs-Reymond.

„Darwin versus Galiani“,

Festrede Berl. Akademie 6. VII. 1876.

s. Reden, Bd. 1 (1886), p. 234.

Auch die Mathematik ist eine Sprache, und zwar nach Bau und Inhalt die vollkommenste Sprache, die es giebt, höher als jede Volkssprache; ja, weil alle Völker sie verstehen, kann sie die Sprache der Sprachen heissen. In ihr spricht sozusagen die Natur selbst, in ihr hat der Schöpfer der Welt geredet und in ihr "redet noch immer ihr Erhalter.

C. Dillimanx.

„Die Mathematik die Fackelträgerin einer neuen Zeit" (Stuttgart 1889), p. 5. 
Les mathématiques ont été de tout temps les adversaires implacables des romans scientifiques.

Arago.

Oeurres, t. 3 (1855), p. $498=$ Werke, Bd. 3 (1855), p. 401.

Tom Pythagoräischen Lehrsatz.

Die Wahrheit, sie besteht in Ewigkeit, Wenn erst die blöde Welt ihr Licht erkannt:

Der Lehrsatz, nach Pythagoras benannt, Gilt heute, wie er galt zu seiner Zeit.

Ein Opfer hat Pythagoras geweiht Den Göttern, die den Lichtstrahl ihm gesandt; Es thaten kund, geschlachtet und verbrannt, Ein Hundert Ochsen seine Dankbarkeit.

Die Ochsen seit dem Tage, wenn sie wittern, Dass eine neue Wahrheit sich enthülle, Erheben ein unmenschliches Gebrülle;

Pythagoras erfüllt sie mit Entsetzen; Und machtlos, sich dem Licht zu widersetzen, Verschliessen sie die Augen und erzittern.

Adelbert r. Chamisso 1835.

s. "Gedichte", z. B. die Ausg. Deutsches Verlagshaus Bong \& Co., p. 412.

Tönt es nicht heute lauter denn je, das Gebrülle aller Dunkelmänner, aller Feinde der freien Meinungsäusserung und Forschung wider den neuen pythagoräischen Lehrsatz, die Lehre Darwins?

L. Boltzmann. "Gustar Robert Kirchhoff", Akad. Festrede Graz 15. XI. 1887 (Leipzig 1888), p. 32. 
Mathesis et ars et scientia dicenda.

L. KRONECKER.

Doctorthese Berlin 1845.

s. Werke, Bd. 1, p. 73.

May not Music be described as the Mathematic of sense, Mathematic as Music of the reason? $\left.{ }^{1}\right)$ the soul of each the same! Thus the musician feels Mathematic, the mathematician thinks Music, - Music the dream, Mathematic the working life - each to receive its consummation from the other when the human intelligence, elevated to its perfect type, shall shine forth glorified in some future Mozart-Dirichlet or Beethoiren-Gauss - a union already not indistinctly foreshadowed in the genius and labours of a Helmholtz!

\section{J. J. Sylvester.}

(,Trilogy"), Philos. Transactions, vol. 154, part III (1864), p. 613; cf. also British Assoc.

Report 1869, Notices and Abstracts, p. 7.

Quoique vous soyez celui de toute l'Europe que je tiens pour le plus grand géomètre, ce ne seroit pas cette qualité-là qui m'auroit attiré ${ }^{2}$ ), mais que je me figure tant d'esprit et d'honnêteté en votre conversation que c'est pour cela que je vous rechercherois.

... pour vous parler franchement de la Géométrie, je la trouve le plus haut exercice de l'esprit: mais en même temps je la connois pour si inutile que je fais peu de différence entre un homme qui n'est que géomètre et un habile artisan. Aussi je l'appelle le plus beau métier du monde, mais enfin ce n'est qu'un métier, et j'ai dit sourent 'qu'elle est bonne pour faire l'essai, mais non pas l'emploi de notre force.

1) Mit genau demselben Ausspruch leitete J. Petzval (1807-1891) regelmäBig seine akustischen Vorlesungen an der Wiener Univers. ein; s. L. Gegenbauer, „Ein vergessener Österreicher","Deutsche Mathem.-Verein. Jahresber. 12 (1903), p. 331.

2) $\mathrm{Zu}$ einer Reise nach Toulouse, dem Wohnorte Fermat's. 
De sorte que je ne ferois pas deux pas pour la Géométrie et je m'assure que rous êtes fort de mon humeur. . . . Je m'y étais mis, il y a un an ou deux, par une raison tout it fait singulière, à laquelle ayant satisfait, je suis au hasard de ne jamais plus 5 penser.

\author{
PASCAL à Fermat. \\ De Bienassis, 10. VIII. 1660. \\ voir Fermat, Oeurres, t. 2 (1894), p. 451 \\ $=$ Blaise Pascal, Oeurres, t. 4 (1779), p. 446/447.
}

Wenn der Giessener Physiker Heinrich Buff die Mathematik lobte, so sagte Liebig: „Nun, sie ist ein Federmesser“. Hierbei lag die Abneigung zu Grunde, die alle morphologischen Denker gegen das rein Formale hegen, und es sollte zugleich angedeutet.werden, dass hilfreiche Rechner ebenso leicht zu finden seien wie Leute, die einem Schriftsteller die Feder schneiden. G. F. KNapp.

s. Münchener Allgem. Zeitg. Beilage 11. III. 1903, p. 450.

La géométrie est la clef de toutes les portes, et je vais travailler à l'acquérir.

Marquise du Châtelet à Frédéric le Grand. Cirey, 27. II. 1739.

voir Oeuvres de Frédéric le Grand, t. 17 (Édition Decker 1851), p. 22.

On a dit que l'analyse mathématique était un instrument. La comparaison peut être admise, pourvu qu'on accorde en même temps que cet instrument, comme le Protée de la Fable, doit sans cesse changer de forme.

Arago.

Oeurres, t. 2 (1854), p. $694=$ Werke, Bd. 2 (1854), p. 573. 
Ein Onkel von mir behauptet, dass man nur irgend jemand aus dem Tollhause oder Irrenhause zu nehmen brauchte, das würde gewiss der beste Mathematiker sein; ich sage dazu und so meint auch Dirichlet, man könne allenfalls behaupten, die Mathematik sei überhaupt eine Verirrung, deshalb braucht aber nicht jede Tollheit mathematischer Natur zu sein. Besagter Onkel, der, wie alle meine Verwandten (ich Glücklicher in ihrer Mitte?) die Menschen nur nach ihrem Werte (des Geldes) schätzt, meinte auch, als ich ihm mit vieler Mühe durch Beispiele die Zerlegbarkeit der Primzahlen $4 \mathrm{n}+1$ in zwei Quadrate und die Nichtzerlegbarkeit derer $4 \mathrm{n}+3$ darthat, „allerdings sei es merkwürdig, aber es gehöre der hyperimaginärste Grad von Verrücktheit dazu, auf so etwas zu verfallen, was ist das schon für Unsinn 'ne Primzahl, nu machen se da noch 'nen Unterschied zwischen $4 \mathrm{n}+1$ und $4 \mathrm{n}+3$, was kommt mer davon heraus;" die Goldmachekunst ist freilich weit besser. Dieser ist noch der geistreichste von meinen Verwandten, nun urteilen Sie über die andern und welche Rolle ich unter ihnen spielen muss.

Eisenstein an M. A. Stern.

Berlin, 10. II. 1848.

s. Abh. zur Gesch. d. Math. 7 (1895), p. 189/190.

. . . la géométrie est une espèce de hochet que la nature nous a jeté pour nous consoler et nous amuser dans les ténèbres.

D'Alembert à Frédéric le Grand.

Paris, 17. IX. 1764.

voir Oeurres de Frédéric le Grand, t. 24 (Édition Decker 1854), p. 385.

D'Alembert, vers la fin de sa vie, songeant à ses premiers travaux, écrivait avec émotion: „Les mathématiques ont été pour moi une maîtresse!"

Joseph Bertrand.

„D'Alembert" (Paris 1889), p. 32. 
Die Mathematik ist doch die angenehmste Wissenschaft; sie und die Astronomie vertreten bei mir Tanzgesellschaften, Konzerte und andere derartige Belustigungen, die ich nur dem Namen nach kenne.

Bessel 1802 als Kaufmannslehrling.

s. „Bessel als Bremer Handlungslehrling“, herausg. v. d. Gesellsch. [Kaufm. Terein] „Union“" (1890), p. 32.

Que mes occupations arithmétiques me rendent heureux dans un temps où je ne rois autour de moi que le malheur et le désespoir! Ce ne sont que les sciences, le sein de sa famille et la correspondance avec ses amis chéris, où l'on puisse se dédommager et se reposer de l'affliction générale. Gauss à Sophie Germain.

Göttingue, 19. I. 1808.

voir Sophie Germain, Oeurres philosophiques, édition de Stupuy (1896), p. 284.

Ich bin zu sehr herabgestimmt und habe nicht genug Lebensfreude, um etwas Litterarisches zu schreiben. Alles im Leben erscheint mir so verblasst und uninteressant. In solchen Augenblicken taugt die Mathematik besser; man freut sich, dass eine Welt so ganz ausserhalb unser selbst existiert. Man ist geneigt, von dem unpersönlichen Stoff zu reden.

S. Kovalevsky 1887.

s. A. Charl. Leffler, „Sonja Koralersky“, Deutsche Ausg., Reclam-Bibl. No. 3297/3298, p. 110.

Die Arbeit an und für sich, das abstrakte Suchen nach wissenschaftlichen Wahrheiten befriedigte Sonja Kovalevsky nicht, sie wollte verstanden werden, wollte, dass man ihr auf halbem Wege entgegenkomme, sie bewundere und ermuntere 
um jedes Fortschrittes willen, den sie machte, jedes neuen Gedankens wegen, der in ihr entstand. Sie wollte ihres Geistes Kind jemand schenken, jemand damit bereichern, nicht bloss die Nenschheit im abstrakten Sinne, sondern ein bestimmtes Individuum, das ihr dafür das seinige geben sollte.

Trotzdem sie Mathematikerin war, schwebten ihr nicht bloss abstrakte Ziele vor, denn sie war leidenschaftlich persönlich in ihrem ganzen Denken und Urteilen. Mittag-Lefffler pflegte oft zu äussern, dass dieses Bedürfnis nach Verständnis eine weibliche Schwachheit bei ihr sei. Wirklich geniale Männer seien nicht auf diese Weise von anderen abhängig. Sie aber behauptete das Gegenteil und führte eine Menge Beispiele ron Männern an, denen die Liebe zu einem Weibe ihre besten Ideen eingegeben habe. Die meisten dieser waren indes Dichter; unter den Gelehrten war es schwieriger, Belege für diesen Satz zu finden. . . . . . Gewiss ist, dass es ihr gelang, eine Menge Beispiele vorzuführen, wie gerade das Gefühl der Einsamkeit das grösste Leid fast aller tiefer angelegten Naturen sei, dass der Fluch auf dem Menschen ruhe, sich immer als das höchste Glück zu träumen, gganz und gar in einem andern Wesen aufgehen zu können und doch immer im Innersten einsam bleiben zu müssen.

A. Charl. Leffler.

„Sonja Kovalevsky“,

Deutsche Ausg., Reclam-Bibl. No. 3297/3298, p. 84/85.

Manche Menschen sind sich selbst genug, ich bin nicht so glücklich, sondern mich foltert die fortwährende Sehnsucht nach Liebe und Zuneigung der Menschen und nach gemütlicheren Verhältnissen, mathematische Beschäftigung ist für mich eine Art Betäubung, um mich vor Melancholie zu retten, so wie für andere Menschen Wein oder Branntwein; daher ist meine Stimmung am düstersten gerade nach Absolvierung eines schwierigen mathematischen Problems, da ich dann recht 


\section{$-78-$}

einsehe, wie sich hierdurch doch garnichts in meiner Lage ändert.

Eisexstein an M. A. Stern.

Berlin, 10. II. 1848.

s. Abh. zur Gesch. d. Math. 7 (1895), p. 190/191.

Plato in Phaedro fingit duplices animas, quarum alteras ait alatas esse, alteris, inquit, decidisse alas. Porro alatas ait volitare in coelum, fruentes congressu colloquioque Dei, et pulcherrimo spectaculo cursuum coelestium, et considerare causas omnium mutationum in inferiore natura, in aëre, in animantium corporibus, in hominum studiis et moribus, in variis imperiorum et civitatum casibus. Atque hae animae toto volitantes coelo captae pulchritudine divinarum rerum, et illius admirandi ordinis et suavitate doctrinae et virtutis, hac una voluptate perpetuo frui cupiunt, nec onerant animos obscoenis voluptatibus, quae perturbant harmoniam virtutis in animis, et obiiciunt caliginem, ne coelestia aspicere possint. At illae animae, quibus alae deciderunt, humi vagantur, et quaerunt voluptates impuras ex rebus terrenis, nec enim illam pulcherrimam lucem rerum coelestium aspiciunt. Etsi autem Plato alas intelligit heroicos impetus ingeniorum, tamen ne illi quidem impetus soli animos subvehunt, sed opus est etiam artibus, quibus illi ipsi impetus attollantur. Sunt igitur alae mentis humanae, Arithmetica et Geometria. Has si alligarerit sibi aliquis praeditus ingenio non sordido, facilime penetrabit in coelum ac libere in coelestium coetu vagabitur, et illa luce ac sapientia fruetur.

Quare illi qui sunt ingeniis non monstrosis praediti, et res optimas maximeque admirandas, et cognitione dignas aspicere cupiunt, has alas sibi addant, videlicet, Arithmeticen et Geometriam.

\section{Philipp Melanchthon 1536.}

„In arithmeticen praefatio Georgii Joachimi Rhetici". v. Corpus Reformatorum, ed. Bretschneider, vol. 11 (Halle 1843), p. 288. 
Un ancien disait que l'Arithmétique et la Géométrie étaient les ailes des Mathématiques; je crois, en effet, qu'on peut dire sans méthaphore que ces deux sciences sont le fondement et l'essence de toutes les sciences qui traitent des grandeurs. Mais non-seulement elles en sont le fondement, elles en sont, pour ainsi dire, encore le complément; car, lorsque l'on a trouvé un résultat, pour pouvoir faire usage de ce résultat, il est nécessaire de le traduire en nombres ou en lignes; pour le traduire en nombres, on a besoin du secours de l'Arithmétique; pour le traduire en lignes, on a besoin du secours de la Géométrie.

\title{
LAGRANGE.
}

„Leçons élémentaires sur les Mathématiques données à l'École Normale en 1795", Leçon seconde. voir Oeurres de Lagrange, t. 7 (1877), p. 198.

Die Mathmatiker sind eine Art Franzosen: redet man zu ihnen, so übersetzen sie es in ihre Sprache, und dann ist es alsobald ganz etwas Anderes.

Goethe.

„Ferneres über Mathematik und Mathematiker." s. Werke, Grosse Weimarische Ausg., Abth. II, Bd. 11 (1893), p. 102.

Purus mathematicus, purus asinus.

\author{
Sprichwort \\ unbekannten Ursprungs.
}

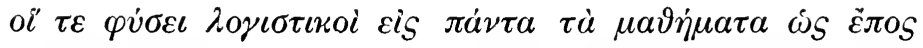

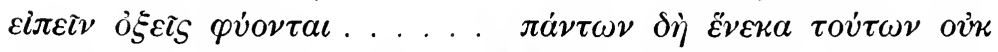

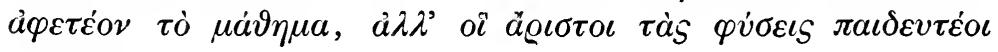
$\dot{\varepsilon} \nu$ av่ $\tau \tilde{\varphi}$.

[Die von Natur zum Rechnen Geschickten entwickeln so ziemlich für alle Wissenschaften Scharfsinn. . . . . daher darf 
diese Wissenschaft nicht vernachlässigt werden, sondern die von Natur Edelsten müssen in ihr unterrichtet werden.]

Plato.

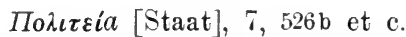

r. Opera II, Bibl. Didotiana 46 (Paris 1883), p. 132, 12-21.

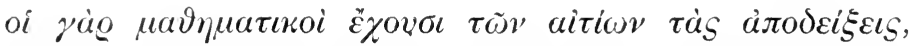

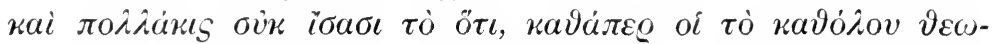

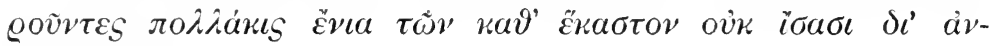

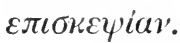

[Die Mathematiker besitzen die Darlegungen der Gründe und wissen oft nicht das Was, gleichwie sie oft, das Ganze betrachtend, einiges von den Einzelheiten nicht wissen, da sie es nicht berücksichtigt haben.]

\section{Aristoteles.}

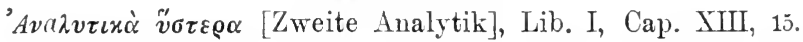
r. Bibl. Didotiana 9, Aristotelis operum rol. I, p. 135, 7-9.

Les géomètres qui ne sont que géomètres ont ... l'esprit droit, mais pourvu qu'on explique bien toutes choses par définitions et principes, autrement ils sont faux et insupportables, car ils ne sont droits que sur les principes bien éclaircis.

\section{PASCAL.}

„Pensées", édition complète de J.-F. Astié (Paris 1883), p. 324.

Blaise Pascal, Newton et d'Alembert, tous trois les plus grands géomètres de l'Europe, ont dit force sottises, l'un dans ses apophthegmes moraux, l'autre dans son Commentaire sur l'Apocalypse, et celui-ci sur la poésie et l'histoire. ${ }^{1}$ ) La géométrie pourrait done bien ne pas rendre l'esprit aussi juste qu'on le lui attribue. Le préjugé favorable à la géométrie en avait fait un axiome; ce n'est pas même un problème après

1) s. Note 3 am Ende des Buches. 
les trois grands géomètres que je viens de citer, et qui ont tous trois si pitoyablement raisonné.

Frédéric le Grand au Marquis d'Argens.

Bettlern, 25. V. 1762.

voir Oeurres de Frédéric le Grand, t. 19 (Édition

Decker 1852), p. 321/322.

Im ïbrigen gefället mir sein dessein [Herausgabe des „Tentamen novae theoriae Musicae ...“(1739) $]^{1}$ ) ganz wohl, weilen aufs wenigste die Theoria musices dadurch perfectionniret und gewiesen wird, dass ein Mathematicus schier alle Wissenschaften auszuführen im Stande ist, da hingegen andere Meister, die nur Practici sind, von ihrer eignen Kunst nicht anderst schreiben als wie ein blinder von der Farb.

JoII. Bernoulli an L. Euler.

11. VIII. 1731.

s. „Correspondance mathém. et phys. de quelques célèbres géomètres du 18ème siècle", t. 2 (1843), p. 10

$=$ J. f. Math. 23 (1842), p. 200.

Die ökonomische Commission [der Berliner Akademie] war der Meinung, eine der nothwendigsten und dringendsten Ausgaben wäre die Vollendung des Baues der Mauer um den botanischen Garten, so wie die Anschaffung einiger Thiere für den Gärtner zur besseren und leichteren Besorgung der Arbeiten; aber Euler widersetzte sich hier mit allen Kräften, und als man ihn um die Gründe seines Verfahrens fragte, erwiederte er ganz unverholen: „es gäbe nichts Unwichtigeres als einen botanischen Garten; die ganze Botanik sei nichts als eine Spielerei und überhaupt nur eine Wissenschaft in der Welt, die Mathematik nämlich."

\section{ThiÉBault.}

„Friedrich der Grosse" [Deutsche Ausg. der "Souvenirs de vingt ans de séjour à Berlin"], Th. 2 (Leipzig 1828), p. 165/166;

s. a. Franz. Ausg., t. 5 (Paris 1804), p. 14.

1) s. Note 4 am Ende des Buches.

A hrens, Scherz und Ernst i. d. Mathem. 
Daran erkenn' ich den gelehrten Herrn!

Was ihr nicht tastet, steht euch meilenfern;

Was ihr nicht fasst, das fehlt euch ganz und gar;

Was ihr nicht rechnet, glaubt ihr, sei nicht wahr;

Was ihr nicht wägt, hat für euch kein Gewicht;

Was ihr nicht münzt, das, meint ihr, gelte nicht.

Mephistopheles.

Goethe, „Faust" II, Akt 1 (Kaiserliche Pfalz).

Tu me crois obsédé par un maurais génie Alcippe, tu te plains de l'étrange manie Qui fait qu'en ma maison devenu prisonnier D'un flot d'X et d'Y je couvre mon papier. Laisse là, me dis-tu, l'algèbre et les formules, Laisse là ton compas, laisse là tes modules C'est un emploi bien triste et des nuits et des jours, Que d'intégrer sans fin et de chiffrer toujours, Apprendrons-nous enfin à quoi servent tes veilles, Ce qu'elles produiront d'étonnantes merveilles, Ft si de tes calculs le magique pouroir, Doit calmer au matin les tristesses du soir? Tu pourrais sembler digne et d'honneur et d'estime, Chacun te saurait gré du zèle qui t'anime, Si sur le prix de l'or tu daignais réfléchir Et faisais faire un pas à l'art de s'enrichir.

\section{Cauchy.}

D'une „Épître d'un géomètre à un jeune poète", lue dans une séance publique des cinq académies. voir J. Bertrand, „Éloges académiques", Nouvelle série (Paris 1902), p. 119.

Ein guter Mathematiker ist ein guter Schachspieler, also dieser jener - ein guter Mathematiker weiss die Differenzialrechnung zehnmal besser als ein elender - und ein guter Differenzialrechenmeister versteht sich so gut als einer aufs 
Deployieren und Schwenken ${ }^{1}$ ) und kann mithin seine Kompagnie (und seine Frau vollends) zu jeder Stunde kommandieren und warum sollte man einem so geschickten, so erfahrnen Offizier seine einzige Tochter nicht geben?

Obristforstmeister v. KNör bei

Jean Paul, „Die unsichtbare Loge", Erster Sektor.

s. Jean Paul, Werke (Ausg. Reimer Berlin), Bd. 1 (1840), p. 4.

Avec toute l'algèbre du monde, on n'est souvent qu'un sot, lorsqu'on ne sait pas autre chose.

Frédéric le Grand à Voltaire.

16. V. 1749.

voir Oeuvres de Frédéric le Grand, t. 22 (Édition Decker 1853), p. 199.

Chose étrange! il paraît que le désir de se faire mieux apprécier par le Roi de Prusse ${ }^{2}$ ) et par la haute société de Berlin qui, à l'exemple du Roi, affectionnait beaucoup les sciences, il parait, dis-je, que ce désir avait engagé Euler à ne livrer à l'Académie de cette ville que des travaux d'application de préférence, et à ne se servir que de la langue française. Or, l'analyse était, comme on le sait, l'admirable instrument qu'il maniait avec le plus d'habileté et de prédilection. Durant cette période, tous ses travaux de pure théorie, rédigés en latin, trouvaient donc un débouché à l'Académie de St.-Pétersbourg qui les accueillait avec empressement.

P. H. Fuss.

„Notice sur la vie et les écrits de Léonard Euler" dans „Correspondance mathém. et phys. de quelques célèbres géomètres du 18 ème siècle“, t. 1 (1843), p. XL. ${ }^{3}$ )

messieurs les géomètres, qui carrent éternellement des courbes inutiles; je les laisse avec leurs points sans étendue

1) Hier Anmerkung Jean Paul's mit Hinweis auf ein Werk, in dem die Differentialrechnung zum $Z_{w e c k}$ der Verwendung bei den oben. genannten militärischen Operationen gelehrt wurde.

2) s. S. 85 .

3) Vgl. a. Euler's Brief an Goldbach, d. d. 4. VII. 1744, ibidem p. 292. 
et leurs lignes sans profondeur, ainsi que messieurs les médecins, qui s'érigent en arbitres de notre vie, et qui ne sont que les témoins de nos maux.

Frédéric le Grand à Voltaire. Potsdam, 17. XII. 1777.

Parmi les géomètres, il n’y a guère eu qu'Archimède et Newton qui aient acquis une véritable gloire, parce qu'ils ont inventé des choses très-difficiles, très-inconnues, et très-utiles; il n'y a point de gloire pour ceux qui ne savent que diviser $a-b$ plus $c$ par $x$ moins $z$, et qui passent leur vie à écrire ce que les autres ont imaginé.

Voltaire à Frédéric le Grand. Ferney, 6. I. 1778.

Copernic, ne vous en déplaise, tiendra aussi son petit coin dans ma bibliothèque, en qualité de Prussien; il pourra trouver place entre Archimède et Newton. Quant à votre Newton, je vous confesse que je n'entends rien à son vide, ni à son attraction; il a démontré avec plus d'exactitude que ses devanciers le mouvement des corps célestes, j’en conviens; mais vous m'avouerez pourtant que c'est une absurdité en forme que de soutenir l'existence du rien. Ne sortons pas des bornes que nous donne le peu de connaissance que nous avons de la matière. A mon sens, la doctrine du vide, et des esprits qui existent sans organes, sont le comble de l'égarement de l'esprit humain. Si un pauvre ignorant de ma classe s'avisait de dire: Entre ce globe et celui de Saturne, ce qui n'a point d'existence existe, on lui rirait au nez; mais le sieur Isaac, ${ }^{1}$ ) qui dit la même chose, a hérissé le tout d'un fatras de calculs que peu de géomètres ont suivi; ils aiment mieux l'en croire sur sa parole, et admettre des contre-vérités, que de se perdre avec lui dans le labyrinthe du calcul intégral et du calcul infinitésimal. Les Anglais ont construit des vaisseaux sur la coupe la plus avan-

1) Newton, der 1705 in den Ritterstand erhoben war, hatte damit bekanntlich Anspruch auf die Anrede mit, ,Sir" und nachfolgendem Vornamen mit oder ohne Familiennamen. 
tageuse que Newton avait indiquée; et leurs amiraux m'ont assuré que ces vaisseaux étaient beaucoup moins bons voiliers que ceux qui sont fabriqués selon les règles de l'expérience. Je voulus faire un jet d'eau dans mon jardin; Euler calcula l'effort des roues pour faire monter l'eau dans un bassin, d'où elle devait retomber par des canaux, afin de jaillir à Sans-Souci. Mon moulin a été exécuté géométriquement, et il n'a pu élever une goutte d'eau à cinquante pas du bassin. Vanité des vanités! vanité de la géométrie!

Frédéric le Grand à Voltaire. 25. I. 1778.

voir Oeuvres de Frédéric le Grand, t. 23 (Édition Decker 1853), p. 417, 419 et 421.

Es ist ganz unglaublich von was für kindischen Besorgnissen und Vorurtheilen, dieser in seinem Fache so grosse Mann [Euler], eingenommen war.

\author{
„J. G. SulzER's
}

Lebensbeschreibung von ihm selbst aufgesetzt"

(Berlin \& Stettin 1809), p. 49.

Votre lettre du 19 de ce mois m'a fait connaitre vos idées sur le fonds prétendu d'une académie des sciences. Mais je crois que, étant accoutumé aux abstractions des grandeurs de l'algèbre vous avez péché contre les règles ordinaires du calcul.

Charlottenbourg, 21. I. 1743.

Moi, qui ne sais point calculer des courbes, je sais pourtant que seize mille écus de recette en valent mieux que treize mille. ${ }^{1}$ ) Potsdam, 16. VI. 1765.

Frédéric le Grand à Euler. voir Oeuvres de Frédéric le Grand, t. 20 (Édition Decker 1852), p. 200 et 209.

1) Es handelt sich hier um Differenzen, die in der Akademie bezüglich der Administration des Kalenderwesens entstanden waren; ent- 
Cette Lettre ${ }^{1}$ ) mortifia tellement M. Euler, qu'il ne roulut plus rester à Berlin.

Le roi posa en question s'il n'avoit pas le droit de le retenir de force; et sur ce qu'on lui objecta que M. Euler étoit Suisse, et que les Suisses avoient des titres qui garantissoient leur liberté, et qui étoient respectés par tous les souverains de l'Europe: „Eh bien, répliqua-t-il, quelques-uns de ses enfans sont nés mes sujets, et je puis au moins les retenir." On ne parvint pas sams peine à le forcer dans ce dernier retranchement; et l'on peut croire que la crainte d'offenser l'impératrice de Russie, ${ }^{2}$ ) contribua peut-être autant à le fléchir que les principes de justice. Mais au moins l'un des fils d'Euler, officier dans l'artillerie, et qui aroit été mis en prison, pour avoir soupé en société avec un habit bourgeois avant d'avoir son congé, fut retenu pendant près d'un an après le départ de ses parens, et peut-être n'auroit-il jamais eu son congé, si Catherine seconde ne l'aroit sollicité elle-même. ${ }^{3}$ )

\section{Thiébault}

„Mes souvenirs de vingt ans de séjour à Berlin," t. 5 (Paris 1804), p. 10/11.

Ayant reçu votre lettre du $\mathbf{1 5}$ de ce mois, par laquelle vous sollicitez, comme vous l'aviez fait par deux lettres précédentes, votre congé, je veux bien vous dire par la présente

hält die von J. G. Sulzer in dessen Autobiographie (Berlin \& Stettin 1809), p. 43 ff. gegebene Darstellung wirklich „die strengste Wahrheit" Sulzer war als Gegner Euler's selbst Partei, jedoch giebt Thiébault „Mes souvenirs de vingt ans de séjour à Berlin", t. 5 (Paris 1804), p. 5 ff. im wesentlichen dieselbe Darstellung - , so verdiente Euler's Verhalten allerdings eine abfällige Beurteilung.

1) Der zuletzt citierte Brief Friedrich's d. Gr. vom 16. VI. 1765.

$\left.{ }^{2}\right)$ Euler war unter den glänzendsten Bedingungen wach Petersburg berufen.

3) Diese ganze Stelle fehlt in der deutschen Ausg. des Werkes (Leipzig 1828). 
que vous me ferez plaisir de vous détester de cette demande et de ne plus m'écrire sur ce sujet.

Potsdam, 17 III 1766.

Je vous permets, sur votre lettre du 30 d'avril dernier, de quitter pour aller en Russie.

Potsdam, 2. V. 1766.

Frédéric le Grand à Euler.

voir Oeuvres de Frédéric le Grand, t. 20

(Édition Decker 1852), p. 210

J'ose assurer V. M. que M. de la Grange remplacera très-bien M. Euler pour les talents et le travail, et que d'ailleurs, par son caractère et sa conduite, il n'excitera jamais dans l'Académie la moindre division ni le moindre trouble. Je prends la liberté de demander à V. M. ses bontés particulières pour cet homme d'un mérite vraiment rare, et aussi estimable par ses sentiments que par son génie supérieur. Je me tiens trop heureux d'avoir pu réussir dans cette négociation, et procurer à V. M. et à son Académie un si excellent sujet. Cet événement répand dans mon âme une satisfaction dont je n'ai pas joui depuis longtemps, et je suis sûr que mon estomac s'en ressentira. ${ }^{1}$ )

D’Alembert à Frédéric le Grand. Paris, 19. V. 1766. voir Oeurres de Frédéric le Grand, t. 24 (Édition Decker 1854), p. 403/404.

Il est bien glorieux pour moi d'avoir pour successeur à Berlin le plus sublime géomètre de ce siècle, et il est certain que je n'aurais pu rendre à l'Académie un plus grand service qu'en prenant mon congé, et, à cet égard, je puis me vanter

3) s. den vorherigen Brief (28. X. 1765), nach dem d'Alembert an einer ,inflammation d'entrailles" litt. 
d'une grande supériorité sur vous, vu que vous ne lưi sauriez jamais rendre un tel service.

Euler à Lagrange.

Saint-Pétersbourg, 24. III. 1775.

voir Oeuvres de Lagrange, t. 14 (1892), p. 241.

Je rous prie de m'expliquer comment rous employez l'analyse en géométrie ... Souvenez-rous au moins que vous arez un disciple très-ignorant, et que ce n'est qu'en vous rabaissant à l'infini que vous pouvez m'instruire. . . . Ma foi est si areugle sur les connaissances que vous me communiquez, que si quelqu'un venait à disputer sur ces matières, pour trancher toute difficulté, je lui répondrais comme les disciples de Pythagore: Il l'a dit.

J'ai sans doute écrit quelque journal d'événements qui, se confondant dans la foule, seront oubliés bientôt. . . Vos ouvrages, mon cher d'Alembert, dureront, quand il ne sera plus question de cette fureur épidémique qui avait gagné les grandes puissances de l'Europe, et dont nous avons pensé être la victime. . . . Voilà la différence de nos travaux; les miens ne dureront qu'un temps, au lieu que les vôtres, semblables aux merveilles d'Égypte, méritent la devise de l'Académie française: A l'éternité.

Frédéric le Grand à d'Alembert (Août 1764.)

- Pythagore, auquel vous me faites l'honneur, Sire, de me comparer, quoique indigne, et avec qui je n'ai rien de commun que de n'oser manger des fèves (à la vérité par de meilleures raisons que lui), ce Pythagore aurait tremblé, s'il eût dû avoir comme moi pour juges de ces écrits Numa, Alexandre et Marc-Aurèle. V. M. prétend que mes rapsodies vivront plus longtemps que les journaux immortels de ses campagnes. J'ai lu, je ne sais en quel endroit, que César annonçait la même chose à un philosophe de son temps, dont il n'est rien venu jusqu'à nous, tandis que les Commentaires 


\section{$-89$}

de César, respectés par dix-huit siècles, sont encore lus et admirés de nos jours.

D'Alembert à Frédéric le Grand. Paris, 17. IX. 1764.

voir Oeuvres de Frédéric le Grand, t. 24 (Édition Decker 1854), p. 382/383 et 385 .

Die Mathematik ist eine gar herrliche Wissenschaft, aber die Mathematiker taugen oft den Henker nicht. Es ist fast mit der Mathematik, wie mit der Theologie. So wie die der letztern Beflissenen, zumal wenn sie in Aemtern stehen, Anspruch auf einen besondern Credit von Heiligkeit und eine nähere Verwandtschaft mit Gott machen, obgleich sehr viele darunter wahre Taugenichtse sind, so verlangt sehr oft der sogenannte Mathematiker für einen tiefen Denker gehalten zu werden, ob es gleich darunter die grössten Plunderköpfe giebt, die man nur finden kann, untauglich zu irgend einem Geschäft, das Nachdenken erfordert, wenn es nicht unmittelbar durch jene leichte Verbindung von Zeichen geschehen kann, die mehr das Werk der Routine, als des Denkens sind.

\section{LICHTENBERG.}

Ausgew. Schriften, Reclam-Bibl. No. 1286-1289, p. 147.

Les géomètres ont un tempérament à eux, et une façon de penser bien plus élevée que les autres hommes.

Frédéric le Grand à d'Alembert.

23. XI. 1765.

voir Oeuvres de Frédéric le Grand, t. 24

(Édition Decker 1854), p. 401/402.

Es mag wahr sein, dass Menschen, die bloss Mathematiker sind, gewisse specifische Fehler haben; aber dass [sic] ist nicht Schuld der Mathematik, sondern gilt von jeder exclusiven Beschäftigung. So ein blosser Sprachgelehrter, ein blosser 
Jurist, ein blosser Soldat, ein blosser Kaufmann u. s. w. Man könnte selbst, solch müssigem Hinundherreden noch beifügen, dass wenn eine gewisse exclusive Beschäftigung oft mit gewissen specifischen Fehlern verbunden ist, sie dagegen auch fast immer von gewissen andern specifischen Fehlern frei ist.

Gauss an Schumacher.

Göttingen, 1.-5. I. 1845.

s. Briefw. Gauss-Schumacher, Bd. 4 (1862), p. 387.

Wilhelm [Weber] kann Gauss jeden Tag so lange er will geniessen. Gauss lebt sehr einsam, und Wilhelm ist zu jeder Stunde willkommen. Gauss ist ein so gesellig gebildeter Mann, dass er in meiner Gegenwart nie von gelehrten Dingen spricht und verlangt, dass ich dabei sein soll; er hat mit uns von 12 bis 5 Uhr von allerlei Sachen gesprochen.

Wilhelm Weber's Schwester [Lina] an den Bruder Ernst Heinrich.

2. VI. 1832.

s. „Wilhelm Weber. Eine Lebensskizze" von Heinr. Weber [Prof. d. Phys. in Braunschweig], (Breslau 1893), p. 24/25 = Deutsche Revue $17_{3}$ (1892), p. 195/196.

Es gibt Fragen, auf deren Beantwortung ich einen unendlich viel höhern Werth legen würde als auf die mathematischen z. B. über Ethik, über unser Verhältniss zu Gott, über unsere Bestimmung und über unsere Zukunft; allein ihre Lösung liegt ganz unerreichbar über uns und ganz ausserhalb des Gebietes der Wissenschaft.

Gauss.

s. Sartorius v. Waltershausen, „Gauss zum Gedächtniss“ (1856), p. 97.

Ich bin seit langer Zeit gewohnt, Ihre Ansichten, mein theuerster Freund, meinen eigenen vorzusetzen, da Sie den 
richtigen Tact aus der Mathematik auf jedes Verhältniss des Lebens zu tragen pflegen.

Schumacher an Gauss.

Altona, 5. VI. 1846.

s. Briefw. Gauss-Schumacher, Bd. 5 (1863), p. 162/163.

Vormittags erschien Gauss regelmässig im literarischen Museum, woselbst er eine grosse Anzahl von Zeitungen durchsah, in denen ihn, ausser den politischen Nachrichten, auch noch insbesondere die Börsennachrichten ansprachen, welche er aufmerksam im Interesse seiner statistischen Speculationen verfolgte. .... Die meisten ehemaligen Studirenden der Georgia Augusta aus dem zweiten Viertel dieses Jahrhunderts werden sich lebhaft das edle Antlitz des grossen Mannes ins Gedächtnis zurückrufen können; denn auf den meisten von ihnen wird sein leuchtendes blaues Auge fragend geruht haben, wenn sie zufällig ein Blatt lasen, nach dem Gauss Verlangen trug, und das sich dann Jeder beeilte dem grossen Manne darzureichen.

F. A. T. Winnecke. „Gauss" (Braunschweig 1877), p. 33.

Gauss wusste auch dem [Zahlen-] Rechnen eine höhere Seite abzugewinnen, indem er der mannigfaltigsten Kunstgriffe sich bediente. Es war ihm dadurch ein Vergnügen was den meisten Menschen eine oft unabweisbare aber stets langweilige Pflicht bildet, und es war einiger Ernst in den scherzhaften Worten, deren wir selbst uns aus einer Vorlesung von Gauss über die Methode der kleinsten Quadrate entsinnen, dass eine gewisse Poesie in der Berechnung von Logarithmentafeln liege. Denselben Vorlesungen gehört die Erinnerung an, dass Gauss, der überall, auch in seinen Vergnügungen, als Mann der Wissenschaft sich fühlte, bei jahrelang fortgesetztem regelmässigem Whistspiele mit den gleichen Freunden stets aufzuschreiben pflegte, wie viele Asse Jeder bei jedem Spiele in 
der Hand gehabt hatte, um an diesen Aufzeichnungen eine erfahrungsmässige Bestätigung des sogenannten Gesetzes der grossen Zahlen in der Wahrscheinlichkeitsrechnung zu gewinnen. Ähnliches bezweckten ohne Zweifel seine Aufzeichnungen der täglichen Coursschwankungen gewisser Staatspapiere an den Hauptbörsen, wenn auch nicht in Abrede gestellt werden will, dass das nächste Interesse beim Vergleichen der Course überhaupt ein persönlich finanzielles gewesen sein mag, da die Gewandtheit, mit welcher Gauss sein Vermögen zu verwalten wusste, fast sprichwörtlich war.

\section{CAntor.}

Artikel „Gauss" in ,Allgem. Deutsche Biographie“, Bd. 8 (1878), p. 444.

Ich glaube, dass unter ähnlichen Verhältnissen ich mich eben so gut [wie Bessel] à la hauteur der kaufmännischen Kenntnisse gestellt haben würde. Ich meinerseits besass bis zu einem vorgerückten Alter in mir selbst nichts, was wie die Welt ist, einen sichern Schatz auch nur gegen den Hungertod hätte geben können, als das Schulmeistern, was mir stets zuwider gewesen ist. $^{1}$ )

Gauss an Schumacher.

Göttingen, 27. VI. 1846.

s. Briefw. Gauss-Schumacher, Bd. 5 (1863), p. 172.

Wie nur bei Wenigen, welche die Geschichte kennt, waren in Gauss der mathematische Tiefsinn mit dem Talente des Beobachters, mit dem genialen praktischen Blicke für Erfindung neuer Beobachtungsmittel, mit der vollendetsten Gewandtheit und unermüdlichen Ausdauer im Rechnen vereinigt. Das machte ihn zum Einzigen seines Jahrhunderts. Deutschland konnte damals, wie die Löwin in der Fabel, sagen, ich habe nur einen geboren, aber dieser ist ein Löwe.

M. A. Stern.

„Denkrede auf Carl Friedrich Gauss,“ Festrede Göttingen Univ. 30. IV. 1877, p. 3/4.

l) s. S. $115-117,119,123$. 
Dem Strome der Weltgeschichte entlang hat Gott unser Herr von Zeit zu Zeit gewisse Marksteine aufgerichtet, welche die verschiedenen Jahrhunderte von einander zu scheiden bestimmt sind. Es sind jene grossen Männer, welche aus dem Dunkel ihrer Umgebung unerwartet hervorgetreten, welche Licht verbreitet und die nach einem fernen Ziele ringende Menschheit ihrem Ideale entgegengeführt haben. Sie sind es gewesen, von denen ein neues religiöses, staatliches und sittliches Leben begonnen, von denen eine höhere Entwicklung der Kunst und Wissenschaft ihren Anfang genommen hat. An der Grenze des verflossenen und dieses Jahrhunderts stehen zwei solcher Marksteine, die wie aus dem reinsten eararischen Marmor von der Hand eines Thorwaldsen gearbeitet erscheinen. Auf dem einen erblickt man den Genius der Poesie, die Fackel des Lichts und der Begeisterung in der einen, den Lorbeerkranz in der andern Hand haltend. Auf dem zweiten Gedenksteine befindet sich der Genius der Nathematik und der Astronomie; gestützt auf den Erdball zeigt er hinauf zum Reich der Gestirne. Die Rückseite desselben führt die schlichte, aber denkwürdige Inschrift:

„Natur, du bist meine Gottheit!

Der Erforschung deiner Gesetze ist mein Leben gewidmet“.1)

- Darunter lesen wir den Namen:

Carl Friedrich Gauss,

geb. zu Braunschweig, den 30. April 1777,

gest. zu Göttingen, den 23. Februar 1855.

W. Sartorius von Waltershausen.

„C. F. Gauss" in „Göttinger Professoren" (8 Vorträge), (Gotha 1872), p. 207/208.

In Göttingen wurde ich mit dem damals dort studierenden Gauss bekannt, mit dem ich noch heute in Freundschaft bin, obgleich weit entfernt mich mit ihm messen zu können. Er

1) Wahlspruch Gauss', s. Note 5 am Ende des Buches. 
war sehr bescheiden und zeigte wenig; nicht drei Tage, wie mit Plato, Jahre lang konnte man mit ihm zusammen sein, ohne seine Grösse zu kennen. Schade, dass ich dieses titellose, schweigsame Buch nicht aufzumachen und zu lesen verstand. Ich wusste nicht, wieviel er wusste, und er hielt, nachdem er meine Art sah, viel von mir, ohne zu wissen, wie wenig ich bin. Uns verband die (sich äusserlich nicht zeigende) Leidenschaft für die Mathematik und unsre sittliche Übereinstimmung, so dass wir oft mit einander wandernd, jeder mit den eigenen Gedanken beschäftigt, stundenlang wortlos waren.

W. BolyaI,

in einer 1840 verfaßten u. der ungar. Akad. übersandten Autobiographie.

s. „Briefw. zw. C. F. Gauss u. W. Bolyai“, herausg. v. Schmidt u Stäckel (1899), p. 178 oder P. Stäckel u. F. Engel, "Gauss, die beiden Bolyai und die nichteuklidische Geometrie", Math. Ann. 49 (1897), p. 152/153.

Gauss ruhte von seiner anhaltenden stillen Arbeit meistens bei mir aus -; sprach nie in voraus, selbst bey fertigen schweigend -; nur einmal sah ich an ihm eine mässige Freude, wo er die kleine Tafel auf welcher er das 17 eck Disqu. Ar. p. 662 [= Werke, Bd. 1 (1870), p. 462] berechnet hat zum Andenken mir gab. - - -

Wir giengen auch zweye zu Fus zu seinen Eltern nach Braunschweig; wo mich wie Gauss nicht in der Stube war, seine Mutter fragte, ob aus ihrem Sohne etwas werde? und auf meine Antwort der erste Mathematiker in Europa, in Thränen zerflos -

W. Bolya an Sartorius von Waltershausen.

Maros-Vásárhely, 13. VII. 1856.

s. „Briefw. zw. C. F. Gauss u. W. Bolyai“, herausg. v. Schmidt u. Stäckel (1899), p. 152. 
Eine Überlieferung berichtet, Laplace habe auf die Frage: „Wer ist der erste Mathematiker Deutschlands?" geantwortet: „Bartels [der Lehrer von Gauss und Lobatschefskij], denn Gauss ist ja der erste Mathematiker der Welt." [?] $\left.{ }^{1}\right)$

\section{A. Wassiluef.}

„N. J. Lobatschefskij“, übers. v. F. Engel, Abh. zur Gesch. d. Math. 7 (1895), p. 209.

Vos Disquisitiones [arithmeticae] vous ont mis tout de suite au rang des premiers géomètres, et je regarde la dernière section ${ }^{2}$ ) comme contenant la plus belle découverte analytique qui ait été faite depuis longtemps.

Lagrange à Gauss.

Paris, 31. V. 1804.

voir Oeurres de Lagrange, t. 14 (1892), p, 299.

Malheureusement, l'étendue de mon esprit ne répond pas à la vivacité de mes goûts, et je sens qu'il y a une sorte de témérité à importuner un homme de génie, lorsqu'on n'a d'autre titre à son attention qu'une admiration nécessairement partagée par tous ses lecteurs.

Le Blanc (pseudonyme de Sophie Germain) à Gauss. (Sans date), [Paris, 1805]:

voir Sophie Germain, Oeurres philosophiques, édition de Stupuy (1896) p. 256.

Einige Schüler von Gauss haben die Arbeit nicht gescheut, die Disquisitiones arithmeticae aus Mangel an Exemplaren von einem Ende bis zum andern abzuschreiben. So wie gewisse Geistliche mit ihrem Gebetbuch umherziehen, pflegt ein grosser

1) Dieselbe Erzäblung findet sich u. a. auch bei Ernst Schering, "Carl Friedrich Gauss und die Erforschung des Erdmagnetismus" (Göttingen 1887), p. 3 (= Göttinger Abhandl. 34 (1887), Math. Cl. 3, p. 3), jedoch wird dort statt Bartels' Joh. Friedr. Pfaff genannt.

2) „De aequationibus, circuli sectiones definientibus". 
Mathematiker unseres Jahrhunderts [Dirichlet] ${ }^{1}$ ) nur in Begleitung eines ganz verlesenen, aus dem Einband gewichenen Exemplars der Disquisitiones arithmeticae auf alle seine Reisen zu gehen.

Sartorius v. Waltershausen. "Gauss zum Gedächtniss" (1856), p. 21.

Dirichlet hat Gauss' Disquisitiones arithmeticae nicht nur einmal oder mehreremal durchstudirt, sondern sein ganzes Leben hindurch hat er nicht aufgehört, die Fülle der tiefen mathematischen Gedanken, die es enthält, durch wiederholtes Lesen sich immer wieder zu vergegenwärtigen, weshalb es bei ihm auch niemals auf dem Bücherbrett aufgestellt war, sondern seinen bleibenden Platz auf dem Tische hatte, an welchem er arbeitete. . . . Dirichlet war der erste, der dieses Werk nicht allein völlig verstanden, sondern auch für andere erschlossen hat.

KunMer.

„Gedächtnissrede auf Gustav Peter Lejeune Dirichlet." s. Berl. Abhandl. 1860 , p. $5 / 6$

$=$ Dirichlet, Werke, Bd. 2, p. 315/316.

Die Disquisitiones arithmeticae gehören der Geschichte an und ich würde in einer neuen Ausgabe mit Ausnahme der Druckfehler nichts ändern.

Gauss.

s. Sartorius v. Waltershausen, „Gauss zum Gedächtniss" (1856), p. 20.

Ihre Auflösung der Aufgabe von den Saecularstörungen ist meines Erachtens die erste wirkliche und bedeutende Entdeckung in der Theorie der himmlischen Bewegungen seit

1) s. den Brief Sartorius' an W. Bolyai vom 12. VIII. 1856 in Briefw. Gauss-Bolyai, herausg. v. Stäckel u. Schmidt (1899), p. 157. 
Clairaut; gegen sie sind die vier Bände der Mécanique Céleste nur Entwickelungen einer alten Theorie. Sie Glücklichster! Welcher Tag wird verbreitet, wo Sie es wollen!

Bessel an Gauss.

Königsberg, 20. V. 1819.

s. Briefw. Gauss-Bessel (1880), p. 302.

Herr von Boguslawski hat mir mitgetheilt, dass Sie mit Ihren Untersuchungen über den Magnetismus der Erde auf einen Sie befriedigenden Punct gekommen sind. Ich kenne die Bedeutung dieses Wortes und wünsche also Glück zu dem allervollständigsten Erfolge, hege auch die Hoffnung, dass Sie ihn nicht länger in Ihrem alleinigen Besitze lassen werden.

Bessel an Gauss.

Königsberg, 4. I. 1839.

s. Briefw. Gauss-Bessel (1880), p. 521.

Die pauca sed matura ${ }^{1}$ ) sind hier [Gauss' Allgemeine Theorie des Erdmagnetismus] goldene Äpfel der Hesperiden, gezeitigt unter der Sonne des Genies, von denen einer mehr werth ist, als eine Sehiffsladung von Borstorfern.

Schumacher an Gauss.

Altona, 24. V. 1839.

s. Briefw. Gauss-Schumacher, Bd. 3 (1861), p. 233.

Wenn Lagrange über die ewige Vergleichung zwischen sich und dem Verfasser der Mécanique céleste in menschlicher Anwandlung mislaunisch wurde, so pflegte er mir zu sagen: „Man sieht klar nur durch ein ganz geöffnetes Thor. Le grand Géomètre fait donner un seul coup et la porte est ouverte, Mr. Laplace donne successivement de petits coups, il en donne

1) s. S. 114.

A hrens, Scherz und Ernst i. d. Mathem. 
trois ou quatre. La porte ne cède qu'un peu et l'on voit mal ou rien par une porte à moitié ouverte!" Der Riesenschlag [für die Theorie des. Erdmagnetismus] ist nun von Göttingen ausgegangen. Die Forderung von Lagrange ist erfüllt ... . ich wïnschte die goldene Zeit heran, wo ein newton ianischer Geist uns von den Fesseln gehäufter Epicykeln befreien und alle Elemente aus einem Princip herleiten würde. Dies Wunder haben Sie vollbracht, mein theurer, hochverehrter Freund: meine Augen haben es noch gesehen.

\section{Al. Humbold' an Gauss.}

Paretz, 18. VI. 1839.

s. K. Bruhns, „Briefe zw. A. v. Humboldt u. Gauss“ (1877), p. 41/42 $=$ E. Schering, „C. F. Gauss und die Erforschung des Erdmagnetismus" (Göttingen 1887), p. 65/66 (= Göttinger Abhandl. 34 (1887), Math. Cl. 3, p. 65/66).

Ihre meisterhafte Behandlung Lder „Dioptrischen Untersuchungen" $\mid{ }^{1}$ ) darf ich nicht hervorheben; sie ist in der Ordnung, denn niemand hat bis jetzt entscheiden können, ob der wesentliche Inhalt, oder die Form in welcher er erscheint, in Ihren Arbeiten am meisten hervortreten.

Besser an Gauss.

Königsberg, 20. I. 1841.

s. Briefw. Gauss-Bessel (1880), p. 531.

Der einzige Mann, der der Berliner Academie neuen Glanz geben kann, heisst Karl Friedrich Gauss.

A. v. Huniboliot 1805 zu Friedrich Wilhelm III. s. Briefw. Gauss-Schumacher, Bd. 3 (1861), p. 29.

Herr von Humboldt machte sich die Hoffnung, Sie bald in Berlin zu sehen .... Es ist in dem so oft unrecht angeklagten Preussen recht gut. . . . Man thut viel für die Wissenschaften, und setzt auch nicht mehr voraus, dass die

1) s. Gauss, Werke, Bd. 5 (1877), p. 243-276. 
Gelehrten allein von den Büchern leben. Ich als alter Preusse würde dann „unser Gauss" - denken Sie sich, mit welchem Stolze! - sagen können.

Besset an Gauss. ${ }^{1}$ )

Königsberg, 24. V. 1810.

s. Briefw, Gauss-Bessel (1880), p. 112.

Humboldt's Brief hat mir viel Freude gemacht, da er die tiefste und reinste Verehrung gegen Sie athmet. Er schreibt mir auch, dass er seit 8 Jahren alles gethan habe, damit Sie Lagranges Zeiten nach Berlin zurückbrächten, bisher sei er aber immer noch auf den Geldpunkt gestossen.

\section{Schumacher an Gauss.}

22. III. (1836).

s. Briefw. Gauss-Schumacher, Bd. 3 (1861), p. 16.

Wir armen Praktiker, womit können wir Sie unterhalten? praktische Armseligkeiten, höchstens mechanisch gebrauchte Zahlen sind die kärglichen Beiträge, die wir der Wissenschaft bringen können.

Lilienthal, 10. XII. 1807.

Was uns anderen so schwer wird, wird Ihnen so leicht; Sie gleiten über die Schwierigkeiten hinweg, daß man sie kaum ahnen sollte.

Lilienthal, 21. I. 1810.

Wie glücklich sind Sie! alles gestaltet sich neu unter Ihren Händen und so, dass man Ihre Schriften nur mit der Überzeugung, dass dadurch die Kraft des menschlichen Geistes erschöpft worden ist, aus der Hand legen kann.

Königsberg, 5. X. 1818.

Bessel an Gauss. ${ }^{1}$ )

s. Briefw. Gauss-Bessel (1880), p. 63/64, 100 u. 280.

1) s. Note 6 am Ende des Buches. 
„Man schreit oft (sagt Ihr Herzog von Cambridge in seiner lebendigen Art sich auszudrücken) gegen Göttingen, so lange wir die Bibliothek und Gauss besitzen, können wir schimpfen lassen." Ich bin einverstanden aber meine Pflicht ist es Ew. kön. Hoheit zu bitten, die Rangordnung der Schätze umzukehren und den ersten Mathematiker unseres Zeitalters, den grossen Astronomen, den geistreichen Physiker zuerst zu nennen.

\section{Al. Huniboldt an Gauss.}

Berlin, 17. II. 1833.

s. K. Bruhns, „Briefe zw. A. v. Humboldt und Gauss" (1877), p. 26

$=$ E. Schering, „C. F. Gauss und die Erforschung des Erdmagnetismus" (Göttingen 1887), p. 52 (= Göttinger Abhandl. 34 (1887), Math. Cl. 3, p. 52).

Darf ich dereinst den Titel Schüler von Gauss führen, so verlange ich nie einen andern.

Schumacher an Gauss.

Altona, 20. IX. 1808.

s. Briefw. Gauss-Schumacher, Bd. 1 (1860), p. 5.

Ich unterschreibe mich wie Dissen ${ }^{1}$ ) mich immer nannte,

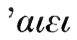

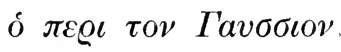

Schumacher an Gauss.

Altona, 3. IV. 1810.

s. Briefw. Gauss-Schumacher, Bd. 1 (1860), p. 35 ; vgl. a. l. c. p. $51,52,90$.

Wir alle zusammen machen gewaltige Forderungen an Mademois. Waldeck [Gauss' Braut], und sie wird schon einen harten Stand haben, wenn sie allen Erwartungen entsprechen will. Vorzüglich werden die Damen, welche Sie kennen, very nice zu befriedigen sein, denn jede hätte Sie gar zu gern ge-

1) Namhafter Philolog in Göttingen. 
habt. In der That, wäre ich ein Frauenzimmer, so wüsste ich wohl wie ich mich jetzt ärgerte.

\section{Schumacher an Gauss.}

Altona, 4. V. 1810.

s. Briefw. Gauss-Schumacher, Bd. 1 (1860), p. 37.

Von den Zusätzen verdanke ich die meisten der freundschaftlichen Güte des Herrn Professor Gauss.

Es wäre thörigt, was dem Geometer Deutschlands zugehört, namentlich ausheben zu wollen - deutlich genug ist des Meisters Stempel auch Seinen Erhohlungen aufgedrückt.

\section{H. C. Schumacher.}

Deutsche Ausg. von Carnot's „Géométrie de Position", II (1810), Vorrede.

Mich verlangt herzlich, den theuersten und werthesten meiner Freunde wiederzusehen, bey dem es mir nur zuweilen vorkömmt, als dürfe ich ihn nicht so lieben, wie ich thue, weil er einem höheren Geschlechte anzugehören scheint.

Altona, 10. VI. 1812.

In welcher glücklichen Zeit lebe ich doch, daß ich mit Ihnen zugleich lebe, und und Sie meinen Freund nennen darf! Ich würde, wenn ich später geboren wäre, alle Ihre Zeitgenossen beneiden.

Altona, 7. IX. 1812.

Mögen Sie in diesem neuen Jahre nur halb so glücklich seyn als Sie es verdienen und Anspruch darauf haben! Sie ganz abzubezahlen scheint mir selbst als Wunsch zu schwer, und niemand weiss das so gut als Ihre Freunde, denen Sie ein Gegenstand der innigsten Liebe sind, ausserdem dass Sie von Allen (also desto mehr von uns) nur als der Gewaltige verehrt werden.

Altona, 23. I. 1813.

Sie können keinen Gegenstand, selbst nicht den unbedeutendsten berühren, ohne durch elegante Behandlung neues 
und unerwartetes Licht darïber zu verbreiten, eine längst bekannte Wahrheit, deren wiederhohlte Bestätigung man aber immer mit freudigem Interesse sieht.

Altona, 4. X. 1843.

Da ich Gott sey Dank noch keine Zeichen eines verrïckten Gehirns gegeben habe, so werden Sie mir leicht glauben, dass ich ....... einen so lächerlichen Wettstreit mit dem Gewaltigen nicht unternehmen werde. Aber so dringend wie möglich bitte ich, machen Sie bald von Ihren Schätzen bekannt.

Kopenhagen, 8. VI. 1816.

\section{Schumacher an Gauss.}

s. Briefw. Gauss-Schumacher, Bd. 1 (1860), p. 89, 93, 96; Bd. 4 (1862), p. 186; Bd. 1, p. 127/128.

Sollte es rathsam sein, gerade mit Dr. Gauss zugleich ïber Materien etwas drucken $\mathrm{zu}$ lassen, die dieser tiefsinnige Analyst schon so lange von allen Seiten untersucht hat und theoretisch und praktisch so genau kennt?

Olbers an Bessel.

Bremen, 9. X. 1806.

s. Briefw. Olbers-Bessel, herausg. v. Erman, Bd. 1 (1852), p. 45; vgl. hierzu l. c. Bd. 2, p. 264.

An Gauss brauche ich wohl keinen Gruss zu bestellen, denn zu dem lieben Gott kann man nur beten und bewundernd emporblicken.

Eisenstein an M. A. Stern 1844.

s. Abhandl. zur Gesch. d, Math. 7 (1895), p. 172.

Det er over al Maade hror de unge Mathematikere her i Berlin og som jeg hører over alt i Tyskland ligesom forguder Gauss. Han er for dem Indbegrebet af al mathematisk Fortræffelighed, men lad være at han vist nok er et stort 
Genie saa er det ligesaa vist at han er [!] ${ }^{1}$ ) et slet Foredrag. Crelle siger at alt hvad Gauss skriver er Gräuel, da det er saa dunkelt at det næsten ikke er mueligt at forstaae det.

(C'est extraordinaire à quel point les jeunes mathématiciens, ici à Berlin, et, à ce que j'entends dire, partout en Allemagne, portent Gauss aux nues, pour ainsi dire. Il est pour eux la substance de toute perfection mathématique, mais s'il est en effet certainement un grand génie, il est tout aussi sûr qu'il rédige mal. Crelle dit que tout Gauss écrit est une horreur, car c'est tellement obscur qu'il n'est presque pas possible de le comprendre.)

Abel à Hansteen.

Berlin, 5. XII. 1825.

Jeg kommer formodentlig til at blive her i Berlin til Enden af Februar eller Marts, og reiser da over Leipzig og Halle til Göttingen (ikke for Gauss Skyld, thi han skal være utaalelig stolt men for Bibliothekets Skyld som skal være fortræffeligt).

(Il est probable que je resterai ici à Berlin jusqu' à la fin de février ou jusqu' en mars, et que je passerai ensuite par Leipzig et Halle pour aller à Göttingen (non pas pour Gauss, car il est, paraît-il, insupportablement orgueilleux, mais pour la bibliothèque qui est, dit-on, magnifique).)

ABEL à Holmboe.

Berlin, 16. I. 1826.

. . jeg gaaer til Göttingen for det forste for at bloquere Gauss, hvis han ikke er altfor stærkt befæstet af Overmod.

(. . j’irai tout d'abord à Göttingen pour faire le blocus de Gauss s'il n'est pas trop fortifié d'orgueil.)

ABEL à Holmboe.

(Paris décembre 1826).

voir „Niels Henrik Abel, Némorial publié à l'occasion du centenaire de sa naissance": "Texte original des lettres", p. 11, 18, 46 et "Correspondance d'Abel" (Traduction française), p. 11/12, 19, 54; voir aussi l. c. (Trad. franç.), p. 12 (lettre IV) et p. 21 (lettre VIII).

1) Zusatz der Herausgeber. 
A bel's Tod, den ich in keiner Zeitung angezeigt gesehen habe, ist ein sehr grosser Verlust für die Wissenschaft. Sollte vielleicht irgendwo etwas die Lebensumstände dieses höchst ausgezeichneten Kopfes betreffende gedruckt sein oder werden, und Ihnen zu Händen kommen, so bitte ich sehr es mir mitzutheilen. Gern hätte icb auch sein Portrait, wenn es irgendwo zu haben wäre.

Gauss an Schumacher.

Göttingen, 19. V. 1829.

s. Briefw. Gauss-Schumacher, Bd. 2 (1860), p. 211

Une tradition, dans notre pays rapporte que Gauss avait dit de ce travail [d'Abel] ${ }^{1}$ ): „Es ist ja ein Gräuel so was zusammenzuschreiben".

.. Que Gauss ait exprimé un blâme sur la rédaction du mémoire ou sur sa rigueur, cela est très vraisemblable, puisqu'A bel, en plusieurs endroits, s'est contenté d'affirmations, là où des démonstrations étaient nécessaires, et sans dire qu'elles avaient été omises faute de place. Il doit y avoir quelque chose de réel à la base de cette tradition. Le mot même "Gränel" se retrouve dans les lettres d'Abel. On serait tenté de croire qu'A bel aurait rapporté l'expression à Crelle, et aurait ainsi provoqué le mot peu réfléchi de ce dernier. ${ }^{2}$ )

L. Sylow.

"Les études d'Abel et ses découvertes"

dans „Niels Heurik Abel, Mémorial publié à l'occasion du centenaire de sa naissance", p. 16.

1) Es handelt sich um das nur in wenigen Exemplaren gedruckte "Mémoire sur les équations algébriques, où l'on démontre l'impossibilité de la résolution de l'équation générale du cinquième degré"; s. N. H. Abel, Oeurres complètes, Ausg. Sylow-Lie (Christiania 1881), t. 1, p. 28-33.

2) s. oben (S. 103) das Citat aus Abel's Brief vom 5. XII. 1825. 
Fast alles, was die Mathematik unseres Jahrhunderts an eigenen wissenschaftlichen Ideen gezeitigt hat, knüpft an Gauss an.

\section{Kronecker.}

Vorles. II, Abschn. 1 (Zahlentheorie), herausg. v. Hensel, Teil I (1901), p. 43.

Den Zeitgenossen schien Gauss vielleicht besonders im höheren Alter eine isolirte Stellung einzunehmen - wir erkennen jetzt, dass er in der Stille dem Genius eines Abel, Jacobi und Riemann voraneilend ${ }^{1}$ ), schon dieselben Bahnen betreten hatte, in denen wir heute wohl vorzugsweise die treibenden Elemente der mathematischen Forschung erblicken. Es wiederholt sich hier nur etwas, was uns eine aufmerksame historische Betrachtung jeder wirklich geistig grossen, über ihre Zeit hinausragenden Persönlichkeit zeigt: dass die Nachwelt immer mehr lernt ihre Verdienste zu würdigen, je mehr sie selber fortschreitend fähig wird, die Bestrebungen jener mit ihrer eigenen Entwickelung zu verknüpfen. Gauss hat zwar auch in seinem Leben voll das Glück genossen, welches die Anerkennung der Zeitgenossen unseren Bestrebungen zu verleihen vermag - uns Epigonen erscheint er aber noch grösser, wenn wir versuchen, ihm in seiner ganzen geschichtlichen Bedeutung gerecht zu werden.

\section{A. Voss.}

„Karl Friedrich Gauss", Vortrag Darmstadt 1877, p. 22/23.

Sie sind ein glücklicher, beneidenswerther Mann! im Hause Glück und Zufriedenheit, ein holdes Weib und ein holder Knabe; auswärts Ruhm und Ehre, Hochachtung zollt Ihnen jeder, innige Zuneigung die, die Sie kennen.

Bessel an Gauss.

Lilienthal, 15. VII. 1807.

s. Briefw. Gauss-Bessel (1880), p. 57.

1) s. Note 7 am Ende des Buches; vgl. a. S. 111/112. 
Wen die holde Göttin der Wahrheit nicht immer flieht, wer eine Braut hat, wie ich habe, und einen Freund, wie Sie sind, der kann sich über Kleinigkeiten schon wegsetzen.

\section{Gauss an Olbers.}

Braunschweig, 3. IX. 1805.

s. „Wilhelm Olbers, Sein Leben und seine Werke", herausg. v. C. Schilling, Bd. 2 (1900), p. 270.

Gauss war von Natur und Vorsehung wahrhaft bevorzugt, demn er hatte von seinem ungewöhnlichen Genie nur Befriedigung, nicht Pein und durchmass sein Leben wie ein majestïtischer Fluss, der keine Stürme kennt, und was mehr ist, er war würdig seines grossen Glückes durch ausgesuchte Herzensgüte und Geradheit des Charakters.

\section{G. RICCI.}

„Anfänge und Entwicklung der neueren Auffassungen der Grundlagen der Geometrie", Antrittsrede Padua 5. XI. 1901. s. Deutsche Mathem.-Verein. Jahresber. 11 (1902), p. 397.

Es ist wahr, mein Leben ist mit Vielem geschmückt gewesen, was die Welt für beneidenswerth hält. Aber glaube mir, lieber Bolyai, die herben Seiten des Lebens, wenigstens, des meinigen, die sich wie der rothe Faden dadurch ziehen, und denen man im höhern Alter immer wehrloser gegenüber steht, werden nicht zum hundersten Theile aufgewogen von dem Erfreulichen. Ich will gern zugeben, dass dieselben Schicksale, die zu tragen mir so schwer geworden ist, und noch ist, manchen andern viel leichter gewesen wären, aber die Gemüthsverfassung gehört zu unserm Ich, der Schöpfer unsrer Existenz hat sie uns mitgegeben, und wir vermögen wenig daran zu ändern. Ich finde dagegen in diesem Bewusstsein der Nichtigkeit des Lebens, was doch jedenfalls der grössere Theil der Menschheit beim Annähern des Ziels anssprechen muss, mir die stärkste 
Bürgschaft für das Nachfolgen einer schönern Metamorphose darbietet.

\author{
Gauss an W. Bolyai. \\ Göttingen, 20. IV. 1848. \\ s. „Briefw. zw. C. F. Gauss u. W. Bolyai“, herausg. \\ v. Schmidt u. Stäckel (1899), p. 132.
}

Le génie de Gauss est essentiellement original. S'il traite une question qui a déjà occupé d'autres savants, il semble que leurs travaux lui soient absolument inconnus. Il a sa manière d'aborder les problèmes, sa méthode propre, ses solutions absolument neuves. Le mérite de ces solutions est d'être générales, complètes, applicables à tous les cas que la question peut embrasser. Malheureusement, l'originalité même des méthodes, un mode particulier de notations, le laconisme exagéré, peut-être affecté, des démonstrations, rendent extrêmement laborieuse la lecture des ouvrages de Gauss.

Les Allemands en général ne sont pas habiles à présenter leurs idées; mais je crois que Gauss a rêvé de réunir, d'abord, tous les défauts de ses compatriotes, en second lieu d'y en ajouter encore qui lui soient propres, afin de s'assurer sur eux une supériorité incontestable. Il a surtout pour les abréviations une prédilection démontante: il représente une chose, très aisée à noter, par un symbole, un groupe de choses analogues par un autre symbole, un groupe de ces groupes par un troisième symbole et ainsi, souvent, jusqu'à la cinquième puissance. ${ }^{1}$ )

Panca sed matura ${ }^{2}$ ), dit-il en parlant de ses ouvrages; il n'en a pas donné pauca, mais il a mis pauca mots dans chacun, trop panca.

\title{
M. MARIE.
}

"Histoire des sciences mathématiques et physiques", t. 11 (Paris 1887), p. 110 et 138.

1) Anscheinend ist hierbei an die Wurzeln der Kreisteilungsgleichung und deren „Perioden" gedacht! Es wird a. a. O. als eine Probe Gauss'scher" Analyse dessen Auflösung der Gleichung $\mathrm{x}^{17}-1=0$ reproduziert.

2) s. S. 114. 
Ich arbeite, wie Sie wissen, langsam, am langsamsten bei derartigen [halb populären] Sachen; ich schäme mich fast zu sagen, wie lange ich an diesen wenigen Seiten geschrieben habe [über „Erdmagnetismus und Magnetometer“, abgedr. in Schumacher's Jahrb. für 1836 = Gauss, Werke, Bd. 5 (1877), p. $315-344]$. - - -

Ich dächte, dass jetzt, nicht sowohl ein nichtmathematischer Gelehrter (denn die sind oft sehr schwer von Begriffen), als z. B. ein verständiger Tischler, der nur ein wenig durch arithmetische Schule gelaufen ist, oder dem man Abkürzungswörter, wie Quadrat und dergleichen vorher erklärt, die Sache müsste begreifen können.

\section{Gauss an Schumacher.}

\section{Göttingen, 9. VII. resp. 28. VII. 1835.}

s. Briefw. Gauss-Schumacher, Bd. 2 (1860), p. 404 u. 406.

Obgleich man von dem, der seinen Gegenstand so tief durchschaut wie Sie, eine klare und scharfe Darstellung desselben erwarten kann, so hat mich doch die, ich möchte sagen unerreichbare Kunst und Einfachheit ${ }^{1}$ ) überrascht, und ich begreife vollkommen wie viel Mühe selbst Ihnen die Form gekostet hat. Ich wüsste Ihrem Aufsatze nichts an die Seite zu setzen, als die kurzen Übersichten, die Lagrange vor seinen Arbeiten von dem bisherigen Stande der Frage zu geben pflegt, und stelle ihn über diese, weil es leichter ist für sein, als für Ihr Publicum zu schreiben.

Schumacher an Gauss.

Altona, 4. VIII. 1835.

s. Briefw. Gauss-Schumacher, Bd. 2 (1860), p. 408.

Der Wunsch, den ich immer bei meinen Arbeiten gehabt habe, ihnen eine solche Vollendung zu geben, ut nihil amplius desiderari possit, erschwert sie mir freilich ausserordentlich,

1) in dem Artikel über Nagnetismus, s. das vorherg. Citat. 
ebenso wie die Nothwendigkeit, heterogener Sachen wegen oft davon abspringen zu müssen. Wenn ich meinen Kopf voll davon habe, stellen Sie sich schwerlich vor, wie angreifend es manchmal für mich ist, Vormittags nach einer schlaflosen Nacht, die ich leider jetzt häufig habe, mich mit Frische in die Sachen hineinzudenken, die ich meinen Zuhörern vorzutragen habe, und nachher wieder mit Lebendigkeit gleich wieder in meinen Meditationen zu Hause zu seyn.

Gauss an Schumacher.

Göttingen, 21. XI. 1825.

In Bezug auf Ihre Arbeiten und den Grundsatz ut nithil amiplius desiderari possit, möchte ich fast wünschen und zum Besten der Wissenschaft wünschen, Sie hielten nicht so strenge daran. Von dem unendlichen Reichthum Ihrer Ideen würde dann mehr uns werden als jetzt, und mir scheint die Materie weit wichtiger als die möglich vollendetste Form, deren diese Materie fähig ist. Doch schreibe ich meine Meinung mit Scheu hin, da Sie gewiss längst das pro und contra möglichst erwogen haben.

Schumacher an Gauss.

Altona, 2. XII. 1825.

Ich habe kaum während einer Periode meines Lebens so angestrengt gearbeitet, und doch vergleichungsweise so wenig reinen Ertrag producirt, wie in diesem Winter. So geht es aber oft bei mathematischen Anstrengungen, wo nicht das Arbeiten, wie das Verfertigen eines Schuhes über einen gegebenen Leisten vollendet werden kann. Ich habe mich zuweilen in diesem Winter Wochen lang, Monate lang mit einer Aufgabe beschäftigt, ohne sie zu meiner Zufriedenheit lösen zu können. Ich war etwas verwundert über Ihre Äusserung als ob mein Fehler darin bestehe, die Materie zu sehr der vollendeten Form hintanzusetzen. Ich habe während meines ganzen wissenschaftlichen Lebens immer das Gefühl gerade vom Gegentheil gehabt, d. i. ich fühle, dass oft die Form vollendeter hätte seyn können, und dass darin Nachlässigkeiten zurückgeblieben sind. 
Denn so werden Sie es doch nicht verstehen, als ob ich mehr für die Wissenschaft leisten würde, wenn ich mich mehr damit begnügte, einzelne Mauersteine, Ziegel etc. zu liefern, anstatt eines Gebäudes, sey es nun ein Tempel oder eine Hütte, da gewissermassen doch das Gebäude auch nur Form der Backsteine ist. Aber ungern stelle ich ein Gebäude auf, worin Haupttheile fehlen, wenn gleich ich wenig auf den äussern Aufputz gebe. . . . . . Höchst drückend fühle ich bei schleunigen Arbeiten meine äusseren Verhältnisse, und das Collegienlesen ist z. B. in diesem Winter unbeschreiblich angreifend für mich gewesen, und Dinge, die an sich leicht sind, werden mir dabei oft sehr schwer.

Gauss an Schumacher. Göttingen, 12. II. 1826.

Es wäre besser, wenn Sie aus dem ungeheuren Schatze Ihrer Erfindungen und Ideen nur soviel als möglich gäben, ohne sich um die Vollendung der Form zu kümmern, und ohne Ihre kostbare Zeit mit der vollkommensten Darstellung Ihrer Erfindungen zu verlieren. Ich glaubte dies Ausfeilen könne ebensogut ein anderer thun, und darin kann ich mich geirrt haben; worin ich mich aber nicht geirrt habe, ist die Behauptung, dass Sie das Erfinden nicht einem andern übertragen können. Jedes Jahr Ihres Lebens mehrt die Ihnen nur verständlichen Andeutungen neuer Ideen.

Schumacher an Gauss.

Altona, 2. XII 1826.

Dasjenige, was man Ausfeilen oder Form mit Recht nennen könnte, ist es doch keineswegs was erheblich aufhält (wenn ich die Sprödigkeit der lateinischen Sprache ausnehme), vielmehr ist es die innige Verkettung der Wahrheiten in ihrem Zusammenhange und eine solche Arbeit ist erst dann gelungen, wenn der Leser die grosse Mühe, die bei der Ausführung Statt gefunden hat, gar nicht mehr erkennt. Ich kann daher nicht leugnen, dass ich keinen recht klaren Begriff davon habe, wie ich meine Arbeiten solcher Art anders, als ich gewohnt bin, aus- 
führen könnte, ohne, wie ich mich schon einmahl ausgedrückt habe, Mauersteine anstatt eines Gebäudes zu liefern. . . . . . . Also, in so fern von wichtigen Gegenständen die Rede ist, etwas im Wesen vollendetes oder gar nichts!

Gauss an Schumacher.

Göttingen, 15. I. 1827.

s. Briefw. Gauss-Schumacher, Bd. 2 (1860), p. $37,41,45 / 46,82 / 83,93 / 94$.

Ich kann es nicht genug an Ihnen, mein geliebter Freund, rühmen, und es wird auch von mehrern meiner Correspondenten dankbar und bewundernd anerkannt, dass Sie stets aus reiner Liebe zur Wissenschaft, sobald Ihnen Ihr Genie und Ihre Forschung eine neue Methode, eine neue Auflösung, eine neue richtigere Ansicht entdeckt hat, diese sogleich bekannt machen, ohne darauf Rücksicht zu nehmen, dass nun Andere Dinge leisten, Fragen auflösen, Rechnungen machen können, die diesen Andern vor Mittheilung Ihrer Entdeckungen zu schwer, ja unmöglich gewesen wären. Gauss scheint mir aber immer erst selbst die schönsten Früchte pflücken zu wollen, zu denen der von ihm aufgefundene und gebahnte Weg hinführt, ehe er Andern denselben zeigt. Ich halte dies für eine kleine Schwachheit des sonst so grossen Mannes, um so weniger zu erklären, da er bei seinem unermesslichen Reichthum an Ideen so Vieles wegzuschenken hat.

\section{Olbers an Bessel.}

Bremen, 25. I. 1825.

s. Briefw. Olbers-Bessel, herausg. v. Erman, Bd. 2 (1852), p. 268.

Ich hasse alles übereilte Publiciren und wünsche immer nur reifes zu geben, und da trifft es sich dann nicht selten, dass wegen dieses oder jenen Umstandes, der, nachdem er erledigt ist, wenige Zeilen füllt, ein wochen- oder monatelanger Aufenthalt entsteht. . . . . [ [Es] ist von jeher mein gewissenhaft befolgter Grundsatz gewesen . . Andeutungen, die auf- 
merksame Leser in jeder meiner Schriften in grosser Menge finden (sehen Sie z. B. meine Disquiss. Arithm. art. 335) ${ }^{1}$ ) stets dann erst zu machen, wenn ich den Gegenstand für mich selbst ganz abgemacht habe, und so werden Sie übersehen, dass der oben erwähnte Fall öfters vorkommen kann, wo um mit gutem Gewissen Eine Zeile schreiben zu können, eine, Monate erfordernde Meditation erfordert wird. Diese Art zu arbeiten kann zuweilen die Folge haben, und hat sie zuweilen gehabt, dass auf Dinge die ich schon seit vielen Jahren besessen habe, später ihrerseits auch andere kommen, und in der Bekanntmachung mir zurorkommen; sie wird vielleicht auch die Folge haben können, dass manches einmahl mit mir ganz untergeht, und ich weiss, dass einige meiner Freunde wünschen, dass ich weniger in diesem Geiste arbeiten möchte: das wird aber nie geschehen; ich kann einmahl an lückenhaftem keine rechte Freude haben, und eine Arbeit an der ich keine Freude habe ist mir nur eine Qual. Möge doch jeder in dem Geiste arbeiten, der ihm am meisten zusagt.

Gauss an Encke.

Göttingen, 18. VIII. 1832.

s. E. Schering, „C. F. Gauss und die Erforschung des Erdmagnetismus" (Göttingen 1887), p. 40/41, auch in Göttinger Abhandl. 34 (1887), Math. Cl. 3 erschienen.

Sie haben nie die Verpflichtung anerkannt, durch zeitige Mittheilung eines dem ganzen angemessenen Theils Ihrer Forschungen die gegenwärtige Kenntniss der Gegenstände derselben zu befördern; Sie leben für die Nachwelt. . . . . . . Wo würden die mathematischen Wissenschaften, nicht allein in Ihrer Wohnung, sondern in ganz Europa jetzt sein, wenn Sie alles ausgesprochen hätten, was Sie ausprechen konnten! Es ist nicht nöthig, diesen Gegenstand weiter zu verfolgen; auch fürchte ich, nur zu wiederholen, was Ihnen hundert Mal gesagt ist.

Bessel an Gauss. Königsberg, 28. V. 1837.

1) Andeutungen besonders über elliptische Transcendenten; s. hierzu Note 7 am Ende des Buches. 
Vorenthalten ${ }^{1}$ ) kann man nur demjenigen vorwerfen, der etwas ganz fertiges d. i. druckfertiges zurückhält, wenn die Publication in seiner Macht steht. Das ist etwas, was ich in meinem Leben noch nicht gethan habe. Es ist zweierlei, mit einem Gegenstande für sich im wesentlichen ganz im reinen zu sein, und ihn für den Druck ausgearbeitet $\mathrm{zu}$ haben. $\mathrm{Zu}$ letzterm brauche ich, da ich eimmal nicht anders als langsam arbeiten kann, Zeit, viel Zeit, viel mehr Zeit, als Sie Sich wohl vorstellen mögen. Und meine 7eit ist vielfach beschränkt sehr beschränkt. Ich brauche ferner dazu (zum Ausarbeiten in diesem Sinne viel mehr als zum ersten Aufsuchen) Heiterkeit des Geistes, und die ist leider nur zu sehr und zu vielfach getrübt. Ich darf daher wohl bitten, billiger über mich zu urtheilen.

Gauss an Bessel.

Göttingen, 28. II. 1839.

Ich habe oft genug Gelegenheit gehabt, das Maximum von Sorgfalt zu bewundern, welches Sie auf Darstellungsart und Form wenden, und auch wohl eingesehen, dass solche Reife nicht mit schneller Aufeinanderfolge der Bekanntmachungen rereinbar ist ..... aber gegen das Maximum hin wachsen die Grössen nur um Quantitäten der zweiten Ordnung, wenn die Zeit um eine Quantitït der ersten wächst. Sollte nicht die Hauptidee selbst, hervortretend in anständiger, wenn auch nicht das Maximum erreichender Darstellung, die Wissenschaft schneller fördern als ihre Vertagung auf die Zeit, welche ihrer allergediegensten Erscheinung günstig ist? ...... Sie sehen gleichgültig zu, wenn Ihnen etwas von Ihrem grossen Reichthum genommen wird; darin haben Sie wirklich nur Ihrer eigenen Ansicht zu folgen, allein Sie können Sich nicht verbergen, dass Sie auch das, was Ihnen nicht genommen wird, in die Gefahr des gänzlichen Verlustes bringen. Sie können

1) Der betr. Brief Bessel's, auf den Gauss hier antwortet, ist einer späteren mutmasslichen Äußerung Gauss' zufolge „wahrscheinlich ein nicht aufbewahrter", s. Briefw. Gauss-Schumacher, Bd. 6 (1865), p. 12, Brief vom 23. XII. 1848; vgl. jedoch a. Briefw. Gauss-Bessel (1880), Vorwort, p. IV. Ahrens, Scherz und Ernst i. d. Mathem. 
also nur erwarten, dass alle Freunde der exacten Wissenschaften ihren eigenen Vortheil gegen ihre Ansicht in die Wage legen; dieses gilt im allgemeinen; die Zeitgenossen haben jedenfalls noch mehr Grund, zu wünschen, dass sie weniger strenge sein möchten. ${ }^{1}$ )

Bessel an Gauss.

Königsberg, 28. VI. 1839.

s. Briefw. Gauss-Bessel (1880), p. 516/517, 524, 526/527.

Übrigens tritt auch hier die oft veranlasste Bemerkung hervor, dass Sie von jeder Art wünschenswerthen Besitzes grossen Reichthum verwahren. Möchte es doch ein Mittel geben, ihn vollständig der Nachwelt zu erhalten.

Bessel an Gauss.

Königsberg, 8. XI. 1843.

s. Briefw. Gauss-Bessel (1880), 1. 564/565.

Pauca sed matura.

Motto auf Gauss's Siegel.

s. z. B. Sartorius v. Waltershausen, "Gauss zum Gedächtniss" (1856), p. 82.

Sie wissen, dass ich langsam schreibe, allein dies kommt hauptsächlich daher, weil ich mir nie anders gefallen kann, als wenn in kleinem Raum möglichst viel ist, und kurz zu schreiben viel mehr Zeit kostet als lang.

Gauss an Schumacher. Göttingen, 2. IV. 1833.

Ich komme wieder auf meinen alten Wunsch zurück, dass es Ihnen gefallen möge, länger und schneller zu schreiben.

1) Gauss nent in einem Brief an Schumacher vom 23. XII. 1848 (s. Briefw. Gauss-Schumacher, Bd. 6 (1865), p. 12) diese Rechtfertigung „mislungen". 
Ich würde ihnen dann sogleich ein neues Petschaft zu überreichen wagen mit Multa nec immatura.

Schumacher an Gauss.

Altona, 6. IV. 1833.

Ich hatte einmal vor, Ihnen ein Siegel stechen zu lassen, mit Ihrem Baume mit wenigen Früchten, und der Umschrift panca, sed matura, aber darunter in der Exergue N.P.I. nemlich Ludwig's des 14 ten Symbolum Nec pluribus impar. Indessen fürchtete ich, Sie würden es nicht gebrauchen.

Schumacher an Gauss.

Altona, 24. VI. 1836.

s. Briefw. Gauss-Schumacher, Bd. 2 (1860), p. $328,330 / 331$; Bd. 3 (1861), p. 75.

Die Art der Darstellung ist in den disquisitiones [arithmeticae], wie überhaupt in den Gaussischen Arbeiten, die Euklidische. Er stellt die Sätze auf und beweist sie, wobei er geradezu mit Fleiss jede Spur der Gedankengänge verwischt, die ihn zu seinen Resultaten geführt haben. In dieser dogmatischen Form ist gewiss auch der Grund dafür zu suchen, dass sein Werk so lange unverstanden blieb und dass es erst der Bemühungen und Forschungen Lejeune-Dirichlets bedurfte $^{1}$ ), um es bei den Nachlebenden zu der vollen Wirkung und Würdigung gelangen zu lassen.

L. Kronecker.

Vorlesungen II, Abschn. 1 (Zahlentheorie), herausg. v. Hensel, Teil I (1901), p. 42.

Gegen das Dociren habe ich einmal eine wahre Abneigung, das perennirende Geschäft eines Professors der Mathematik ist doch im Grunde nur, das ABC seiner Wissenschaft zu lehren; aus den wenigen Sčhülern, die einen Schritt weiter gehen, und gewöhnlich, um in der Metapher zu bleiben, beim Zusammenlesen bleiben, werden die meisten nur Halbwisser; denn die

1) vgl. S. 96. 
selteneren Anlagen wollen sich nicht durch Vorlesungen bilden lassen, sondern bilden sich selbst. Und mit diesen undankbaren Arbeiten verliert der Professor seine edle Zeit. Ich habe es bei meinem vortrefflichen Freunde [Joh. Friedr.] Pfaff gesehen, bei dem ich einmal ${ }^{1}$ ) ein paar Monate war, wie wenige fragmentarische Stunden er zu eigenen Arbeiten übrig hat von den publicis, privatis, privatissimis, den Vorbereitungen dazu und anderen mit dem Amte eines Professors verbundenen Beschäftigungen. Die Erfahrung scheint dies auch zu bestätigen. Ich weiss keinen Professor, der wirklich viel für die Wissenschaft gethan hätte, als den grossen Tobias Mayer, und dieser galt zu seiner Zeit für einen schlechten Professor. Ebenso, wie unser Freund Zach es öfters angemerkt hat, in unseren Tagen sind diejenigen, die das Beste für die Astronomie thun, nicht die besoldeten Universitätslehrer, sondern sogenannte Dilettanten, Ärzte, Juristen etc.

Und bei der Ansicht, wenn die Farben vielleicht auch etwas zu dunkel sein sollten, würde auch ich unendlich lieber das letztere sein, als das erstere, wenn ich nur unter beiden die Wahl hätte. Ich würde mit tausend Freuden ein ungelehrtes Amt annehmen, zu dem Arbeitsamkeit, Akkuratesse, Treue u. dgl. ohne Fakultätskenntnisse hinreichend sind, und das nicht Rang oder Einfluss, sondern nur eine gemächliche Lage und hinreichende Musse gäbe, um meinen Göttern opfern zu können. So hoffe ich z. B. die Redaktion der Volkszählungen, Geburtsund Sterbelisten in hiesigen Landen zu bekommen, nicht als Amt, sondern zu meinem Vergnügen und zur Satisfaktion, mich für die Vortheile, die ich hier geniesse, einigermaassen nützlich zu machen.

\section{Gauss an Olbers.}

Braunschweig, 26. X. 1802.

s. "Wilhelm Olbers, Sein Leben und seine Werke", herausg. v. C. Schilling, Bd. 2 (1900), p. 105/106.

1) $1799 / 1800$ in Helmstedt. 
Zu den zersplitternden Geschäften gehören besonders meine Vorlesungen, die ich in diesem Winter zum ersten Male halte, und die mich eben deswegen jetzt viel mehr Zeit kosten, als mir lieb ist. Ich hoffe indess, dass das zweite Mal dieser Zeitaufwand viel geringer sein werde, sonst würde ich mich damit nie aussöhnen können. . . . . . selbst praktische [astronomische] Arbeiten müssen doch weit mehr Satisfaction geben, als wenn man ein Paar mittelmässige Köpfe mehr bis zum B bringt, die sonst beim A stehen geblieben wären!

Gauss an Bessel.

Göttingen, 4. XII. 1808.

Ich lese in diesem Winter zwei Collegia für drei Zuhörer, wovon einer nur mittelmässig, einer kaum mittelmässig vorbereitet ist, und dem dritten sowohl Vorbereitung als Fähigkeit fehlt. Das sind nun einmal die onera einer mathematischen Profession.

Gauss an Bessel.

Göttingen, 7. I. 1810.

das Lesen hat seine unangenehme Aussenseite verloren: ich lese ziemlich gern und noch immer vor einem vollen Auditorio.

Bessel an Gauss.

Königsberg, 26. VIII. 1810.

s. Briefw. Gauss-Bessel (1880), p. 97, 107, 122.

Mein Collegium [das erste überhaupt], welches ich publice vor vielen Zuhörern lese, macht mir wenig oder gar keine Mühe, denn ich lese ganz frei und notire mir nur kurz die Punkte, über die ich in der Stunde etwas zu sagen denke; das ist für das halbe Jahr die Arbeit zweier Tage gewesen. Umständlichere Ausarbeitungen, die ich früher unternahm, habe ich gleich verworfen.

Bessel an Olbers.

Königsberg, 8. VII. 1810.

s. Briefw. Olbers-Bessel, herausg. v. Erman, Bd. 1 (1852), p. 231. 
Ich hörte im Winter [1823/24] Kollegien bei dem berühmten Astronomen Bessel..... Das Kolleg bei Bessel war in der ersten Stunde am Anfange des Semesters überfüllt. Man konnte kaum einen Sitzplatz bekommen. Schon in der dritten Stunde war daran kein Mangel und in der zweiten Woche reduzirte sich die Zahl der Zuhörer auf wenig über ein halbes Dutzend. Der Grund lag darin, dass Bessel sehr bedeutende mathematische Kenntnisse voraussetzte, ohne welche niemand seinem Vortrage folgen konnte; dann auch darin, dass ihm jeder Massstab für leicht und schwer fehlte. Sobald die Zahl der Zuhörer eine sehr kleine geworden war, wurde er ohne weiteres unterbrochen, wenn seine Schlussfolgerungen durch Übergehung aller Zwischensätze unverständlich blieben. Er antwortete dann stets: „Wenn Sie das nicht verstehen, so sollten Sie gar nicht in mein Kolleg kommen", ergänzte aber doch das Fehlende. Ein anderesmal hielt er sich bei einem Punkt auf, der uns allen ganz klar war, und wunderte sich, dass wir dies zu erkennen gaben. Man durfte aber unmittelbar hinter Bessel kein anderes Kolleg besuchen, sondern musste sofort nach Hause gehen und den Vortrag nach den gemachten kurzen Notizen gründlich durcharbeiten, sonst war es unmöglich, der Stunde zu folgen. Die Besselsche Einleitung in die allgemeine Arithmetik war eine vollständig philosophische Abhandlung über Raum und Grössen. Bessel meinte ganz ernstlich, wer die Méchanique [sic] céleste von Laplace nicht gelesen habe, könne sich nicht zu den gebildeten Menschen zählen.

Hans Victor von Unruh, [der bekannte Parlamentarier und Ingenieur]. „Erinnerungen aus dem Leben", herausg. von H. v. Poschinger (1895), p. 29.

Ein Beispiel vom Vorwalten der analytischen Methode bietet die 1788 erschienene Mécanique céleste [sic] von Lagrange. KARL vON RAUMER.

„Geschichte der Pädagogik“, 4. Aufl., Th. III (1873), p. 313 Anm. 
Alle Vorlesungen bei Gauss hörte Encke gern; mehrfach nennt er sie [in Briefen] seine herrlichste Beschäftigung, Gauss einen göttlichen Lehrer, dessen Theorie der Störungen himmlisch, und als er die letzte Stunde am Montag den 5. April 1813 gehabt, drückt er Gerling brieflich sein Bedauern aus; es sei ihm eine traurige Empfindung gewesen und sein Trost sei nur, dass Alles in der Welt endlich ein Ende nehmen müsse.

\title{
C. BRuhns.
}

„Johann Franz Encke" (Leipzig 1869), p. 19.

. Diesen Winter habe ich leider ein Collegium mit einem höchst einfältigen Schüler.

\author{
Gauss an Bessel. \\ Göttingen, 21. XI. 1811.
}

Ich habe diesen Winter zwei Collegia zu lesen und habe ein paar geschickte junge Leute zu Zuhörern.

Gauss an Schumacher.

Göttingen, 6. I. 1811.

Das Collegienlesen für Schüler, die sich nicht auszeichnen ist ein sehr lästiges undankbares Geschäft.

Gauss an Bessel.

Göttingen, 27. I. 1816.

s. Briefw. Gauss-Bessel (1880), p. 154 u. 232;

Briefw. Gauss-Schumacher, Bd. 1 (1860), p. 62.

Unter allen grösseren und kleineren Werken von Gauss ist keines, welches nicht in dem betreffenden Fache einen wesentlichen Fortschritt durch neue Methoden und neue Resultate begründete; sie sind Meisterwerke, welche denjenigen Charakter der Klassicität an sich tragen, welcher dafür bürgt, dass sie für alle Zeiten, nicht bloss als Monumente der geschichtlichen Entwickelung der Wissenschaft erhalten, sondern auch von den künftigen Generationen der Mathematiker aller Nationen, als Grundlage jedes tiefer eingehenden Studiums und als reiche 
Fundgrube fruchtbarer Ideen werden benutzt und mit Fleiss studirt werden. Bei diesen ausserordentlichen Vorziigen der Gaussischen Schriften, ja zum Theil sogar wegen derselben, war ihre Einwirkung auf die mathematischen Studien, namentlich in Deutschland, längere Zeit hindurch nur eine äusserst geringe. . . . . . . . In der Darstellung haben alle Gaussischen Schriften diejenige vollendete Klarheit und Bestimmtheit, welche bei einem vollständig eingehenden Studium selbst die Möglichkeit von Missverständnissen ausschliesst, aber diese Darstellung, so wie auch die Methoden selbst, sind nicht darauf berechnet das Studium der Gaussischen Schriften zu erleichtern. Dieser Charakter seiner Schriften war auch mit Gauss eigenem Charakter in vollem Einklange. Auf seiner erhabenen Stellung in der Wissenschaft, auf welcher er längere Zeit ganz isolirt stand, besass er eine so grosse Autarkie, dass er das Bedürfniss andere zu sich heranzuziehen und heranzubilden kaum empfand. Er hatte keine Freude am Dociren. - - -

E. E. Kumaier.

Rektoratsrede Berlin Univ. 3. VIII. 1869, p. 8 u. 9.

Während die Gaussischen Schriften nur schwer dem Studium zugängig sind, fand das gerade Gegentheil in den Gaussischen Vorlesungen statt. Hiervon sind alle freudig überrascht gewesen, welche gleich mir das Glïck gehabt haben Vorlesungen von Gauss beizuwohnen, denn da war alles Klarheit und Licht von Anfang bis zu Ende. Gauss liebte es nicht, dass in seinen Vorlesungen auch nur ein Wörtchen niedergeschrieben wurde, er forderte Zuhörer im strengen Sinne dieses Wortes. Vor diesen Zuhörern öffneten sich sodann aber die Schleusen seines Geistes, er liess sie einen Einblick thun in die innere Werkstätte, aus welcher so Grosses hervorgegangen war, und indem er alle Pforten erschloss und alle Wege gangbar machte, gab er in seinen Vorlesungen gerade dasjenige, was als vermittelndes Element zwischen dem Leser und dem Schriftsteller in seinen Werken vermisst wird. Gauss konnte eine Stunde lang darüber reden, weshalb er einen gewissen Weg, 
der scheinbar zur Sache führte, nicht einschlage, und war dann wohl am Ende der Stunde selbst verwundert darüber, dass in der Sache kein Schritt vorwärts geschehen war. Die Zuhörer, deren kleine Zahl gewöhnlich um Einen Tisch Raum fand, lauschten den Worten des Meisters mit ungetheilter Aufmerksamkeit.

\section{Th. Wittsstein.}

„Gedächtnissrede auf Carl Friedrich Gauss"(Hannover 1877),p. 18.

Ich habe niemals in meinen Vorlesungen dictirt, auch selbst kein Heft ausgearbeitet, sondern spreche ganz frei weg; was dabei an rhetorischem Schmuck verloren wird, findet wohl vollen Ersatz in grösserer Lebendigkeit. In der Regel schreiben meine Zuhörer in den Stunden nichts auf, haben sie aber selbst Eifer, so bringen sie nachher zu Hause das Gelernte zu Papier, wie ich das auch von vielen namentlich weiss.

Gauss an Schumacher.

Göttingen, 27. XII. 1842.

s. Briefw. Gauss-Schumacher (1860-1865), Bd. 4, p. 105; vgl. a. ib. Bd. 5 , p. $77 / 78$.

Es ist allerdings wahr, dass ich für Alles was von Ihnen kommt im voraus eingenommen bin, aber diese Praevention ist nur ein Resultat der vieljährigen Erfahrung, dass nichts aus Ihren Händen (selbst durch die 3te Hand) kommt, ${ }^{1}$ ) in dem man nicht den unguem leonis erkennt.

Schumacher an Gauss. Altona, 29. XII. 1842.

s. Briefw. Gauss-Schumacher, Bd. 4 (1862), p. 109.

Die Vorlesungen über practische Astronomie und über verschiedene Theile der Mathematik, namentlich über die

1) Eis handelt sich um die Ausarbeitung einer Gauss'schen Vorlesung durch einen Studenten. 
Methode der kleinsten Quadrate, schienen Gauss im vorgerückten Alter mehr Freude als in seinen frühern Lebensjahren zu machen, und wenn er auch anfangs über diese ihm aufgebürdete Last beim Beginn eines jeden neuen Semesters klagte, so war er doch bald mit grosser Lebendigkeit und merkwürdiger geistiger Energie mitten in der Sache.

Sartorius v. Waltersilausen. „Gauss zum Gedächtniss" (1856), p. 67.

Gauss liebte es, bei seinen Vorlesungen auch vielfach Beispiele rechnen zu lassen, und gab Arbeiten auf, deren Resultat er öfter publicirte. Viele Rechnungen liess er durch Gerling, Nicolai, Encke und Andere ausführen, sodass Nicolai sich einmal in einem Briefe an Encke darüber aufhält, indem er Encke zu der glücklichen Vollendung des kleinen Rechenexempels (die Vorausberechnung des Laufes der Pallas) gratulirt, welches der schlaue Gauss ihm des Beispiels wegen aufgegeben hatte.

\section{Bruhns.}

„Johann Franz Encke“ (Leipzig 1869), p. 21.

Sie wissen vielleicht, dass es immer sehr zweifelhaft ist, ob meine Vorlesungen zu Stande kommen; wo wohnen Sie? bei dem Barbier Vogel? Nun, das trifft sich ja glücklich, denn der ist auch mein Barbier, durch ihn werde ich Sie benachrichtigen.

Gauss zu R. Dedekind 1850.

s. „Festschr. zur Feier des 150-jähr. Bestehens d.

Kgl. Ges. d. Wiss. z. Göttingen" (Berlin 1901), p. 47/48.

In seinen späteren Jahren war Gauss nur schwer dazu zu bewegen, ein Colleg zu lesen; jedoch war er, unter Beobachtung aller Formen, stets dem strebenden Studirenden zugänglich. Der Schreiber dieser Zeilen gedenkt nicht selten mit dankbarer 
Erinnerung mancher halben Stunde aus den Jahren 1853 und 1854, die der grosse Mann in anregender und wesentlich fördernder Belehrung dem Anfänger widmete, welchem er gestattet hatte, mit Fragen bei dem Selbststudium der Theoria motus ihn zu behelligen, ein Thema, auf das glücklicherweise diese Erlaubniss nicht beschränkt blieb.

F. A. T. Winnecke.

„Gauss" (Braunschweig 1877), p. 21.

Meiner Einsicht nach ist Unterricht hei solchen Köpfen, die nicht etwa nur eine Masse von Kenntnissen einsammeln wollen, sondern denen es hauptsächlich daran liegt, ihre eigenen Kräfte zu üben, um selbst zur Erweiterung der Wissenschaften beizutragen, sehr unzweckmässig: einen solchen muss man nicht bei der Hand fassen und zum Ziele führen, sondern nur von Zeit zu Zeit ihm Winke geben, um sich selbst auf dem kürzesten Wege hin zu finden.

GAuss an Schumacher.

Göttingen, 2. X. 1808.

s. Briefw. Gauss-Schumacher, Bd. 1 (1860), p. 6.

Während eines mehrjährigen Aufenthaltes in Göttingen sah v. Staudt sich nicht bloss durch die Lehre des grossen Mathematikers Gauss gefördert, sondern von des verehrten Meisters Anerkennung und Lob beglückt. Man erzählt sich, dass dieser, wenn er die Lösung einer von ihm gestellten Aufgabe aus den Händen des lieben Schülers entgegennahm, ihm dagegen seine eigene Bearbeitung übergab, mit der heiteren Bemerkung, er rechne auf gegenseitige Befriedigung.

\section{v. Martius.}

„Nekrolog auf Christian von Staudt", München Akad.,

Sitzungsber. 1868, I, p. 429

$=$ Arch. Math. Phys. 49 (1869), Liter. Ber. CLXXXXIII, p. 2. 
Nachdem Möhius 1813 ein grösseres . . Reisestipendium zuerkannt worden war, ging er nach Göttingen, wo er ein Jahr lang als Schüler von Gauss verweilte, dann wandte er sich nach Halle, um bei Joh. Friedr. Pfaff ein Privatissimum über Integralrechmung zu hören. Die gewöhnlichen mathematischen Vorlesungen, welche damals an Universitäten gehalten wurden, bewegten sich nämlich fast nur im Gebiete der Elemente der Wissenschaft und ins Besondere waren damals öffentliche Vorlesungen über Differential- und Integralrechnung nicht üblich. Mollweid $\mathrm{e}^{\mathrm{l}}$ ) hat es stets für unmöglich erklärt, einer grösseren Schülerzahl diese Disciplinen zu lehren und erst unter seinem Nachfolger ${ }^{2}$ ) ist in Leipzig die höhere Analysis in das Gebiet der Vorlesungsgegenstände eingetreten.

\section{Heinkicil Gretschel.}

„August Ferdinand Moebius", Arch. Wath. Phys. 49 (1869), Liter. Ber. CLXXXXV, p. 3.

La géométrie n'est pas aussi cultivée en Allemagne que dans les autres pays de l'Europe. On prétend que les Germains n’ont point de têtes géométriques, ce qui certainement est faux: les noms de Leibniz et de Copernic prouvent le contraire. La cause en est, ce me semble, que cette science manque d'encouragement et surtout de professeurs assez habiles pour l'enseigner.

Frédéric le Grand ${ }^{3}$ ).

"Lettre sur l'éducation."

voir Oeurres de Frédéric le Grand, t. 9

(Édition Decker 1848), p. 119.

Neumann begann [als Student in Berlin] neben den Vorlesungen über Mineralogie privatim Mathematik zu treiben privatim, denn an der Berliner Universität wurden damals

1) Prof. math. 1814-1825.

2) Drobisch.

3) s. Note 8 am Ende des Buches. 
[1818] Vorlesungen über Mathematik nicht gehalten. Zwar war ein Lehrer für Mathematik vorhanden - ich glaube Tralles -, aber derselbe weigerte sich, Vorlesungen zu halten, "weil es nicht lohne“. Neumann hat später mit vielem Humor die Geschichte eines Versuchs erzählt, an dem er betheiligt war, diesen seltsamen „Universitätslehrer“ zu einer Vorlesung über Differentialrechnung zu bewegen. Tralles hätte die erste und zweite Stunde nur Formeln angeschrieben, ohne ein Wort zu sprechen, und als in der dritten Stunde einzig noch die Petenten anwesend waren, die „Vorlesung" mit den Worten geendet: "Sie sehen, es lohnt nicht".

\section{W. Volixt.}

„Zur Erinnerung an F. F. Neumann", Göttinger Nachr. 1895, Math.-phys. Kl., p. 251.

Wenn Gauss seit dem Anfange dieses Jahrhunderts die neuere Mathematik in Deutschland gleichsam erst einführte, und namentlich einen Theil gewissermassen erst schuf, dem er selbst einen entschiedenen Vorzug vor allen andern beilegte, so haben Jacobi und Dirichlet das Verdienst, in Preussen diese bis dahin noch nicht zur vollständigen Blüthe gelangte Richtung für die jüngeren Kräfte zugänglich und fruchtbar gemacht zu haben. - -

\section{ENCKe.}

Beantwortung von Kummer's Antrittsrede i. d. Berliner Akademie. s. Berl. Monatsber. 1856, p. 382 .

Jacobis erste Vorlesung über die Theorie der krummen Oberflächen und Curven doppelter Krümmung, ${ }^{1}$ ) welche weder hier noch an anderen deutschen Universitäten bis dahin gehalten worden war, kann als Anfang der allgemeinen Neu-

1) Berlin W. S. $1825 / 1826$; s. das von L. Kronecker mitgeteilte „Verz. der Vorles., welche Jacobi a. den Univ. zu Berlin u. Königsberg gehalten hat" in Jacobi, Werke, Bd. 7, p. 409. 
gestaltung des mathematischen Universitäts-Unterrichts angesehen werden, welche seitdem in's Leben getreten ist.

E. E. Kummer.

(,,Über die Fortbildung und Verbreitung der mathem. Wissenschaften unter Friedrich Wilhelm III."),

Rektoratsrede Berlin Univ. 3. VIII. 1869, p. 10.

Die Vorlesungen Jacobi's bewegten sich sämmtlich ausserhalb des Gebietes der Lehrbïcher in den Tiefen der Wissenschaft und umfassten nur solche Theile derselben, in denen er selbst schaffend aufgetreten war, mit der Tendenz die Gedanken an Stelle der Rechnung zu setzen. Indem er die jeder Theorie zu Grunde liegenden leitenden Gedanken festzustellen sich bemühte, entwickelte er seinen Zuhörern die Probleme mit einer Einfachheit, die Ähnliches zu erfinden hoffen liess. ${ }^{1}$ )

OtTo Hesse 1853.

s. Ges. Werke (1897), p. 713.

Wenn es die immer mehr hervortretende Tendenz der neueren Analysis ist Gedanken an die Stelle der Rechnung zu setzen, so giebt es doch gewisse Gebiete, in denen die Rechnung ihr Recht behält. Jacobi, der jene Tendenz so wesentlich gefördert hat, leistete vermöge seiner Meisterschaft in der Technik auch in diesen Gebieten Bewundernswürdiges. - _ - -

Jacobi hat mit Euler wie durch Vielseitigkeit und Fruchtbarkeit so auch darin die grösste Ähnlichkeit, dass ihm alle Hülfsmittel der Wissenschaft immer gegenwärtig waren und jeden Augenblick zu Gebote standen.

\section{DIRICHLET.}

"Gedächtnissrede auf Carl Gustav Jacob Jacobi.“

s. Abhandl. d. Berl. Akad. 1852 , p. 19 u. 26

$=$ Dirichlet, Werke, Bd. 2, p. 245 u. 252

$=$ Jacobi, Werke, Bd. 1, p. 21 u. 27.

1) s. Note 9 am Ende des Buches. 
Die physikalischen Autoritäten waren geneigt die Richtigkeit des Gesetzes [von der Erhaltung der Kraft] zu leugnen und in dem eifrigen Kampfe gegen Hegel's Naturphilosophie, den sie führten, auch meine Arbeit für eine phantastische Speculation zu erklären. Nur der Mathematiker Jacobi erkannte den Zusammenhang meines Gedankenganges mit dem der Mathematiker des vorigen Jahrhunderts, interessirte sich für meinen Versuch und schützte mich vor Missdeutung. ${ }^{1}$ )

\section{H. von Helmholtz.}

s. „Anspr. u. Red. geh. bei der Helmholtz-Feier 2. XI. 1891“, p. 52.

Jakoby ${ }^{2}$ ) hat uns in den letzten Wochen viel besucht. Was kann der grob sein! - Eigentlich haben die groben Leute ganz recht, wenn sie, wie Jakoby, doch noch etwas daneben sind, denn wenn sie sich einmal zu anderer Leute Alltagshöflichkeit herablassen, kann sich ihr Auditorium gar nicht vor Wonne fassen und sperrt Maul und Nase auf und bedankt sich schönstens. Ich hätte ihn wohl mit Schönlein [dem berühmten Arzt] zusammen sehen mögen, wer da das gröbste Wort behalten hätte. . . . . Gott bewahre mich, so krank zu werden, wie ich es sein müsste, um Schönlein zu consultiren.

Fanny Hensel an ihre Schwester Rebecka Dirichlet. Berlin, 27. VII. 1843.

Ein Talent, das Du schwerlich in Jakoby vermuthet hast, ist das, mit einiger Prätention, aber sehr gut vorzulesen ... man hat [bei ihm] viel zu überwinden, um zu einem ausgezeichneten Geist zu gelangen, aber das ist er wirklich in jeder Hinsicht.

Rebecka Dirichlet an Fanny Hensel. Florenz, 26. IX. 1843.

Jakoby's sind mir ein überaus angenehmer Gewinn; sein iiberlegener Geist zeigt sich in jeder Art, und da er uns gern

1) s. Note 10 am Ende des Buches.

2) der Mathematiker C. G. J. Jacobi. 
zu haben scheint, benimmt er sich gegen uns aufs Liebenswürdigste; unter Anderm kann man nicht mit mehr Verständniss Musik hören, als er.

Fanny Hexsei, geb. Mendelssohn-Bartholdy. [Anscheinend aus dem Tagebuche], Ende April 1846.

\section{s. S. Hensel, „Die Familie Nendelssohn“, Bd. 2 (Berlin 1884), p. 218, 239 u. 364365.}

Der röthliche Mathematiker [Jacobi|, der durch Inconsequenz, ewiges Schwanken und orientalisches Benehmen uns viel Sorge gemacht hat, ist nun glücklicher Weise für unsere Academie erhalten ..... Er hat tugendhafte Verheissungen ... unterzeichnet, wird keine rothen Ordensbänder mehr bei Beerdigungen tragen, und hat erwiesen, dass auch er Alles gethan habe, um den Staat zu retten!

Ar. von Humboli't an Schumacher 1850.

Ich kriege jetat wieder mein um mehrere hundert Thaler vermehrtes altes Gehalt und zwar vom Zeitpunkt an (1. Oct. 49), wo man mich zu philosophischer Entsagung nöthigen wollte. Lernen Sie daher, dass man keinem Mathematiker ungestraft ein Leid thun darf.

C. G. J. JАCOBI an Schumacher 1850.

s. Briefw. Gauss-Schumacher, Bd. 6 (1865), p. 68/69 u. 72.

Sie würden mich sehr verbinden, wenn Sie mir etwas näheres über diesen wie es scheint sehr talentvollen jungen Mann [Jacobi], auch über seine persönlichen Verhältnisse anzeigen wollten.

Gauss an Bessel.

Göttingen, 20. XI. 1826. 
$\mathrm{Jacobi}$ ist gewiss talentvoll, allein er hat sich hier fast alle zu Feinden gemacht, weil er, als er hier ankam, jedem etwas unangenehmes sagte: den geborenen Koenigsbergern versicherte er, dass er seinen hiesigen Aufenthalt als ein Exil betrachte, den Philosophen lobte er Hegel, den Philologen Böckh, alles auf eine Art die man ihm nicht verzeihen will. Doch hoffe ich, dass solche kleine Albernheiten bald nicht mehr werden erwähnt werden. Mir ist er immer als ein artiger junger Mann erschienen.

\author{
Besser an Gauss. \\ Königsberg, 12. XII. 1826.
}

s. Briefw. Gauss-Bessel (1880), p. 463 u. 469.

Mein unvergesslicher Mathematiker Jacoby sagte mir, als ich mein erstes Buch hatte drucken lassen: „es ist eine ungeheure Stufe, die man erstiegen hat".

\title{
LeHrS
}

[bedeutender Königsberger Philolog u. Freund C.G.J.Jacobi's] an seinen Freund Wilh. Tobias.

Königsberg, 6. V. 1874.

s. „Ausgew. Briefe von und an Chr. A. Lobeck und K. Lehrs", herausg. v. Arthur Ludwich, Th. II (Leipzig 1894), p. 933.

Jacobi wird wahrscheinlich die ganze Sache ${ }^{1}$ ) liegen lassen, und so würde dann (Jacobi's eigne Worte) die Mécanique céleste in dem état pitoyable bleiben, dans lequel Laplace l'a laissée.

\section{Schumacher an Gauss.}

Altona, 31. I. 1842.

s. Briefw. Gauss-Schumacher, Bd. 4 (1862), p. 56.

Frankreich schien [zu Napoleon I. Zeit], wie in den Waffen, so auch in der Mathematik unüberwindlich. Nachdem es nun

1) Rechnungen aus der Störungstheorie.

Ahrens, Scherz und Ernst i. d. Mathem. 
aber auf dem Kriegsfelde glücklich besiegt worden, haben wir, wie in der Sage von der Hunnenschlacht die Schatten in den Lüften fortkämpften, in den Regionen des Gedankens weitergekämpft, unterstützt von der heiligen Allianz mit dem Geiste, die Preussen geschlossen, und manchen glorreichen Sieg in den Wissenschaften erstritten. Und so rühmen wir uns auch in der mathematischen Wissenschaft, nicht mehr die zweiten zu sein.

$$
\text { C. G. J. JАСОBI } 1846 .
$$

Opuscula mathem. I, Widmung an Friedrich Wilhelm IV. = Jacobi, Werke, Bd. 7 (1891), p. 374.

Der Brief von La Grange über Laplace's mögliche Versetzung nach Berlin unter Friedrich dem II. hat Sie gewiss interessirt. Es herrscht dazu ein liebenswürdiger Ton in dem Briefe. So möchte jetzt nicht eine Correspondenz zwischen Poisson, Cauchy und unserem incisiven Jacobi aussehen. Andere Zeiten, andere Racen, auch andere Dimensionen!

Al. Huniboldt an Gauss.

Berlin, 21. II. 1840.

s. K. Bruhns, ,Briefe zw. A. v. Humboldt und Gauss" (1877), p. 48.

Vorige Woche führte Alerz [Arzt in Rom] Dirichlet und Jakoby ${ }^{1}$ ) zur Lady Somerville; da kamen sie Beide ganz aufgeregt zurück, die berühmte blue stocking hatte nämlich garnichts von Jakoby gewusst, nur von seinem Bruder, der ihr eine galvanisch vergoldete Medaille übersandt ${ }^{2}$ ) und von nichts als monsieur votre frère gesprochen, worüber seine Eitelkeit sehr verletzt war; aus lauter Grimm war er aber rasend witzig und geistreich, wir kamen den ganzen Abend nicht aus dem Lachen. - Freitag früh trinken Dirichlet und Jakoby bei Papstens Kaffee.

Rebecka Dirichlet an ihre Schwester Fanny Hensel. Rom, 27. XII. 1843.

1) s. S. 127 Anm.

2) Moritz Hermann J., der bekannte Erfinder der Galvanoplastik. 
Dirichlet warsehr enchantirt von Papsten [Gregor XVI.], er hat sich über eine halbe Stunde mit ihnen unterhalten über lauter mathematische Gegenstände und Personen und viel besser Bescheid gewusst als Lady So merville; sie meinen, er hätte sich präparirt. Es muss doch schön gewesen sein, Dirichlet auf den Knien den Pantoffel und Jakoby als Ketzer die Hand küssen zu sehn.

Dieselbe an dieselbe.

s. S. Hensel, „Die Familie Mendelssohn“, Bd. 2

(Berlin 1884), p. 272 u. 276.

Jacobi ist herrlich geheilt. Ich glaube er bleibt hier. Da er dem Könige doch sehr viel kosten muss (2500--3000 Thlr., fast so viel wie ein Schelling'scher Philosoph oder ein Missionair), so ist es rühmlicher das Ornament in der Hauptstadt zu besitzen. Er ist heiter und miles gloriosus, neben sich im Triumvirate nur 2 anerkennend, Gauss und Cauchy, - tout le reste lui parait de la vermine. Ich liebe diese Ausschliessungen nicht.

AL. Yon Humbolut an Schumacher.

3. VII. 1844.

s. Briefw. Gauss-Schumacher (1860-1865), Bd. 4, p. 267; s. a. ibidem Bd. 5, p. 205.

Cauchy, Gauss und Jacobi haben nur in Einzelheiten gearbeitet und es hat ihnen jener Sinn für logische und systematische Eleganz gefehlt, der in einem Lagrange die Beschränkung auf Stückwerk überwand und ihn für die antike Strenge eintreten liess. Ja es hat ihnen überall der Geist des 18. Jahrhunderts mit seiner logisch aufklärenden Richtung gefehlt; denn der Legitimist und Jesuitenlehrer Cauchy und der religiös beengte, ${ }^{\text {i) }}$ auf Fïrstenprotection und den Hofrathstitel eitle

1) vgl. S. 29 (Eugen Dühring). 
Maurersohn Gauss stellten wahrlich keine Typen im Sinne des 18. Jahrhunderts vor. Bei Jacobi aber stand die Neigung für einzelne isolirte Verïstelungen, um nicht zu sagen für das Abgerissene der Wissenschaft, dem abschliessenden Denken, wie es ein Lagrange geübt hatte, antipathisch entgegen, und es dürfte kein geringer Theil hievon auf die Raceneigenschaft zu verrechmen sein, obwohl diese bei einer Capacität nicht so übel wirken konnte, wie bei den stark jüdisch gemischten Epigonen von heute, unter denen der Sinn für das Ganze, für strenge Grundlegung und für klare Begriffsfassung vollends abhanden gekommen ist. - - - - - - - - - Überhaupt kann das 19. Jahrhundert auch in der formellen Behandlung der Mathematik als eine Zeit des Rückschlags gegen bessere Bestrebungen und als eine Phase der Erschlaffung des logischen und systematischen Geistes angesehen werden. Der vorangehende Aufschwung, wie er besonders durch Lagrange vorgestellt ist, hatte das Ziel noch nicht völlig erreicht, und da man nichts unmittelbar Übernehmbares blos weiter zu geben hatte, so ist nıan natürlich wieder nach rückwärts gelangt.

\section{Euger Dühring.}

„Kritische Gesch. der allgem. Principien der Mechanik", 2. umgearb. Aufl. der im nächsten Citat erwähnten Preisschrift (1877), p. 504/505.

Den angenehmen Eindruck des Ganzen vollendet eine sehr einfache aber an glücklichen Wendungen reiche Schreibart, die warme Anerkennung jedes Verdienstes, die erklärende Entschuldigung des Misslungenen und die vornehme Schonung, mit der über das Verkehrte hinweggegangen wird. - - -

Aus dem Urteil der GötTringer philos. Facultät über Eugen Dühring's Preisschrift [s. das vorhergehende Citat]. s. Göttinger Nachr. 1872, p. 152. 
Abel sender hermed en Afhandling om elliptiske Transcendenter, som han beder trykket saasnart mueligt, da Jacobi træder ham i Hælene, og han forgangen, da jeg flyede hem de sidste Nummer af Astron. Nachr. blev ganske bleeg, og maatte löbe til Conditoren og tage en bitter Snaps for at forvinde Alterationen. Han har i flere Aar været i Besiddelse af en almindelig Methode, som han her meddeler, og som omfatter mere end Jacobi's Sætninger.

[A bel sendet hiermit eine Abhandlung über elliptische Transzendenten, die er so bald wie möglich zu drucken bittet, da Jacobi ihm auf den Fersen folgt, und er neulich, als ich ihm die letzte Nummer der Astron. Nachr. hinreichte, ganz bleich wurde und zum Conditor laufen und einen bitteren Schnaps nehmen musste, um seine Erregung zu verwinden. Er ist seit mehreren Jahren im Besitz einer allgemeinen Methode gewesen, die er hier mitteilt und die mehr umfasst als Jacobi's Sätze.] **[HaNsteEn $]^{1}$ ) an Schumacher.

[7usatz Schumacher's (Altona, 6. VI. 1828) bei der Mitteilung des Vorstehenden an Gauss]:

„Wenn Sie einmal Ihre Untersuchungen ${ }^{2}$ ) bekannt machen, wird es ihm wahrscheinlich noch mehr an Schnaps kosten."

s. Briefw. Gauss-Schumacher, Bd. 2 (1860), p. 179.

La déduction d' $\mathrm{Abel}^{3}$ ) est au-dessus de mes éloges comme elle est au-dessus de mes propres travaux.

$J_{A C O B I}$ à Legendre.

Königsberg, 9. IX. 1828.

voir "Correspondance mathém. entre Legendre et Jacobi,“ éd. par Borchardt, J. f. Math. 80 (1875), p. 241

$=$ Jacobi, Werke, Bd. 1, p. 423.

1) s. in "Niels Henrik Abel, Mémorial publié à l'occasion du centenaire de sa naissance": Elling Holst, „Introduction historique", p. 96.

2) s. Note 7 am Ende des Buches.

3) s. Abel, „Solution d'un problème général concernant la transformation des fonctions elliptiques," Astron. Nachr. 6, No. 138 (1828) $=$ Oeurres compl., édition de Sylow etLie, t. 1(Christiania 1881), p. 403-428. 
('ertainement M. Jacohi va perfectionner à un degré inesperé non seulement la théorie des fonctions elliptiques mais encore les mathématiques en général. Je l'estime on ne peut plus.

ABes ì Legendre.

Christiania, 25. XI. 1828.

voir „Niels Henrik Abel, Mémorial publié à l’occasion du centenaire de sa naissance": „Correspondance d'Abel", p.90.

Tous serez sans doute dignes l'un de l'autre par la noblesse de vos sentimens et par la justice que vous vous rendrez réciproquement.

\section{Le GeNDRe à Abel.}

Paris, 25. ‥ 1828.

voir "Niels Henrik Abel, Mémorial publié à l'occasion du centenaire de sa naissance": „Correspondance d'Abel", p. 78.

Peu de jours après l'envoi de ma dernière lettre, j’appris la triste nouvelle de la mort d'Abel. Notre Gouvernement l'avait appelé à Berlin, mais l'appel ne l'a pas trouvé parmi les vivants. L'espérance que j’avais conçue de le trouver à Berlin a été si cruellemeut déçue. Les vastes problèmes qu'il s'était proposés, d'établir des critères suffisants et nécessaires pour qu'une équation algébrique quelconque soit résoluble, pour qu'une intégrale quelconque puisse être exprimée en quantités finies, son invention admirable de la propriété générale qui embrasse toutes les fonctions qui sont des intégrales de fonctions algébriques quelconques, etc., etc., marquent un genre de questions tout à fait particulier, et que personne avant lui n'a osé imaginer. Il s'en est allé, mais il a laissé un grand exemple.

\section{JACOBI à Legendre.}

Potsdam, 14. VI. 1829.

voir "Correspondance mathématique entre Legendre et Jacobi," éd. par Borchardt, J. f. Math. 80 (1875), p. 265,266

$=$ Jacołi, Werke, Bd. 1, p. 447. 
Legendre hat ein zweites Supplement herausgegeben, wo er in der Einleitung so von Abel spricht, dass es den Anschein hat, er setze ihn Jacobi'n nach. ${ }^{1}$ ) Ich weiss von Ihnen, dass grade das umgekehrte der Fall ist.

\section{Schumacher an Gauss.}

12. V. 1829.

s. Briefw. Gauss-Schumacher, Bd. 2 (1860), p. 210.

Malgré les hommages si largement rendus, malgré les sympathies les plus chaleureuses et les plus unanimes pour notre compatriote, il s'est maintenu une certaine confusion dans l'opinion générale relativement à la question de propriété. Cette manière de voir a particulièrement son expression dans l'admission d'une singulière communauté de propriété - grâce à laquelle, il est vrai, tant de choses se perdent et tant d'équivoques se produisent. Même dans l'honneur que l'on rend si volontiers à notre géomètre, il se mêle un certain vague. L'éloge est exprimé par de grands mots, qui peuvent sonner harmonieusement, mais qui seront oubliés un jour si la réalité qu'ils doivent exprimer, et qui appartient à $\mathrm{Abel}$ sans partage, ne peut lui être restituée.

Son contemporain et survivant, si hautement distingué, a eu le bonheur de travailler dans un grand pays, au milieu de relations plus étendues. La nouvelle doctrine et les nouvelles idées y poussaient des racines et se développaient rapidement. La période de l'indifférence, qui dura autant que la vie d'A bel, était passée. L'intelligence de cette théorie pénétrait dans des cercles de plus en plus vastes, et à la tête d'une nombreuse troupe d'élèves marchait Jacobi, comme le chef du nouveau mouvement. Abel, dans son étroite et lointaine patrie, était abandonné à lui-même et à son petit noyau d'intimes. Il n'avait pas, lui, une foule de disciples autour de lui; ses élèves,

1) Gemeint ist wohl das anscheinend zugleich mit dem 2. Supplement erschienene "Avertissement" zu dem dritten (die Supplemente enthaltenden) Bande des "Traité des fonctions elliptiques et des intégrales Eulériennes" (Paris 1825-1828); vgl. jedoch auch die Finleitung zu dem 1. Supplement. 
nous pouvons le dire, c'était le petit nombre de ses anciens maîtres; c'était le grand Gauss ${ }^{1}$ ); c'était, dans une sphère, certainement moins élevée, mais néanmoins très éclatante, Jacobi. Il y avait aussi là le vieux Legendre - bien qu'il lui semblât assez difficile de suivre la bannière révolutionnaire du novateur ${ }^{2}$ ).

\section{C.-A. BJerknes.}

„Niels-Henrik Abel", Traduction française

(Paris 1885), p. 120/121.

Abel étant mort.., Jacobi se trouva pendant longtemps avoir la réputation d'un homme, qui, dans les mêmes proportions que lui, avait déterré une part essentielle du trésor sur lequel le dix-neuvième siècle a vécu en mathématiques. Maintenant que nous avons un certain recul de temps, les compatriotes de Jacobi eux-mêmes donnent le nom d'Abel aux sommets les plus escarpés et aux profils les plus audacieux de la région mathématique mouvementée qui fut alors découverte. Mais A bel mourut, et Jacobi, jeune géant lui-même, prit sur ses épaules les idées de tous deux, et les porta plus loin.

\section{Elling Holst.}

„Niels Henrik Abel, Mémorial publié à l'occasion du centenaire de sa naissance": „Introduction historique", p. 94.

La trop brève carrière de ces deux mathématiciens [A bel et Galois] se développa au moment où une ère nouvelle commençait, tant en analyse qu'en géométrie. - - - -

A la tête des savants de cette nouvelle époque, Gauss et Cauchy, Abel et Galois doivent être mis en première ligne; et s'il est juste de nommer, immédiatement après ces génies créateurs, Jacobi, dont le talent brillant s'est attaqué [attaché] à tant de branches des mathématiques, à mon avis, pour l'originalité, la puissance et la profondeur, il ne saurait toute-

1) s. S. 105, sowie Note 7 am Ende des Buches.

2) s. Note 11 am Ende des Buches. 
fois être comparé aux quatre mathématiciens cités plus haut. Parmi les successeurs de ces maîtres, Riemann occupe une place particulièrement brillante, bien que l'exposition de ses idées, si originales, si profondes et si fécondes, manque souvent de la clarté, parfois même de la rigueur désirables.

S. LIE.

„Influence de Galois sur le développement des mathématiques," Le centenaire de l'école normale (1895), p. 482.

Sophus Lie joignait malheureusement, comme Galois, au génie qui fait tout pardonner, le grave défaut, de manquer de clarté. - - - -

Jaco bi est, suivant de bons juges, le plus illustre géomètre du siècle; pour quelques-uns même, le plus grand qui ait jamais existé. Il n'est pas, suivant Sophus Lie, comparable à Galois. De telles appréciations ne peuvent se discuter.

\section{J. Bertrand.}

„Éloges académiques", Nouvelle série (Paris 1902), p. 344 et 345.

Abel, der gewohnt war, überall den höchsten Standpunkt zu nehmen - - - -

\section{Weierstrass.}

Antrittsrede in d. Berliner Akademie.

s. Berl. Monatsber. 1857, p. 349.

Ce qui surtout est la marque d'Abel, outre sa richesse d'idées, c'est son effort vers la rigueur absolue, la grande généralité avec laquelle il pose les problèmes, et sa manière de les épuiser. Une autre particularité encore, c'est qu'il ne se sert dans son exposition que de moyens si simples; il semble que tout sorte aisément du bon choix de la position du problème. Les deux mémoires sur la transformation [des fonctions elliptiques] et celui sur les équations abéliennes me paraissent typiques à cet égard. Il n'a pourtant pas obtenu ses résultats 
nouveaux sans se servir de nouveaux instruments, comme, par exemple, l'irréductibilité des équations algébriques et la résolvante de Galois. Mais il n'y consacre que peu de mots et n'insiste pas sur leur grande valeur pour l'analyse en général. . . . . . Il montre en tout cela la même simplicité et la même modestie que dans toute sa vie; et pourtant il était et devait être conscient de sa propre valeur. - - - - - - -

Chacune des théories dont il s'est occupé dans ses dernières années porte en effet la marque durable de sa main. Son nom est devenu populaire parmi les mathématiciens; il n'y a aucun nom dont on se soit servi si volontiers que celui d'A bel, toutes les fois qu'il s'est agi de désigner des théories ou des idées nouvelles. Cela est caractéristique; car l'adjectif a bélien n'a été employé que pour des idées et des théories, créées par A bel lui-même, ou basées sur ses découvertes.

\section{Sylow.}

"Les études d'Abel et ses découvertes", p. 58/59 dans „Niels Henrik Abel, Mémorial publié à l'occasion du centenaire de sa naissance".

Auffallender Weise hat eine so wichtige Function noch keinen andern Namen, als den der Transcendente $\Theta$, nach der zufälligen Bezeichnung, mit der sie zuerst bei Jacobi erscheint, und die Mathematiker würden nur eine Pflicht der Dankbarkeit erfüllen, wenn sie sich vereinigten ihr Jacobi's Namen beizulegen.

\section{Dirichlet.}

„Gedächtnissrede auf Carl Gustar Jacob Jacobi.“

s. Abhandl. d. Berliner Akad. 1852, p. 13

= Dirichlet, Werke, Bd. 2, p. 239

$=$ Jacobi, Werke, Bd. 1, p. 14/15.

Steiner sprach Jacobi zu Ehren, der mit Hülfe des willkürlichen Parameters in einem Curvenbüschel ihm verschiedene wichtige Aufgaben löste, in der Vorlesung voller Bewunderung 
von der Brauchbarkeit des Judencoeffizienten, und einem jüngeren Mathematiker, der ihm einmal mit einem Satze aus der Determinantentheorie aushelfen konnte, rief er mit einem unbeschreiblichen Blicke, der aus Misstrauen und Anerkennung: seltsam gemischt war, zu: „So-0-o, der Schlingel versteht auch Determinanten?“

\section{F. Geiser.}

„Zur Erinnerung an J. Steiner" (1874), p. 29.

Je vois sans regret les fonctions $s n, c n$ et $d n$ reléguées au second plan; mais qu'il me soit permis de plaider un peu en faveur de la fonction $\Theta$; la série si simple qui la représente met presque immédiatement en évidence ses propriétés essentielles, et cet avantage me semble compenser bien des inconvénients; je crois done qu'elle ne tardera pas, après une disgrâce passagère, à reprendre son rang à côté de la fonction $\sigma$.

H. Poincaké.

„Notice sur Halphen,“ Journal de l'école polytechnique, (cah. 60 (1890), p. 158.

Der Tod, welcher Jacobi so früh ..... von der Arbeit hinweggenommen, hat der Wissenschaft die grossen Bereicherungen nicht gegönnt, die sie von Jacobi's nie ermüdender Thätigkeit noch erwarten durfte.

Dirichlet.

„Gedächtnissrede auf Carl Gustav Jacob Jacobi.“ s. Abhandl. Berl. Akad. 1852, p. 26

$=$ Dirichlet, Werke, Bd. 2, p. 252

$=$ Jacobi, Werke, Bd. 1, p. 28.

Jacobi's so unerwartetes Hinscheiden ... . . ist ein grosser Verlust für die Wissenschaft. So schmerzhaft mir auch, schon wegen seiner früheren Verhältnisse zu dem Könige, das ganz zwecklose und kindische Benehmen zu einer andern Zeit [war], 
so habe ich ausdauernd und mit Erfolg die Schritte gethan, die ihn Preussen erhalten konnten. Meinen inneren Gefühlen ist er nie nahe gewesen. Sein Tod regt mich nur an endlich einmal dem talentvollen [, trefflichen leider! kränkelnden, mir immer gleich lieben, von den Berliner mathematischen Grossmächten wenig gepflegten" (Brief rom 23. III. 1847, 1. c. p. 54)] Eisenstein eine geziemende Anerkennung zu verschaffen.

\section{Al. Hunbold' an Gauss.}

Berlin, 22. II. 1851.

s. K. Bruhns, ,Briefe zw. A. v. Humboldt und Gauss" (1877), p. 56.

Allerdurchlauchtigster, Grossmächtigster König,

Allergnädigster König und Herr!

Sire, in tiefster Unterthänigkeit naht sich die synthetische Geometrie den Stufen Höchstdero erhabenen Thrones, um in demüthigster Ehrfurcht ihr Schärflein zu den neusten Entdeckungen der allgewaltigen Analysis niederzulegen.

Ew. Majestät wollen es aber auch nicht ungnädig vermerken, wenn ich mich unterthänigst unterfange, von der Anziehung des Ellipsoids zu sprechen!

Ich seh', o $\operatorname{Rex}^{1}$ ), Deinen gnädigen Blick, der von Mitleid, Verachtung und Neugier gemischt, mir zu reden gebeut. Wohlan denn, ich erdreiste mich zu reden. - - - -

Nun adieu Rex! Glück im neuen Jahr! Der Herr lasse sein Antlitz über dem Deinigen leuchten und segne Dein Haus! Amen.

Berlin, den 31. Dec. 1833.

Dein treuer

Jakob.

Anfang und Ende eines Briefes von Steiner an Jacobi, mitgeteilt von E. Jahnke, Arch. Math. Phys. (3) 4 (1903), p. $269 / 270$ u. 275 .

1) Steiner pflegte auch sonst Jacobi „Rex“ zu nennen, während Dirichlet „Marquis" hiess (s. z. B. den auf S. 143 citierten Briefwechsel Steiner-Schläfli, p. 239 resp. 96). 
Der synthetischen Geometrie widmete sich Steiner mit unermüdlicher und ausschliesslicher Thätigkeit, bis zu dem Grade der Schwärmerei, dass er es wie eine Schmach der Synthesis aufnahm, wenn bisweilen die Analysis, deren Macht er nicht unterschätzte, gleiche oder gar weitergreifende Resultate brachte.

Otтo Hesse.

„Jakob Steiner“, J. f. Math. 62 (1863), p. 200.

Der Analysis muss durch die Forschungen der Synthesis vorerst die richtige Grundlage gegeben werden, auf der sie sodann, ihre Kraft entfaltend, mit Erfolg weiter bauen kann; wie dies überhaupt in der Geometrie meist geschah, ohne dass man es immer eingestand.

\author{
J. STEINER. \\ „Über Maximum und Minimum" etc. \\ s. Werke, heransg. v. Weierstrass, Bd. 2 (1882), p. 181.
}

Wenn nun wirklich in diesem Werke gleichsam der Gang, den die Natur befolgt, aufgedeckt wird, so werden alle hier synthetisch entwickelten Resultate sich natürlicher Weise auch durch analytische Hülfsmittel auffinden lassen, was meines Erachtens durchaus nichts Überraschendes in sich tragen kann. Der Analyst, der dieses ausführt, hat nicht mehr als seine Pflicht gethan, wenn er jeden Fortschritt der Wissenschaft benutzt und sich denselben so zur Lehre dienen lässt, dass seine Methode darnach vervollständigt wird. Auch ist es recht eigentlich seine Sache, jene Resultate zu verallgemeinern, und ich sollte meinen, dass seine Arbeit nicht an ihrem Werthe verlieren würde, wenn er es unterliesse, gegen seinen Wegweiser sich vornehm zu geberden.

\title{
J. Steiner.
}

„System. Entw. d. Abhäng. geometrischer Gestalten von einander," Vorrede.

s. Werke, herausg. v. Weierstrass, Bd. 1 (1881), p. 234. 
Mit den Namen Plücker und Steiner ist für die deutsche Geometrie die Trennung in sogenannte analytische und synthetische Geometrie gegeben, welche bis in die allerneueste Zeit die Geometer in zwei getheilte Lager gespalten hat. Steiner hatte in der unmittelbaren geometrischen Anschauung das hinreichende Hülfsmittel und den einzigen Gegenstand seiner Erkenntniss erblickt, während Plücker in der Identitüt der analytischen Operation und der geometrischen Construction die Quelle seiner Beweise suchte und geometrische Wahrheit nur als eins der vielen denkbaren Gegenbilder analytischer Beziehung betrachtete. Und doch sind die Ziele, welche die beiden Forscher verfolgten, in vieler Hinsicht nahe verwandt: der Fortschritt der Wissenschaft hat es inzwischen ermöglicht, fast an allen Stellen zwischen den formal geschiedenen Betrachtungsweisen den Übergang zu bewerkstelligen. Das gemeinsam Characteristische: die projectivische Anschauung und die Anlehnung an die Begriffsbildung der Algebra tritt je länger je mehr in den Vordergrund, und die Bevorzugung der einen oder anderen Ausdrucksweise erscheint als etwas verhältnissmässig Nebensächliches.

Nachruf auf Clebscin von einigen seiner Freunde. Math. Ann. 7 (1874), p. 12.

Può anzi dirsi che per lui [Cayley], come per qualche altro fra $\mathrm{i}$ più eminenti geometri, a ciascun nuovo risultato ottenuto nella analisi rispondesse un nuovo risultato geometrico, e reciprocamente. E ciò spiega per quali ragioni sopra una stessa quistione geometrica egli ritornasse più volte, e vi ritornasse appunto quando, scoperte analitiche di sorgente specialmente propria, od anche d'altri, prestavano a lui uno strumento più potente di indagine.

Brioscill.

"Notizie sulla vita e sulle opere del Cayley", Atti della Accademia dei Lincei (5) 4,1 (1895), p. 182. 
Ihren Gruss habe ich Steiner überbracht. Den kleinen Krieg mit ihm dürften Sie weniger ernst nehmen. Er ist zu sehr Hypochonder, als dass er von dem abgehen sollte, was er sich einmal in den Kopf gesetzt hat. Er scheint sich schon durch Ihre früheren Arbeiten verletzt zu fühlen, wie überhaupt durch die der Analytiker, und es ist besonders Plücker, den er gründlich hasst, und welcher wohl seine geringe Zärtlichkeit für die Analysis hervorgerufen hat. Übrigens dürfte Steiner seine wichtigsten Entdeckungen immer publicirt haben und nur neue Methoden für die Behandlung bekannter Probleme in seinen Manuskripten zurückbehalten. Von dem Probleme der Wendepunkte für Curven vierten Grades hat er oft gesagt, dass er daraus „nichts Gescheidtes“ machen kann. Es scheint überhaupt die Synthesis der Gränze nahe zu sein, wo sie ohne die Hülfe der Analysis nicht weiter kommen kann. Vielleicht irre ich mich, wenn ich aus dem Umstande, das Steiner recht gerne analytische Resultate benutzt, einige Gründe für die Behauptung entnehmen kann.

\section{S. Aroniold an Hesse.}

Berlin, 18. XII. 1849.

s. S. Gundelfinger, „Drei Briefe Aronholds an Hesse“, J. f. Math. 124 (1902), p. 64.

Ich kann leider nicht mehr wie früher arbeiten; die Phantasie ist fast ganz erloschen, das erschlaffte Gangliensystem wirkt auf das Gehirn, so dass ich beim besten Willen, etwas zu thun, immer einschlafe, sobald ich die Augen zumache, um die Gegenstände anzuschauen. Der kleine Jüd ${ }^{1}$ ) stände mir wohl zur Hand, aber was habe ich davon; was sich durch Rechnung von selbst Weiteres einstellt, theilt er mir nicht mit, und was von mir stammet, wird mir wenig verdankt,

1) Aronhold, den Steiner sonst auch ,den Schmützer" nannte (s. z. B. l. c. p. 239). 
wird als aus der Rechnung hervorgehend benützt, ohne des Urhebers zu gedenken.

STELNer an Schläfli.

Marienbad, 31. VII. 1851.

s. J. H. Graf, „Wer Briefwechsel zwischen Jakob Steiner und Ludwig Schläfli", Bern Mitth. Naturf. Ges. 1896, p. 74.

Sitôt qu'un auteur ingénieux a su parvenir à quelque vérité nouvelle, n'est-il pas à craindre que le calculateur le plus stérile ne s'empresse d'aller vite la rechercher dans ses formules, de la découvrir une seconde fois, et à sa manière, qu'il dit être la bonne et la véritable; de telle sorte qu'on ne s'en croie plus redevable qu'à son analyse, et que l'auteur lui-même, quelquefois peu exercé, ou même étranger à ce langage et à ces symboles sous lesquels on lui dérobe ses idées, ose à peine réclamer ce qui lui appartient, et se retire presque confus, comme s'il avait mal inventé ce qu'il a si bien découvert? Singulier artifice, que je n'ai pas besoin de caractériser davantage, mais qu'il est bon de signaler comme un des plus nuisibles aux progrès des sciences, parce qu'il est sans contredit un des plus propres à décourager les inventeurs!

\section{Poinsot.}

„Théorie nouvelle de la rotation des corps", Journal de mathém. pures et appliquées 16 (1851), p. 89.

On se tromperait en croyant que la géométrie, dans ses moyens de procéder à la recherche de la vérité, doit avoir des bornes posées seulement par la nature de cette science, et non par la nature même des choses. . . Si la géométrie ne peut pas s'élever à des questions d'un certain ordre, l'analyse doit donc également se trouver insuffisante pour atteindre à leur solution. Ainsi, de même que la géométrie ne peut pas offrir de moyens généraux pour décrire complettement les lignes de courbure des surfaces supposées quelconques, l'analyse ne 
présentera pas non plus de méthode qui s'étende à la fois aux surfaces de tous les genres et qui puisse offrir, dans tous les cas, l'équation immédiate de leurs lignes de courbure. Cette équation, effectivement, ne pouvant être obtenue que par l'intégration de fonctions dépendantes de la forme qu'affecte la surface, l'intégration demeure impossible tant que la surface, et par conséquent ces fonctions ne cessent pas d'être générales et indéterminées.

\section{DUPIN.}

„Développements de géométrie“ (Paris 1813), p. 236/237.

Plücker hat die Grundgedanken der neueren Geometrie in die Algebra hinübergetragen. Aber seine Interessen waren nach vielen Seiten begrenzt. . . . Die synthetische Richtung, wie sie Steiner vertrat, konnte und mochte Plücker durchaus nicht gelten lassen ${ }^{1}$ ), er betrachtete sie als überflüssig, wo nicht als schädlich. Und auch in der Analysis hatte er starke Antipathien. Seine Hauptleistung war es gewesen, das unnötige Rechnen in der analytischen Geometrie zu vermeiden und aus den zweckmässig zusammengezogenen Formeln heraus die Schlussresultate unmittelbar abzulesen. Um so weniger mochte er sich mit Hesse befreunden, der, als Schüler Jacobis, das Rechnen auch in der Geometrie wieder zu Ehren brachte und mit Virtuosität übte.

Felix Klein.

Antrittsrede Leipzig Univ. 25. X. 1880.

s. Zeitschr. math. naturw. Unterr. 26 (1895), p. 537.

Gleich nach der Berufung nach Bonn bei dem Tode v. Münchow's war Plücker auch das Directorium des physikalischen Cabinetes übertragen worden. Da Plücker jedoch bis dahin nur im Gebiete der Mathematik schriftstellerisch aufgetreten war, so wurde ihm vorgeworfen, er sei überhaupt kein Physiker und es wurde im Jahre 1839 der mit den

1) vgl. S. 143 (Aronhold an Hesse).

A hrens, Scherz und Ernst i. d. Mathem. 
Zwecken seiner Versetzung völlig unbekannte Radicke von Berlin als Professor nach Bonn geschickt mit dem ausgesprochenen Zwecke, Plücker als Physiker zu verdrüngen. Letzterem wurde, indem man seine Qualification als Physiker in Zweifel zog, zugemuthet, er möge das Directorium des physikalischen Cabinetes mit Radicke theilen ..... Es ist selbstverständlich, dass Plücker diese Zumuthungen, als seiner Ehre widersprechend, ablehnte... Hierauf wandte sich Plücker mit dem grössten Eifer und mit den besten Erfolgen physikalischen Untersuchungen zu. - - - -

Hatte nun Plücker sicher durch seine Arbeiten nachgewiesen, dass er ein Physiker ersten Ranges war, so dass gegen ihn ein Vorwurf in dieser Richtung ein Hieb gewesen wäre, der den Führenden selbst getroffen haben würde, so war er inzwischen (1853) grade von der umgekehrten Seite angegriffen worden. Ein Mathematiker in Bonn stellte die Behauptung auf, Plücker sei kein Mathematiker, wahrscheinlich in der Voraussetzung, die analytische Geometrie gehöre nicht in das Gebiet der Mathematik; dieser Behauptung fügte er noch als Erläuterung hinzu, er selbst docire die "schönen" Theile der Mathematik. Zunächst sah Plücker in Folge dieser Intriguen sich gezwungen, sein Amt als Director der wissenschaftlichen Prüfungs-Commission, das er siebzehn Jahre mit dem segensreichsten Erfolge verwaltet hatte, niederzulegen, und aus dieser Commission auszuscheiden. Heine wurde an seiner Stelle Examinator der Mathematik. Ein Versuch auch die Stelle eines Professor ordinarius zu erlangen glückte jedoch nicht, indem hier eine bedeutende Concurrenz [der von der Fakultät und von Plücker selbst empfohlene A. Beer] eintrat.

\section{A. Dronke.}

„Julius Plücker" (Bonn 1871), p. 13 u. 16/17.

Heutzutage wird kein Zweifel mehr darïber sein, dass die Principien der Analytiker [Moebius, Plücker etc.] die weiter- 
tragenden gewesen sind. In der That fehlte den Synthetikern [Steiner, Chasles u. a.] zweierlei, um eine allgemeine Theorie der algebraischen Gebilde in Angriff zu nehmen: einerseits die Definition der imaginären Elemente, andererseits eine Begriffsbestimmung des Algebraischen überhaupt. Nun hat man ja später Beides .... in synthetischer Form entwickelt, aber man hat dabei das eigentliche Princip der synthetischen Geometrie zur Seite schieben müssen. Dieses Princip, wie es in der Theorie der geradlinigen Figuren und der Gebilde zweiten Grades so glänzend hervortritt, ist die unmittelbare Beweiskraft der anschauungsmässigen Construction.

Felix Klein.

s. Autogr. Vorles. üb. „Riemann'sche Flächen“, S. S. 1892, Zweiter Abdruck (Göttingen 1894), I, p. 231/232.

Il est impossible d'étudier les OEuvres des grands mathématiciens et même celles des petits, sans remarquer et sans distinguer deux tendances opposées, ou plutôt deux sortes d'esprits entièrement différents. Les uns sont avant tout préoccupés de la logique; à lire leurs Ouvrages, on est tenté de croire qu'ils n'ont avancé que pas à pas ... Les autres se laissent guider par l'intuition et font du premier coup des conquêtes rapides, mais quelquefois précaires - - -

Ce n'est pas la matière qu'ils traitent qui leur impose l'une ou l'autre méthode. Si l'on dit souvent des premiers qu'ils sont des analystes et si l'on appelle les autres géomètres, cela n'empêche pas que les uns restent analystes même quand ils font de la Géométrie, tandis que les autres sont encore des géomètres, même s'ils s'occupent d'Analyse pure. C'est la nature même de leur esprit qui les fait logiciens ou intuitifs, et ils ne peuvent la dépouiller quand ils abordent un sujet nouveau.

Ce n'est pas non plus l'éducation qui a développé en eux l'une des deux tendances et qui a étouffé l'autre. On naît 
mathématicien, on ne le devient pas, et il semble aussi qu'on naît géomètre, ou qu'on naît analyste.

H. Poincaré.

„Du rôle de l'intuition et de la logique en mathématiques." voir C. R. du deuxième congrès intern. des mathém. Paris 1900 (Paris 1902), p. 115/116.

Depuis son enfance, Chasles ne buvait que de l'eau..... Un géomètre illustre a trouvé plaisant à dire: „Si M. Chasles buvait du vin, il ferait peut-être du calcul intégral!“

J. Bertrand.

„Éloges académiques“, Nouvelle série (Paris 1902), p. 40.

. . it must be said that the degree of exactness of the intuition of space may be different in different individuals, perhaps even in different races. It would seem as if a strong naïve space-intuition were an attribute pre-eminently of the Teutonic race, while the critical, purely logical sense is more fully developed in the Latin and Hebrew races. A full investigation of this subject, somewhat on the lines suggested by Francis Galton in his researches on heredity, might be interesting.

Felix Klein.

„The Evanston colloquium", Lectures on mathematics Evanston Jll. 1893 (New York 1894), p. 46.

Die Kraft der geometrischen Vorstellung hat der ganzen Persönlichkeit [Steiners] ein eigenes Gepräge auch über die wissenschaftliche und paedagogische Thätigkeit hinaus gegeben. Sie befähigte Steiner in hohem Maasse, plastische Kunstwerke zu beurtheilen, wie sich namentlich auf seiner italienischen Reise [1843] zeigte, da er ohne Anleitung bei verstümmelten 
Statuen die Intentionen des Bildhauers aufzufinden und die angebrachten Restaurationen in Bezug auf ihre Richtigkeit mit einem Scharfsinn zu prüfen verstand, der von Sachkennern in hohem Maasse anerkannt wurde.

\section{F. Geiser.}

„Zur Erinnerung an J. Steiner“ (1874), p. 30/31.

Clebsch war in erster Linie Algebraiker, und allen seinen Arbeiten gemeinsam ist die vollendete Beherrschung des algebraischen Apparates. Ihr zur Seite stellt sich in den späteren Untersuchungen die klare geometrische Auffassung, vermöge deren jeder Schritt, den die Rechnung vollführt, zu einem anschaulichen Verständnisse gebracht wird. Aber es ist nicht die concrete Art, die räumlichen Verhältnisse zu sehen, wie wir sie bei manchen anderen Geometern finden; die geometrische Anschauung ist ihm mehr Symbol und Orientierungsmittel für die algebraischen Probleme, mit denen er sich beschäftigt. - -

Clebsch hat Jacobi nicht persönlich gekannt, aber er hat dessen Werke mit Vorliebe studirt und sich später geradezu gelegentlich als Schüler desselben bezeichnet.

Nachruf auf Clebsch von einigen seiner Freunde. Math. Ann. 7 (1874), p. $3 / 4$ u. 7.

Sicherlich wird man Lie .... . in erster Linie nicht als Analytiker, sondern als Geometer zu bezeichnen haben. Aber seine Anschauung bezieht sich . . nur auf das Verhalten von Gebilden im Unendlich-Kleinen oder begrenzten Gebiete. Das concrete Bild einer Gesammt-Mannigfaltigkeit in ihrer Gestalt, d. h. in ihren endlichen Formenbeziehungen, deren Wechsel bei variabeln Parametern, die Realitätsfragen überhaupt, haben Lie nie interessiert. In jenem Gebiet aber, das in den geometrischen Elementen und Operationen nur eine Versinnlichung, 
eine Zusammenfassung von ganz abstracten Begriffshildungen, oft von den complicirtesten, sieht, war Lie Meister. - - -

Die begriffliche Auffassung selbst ist für Lie auch späterhin immer das Primäre geblieben; sie ist es, welche Lie, wie in den Untersuchungen über Differentialgleichungen, so auch in seinen späteren Forschungen, in der Gruppentheorie und ihren vielgestaltigen Anwendungen, leitet; und die unmittelbare Zuriickführung auf die Grundbegriffe allein ist es, welche ihn in seinen Schlïssen befriedigt.

Der Gruppen- und Invarianten-Gedanke war Lie nicht nur ein methodischer Gesichtspunkt, von dem aus er das ganze alte Gebiet der Mathematik neu durchdenken wollte, sondern auch das Element, das nach und nach alle mathematische Wissenschaft durchdringen oder einheitlich verbinden sollte.

Sein ganzes Denken, wie sein Wissen, stand weit ab von jener Richtung, welche, durch grosse Forscher gepflegt, als „Praecisionsmathematik“ im Laufe dieses Jahrhunderts allmählich emporgestiegen ist und am Ende desselben fast die allein herrschende zu sein scheint: gerade ihr gegenüber steht Lie da wie rückweisend auf die früheren Classiker der infinitesimalen und projectiven Geometrie, als lebendiges Zeugniss, dass auch deren Gedankengebiet unter dem Einfluss eines naiv-schöpferischen Genies, das beide Geometrien vereint, wieder fruchtbar werden und zu unabsehbaren Neuentwickelungen, neu nach Inhalt und Tragweite, gelangen kann.

\section{NoEther.}

"Sophus Lie",

Math. Ann. 53 (1900), p. 1415, 18/19, 39, 39/40.

During a conversation with the writer in the last weeks of his life, Sylvester remarked as curious that notwithstanding he had always considered the bent of his mind to be rather analytical than geometrical, he found in nearly every case that 
the solution of an analytical problem turned upon some quite simple geometrical notion, and that he was never satisfied until he could present the argument in geometrical language.

P. A. M[aC Mahon].

London Roy. Soc. Proc. 63 (1898), p. XVII.

Man muss nichtwähnen, dass Weierstrass den von Riemann [für das Studium der algebraischen Functionen und ihrer Integrale] betretenen Weg missachtete; er hat es ja selbst ausgesprochen, dass dem Forscher jeder Weg frei steht, dass es sich aber um die Begründung handelt. ${ }^{1}$ ) Jedenfalls war er von Bewunderung erfüllt für die Leistungen seines dem Leben nur $\mathrm{zu}$ früh entrissenen Rivalen. - -

\section{E. LAMPE.}

"Karl Weierstrass",

Gedächtnisrede Berlin Phys. Ges. 5. III. 1897, p. 13

$=$ Deutsche Mathem.-Verein. Jahresber. 6, 1897, p. 35.

La méthode de Riemann [dans la théorie des fonctions analytiques] est avant tout une méthode de découverte, celle de Weierstrass est avant tout une méthode de démonstration.

\section{H. Poincaré.}

"L'oeuvre mathématique de Weierstrass", Acta mathem. 22 (1899), p. 7.

C'est l'intuition du nombre pur, celle des formes logiques pures qui éclaire et dirige ceux que nous avons appelés analy stes.

C'est elle qui leur permet non seulement de démontrer, mais encore d'inventer. C'est par elle qu'ils aperçoivent d'un coup d'oeil le plan général d'un édifice logique, et cela sans que les sens paraissent intervenir.

1) s. das Citat auf S. 66. 
En rejetant le secours de l'imagination, qui ... n'est pas toujours infaillible, ils peuvent avancer sans crainte de se tromper. Heureux donc ceux qui peuvent se passer de cet appui! Nous devons les admirer, mais combien ils sont rares!

Parmi les analystes, il y aura donc des inventeurs, mais il y en aura peu.

\section{H. Poincaré.}

"Du rôle de l'intuition et de la logique en mathématiques." voir $\mathrm{C} . \mathrm{R}$. du deuxième congrès intern. des mathém. Paris 1900 (Paris 1902), p. 129.

Ich bedaure jeden Mathematiker herzlich, dem die Natur kein lebhaftes Raumvorstellungsvermögen verliehen hat.

Felix Klein.

„C̈ber Aufgabe und Methode des mathematischen Unterrichts an den Universitäten", Vortr. Naturf.-Vers. Düsseldorf 1898.

s. Deutsche Mathem.-Verein. Jahresber. 7, 1897/1898, p. 137.

Die Analysis zieht einem die Schlafkappe über den Kopf. Bei uns heisst es: Augen aufsperren, dann sieht man die Sachen auch.

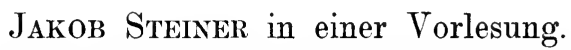

s. M. Cantor in „Allg. Deutsche Biogr.“, Bd. 35 (1893), p. 702.

Der Aufsatz von La Place in der Conn. des tems 1816 ist meinem Urtheile nach dieses grossen Geometers ganz unwürdig. Ich finde zwei verschiedene, sehr arge Missgriffe darin. ${ }^{1}$ ) Ich hatte mir bisher immer vorgestellt, dass bei den Geometern vom ersten Range der Kalkul immer nur das Kleid

1) s. die ausführlichere Besprechung bei Gauss, Werke, Bd. 6 (1874), p. $581-583$. 
sei, in dem sie das, was nicht durch Kalkul, sondern durch Meditation über die Sache selbst geschaffen, vorführen ${ }^{1}$ ). - Gauss an Olbers.

Göttingen, 31. XII. 1831.

s. "Wilhelm Olbers, Sein Leben und seine Werke", herausg. v. C. Schilling, Bd. 2 (1900), p. 567.

Gauss bemerkte ein Mal, dass manche der namhaftesten Mathematiker, Euler sehr oft, selbst mitunter Lagrange ${ }^{2}$ ), dem Calcul zu sehr vertrauend sich nicht in jedem Augenblick vom Gang ihrer Untersuchungen hätten Rechenschaft geben können. Er könne dagegen von sich behaupten, dass er bei jedem Schritt den er gethan immer den Zweck und das Ziel seiner Operationen genau vor Augen gehabt habe, ohne vom Wege abzukommen. Dasselbe sei auch von Newton zu sagen.

Sartorius v. Waltershausen.

„Gauss zum Gedächtniss" (1856), p. 80.

Pour peu que l'objet qu'on poursuit soit compliqué, les méthodes algébriques forcent de l'oublier totalement, pour ne songer qu'à leurs formules; la route qu'on suit est assurée; mais le but où l'on veut arriver, le point d'où l'on est parti, disparaissent également aux regards du géomètre; et il a fallu longtemps du courage pour oser perdre la terre de vue, et s'exposer sur la foi d'une science nouvelle.

Condorcet.

Oeurres, Édition d'Arago, t. 3 (1847), p. 8.

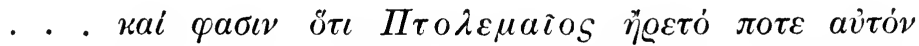

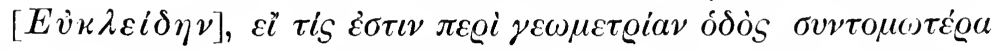

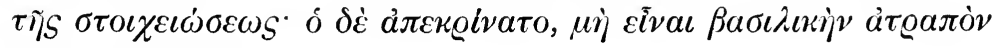
$\varepsilon \dot{\pi} i \quad \gamma \varepsilon \omega \mu \varepsilon \tau \varrho i \alpha \nu$.

[. . . und man sagt, dass Ptolemäus ihn [Euklid] einmal gefragt habe, ob es nicht für die Geometrie einen

1) vgl. S. 44 (Gauss an Schumacher).

2) s. Note 1 am Ende des Buches. 
kürzeren Weg als die Unterweisung in den „Elementen“ gebe; er aber antwortete, es gebe zur Geometrie hin keinen Richtsteig für Könige.]

Proclus Diadochus.

„In primum Euclidis elementorum librum commentarii“", ed. Friedlein (1873), p. 68.

Es ist eine saure Arbeit, die ich gethan habe, und eine saure Arbeit, in der ich begriffen bin. Nicht Fleiss und Gedächtniss sind es, die hier zum Ziele führen, sie sind hier die untergeordnetsten Diener des sich bewegenden reinen Gedankens. Aber hartnäckiges, hirnzersprengendes Nachdenken erheischt mehr Kraft als der andauerndste Fleiss. Wenn ich daher durch stete Übung dieses Nachdenkens einige Kraft darin gewonnen habe, so glaube man nicht, es sei mir leicht geworden, durch irgend eine glückliche Naturgabe etwa. Saure, saure Arbeit hab' ich zu bestehen, und die Angst des Nachdenkens hat oft mächtig an meiner Gesundheit gerüttelt. - - - -

C. G. J. JАCOBI (20-jährig) in einem Brief a. d. J. 1824. s. Werke, Bd. 1, p. 23 (Dirichlet, „Gedächtnissrede auf Jacobi“).

Sie erinnern Sich . . vielleicht meiner Klagen über einen Satz, der . . . . . alle meine Bemühungen, einen genügenden Beweis zu finden, vereitelt hatte. Dieser Satz ist schon in meinen Disq. [arithm.] pg. 636 ${ }^{1}$ ) angedeutet . . . . . . . . die Bestimmung eines Wurzelzeichens ist es gerade, was mich immer gequält hat. Dieser Mangel hat mir alles Übrige, was ich fand, verleidet; und seit 4 Jahren wird selten eine Woche hingegangen sein, wo ich nicht einen oder den andern vergeblichen Versuch, diesen Knoten zu lösen, gemacht hätte besonders lebhaft nun auch wieder in der letzten Zeit. Aber alles Brüten, alles Suchen ist umsonst gewesen, traurig habe

1) In den gesammelten Werken von Gauss Bd. I, S. 442-443 [Anm. des Herausgebers C. Schilling]. 
ich jedesmal die Feder wieder niederlegen müssen. Endlich vor ein paar Tagen ist's gelungen - aber nicht meinem mühsamen Suchen, sondern bloss durch die Gnade Gottes möchte ich sagen. Wie der Blitz einschlägt, hat sich das Räthsel gelöst: ich selbst wäre nicht im Stande, den leitenden Faden zwischen dem, was ich vorher wusste, dem, womit ich die letzten Versuche gemacht hatte - und dem, wodurch es gelang, nachzuweisen. Sonderbar genug erscheint die Lösung des Räthsels jetzt leichter als manches andere, was mich wohl nicht so viele Tage aufgehalten hat, als dieses Jahre, und gewiss wird niemand, wenn ich diese Materie einst vortrage, von der langen Klemme, worin es mich gesetzt hat, eine Ahnung bekommen.

\section{Gauss an Olbers.}

Braunschweig, 3. IX. 1805.

s. "Wilhelm Olbers, Sein Leben und seine Werke“, herausg. v.

C. Schilling, Bd. 2 (1900), p. 268,269

= Schering, "Carl Friedrich Gauss' Geburtstag nach hundertjähriger Wiederkehr" (Göttingen 1877), p. 14.

.....Archimedes casu venit in balineum ibique cum in solium descenderet, animadvertit quantum corporis sui in eo insideret tantum aquae extra solium effluere. idque cum eius rei rationem explicationis ostendisset, non est moratus sed exsiluit gaudio motus de solio et nudus vadens domum versus significabat clara voce invenisse quod quaereret. nam currens identidem

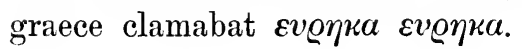

\section{ViTRUV.}

De architectura lib. 9, ed. Rose (1899), p. 212;213.

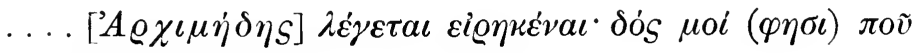

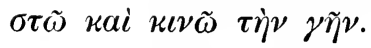

.... [[A rchimed es] soll ausgerufen haben: gieb mir (spricht er), wo ich stehen kann, und ich bewege die Erde.]

\section{Pappus.}

Collection. mathem. liber VIII. v. edit. Fr. Hultsch, vol. 3 (1878), p. 1060. 
Im Sommer 1852 hörte ich in Berlin bei Dirichlet eine Vorlesung über bestimmte Integrale. Er trug uns eines Tages die Lehre von dem Diskontinuitätsfaktor vor, eine seiner glänzendsten Erfindungen. Am Schlusse seiner Auseinandersetzung bemerkte er: „Das ist ein ganz einfacher Gedanke, aber“ - und dabei strich er schmunzelnd seinen Bart - „wenn man ihn nicht hat, so hat man ihn eben nicht!"

Moritz Cantor.

"Phantasie und Nathematik,"

Frankf. Zeitg. 4. III. 1903, No. 63

[dort angegeben als abgedruckt aus „Deutsche Revue", herausg. v. R. Fleischer, März 1903].

In den letzten zwei Monaten habe ich mich viel mit eigenen mathematischen Speculationen beschäftigt, die mir viel Zeit gekostet, ohne dass ich eigentlich mein erstes Ziel bisher erreicht hatte. Immer wurde ich von den vielfach sich kreuzenden Aussichten von einer Richtung in eine andere gelockt, mitunter auch von Irrlichtern, wie dies bei mathematischen Speculationen nichts Seltenes ist.

Gauss an W. Weber.

21. V. 1843.

s. "Wilhelm Weber. Eine Lebensskizze" von H. Weber [Prof. d. Phys. in Braunschweig], (Breslau 1893), p. 77 $=$ Deutsche Revue $17_{4}$ (1892), p. 99.

Die Mathematik allein kann nicht glücklich machen und sie ist so schwierig, dass man nur selten die Genugthuung hat, einen Schritt vorwärts zu kommen.

Eisenstein an M. A. Stern.

Berlin, 20. IV. 1846.

s. Abh. zur Gesch. d. Math. 7 (1895), p. 183. 
Die arithmetischen Begriffe und Beweismethoden erfordern zu ihrer Auffassung und völligen Beherrschung einen hohen Grad von Abstractionsfähigkeit des Verstandes, und dieser Umstand wird bisweilen als ein Vorwurf gegen die Arithmetik geltend gemacht. Ich bin der Meinung, dass alle die anderen Wissensgebiete der Mathematik wenigstens einen gleich hohen Grad von Abstractionsfähigkeit des Verstandes verlangen vorausgesetzt, dass man auch in diesen Gebieten die Grundlagen iiberall mit derjenigen Strenge und Vollständigkeit zur Untersuchung zieht, welche thatsächlich notwendig ist.

D. HiLberT.

„Die Theorie der algebr. Zahlkörper“, Vorwort, p. II in Deutsche Mathem.-Verein. Jahresber. 4, 1894/1895.

Seit mehreren Monaten sind es gewisse Untersuchungen aus der höheren Arithmetik, auf die ich wiederum zurückgekommen bin, und die mich schon seit beinahe 12 Jahren geplagt haben. Sie gehören zu der Gattung derjenigen, wo man nicht im voraus sagen kann: diess will ich thun, sondern wo, vielleicht nach 999 misslungenen Versuchen, eine glückliche $1000^{\text {te }}$ Combination zum Ziele führt. Jetzt habe ich zwar das Ziel erreicht, doch immer noch auf einem nicht genug kurzen Wege. Der Gegenstand ist die Theorie der biquadratischen Reste.

$$
\text { Gauss an Bessel. }
$$

Göttingen, 23. XII. 1816.

s. Briefw. Gauss-Bessel (1880), p. 247.

Une fois, je vis passer Laplace près d'une heure, à tâcher de ressaisir la chaîne de raisonnements qu'il avait cachée [dans sa Mécanique céleste] sous ce mystérieux symbole: Il est aisé de voir.

Biot.

„Mélanges scientifiques et littéraires", t. 1 (Paris 1858), p. 3. 
Wenn man nun versucht,..... so kann man . . mittelst Ihrer weiten Fassung des Satzes . . den schönsten Unsinn herausbringen. Also noch einmal: Haben Sie die Güte, für Ihren interessanten Satz einen logisch gegliederten Beweis zu geben, zu dem ich von ganzem Herzen Ja und Amen sagen kann.

Ich kann nicht recht begreifen, warum Sie wieder darauf zurückkommen; Sie repetiren damit nur die einfachsten analytischen Hülfsmittel, die für andere Untersuchungen dienen mögen, aber an sich nicht werth sind, viel besprochen $\mathrm{zu}$ werden. - . - - - -

Antwort verspätet wegen Examenreisen, und daherigen beim Witterungsumschlag vom 20. April zugezogenen Katarrh. Leider nur aus Pflicht geschrieben, sonst unfühig in diese verhexten Zerfällungen einzutreten, bei denen ich kein treibendes Interesse verspüre.

SCHLÄFLI an Steiner.

Bern, 1. V. 1855.

\section{Gestrenger Signore!}

Ihr ungnädiges Gripp-Schreiben nimmt mir fast den Nuth neue Fragen zu stellen. Sie haben keinen Massstab für die Verstandes- und Gedächtnissschwäche Anderer, urtheilen bloss nach sich, daher keine Nachsicht. Bei meiner Erschlaffung kann ich nicht immer mit hohen Sätzen rumpeln; zudem besteht meine Grösse ja nur im Kleinen, im sorgfältigen allseitigen Erforschen desselben; und wahrlich ich sage Euch, wer nur Grosses fressen will, sieht das Gras nicht wachsen, kann die Welt nicht unmittelbar belehren. Die meisten Menschen steigen vom Kleinen aufwärts; es giebt wenige Bevorzugte, die Alles auf einmal und von Oben herab verschlingen, es sind Elephanten, mehr als der alte Mezzo-Elefanti in Rom war.

Anfang der Antwort Sternen's an Schläfli.

$$
\text { 7.-12. V. } 1855 \text {. }
$$

s. J. H. Graf, „Der Briefwechsel zwischen Jakob Steiner und Ludwig Schläfli," Bern Mittheil. Naturf. Gesellsch. 1896, p. 234,236 u. 238. 
Euler calculait sans aucun effort apparent, comme les hommes respirent, comme les aigles se soutiennent dans les airs.

Arago.

Oeuvres, t. 2 (1854), p. 133/184=Werke, Bd. 2 (1854), p. 109.

Euler's Art zu arbeiten war in der Hauptsache die, dass er zunächst seine Kräfte auf ein spezielles Problem konzentrierte, und so zu einer speziellen Auflösungsmethode gelangte. . . . . Er war eine wesentlich konkrete Natur, die sich mit wirklicher Liebe und Begeisterung dem Stoffe hingab und sich von ihm treiben liess. Man hat ihn die "lebendige Analysis" "1) genannt, und ich möchte sagen: Euler stand mit den Problemen „auf Du und Du“. Daher geht durch alle seine Schriften ein warmer lebendiger Zug . . . . . Mit behaglicher Breite . . erzählt er, was ihn seine Untersuchungen gelehrt haben - und so lesen sich, wie man gesagt hat, seine Schriften, wie "Novellen“.

\section{Hermann Hankel.}

„Die Entwickelung der Mathematik in den letzten Jahrhunderten," Akadem. Vortrag Tübingen 29. IV. 1869, 2. Aufl. (Tübingen 1884), p. 13/14.

Als eines Morgens aus einer Klasse [des Braunsberger Gymnasii] grosser Lärm gehört wurde, stellte sich heraus, dass Weierstrass, der die Stunde zu geben hatte, nicht erschienen war. Der Director begab sich persönlich in die Wohnung von Weierstrass; auf das Anklopfen tönte von innen das Herein, und drinnen sass Weierstrass, obschon es lichter Tag war, im verdunkelten Zimmer bei herabgebrannter Lampe. Er hatte die Nacht hindurch gearbeitet und den Anbruch des Tages nicht gemerkt. Vom Director darauf aufmerksam gemacht und auf die ihn erwartenden lärmenden Schüler hingewiesen, er-

1) s. Note 12 am Ende des Buches. 
widerte er nur, er könne seine Arbeit nicht unterbrechen; denn er sei einer wichtigen Entdeckung auf der Spur, die in der Wissenschaft Aufsehen machen werde.

E. LAMIPE.

„Karl Weierstrass",

Gedächtnisrede Berlin Phys. Ges. 5. III. 1897, p. 17

$=$ Deutsche Mathem.-Verein. Jahresber. 6, 1897, p. 38/39; vgl. a. W. Killing, "Karl Weierstrass", Natur und Offenbarung 43 (1897), p. 716/717.

Lambert beschloss zu der [Antritts-]Rede [in der Berliner Akademie] die Lösung einer wichtigen Frage über die Zurückwerfung der Lichtstrahlen zu nehmen. Hierzu musste er jedoch noch einige Experimente machen und es fehlte ihm an einem dazu benöthigten grossen Spiegel, denn er selbst besass nur ein Taschenspiegelchen, kaum gross genug um seine Perücke davor aufsetzen zu können. Sein Entschluss war indess bald gefasst: an einem hellen Tage ging er auf ein Caffeehaus an der Ecke der breiten Strasse, dem Schloss gegenüber, wo gerade einige Officiere und andere Personen eine Parthie Tarock spielten. Als er eintrat grüsste er sie nach seiner Art, d. $h$. ohne sie weiter anzusehen und indem er den Kopf in einer Diagonallinie von einer Seite zur andern bewegte; nach dieser wunderlichen Höflichkeitsbezeugung schritt er aber ohne sich aufzuhalten, einem grossen für seine Absichten wohlplacirten Spiegel zu, zog den Degen, richtete die Spitze desselben gegen den Spiegel, hieb in die Kreuz und Quere, trat bald vor und bald zurück, stand dann wieder einige Augenblicke nachsinnend still, begann hierauf das Spiel von neuem und trieb es so wohl eine halbe Stunde lang zur ungemeinen Verwunderung der Anwesenden, die ihre Karten hingelegt hatten, ihn in einem weiten Kreise umstanden und einen Wahnsinnigen in ihm zu sehen glaubten. Nachdem er aber seinen Versuch gehörig gemacht, steckte Lambert seinen Degen ruhig wieder ein, sah die ihn Umstehenden mit einem gleichgültigen Blick an und 
ging nun eine Denkschrift zu verfassen welche die Bewunderung aller Gelehrten erregte.

\section{Thiébault.}

"Friedrich der Grosse" [Deutsche Ausg. der "Souvenirs de vingt ans de séjour à Berlin"], Th. 2 (Leipzig 1828), p. 170/171;

s. a. Franz. Ausg., t. 5 (Paris 1804), p. 28-30.

Die Klarheit und Bestimmtheit seines [Dirichlet's] Denkens und die ungewöhnliche Kraft seines Gedächtnisses, vermöge deren er das einmal Gedachte und Erforschte zu jeder Zeit vollkommen gegenwärtig behielt, machten ihm den Gebrauch der Feder beim Arbeiten fast ${ }^{*}$ ganz entbehrlich. Er hatte auch nicht eine besondere Ruhe oder Musse dazu nöthig, sondern konnte auf Spaziergängen, auf Reisen, bei musikalischen Unterhaltungen und überhaupt in allen Lagen, wo er nicht selbst zu sprechen oder zu handeln nöthig hatte, seine tiefen Speculationen mit demselben Erfolge fortsetzen als an seinem Schreibtische. Als Beispiel hierfür kann ich anführen, dass er die Lösung eines schwierigen Problems der Zahlentheorie, womit er sich längere Zeit vergeblich bemüht hatte, in der Sixtinischen Kapelle in Rom ergründet hat, während des Anhörens der Ostermusik, die in derselben aufgeführt zu werden pflegt.

KuMner.

"Gedächtnissrede auf Gustav Peter Lejeune Dirichlet." s. Abhandl. Berl. Akad. 1860, p. 34

$=$ Dirichlet, Werke, Bd. 2, p. 343 .

Über eine Erscheinung sich zu wundern ist in der Mathematik wie in anderen Gebieten oft schon eine halbe Entdeckung.

\section{DIRICHLET.}

„Gedächtnissrede auf Carl Gustav Jacob Jacobi.“

s. Abhandl. Berliner Akad. 1852, p. 8

$=$ Dirichlet, Werke, Bd. 2, p. 233

$=$ Jacobi, Werke, Bd. 1, p. 10. 
Le génie, dans les sciences d'observation, se réduirait-il à la faculté de dire à propos, Pourquoi?

Arago.

Oeuvres, t. 1 (1854), p. $454=$ Werke, Bd. 1 (1854), p. 364.

Es ist, so zu sagen, ein wissenschaftlicher Takt, welcher die Mathematiker bei ihren Untersuchungen leiten und sie davor bewahren muss, ihre Kräfte auf wissenschaftlich wertlose Probleme und abstruse Gebiete zu wenden, ein Takt, der dem a s sthetischen nahe verwandt, das einzige ist, was in unserer Wissenschaft nicht gelehrt und gelernt werden kann, aber eine unentbehrliche Mitgift eines Mathematikers sein sollte.

Hermane Hankel.

„Die Entwickelung der Mathematik in den letzten Jahrhunderten", Akad. Vortrag Tübingen 29. IV. 1869, 2. Aufl. (Tübingen 1884), p. 21.

Croit-on . . que les logiciens ${ }^{1}$ ) ont toujours procédé du général au particulier, comme les règles de la logique formelle semblaient les y obliger? Ce n'est pas ainsi qu'ils auraient pu étendre les frontières de la Science; on ne peut faire de conquête scientifique que par la généralisation.

H. Poincaré.

„Du rôle de l'intuition et de la logique en mathématiques." voir C. R. du deuxième congrès intern. des mathém. Paris 1900

(Paris 1902), p. 127.

Oportet nobis servire voces geometricas analogiae: plurimum namque amo analogias fidelissimos meos magistros, omnium naturae arcanorunn conscios: in geometria praecipue suspiciendos, dum infinitos casus interjectos intra sua extrema mediumque

l) vgl. $S_{2} 147 / 148$. 
quantumvis absurdis locutionibus concludunt, totamque rei alicujus essentiam luculenter ponunt ob oculos.

\section{J. KEPLER.}

"Ad Vitellionem Paralipomena", Caput IV. 4. v. Opera, ed. Frisch, 2, p. 187.

Afin de parvenir dans les sciences physiques et mathématiques à la connaissance de la vérité, il faut d'abord étudier les faits, multiplier les observations, puis chercher à les lier les unes aux autres par des formules, et à reconnaître ainsi les lois particulières qui régissent une certaine classe de phénomènes. Ce n'est qu'après avoir établi ces lois particulières qu'on peut généralement espérer de constater et de découvrir les lois plus générales qui complètent les théories, en rattachant à un même principe une multitude de phénomènes en apparence très-divers.

\section{Augustin Cauchy.}

„Sept leçons de physique générale“, Turin 1833, avec appendices par l'abbé Moigno (Paris 1868), p. 14/15.

Ich bin im Stande gewesen einige mathematisch[-]physikalische Probleme zu lösen, und darunter sogar solche, an welchen die grossen Mathematiker seit Euler sich vergebens bemüht hatten, z. B. die Fragen wegen der Wirbelbewegungen und der Discontinuität der Bewegung in Flüssigkeiten, der [die] Frage über die Schallbewegung an den offenen Enden der Orgelpfeifen u. s. w. Aber der Stolz, den ich über das Endresultat in diesen Fällen hätte empfinden können, wurde beträchtlich herabgesetzt dadurch, dass ich wohl wusste, wie mir die Lösungen solcher Probleme fast immer nur durch allmählich wachsende Generalisationen von günstigen Beispielen, durch eine Reihe glïcklicher Einfälle nach mancherlei Irrfahrten gelungen waren. Ich musste mich vergleichen einem Bergsteiger, der ohne den Weg zu kennen, langsam und mühselig hinaufklimmt, oft umkehren 
muss, weil er nicht weiter kann, bald durch Überlegung, bald durch Zufall neue Wegspuren entdeckt, die ihn wieder ein Stück vorwärts leiten, und endlich, wenn er sein Ziel erreicht, zu seiner Beschämung einen königlichen Weg findet, auf dem er hätte herauffahren können, wenn er gescheidt genug gewesen wäre den richtigen Anfang zu finden. In meinen Abhandlungen habe ich natürlich den Leser dann nicht von meinen Irrfahrten unterhalten, sondern ihm nur den gebahnten Weg beschrieben, auf dem er jetzt ohne Mühe die Höhe erreichen mag.

\section{H. von HeLmholtz.}

s. "Anspr. u. Red. geh. bei der Helmholtz-Feier 2. XI. 1891" (Berlin 1892), p. 54.

„En causant un jour avec mon frère défunt [C. G. J. Jacobi] sur la necessité de contrôler par des expériences réitérées toute observation, même si elle confirme l'hypothèse, il me raconta avoir découvert un jour une loi très-remarquable de la théorie des nombres, dont il ne douta guère qu'elle fùt générale. Cependant par un excès de précaution ou plutôt pour faire le superflu, il voulut substituer un chiffre quelconque réel aux termes généraux, chiffre qu'il choisit au hasard ou, peut-être, par une espèce de divination, car en effet ce chiffre mit sa formule en défaut; tout autre chiffre qu'il essaya en confirma la généralité. Plus tard il réussit à prouver que le chiffre choisi par lui par hasard, appartenait à un système de chiffres qui faisait la seule exception de la règle.

Ce fait curieux m'est resté, dans la mémoire, mais comme il s'est passé il y a plus d'une trentaine d'années, je ne rappelle plus des détails."

\section{H. JACOBI ${ }^{1}$ ) à J. J. Sylvester.}

Exeter, 24. VIII. 1869.

voir British Assoc. for the advancement of Science Report 1869, Notices and Abstracts, p. 9.

1) s. S. 130, Anm. 2 . 
Un jour en l'année 1666, Newton retiré à la campagne, et voyant tomber des fruits d'un arbre, à ce que m'a conté sa nièce, ( $\left.\mathrm{M}^{\mathrm{me}} \mathrm{Conduit}\right)$ [,] se laissa aller à une méditation profonde sur la cause qui entraîne ainsi tous les corps dans une ligne, qui, si elle était prolongée, passerait à peu près par le centre de la terre.*

* Un étranger demandait un jour à Newton comment il avait découvert les lois du système du monde. En y pensant sans cesse, répondit-il. C'est le secret de toutes les grandes découvertes: le génie dans les sciences ne dépend que de l'intensité et de la durée de l'attention dont la tête d'un homne est susceptible,

\section{VolTAIRE.}

„Elemens de philosophie de Newton", troisième partie, chap. 3. voir Oeurres de Voltaire, t. 31 (Gotha 1786), p. 175.

J'admire la constance avec laquelle vous êtes capable de poursuivre un même objet pendant un si long temps; pour moi j'ai ce malheur que, à force de remanier la même matière, j'en prends à la fin un si furieux dégoût, qu'il m'est comme impossible d'y revenir encore - - -

LAGrange à d'Alembert

Berlin, 15. VII. 1769.

Vous êtes étonné que j'aie la patience de revenir si souvent aux mêmes objets. Ce n'est que par ce moyen que j'ai pu faire en ma vie quelque chose de passable, car il n'est pas trop dans la nature de mon esprit de m'occuper de la même chose fort longtemps de suite; je la laisse bientôt, mais je la reprends ensuite autant de fois qu'il me vient en fantaisie, sans me rebuter, et, pour l'ordinaire, cette opiniâtreté éparpillée me réussit, lorsque souvent je n'aurais rien gagné par une opiniâtreté trop longtemps continue.

D'Alember'T à Lagrange. Paris, 7. VШI. 1769. 
A Dieu ne plaise que je désapprouve l'espèce d'opiniâtreté que vous mettez dans vos recherches; je sais que c'est le moyen de réussir dans tout ce qu'on se propose, et je me rappelle toujours d'avoir ouï dire que Newton répondait à ceux qui lui demandaient comment il avait pu trouver le șrstème du monde que ce n'était qu'à force d'y avoir pensé. ${ }^{1}$ )

LAGRANGe à d'Alembert. Berlin, 12. IX. 1769.

voir Oeurres de Lagrange, t. 13 (Paris 1882), p. 138, 146, 149.

Man muss nie denken, dieser Satz ist mir zu schwer, der gehört für grosse Gelehrte, ich will mich mit den andern hier beschäftigen; das ist eine Schwachheit, die leicht in eine völlige Unthätigkeit ausarten kann. Man muss sich für nichts zu gering halten.

\section{LichTENBERG.}

Ausgew. Schriften, Reclam-Bibl. No. 1286-89, p. 195.

Essayez et vous verrez que cette théorie ${ }^{2}$ ) est plus claire qu'on ne le croit généralement.

Monge ì Arago 1809.

voir Arago, Oeurres, t. 1 (1854), p. 96

$=$ Werke $\mathrm{Bd} .1$ (1854), p. 75.

So weit meine Erfahrung reicht, kamen günstige Einfälle nie dem ermüdeten Gehirne und nicht am Schreibtisch..... Oft waren sie . . . des Morgens beim Aufwachen da, wie auch Gauss (s. Werke V, p. 609: Das Inductionsgesetz. [Gefunden 1835. Januar 23. Morgens 7 u v. d. Aufst.]) angemerkt hat. Besonders gern aber kamen sie bei gemächlichem Steigen über

1) s. S. 165 .

2) die Theorie der partiellen Differentialgleichungen. 
waldige Berge in sonnigem Wetter. Die kleinsten Mengen alkoholischen Getränks aber schienen sie zu verscheuchen.

H. von HeLMHOLT\%.

s. "Anspr. u. Red. geh. bei der Helmholtz-Feier 2. XI. 1891" (Berlin 1892), p. 55.

Der Akademiker Se ebeck erzählte [Wilh.] Weber [1828] bei einem Gespräch über die Beschäftigung mit mathematischen Gegenständen einen interessanten Ausspruch von Gauss, den derselbe gegen Pfaff geäussert hätte, er (Gauss) habe gerade, wenn er sich in einem Zustand befinde, wo er an allem Erfolg zu verzweifeln glaube, viele der besten Ideen gehabt.

Heinrich Weber [Prof. d. Phys. in Braunschweig].

"Wilhelm Weber. Eine Lebensskizze“ (Breslau 1893), p. 15

$=$ Deutsche Revue, herausg. v. R. Fleischer, $17{ }_{3}$ (1892), p. 191.

Gauss äusserte sich einmal, dass ihm in seiner Jugend die Gedanken in solcher Fülle ununterbrochen zugeströmt seien, dass er ihrer kaum hätte Herr werden und nur einen Theil derselben aufzeichnen können.

Sartorius v. Waltershausen. „Gauss zum Gedächtniss" (1856), p. 78.

Das ist eben der Vorzug unserer grossen Männer, dass sie nicht nur die Fülle der Gedanken haben, sondern dass diese ihnen auch kein Hinderniss ist für deren Ausbeutung.

Kronecker an Dirichlet.

Ilsenburg, 1. VIII. 1858.

s. Dirichlet, Werke, Bd. 2, p. 410

$=$ Göttinger Nachr. 1885, p. 382. 


\section{$-168$}

In der Theorie der Thetafunctionen ist es leicht, eine beliebig grosse Menge ron Relationen aufzustellen, aber die Schwierigkeit beginnt da, wo es sich darum handelt, aus diesem Labyrinth ron Formeln einen Ausweg zu finden. Die Beschäftigung mit jenen Formelmassen scheint auf die mathematische Phantasie eine verdorrende Wirkung auszuüben. Naucher der bedeutenden Forscher, deren zäher Beharrlichkeit es gelang, die Theorie der Thetafunctionen von zwei, drei oder vier Variabeln zu fördern, ist nach den hervorragendsten Proben glänzendster analytischer Begabung auf lange Zeit oder für inmer verstummt. Ich ${ }^{1}$ ) habe jener Lähmung der mathematischen Schaffenskraft dadurch Herr zu werden versucht, dass ich immer wieder an dem Jungbrunnen der Arithmetik Erholung gesucht habe.

G. Frobenius.

Antrittsrede in der Berliner Akademie. s. Berl. Sitzungsber. 1893, p. 628 .

Rosenhain wurde im März 1857 als ausserordentlicher Professor nach Königsberg berufen und verblieb bei im Ganzen geringen Lehrerfolgen und vollkommener schriftstellerischer Unthätigkeit in dieser Stellung bis zum Frühjahre 1885, wo er von der Verpflichtung Vorlesungen zu halten, entbunden nach Berlin zog. Rosenhain war zweifellos ein hochbegabter Geist, von vielseitiger Ausbildung, tüchtiger Musiker, mit vielen neueren Sprachen vollkommen vertraut, anregender Gesellschafter, aber ohne den Trieb zu arbeiten und zu schaffen. Die Wissenschaft hatte nach seinen Anfängen das Recht weit mehr ron ihm zu verlangen, als er geleistet hat.

M. CANTOR.

Artikel "Johann Georg Rosenhain" in „Allg. Deutsche Biographie“, Bd. 29 (1889), p. 209.

1) vgl. S. 54 . 
Jeg maa altid have ... enkelte Dovenskabs Perioder, for at kunne kile paa igjen med fornyet Kraft.

(Il me faut toujours des périodes de paresse, pour pouvoir prendre de nouveau mon élan avec des forces nouvelles.)

ABes à Holmboe.

Vienne, 16. IV. 1826.

voir „Niels Henrik Abel, Mémorial publié à l'occasion du centenaire de sa naissance": „Texte original des lettres“, p. 25 et „Correspondance d'Abel“(Traduction française),p. 27.

Sonja Kovalevsky behauptete oft, dass sie nie so glücklich wäre, als während der Perioden völliger Faulheit, wo es ihr eine Anstrengung erschien, sich von dem Lehnstuhle zu erheben, auf welchen sie niedergesunken war, und der nächstbeste unterhaltende Roman, eine rein mechanische Handarbeit, sowie Cigaretten und Thee die ganze Beschäftigung ausmachten, deren sie bedurfte. Es war vermutlich ein grosses Glück für sie, dass sie dieses Vermögen besass, auf die allzu anstrengende Gehirnarbeit und die unablässige geistige Erregung, der sie sich mitunter überliess, eine Reaktion eintreten zu lassen.

\section{A. Charl. Leffler.}

$$
\text { "Sonja Kovalevsky", }
$$

Deutsche Ausg., Reclam-Bibl. No. 3297/3298, p. 83.

Jeder productive Mensch grossen Stiles, dessen Schaffensperiode nach Jahren zählt, wird andererseits in Zuständen von Unproductivität schmachten müssen, die ebenfalls nach Jahren zählen; so will's das Gesetz seiner Ebbe und Fluth. Da beisst es seinen Glauben an sich selbst mit den Zähnen festhalten! und Manchem wurde dabei ein Zahn mit ausgerissen. Es ist eine höchst vornehme Art von Selbstbewusstsein, die hier vom Menschen gefordert wird, ein Höhenüberblick, der eine lange Reihe von Gipfeln und Thälern als nothwendige Einheit zu- 
sammenfasst, eine Gluth und Ekstase der dramatischen Illusion, die ïber lange Zwischenacte voll eisiger Alltags-Ernüchterung vorhält. Dieses tiefe und centrale Selbstbewusstsein würde genügen, den urschöpferischen Künstler von uns kleinen Halbkünstlern und Variablen „kurzer Periode“ zu unterscheiden von uns, die jedes augenblickliche Misslingen zweifelsüchtig und muthlos macht, und die spätestens alle sechs Tagewerke einmal zu sich Bravo sagen müssen.

Paul Mongré. ${ }^{1}$ )

„Sant' Ilario. Gedanken aus der Landschaft Zarathustras" (Leipzig 1897), p. 193/194.

Ma tête est devenue presque absolument incapable de travail, quoique d'ailleurs le fond de ma santé ne soit pas mauvais. Dites-moi, je vous prie, des nouvelles de vos travaux et de ce que vous faites; je suis comme les vieux gourmands, qui, ne pouvant plus digérer, ont encore du plaisir à voir manger les autres.

\section{D'Alember't à Lagrange.}

Paris, 7. VIII. 1767.

voir Oeuvres de Lagrange, t. 13 (Paris 1882), p. 99.

Les journées de M. de la Grange étoient réglées et remplies sur un plan très-uniforme. Ses matinées étoient consacrées à sa correspondance et à la lecture; occupations qu'il plaçoit aux heures où l'on peut être distrait, parce qu'elles peuvent être interrompues sans de graves inconvéniens. Immédiatement après son dîner, il donnoit quelques heures aux visites qu'il avoit à faire, et à une promenade qu'il faisoit seul, parce qu'il croyoit devoir marcher vite pour que cet exercice lui fût plus salutaire. A six heures du soir, il rentroit dans son cabinet, et s'y renfermoit de manière à n'y pas être troublé jusqu'à minuit, où il prenoit quelques tasses de thé au lait avant de

1) s. S. 69 unten. 
se coucher. C'est dans ces six heures d'une profonde solitude, qu'il a fait les immenses travaux dont les Mémoires de l'académie sont remplis, et qui lui assurent une si glorieuse réputation. Que dirai-je de plus? Philosophe toujours égal, toujours sage et toujours tolérant, réunissant à son génie pour les mathématiques, des connoissances aussi étendues que variées sur les diverses branches de la littérature, ayant dans le caractère une aménité douce et naturelle, il a été chéri et singulièrement respecté par ceux qui l'ont connu, et vivement regretté par ceux dont la destinée l'a séparé.

\section{ThiéBault.}

„Mes souvenirs de vingt ans de séjour à Berlin", t. 5 (Paris 1804), p. $41 / 42$.

On sait que Lagrange, dans les derniers temps de sa vie, fatigué des recherches d'Analyse et de Mécanique, qui lui assurent pourtant une gloire immortelle, s'était livré à d'autres études et avait abandonné, pendant quelque temps du moins, les Mathématiques pour la Chimie, pour la Physique, pour les spéculations philosophiques. Cet état d'esprit de Lagrange, nous le rencontrons presque toujours à certains moments de la vie des plus grands savants. Les idées nouvelles qu'ils ont contribué à introduire dans la Science leur paraissent suffisamment développées; ils ont rempli leur tâche et éprouvent le besoin de tourner vers des sujets tout nouveaux l'activité de leur esprit. A l'époque de Lagrange, le programme de recherches et de travaux ouvert aux géomètres par la découverte du Calcul infinitésimal paraissait bien près d'être épuisé. Des équations différentielles plus ou moins compliquées à intégrer, quelques Chapitres à ajouter au Calcul intégral, à la Mécanique, et il semblait que l'on allait toucher aux bornes mêmes de la Science tout entière. Poisson, Fourier, Cauchy s'occupaient de créer des voies nouvelles pour les recherches d'Analyse, en étudiant l'application des théories mathématiques à la Physique et à la Mécanique moléculaire. 
La Géométrie moderne, et ce sera pour elle un immortel honneur, est venue changer entièrement ces conditions en offrant aux travaux une voie nouvelle, et surtout en nous montrant par des succès éclatants que, même dans le sujet le plus simple, il y a toujours quelque chose à faire pour un esprit ingénieux et inventif.

\section{Gaston Darboux.}

(„Notice sur les travaux de M. Chasles"), Bull. des sc. mathém. et astronom. (2) 4 (1880), p. 437.

Peut donc qui voudra, dans l'état actuel de la science, généraliser et créer en Géométrie;le génie n'est plus indispensable pour ajouter une pierre à l'édifice.

\section{Chasles.}

„Aperçu historique" (1837), p. 269

= Deutsche Ausg. von Sohncke (1839), p. 267.

Euklid hat einst seinem Könige Ptolemäus .... erwidert: „Es giebt keinen Königsweg zur Mathematik."1) - In einem höheren Sinne des Wortes ist die neuere Geometrie dieser Königsweg!

Hermane Hankel.

„Projektivische Geometrie“ (Leipzig 1875), p. 33.

Es kann die Forschung von bestimmten Problemen ausgehen, deren Wichtigkeit sie erkannt hat, deren Lösung mit allen Kräften mehr oder weniger direct angestrebt wird. Aber ebenso berechtigt ist die andere Art der Forschung, welche sich nur das Gebiet ihrer Thätigkeit wählt, in diesem aber freie Umschau hält, und, entgegengesetzt der ersten, nach Problemen späht, deren Lösung sich ermögliche. Über den relativen Werth dieser Forschungsmethoden werden verschiedene Individualitäten

1) s. S. $153 / 154$. 
immer verschiedener Ansicht sein. Wenn die erstere zu grösserer Vertiefung führen kann, so ist sie auch der Unfruchtbarkeit nur zu leicht ausgesetzt. Der andern schuldet man Dank für die Erwerbung grosser und neuer Gebiete; wobei denn im Einzelnen Vieles der erstern Methode zu ergründen und zu begrenzen verbleiben mag.

\section{Clebsch.}

"Zum Gedächtniss an Julius Plücker",

gel. i. d. Kgl. Ges. d. Wiss. Göttingen 2. XII. 1871.

s. Göttinger Abhandl. 16, 1871 (Göttingen 1872), Math. Cl., p. 6.

On peut . reprocher à Cauchy de n'avoir pas rempli complétement la mission que le ciel lui avait donnée, parce qu'il n'a pas su se borner, parce qu'il ne savait pas apprécier la valeur relative des produits de son esprit, parce qu'un entraînement irrésistible le faisait passer sans cesse d'un sujet à l'autre, et le condamnait fatalement à renvoyer à la séance suivante une fin qu'un nouveau travail aussi brillant, mais autant mutilé, ajournait indéfiniment. ${ }^{1}$ )

\section{L'abbé Moigno.}

„Sept leçons de physique générale par Augustin Cauchy", Turin 1833, avec appendices par l'abbé Moigno

(Paris 1868), p. XI/XII.

Ce qui me frappe dans la carrière mathématique de Weierstrass, c'est la remarquable unité de la pensée, persistant à travers l'étendue et la variété de son oeuvre.

Dès le début, il s'est proposé un but bien déterminé, il a créé des méthodes pour l'atteindre; et, s'il a essayé quelquefois ces méthodes sur d'autres problèmes, il n'a jamais perdu de vue l'objet final de ses recherches.

H. Poincaré.

„L'oeuvre mathématique de Weierstrass", Acta mathem. 22 (1899), p. 1.

1) s. Note 13 am Ende des Buches. 
Il semble que l'on puisse aujourd'hui distinguer, chez les mathématiciens, deux tendances d'esprit différentes. Les uns se préoccupent principalement d'élargir le champ des notions connues; sans se soucier toujours des difficultés qu'ils laissent derrière eux, ils ne craignent pas d'aller en avant et recherchent de noureaux sujets d'études. Les autres préfèrent rester, pour l'approfondir davantage, dans le domaine de notions mieux élaborées; ils veulent en épuiser les conséquences, et s'efforcent de mettre en évidence dans la solution de chaque question les véritables éléments dont elle dépend. C'es deux directions de la pensée mathématique s'observent dans les différentes branches de la Science; on peut dire toutefois, d'une manière générale, que la première tendance se rencontre le plus souvent dans les travaux qui touchent au Calcul intégral et à la théorie des fonctions; les travaux d'Algèbre moderne et de Géométrie analytique relèvent surtout de la seconde. C'est à celle-ci que se rattache principalement l'oeuvre d'Halphen: ce profond mathématicien fut avant tout un algébriste.

\section{E. Picard.}

"Notice sur la vie et les travaux de Georges-Henri Halphen," Paris C. R. de l'acad. des sc. 110 (1890), p. 489/490.

Si les mathématiciens s'abandonnaient tous à la première tendance, la Science ne tarderait pas à s'encombrer d'une foule de méthodes pratiquement inapplicables et les savants s'habitueraient trop rite à se contenter à bon marché. Ceux qui sont au courant de l'état actuel des Mathématiques ne jugeront peut-être pas que cette crainte soit sans fondement.

\section{H. Poincaré.}

"Notice sur Halphen", Journal de l'école polytechnique, cah. 60 (1890), p. 143. 
Bertrand, Darboux, and Glaisher have compared Cayley to Euler, alike for his range, his analytical power, and, not least, for his prolific production of new views and fertile theories. There is hardly a subject in the whole of pure mathematics at which he has not worked. - - -

In several of the notices that appeared at his death he was described as a great explorer. Such he undoubtedly was, but he was more. He not merely discovered new countries but he also opened them up, so that others were able to enter into some possession of those regions without undergoing the difficulties that he had overcome. And if the metaphor may be carried further, he had the restlessness of the explorer: he could not long remain satisfied with an achievement concluded, but must try his fortune again and elsewhere.

A. R. F[orsyti ].

London Roy. Soc. Proc. 58 (1895), p. XXI.

$\grave{\mathrm{E}}$ inutile il ripetere che il metodo originale del Cayley di considerare i vari aspetti dei problemi da lui studiati predomina in tutti quegli scritti; che se ciò deve ascriversi a merito suo, è però d'altra parte una conseguenza di questo fatto la non corrispondente influenza che essi ebbero nel movimento normale di questa parte delle matematiche.

Brioschi.

„Notizie sulla vita e sulle opere del Cayley", Atti della Accademia dei Lincei (5) 4,1 (1895), p. 182.

On ne peut pas affirmer qu'il eût été donné à Laplace de créer une science entièrement nouvelle, comme l'ont fait Archimède et Galilée; de donner aux doctrines mathématiques des principes originaux, et d'une étendue immense, comme Descartes, Newton et Leibnitz; ou, comme Newton, de transporter le premier dans les cieux, et d'étendre à tout l'univers la dynamique terrestre de Galilée: mais Laplace était né 
pour tout perfectionner, pour tout approfondir, pour reculer toutes les limites, pour résoudre ce que l'on aurait pu croire insoluble. Il aurait achevé la science du ciel, si cette science pouvait être achevée.

\section{Fourier.}

"Éloge historique de M. le Marquis de Laplace". voir Mém. de l'Acad. des Sc. de Paris, 10, 1831,

Histoire, p. XC/XCI.

Kirch h off trieb der Geist nicht, absolut Neues aufzusuchen, und die in so mannigfacher Hinsicht interessanten, in neuester Zeit erschlossenen Gebiete, in welchen uns die Thatsachen noch keine Möglichkeit des Verständnisses und der exacten Behandlung bieten, reizten ihn nicht zur Bebauung. Seine Gabe war nicht das Anfangen, sondern das Vollenden. Es ist gewiss bezeichnend für seine Arbeiten, für seine Neigung, nur von den sichergestelltesten Grundlagen und nur in völlig mathematischer Strenge die Entwickelung fortzuführen, dass er wohl fast niemals gezwungen gewesen ist, auch nur in Kleinigkeiten sich selbst zu berichtigen oder berichtigen zu lassen.

Und nicht weniger wie von seinen theoretischen Arbeiten gilt dies von seinen experimentellen; Kirchh off hat, verglichen mit andern Fachgenossen, wenig beobachtet - aber wo er mit der Beobachtung einsetzt, nachdem die bezügliche Erscheinung ihm theoretisch vollkommen aufgeklärt ist, bietet er ein Meisterwerk an Durcharbeitung der Methode und an Gewissenhaftigkeit der Anwendung.

\section{W. VoIGT.}

„Zum Gedächtniss von G. Kirchhoff“, p. 9 in Göttinger Abhandl. 35 (1888).

Ich betrachte es als meine Lebensaufgabe, durch möglichst klare, logisch geordnete Ausarbeitung der Resultate der alten classischen [atomistischen] Theorie, soweit es in meiner Kraft 
steht, dazu beizutragen, dass das viele Gute und für immer Brauchbare, das meiner Überzeugung nach darin enthalten ist, nicht einst zum zweitenmale entdeckt werden muss, was nicht der erste Fall dieser Art in der Wissenschaft wäre.

\section{Boltzinann.}

„Über die Entwieklung der Methoden der theoretischen Physik in neuerer Zeit", Vortrag Naturf.-Vers. München 1899. s. Deutsche Mathem.-Verein. Jahresber. 8, 1899, p. 76.

Selbst wenn ich, um mit Homer zu reden, ein ehernes Herz und eine tausendfältige Zunge ${ }^{1}$ ) hätte, würde ich nicht die Begeisterung schildern können, in die mich die grossartigen und genialen Entdeckungen dieses ungeheuren Kopfes [Dirichlet] nicht nur in einem, sondern fast in allen Gebieten der Nathematik versetzt haben; wie sich immer in den verwickeltsten Theorien ein einfacher schoener und klarer Hauptgedanke zum Grunde legt, an den sich das Ganze wie um einen Mittelpunkt anreiht, wie er immer den wahren Kern herauszufinden weiss, so dass man, wie man auch nachher den Gegenstand anders drehen mag, doch einsieht, das war es, worauf es ankam, so und nicht anders musste es gemacht werden.

\section{Eisenstein}

[20-jährig, 1849] in seinem Curriculum vitae.

s. $\Lambda$ bhandl. zur Gesch. d. Math. 7 (1895), p. 158.

Ich sehe endlich ein, dass ich meinem Ideal nicht mehr nachstreben kann und darf, nämlich auch nur eine - wenn anch in niedrigeren Regionen sich bewegende - doch so in sich vollendete Arbeit zu liefern, wie die Ihrigen Alle sind. Es ist einmal mein Kreuz, dass ich immer auf Gebiete geführt werde, die bessere geistige Kräfte erfordern, als mir von der Natur verliehen sind, und die mehr Zeit in Anspruch nehmen

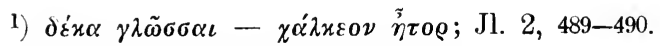

Ahrens, Scherz und Ernst i. d. Mathem. 
als die ist, auf welche ich bei meinem doch eigentlich sehr unzuverlässigen Körper zu rechnen habe.

Kronecker an Dirichlet.

Berlin, 2. XII. 1857.

s. Dirichlet, Werke, Bal 2, p. 405

$=$ Göttinger Nachr. 1885, p. 377378.

So hervorragend auch Kronecker's Leistungen auf den verschiedensten Gebieten der Grössenforschung sind, so reicht er doch in der Analysis an Cauchy und Jacobi, in der Functionentheorie an Riemann und Weierstrass, in der Arithmetik an Dirichlet und Kummer, in der Algebra an Abel und Galois nicht ganz heran Aus diesem Grunde ist seine Bedeutung nicht selten von solchen Gelehrten unterschätzt worden, deren Studien sich nur auf einzelne dieser Disciplinen erstreckten.

\author{
G. Frobenius. ${ }^{1}$ ) \\ "Gedächtnissrede auf L. Kronecker." \\ s. Berl. Abh. 1893, p. 3.
}

Da ich mich zumeist dadurch so unabhängig fühle, dass mich keine Spur irgend welchen Ehrgeizes quält, da ich vielmehr einzig und allein meine Freude in der Erkenntniss des Wahren habe, so kommt es mir wenig darauf an, wozu ich grade meine Zeit verwende, wenn ich sie nur überhaupt gut benutze.

Kronecker an Dirichlet.

Berlin, 17. V. 1857.

s. Dirichlet, Werke, Bd. 2, p. 398

$=$ Göttinger Nachr. 1885, p. 371.

J'ai toujours envisagé la Géométrie comme un objet d'amusement plutôt que d'ambition, et je puis vous assurer que je jouis beaucoup plus des travaux des autres que des miens, dont je suis toujours mécontent; vous voyez par lì que, si

1) s. a. S. 62 . 
vous êtes exempt de jalousie par vos propres succès, je ne le suis pas moins par mon caractère.

\section{LAGRANGE à Laplace.}

Berlin, 1. IX. 1777.

voir Oeurres de Lagrange, t. 14 (1892), p. 71 ; voir aussi 1. c. p. 98.

J'ai tousjours cultivé les mathématiques par gout plustot que par le désir d'une vaine réputation, dont je ne fais aucun cas. Mon plus grand amusement est d'étudier la marche des inventeurs, de voir leur génie aux prises avec les obstacles qu'il ont rencontrés et qu'ils ont sçu franchir; je me mets alors à leur place et je me demande comment je m'y serois pris pour surmonter ces mesmes obstacles, et quoique cette substitution n'ait le plus souvent rien que d'humiliant pour mon amour propre, cependant le plaisir de jouir de leur succès me dédomage amplement de cette petitte humiliation. Si je suis assez heureux pour ajouter quelque chose à leurs travaux, j'en attribüe tout le mérite à leurs 1 ers efforts, bien persuadé que dans ma position ils auroient été beaucoup plus loin que moi. Vous voyez par là, mon cher confrère, que personne ne lit vos ouvrages avec plus d'attention et ne cherche mieux à faire son profit que moi; aussi personne n'est plus disposé à vous rendre une justice plus entière, et je vous prie de me regarder comme un de ceux qui vous aiment et qui vous admirent le plus.

LAPLACE à d'Alembert.

$$
\text { Paris, 15. XI. } 1777 .
$$

voir Bull. des sc. mathém. et astronom. (2) 3 (1879), p. 218 $=$ Bullettino di bibliografia e di storia delle scienze mat. e fis. 19 (1886), p. 160;161.

Poinsot avait écrit dans son mémoire [intitulé Théorie générale de l'équilibre et du mouvement des Systìmes]: „MM. Lagrange et Laplace ont les premiers.... “.1)

1) Diese Stelle finde ich nicht, vielmehr beginnt die citierte $A b-$ handlung mit den Worten (Journal de l'école polytechnique, 13ième cahier, 
Les travaux de Laplace dominaient alors toutes les parties de la science; mais, à parler franchement, sur les principes généraux de l'équilibre et du mouvement des systèmes, il n'avait rien proposé d'original. Lagrange fut froissé. „Pourquoi, dit-il a Poinsot, avez-vous associó le nom de Laplace au mien? Vous m'avez choqué!“ ..... „Je n’avais pas, répondit Poinsot, cité d'autre nom que le vôtre. J'ai montré à un ami la première rédaction du mémoire. - Tu veux présenter à l'Académie, m'a-t-il dit, un mémoire de mécanique sans citer Laplace! Tu n'auras pas de rapport! - La première ligne est écrite de sa main. C'est lui qui y a introduit le nom de votre illustre confrère." Lagrange sourit. _ - -

L'ami de Poinsot... connaissait le monde académique d'alors, et n'y avait pas grand mérite. Laplace régnait sans partage. Lagrange, pour les bons juges, était au moins son égal, mais il avait pour maxime, comme plus tard Poinsot, de ne se mêler de rien; il écartait les importuns, mais ne protégeait personne. Les maladroits seuls le flattaient. Il était habile au contraire de louer Laplace très haut. ${ }^{1}$ ) On saisissait toutes les occasions, souvent même on les faisait naître.

\section{J. Bertrand.}

„Éloges académiques", Nouvelle série (Paris 1902), p. 9/10.

tome 6 (1806), p. 206): „Le principe des vitesses virtuelles est devenu, comme on sait, la loi fondamentale de la mécanique. C'est sur cette loi que reposent aujourd'hui les meilleurs ouvrages que nous ayons dans la science, la Mécanique de M. Lagrange, où la grande fécondité du principe parut d'abord [hierzu eine Anmerkung, in der die Bedeutung von Lagrange's "Mécanique analytique" gewürdigt und dieses Werk übrigens wesentlich anders beurteilt wird als z. B. in dem auf S. 22/23 dieses Buches eitierten Worte Poinsot's], et la Mécanique céleste de M. Laplace; deux productions du premier ordre, et qui mesurent, pour ainsi dire, la hauteur où les sciences exactes se sont élevées de nos jours."

l) vgl. S. 97/98 (Humboldt an Gauss). 
Ausser La Place führen in den Sitzungen des Bureau des longitudes, die mir weit interessanter sind, als die Sitzungen des Instituts, mehrentheils La Grange, Delambre, Poisson, Prony, Rossel, Arago und Biot das Wort: aber Alles beugt sich vor der imponirenden Grösse von La Place.

OLbers an Bessel.

Paris, 10. VII. 1812.

s. Briefw. Olbers-Bessel, herausg. v. Erman, Bd. 1 (1852), p. 337.

L'astronome Lemonnier ${ }^{1}$ ) ayant remarqué, dans un Mémoire qui remporta un prix de Mécanique à l'Académie des Sciences, des développemens d'analyse mathématique fort supérieurs à la capacité connue du concurrent, soupçonna M. Lagrange d'en être l'auteur. Celui-ci s'excusait d'avoir fait gagner le prix à ce mécanicien, en disant que c'était un bon père de famille. On le connaissait capable d'enfreindre ainsi les réglemens.

\section{Virey et Potel.}

„Précis historique sur la vie et la mort de Joseph-Louis Lagrange" (Paris 1813), p. 15.

Indtil dette Øjeblik har jeg kun gjort Bekjendtskab med Legendre, Cauchy og Hachette ..... og Herrn Le-jeune Dirichlet en Preusser, som forleden Dag kom op til mig da han ansaae mig for sin Landsmand. Det er en meget skarpsindig Mathematiker. Han har i Forening med Legendre beviist Umueligheden af at opløse $i$ hele Tal Ligningen $\mathrm{x}^{5}+\mathrm{y}^{5}=\mathrm{z}^{5}$ og andre smukke Ting. - Legendre er en overmaade forekommende Mand men ulykkeligviis steinalt, Cauchy er fou, og der er ingen Udkomme med ham, omendskjøndt han er den Mathematiker som for nærværende Tid weed hvorledes Mathematiken skal behandles. Hans Sager ere fortræffelige men han skriver meget utydelig. I Førstningen forstod jeg næsten ikke et Gran af hans Arbeider nu gaaer

1) von 1792 an der Schwiegervater von Lagrange. 
det bedre. . . . . Cauchy er umaadelig catholsk og bigott. En saare forunderlig Ting for en Mathematiker. . Laplace skriver vel ikke mere ..... Jeg har seet ham ofte i Institutet. Han seer rask og liden ud men har den samme Feil som Haltefanden beskylder Zambullo for nemlig la mauvaise habitude de couper la langue aux gens. ${ }^{1}$ ) Poisson er en kort Mand med en pæn lille Mave. Han forer sit Legene med Værdighed. I ligemaade Fourier. - - -

(Jusqu'à présent je n'ai fait connaissance qu'avec Legendre, Cauchy et Hachette.... et Herr Le-jeune Dirichlet, un prussien, qui l'autre jour est venu me trouver, me considérant comme un compatriote. C'est un mathématicien très sagace. Il a démontré en même temps que Legendre l'impossibilité de résoudre en nombres entiers l'équation $\mathrm{x}^{5}+\mathrm{y}^{5}=\mathrm{z}^{5}$ et d'autres jolies choses. - Legendre est un homme extrêmement aimable, mais par malheur „vieux comme les pierres“. Cauchy est fou et il n'y a rien à faire avec lui, bien qu'il soit en ce moment le mathématicien qui sait comment il faut traiter les mathématiques. Ses travaux sont excellents, mais il écrit d'une manière très confuse. Au commencement je ne comprenais presque rien à ce qu'il écrit, maintenant ça va mieux. . . . Cauchy est extrêmement catholique et bigot. Chose bien étrange pour un mathématicien. . . L Laplace n'écrit plus guère. ... Je l'ai vu souvent à l'Institut. Il a l'air alerte et petit, mais il a le défaut que le diable boiteux reproche à Zambullo, c'est-à-dire „la mauvaise habitude de couper la langue aux gens". Poisson est un petit homme avec un joli petit ventre. Il porte son corps avec dignité. De même Fourier. - - - )

N. H. ABel à Holmboe.

Paris, 24. X. 1826.

voir „Niels Henrik Abel, Mémorial publié à l'occasion du centenaire de sa naissance": „Texte original des lettres", p. 41 ou "Correspondance d'Abel" (Traduction française), p. $45 / 46$.

1) Dans le célèbre roman de Lesage, Le diable boiteux [Anm. des Herausgeber, s. l. c.: „Correspondance d'Abel“, p. 123]. 
Lorsque l'Académie perdit Lagrange en 1813, Ampère, Cauchy et Poinsot briguèrent l'honneur de lui succéder: Poinsot fut élu. . . . S. Si, dans cette élection, nous voulons signaler le trait le plus étrange, bornons-nous à rappeler qu'en classant les candidats par ordre de mérite, la section de géométrie, présidée par Laplace, n'accorda le premier rang, ni à Cauchy, ni à Ampère, ni à Poinsot, mais à un concurrent dont j'oublie le nom.

\section{J. Bertikani.}

„Éloges académiques", Nouvelle série (Paris 1902), p. 16.

Cultivant les sciences sans ambition, sans intrigue et seulement pour mon plaisir, le suffrage de la multitude m'est entièrement indifférent. - $-\ldots$

LAPLACE à Lagrange.

Paris, 23. XI. 1780.

voir Geuvres de Lagrange, t. 14 (1892), p. 99.

Es ist nicht das Wissen, sondern das Lernen, nicht das Besitzen sondern das Erwerben, nicht das Da-Seyn, sondern das Hinkommen, was den grössten Genuss gewährt. Wenn ich eine Sache ganz ins Klare gebracht und erschöpft habe, so wende ich mich davon weg, um wieder ins Dunkle zu gehen; so sonderbar ist der nimmersatte Mensch, hat er ein Gebäude vollendet so ist es nicht um nun ruhig darin zu wohnen, sondern um ein andres anzufangen. So stelle ich mir vor muss dem Welteroberer zu Muthe seyn, der nachdem ein Königreich kaum bezwungen ist, schon wieder nach andern seine Arme ausstreckt.

Gauss an W. Bolyai.

Göttingen, 2. IX. 1808.

s. „Briefw. zw. C. F. Gauss u. W. Bolyai“, herausg. v. Schmidt u. Stäckel (1899), p. 94. 
Si je n'étais pas devenu général en chef et l'instrument du sort d'un grand peuple, j'aurais couru les bureaux et les salons pour me mettre dans la dépendance de qui que ce fût, en qualité de ministre ou d'ambassadeur? Non, non! je me serais jeté dans l'étude des sciences exactes. J'aurais fait mon chemin dans la route des Galilée, des Newton. Et puisque j'ai réussi constamment dans mes grandes entreprises, eh bien, je me serais hautement distingué aussi par des travaux scientifiques. J'aurais laissé le souvenir de belles découvertes. Aucune autre gloire n'aurait pu tenter mon ambition.

NAPOLÉON I ${ }^{\mathrm{er}}$.

roir Arago, Oeuvres, t. 1 (1854), p. 250

= Werke, Bd. 1 (1854), p. 198.

Le général Bonaparte, récemment revenu d'Égypte, assistait ce jour-là à la séance [de l'Académie] comme membre de la section de mécanique. Il vint avec les autres; soit de lui-même, à titre de mathématicien dont il se faisait fort, ou parce que Monge l'amena, pour lui faire les honneurs d'un travail issu de sa chère École polytechnique [Mémoire de Biot sur les équations aux différences mêlées, Mém. Sav. Étrang. I, 1805, p. 296]; à quoi le général répondit: „Je reconnais bien cela aux figures" [tracées déjà auparavant sur le tableau]. Je pensai qu'il était bien habile de les reconnaître, puisque, hormis M. Laplace, personne encore ne les avait vues.

Biот.

"Mélanges scientifiques et littéraires", t. 1 (Paris 1858), p. 7.

Les géomètres ont un grand avantage: leur gloire ne dépend que d'un petit nombre de juges dignes de l'être. Le suffrage de ces juges leur suffit, et la voix de la multitude ne pourrait les en dédommager.

Condorcet.

Oeuvres, Édition d'Arago, t. 2 (1847), p. 147. 
„Ed. Heine war einer von denen, für die man publicirt; denn es sind doch nur Wenige, an die man als an Leser von Urtheil und Nachsicht beim Niederschreiben seiner Geistesproducte denkt.“

\author{
Paul du Bors-Reymond an Kronecker. \\ Tübingen, 3. XI. 1881. \\ s. J. f. Math. 104 (1889), p. 354.
}

Gauss glaubte in der Übersetzung von Arago's Werken mit Freuden Ihre Hand da zu erkennen, wo die Zahl derjenigen Männer besprochen wird, denen über exacte Untersuchungen ein endgültiges Urtheil zustehe: die Zahl werde im Original auf etwa 10, in der Übersetzung auf etwa 8 angegeben: er meinte diese Beschränkung, die ihm ganz besonders zusagte, könne nur von $E[w]$. E[xcellenz] ausgegangen sein. $\left.{ }^{1}\right)$

BaUm [Prof. d. Chirurgie in Göttingen u. Gauss' Arzt] an Alex. v. Humboldt.

Göttingen, 28. V. 1855.

s. K. Brubns, „Briefe zw. Alex. v. Humboldt u. Gauss" (Leipzig 1877), p. 75.

Nur wenn ich Zeit habe, etwas für mich zu verarbeiten und in succum et sanguinem zu verwandeln, kann ich mir ein Urtheil erlauben.

Gauss an Schumacher.

Göttingen, 12. II. 1837.

s. Briefw. Gauss-Schumacher, Bd. 3 (1861), p. 153/154.

1) In dem franz. Original der Oeuvres, t. 1, p. 289 heisst es: „de huit à dix", in der deutschen Ausg. Hankels (mit einer Einleitung von Humboldt), Bd, 1, p. 229 dagegen: „sieben bis acht". Nach Karl Bruhns, "Alexander von Humboldt", Bd. 2 (1872), p. 173 nahm Humboldt diese Änderung „mit Dirichlet's Beirath" vor. 
Lorsque, en traitant un sujet de mathématiques pures ou appliquées, un géomètre arrive à des résultats en désaccord avec ceux que ses prédécesseurs ont obtenus, il se doit ì lui-même d'expliquer la cause de ces différences.

$$
\begin{gathered}
\text { AkaGo. } \\
\text { Oeuvres, t. } 2 \text { (1854), p. } 638 \\
=\text { Werke, Bd. } 2 \text { (1854), p. } 524 .
\end{gathered}
$$

Bei Gegenständen, mit denen ich mich noch nicht lange beschäftigt habe, bin ich gegen meine eigenen Ansichten, zumal wenn sie einem Laplace widersprechen, misstrauisch.

\section{Gauss an Bessel.}

Göttingen, 20. XI. 1826.

s. Briefw. Gauss-Bessel (1880), p. 463.

Wenn ich [bei Bessel] wo Anstoss nehme, bin ich immer erst lange misstrauisch gegen mein eignes Urtheil.

Gauss an Schumacher.

Göttingen, 9. I. 1841.

s. Briefw. Gauss-Schumacher, Bd. 4 (1862), p. 3.

L'impatience me prend aussi bien qu'à vous et, quoique je sois encore au lit, je ne puis m'empêcher de vous dire que je reçus hier ... votre lettre sur les partis ${ }^{1}$ ), que j'admire si fort que je ne puis vous le dire..... j'en suis tout satisfait, car je ne doute plus maintenant que je ne sois dans la vérité, après la rencontre admirable où je me trouve avec vous.

PASCAL à Fermat.

29. VII. 1654.

Nos coups fourrés continuent toujours et je suis aussi bien que vous dans l'admiration de quoi nos pensées s'ajustent si exactement qu'il semble qu'elles aient pris une même route et

1) Verteilung des Einsatzes bei abgebrochenem Spiel. 
fait un même chemin. . . je suis persuadé que le vrai moyen pour s'empêcher de faillir est celui de concourir avec vous. Mais, si j'en disois davantage, la chose tiendroit du compliment, et nous avons banni cet ennemi des conversations douces et aisées.

Fermat à Pascal.

Toulouse, 29. VIII. 1654.

voir Fermat, Oeuvres, t. 2 (1894), p. 289/290 et 307/309

= Blaise Pascal, Oeuvres, t. 4 (1779), p. 412 et 435/436.

Je n'ai pas eu moins de joie de recevoir la Lettre par laquelle vous me faites la faveur de me promettre votre amitié, que si elle me venoit de la part d'une maitresse dont j'aurois passionnément desiré les bonnes grâces.

Descaites à Fermat.

27. VII. 1638.

voir Fermat, Oeuvres, t. 2 (1894), p. 163.

Die Fachgenossen und das Publikum urtheilen über ein wissenschaftliches oder Kunstwerk nach dem Nutzen, der Belehrung oder Freude, die es ihnen gebracht hat. Der Autor ist meist geneigt seine Werthschätzung nach der Mühe anzusetzen, die es ihm gemacht hat; und diese beiden Arten der Schätzung treffen selten zusammen. - - - - - - -

Der Augenspiegel ist wohl die populärste meiner wissenschaftlichen Leistungen geworden, aber ..... dabei hat das Glück eigentlich eine unverhältnissmässig grössere Rolle gespielt, als mein Verdienst. - - - - - - - -

Für meine äussere Stellung vor der Welt war die Construction des Augenspiegels sehr entscheidend. Ich fand fortan bei Behörden und Fachgenossen bereitwilligste Anerkennung und Geneigtheit für meine Wünsche. - - - -

\section{H. von HeLmholtz.}

s. „Anspr. u. Red. geh. bei der Helmholtz-Feier 2. XI. 1891“ (Berlin 1892), p. 48, 52 u. 53. 
Jeder, der die Idee einer Wissenschaft in sich trägt, kann nicht anders als die Dinge darnach abschätzen, wie sich der menschliche Geist in ihnen offenbart: nach diesem grossen Massstab muss ihm daher manches als geringfügig vorkommen, was den andern ziemlich preiswürdig erscheimen kamm.

\section{G. J. JАCOBI}

(20-jährig) in einem Brief a. d. J. 1824.

s. Werke, Bd. 1, p. 24 (Dirichlet, „Gedächtnissrede auf Jacobi“).

Wer die Schwierigkeiten kennt, die mathematischen Formeln zu finden, welche die Naturerscheinungen genau zu beschreiben und voraus zu berechnen erlauben, der begreift, dass dieses Ziel nur durch schrittweises Vordringen erreicht werden kann, und schätzt den Vortheil jeder Vervollkommnung der mathematischen Methode, wenn er auch zugiebt, dass Dirichlet die Grösse einer Entdeckung zu ausschliesslich nach dem dabei aufgewandten mathematischen Scharfsinne taxirte, welcher die Berechnung der Classenzahl aller quadratischen Formen als die grösste Entdeckung unseres Jahrhunderts gepriesen haben soll.

\section{Boltzmann.}

"Gustav Robert Kirchhoff," Akadem. Festrede Graz 15. XI. 1887 (Leipzig 1888), p. 28.

Der Werth einer mathematischen Disciplin ist nach ihrer Anwendbarkeit auf empirische Wissenschaften zu schätzen.

C. Runge.

Doctorthese Berlin 23. VI. 1880.

Der Werth einer mathematischen Disciplin kann nicht nach ihrer Anwendbarkeit auf empirische Wissenschaften bemessen werden.

F. Rudio.

Doctorthese Berlin 23. VI. 1880. 


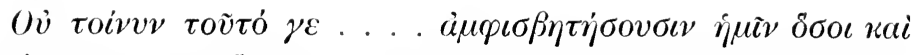

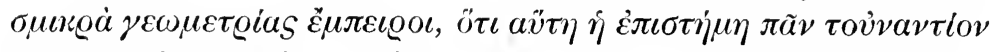

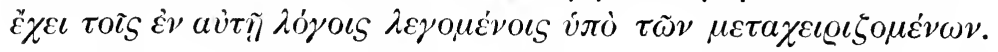

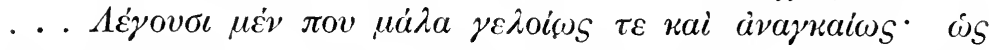

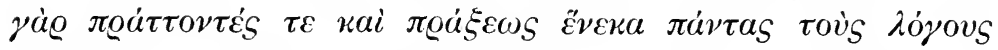

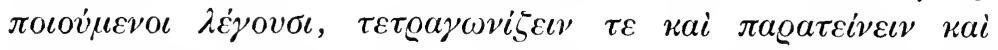

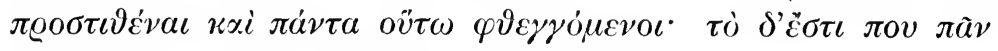

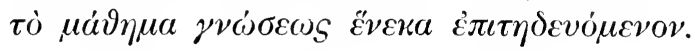

[Das nun werden .... uns wohl auch diejenigen, die der Geometrie nur wenig kundig sind, nicht bestreiten, dass es um diese Wissenschaft gerade entgegengesetzt bestellt ist wie in den Reden derjenigen, die sie betreiben, über sie. . . . Sie reden nämlich ziemlich lächerlich und notdürftig; denn sie sprechen, als ob sie etwas ausführten und eines Geschäftes wegen alle die Worte machten, wenn sie reden von quadrieren, beschreiben, ansetzen und ähnlichem mehr; die ganze Wissenschaft wird doch nur der Erkenntnis wegen betrieben.]

PLATO.

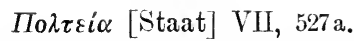

v. Opera II, Bibl. Didotiana 46 (Paris 1883), p. 132, 41-50.

M. de Castillon le père, académicien de la classe de mathématiques, aimant à travailler à divers instruments, sur-tout aux heures où se fait la digestion, et ayant entrepris de fabriquer une grande lunette, vint demander à M. Euler son directeur, s'il feroit bien de suivre par rapport aux verres, les calculs contenus dans un Mémoire que ce MI. Euler avoit donné l'année précédente, sur le maximum de la concavité ou convexité convenable de ces sortes de verres. . . „Gardez-vous en bien!“ lui répondit son directeur: „ces calculs ne vous conduiroient qu'à de faux résultats; mais attendez jusqu'à l'année prochaine; alors on imprimera un autre Mémoire auquel je travaille à présent, et où vous trouverez les véritables règles à suivre dans ce travail.“ - „Mon cher directeur", reprit M. de Castillon, „permettez-moi de vous demander pourquoi vous avez fait 
imprimer votre premier Mémoire, puisque vous saviez qu'il conduisoit à de faux résultats, et que vous aviez l'intention d'en donner un second qui seul rempliroit votre objet?" „Vous êtes dans l'erreur, mon ami, si vous croyez que mon premier Mémoire soit inutile: il est an contraire très-précieux, parce qu' indépendamment de son objet, il contient des calculs qui par leur marche et leur application, deviennent autant de modiles, autant de formules neuves; en un mot, songez bien que ce sont toujours des calculs, et des calculs d'un mode nouveau. Non, non, ce Mémoire n'est pas inutile; il s'en faut bien!"

\title{
Thinébault.
}

"Mes souvenirs de vingt ans de séjour à Berlin", t. 5 (Paris 1804), p. 14-16.

M. Euler paraissait quelquefois ne s'occuper que du plaisir de calculer, et regarder le point de mécanique ou de physique qu'il examinait, seulement comme une occasion d'exercer son génie et de se livrer à sa passion dominante. Aussi les savants lui ont-ils reproché d'avoir quelquefois prodigué son calcul à des hypothèses physiques, ou même à des principes métaphysiques dont il n'avait pas assez examiné, ou la vraisemblance ou la solidité.

\author{
CONDORCET. \\ „Éloge de M. Euler." \\ voir Condorcet, Oeuvres, Édition d'Arago, t. 3 (1847), p. 27/28.
}

On ne peut trop désirer que le domaine de la Géométrie s'étende. C'est dans cette vue que je me suis un peu livré ì la Physique, et je ne désespère pas de déterminer quelques nouveaux objets physiques, assez bien pour y appliquer l'Analyse.

LAPLACE à Lagrange.

Paris, 11. II. 1784 .

voir Oeuvres de Lagrange, t. 14 (1892), p. 130. 
Neumann war.... in der Mathematik wesentlich Autodidact; ${ }^{1}$ ) und so sehr er in einzelnen Fällen mathematische Entwickelungen um ihrer selbst willen gepflegt hat, im Ganzen war ihm die Analyse doch nur die Sprache für die Gesetze der Erscheinungen. Demgemäss sind seine mathematischen Entwickelungen häufig nicht den Gesichtspunkten der äussersten Eleganz und Allgemeinheit entsprechend, die der reine Mathematiker verfolgt; ihm war es wichtiger, dass das Problem wirklich physikalisches Interesse bot, dass bei der Lösung in jedem Moment die Beziehung zu den behandelten Erscheinungen klar erkennbar war, und dass das Resultat die Anwendung auf die Beobachtung gestattete. Von diesem Gesichtspunkt aus betrachtet ist seine Behandlungsart der in Vorlesungen und Seminaren vorgetragenen Probleme eine mustergültige und hat im hohen Grade vorbildlich gewirkt.

W. Voigt.

„Zur Erinnerung an F. E. Neumann“, Göttinger Nachr. 1895, Math.-phys. Kl., p. 255.

J'ai lu avec plaisir le Rapport de M. Poisson sur mon Ouvrage [Fundamenta nova theoriae functionum ellipticarum], et je crois pouvoir en être très-content; ..... Mais M. Poisson n'aurait pas dû reproduire dans son Rapport une phrase peu adroite de feu M. Fourier, où ce dernier nous fait des reproches, à Abel et à moi, de ne nous pas être occupés de préférence du mouvement de la Chaleur. Il est vrai que M. Fourier avait l'opinion que le but principal des mathématiques était l'utilité publique et l'explication des phénomènes naturels; mais un philosophe comme lui aurait dû savoir que le but unique de la science, e'est l'honneur de l'esprit humain, et que sous ce titre, une question des nombres vaut autant qu'une question du système du monde.

JACOBI à Legendre.

Königsberg, 2. VII. 1830.

voir „Correspondance mathém. entre Legendre et Jacobi“, éd. par Borchardt, J. f. Math. 80 (1875), p. 272/273

$=$ Jacobi, Werke, Bd. 1, p. 454/455.

1) s. S. $124 / 125$. 
Dans une Académie des sciences, si elle apprécie convenablement son mandat, l'auteur d'une découverte n'est jamais exposé à cette question décourageante qu'on lui adresse si souvent dans le monde: à quoi bon? Là, chacun comprend que la vie animale ne doit pas être la seule occupation de l'homme; que la culture de son intelligence, qu' une étude attentive de cette variété infinie d'êtres animés et de matières inertes dont il est entouré, forment la plus belle partie de sa destinée. Arago.

Oeurres, t. 1 (1854), p. 167/168=Werke, Bd. 1 (1854), p. 134.

Ich fürchte nicht, dass man mir vorwerfe, es werde die Bedeutung, welche die Mathematik als reine Wissenschaft mit rollstem Rechte beansprucht, herabgesetzt, wenn ich sie ganz besonders auch darum hochstelle, weil durch sie allein ein wahrhaft befriedigendes Verständniss der Natur-Erscheinungen vermittelt wird. Niemand zwar kann bereitwilliger als ich es anerkennen, dass man den Zweck einer Wissenschaft nicht ausserhalb derselben suchen darf, und dass es nicht nur ihre Würde beeinträchtigen, nein, dass es geradezu an ihr sich versündigen heisst, wenn man, statt sich ihr mit vollster Liebe und Hingebung zu widmen, nur Dienste ron ihr verlangt, nur sie brauchen will für irgend eine andere Disciplin oder für die Bedürfnisse des Lebens, und darum wohl gar sich vermisst, der weiterschreitenden Forschung ihren Weg vorzeichnen zu wollen, und jede Richtung verwirft, die nicht sofort zu praktisch verwerthbaren Resultaten zu führen scheint. Ich meine aber, es muss das Verhältniss zwischen Mathematik und Naturforschung etwas tiefer aufgefasst werden, als es geschehen würde, wenn etwa der Physiker in der Mathematik nur eine wenn auch unentbehrliche Hülfs-Disciplin achten, oder der Nathematiker die Fragen, die jener ihm stellt, nur als eine reiche Beispiel-Sammlung für seine Methoden ansehen wollte.

W EIERSTRASS.

Antrittsrede in d. Berliner Akademie. s. Berl. Monatsluer. 1857, p. 350 . 
Die Nathematik hat auch als Hülfswissenschaft, namentlich in ihren Anwendungen auf die Natur, manche grossartige Triumphe gefeiert, und es ist nicht zu leugnen, dass sie diesen hauptsächlich die allgemeine Achtung verdankt in welcher sie steht, aber ihre höchste Blüthe kann sie nach meinem Dafürhalten nur in dem ihr eigenen Elemente des abstrakten reinen Quantums entfalten, wo sie unabhängig von der äusseren Wirklichkeit der Natur nur sich selbst zum Zwecke hat.

E. E. Kummer.

Antrittsrede in d. Berl Akademie.

s. Berl. Monatsber. 1856, p. 378/379.

Es würde schwarzer Undank gegen Euler und Lagrange sein, wollte der Astronom nicht ... bekennen, dass seine Sympathien bis heute noch besonders warm der ersten der beiden Blütheperioden der Mathematik in der Berliner Akademie zugewandt sind, und dass ich darum nicht ohne einige Einschränkung mir aneignen kann, was an diesem Tisch aus höchstverehrtem, jetzt eben für immer geschlossenem Munde [Kummer $\uparrow 14$. V. 1893] zu Gunsten der rein theoretischen Forschung gesagt ist.

\section{Auwers.}

Antwort auf die Antrittsrede von Frobenius in d. Berl. Akademie 29. VI. 1893.

s. Berl. Sitzungsber. 1893, p. 631.

Gauss hat es nicht verschmäht, selbst mit dem Theodoliten praktisch zu arbeiten, und hat dann eben auf Grund dieser Arbeiten praktische Präcisionsmethoden geschaffen, die Gemeingut der gesamten Culturwelt geworden sind und mit denen er der Menschheit eines der wertvollsten Geschenke gemacht hat. So ist die Geodäsie zu einem Muster für den rationellen Anwendungsbetrieb einer theoretischen Wissenschaft geA hrens, Scherz und Ernst i. d. Mathem. 
worden. ..... Jeder Feldmesser ist mit einem Tropfen Gaussischen Oles gesalbt.

\section{G. Hauck.}

„Wirkung der neuen preussischen Prüfungsordnung für Lehramtscandidaten auf den Universitätsunterricht", CorreferatJahresvers. d. Deutschen Mathem.-Verein. München 1899.

s. Deutsche Mathem.-Verein. Jahresber. 8, 1899(Leipzig 1900), p. 108.

Alle Messungen in der Welt wiegen nicht ein Theorem auf, wodurch die Wissenschaft der ewigen Wahrheiten wahrhaft weiter gebracht wird.

Gauss an Bessel.

Göttingen, 14. III. 1824.

s. Briefw. Gauss-Bessel (1880), p. 428.

I am not making before you a defence of mathematics, but if I were I should desire to do it - in such manner as in the „Republic“ Socrates was required to defend justice quite irrespectively of the worldly advantages which may accompany a life of virtue and justice, and to shaw that, indepedently of all these, justice was a thing desirable in itself and for its own sake - not by speaking to you of the utility of mathematics in any of the questions of common life or of physical science. Still less would I speak of this utility before, I trust, a friendly audience, interested or willing to appreciate an interest in mathematics in itself and for its own sake. I would, on the contrary, rather consider the obligations of mathematics to these different subjects as the sources of mathematical theories now as remote from them, and in as different a region of thought - for instance, geometry from the measurement of land, or the Theory of Numbers fron arithmetic - as a river at its mouth is from its mountain source

Arthur Cayley.

Address Southport September 1883.

cf. Report of the fifty-third meeting of the British Assoc for the advancement of Science, p. $4 / 5$. 
La quadrature d'une courbe n'est bonne à rien; et l'idée d'aller mal mesurer un degré du méridien, pour savoir si le pôle est allongé de quatre ou cinq lieues ${ }^{1}$ ), est une idée si romanesque, que toutes les mesures ont été différentes dans tous les pays. Un bon ingénieur vaut mieux que tous ces calculateurs de fadaises difficiles.

Voltaire à Frédéric le Grand. Ferney, 2. I. 1775.

Vous avez raison de trouver la géométrie pratique préférable ì la transcendante. L'une est utile et nécessaire, l'autre n'est qu'un luxe de l'esprit. Cependant ces sublimes abstractions font honneur à l'esprit humain, et il me semble que les génies qui les cultivent se dépouillent de la matière autant qu'il est en eux, et s'élèvent dans une région supérieure à nos sens. J'honore le génie dans toutes les routes qu'il se fraye, et quoiqu'un géomètre soit un sage dont je n'entends pas la langue, je me plains de mon ignorance, et je ne l'en estime pas moins.

Frédéric le Grand à Voltaire. Potsdam, 27. I. 1775 .

voir Oeuvres de Frédéric le Grand, t. 23 (Édition Decker 1853), p. 303 et $306 / 307$.

If in the range of human endeavour after sound knowledge there is one subject that needs to be practical, it surely is Medicine. Yet in the field of Medicine it has been found that

1) Dies zielt auf Voltaire's alten Gegner Maupertuis ( $\dagger$ 1759), der bekanntlich Leiter der Gradmessung in Lappland gewesen war. Ganz unzweifelhaft geht dies aus dem Folgenden hervor, wo Voltaire spricht von „un géomètre chimérique et calomniateur. Je mourrai le coeur percé du mal qu'il m'a fait en m'éloignant de vous". Bekanntlich fiel Voltaire durch sein Pamphlet gegen Maupertuis, den „Docteur Akakia“, in Ungnade bei seinem königlichen Freunde, der den Praesidenten seiner Akademie schützen zu sollen glaubte (s. hierfür insbesondere aus dem Briefwechsel Friedrich's mit Maupertuis, herausg. v. R. Koser in 33d. 72 der „Publicationen aus den K. Preuss. Staatsarchiven" (Leipzig 1898), p. 284-291). 
branches such as biology and pathology must be studied for themselves and be developed by themselves with the single aim of increasing knowledge; and it is then that they can be best applied to the conduct of living processes. So also in the pursuit of mathematics, the path of practical utility is too narrow and irregular, not always leading far. The witness of history shows that, in the field of natural philosophy, mathematics will furnish more effective assistance if, in its systematic development, its course can freely pass beyond the ever-shifting domain of use and application.

\section{A. R. Forsyth.}

Address Toronto 1897.

cf. Report of the 67 th meeting of the British Assoc. for the advancement of Science, p. 546.

Wem konnte es einfallen, als Galvani Froschschenkel mit verschiedenartigen Metallen berührte und sie zucken sah, dass 80 Jahre später Europa mit Drähten durchzogen sein würde, welche Nachrichten mit Blitzesschnelle von Madrid nach Petersburg trugen mittels desselben Vorgangs, dessen erste Aeusserungen der genannte Anatom beobachtete! - - - - -

Wer bei der Verfolgung der Wissenschaften nach unmittelbarem praktischen Nutzen jagt, kann ziemlich sicher sein, dass er vergebens jagen wird. Vollständige Kenntniss und vollständiges Verständniss des Waltens der Natur- und Geisteskräfte ist es allein, was die Wissenschaft erstreben kann. Der einzelne Forscher muss sich belohnt sehen durch die Freude an neuen Entdeckungen, als neuen Siegen des Gedankens über den widerstrebenden Stoff, durch die ästhetische Schönheit, welche ein wohlgeordnetes Gebiet von Kenntnissen gewährt, in welchem geistiger Zusammenhang zwischen allen einzelnen Theilen stattfindet, eines aus dem andern sich entwickelt und alles die Spuren der Herrschaft des Geistes zeigt; er muss sich belohnt sehen durch das Bewusstsein, auch seinerseits zu dem wachsenden Capital des Wissens beigetragen zu haben, 
auf welchem die Herrschaft der Menschheit über die dem Geiste feindlichen Kräfte beruht.

\section{HeLMHOLTZ.}

„Über das Verhältniss der Naturwissenschaften zur Gesammtheit der Wissenschaft", Prorectoratsrede Heidelberg 22. XI. 1862.

s. Vorträge u. Reden, Bd. 1 (1884), p. 142/143.

Wird das, was uns Theoretiker jetzt interessiert, später noch einmal in allgemeinerem Sinne verwendbar werden? Ich habe oft, und ich darf sagen: mit ehrlichem Zweifel darüber nachgedacht; aber ich bin schliesslich, je länger ich es that, desto mehr in meiner optimistischen Überzeugung gefestigt worden. Unsere Theorieen sind nicht überflüssig, ein eitles Spiel der Phantasie. Es handelt sich nur um gewisse Schwierigkeiten, die sich ihrer Verbreitung und Verwertung entgegenstellen

F. KLeIN.

Antrittsrede Leipzig Univ. 25. X. 1880.

s. Zeitschr. math. naturw. Unterr. 26 (1895), p. 536.

Selbstverständlich giebt es viele Gebiete, wo die wissenschaftliche Erkenntniss der Technik vorangegangen oder wenigstens die Wege gewiesen hat, wie die Chemie, Kältetheorie, Elektrizitätslehre, u. s. w. Die Regel ist aber, dass die theoretische Naturforschung der Technik nachgefolgt ist, dass erst eine technische Anwendung naturwissenschaftliche Einsicht gebracht hat.

A. RiedLer.

„Unsere Hochschulen und die Anforderungen des zwanzigsten Jahrhunderts", 3. Aufl. (Berlin 1898), p. 48.

Il n'est pas douteux que, depuis le commencement des siècles, l'industrie n'ait été redevable à la science de progrès nombreux et considérables, et que la pratique n'ait reçu de la 
théorie des secours efficaces. Mais il ne serait pas juste de ne pas tenir compte de ce que, par réciprocité, la science doit à l'industrie, directement ou indirectement. Souvent des problèmes indiqués par celle-ci ont été le point de départ de recherches scientifiques considérables; souvent aussi les fabriques, les usines ont fourni de puissants moyens d'étude qui faisaient défaut dans les laboratoires et dont la science a largement profité. Les travaux du savant Alsacien dont la mort a récemment attristé ses amis, les travaux de G.-A. Hirn, sont un exemple frappant de cette pénétration réciproque de deux ordres d'idées que l'on considère quelquefois, à tort, comme opposés - - - -

Attaché comme ingénieur à une importante manufacture au Logelbach, il eut, au début surtout, à s'occuper de questions absolument pratiques et à rechercher des améliorations rendues nécessaires par des besoins nouveaux, pour maintenir cette manufacture au niveau des progrès généraux de l'industrie.

Mais de ces recherches spéciales, il fut conduit à l'examen des questions théoriques qui s'y rattachaient, puis à l'étude de sujets dépendant de la science pure; il sortit même des limites de celle-ci pour aborder la philosophie ou mieux, peut-être, la métaphysique de la science. Il y a là un curieux exemple d'une succession de travaux qui, sans sortir d'un ordre de phénomènes déterminé, s'étend de la pratique pure aux notions les plus abstraites, mais entre lesquels on peut suivre presque toujours la filiation des idées.

\section{C.-M. Gariel.}

"Les travaux de G.-A. Hirn“, Revue scientifique 45 (1890), p. 193.

Now it seems to me that the difference between scientific and merely technical thought $\ldots \ldots \ldots \ldots \ldots \ldots$ is just this: Both of them make use of experience to direct human action; but while technical thought or skill enables a man to deal with the same circumstances that he has met with 


\section{- $199-$}

before, scientific thought enables him to deal with different circumstances that he has never met with before.

\section{W. K. ClifFord.}

"On the aims and instruments of scientific thought", Lecture delivered before the British Association for the advancement of Science, Brighton 19. VIII. 1872.

cf. Clifford, „Lectures and essays", vol. 1 (London 1879), p. 128.

Die Mathematiker und ihre Anhänger machen von der Thatsache, dass es eine Reihe von Wissensgebieten, darunter den Naschinenbau, giebt, welche Gelegenheit zu reichlichen mathematischen Betrachtungen bieten, ohne weiteres den grossen Gedankensprung und gehen von der Möglichkeit mathematischer Berechnungen über zur Zweckmässigkeit oder gar Notwendigkeit mathematischer Behandlung aller Ingenieuraufgaben.

In der Regel finden wir in den verschiedensten Zeiten und bei den verschiedensten Berufen die oben geschilderte Erscheinung.

So spricht man in der Geschichte der Forstwissenschaft von einer Periode der Mathematiker, und die heutigen Forstmänner sind glücklich in dem Bewusstsein, dass diese Zeit der Verirrung längst hinter ihnen liegt. In der Entwicklung der Forstwirtschaft stellt diese Zeit eine der unfruchtbarsten dar, und wenn wir heute lesen, in welch naiver Weise jene Mathematiker unserem schönen deutschen Walde mit ihrem Formelkram auf den Leib rückten, so überkommt den Leser unwillkürlich ein mitleidiges Lächeln. Die unglaublichsten Dinge wollten jene Herren berechnen, und was sich an forstwissenschaftlichen Fragen nicht in ein mathematisches Gewand hineinzwängen liess, das liessen sie „als nicht geeignet zur wissenschaftlichen Behandlung" einfach weg - -

P. voN Lossow.

„Zur Frage der Ingenieurausbildung“, Zeitschr. d. Ver. deutscher Ingenieure, Bd. 43, No. 13 (1899), p. 360. 
Manch' überraschende Erfindung stellt sich bei näherer Prüfung ihrer Genesis als Folge planmässig fortgesetzter Forschungsarbeit und keineswegs als unvermittelter Einfall heraus. Jahrelang in Archiven schlummernde wissenschaftliche Arbeiten erlangen plötzlich eminente, praktische Wichtigkeit. Das Wort Dubois-Reymond's, dass es kaum eine noch so weltabgelegene wissenschaftliche Untersuchung gebe, die nicht im Laufe der Zeit praktischer Anwendung fähig wäre ${ }^{1}$ ), bewahrheitet sich. Vor allem aber wird die Wissenschaft seitens der Technik geschätzt als kritische Leuchte, die eine klare Sichtung der ungeheuren Zahl von Möglichkeiten und Unmöglichkeiten gestattet. Sie bewahrt uns vor Vergeudung immenser Mittel und geistiger Arbeit an fruchtlose Versuche. Gleiche Fühigkeiten vorausgesetzt, verleiht die wissenschaftliche Ausbildung dem Techniker, rein durch die Schärfung des kritischen Urteiles, eine grosse Überlegenheit über die reinen Praktiker.

\section{A. Strodola.}

"Über die Beziehungen der Technik zur Mathematik." s. Verhandl. d. 1. intern. Mathem.-Congr. Zürich 1897 (Leipzig 1898), p. 261

$=$ Zeitschr. d. Ver. deutscher Ingenieure, Bd. 41, No. 44 (1897), p. 1257.

Der praktische Ingenieur pflegt die Differential- und Integralrechnung möglichst schnell an den Nagel zu hängen und sich auf elementare Methoden zu beschränken. Fragen Sie nach, so werden 99 unter 100 dies zugestehen. . . . A ber gerade die elementaren Methoden die der Praktiker gebraucht, werden auf vielen technischen Hochschulen vernachlässigt. Dort hört er alles in hoch-

1) s. E. du Bois-Reymond, Reden, 1. Folge (1886), p. 147/148, wo für ähnliche Aussprüche Fontenelle, Cuvier und Friedrich Heinrich Jacobi citiert werden; s. a. ibid. p. 273. 
wissenschaftlichem Gewande, er lernt es so, wie er es später nicht verwerten kann.

\section{HOLZMÜLLER.}

„Über die Beziehungen des mathem. Unterr. zum IngenieurWesen und zur Ingenieur-Erziehung." Vortr. Vers. des Vereins zur Förderung d. Unterr. i. d. Mathem. u. d. Naturw. Elberfeld 1896. s. Zeitschr. math. naturw. Unterr 27 (1896), p. 478.

So lange ... der Techniker entwerfend und praktisch construirend vorgeht oder bereits fertige theoretische Anschauungen für den Einzelfall benutzt, wird er immer mit den allerelementarsten mathematischen Mitteln auskommen und seine eigentliche geistige Thätigkeit vorwiegend in der Richtung des Combinirens und in geschickter Anpassung an die mannigfachen und oft schwer zu erfüllenden Anforderungen der Praxis nach Massgabe seiner persönlichen Erfahrungen bethätigen. Diese Betriebsmathematik ist seit einem Jahrhundert und jedenfalls durch Euler in dem hier in Betracht kommenden Umfange abgeschlossen. Aber sobald die Notwendigkeit eintritt, umfassendere Probleme theoretisch durchzuarbeiten - wir haben hier in erster Linie die kinetischen im Auge -, die zugleich neue Gesichtspunkte für den Gang der experimentellen Forschung liefern sollen, wird in manchen Fällen eine lebendige Kenntnis der Ideen eines Lagrange, Gauss oder Riemann entschieden förderlicher sein, als ein mühsames Herumsuchen in Integraltafeln und Ingenieurkalendern. Wir dürfen jedoch hierbei nicht vergessen, dass gerade diese Schöpfer der modernen Mathematik der eigentlichen Technik ferngestanden haben, und dass infolgedessen die Übersetzung ihrer genialen Ideen in die hausbackene Form, in der sie hier gebraucht werden, eine Aufgabe bildet, deren geschickte und zweckmässige Lösung durchaus nicht leicht ist. Nur Poncelet macht eine Ausnahme, da er auf beiden Gebieten gleich heimisch war. Von ihm haben wir die schoene Methode der Linearapproximationen, die es ermöglicht, 
ungefüge analytische Ausdrücke durch äusserst einfache zu ersetzen, so dass die Durchführung des betreffenden Problems ohne Anwendung transcendenter Functionen erfolgen kann.

K. Heun.

"Die kinetischen Probleme der wissenschaftlichen Technik", Deutsche Mathem.-Verein. Jahresber. 9 (1901), p. 118/119.

Der Wert eines weit gehenden Mathematikunterrichtes für Ingenieure wird heutzutage vielfach arg überschätzt; und doch sind keine beweiskräftigen Fälle bekannt geworden, dass ein Ingenieur als solcher deshalb Hervorragendes geleistet hat, weil er ein tüchtiger Mathematiker war, und ebenso wenig war es schon da, dass ein Ingenieur nur deshalb in seinem Beruf unfähig war, weil er mangelhafte mathematische Kenntnisse hatte.

Im Gegenteil, inbezug auf die Art und Weise des Denkens unterscheidet sich der Mathematiker vom Ingenieur grundsätzlich. . . . . Jeder Konstruktionslehrer weiss, welche Mühe es kostet, in den Köpfen der Studirenden, nachdem sie sich zwei Jahre lang fast ausschliesslich mit mathematischen Studien beschäftigen mussten, eine gewisse Revolution hervorzurufen,

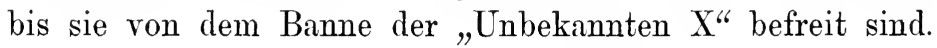

\section{P. von Lossow.}

„Zur Frage der Ingenieurausbildung“, Zeitschr. d. Ver. deutscher Ingenieure, Bd. 43, No. 13 (1899), p. 357.

An polytechnischen Schulen sollte es nicht genügen den Techniker, der später selbständig Projecte ausarbeiten soll, der die Tragweite aller Regeln und Formeln die er anwendet kennen, und seine Projecte räumlich durchschauen soll, mit graphischen Constructionsrecepten gefüttert zu haben, sondern der Zweck sollte sein, vor allem denkende Menschen zu bilden, welche auszuführende Projecte rüumlich mathematisch überblicken. Dies kann nur auf Grundlage einer guten mathematischen Vor- 
bildung geschehen, und für Ingenieure sind namentlich die geometrischen Disciplinen besonders wichtig, weil sie mehr als alle andern das räumliche Anschauungsvermögen ausbilden. Wohl ist es Sitte, ausgezeichnete Mathematiker und Geometer an die polytechnischen Schulen zu berufen, allein können diese sich da wohl fühlen, wenn die Herrn Professoren der praktischen Fächer nie die mathematische Bildung ihrer Zuhörer in Anspruch nehmen ... Kann unbenützte Vorbereitung nach-

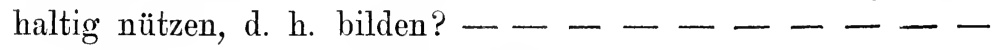

Berlin besitzt zur Zeit wohl die erste mathematische Facultät der Welt, allein leider sitzt sie abseits an der Universität, und bildet für die Realgymnasien, die technische Anstalten sind, Oberlehrer, die von der Technik nichts verstehen. Und schliesslich versteht der Techniker zu wenig Mathematik, wird zu wenig von ihr durchdrungen, weil er mit Denjenigen nicht in Berïhrung kommen kann, die nach den höchsten Zielen streben. Nie hätte ein Cremona in Berlin entstehen können, der hoch oben auf der mathematischen Stufenleiter stehend, auf dem praktischen Gebiete auch etwas leistet; nie wird ein an einer Universität gebildeter Mathematiker einen Kräfteplan zeichnen - - -

\section{Culumans.}

„Die Graphische Statik“ (Zürich 1875), p. VII u. IX/X.

Weil die technischen Professoren gefunden haben, dass sie mit den ihrerseits geplanten Vervollkommnungen des Unterrichts auf ihren eigenen Wissenschaftsgebieten nicht durchdringen, den an sie herantretenden gewaltig gestiegenen Anforderungen kaum länger gerecht werden können, wenn nicht in der Gesamtorganisation des Unterrichts Änderungen eintreten, haben sie den Mathematikunterricht als das Gebiet bezeichnet, auf dem reformiert werden muss. Wer steht dafür ein, dass man nicht demnächst auch von den Naturwissenschaften ein Gleiches behauptet? - - - - 
Man scheint auf technischer Seite das Gefühl gehabt zu haben, dass es nicht gerade leicht sein werde, den Anteil der höheren Mathematik am Ingenieurstudium erheblich zu reduzieren und hat es vielleicht darum für strategisch richtig gehalten, einen recht kräftigen Vorstoss gegen dieselbe und gegen die Mathematiker auszuführen. Man hat nämlich erklärt, dass für den Ingenieur die Erwerbung weitgehender mathematischer Kenntnisse nicht nur völlig überflüssig, sondern unter Umständen sogar schädlich sei, ferner dass das Studium der Ingenieurwissenschaften auch nicht entfernt in dem von den Mathematikern behaupteten Maasse vom Studium der höheren Mathematik abhängig sei.

Natürlich konnte es den praktisch denkenden Ingenieuren nicht entgehen, dass die Durchführung ihrer Vorschläge sehr erleichtert, die ganze Erörterung der leidigen Frage mit einem Schlage bedeutend rereinfacht werden würde, wenn die Lehrer der Mathematik (selbstverständlich nur an den technischen Hochschulen) unter die Vormundschaft der technischen Professoren gestellt würden. Daher der Kampfesruf: „Die technischen Hochschulen den Technikern!“

\section{E. Papperitz.}

"Die Nathematik an den deutschen technischen Hochschulen" (Leipzig 1899), p. 15, 8, 10.

Es ist hier vielleicht zweckmässig, hervorzuheben, dass das Menschenmaterial, aus dem die in der ausführenden Technik wirklich hervorragend tüchtigen Ingenieure hervorgehen, abgesehen von Ausnahmen, keine Neigung zu haben pflegt, die Mathematik um ihrer selbst willen zu studieren.

\section{BaCH.}

Besprechung von E. Papperitz, „Die Mathematik an den deutschen technischen Hochschulen", Hochschul-Nachrichten, Jahrg. 10 (1900), p. 120. 
Die Naturwissenschaften und die Kunst bilden die ideale Grundlage, auf welcher die technischen Wissenschaften in selbständiger und eigenartiger Entwicklung ihrer praktischen Aufgaben ruhen. - Diese drei Gebiete nach ihrem Inhalt und nach ihrer Bedeutung für die Gegenwart zu erfassen, sie auszubauen und in lebendige Beziehung zu einander zu setzen, das ist die wesentlichste und bedeutsamste Aufgabe, welche, eine ideale und praktische zugleich, die kommende Zeit unsern technischen Hochschulen stellt.

\section{W. Dyck.}

„Über die Beziehungen zwischen künstlerischem und wissenschaftlichem Erfassen der Natur", Festrede München T'echn. Hochsch. 16. I. 1901.

s. Münchener Allg. Zeitg. Beilage 12. II. 1901, No. 35, S. 5.

Wenn die Sprache der Niederschlag der überlieferten und fortschreitenden Gedankenwelt ist, dann ist es sehr bezeichnend, welchen Begriff die gewöhnliche und welchen die gelehrte Auffassung mit dem Worte "Technik" verbindet. "Techne" heisst „Kunst“, „technikos": „kunstmässig“, „kunstgerecht“, und als "Techniker" müsste sinngemäss der Kenner und Ausüber der Kunst, als „Technologie“, wörtlich übersetzt und dem Sinne nach, die Kunstlehre bezeichnet werden. Der gewöhnliche Sprachgebrauch weicht auch von solchem Sinne wenig ab. Nun sehe man aber in unseren gelehrten Wörterbüchern nach! Da steht bei „Technik“: „äusserliche Kunstfertigkeit, Gewerbe thätigkeit, Hand griff, Behandlung des materiellen, äusserlichen Theils der Kunst“, und „technisch“ ist: „handwerksmässig, gewerblich“, „Technologie“: „Gewerbekunde, Handwerksbeschreibung". So legt unsere Gelehrsamkeit in alles, was das Wesen der Technik ausmacht, einen erniedrigenden Sinn, von dem andere Nationen nichts wissen.

Mit dem Worte „Ingenieur" ist Gleiches bisher noch nicht gelungen. Seine Wurzeln führen zu deutlich auf hochstehende Dinge zurïck, auf: Kriegsmaschine, scharfsinnige Erfindung, 
geistreicher, scharfsinniger Mensch, trefflicher Kopf, „fein berechnender Mensch“(Grimm), „eing ebären“, „einpflanzen“, sinnreich, erfinderisch u. s. w., alles Begriffe, die das Wesen der Ingenieurthätigkeit vortrefflich kennzeichnen.

\section{A. Riedler.}

„Unsere Hochschulen und die Anforderungen des zwanzigsten Jahrhunderts", 3. Aufl. (Berlin 1898), p. 99/100.

Es entsteht eine Schule [die École polytechnique], in welcher Alle, welche sich dem Land- und Wasserbau, dem Hüttenwesen, der Artillerie, dem Genie, der Marine widmen, die Weihe einer höheren mathematischen Vorbildung erhalten, die das Seminar aller Lehrer der Mathematik, Physik und Chemie für ganz Frankreich ist, aus der alle Mitglieder der jetzigen Akademie der Wissenschaften in diesen Fächern hervorgegangen sind. Hier soll diejenige Bildung ertheilt werden, die den Staatsdiener über die gemeine Routine erhebt und ihn vor dem Versinken in's Handwerksmässige bewahrt; die hier erworbene Einsicht durchdringt die Fabriken und alle Gewerbzweige des Handwerkers. Die ersten Gelehrten Europa's, wie sie sich nur in einer Welthauptstadt vereinigt finden, stehen ihr vor; es ist eine Schule ohne Vorbild und ohne Nachbild in Europa.

\section{G. J. JACOBI.}

„Über die Pariser polytechnische Schule“,

Vortr. Königsberg Phys.-ökonom. Gesellsch. 22. V. 1835.

s. Werke, herausg. v. Weierstrass, Bd. 7 (1891), p. 356.

Ich bedauere die Trennung von Universität und technischer Hochschule lebhaft; ich möchte die Hochschule, gewissermaassen als ein Seitenstück zur medicinischen Facultät, als technische Facultät der Universität angegliedert sehen.

„Über den Plan eines physikalisch-technischen Instituts an der Universität Göttingen",

Vortr. Hannover Ingenieurverein 6. XII. 1895. 
Universität und technische Hochschule verfolgen nicht nur zusammengehörige Zielpunkte, sondern, wenn sie ihre Interessen richtig verstehen, sehen sie sich immer mehr auf einander angewiesen; sie müssen um ihrer selbst willen daran gehen, Arbeitsmethoden, Auffassungen, Kenntnisse, schliesslich auch Persönlichkeiten von einander zu entlehnen. ...... Die technischen Hochschulen brauchen zur Entwickelung ihres Specialunterrichts Einrichtungen nach Art der Universitäten, diese letzteren wieder dürfen gegenüber den Fortschritten des Ingenieurwesens nicht länger die unbeteiligten Zuschauer bilden.

"Universität und technische Hochschule",

Vortr. Naturf.-Vers. Düsseldorf 1898.

Die Universitäten sollen nicht etwa jetzt in ein neues Extrem verfallen und ihren Zuhörern ausschliesslich eine Kenntnis der angewandten Mathematik vermitteln, sondern das Ziel muss eine allseitige mathematische Durchbildung des Studierenden sein.

„Allgemeines über angewandte Nathematik", Vortr. Feriencurs für Oberlehrer, Göttingen 1900.

\section{Fetix Klein.}

s. Klein u. Riecke, „Über angewandte Mathem. u. Phys.“" (1900), p. 214, 241/242, 24.

Wenn es im Interesse der Universität liegt, dann muss sie die ganze technische Hochschule aufnehmen und damit ein Jahrhundert der Entwickelung der technischen Wissenschaften nachholen. Was die technische Hochschule dabei empfangen kann, ist wenig gegenüber dem, was der Universität fehlt und die wissenschaftliche Technik ihr bringen kann.

A. RIEDLER.

„Unsere Hochschulen und die Anforderungen des zwanzigsten Jahrhunderts", 3. Aufl. (Berlin 1898), p. 98. 
Unseres Erachtens werden die Bestrebungen, specifisch technische Fächer an den Universitäten einzubürgern, ganz von selbst an der Macht der Verhältnisse scheitern. Sie werden auch mit Hülfe von Prüfungsordnungen nicht durchzusetzen sein. Sie können aber eine andere Wirkung haben. Sie können nämlich dazu dienen, Gegensätze, die angeblich beseitigt werden sollen, noch zu verschärfen. Schwerlich wird die Sympathie unserer Techniker dadurch zu gewinnen sein, dass auch für ihre Anstalten „die freieste und weitestgehende Entwickelung“ [F. Klein, Physik. Zeitschr. 1, 1899/1900, p. 145] gefordert wird. Hat doch auch die Technische Hochschule wichtigere Aufgaben, als die, Einrichtungen der Schwesteranstalt nachzuahmen.

E. STUDY.

„Einige Bemerkungen zu der neuen preussischen Prüfungsordnung [für Lehramtskandidaten]".

s. Deutsche Mathem.-Verein. Jahresber. 8, 1899, p. 134.

Die Bestrebungen der Universität Göttingen, ein Institut für Ingenieurwissenschaften zu schaffen, sind ... ein Beleg dafür, wie einseitig die wissenschaftliche Technik in Universitätskreisen aufgefasst wird. - $-\ldots$

Ein einseitig theoretisches Bildungsinstitut kann, sei es auf militärischem, technischem oder wirthschaftlichem Gebiete, weder die Kämpfer in der Front noch die Leiter des Kampfes und am wenigsten die Generalstabsoffiziere ${ }^{1}$ ) ausbilden, höchstens Schlachtenbummler.

\section{A. RIEDLER.}

„Unsere Hochschulen und die Anforderungen des zwanzigsten Jahrhunderts" (Berlin 1898), p. 101 u. 103.

Wirken wir dahin, dass die mathematische Wissenschaft an unseren [technischen] Hochschulen reicher kultiviert wird, nicht für das Gros, aber für eine kleine Zahl auserlesener Studierender, und es wird mit den Jahren dahin

1) vgl. F. Klein, „Über die Neueinrichtungen für Electrotechnik und allgemeine technische Physik an der Universität Göttingen" (Leipzig 1900), p. $14 / 15$. 
kommen, dass wir Mathematiker haben, welche die Technik kennen, oder Ingenieure, welche mathematische Vorlesungen halten können.

\section{R. Fricke.}

„Über den mathematischen Hochschuluuterricht", Vortrag Braunschweig Ver. f. Naturwiss. 19. III. 1902.

s Deutsche Mathem.-Verein. Jahresber. 11 (1902), p. 240/241.

Ein Ingenieur, der ein Mathematikus ist, ist wohl nicht per pleonasmum gesagt.

Lichtenberg an Kästner.

Stade, 24. V. 1773.

s. Lichtenbergs Briefe, herausg. v. Leitzmanu und Schüddekopf, Bd. 1 (1901), p. 129.

There is no useful mathematical weapon, which an engineer may not learn to use.

\section{LORD Kelvin.}

cf. Perry, "The calculus for engineers" (London 1899), p. 5 $=$ Deutsche Ausg. von R. Fricke u. F. Süchting (Leipzig 1902), p. VIII.

Das mathematische Denken und nicht die Formel ist für den Techniker die Grundlage seiner Bildung, die er auch da braucht, wo es sich nicht um speziell mathematische Probleme handelt. Lücken im Fachstudium können in der Praxis oft ausgef üllt werden, Lücken in der Grundlage der Bildung nur schwer. Wird die Mathematik [an den technischen Hochschulen] von Technikern gelesen, so werden die anderen grundlegenden Wissenschaften, wie Physik und Mineralogie nachfolgen, die allgemein bildenden Wissenschaften fortfallen und die technischen Hochschulen die Fühlung mit den Universitäten verlieren.

\section{Krause.}

„Über die Entwickelung der höheren Analysis", Rektorats-Rede Dresden Techn. Hochsch. 1894

s. Jahres-Ber. d. Techn. Hochsch. Dresden 1893/1894, p. 25. 


\section{$-210-$}

Die Universitäten haben in Preussen das Monopol der Lehrerausbildung, selbstverständlich auch für die Lehrer der darstellenden Geometrie, also eines Faches, welches die herrschende Schule gar nicht kennt. Gleichwohl ist es den technischen Hochschulen verwehrt, solche Lehrer auszubilden. Der Kandidat, der in der gymnasialen mathematischen Ausbildung in keine Beziehung zu Raumvorstellung gebracht wurde, bezieht vorschriftsmässig die Universität, hört dort, was die Mathematiker etwa über Funktionentheorie u. s. w. zu lesen belieben, und dann wird er eben Lehrer der darstellenden Geometrie. Es ist ja ein Leichtes, sich „hineinzuarbeiten“. Hat er als Mathematiker vollends gar keine Ahnung vom Ausdruck der Vorstellung, von dem was nach gymnasialer Auffassung ,Zeichnen“ genannt wird, nun dann fehlt ihm nach gleicher Auffassung nur die „Handfertigkeit“. Das ist kein Hinderniss! Da wird das „Zeichnen“ dem „Zeichenlehrer“ übertragen, der ja für die „Handfertigkeit“ da ist, und der Mathematiker lehrt die geometrische Theorie dazu, oder es wird, innerer Überzeugung folgend, gleich die ganze darstellende „Geometrie“ dem Zeichenlehrer übertragen. Daraus erwachsen Zustände, welche für die Ingenieur-Erziehung die traurigsten Folgen haben.

\section{A. Riedler.}

„Zur Frage der Ingenieur-Erziehung“,

Volkswirthschaftl. Zeitfragen, Heft 126 (Berlin 1895), p. 20.

Ist es nicht eine ebenso würdige Aufgabe der Mathematik, richtig zu zeichnen, wie die, richtig zu rechnen?

\section{Felix KLeIN.}

„Über die Beziehungen der neueren Mathematik zu den Anwendungen", Antrittsrede Leipzig Univ. 25. X. 1880. s. Zeitschr. math. naturw. Unterr. 26 (1895), p. 540.

Avant d'avoir entendu Monge, je ne savais pas que je savais la géométrie descriptive.

LaGrange.

voir Arago, Oeuvres, t. 2 (1854), p. 44 (

$=$ Werke, Bd. 2 (1854), p. 357 
In ihrem mathematischen Theile kann die Géométrie descriptive dazu dienen, Solche, die zu abstracten mathematischen Vorstellungen und Beweisen weniger fähig oder gebildet sind, auf dem Wege des praktischen Zeichnens mit einer Menge nützlicher mathematischer Wahrheiten vertraut zu machen.

$$
\text { C. G. J. Јасові. }
$$

„Über die Pariser polytechnische Schule“, Vortr. Königsberg Phys.-ökonom. Gesellsch. 22. V. 1835. s. Werke, herausg. v. Weierstrass, Bd. 7 (1891), p. 357.

Bei der Enthüllung des Gauss-Weber-Denkmals in Göttingen ist mit Recht darauf hingewiesen worden ${ }^{1}$ ), dass die Naturwissenschaften sich nicht mehr allein auf die reine um ihrer selbst willen betriebene Wissenschaft beschränken dürften, sondern Anwendung und Verwerthung suchen müssten. Das ist ein vollständiger Bruch mit der Überlieferung und das Einlenken in die vielgestaltige wissenschaftliche Thätigkeit, die unser Arbeitsfeld ist. Wir können diese Richtungsänderung nur mit Genugthuung begrüssen.

Indem Nernst die Wissenschaft nicht bloss um ihrer selbst willen betrieb, sondern an die deutsche Industrie herantrat und mit ihrer Hülfe seine Idee ausgestaltete, bewies er, dass er die Wissenschaft in ihren vielfältigen Beziehungen zum Leben richtig erfasst hat und leistete er der Wissenschaft selbst einen Dienst. Sein grosser Vorgänger Weber liess es beim ersten unvollkommenen Schritte bewenden, und so ist es gekommen, dass seine Idee, um in brauchbarer Gestalt zu uns zu kommen, den Umweg über das Ausland machen musste, das nunmehr selbst das deutsche wissenschaftliche Verdienst bestreitet.

A. Riedler. ${ }^{2}$ )

„Die technischen Hochschulen und ihre wissenschaftlichen Bestrebungen", Rektoratsrede Berlin Techn. Hochsch. 1. VII, 1899 (Berlin 1899), p. 10.

1) Bei der am 17. VI. 1899 vollzogenen Enthüllung wurde die Weiherede, welche - soviel mir bekannt - gedruckt nicht erschienen ist, von W. Voigt gehalten.

2) Die beiden Abschnitte dieses Citats sind von mir hier in ihrer Reihenfolge vertauscht. 
„Wir können hierbey eine . . . bisher in ihrer Art einzige Anlage nicht unerwähnt lassen, die wir unserm Hn Professor Weber verdanken. Dieser hatte bereits im vorigen Jahre von dem Plysikalischen Cabinet aus über die Häuser der Stadt hin bis zur Sternwarte eine doppelte Drahtverbindung geführt, welche gegenwärtig von der Sternwarte bis zum Magnetischen Observatorium fortgesetzt ist.

.. Die Leichtigkeit und Sicherheit, womit man durch den Commutator die Richtung des Stroms und die davon abhängige Bewegung der Nadel beherrscht, hatte schon im vorigen Jahre Versuche einer Anwendung zu telegraphischen Signalisierungen veranlasst, die auch mit ganzen Wörtern und kleinen Phrasen auf das vollkommenste gelangen. Es leidet keinen Zweifel, dass es möglich seyn würde, auf ähnliche Weise eine unmittelbare telegraphische Verbindung zwischen zweyen eine beträcbtliche Anzahl von Meilen von einander entfernten Örtern einzurichten: allein es kann natürlich hier nicht der Ort seyn, Ideen über diesen Gegenstand weiter zu entwickeln."

$$
\text { C. F. Gacss. }
$$

Göttinger Gelehrte Anzeigen 9. VIIl. 1834, Stück 128, p. 1272-1274 $=$ Werke, Bd. 5 (1877), p. 524/525.

Darf ich Ihnen vertraulich sagen, was mir selbst bei meinen Arbeiten die meiste Satisfaction giebt, so sind es viel mehr die theoretischen Eroberungen im Gebiet des Electromagnetismus, als die in dem des reinen Magnetismus. In andern äussern Verhältnissen als die meinigen sind, liessen sich wahrscheinlich auch für die Societät wichtige und in Augen des grossen Haufens glänzende praktische Anwendungen daran knüpfen. . . . . . . Könnte man darauf Tausende von Thalern wenden, so glaube ich, dass z. B. die Electromagnetische Telegraphie zu einer Vollkommenheit und zu einem Maassstabe gebracht werden könnte, vor der die Phantasie fast erschrickt. Der Kaiser von Russland'könnte seine Befehl 
ohne Zwischenstation in derselben Hinute von Petersburg nach Odessa, ja vielleicht nach Kiachta geben - - - - - -

Gacss an Schumacher.

Göttingen, 6. VIII. 1835.

s. Briefw. Gauss-Schumacher, Bd. 2 (1860), p. 411/412.

Vor einigen Tagen habe ich zum ersten Mahle probirt, ob die durch meinen Inductor mit 3500 Windungen erzeugten Ströme auch den menschlichen Körper zu durchdringen stark genug sind. Gegen meine Erwartung hat sich diese Frage bejahend beantwortet. Lässt man den Strom durch die benetzten Hände gehen, so ist der Strom, dessen Dasein das Magnetometer zeigt, und seine Stärke scharf misst, noch zu schwach, um gefühlt zu werden; allein durch die Lippen oder Zunge geleitet, ist er etwa viermahl stärker, sehr merklich zu fühlen, ja zu schmecken....... Man würde selbst diese Methode zum Telegraphiren brauchen können, und die Depesche, welche S. Maj. aller Reussen in Petersburg abspielen lassen wollte, würde in demselben Augenblick in Odessa geschmeckt werden können. Wollte man eine mehrfache Kette ziehen, und zugleich eine correspondirende Anzahl Schmecker am andern Ende aufstellen, wozu man auch blinde Invaliden brauchen könnte, die nur jedesmahl, wo Ihnen [sic] zu schmecken gegeben wird, die Hand in die Höhe zu heben hätten, während ein Secretär die aufgehobenen Hände protocollirte, so würde selbst nach dieser Methode sehr schnell telegraphirt werden können.

Gauss an Schumacher. Göttingen, 13. IX. 1835.

Nach Ihrer Beschreibung fühle ich keinen sonderlichen Appetit zu Ihrem magnetischen Gerichte. Wenigstens, so scheint es, muss man in dem Genusse mässig seyn.

Schumacher an Gauss. Altona, 18. IX. 1835.

s. Briefw. Gauss-Schumacher, Bd. 2 (1860), p. $417 / 418$ u. 418. 
Gauss war keineswegs gegen diesen, jetzt in unsere Lebensverhältnisse so tief eingreifenden Erfolg seiner Forschungen [magnetelectrische Telegraphie] gleichgültig. Nur wollte er den Werth der wissenschaftlichen Untersuchungen nicht nach der Grösse des Gewinns abgeschätzt wissen, den die materiellen Interessen daraus ziehen. Die Wissenschaft, äusserte er einmal im Gespräche, soll die Freundin der Praxis sein aber nicht ihre Sclavin, sie soll ihr schenken aber nicht ihr dienen. Hiermit übereinstimmend sagt er in der Einleitung zu seiner Theorie des Erdmagnetismus „die Wissenschaft, wenn gleich gern auch den materiellen Interessen förderlich, lässt sich doch nicht auf diese beschränken, sondern fordert für alle Elemente ihrer Forschung gleiche Anstrengung. “1)

\section{A. Stern.}

„Denkrede auf Carl Friedrich Gauss", Festrede Göttingen Univ. 30. IV. 1877, p. 15.

Thou, nature, art my goddess; to thy law My services are bound - -

Shakespeare.

King Lear, Act I, Scene II [Wahlspruch Gauss', jedoch mit der Änderung „laws statt des Shakespeare'schen „law". $\left.\left.{ }^{2}\right)\right]$

Gauss war überall bemüht der Mathematik eine neue Seite für ihre Anwendung zu eröffnen. Er führte daher die mannigfaltigsten Zahlenregister, die in kleinen Büchern auf die sauberste und pünktlichste Weise eingetragen wurden. So z. B. hatte er ein Verzeichniss der Lebensdauer der neisten bedeutenden Männer und namentlich seiner verstorbenen Freunde in Tagen berechnet; dann führte er über die monatlichen Einnahmen der Hannoverschen Eisenbahnen ein Register; ein

1) s. Gauss, Werke, Bd. 5 (1877), p. 121, wo es jedoch , dem materiellen Interesse" und dementsprechend ,auf dieses" heisst.

2) s. S. 93 . 
anderes über die Schrittdistanzen von der Sternwarte nach Orten die er öfter zu besuchen pflegte ${ }^{1}$ ) $-\ldots$

SARTORIUS V. WaLtershiaUSEN. „Gauss zum Gedächtniss" (1856), p. 89.

Es ist übermorgen der Tag, wo Sie, mein hochverehrter Freund, in ein Gebiet übergehen, in welches noch keiner der Koryphäen der exacten Wissenschaften eingedrungen ist, der Tag, wo Sie dasselbe Alter erreichen, in welchem Newton seine durch 30766 Tage gemessene irdische Laufbahn geschlossen hat.

$$
\text { C. F. Gauss an A. v. Humboldt. }
$$

Göttingen, 7. XII. 1853.

s. K. Bruhns, „Briefe zw. A. v. Humboldt und Gauss“

(Leipzig 1877), p. 68

$=\mathrm{K}$. Bruhns, „Alexander von Humboldt", Bd, 2 (1872), p. 172.

Ein Professor Dr. Sch .... in H ..... hat ausgerechnet, dass am 29. April d. J. Vormittags um 10 Uhr 40 Minuten genau eine Milliarde Minuten seit Beginn unserer Zeitrechnung verflossen war.

Wie viel Tausende von Minuten muss dieser Gelehrte zu verlieren haben!

\section{Ḱladderadatscii.}

4. V. 1902.

In wenigen Tagen, am letzten dieses Monats, vollenden Sie das 67. Jahr ihres [sic!] thatenreichen, über so viele Gebiete Licht spendenden und glanzvollen Lebens! Ich bringe Ihnen dazu meine wärmsten Glïckwünsche dar. . . . . Die Zahl 67 ist die letzte jener drei kritischen Zahlen im ersten Hundert, für welche der Beweis jenes berïhmten Fermat'schen Satzes, der die grössten Mathematiker so lange beschäftigt hat, noch nicht erbracht ist. Die grössten arithmetischen Fährlichkeiten des ersten Hundert Ihrer Lebensjahre haben Sie also bei Empfang dieser Zeilen glücklich überstanden, und ich wïnsche

1) vgl. S. 91/92 (M. Cantor). 
Ihnen von Herzen, dass Sie alle physischen Fährlichkeiten ebenso sicher und leicht ïberstehen mögen! Dieser 31. August ist der erste Ihrer Geburtstage, den Sie in dem neuen Lebensstadium, in welches Sie das neue von Ihnen übernommene Amt geführt hat, begehen. Mögen Sie darin volle Befriedigung finden und eine lange Reihe von Jahren zum Segen der Wissenschaft Ihre grossartige Wirksamkeit ausüben, dabei aber auch der Universität - wie Sie es zugesagt haben und wie es jener Erlass betreffend "die Erhaltung der Kraft Helmholtz" gestattet $^{1}$ ) - Ihre segensreiche Lehrthätigkeit aufs Intensivste widmen. Nach diesem Stadium, in welchem Sie ausschliesslich mathematische Physik vortragen, wird - ich bin ebenso von dem Wunsche als der Überzeugung beseelt - ein Stadium nachkommen, in welchem Sie sich der reinen Mathematik zuwenden und auch dahinein die Leuchte Ihres Geistes tragen. Sie haben ja an vielen Stellen schon angesetzt, und es ist - wie mir schon lange scheint - nur die Consequenz des in der Geschichte der Wissenschaften wohl einzig dastehenden Entwickelungsganges Ihres wissenschaftlichen Lebens, dass es von der rechten Seite praktischst-wissenschaftlicher Medicin beginnend, durch die Physiologie zur experimentellen und theoretischen Physik weiterschreitend, schliesslich auf der äussersten Linken in der ganz abstracten „reinen“ Mathematik anlangt. Der Reichthum praktischer Erfahrung, gesunder und interessanter Probleme, den Sie der Mathematik zubringen, wird derselben - wie im vorigen Jahrhundert die Arbeit der Astronomen - sicher dann eine neue Richtung und damit einen neuen Aufschwung geben; denn die einseitig nur in sich selbst gekehrten mathematischen Speculationen führen in sterile Gebiete. Also, kommen Sie auch zu uns hinüber, hochverehrter Freund, und drücken Sie auch in die Bahnen der reinen

1) Über die Bemühungen der Berliner philos. Fakultät, sich ihr Mitglied H. von Helmholtz trotz dessen Ernennung zum Praesidenten der phys.-techn. Reichsanstalt zu erhalten, und ihre diesbezügliche Eingabe an den preuss. Cultusminister in dem von Kronecker und Ed. Zeller vorgeschlagenen Wortlaut s. Königsberger, l. c., Bd. 2 (1903), p. 352-354. 
Mathematik die unvergänglichen Spuren Ihrer originellen und kühnen Forschungsschritte, damit auch dort die Pfade der $\mathrm{Zu}$ kunft damit bezeichnet werden.

\section{Kronecker an H. von Helmholtz.}

28. VIII. 1888.

§. Königsberger, „Hermann von Helmholtz“, Bd. 3 (1903), p. 4/5.

Nur danm interessirten Helmholtz auch mathematische Untersuchungen an sich, wenn es sich um die Aufsuchung der Grundlagen und Axiome mathematischer Disciplinen handelte, und so hat er in der That darauf bezügliche Forschungen für die drei grossen Gebiete der Mathematik, die Geometrie, Arithmetik und Mechanik angestellt, die für die Erkenntnisstheorie, sowie für die gesammte Entwicklung der mathematischen Physik bahnbrechend gewesen sind; aber auch hier gaben ihm wieder im Gegensatz zu ähnlichen oder ganz gleichgerichteten Untersuchungen anderer ausgezeichneter Mathematiker stets die Beobachtung und Erfahrung den festen Boden und eine sichere Richtschnur für seine Wege, auf denen er zu den abstractesten mathematischen Wahrheiten gelangte. ${ }^{1}$ )

\section{LEO KÖNIGSBERGier.}

„Hermann von Helmholtz's Untersuchungen über die Grundlagen der Mathematik und Mechanik" (Leipzig 1896), p. 3.

Die Mathematik ist doch nicht allein wegen der Naturwissenschaft da: Auch wenn man ihr kein selbständiges Existenzrecht zugestehen will, so haben die Psychologie, die Logik, die Erkenntnistheorie Ansprüche an sie; und sie verlangen, dass die Elemente unsrer 7ahl- und Raumvorstellungen bis in ihre Konsequenzen verfolgt und die dabei sich unerbittlich ergebenden Paradoxieen blossgelegt werden.

\section{H. BuRKhaRdT.}

„Mathematisches und naturwissenschaftliches Denken“,

Antrittsvorl. Zürich Univ. 6. XI. 1897.

s. Münchener Allg. Zeitg. Beilage 22. XI. 1897

$=$ Deutsche Mathem.-Verein. Jahresber. 11 (1902), p. 54.

1) vgl. S. 29 (Leo Königsberger). 
Vous ne serez jamais qu'un philosophe, et qu'est-ce qu'un philosophe? C'est un fou qui se tourmente pendant sa vie, pour qu'on parle de lui lorsqu'il n'y sera plus.

La Nourrice de D'Alembert à celui-ci, déjà homme célèbre. voir Condorcet, Oeurres, Édition d'Arago, t. 3 (1847), p. 55; voir aussi „Nouv. Biographie universelle“, t. 1 (Paris 1852), p. 759.

Il me semble que chaque pays a presque sa Métaphysique particulière comme sa langue.

LAGRANGe à d'Alembert.

Berlin, 27. I. 1778.

voir Oeuvres de Lagrange, t. 13 (Paris, 1882), p. 336.

Dirichlet pflegte von der Philosophie zu sagen, es sei ein wesentlicher Mangel derselben, dass sie keine ungelösten Probleme habe wie die Mathematik, dass sie sich also keiner bestimmten Grenze bewusst sei, innerhalb deren sie die Wahrheit wirklich erforscht habe und über welche hinaus sie sich vorläufig bescheiden müsse, nichts zu wissen. Je grössere Ansprüche auf Allwissenheit die Philosophie machte, desto weniger vollkommen klar erkannite Wahrheit glaubte er ihr zutrauen zu dürfen, da er aus eigener Erfahrung in dem Gebiete seiner Wissenschaft wusste, wie schwer die Erkenntniss der Wahrheit ist, und welche Mühe und Arbeit es kostet, dieselbe auch nur einen Schritt weiter zu fördern.

\section{KUMMER.}

„Gedächtnissrede auf Gustav Peter Lejeune Dirichlet." s. Abhandl. d. Berl. Akad. 1860, p. 31 $=$ Dirichlet, Werke, Bd. 2, p. 340 .

Verworrenheiten in Begriffen und Definitionen sind nirgends mehr zu Hause, als bei Philosophen, die keine Mathematiker sind..... Sehen Sie sich doch nur bei den heutigen Philosophen um, bei Schelling, Hegel, Nees von Esenbeck und Consorten, stehen Ihnen nicht die Haare bei ihren Definitionen zu Berge. Lesen Sie in der Geschichte der alten Philosophie, was die damaligen Tagesmänner Plato und andere (Aristoteles 
will ich ausnehmen) für Erklärungen gegeben haben. Aber selbst mit Kant steht es oft nicht viel besser; seine Distinction zwischen analytischen und synthetischen Sützen ist meines Erachtens eine solche, die entweder nur auf eine Trivialität hinauslïuft oder falsch ist.

Gauss an Schumacher.

Göttingen, 1. XI. 1844.

s. Briefw. Gauss-Schumacher, Bd. 4 (1862), p. 337; vgl. a. ibid. p. 309.

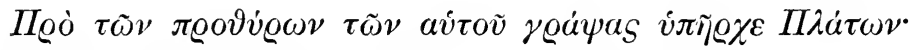

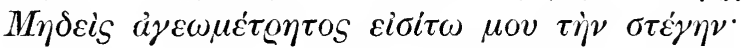

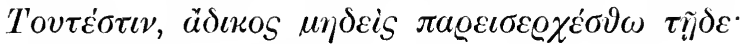

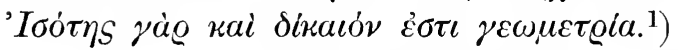
[Vor seine Thür schrieb Plato gleich zu Anfang: . Niemand komme, unkundig der Geometrie, mir unter mein Dach; das heisst: kein Ungerechter komme heimlich hier hinein; denn Gleichheit und Recht ist Geometrie.]

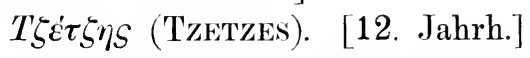

Historiarum var. Chiliades VIII, 972-975, ed. Kiessling (Leipzig 1826), p. 322.

Veritas $^{2}$ ) humanum genus in aeternum lateret, nisi mathesis, quae non circa fines, sed tantum circa figurarum essentias et proprietates versatur, aliam veritatis normam hominibus ostendisset.

\section{SPINOZA.}

„Ethica“, Pars I, Propos. 36, App.

s. z. B. Ginsberg'sche Ausg. (Leipzig 1875), p. 111.

Eine reine Naturlehre über bestim m te Naturdinge (Körperlehre und Seelenlehre) ist nur vermittelst der Mathematik möglich, und da in jeder Naturlehre nur so viel eigentliche Wissenschaft angetroffen wird, als sich darin Erkenntniss a priori befindet,

1) Über ältere Belege für jene platonische Inschrift s. Note 14 am Ende des Buches.

2) hinsichtlich der „causae finales" 
so wird Naturlehre nur so viel eigentliche Wissenschaft enthalten, als Mathematik in ihr angewandt werdèn kann.

\section{KANT.}

„Metaphys. Anfangsgründe der Naturwissenschaft", Vorrede. s. J. H. von Kirchmann's Philos. Bibl., Bd. 48, p. 171.

Goethe hatte den treuen, sich hingebenden, objektiven Blick in die Natur der Sachen; Neuton war bloss Mathematiker, stets eilig nur zu messen und zu rechnen, und zu dem Zweck eine aus der oberflächlich aufgefassten Erscheinung zusammengeflickte Theorie zum Grunde legend. Dies ist die Wahrheit: schneidet Gesichter wie ihr wollt!

A. Schopenhauer.

- „Parerga und Paralipomena“, Bd. 2, Kap. VII

(„Zur Farbenlehre“), § 108.

s. Werke, herausg. v. J. Frauenstädt, 2. Aufl., Bd. 6 (1888), p. 212.

Newton hatte durch eine künstliche Methode seinem Werk [der Optik」 ein dergestalt strenges Ansehn gegeben, dass Kenner der Form es bewunderten und Laien davor erstaunten. Hiezu kam noch der ehrwürdige Schein einer mathematischen Behandlung, womit er das Ganze aufzustutzen wusste.

Gonthe.

„Zur Farbenlehre. Polemischer Theil."

s. Werke, Grosse Weimarische Ausg., Abth. II, Bd. 2 (1890), p. 4/5 $=$ Goedeke'sche 10-bändige Ausg., Bd. 10, p. 230.

Nun so wäre denn endlich die Untersuchung in die Geheimnisse der Mathematik gehüllt, damit doch ja niemand so leicht wage sich diesem Heiligthum zu nähern.

GowTHE über Newtons „Optik“.

"Materialien zur Geschichte der Farbenlehre."

8. Werke, Grosse Weimarische Ausg., Abth. I, Bd. 4 (1894), p. 127

$=$ Goedeke'sche 10-bändige Ausg., Bd. 10, p. 517. 
Nature and Nature's laws lay hid in night;

God said, Let Newton be! and all was light. ${ }^{1}$ )

Alexander Pope.

ef. Poetical works (Edinburgh 1780), vol. 3, p. 253.

Newton, avec lequel L'Hôpital n'eut pas de relations directes, était l'objet de toute son admiration. Aimant à questionner ceux qui avaient eu l'honneur de voir un si grand homme, il s'étonnait, dit-on, dans son naif enthousiasme, que, soumis aux lois de l'humanité, l'auteur du livre des Principes pût manger, boire et dormir comme les autres hommes.

\section{J. Bertrand.}

„L'Académie des sciences et les académiciens de 1666 à $1793^{4}$ (Paris 1869), p. 240.

Sir Isaac Newton, a little before he died, said: „I don't know what I may seem to the world, but, as to myself, I seem to have been only like a boy playing on the sea shore, and diverting myself in now and then finding a smoother pebble or a prettier shell than ordinary, whilst the great ocean of truth lay all undiscovered before me.

\section{The Rev. J. Spence.}

„Anecdotes, observations, and characters, of books and men.

Collected from the conversation of Mr. Pope, and other eminent persons of his time", Second edit. by S.W. Singer(London 1858), p. 40.

Dieu parle, et le chaos se dissipe à sa voix:

Vers un centre commun tout gravite à la fois.

Ce ressort si puissant, l'ame de la nature,

Etait enseveli dans une nuit obscure:

1) in der von Brandes besorgten deutschen Ausg. (1833) von Brewster, "The Life of Sir Isaac Newton" folgendermassen übersetzt (1. c. p. 288):

„Natur und der Natur Gesetz waren in Nacht gehüllt;

Gott sprach: Es werde Newton! und das All ward lichterfüllt." 
Le compas de Newton, mesurant l'univers, Lève enfin ce grand voile, et les cieux sont ouverts.

Confidens du Très-Haut, substances éternelles, Qui brûlez de ses feux, qui couvrez de vos aîles Le trône où votre maître est assis parmi vous, Parlez, du grand Newton n'étiez-vous point jaloux?

VOLTAIRE.

„Épitre à madame la marquise du Châtelet, sur la philosophie de Newton."

voir Voltaire, „Mélanges philosophiques, littéraires, historiques .....", t. 1 (Genève 1771), p. 9 $=$ Oeuvres de Voltaire, t. 13 (Gotha 1785), p. 95.

J'aime beaucoup la philosophie et les vers. Quand je dis philosophie, je n'entends ni la géométrie ni la métaphysique. La première, quoique sublime, n'est point faite pour le commerce des hommes; je l'abandonne à quelque rêve-creux d'Anglais: qu'il gouverne le ciel comme il lui plaira, je m'en tiens à la planète que j'habite.

Frédéric le Grand à Voltaire.

Potsdam, 13. II. 1749.

voir Oeuvres de Frédéric le Grand, t. 22 (Édition Decker 1853), p. $181 / 182$.

Über die Polarisation des Lichts haben die Franzosen nichts als unsinnige Theorien, aus der Undulation und der homogenen Lichter = Lehre, nebst Rechnungen, die sich auf nichts gründen. Stets sind sie eilig, nur zu messen und zu rechnen, halten es für die Hauptsache und le calcul! le calcul! ist ihr Feldgeschrei. Aber ich sage: où le calcul commence, l'intelligence des phénomênes cesse: während Einer blosse Zahlen und Zeichen im Kopfe hat, kann er nicht dem Kausalzusammenhang auf die Spur kommen. Das Wieviel und Wiegross hat für praktische Zwecke Wichtig- 
keit: in der Theorie aber kommt es hauptsächlich und zunächst auf das Was an.

\section{A. Schopenhauer.}

„Parerga und Paralipomena“, Bd. 2, § 80.

s. Werke, herausg. v. J. Frauenstädt, 2. Aufl, Bd. 6 (1888), p. 128; vgl. a. ibidem, Bd. 1 (1888), p. 77 („Über d. vierf. Wurz. d. Satzes v. Gr.", Kap. IV, § 21).

Wie einer solchen Wissenschaft, die uns von Stufe zu Stufe bis zu ganz bestimmten Realprincipien hinaufführt, der philosophische Werth abgesprochen werden kann, das scheint schwer begreiflich. Hat doch diese Wissenschaft (aus der Anwendung ihrer Principien auf die Wirklichkeit) unbekannte Planeten a priori entdeckt. Und das ist mehr werth als allgemeine Redensarten!

\section{O. Liebmann.}

„Zur Analysis der Wirklichkeit", 3. Aufl. (1900), p. 288 („,̈ber den philosophischen Werth der mathematischen Naturwissenschaft").

Daguerre's Erfindung, wenn nicht etwan, wie Einige behaupten, der Zufall viel dazu beigetragen hat, daher Arago die Theorie dazu erst hinterher ersinnen musste, ist hundert Mal scharfsinniger, als die so bewunderte Entdeckung des Leverrier.

\section{A. Schopenhauer.}

„Parerga und Paralipomena“, Bd. 2, Kap. VI, § 82.

s. Werke, herausg. v. J. Frauenstädt, 2. Aufl., Bd. 6 (1888), p. 135.

\section{Archimedes und der Jüngling. ${ }^{1}$ )}

$\mathrm{Zu}$ Archimedes kam ein wissbegieriger Jüngling, Weihe mich, sprach er zu ihm, ein in die göttliche Kunst,

1) nach Schiller's Gedicht' „Archimedes und der Schüler", s. z. B „Schillers sämtl. Schriften", hist.-krit. Ausg. v. K. Goedeke, Th. 11 (1871), p. 92 . 
Die so herrliche Dienste der Sternenkunde geleistet, Hinter dem Uranos noch einen Planeten entdeckt. Göttlich nennst Du die Kunst, sie ist's, versetzte der Weise, Aber sie war es, bevor noch sie den Kosmos erforscht, Ehe sie herrliche Dienste der Sternenkunde geleistet, Hinter dem Uranos noch einen Planeten entdeckt.

Was Du im Kosmos erblickst, ist nur der Göttlichen Abglanz, In der Olympier Schaar thronet die ewige Zahl.

\section{G. J. JACoBI an A. v. Humboldt.}

s. L. Kronecker, „Über den Zahlbegriff“ in „Philos. Aufs., Eduard Zeller z. s. 50-jähr. Doctor-Jubil. gewidmet"

(Berlin 1887), p. 264

$=$ J. f. Math. 101 (1887), p. 338

= Kronecker, Werke, herausg. v. Hensel, Bd. 3, 1 (1899), p. 252.

\section{An die Astronomen. ${ }^{1}$ )}

Schwatzet mir nicht soviel ron Nebelflecken und Sonnen,

Ist die Natur nur gross, weil sie zu zählen euch gibt? Euer Gegenstand ist der erhabenste freilich im Raume,

Aber Freunde, im Raum wohnt das Erhabene nicht. SCHILLER.

s. "Sämtl. Schriften", hist.-krit. Ausg. v. K. Goedeke, Th. 11 (1871), p. 188.

Christian Wolf war als Philosoph auch zugleich ein recht guter Mathematiker, welches würklich ein sehr bemerkenswerther Umstand ist, weil nicht alle Philosophen sich dieses Vortheils rühmen können. Zwar ist umgekehrt die Sache so merkwürdig nicht; denn wenn man annehmen will, dass die Mathematik sein Hauptfach gewesen sey, so ist wol gewis, dass das Verdienst, ein Philosoph gewesen zu seyn, nicht mehr so wichtig bleibt.

„Nachrichten von dem Leben und den Frfindungen der berühmtesten Mathematiker" (Münster 1788), p. 304/305.

1) Eine etwas abweichende Lesart dieses Epigramms, sowie eine Conjektur bezüglich seiner Auslegung s. bei E. Boas, „Schiller und Goethe im Xenienkampf" (Stuttgart \& Tübingen 1851), Th. I, p. 267 u. 117/118. 
[Für die geistige Welt] tritt uns als das Wahre und desswegen auch als das allein Fruchtbare die von unserem scharfsinnigen und tief denkenden Landsmanne Gall aufgestellte Geisteslehre, die sogenannte Phrenologie, entgegen - - - -

Wer das Schlussvermögen in hohem Grade besitzt, wird in hohem Grade angenehm erregt durch eine vollkommen folgerichtige sprunglose Kette von Schlüssen, wie sie z. B. die mathematischen Wissensschaften liefern, wogegen ihn jeder Fehler im Schliessen lebhaft schmerzt. - -

Auf welchen Gegenstand sich das Schlussvermögen richtet, hängt von den übrigen Geistesvermögen ab. Bei grossem Zahlen- oder Raumsinne wird ein Mathematiker oder Geometer entstehen, bei Vergleichungsvermögen und vielseitigen Geisteskräften ein Philosoph, ein Weltweiser. Mathematik und Philosophie wurden häufig von denselben Männern, wie z. B. von Descartes und Leibnitz, mit grossem Erfolge getrieben.

Der Sitz des Schlussvermögens ist zu beiden Seiten des Vergleichungsvermögens unter dem oberen Theile der Stirne.

Der Sitz des Raumsinnes bei dem Menschen ist über dem inneren Ende der Augenbrauen zu beiden Seiten des Gegenstandssinnes und erstreckt sich in eigenthümlichem Bogen nach oben und aussen. - $-\ldots$

Der Sitz des Zahlensinnes ist an dem äussersten Ende der Augenbrauen. Ist er ausgebildet, so zeigt sich diese Stelle gewölbt und der äussere Brauenbogen abwärts gedrückt. ${ }^{1}$ )

CHr. Wiener.

„Die Grundzüge der Weltordnung" (Leipzig \& Heidelberg 1863), Vorwort, p. IV; p. 305/306; p. 296; p. 299.

Der allgemeine Grund dafür, dass mathematisches und philosophisches Talent sich oft vereint finden, liegt darin, dass es nur die eine Befähigung und Neigung für das rein abstrakte Denken ist, welcher die beiden verschiedenen Wege der mathematischen so wie der philosophischen Speculation gleichmässig

1) vgl. S. 242/243 (P. J. Moebius und Rud. Wagner).

A hrens, Scherz und Ernst i. d. Mathem. 
offen stehen; ob ein mit diesem Talente vorzugsweise begabter wissenschaftlicher Forscher sich mehr der einen oder der andern dieser verwandten Wissenschaften zuwendet, oder ob er einer derselben sich ganz ergiebt, scheint mehr nur von äusseren Bedingungen abhängig zu sein.

E. E. Kunmer.

(,C̈ber die Beziehungen Friedrichs des Grossen zur Mathematik und zu den Mathematikern seiner Zeit"),

Festrede Berlin Akad. 26. I. 1865.

s. Berl. Monatsber. 1865, p. 74 .

On demandoit un jour: Qu'est ce qu'un métaphysicien? Un géométre répondit: C'est un homme qui ne sçait rien. Les chymistes, les physiciens, les naturalistes, et tous ceux qui se livrent à l'art expérimental, non moins outrés dans leur jugement, me paroissent sur le point de vanger la métaphysique, et d'appliquer la même définition au géométre. Ils disent: A quoi servent toutes ces profondes théories des corps célestes, tous ces énormes calculs de l'astronomie rationelle, s'ils ne dispensent point Bradley ou le Monnier d'observer le ciel? Et je dis heureux le Géométre en qui une étude consommée des sciences abstraites n'aura point affoibli le goût des beaux arts, à qui Horace et Tacite seront aussi familiers que Newton, qui sçaura découvrir les propriétés d'une courbe et sentir les beautés d'un poëte, dont l'esprit et les ouvrages seront de tous les tems, et qui aura le mérite de toutes les académies! Il ne se verra point tomber dans l'obscurité; il n'aura point à craindre de survivre à sa renommée.

\section{DiDEROT.}

„Pensées sur l'interpretation de la nature“, III (1754), p. 4/5.

Wir Mathematiker auch sind echte, berufene Dichter; Uns liegt noch der Beweis für das Gedichtete ob!

\section{KRONECKER}

an den Mathem. Verein der Univ. Berlin zu dessen 29. Stiftungsfest, December 1890.

s. Ber. d. Mathem. Vereins Ostern 1890-Ost. 1891, p. 9 
Huyghens, ce philosophe Hollandais qui découvrit en France une lune de Saturne, s'attacha aussi à observer Mlle. Ninon Lenclos. Elle métamorphosa un moment le mathématicien en galant et en poète. Il fit pour elle ces vers qui sont un peu géométriques ${ }^{1}$ ):

Elle a cinq instrumens dont je suis amoureux,

Les deux premiers ses mains, les deux autres ses yeux.

Pour le plus beau de tous, le cinquiéme qui reste,

Il faut être fringant et leste.

VOLTAIRE.

„Lettre sur Mademoiselle de Lenclos."

voir „Mélanges philosophiques, littéraires, historiques ....", t. 2 (Genève 1771), p. 390

$=$ Oeuvres de Voltaire, t. 49 (Gotha 1787), p. 247/248.

Je scavois tresbien qui estoit Mademoiselle de l'Enclos, et Monsieur de Boisrobert ${ }^{2}$ ) m'a plus de trois fois promis de m'y mener, mais tousjours il y est venu quelque empeschement. Il me monstra un tableau ou elle estoit peinte toute nuë, lors qu'elle estoit encore raisonnable, mais a cet heure elle est passée, et converse, à ce qu'on dit, arec les femmes honnestes apres que l'age luy a fait quiter son premier mestier. Mon Pere la voit assurement pour l'amour de la musique et je n'en veux point avoir d'autre pensee.

Christiana Huygrans (à Lodewijk Huygens). 27. VII. 1662 .

voir Oeuvres compl., t. 4 (La Haye 1891), p. 183/184.

\section{Sylvester's „Spring's Début.“}

Spring's Début, which is a fantastic poem in praise of one Mary Winn, of Baltimore, consists of two

1) Nicht Chr. Huygens, sondern dessen häufig als Dichter aufgetretener Vater Constantin H. soll der Verfasser dieser Verse gewesen sein; vgl. hierzu das folgende Citat, sowie J. Bosscha, ,Christian Huygens", Discours Amsterdam Univ. 8. VII. 1895 (Extrait des Archives Néerlandaises, t. 29), p. $44 / 45$.

2) François le Metel de Boisrobert (1592-1662), Abbé in Paris und später „Aumônier du Roi“ (s. Huygens, Oeuvres, t. 2 (1889), p. 377). 
hundred and thirteen seven-syllabled lines, all ending in rhymes or assonances to this lady's name. Here, for example, is a passage of parallels to the accomplished lady:

„Fine as point-lace or Mechlin,

Circe - but more feminine,

Pamela, artless genuine,

Like loved child of Cymbelin' -

Sainted, sweet-souled Imogen,

Not more lively, dear Nell Gwynne,

Dreamier nocturne of Chopin,

Swan-song of weird Lohengrin,

Calmer aureoled Capuchin

Niched beneath stone baldaquin,

Loved of the Dryads Evelyn,

Daintier touch of Maturin,

Purer rill of tears, Undine,

Shedde to undoe each staine of sinne,

Prouder, self-ruled Catherine

Fre rose might of Mazarin

Or who broke to rein Berlin

High autocrator Tzarin,

Pious as Evangeline."1)

cf. The Academy 51 (27. III. 1897), p. 358

$=$ Johns Hopkins Univ. Circulars 16 (1897), p. 56/57.

Gauss behauptete [als Student in Göttingen], Kästner sei der erste Mathematiker unter den Dichtern und der erste Dichter unter den Mathematikern.

\section{W. SCHUR.}

„Beitr. zur Gesch. d. Astronomie in Hannover."

s. „Festschr. zur Feier des 150-jähr. Bestehens d. Kgl. Ges. d. Wiss. z. Göttingen" (Berlin 1901), p. 125; s. a. L. Hänselmann, „Karl Friedrich Gauss" (Leipzig 1878), p. 31.

1) Einige Erläuterungen zu den vorstehenden Versen s. in Note 15 am Ende des Buches. 
Kästner hatte einen ganz eminenten Mutterwitz, aber sonderbar genug, er hatte ihn bei allen Gegenständen ausserhalb der Mathematik, er hatte ihn sogar, wenn er über Mathematik (im Allgemeinen) sprach, aber er wurde oft ganz davon verlassen innerhalb der Mathematik.

GAuss an Schumacher.

Göttingen, 22. XII. 1845.

s. Briefw. Gauss-Schumacher, Bd. 5 (1863), p. 95/96.

Plus que les autres sciences, les mathématiques exigent de ceux qui sont appelés à augmenter par de nouvelles conquêtes le domaine du savoir, une imagination puissante. La clarté de la pensée n'a jamais, à elle seule, fait de découvertes. La meilleure oeuvre du mathématicien est de l'art, un art élevé, parfait, hardi comme les rêves les plus secrets de l'imagination, clair et limpide comme la pensée abstraite. Le génie mathématique et le génie artistique se touchent, et il faudrait même expliquer pourquoi ces deux sortes de génies se développent si rarement chez le même homme. Sophie Kovalevsky avait dès sa jeunesse hésité entre les mathématiques et la littérature.

G. Mittag-Leffler.

„Sophie Kovalevsky“, Acta mathem. 16, 1892/1893, p. 388/389.

Ich gewöhnte mich daran, von den einzelnen Sätzen ab schärfer in den Zusammenhang zu dringen und ganze Theorien als eine Einheit aufzufassen. So ging mir die Idee des mathematisch Schoenen auf. Es giebt ein solches mathematisch Schoene, ebenso wie ein aesthetisch Schoenes... . . Es giebt auch einen mathematischen Takt oder Geschmack, der der Untersuchung gleich von vorn herein ansieht, ob sie zu einem Resultate führen werde oder nicht, und die Betrachtungen und Entwickelungen demgemäss leitet.

\section{Eisenstein}

[20-jährig, 1843] in seinem Curriculum vitae. s. Abhandl. zur Gesch. d. Math. 7 (1895), p. 157. 
Comme on dit beanté poétique, on devrait aussi dire beauté géométrique et beanté médicinale. Cependant on ne le dit point, et la raison en est qu'on sait bien quel est l'objet de la géométrie et qu'il consiste en preuve, et quel est l'objet de la médecine et qu'il consiste en la guérison, mais on ne sait pas en quoi consiste l'agrément qui est l'objet de la poésie. On ne sait ce que c'est que ce modèle naturel qu'il faut imiter, et à faute de cette connaissance, on a inventé de certains termes bizarres, „siècle d'or, merveille de nos jours, fatal“, ... etc; et on appelle ce jargon beauté poétique.

Mais qui s'imaginera une femme sur ce modèle-là, qui consiste à dire de petites choses avec de grands mots, verra une jolie demoiselle toute pleine de miroirs et de chaînes dont il rira, parce qu'on sait mieux en quoi consiste l'agrément d'une femme, que l'agrément des vers.

\section{Blatise Pascal.}

„Pensées“, Édition complète de J.-F. Astié (Paris 1883), p. 183/184.

Souvent, les personnes qui n'ont pas eu l'occasion de faire ample connaissance avec les mathématiques les confondent avec l'arithmétique ${ }^{1}$ ) et les considèrent comme une science sèche et aride. En réalité les mathématiques exigent beaucoup d'imagination, et l'un des plus grands mathématiciens de notre siècle ${ }^{2}$ ) a pu dire avec raison qu'il est impossible d'être bon mathématicien, si en même temps l'on n'est pas un peu poète. - _ _ -

En ce qui me concerne personnellement, je ne saurais dire ce que j'aime le plus des mathématiques ou des lettres. Dès que ma tête est fatiguée des spéculations abstraites, je me sens attirée vers l'observation de la vie et disposée à prendre la plume. A d'autres moments, tout dans la vie me semble mesquin, insignifiant, et je me réfugie dans la contemplation des

1) = Rechnen.

2) Weierstrass, s. Note 16 am Ende des Buches; vgl. jedoch a. Kronecker, s. 73 u. 226. 
lois immuables et éternelles de la science. ${ }^{1}$ ) Peut-être aurais-je pu faire mieux dans chacune de ces deux sphères, si je m'étais adonnée exclusivement à l'une ou à l'autre? Mais que voulez-vous, je n'ai jamais eu le courage de choisir entre les mathématiques et les lettres.

\section{Sophie Kovalevsky}

à la romancière russe, $M^{\mathrm{me}}$ Choubleski. voir Rebière, "Les femmes dans la science"

(Paris 1897), p. 164 et 165.

Ich bin von der tiefen inneren Verwandtschaft der Kunst und der Wissenschaft überzeugt. Auch die Kunst sucht uns Wahrheiten zu verkünden, psychologische Wahrheiten, wenn auch in ganz anderer Form, in der Form sinnlicher Erscheinungen und nicht in der Form des Begriffes. Aber schliesslich wird sich bei vollendeter Erscheinung ja auch die begriffliche Fassung finden müssen, und beide werden schliesslich vereint zusammen wirken.

\section{H. yon Hesmholitz.}

s. „Anspr. u. Red. geh. bei der Helmholtz-Feier 2. XI. 1891" (Berlin 1892), p. 16/17.

Eodem modo literis atque arte animos delectari posse.

\section{G. Cantor.}

These der Habilitationsschrift Halle 1869.

Les adeptes des mathématiques y trouvent des jouissances analogues à celles que donnent la peinture et la musique. Ils admirent la délicate harmonie des nombres et des formes; ils s'émerveillent quand une déconverte nouvelle leur ouvre une perspective inattendue; et la joie qu'ils éprouvent ainsi n'a-t-elle pas le caractère esthétique, bien que les sens n'y prennent aucune part? - - - -

C'est pourquoi je n'hésite pas à dire que les mathématiques méritent d'être cultivées pour elles-mêmes et que les théories

1) vgl. a. S. 76 (S. Kovalevsky), sowie jedoch a:S. 243 (A. Charl. Leffler). 
qui ne peuvent être appliquées à la physique doivent l'être comme les autres.

\section{H. Poincaré. ${ }^{1}$ )}

"Sur les rapports del'analyse pure et de la physique mathématique." voir Verhandl. d. 1. intern. Mathem.-Congr. Zürich 1897

(Leipzig 1898), p. 82.

To a missing member

of a family group of terms in an algebraical formula.

Lone and discarded one! divorced by fate, Far from thy wished-for fellows - whither art. flown?

Where lingerest thou in thy bereaved estate, Like some lost star, or buried meteor stone? Thou mindst me much of that presumptuous one Who loth, aught less than greatest, to begreat, From Heaven's immensity fell headlong down To live forlorn, self-centred, desolate: Or who, new Heraclid, hard exile bore, Now buoyed by hope, now stretched on rack of fear, Till throned Astraea, wafting to his ear Words of dim portent through the Atlantic roar, Bade him ,the sanctuary of the Muse revere And strew with flame the dust of Isis' shore".

J. J. Sylvester.

Inaugural lecture, delivered before the University of Oxford 12. XII. 1885. cf. Nature 33 (7. I. 1886), p. 228.

In dem Reiche des Mathematischen herrscht eine eigenthümliche Schönheit, welche nicht sowohl mit der Schönheit der Kunstwerke, als vielmehr mit der Schönheit der Natur übereinstimmt und welche auf den sinnigen Menschen, der das

1) vgl. S. 243 ein Wort von demselben Autor. 
Verständniss dafür gewonnen hat, ganz in ähnlicher Weise einwirkt, wie diese.

E. E. KuMMer.

(,Über einige mathematische und philosophische Grundanschauungen Leibnizens, an dem Beispiele der Leibnizischen

Reihe für die Quadratur des Cirkels betrachtet"),

Festrede Berlin Akad. 4. VII. 1867.

s. Berl. Monatsber. 1867, p. 395.

Wenn aufgegeben wäre: dass schiefe Flächen in verschiedenen Neigungen gegen den Horizont, doch von solcher Länge angeordnet würden, damit frei herabrollende Körper darauf gerade in gleicher Zeit herabkämen, so wird ein Jeder, der die mechanischen Gesetze versteht, einsehen, dass hierzu mancherlei Veranstaltung gehöre. Nun findet sich aber diese Einrichtung im Cirkel von selber mit unendlich viel Abwechslung der Stellungen, und doch in jedem Falle mit der grössten Richtigkeit. Denn alle Sehnen, die an den Verticaldurchmesser stossen, sie mögen von dessen oberstem oder unterstem Puncte ausgehen, nach welchen Neigungen man auch will, haben insgesammt das gemein: dass der freie Fall durch dieselbe in gleichen Zeiten geschieht. Ich erinnere mich, dass ein verständiger Lehrling, als ihm dieser Satz mit seinem Beweise von mir vorgetragen wurde, nachdem er Alles wohl verstand, dadurch nicht weniger wie durch ein Naturwunder gerührt wurde. Und in der That wird man durch eine so sonderbare Vereinigung vom Mannigfaltigen nach so fruchtbaren Regeln in einer so schlecht [ = schlicht] und einfältig scheinenden Sache, als ein Cirkelkreis ist, überrascht und mit Recht in Bewunderung gesetzt. Es ist auch kein Wunder der Natur, welches durch die Schönheit oder Ordnung, die darin herrscht, mehr Ursache zum Erstaunen gäbe, es müsste denn seyn, dass es deswegen geschähe, weil die Ursache derselben ja nicht so deutlich einzusehen ist, und die Bewunderung eine Tochter der Unwissenheit ist. ImManUel Kant.

„Der einzig mögliche Beweisgrund zu einer Demonstration des Daseyns Gottes."

s. Werke, herausg. v. Rosenkranz u. Schubert, Th. I (Leipzig 1838), p. 199200. 
Das Streben nach universeller Bildung allein war es nicht, was die grossen Meister der Renaissance, wie Brunellesco, Lionardo da Vinci, Raphael, Michelangelo und namentlich auch Albrecht Dürer, mit unwiderstehlicher Macht zu den mathematischen Wissenschaften hinzog. Sie waren sich bewusst, dass, bei aller Freiheit individueller Phantasie, auch die Kunst ein Gesetz der Nothwendigkeit kennt und umgekehrt, bei aller Strenge des logischen Aufbaus auch die Mathematik dem Gesetze der Schönheit folgt.

\section{F. Rudio.}

„Über den Antheil der mathematischen Wissenschaften an der Kultur der Renaissance", Heft 142 der Virchow-Holtzendorff'schen Sammlung gemeinverst. wiss. Vortr., p. 19.

Unter den ... Abhandlungen Kirchhoff's sind einige von ungewöhnlicher Schönheit. Schönheit höre ich Sie da fragen; entfliehen nicht die Grazien, wo Integrale ihre Hälse recken, kann etwas schön sein, wo dem Autor auch zur kleinsten äusseren Ausschmückung die Zeit fehlt? - Doch -; gerade durch diese Einfachheit, durch diese Unentbehrlichkeit jedes Wortes, jedes Buchstaben, jedes Strichelchens kömmt der Mathematiker unter allen Künstlern dem Weltenschöpfer am nächsten; sie begründet eine Erhabenheit, die in keiner Kunst ein Gleiches, - Aehnliches höchstens in der symphonischen Musik hat. Erkannten doch schon die Pythagoräer die Aehnlichkeit der subjectivsten und der objectivsten der Künste. Ultima se tangunt. Und wie ausdrucksfähig, wie fein charakterisirend ist dabei die Mathematik. Wie der Musiker bei den ersten Tacten Mozart, Beethoven, Schubert erkennt, so würde der Mathematiker nach wenig Seiten, seinen Cauchy, Gauss, Jacobi, Helmholtz unterscheiden. Höchste äussere Eleganz, mitunter etwas schwaches Knochengerüste der Schlüsse charakterisirt die Franzosen, die grösste dramatische Wucht die Engländer, vor Allen Max w ell. Wer kennt nicht seine dynamische Gastheorie? - Zuerst entwickeln sich majestätisch die Varia- 
tionen der Geschwindigkeiten, dann setzen von der einen Seite die '/ustands-Gleichungen, von der anderen die Gleichungen der Centralbewegung ein, immer höher wogt das Chaos der Formeln; plötzlich ertönen die vier Worte: „Put $n=5$ “. Der böse Dämon $V$ verschwindet, wie in der Musik eine wilde, bisher alles unterwühlende Figur der Bässe plötzlich verstummt; wie mit einem Zauberschlage ordnet sich, was früher unbezwingbar schien. Da ist keine Zeit zu sagen, warum diese oder jene Substitution gemacht wird; wer das nicht fühlt, lege das Buch weg; Maxwell ist kein Programmmusiker, der über die Noten deren Erklürung setzen muss. Gefügig speien nun die Formeln Resultat auf Resultat aus, bis überraschend als Schlusseffect noch das Wärme-Gleichgewicht eines schweren Gases gewonnen wird und der Vorhang sinkt.

.... Kirchhoff's ganze Richtung war eine andere, und ebenso auch deren treues Abbild, die Form seiner Darstellung ... Ihn charakterisirt die schärfste Präcisirung der Hypothesen, feine Durchfeilung, ruhige mehr epische Fortentwicklung mit eiserner Consequenz ohne Verschweigung irgend einer Schwierigkeit, unter Aufhellung des leisesten Schattens. Um nochmals zu meiner Allegorie zurück zu greifen, er glich dem Denker in Tönen: Beethoven. - Wer in Zweifel zieht, dass mathematische Werke künstlerisch schön sein können, der lese seine Abhandlung über Absorption und Emission [s. „Gesammelte Abhandlungen" (Leipzig 1882), p. 571-598] oder den der Hydrodynamik gewidmeten Abschnitt seiner Mechanik.

L. Bolizimann.

„Gustar Robert Kirchhoff", Akadem. Festrede Graz 15. XI. 1887 (Leipzig 1888), p. 28-30.

Mein Grossvater [A. F. Moebius] sagte, die Mathematik sei eigentlich sehr poetisch.

P. J. Moebius.

„Über die Anlage zur Mathematik“ (Leipzig 1900), p. 116. 
Comment quitter [la psychologie,] un pays plein de fleurs et d'eaux vives; comment quitter des ruisseaux, des bocages, pour les déserts brûlés par les rayons de ce soleil mathématique, qui, répandant sur les objets la plus vive lumière, les flétrit, les dessèche jusqu'à la racine! - _ -

Ampìre à ses amis Lyonnais. voir Arago, Oeuvres, t. 2 (1854), p. 37

$=$ Werke, Bd. 2 (1854), p. 31/32.

Mathematik und Musik, der schärfste Gegensatz geistiger Thätigkeit, den man auffinden kann, und doch verbunden, sich unterstützend, als wollten sie die geheime Consequenz nachweisen, die sich durch alle Thätigkeiten unseres Geistes hinzieht und die uns auch in den Offenbarungen des künstlerischen Genius unbewusste Aeusserungen geheimnissvoll wirkender Vernunftmässigkeit ahnen lässt.

\section{HeLmHoLTz.}

„Über die physiologischen Ursachen der musikalischen Harmonie", Vorles. Bonn Winter 1857.

s. Vorträge u. Reden, Bd. 1 (1884), p. 82.

Tüchtige Mathematiker haben meistens ein gutes musikalisches Gehör, wogegen grosse Komponisten sich mit der Mathematik nicht leicht befreunden.

\section{W. Preyer.}

„Über den Ursprung des Zahlbegriffs" etc. in Festschrift zu Helmholtz' 70. Geburtstag (1891), p. 12.

On a dit que Lagrange n'était pas insensible aux charmes de la musique. En effet, quand une réunion était nombreuse, il n'était pas fâché qu'un concert vînt interrompre les conversations et fixer toutes les attentions. Dans une de ces occasions, je lui demandais ce qu'il pensait de la musique: Je l'aime parce qu'elle m'isole; j'en écoute les trois 
premières mesures, à la quatrième je ne distingue plus rien, je me livre à mes réflexions, rien ne m'interrompt, et c'est ainsi que j'ai résolu plus d'un problême difficile.

\section{Delambre.}

"Notice sur la vie et les ourrages de M. Lagrange," Mém. de la classe des sciences mathém. et phys. de l'Institut de France 13, 1812, Histoire de la classe mathém., p. LXXV $=$ Oeurres de Lagrange, t. 1 (1867), p. XLVII/XLVII.

Abel n'était pas musicien. Il n'avait pas pris part à l'enseignement du chant à l'école. Une anecdote caractéristique nous est parvenue du temps où il était étudiant. Un ami très musicien jouait du piano pour un cercle de camarades. Abel suivit le jeu avec une attention plus vive que d'habitude. Lorsque le morceau fut fini, l'exécutant éprouva cette déception d'apprendre qu'Abel avait été absorbé par ce problème mathématique de combinaisons: „trouver une relation entre les nombres de fois que chaque touche est frappée par chaque doigt du joueur."

\section{Elling Holst.}

"Niels Henrik Abel, Mémorial publié à l'occasion du centenaire de sa naissance": „Introduction historique", p. 57/58.

Sophie Kovalevsky äusserte einst, dass sie ihr ganzes mathematisches Talent darum geben würde, wenn sie gut singen könnte.

Sophie von Adelung [eine Verwandte von S.Kovalevsky].

s. Deutsche Rundschau, Bd. 89 (Okt.-Dec. 1896), p. 405.

Eine schoene Frucht hat mir die Astronomie gebracht. Henriette Sontag ist bei mir gewesen und ich habe mit ihr einige Zärtlichkeiten gewechselt. Sie konnte nicht begreifen, wie ich allerlei ausrechnen könne; ich dagegen nicht, wie sie so 
singen könne. Darauf hat sie mich versichert, sie wolle mich eher zu einer Sontag machen als ich sie zu einem Astronomen; ich meinestheils zweifelte an der Möglichkeit der ersten Verwandlung, selbst wenn sie auch nicht gleich zum Extrem gebracht werden solle u. s. w.

Bessel an Olbers.

Königsberg, 5. XII. 1830.

s. Briefw. Olbers-Bessel, herausg. v. Erman, Bd. 2 (1852), p. 351.

Helmholtz soll sich in seiner gewohnten witzigen Weise einem Bekannten gegenüber, der ihn mit seiner Bayreuthschwärmerei aufzog, folgendermassen geäussert haben: „Siehst Du, wenn ich mich so recht in "Parzival" oder "Tristan" ausgeschwelgt habe, so geht mir das Integriren einer Differentialgleichung noch einmal so leicht von statten!"

S. S. Epstein.

"Hermann von Helmholtz als Mensch und Gelehrter"

(Stuttgart etc. 1896), p. 50

$=$ Deutsche Revue, herausg. v. R. Fleischer, $21_{2}$ (Mai 1896), p. 198.

Comme Vandermonde ne pouvoit cesser d'être géomètre, il ne s'occupa pas long-temps de musique sans calculer les moyens qu'elle emploie, observer les usages autorisés par les grands succès, simplifier ces procédés par l'analyse, comparer les résultats de ces réductions, tirer de ces résultats des formules générales, et présenter les règles de l'art. - - -

Il rapporte les manières de procéder adoptées jusqu'à lui à deux règles principales, qui par-là lui paroissent établies sur des effets avoués par tous les musiciens. Ces deux règles générales, l'une sur la succession des accords, l'autre sur l'arrangement des parties, dépendent elles-mêmes d'une loi plus élevée, qui, selon Vandermonde, doit régir toute l'harmonie. ...... Il obtint l'approbation de trois hommes fameux, et, pour ainsi dire, des représentans des trois grandes écoles 
d'Allemagne, de France et d'Italie; de Gluck, de Philidor et de Piccini.

LACEPÈDE.

"Notice sur la vie et les ouvrages de Vandermonde," Mém. de l'institut national (sciences math. et phys.) 1, an VI $[=1898]$, p. XXIII et XXIV.

Vous savez qu'au spectacle un certain fils d'Euclide S'avisa d'égayer son cerveau trop aride;

Sans entendre, sans voir et même sans parler,

Il se mit, en rêvant, d'abord à calculer

Les effets de la voix, l'espace de la salle,

Le théâtre, l'optique et le grand cintre ovale;

Cela fait, ne trouvant rien de touchant pour lui,

Et se sentant glacé de dégoût et d'ennui,

Sans qu'il eût vu finir un acte (est-il croyable?),

Il sortit brusquement, donnant le tout au diable. ${ }^{1}$ )

Frédéric le Grand.

"Sur les plaisirs."

voir Oeuvres de Frédéric le Grand, t. 10 (Édition Decker 1849),p. 169.

Das mathematische Talent hat mit dem musikalischen Talent, mit welchem es oft . . vereint gefunden wird, das gemein, dass es schon in früher Jugend sich verräth, wovon auf der einen Seite Blaise Pascal, auf der anderen Mozart bekannte Beispiele sind.

Emil du Bois-Reymond.

„Gedächtnissrede auf Hermann von Helmholtz", p. 4 in Berl. Abhandl. 1896.

Ich konnte eher als sechsjähriger Knabe den Beweis eines mathematischen Satzes verstehen, als dass man in der

1) Nach Preuss, dem Herausgeber der Werke Friedrich's (s. l. c., t. 20, p. XXII) bezieht sich diese Stelle auf L. Euler. 
Stube die Mütze von dem Kopf nehmen oder dass man das Fleisch nicht mit der Gabel zerreissen, sondern mit dem Messer zerschneiden müsse. Indem ich von allen Dingen erst den Grund wissen wollte, machte ich durch meine Widersetzlichkeit meinen Eltern vielen Kummer.

Eisenstein

[20-jährig, 1843] in seinem Curriculum vitae.

s. Abhandl. zur Gesch. d. Math. 7 (1895), p. 150.

Sie würden mich sehr verbinden, wenn Sie mir mitteilten, ob und in welcher Weise man bei Ihnen einen Verleger für Tänze (Polkas, Walzer) findet; ich habe mehrere dergleichen komponiert, die hier allgemeinen Beifall finden, aber es ist schwer, hier einen Verleger zu gewinnen, wenn man nicht schon Komponist von Ruf ist.

Eisenstein an M. A. Stern.

Berlin, 14. I. 1850.

s. Abhandl. zur Gesch. d. Math. 7 (1895), p. 201.

Gauss pflegte oft scherzweise zu sagen, er habe früher rechnen als sprechen können.

SARTORIUS v. Waltershausen. „Gauss zum Gedächtniss" (1856), p. 11.

À 15 ans Lagrange professait, dans une école d'artillerie, les mathématiques, devant des élèves dont aucun n'était aussi jeune que lui.

VIREY et Potel.

„Précis historique sur la vie et la mort de Joseph-Louis Lagrange" (Paris 1813), p. 4.

Un jeune homme [Lagrange], s'emparant d'un sujet traité par Newton, Taylor, [Jean] Bernoulli, d'Alembert 
et Euler [problème des cordes vibrantes], paraît tout à coup au milieu de ces grands Géomètres comme leur égal, comme un arbitre qui, pour faire cesser une lutte difficile, leur montre à chacun, en quoi ils ont raison, en quoi ils se sont trompés, les juge, les réforme, et leur donne la véritable solution qu'ils ont entrevue sans y pouvoir atteindre.

\section{Delambre.}

„Notice sur la vie et les ouvrages de M. Lagrange,“ Mémoires de la classe des sc. mathém. et phys. de l'Institut de France 13, 1812, Histoire, p. XXXVIII $=$ Oeurres de Lagrange, t. 1 (1867), p. XIII.

Clairant fut un enfant merveilleusement précoce..... il avait lu, à l'âge de dix ans, l'Analyse démontrée de Guinée et le Traité des sections coniques du marquis de l'Hôpital. Vers le milieu de sa treizième année, il composa un mémoire sur les propriétés de quelques courbes nouvelles, qui, présenté à l'Académie des Sciences et approuvé par elle, fut imprimé à la suite d'un travail de son père, dans le recueil intitulé: Miscellanea Berolinensia [t. 4]. - - - -

A l'âge de seize ans, Clairaut avait terminé un traité sur les courbes à double courbure [Paris 1731] . . . l'Académie des Sciences l'accueillit avec faveur et présenta peu de temps après le jeune auteur comme second candidat à la place de membre adjoint pour la mécanique. . . . . Le roi . . . laissa la place vacante pendant deux ans entiers, et, lorsque Clairaut eut atteint l'âge de dix-huit ans, ce fut lui qu'il choisit en le dispensant de la règle qui fixait à vingt ans la limite d'âge des académiciens.

J. Bertrand.

„Éloges académiques", Nouvelle série (Paris 1902), p. 232 et $233 / 234$.

Pour apprendre les Mathématiques, Neuton n'étudia point Euclide, qui lui parut trop clair, trop simple, indigne de lui Ahrens, Scherz und Ernst i. d. Mathem. 
prendre du temps; il le savoit presque avant que de l'avoir lû, et un coup d'oeil sur l'énoncé des Théorêmes les lui démontroit. Il sauta tout d'un coup à des Livres tels que la Géométrie de Descartes, et les Optiques de Kepler. On lui pourroit appliquer ce que Lucain a dit du Nil, dont les Anciens ne connoissoient point la source, Qu'il n'a pas été permis aux hommes de voir le Nil foible et naissant.

Fontenelle.

„Eloge de M. Neuton."

voir Oeurres de Fontenelle, t. 6 (1758), p. 328.

Das mathematische Organ besteht in einer abnormen Bildung der Stirnecke, die auf Vergrösserung des von der Stirnecke umschlossenen Raumes hinausläuft. ${ }^{1}$ ) - - - -

Geht man aus der mathematischen Section bei einer Naturforscherversammlung in die Section der Physiker, so fällt der Unterschied der Köpfe ohne Weiteres auf, geht man gar in eine medicinische Section, so glaubt man gar eine andere Menschenrasse zu sehen.

P. J. Moebius.

„Über die Anlage zur Mathematik“ (Leipzig 1900), p. 140 u. $145 / 146$.

Die Gehirne unserer beiden grossen Mathematiker ${ }^{2}$ ) [Gauss und Dirichlet] sind mit sehr reichen und tiefen Windungen versehen; sie gehören $\mathrm{zu}$ den reichsten, die ich bis jetzt beobachtet habe. Besonders reich und ansehnlich sind die Stirnwindungen. Specifische Formen und Anordnungen kommen aber nicht vor. Es ist schade, dass das Gehirn von La Place, das im Besitze Magendies ${ }^{3}$ ) sich befand und dessen jetzigen Aufbewahrungsort ich nicht kenne, zur Vergleichung nicht benutzt werden konnte. Es würde sich freilich wohl daran eben

1) vgl. S. 225 (Chr. Wiener).

2) Die Gehirne befinden sich in den Sammlungen des physiologischen Instituts der Universität Göttingen.

3) Der berühmte Physiolog war Laplace's Arzt. 
so wenig ein für die mathematische Begabung charakteristisches specifisches Formelement herausgestellt haben.

\section{Rudolph Wagner}

[Prof. d. Physiologie u. vergleichenden Anatomie in Göttingen]. „Vorstudien zu einer künft. wissensch. Morphologie" etc., Göttinger Abhandl. 11, 1860, Phys. Cl., p. 80.

Le savant digne de ce nom, le géomètre surtout, éprouve en face de son oeurre la même impression que l'artiste; sa jouissance est aussi grande et de même nature. Si je n'écrivais pas pour un public amoureux de la Science, je n'oserais pas m'exprimer ainsi; je redouterais l'incrédulité des profanes. Mais ici je puis dire toute ma pensée. Si nous travaillons, c'est moins pour obtenir ces résultats positifs, auxquels le vulgaire nous croit uniquement attachés, que pour ressentir cette émotion esthétique et la communiquer à ceux qui sont capables de l'éprouver.

\section{H. Poincaré. ${ }^{1}$ )}

„Notice sur Halphen“, Journal de l'école polytechnique, cah. 60 (1890), p. 143.

Nangel an Freude während der Arbeit....... hatte Sonja [Kovalevsky] immer, wenn sie mit wissenschaftlichen Untersuchungen beschäftigt war. ${ }^{2}$ ) Sie ging immer bis zu jenem Übermass, das sie ausser stand setzte, nicht bloss vom Leben, sondern auch von der Arbeit selbst irgend etwas zu geniessen; der Gedanke wurde ihr Tyrann anstatt ihr Diener, und keine Freude am Schaffen gab es bei ihr in solchen Zeiten.

Ganz verschieden erging es ihr bei ihren späteren litterarischen Arbeiten; diese machten ihr die grösste Freude und versetzten sie in die gehobenste Stimmung.

A. Chart. Leffler.

"Sonja Kovalevsky",

Deutsche Ausg., Reclam-Bibl. No. 3297-3298, p. 33.

1) vgl. a. S. 231/232 ein Wort von demselben Autor.

2) vgl. S. $76 / 77$. 
Ein Alter ${ }^{1}$ ) vergleicht die Mathematiker mit den Lotophagen Wer einmal, sagt er, die Süssigkeit der mathematischen Ideen gekostet, kann nicht mehr davon ablassen.

C. G. J. JACOBI an A. v. Humboldt.

s. Kronecker, ,Über den Zahlbegriff“' J. f. Math. 101 (1887), p. 337.

Ce qu'il y a de plus fâcheux pour moi, e'est que la Géométrie est la seule occupation qui m'intéresse véritablement, sans qu'il me soit permis de m'y livrer. Tout ce que je fais de littérature, quoique très-bénignement accueilli (à ce qu'il me semble) dans nos séances publiques de l'Académie française, n'est pour moi que du remplissage et une espèce de pis-aller.

\section{D'AleMbert à Lagrange.}

Paris, 22. IX. 1777.

voir Oeuvres de Lagrange, t. 13 (Paris 1882), p. 331; voir aussi ibidem p. 261, 267, 294, 349, 367.

Le bon ou mauvais discours que j'ai fait à l'Académie française a eu plus de succès qu'il n'en méritait, mais j'aimerais bien mieux résoudre des problèmes difficiles, quand même on n'en parlerait pas. Je le dis tous les jours à nos beaux esprits, qui en sont tout étonnés.

\section{D'Alembert à Lagrange.}

Paris, 30. III. 1778.

voir Oeurres de Lagrange, t. 13 (Paris 1882), p. 338; voir aussi ibidem p. 290, 341/342.

For many years I have in the hours of leisure granted me, given much study to the Life and Works of Francis Bacon, who in my eyes is one of the greatest geniuses of Christianity. By this I have become persuaded, that the opinion so

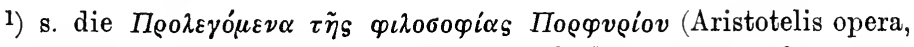
vol. 4: Scholia in Aristotelem, ed. Brandis (Berlin 1836), p. 8), wo es

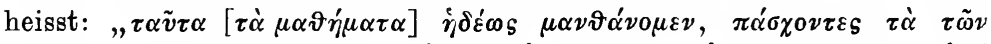

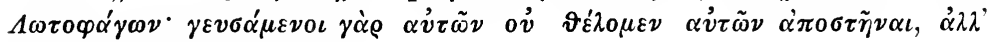

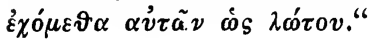


ridiculed by most scholars, of Francis Bacon being the writer of the Shakespearian Dramas, is founded on truth - -

Dr. phil. George Cantor, Mathematicus. "Resurrectio Divi Quirini Francisci Baconi"

(Halle 1896), Introduction.

Die Shakespeare-Baconfrage ist, wie der Spiritismus, vielleicht ein Gipfel im Hochgebirge der Absurdität. Man entschliesst sich, Jemandem eine, zwei, drei Voraussetzungen wider den bon sens zuzugeben, man zwingt sein Gehirn, mit den Schwingungen eines Querkopfgehirns parallel zu schwingen - aber das hilft noch nichts; innerhalb des Unsinnigen kommt erst das Unsinnigste, die Narrheit potenzirt sich. Es giebt Systeme, die auf einem falschen Grunde wenigstens richtig aufgebaut sind, man tritt um den Preis eines sacrifizio dell' intelletto ein, darf dann aber wieder seine Vernunft gebrauchen. Und es giebt andere, die auf Schritt und Tritt den Unsinn nicht loswerden, Systeme, die es mit einer einmaligen Gehirnverrenkung keineswegs bewenden lassen; die Vernunft wird von unten auf gerädert - gliedweise und fingerweise verstümmelt.

Paul Mongré. ${ }^{1}$ )

"Sant' Ilario. Gedanken aus der Landschaft Zarathustras" (Leipzig 1897), p. 206.

Les poëtes surtout furent indignés d'être jugés par un géomètre [D'A lembert, „Réflexions sur la poésie et sur l'histoire“]. La sécheresse des mathématiques leur semblait devoir éteindre l'imagination; et ils ignoraient sans doute qu'Archimède et Euler en ont mis autant dans leurs ouvrages, qu'Homère ou l'Aristote en ont montré dans leurs poésies.

\section{CONDORCET.}

„Éloge de M. D'Alembert."

voir Condorcet, Oeuvres, Édition d'Arago, t. 3 (1847), p. 83.

1) s. S. 69 unten. 
Le peuple versificateur est bien à plaindre d'être foudroyé par des curvilignes. Cependant il ne se croit pas perdu, et il est persuadé que trente bons vers font plus de plaisir au public que tout le calcul des éphémérides ne lui en fera jamais. . . . . . . qu'ils se barbouillent d'algèbre, qu'ils pâlissent sur leur calcul infinitésimal, qui est la chose la plus amusante du monde; mais qu'ils s'abstiennent en même temps de porter la guerre dans une province voisine dont ils ne connaissent ni les lois ni les coutumes, et où ils embrouilleraient tout sous le prétexte spécieux de réformer les abus.

Messieurs les géomètres voudraient être les maîtres du genre humain. Ils se saisissent de la raison comme si eux seuls avaient des droits sur elle; ils vous parlent avec emphase de l'esprit philosophique, comme si on ne pouvait le posséder que par $a b$ minus $x$, et cent choses pareilles. Qu'ils sachent que la raison appartient à tous les états de la vie, et que l'analyse, la méthode et le jugement sont aussi nécessaires au poëte qu'au calculateur. - -

Un certain géomètre qui a perdu un oeil en calculant [Euler] s'avisa de composer un menuet par a plus $b$. Si on l'avait joué devant le tribunal d'Apollon, le pauvre géomètre courait risque d'être écorché vif comme Marsyas. - -

Ces sauvages mesurent tout avec la même toise, le théorème comme une épigramme, et ils voudraient soumettre à l'algèbre l'Art poétique de Despréaux [Boileau] comme le calcul des forces vives. Qu'ils apprennent qu'on ne calcule ni le sentiment ni le plaisir. Qu'ils se défient de leurs organes engourdis par l'opium du calcul intégral et infinitésimal. - - -

Pour eux, ils ont le privilége d'avancer autant de paradoxes qu'il leur plaît. Tout sophisme est sanctifié par l'esprit géométrique.

... . quand il n'y aura plus de poëme, le public s'amusera avec des courbes de toutes les espèces. Les dames calculeront les précessions équinoxiales à leur toilette. Les propos de ruelles rouleront sur les angles d'incidence et de réflexion, sur les sections coniques, et sur toute l'algèbre de l'univers. J'ose 
cependant avertir nos législateurs curvilignes que cet heureux temps n'arrivera pas, ou que, s'il arrive, il ne durera guère.

On nous accuse que nous nous parons des plumes de la mythologie. Prouvez-leur que leur Newton est un plagiaire qui a pris son calcul du mouvement des planètes de Huyghens, son attraction des vertus plastiques ou vertus occultes des platoniciens, qui a pris le vide d'Épicure, et a donné de l'existence au rien, et, ce qui pis est, qui a calculé le rien. Voici comment. Toutes les planètes nagent dans le vide; or, la distance qu'il y a d'une planète à l'autre est calculée; par exemple, on compte trois millions de lieues d'ici à Jupiter. Voilà donc trois millions de riens calculés; or, ce qu'on calcule existe; donc le rien ne peut pas exister. C'est en les attaquant que vous les réduirez à vous offrir la paix, et les conditions seront que désormais personne ne parlera que de ce qu'il entend bien, et qu'on se gardera de donner des règles sur les arts sans en avoir étudié la matière $-\ldots$

FrédÉric le Grand.

„Réflexions sur les réflexions des géomètres sur la poésie (Doutes sur les réflexions de M. d'Alembert ${ }^{1}$ ) sur la poésie)." voir Oeurres de Frédéric le Grand, t. 9 (Édition Decker 1848), p. $63-65,68,72-74$.

Das ideale Drama zu n Personen muss enthalten: n Persönlichkeiten, $\frac{\mathrm{n}(\mathrm{n}-1)}{2}$ dialogische Beziehungen (Unterdramen zu je Zweien), $\frac{\mathrm{n}(\mathrm{n}-1)(\mathrm{n}-2)}{6}$,dreieckige" Verhältnisse (Unterdramen zu je Dreien) und so fort, oder, wie man in der Arithmetik sagt, Unionen, Binionen, Ternionen in der überhaupt möglichen Anzahl. Also innere Vollständigkeit und Erschöpfung aller denkbaren Combinationen; keiner der Handelnden soll nur einseitig wirken oder einseitig empfangen ..... Man sieht,

1) s. das vorhergehende Citat. 


\title{
$-248-$
}

wie sehr der ernsthafte Dramatiker bemüht sein wird, sein $n$ zu verkleinern.

\author{
Paul Mongré. ${ }^{1}$ )
}

"Sant' Ilario. Gedanken aus der Landschaft Zarathustras" (Leipzig 1897), p. 201/202.

Jeg veed ingen større Fornøielse end end $[\mathrm{sic}]^{2}$ ) at see et Stykke af Moliere hvori Mlle Mars spiller. Jeg er virkelig ganske henrykt; Hun er 40 Aar men spiller dog meget unge Roller.

(Je ne connais pas de plus grand plaisir que de voir une pièce de Molière où joue Mlle Mars. Alors je suis tout à fait ravi; elle a 40 ans, mais elle joue tout de même des rôles très jeunes.)

\section{N. H. Aber à Holmboe.}

Paris, 24. X. 1826.

voir „Niels Henrik Abel, Mémorial publié à l'occasion du centenaire de sa naissance": "Texte original des lettres", p. 43 et "Correspondance d'Abel" (Traduction française), p. 47/48.

Ch. F. D. de Villers [Prof. der Philologie in Göttingen erfreute Jean Paul [in einem Briefe aus dem Jahre 1813. durch die Nachricht, dass der berühmte Mathematiker uni Astronom K. Fr. Gauss zu seinen „wärmsten Anbetern“ ge höre und ihn beinahe so leidenschaftlich lese und liebe wit er selbst.

\section{P. NeRrLich.}

„Jean Paul" (1889), p. 543

Gauss und Jean Paul haben sich gegenseitig verehr aber nie gesehen.

In Goethes Schreib- und Denkungsweise konnte sich de grosse Mathematiker viel weniger versetzen und obgleich e*

1) s. S. 69 unten.

2) Zusatz der Herausgeber. 
den Dichter ohne Zweifel in allen seinen Werken kannte, so hat er ihn doch nur unvollständig befriedigen können: er sei ihm an Gedanken zu arm und seine lyrische Poesie deren Werth und vollendete Form er nicht verkannte, schlug er nicht sehr hoch an. Noch weniger sagte ihm Schiller zu, dessen philosophische Ansichten ihm mitunter vollständig zuwider waren.

Sartorius v. Waltersifausen.

"Gauss zum Gedächtniss" (1856), p. 92/93.

On s'étonne quelquefois de la perfection de notre langue; on vante sa précision et sa clarté; on admire son ordre méthodique et sa logique rigoureuse; mais il ne faut pas oublier qu'elle est, en grande partie, l'oeuvre de quelques écrivains de génie dont le jugement s'était mûri, dont le goût était devenu plus sûr par l'étude des sciences exactes ou philosophiques. N'est-ce pas, en effet, par les écrits de Descartes et de Pascal que la langue française a été définitivement fixée quant à ses principes et à ses règles? Leurs traditions n'ont pas été interrompues, et, de nos jours encore, les ouvrages des Laplace, des Biot, des Cuvier, des Arago, peuvent être cités comme des modèles de bonne littérature.

\section{C.-A. Valson.}

„La vie et les travaux du baron Cauchy" (Paris 1868), t. 1, p. 155; voir aussi du même: „Les savantš illustres du XVIe et du XVII e siècle“, t. 2 (1880): „Pascal", p. 73/74.

In 234 meist ganz kurzen Briefen behandelt Euler die wichtigsten Gebiete der Astronomie, der mathematischen und physikalischen Geographie, der Physik und der Philosophie und zwar in einer so klaren, lichtvollen, ich möchte fast sagen liebenswürdigen Sprache, dass dieselben noch heute als Muster populärer Darstellung bezeichnet werden dürfen.

F. Rudio.

"Leonhard Euler",

Schweizer öffentl. Vorträge, Bd. 8, Heft II (1884), p. 19/20. 
M. Euler n'aurait pas dû publier pour son honneur ses Lettres à une princesse d'Allemagne.

2. VI. 1769.

Les Lettres de M. Euler à une princesse d'Allemagne vous amuseront peut-être par les sorties que vous y trouverez contre les esprits forts.

$$
\text { 15. VII. } 1769 .
$$

Les Lettres de M. Euler n'ont d'autre mérite que d'être sorties de la plume d'un grand géomètre.

2. VIII. 1769.

LAGRANGE à d'Alembert.

... suivant ce que vous m'en dites, e'est son Commentaire sur l'Apocalypse. ${ }^{1}$ ) Notre ami Euler est un grand analyste, mais un assez mauvais philosophe.

16. VI. 1769.

Vous avez bien raison de dire qu'il n'êut pas dû faire imprimer cet Ouvrage pour son honneur. Il est incroyable qu'un aussi grand génie que lui sur la Géométrie et l'Analyse soit en Métaphysique si inférieur au plus petit écolier, pour ne pas dire si plat et si absurde, et c'est bien le cas de dire: Non omnia eidem Dii dedere. ${ }^{2}$ )

7. VIII. 1769.

D'AleMbert à Lagrange.

voir Oeuvres de Lagrange, t. 13 (Paris 1882), p. $132,143,145,135,147 / 148$.

La langue des calculs a aussi son style, et tous les auteurs ne l'écrivent pas avec le même degré de perfection..... Les

1) „Allusion à l'Ourrage de Newton sur l'Apocalypse' [Note de l'éditeur L. Lalanne].

2) Livius, Ab urbe condita liber 22, cap. 51, 4: „tum Maharbal: ,non omnia nimirum eidem dii dedere: vincere scis Hannibal, victoria uti nescis'." 
formules remplacent la phrase; elles peuvent être plus ou moins élégantes. L'analyse parle aux yeux. ${ }^{1}$ ) Ainsi, au lieu de l'harmonie ou de l'accord entre les sons, elle doit présenter entre ses divers éléments des rapports d'ordre et de simplicité faciles à saisir au premier coup d'oeil. Les personnes initiées à ce genre de discours trouvent bien certainement dans la contemplation des formules une sorte de charme qui les entraîne vers l'étude.

\section{Sophie Germain.}

"Considérations générales sur l'état des sciences et des lettres." voir Oeuvres philosophiques, édition de Stupuy (1896), p. 86/87.

Wir können getrost sagen, dass die ganze Form des modernen mathematischen Denkens von Euler geschaffen worden ist. Man nehme einen Schriftsteller unmittelbar vor Euler, man hat die grösste Mühe nur der Ausdrucksweise des Autors zu folgen, weil derselbe es noch nicht verstanden hat, die Formeln durch sich selbst sprechen zu lassen. Diese Kunst hat erst Euler gelehrt.

\section{F. Rudio.}

„Leonhard Euler",

Schweizer öffentl. Vorträge, Bd. 8, Heft III (1884), p. 17.

Ce que je vois de vous m'enchante de plus en plus par la finesse des idées et par l'élégance de l'analyse. Comme ce dernier point a souvent été négligé par les grands géomètres, je m'y suis principalement attaché depuis quelque temps, et je me réjouis infinement que d'autres en fassent de même, surtout lorsqu'ils y réussissent aussi bien que vous.

LAGRANGe à Laplace.

Berlin, 5. VIII. 1783.

voir Oeurres de Lagrange, t. 14 (1892), p. 127.

1) s. Note 17 am Ende des Buches. 
Lagrange, wie fast alle Analysten der neuern Zeit, trifft zuweilen der Vorwurf, beim Spiel der Zeichen nicht immer die Sache lebendig gegenwärtig zu haben. ${ }^{1}$ )

GaUss an Schumacher.

Göttingen, 29, I. 1829.

s. Briefw. Gauss-Schumacher, Bd. 2 (1860), p. 200.

On raconte qu'un jeune homme abordant le calcul différentiel y rencontrait des contradictions qui, s'il est mal enseigné, peuvent réellement s'y trouver. Il osa consulter d'Alembert, illustre déjì et, comme disait Diderot, coryphée admiré des sciences mathématiques. La réponse est restée célèbre: „Allez en avant, la foi vous viendra."

Ce mot brillant, mais dépourvu de toute vérité, explique assez bien les défauts de d'Alembert. Il se réserve d'éclairer chaque page par la lecture de la suivante; c'est ce qu'on appelle manquer de méthode.

\author{
Joseph Bertrand. \\ „D'Alembert" (Paris 1889), p. 56/57.
}

Son principe est qu'un homme de lettres qui cherche à fonder son nom sur des monumens durables, doit être fort attentif à ce qu'il écrit, assez à ce qu'il fait, et médiocrement à ce qu'il dit. M. d'Alembert conforme sa conduite à ce principe; il dit beaucoup de sottises, n'en écrit guères, et n'en fait point.

\title{
D'AleMbert.
}

„Portrait de l'auteur, fait par lui même“, 1760. voir Oeurres posthumes de d'Alembert, t. 1 (Paris 1799), p. 32 $=$ J. Bertrand, ,D'Alembert" (Paris 1889), p. 191/192.

Je déclare sans peine qu'il doit y avoir beaucoup de fautes dans mon Ouvrage; je ne connois aucun Livre d'Astronomie où

1) vgl. S. 152/153, sowie S. 44. 
il n'y en ait pas plusieurs; Optimus ille est qui minimis urgetur, (Horat. Sat. I. 3 [68/69]). Ainsi les plus habiles Astronomes seront obligés de m'excuser, Aequum est peccatis veniam poscentem reddere rursus [ibidem, 74/75]. La mémoire nous trompe, le calcul nous égare à tout moment, la chaleur de la composition nous entraîne; enfin, on ignore souvent des choses qu'il auroit fallu scavoir. D'ailleurs je n'ai pas pris beaucoup de peine pour chercher mes fautes; il y avoit trop de temps à perdre et trop d'ennui à éprouver; j'écris pour mon amusement, et j'y renoncerois si j'étois obligé de mettre dans mes Ecrits cette rigoureuse exactitude si ennuyante pour un Auteur, et qui fait souvent, dit-on, tout le sublime des Sots.

DE LA LANDE.

"Astronomie" (Paris 1764), Préface, p. XV.

Mes occupations se réduisent à faire de la Géométrie tranquillement et dans le silence. Comme je ne suis pas pressé et que je travaille plus pour mon plaisir que par devoir, je fais comme les grands seigneurs qui bâtissent: je fais, je défais et je refais plusieurs fois, jusqu'à ce que je sois passablement content de mon travail, ce qui néanmoins arrive très-rarement.

LAGRANGe à d'Alembert.

Berlin, 1. I. 1781.

voir Oeurres de Lagrange, t. 13 (Paris 1882), p. 360/361.1)

Lorsque M. Euler publiait un mémoire sur un objet nouveau, il exposait avec simplicité la route qu'il avait parcourue, il en faisait observer les difficultés ou les détours; et, après avoir fait suivre scrupuleusement à ses lecteurs la marche de son esprit dans ses premiers essais, il leur montrait ensuite comment il était parvenu à trouver une route plus

1) s. a. ibidem p. 132: Brief an d'Alembert, 2. VI. 1769; sowie Oeuvres, t. 14 (1892), p. 85: Brief an Laplace, 5. VII. 1779. 
simple: on voit qu'il préférait l'instruction de ses disciples à la petite satisfaction de les étonner, et qu'il croyait n'en pas faire assez pour la science, s'il n'ajoutait, aux vérités nouvelles dont il l'enrichissait, l'exposition näve des idées qui l'y avaient conduit.

Souvent Euler aime mieux ne pas faire l'histoire de ses pensées, que de s'exposer au soupçon d'en avoir donné un roman ingénieux, et fait après coup.

Condorcet. „Éloge de M. Euler.“

voir Condorcet, Oeurres, Édition d'Arago, t. 3 (1847), p. 25/26 et 12.

Je trouve que M. Frenicle se tient fort caché et ne veut pas décourrir son artifice. Je n'en fais pas de même, car vous savez assez avec quelle liberté j'étale toutes mes pensées.

Feriatit à Mersenne.

Toulouse, 10. VIII. 1638.

voir Fermat, Oeurres, t. 2 (1894), p. 165.

. . . andere, zu denen auch ich gehöre, möchten überhaupt alle Kunstgriffe aus der Mathematik verbannen, weil für sie jeder Kunstgriff etwas unnatürliches und daher unbefriedigendes hat.

\section{F. ENGEL.}

„Der Geschmack in der neueren Mathematik", Antrittsvorles. Leipzig Univ. 24. X. 1890 (Leipzig 1890), p. 7.

Cauchy ne pouvait souffrir en effet qu'une idée nouvelle restât captive dans son esprit, et l'intervalle de huit jours qui sépare les séances de l'Académie lui semblait quelquefois bien long, telle était son impatience de communiquer à ses collègues tout ce qui, dans ses recherches, pouvait intéresser la science. ${ }^{1}$ )

$$
\text { C.-A. VALSON. }
$$

„La vie et les travaux du baron Cauchy" (Paris 1868), t. 1, p. 252.

1) vgl. S. 173 (Moigno). 
Enfin, et que notre illustre confrère [Cauchy] prenne cette observation en bonne part, le public en général et le public scientifique en particulier, jugeant du présent par le passé, ne croient pas qu'il ait été donné à personne de faire une découverte par semaine. Si ses productions paraissaient à de plus grands intervalles, les géomètres auraient le temps de les mieux juger. ${ }^{1}$ )

Arago.

voir Oeurres, t. 2 (1854), p. $640=$ Werke, Bd. 2 (1854), p. 525.

Plus de sept cents mémoires ......, puis des ouvrages d'une importance capitale ....... sont le témoignage de la prodigieuse activité scientifique de Cauchy et de la fécondité de son génie.

Ch. Hermite.

Discours 5. VIII. 1889.

voir "Inauguration de la Nouvelle Sorbonne" (Paris, Delalain frères), p. 28.

Es ist zu beklagen, dass den jungen französischen Forschern die Akademie ein zu verlockendes Ziel ist. Jede Woche einen Artikel in die Comptes rendus zu bringen, der wirklich Werth hat, das ist doch unmöglich.

Weierstrass an S. Kowalevski.

11. IV. 1882.

s. Mittag-Leffler, „Une page de la vie de Weierstrass", Compte rendu du deuxième congrès intern. des mathém. Paris 1900 (Paris 1902), p. 146.

1) Dies Citat entstammt der Gedächtnisrede auf Poisson, welche in den "Oeurres d'Arago" als "oeurre posthume" bezeichnet ist, welche jedoch auszugsweise in der Sitzung der Acad. des sc. 16. XII. 1850 verlesen war und zwar von Laugier im Namen Arago's, der durch Krankheit behindert war; s. C. R., t. 31 (1850), II, p. 840 . 
Lagrange schied [von der Berliner Akademie] mit einem Witzwort. Da er, aus der letzten Sitzung kommend, lange auf seinen Wagen warten musste, rief er aus: „Es ist leichter, in die $\Lambda$ kademie hinein, als aus ihr heraus zu gelangen“.

Ludwig Geiger.

„Berlin 1688-1840. Geschichte des geistigen Lebens der preussischen Hauptstadt", Bd. 2 (1895), p. 31.

Als Encke 25 Jahre Akademiker war, las er am 1. August 1850 in der Akademie eine Abhandlung über die Zusammensetzung der Berliner akademischen Abhandlungen, worin er die mathematische Abtheilung einigemal ganz allein aus seinem kleinen Beitrage bestehend vorfindet. In den Monatsberichten sind zwar mehrere kleine Abhandlungen von Mathematikern, doch glaubt er auch in den Abhandlungen Lagrange's Wahlspruch, „den Geist immer in Athem halten zu müssen“, wünschen zu können.

.... Dieser Vortrag verursachte ihm viele Verdriesslichkeiten und Unannehmlichkeiten und machte besonders den Bruch zwischen ihm und Jacobi unheilbar.

\section{Bruhns.}

„Johann Franz Encke" (Leipzig 1869), p. 317/318.

Le sieur de la Grange doit arriver à Berlin ..... ., et je dois à vos soins et à votre recommandation d'avoir remplacé dans mon Académie un géomètre borgne par un géomètre qui a ses deux yeux, ce qui plaira surtout fort à la classe des anatomistes. - - - - - - - - - M. Euler qui aime à la folie la grande et la petite Ourse, s'est approché du nord pour les observer plus à son aise. Un vaisseau qui portait ses $x$, $z$, et son $k k$, a fait naufrage; tout a été perdu, et c'est dommage, parce qu'il y aurait eu de quoi remplir six volumes in-folio de mémoires chiffrés d'un bout à 
l'autre, et l'Europe sera vraisemblablement privée de l'agréable amusement que cette lecture lui aurait donnée.

Frédéric le Grand à d'Alembert.

26. VII. 1766.

voir Oeuvres de Frédéric le Grand, t. 24 (Édition Decker 1854), p. 407.

M. Euler s'étoit engagé plus d'une fois envers le Comte Orlof, de fournir à l'Académie [de Pétersbourg] assez de mémoires, pour remplir les Actes jusqu'à vingt ans après sa mort.

N. Fuss.

„Eloge d'Euler" (1783), p. 62.

Und er hat mehr gehalten als er versprochen hatte. Denn seine Arbeiten haben bis zum Jahre 1823, also 40 Jahre lang nach seinem Tode, die Memoiren der Petersburger Academie geschmückt, die dann noch im Archiv zurückgebliebenen Abhandlungen wurden 1830 veröffentlicht, und als man im Jahre 1843, also 60 Jahre nach Eulers Tode, bei Gelegenheit einer Zusammenstellung seiner Arbeiten geglaubt hatte, nun endlich das Riesenvermächtniss bewältigt zu haben, da fanden sich plötzlich noch über 50 ungedruckte Abhandlungen vor, die man trotzdem iibersehen hatte.

\section{F. Rudio.}

„Leonhard Euler",

Schweizer öffentl. Vorträge, Bd. 8, Heft III (1884), p. 11

Wie sagte doch boshaft oder berechnend ein Kollege: Die [auf dem Züricher Kongress 1897 behandelte] Frage der [mathem.] Bibliographie wäre gelöst, wenn sämtliche mathematische Schriften auf neutralem Boden zu einem grossen Autodafé vereinigt würden à la Omar dem Kalifen, dann liesse sich wieder auf anständigem Fuss punkto Honorar mit den Herren Verlegern verhandeln! Das gäbe ein Wettrennen, da 
wäre es eine Lust, Bücher zu schreiben! Es wird nicht sein sollen!

\section{J. H. GraF.}

„Rückblicke und Ausblicke bei Anlass des ersten intern.

Mathematikerkongresses . . in Zürich."

s. Zeitschr. math. naturw. Unterr. 28 (1897), p. 555 [dort angegeben als abgedr. aus dem Berner „Bund“ (16. VIII. 1897)].

Was gedruckt werden soll ist gewöhnlich mehr als einmal geschrieben. Briefe dagegen concipire ich höchst selten, ich glaube in meinem ganzen Leben kaum ein Dutzend Briefe vorher concipirt zu haben.

Gauss an Schumacher.

Göttingen, 12. X. 1840.

s. Briefw. Gauss-Schumacher, Bd. 3 (1861), p. 408/409.

Bei meinen Arbeiten über die auflösbaren Gleichungen ${ }^{1}$ ) habe ich die Erfahrung gemacht, dass ich die Dinge erst eine lange Zeit wärmen muss, ehe sie die für die Publication erforderliche Reife erhalten.

KroneCKer an Dirichlet.

Berlin, 3. III. 1856.

s. Dirichlet, Werke, Bd. 2, p. 393

$=$ Göttinger Nachr. 1885, p. 366 .

Ich glaube nicht, dass ich je die letzte Correctur einer $\mathrm{Ab}$ handlung beendet hatte, ohne nicht 24 Stunden später schon wieder einige Punkte gefunden zu haben, die ich hätte besser oder vollständiger machen können.

\section{H. von Helmholtz.}

s. „Anspr. u. Red. geh. bei der Helmholtz-Feier 2. XI. 1891“ (Berlin 1892), p. 56/57

= L. Königsberger, „Hermann von Helmholtz“, Bd. 3 (1903), p. 99.

1) Gemeint sind hier wohl vor allem die in den Berliner Ber. 1853, p. 365 ff. abgedruckten, sowie die bereits damals angestellten, jedoch erst 1861, Berl. Ber., p. 609 ff. mitgeteilten Untersuchungen. 
Je n'ai jamais terminé un travail sans regretter la façon dont je l'avais rédigé ou le plan que j’avais adopté. Voilà une impression qu'Halphen n'a jamais connue.

H. Poincaré.

„Notice sur Halphen", Journal de l'école polytechnique, cah. 60 (1890), p. 144.

Sylvester's handwriting was bad, and a trouble to his printers. His papers were finished with difficulty. No sooner was the manuscript in the editor's hands than alterations, corrections, ameliorations and generalisations would suggest themselves to his mind, and every post would carry further directions to the editors and printers.

$$
\text { P. A. Mac Mahon. }
$$

„James Joseph Sylvester", Nature 55 (1897), p. 494

$=$ Johns Hopkins University Circulars 16 (1897), p. 27.

Sylvester wurde von der ihn gerade beschäftigenden Materie so völlig beherrscht, dass er je in ihr nicht nur die Spitze alles Wichtigen, Merkwürdigen und Zukunftsreichen sah und sie als solche bezeichnete, sondern dass sie ihm auch die Phantasie und die Imaginationsgabe in noch höherem Masse erregte, als die Reflexionskraft — so sehr, dass er die Fähigkeit, seinen Stoff sachlich zu meistern, oder gar ihn geordnet darzustellen, niemals gewinnen konnte. . . Keine seiner Arbeiten zeigt das Verlangen, den Gegenstand erst nach allen Seiten zu vertiefen und ausreifen $\mathrm{zu}$ lassen: jede blosse Vermuthung, häufig das während des Druckes Concipirte, völlig Unreife oder Falsche wird mit grösster Sorglosigkeit, immer unter völliger Litteraturunkenntniss, im Moment des Entstehens in die Öffentlichkeit hinausgeworfen, ohne dass je eine Spur von Selbstkritik gewaltet hat. - - - -

Sylvester war kein harmonisch veranlagter oder ausgeglichener Geist, sondern ein instinctiv schaffender schöpferischer Kopf, ohne Selbstzucht. ... . Ihn leitet ein, durch sehr lange Beschäftigung mit den Formenprocessen ausgebildeter 
algebraischer Sinn und führt ihn treffsicher $\mathrm{zu}$ allgemeinen Grundwahrheiten, die im Einzelfall verdeckt liegen. Der Mangel an Systematik wird hier durch den Vorzug der Freiheit von rein mechanischer Gedankenthätigkeit ausgeglichen.

M. NoETher.

„James Joseph Sylvester", Mathem. Ann. 50 (1898), p. 155/156.

Let him [the author] be permitted also in all humility to add ...., that in consequence of the large arrears of algebraical and arithmetical speculations waiting in his mind their turn to be called into outward existence, he is driven to the alternative of leaving the fruits of his meditations to perish (as has been the fate of too many foregone theories, the still-born progeny of his brain, now for ever resolved back again into the primordial matter of thought), or venturing to produce from time to time such imperfect sketches as the present, calculated to evoke the mental cooperation of his readers, in whom the algebraical instinct has been to some extent developed, rather than to satisfy the strict demands of rigorously systematic exposition.

\section{J. J. Sylvester.}

„Sequel to the theorems communicated by Mr. Cayley relating to ,Canonic roots" ",

Philosophical Magaz. (4) 25 (1863), p. 460

Greifen wir, um ein Exempel vor Augen zu naben, aus dem vorigen Jahrhundert die Periode heraus, welcher Albrech1 v. Haller den Stempel seines Geistes aufgedrückt hat, sc dürfen wir dreist behaupten, dass es damals keinen Botanike: gab, der nicht seinen Plinius, keinen Mediciner, der nich seinen Hippokrates, keinen Mathematiker, der nicht seineri Euklid von innen und aussen kannte. . . . . . . Heute ha sich dieses Verhältniss so ganz geändert, dem Studirendes. bleibt die Vergangenheit seiner Specialwissenschaft eine terr, incognita, und man darf noch froh sein, wenn er geschichtlich: 
Studien nur als überflüssig, nicht auch als geradezu schädlich zu betrachten gelernt hat.

Für die Mathematik hat Lagrange, für das Gesammtgebiet der kosmisch - tellurischen Disciplinen Alexander v. Humboldt, für die beschreibende Naturwissenschaft Cuvier den Nachweis erbracht, dass nur durch historische Rückblicke die einzelnen Probleme unter einander in Verbindung gesetzt, iiberhaupt klar in ihrer Stellung zum grossen Ganzen erfasst werden können. - - -

\section{S. GÜNTHER.}

„Die Ziele und Resultate der neueren mathematisch-historischen Forschung", Vortrag Naturf.-Vers. Graz 1875.

s. Zeitschr. math. naturw. Unterr. 7 (1876), p. 160 [dort angegeben als abgedruckt aus dem "Tageblatt" der Naturf.-Vers.].

J'admets que l'Histoire des Mathématiques modernes aura besoin d'être étudiée dans divers Volumes destinés chacun ì l'Histoire détaillée d'une branche spéciale.

\section{CAntor.}

"Sur l'Historiographie des Mathématiques."

voir Compte rendu du deuxième congrès intern. des mathém. Paris 1900 (Paris 1902), p. 41/42.

Now there is this reason for grounding the order of exposition upon the historical sequence of discovery, that by so doing we are most likely to present each new form of truth to the mind, precisely at that stage at which the mind is most fitted to receive it, or even, like that of the discoverer, to go forth to meet it. Of the many forms of false culture, a premature converse with abstractions is perhaps the most likely to prove fatal to the growth of a masculine vigour of intellect.

George Boole.

„A treatise on differential equations", 4. ed. (London 1877), Preface, p. VI. 
Sylvester führt bei jeder Begriffsmodification, jedem Einzelprocess neue Namen ein, bestimmt, die Anschaulichkeit der Begriffe und ihre sprachliche Beherrschung zu heben, aber in der Übertreibung ihrer Menge und ihrer Wichtigkeit, wie durch den fortwährenden Wechsel in das Gegentheil umschlagend.

M. Noether.

„James Joseph Sylvester“, Mathem. Ann. 50 (1898), p. 136.

Perhaps I may without immodesty lay claim to the appellation of the Mathematical Adam, as I believe that I have given more names (passed into general circulation) of the creatures of the mathematical reason than all the other mathematicians of the age combined.

\author{
J. J. Sylvester. \\ Nature 37, 1887/1888, p. 152.
}

Weierstrass erzählte zu Anfang der sechziger Jahre, er hätte die Arbeiten Sylvesters aus der algebraischen Formenlehre so lange aufmerksam verfolgt, bis dieser mit hebräischen Buchstaben zu rechnen angefangen hätte; da wäre ihm der Kram zu bunt geworden, und er hätte sich nicht mehr darum bekümmert.

E. LAMPE.

"Arthur Cayley $\dagger$ und James Joseph Sylvester $\dagger$. Nachruf" Naturw. Rundschau 12 (1897), p. 361.

Ich habe es von jeher mir zur Pflicht gemacht, auf dit Reinheit der mathematischen Begriffsbestimmungen zu halten was man freilich bei den meisten Geometern neuerer Zeit of 1 vermisst.

Gauss an Schumacher.

Göttingen, 26. X. 1840.

s. Briefw. Gauss-Schumacher, Bd. 3 (1861), p. 415 
Bei manchen Definitionen.., die man in mathematischen Schriften findet, möchte man an den Rand schreiben:

„Was man nicht recht beweisen kann,

Das sieht man als Erklärung an."

\section{G. Frege.}

„Über die Grundlagen der Geometrie", Deutsche Mathem.-Verein. Jahresber. 12 (1903), p. 321.

Die ersten Begriffe, mit denen eine Wissenschaft, welche es auch sei, beginnt, müssen klar und auf die kleinste "Zahl zurückgeführt sein. Nur dann können sie für das Lehrgebäude eine feste und genügende Grundlage bilden. Begriffe dieser Art werden durch die Sinne erworben; auf angeborene darf man sich nicht verlassen.

\section{LOBATSCHEFSKIJ.}

„Über die Anfangsgründe der Geomtrie.“

s. F. Engel, „N. I. Lobatschefskij“ (Leipzig 1899), p. 2.

Es liegt nicht in Cayley's Art, die Begriffe bis in ihre letzten Elemente zu analysiren, ... Meister aber ist er in der empirischen Verwendung des Materials: wie er es in einem abstracten Gedanken vereinigt, diesen generalisirt und dem rechnenden Versuch unterwirft, wie dann aus den neugewonnenen Daten mit einem Schlage die allgemeine umfassende Idee erscheint, an deren nachträglicher Bewährung durch die verificirende Rechnung Jahre der Arbeit gesetzt werden. So ist Cayley der Naturforscher unter den Mathematikern. - -

Stil und Darstellungsweise Cayley's entsprechen völlig dieser Schaffensart; ersterer ist schlicht und sachlich, giebt ohne viel Reflexionen die Resultate und die Rechnungen wieder und ist in den Bezeichnungen oft plastisch; letztere bringt gewöhnlich zunächst einen rechnerisch ausgeführten speciellen Fall, dann ein allgemein ausgesprochenes Resultat, zuletzt dessen Verification. 
..... Jedenfalls sind die Arbeiten so niedergeschrieben, wie sie entstanden sind, mit weniger Rücksicht auf das Wie, als auf das Was.

\section{Noether.}

„Arthur Cayley“, Mathem. Ann. 46 (1895), p. 479/480.

When Cayley had reached his most advanced generalisations he proceeded to establish them directly by some method or other, though he seldom gave the clue by which they had first been obtained: a proceeding which does not tend to make his papers easy reading. - _ - - -

His literary style is direct, simple and clear. His legal training had an influence, not merely upon his mode of arrangement but also upon his expression; the result is that his papers are severe and present a curious contrast to the luxuriant enthusiasm which pervades so many of Sylvester's papers. He used to prepare his work for publication as soon as he had carried his investigations in any subject far enough for his immediate purpose ... A paper once written out was promptly sent for publication; this practice he maintained throughout his life..... The consequence is that he has left few arrears of unfinished or unpublished papers; his work has been given by himself to the world.

\section{A. R. F[orsyth].}

London Roy. Soc. Proc. 58 (1895), XXIII and XXIV.

. gewiss ist, dass wenn meine Lage $^{1}$ ) immer die nämliche bleibt, ich den grössern Theil meiner früheren theoretischen Arbeiten, denen noch, der einen mehr, der anderen weniger an der Vollendung fehlt, und die ron solcher Art sind, dass Vollendung sich nicht erzwingen lässt wenn man

1) Gemeint ist die amtliche Tätigkeit in Göttingen; es handelte sich damals um eine Berufung G's nach Berlin. 
eben will, mit in's Grab nehmen werde. Denn etwas unvollendetes kann und mag ich einmal nicht geben.

Gauss an Bessel.

Göttingen, 15. XI. 1822.

s. Briefw. Gauss-Bessel (1880), p. 410.

Au lieu de poursuivre obstinément une perfection idéale, qu'il n'est pas donné à l'homme d'atteindre, les observateurs feront sagement de mettre le public, le plus tôt possible, dans la confidence de leurs travaux.

\section{Arago.}

Oeuvres, t. 1 (1854), p. $353=$ Werke, Bd. 1 (1854), p. 283.

Als in den sechziger Jahren bei der Veröffentlichung des Nachlasses von Gauss in den gesammelten Werken desselben ein jüngerer Mathematiker bemerkte, nun seien ja auch die nachlässigen Werke von Gauss zugänglich geworden, sprach sich Kummer, der dem Witzworte schmunzelnd sein Ohr geliehen hatte, dahin aus, er werde dafür sorgen, dass von ihm nichts Nachlässiges vorgefunden werde, und er werde aufhören, etwas zu veröffentlichen, sobald er die Abnahme seiner geistigen Kräfte spüre. Diesem Vorsatze ist er treu nachgekommen - -

\section{E. Lampe.}

„Nachruf für Ernst Eduard Kummer“,

Deutsche Mathem.-Verein. Jahresber. 3, 1892/1893, p. 14

$=$ Naturw. Rundschau 8 (1893), p. 361.

Immer nehme ich mir vor, nichts mehr drucken zu lassen, da ich meine Altersschwäche [im 81sten Lebensjahre] nur zu sehr fühle, und immer werde ich wieder verlockt, diesen so vernünftigen Vorsatz wieder zu übertreten.

Olbers an Bessel.

Bremen, 9. I. 1839.

s. Briefw. Olbers-Bessel, herausg. v. Erman, Bd. 2(1852), p. 434/435. 
Je crois devoir m'excuser, vis-à-vis du lecteur, de lui livrer un mémoire aussi maussadement, je puis même dire, aussi inintelligiblement rédigé [J. Liouville, „Mémoire sur la théorie analytique de la chaleur"]. . ... je crus trouver dans le double titre d'ingénieur et d'ancien élève de l'École polytechnique une garantie suffisante du talent de rédaction de l'auteur, et j'envoyai de suite l'ouvrage à l'impression. - - - - -

Je ne prétends contester aucunement la capacité mathématique de M. Liouville; mais à quoi sert cette capacité, si elle n'est accompagnée de l'art de disposer, de l'art de se faire lire, entendre et goûter.

Je désire bien vivement que M. Liouville se venge prochainement des reproches un peu sévères peut-être que, bien à regret, sans doute, je me trouve contraint de lui adresser aujourd'hui, en publiant quelque mémoire que l'on puisse lire à peu près comme on lit un roman; mais la vérité est que je le désire beaucoup plus que je ne l'espère. Une longue expérience m'a prouvé que le mal dont il est atteint est un mal à peu près incurable.

\section{J. D. G[ERGOnNe].}

Annales de mathém. pures et appliquées 21, 1830/1831, p. 181.

Ehe ich schliesse, muss ich den Vorwitz des Setzers oder Correctors rügen, der am Ende des ersten Theils, statt der Worte: „sonst habe ich nur folgende erhebliche Schreib- und Druckfehler bemerkt":

sich unterfing

„sonst habe ich nur folgende erhebliche Schreibfehler bemerkt:"

abzudrucken, auch gegen meine, und meines Herrn Verlegers Erinnerung fortfuhr, das $k_{i}$ an die Stelle des $c$ zu setzen.

H. C. Schumacher.

Deutsche Ausg. von Carnot's „Géométrie de Position“, II (Altona 1810, Hammerich), Vorrede. 
Il existe dans les mains des élèves de nos grandes écoles, un Traité de calcul différentiel et de calcul intégral, publié sans nom d'auteur, sans titre et sans table de matières; l'imprimeur, après de nombreuses tentatives, avait fini par comprendre que notre Ampère ne lui fournirait jamais les quelques lignes qui eussent été nécessaires pour donner au nouveau livre la forme que tous les livres ont eue, depuis le temps de Gutenberg.

Arago.

Oeurres, t. 2 (1854), p. 105/106 = Werke, Bd. 2 (1854), p. 86.

Meines Wissens giebt es nur eine mathematische Zeitschrift, die lange Zeit nach dem Tode eines Mathematikers denselben noch als Mitglied der Redaktion angiebt, nämlich die Acta Mathematica (siehe die zweite Seite des Umschlages des am 15. December 1901 erschienenen Doppelheftes 25 : 1-2, wo unter „Redaction" auch der Name „S. Lie“ vorkommt).

G. Eneströ̈r.

„Wie soll ein Mathematiker-Kalender zweckmässig bearbeitet werden?", Bibl. mathem. (3) 3 (1902), p. 233.

Hand in Hand mit der Gleichgültigkeit gegen die formale Seite der Sprache geht bei den Deutschen die Vernachlässigung des Stils.

Um bei den deutschen Naturforschern stehen zu bleiben, wie viele unter ihnen giebt es denn, welchen der Gedanke, dass man auf die Darstellung Fleiss verwenden müsse, und dass eine wissenschaftliche Abhandlung ein Kunstwerk sein könne wie eine Novelle, nicht als wunderliche Grille erscheint? . . . . . . Unbekümmert um die äussere Erscheinung treten sie im Schlafrock vor die Öffentlichkeit, und, was kaum minder schlimm ist, die Öffentlichkeit ist es zufrieden. Ja sie suchen etwas darin, äusserer Hülfsmittel sich zu entschlagen, als ob die Wahrheit unter gefälliger Form litte, als ob formale Durchbildung eines Gedankengefüges nicht der sicherste Weg wäre, übersehene 
Lücken und Fehler aufzudecken, und als ob nicht gerade die Hochmeister des Gedankens, beispielsweise ein Gauss, ihre Arbeiten auch äusserlich mit der grössten Sorgfalt vollendet hätten. Je hastiger gegenwärtig die wissenschaftliche Production, um so grösser die stilistische Verwilderung. Lehrreich ist zu beobachten, dass sie weniger bei den Forschern um sich greift, deren Gegenstand strenges Denken erheischt, bei den Physikern, mehr bei denen, die am anderen Ende der Reihe stehen, den Medicinern. Namentlich treiben diese einen nicht genug zu tadelnden Missbrauch mit Fremdwörtern.

Emil de Bois-Reymond.

„Über eine Kaiserliche Akademie der deutschen Sprache“, Festrede i. d. Berl. Akademie 26. III. 1874.

s. Reden, 1. Folge (1886), p. 159-161.

Ich kenne Niemand, der das Talent sich scharf und kurz auszudrücken in so hohem Grade wie Sie besitzt.

Schumacher an Gauss 21. VII. 1836.

Das Compliment, welches Sie . . mir wegen meines Talents, mich kurz und scharf auszudrücken, machen, muss ich wenigenst in so fern von einer andern als der deutschen oder höchstens noch lateinischen Sprache ${ }^{1}$ ) die Rede ist, doch von mir ablehnen.

Gauss an Schumacher. Göttingen, 24. VII. 1836.

s. Briefw. Gauss-Schumacher, Bd. 3 (1861), p. 89 u. 94.

In einer fremden Sprache kostet die Aufmerksamkeit auf die Diction, so dass ich selbst mit Rundung und Natürlich-

1) es handelte sich für Schumacher hier um die englische Sprache. 
keit ganz zufrieden bin, mir immer wenigstens eben so viel Zeit, wie die Sache selbst.

Gauss an Schumacher.

Göttingen, 4. II. 1821.

s. Briefw. Gauss-Schumacher, Bd. 1 (1860), p. 204.

Mein Werkchen über die Bestimmung der Planetenbahnen u. s. w. [die "Theoria motus corporum coelestium"] . . . . naht sich seiner Vollendung. .... . Bey den jetzigen Zeitumständen, fürchte ich, wird es vielleicht in Deutschland einige Mühe kosten, einen soliden Verleger zu finden.

C. F. Gauss [an Frhn. F. von Zach].

Braunschweig, 3. I. 1807.

s. von Zach's Monatl. Correspondenz zur Beförderung der Erd- u. Himmels-Kunde 15 (1807), p. 153.

Ich habe mit Leidwesen in der Monatlichen Correspondenz gesehen, dass es Ihnen bei den jetzigen Umständen Schwierigkeiten macht, einen Verleger zu Ihrem Werke über die Planeten-Bahnen zu finden.

Bessel an Gauss. Lilienthal, 26. III. 1807.

Mein Büchlein über die Planetenbahnen wird nun hoffentlich bald an's Licht kommen können. Perthes ist willig, Hebeammendienst dabei zu thun, und ich denke, wir wollen über die Bedingungen leicht einig werden. Bloss die Übersetzung in's Lateinische verursacht noch einigen Aufenthalt, ich verdenke es indess dem Buchhändler nicht, dass er seines Vortheils wegen darauf besteht.

Gauss an Bessel.

Braunschweig, 12. V. 1807.

s. Briefw. Gauss-Bessel (1880), p. 50 u. $55 / 56$.

Le latin n'a pas de termes pour exprimer les vérités mathématiques et physiques qui manquaient aux anciens - - - 
. . il faut avouer que ce n'est plus guère la peine d'écrire les livres de sciences dans une langue morte, à laquelle il faut toujours ajouter des expressions inconnues à l'antiquité, et qui peuvent causer de l'embarras. Le français, qui est la langue courante de l'Europe, et qui s'est enrichi de toutes ces expressions nouvelles et nécessaires, est beaucoup plus propre que le latin à répandre dans le monde toutes ces connaissances nouvelles.

VolTAIRE.

„Éloge historique de Madame la Marquise du Châtelet." voir Voltaire "Mélanges philosophiques, littéraires,

historiques ......", t. 1 (Genève 1771), p. 4

$=$ Oeurres de Voltaire, t. 47 (Gotha 1787), p. 75.

Sermo latinus parum idoneus est disputationi de rebus physicis habendae.

\title{
C. Neumann.
}

These der Habilitationsschr. Halle 1858.

Lingua latina ad problemata recentioris mathesis tractanda non est apta.

G. Valentin.

Doctorthese Berlin 1879.

Für die mathematischen Wissenschaften wäre die Wiedereinführung einer allgemeinen Gelehrtensprache, etwa des Lateinischen, von grossem Nutzen.

\author{
K. Th. Vahlen. \\ Doctorthese Berlin 1893.
}

In den Zeiten, wo ich die meisten meiner Arbeiten lateinisch zu schreiben hatte, musste ich sehr oft den mir vorschwebenden Gedanken erst lange hin und her wenden, bis ich eine einigermassen genügende und doch oft keineswegs mich ganz befriedigende Wendung gefunden hatte. Doch kommt dergleichen nie vor, so lange man sich bloss im rein mathematischen (ich 
möchte sagen im technisch-mathematischen) Felde bewegt, sondern hauptsächlich, wo man den Gegenstand und das Charakteristische seines Wesens aus einem höheren gleichsam, philosophischen - wie Lagrange zu sagen pflegte metaphysischen - Standpunkte betrachtet.

Gauss an Schumacher.

Göttingen, 4. XII. 1849.

s. Briefw. Gauss-Schumacher, Bd. 6 (1865), p. 50/51.

Ich werde nie wieder über wissenschaftliche Dinge Latein schreiben.

Gauss an Schumacher.

Göttingen, 29. IV. 1845.

s. Briefw. Gauss-Schumacher, Bd. 4 (1862), p. 437.

In Königsberg .... war der Philolog Lobeck . . auch Professor eloquentiae und als solcher auch Redacteur des Lectionskataloges. Dieses Geschäft schien ihm aber so wenig würdig, darauf Zeit zu verwenden, dass er es nicht selbst besorgte, sondern, wie man sagte, irgend einem Studenten überliess. Unsere Lectionskataloge gewannen dadurch den Character einer classischen Nachlässigkeit. Da erschienen Kataloge für ein Sommer-Semester, welches am 25. October beginnen werde. Da wurde eine Vorlesung, welche für den Deutschen L.-K. von Professor [Franz] Neumann so angegeben war: Über die Fortpflanzung der Wärme in den Mineralien, im Lateinischen, angekïndigt: de vermium in corporibus solidis propagatione (im Sommer 1831). Der unfindbare Übersetzer hatte also Würmer für Wärme gelesen. Ich war grade zugegen, als Professor Neumann seinem Collegen Lo beck Vorstellungen über diese Unachtsamkeit machte. „Ja, warum kündigen die Herren ihre Vorlesungen nicht in beiden Sprachen zugleich an?" war die einzige Antwort, die er erhalten konnte. Schrieb man aber Lateinisch, so wurde natïrlich die Wortkritik geltend gemacht und man war nie ganz sicher, was daraus 


\section{$-272$}

wurde. Den Ausdruck in mineralibus hätte Lobeck schwerlich stehen lassen, aber ob Wärme oder Würmer sich in den Mineralien fortpflanzen, schien ihm sehr gleichgültig, da die Classiker sich um dergleichen nicht bekümmert hatten.

Kard Ernst von Baer [Prof. d. Zoologie]. „Nachrichten über sein Leben und seine Schriften" (Petersburg 1866), p. 261/262.

Le doyen du Collège royal, en 1566 , poursuivait devant le Parlement la révocation du professeur de mathématiques, alléguant dans sa requête que, notoirement ignorant de la langue grecque, Jacques Charpentier ne pouvait enseigner Euclide.

\section{J. Bertrand.}

„Éloges académiques" (Paris 1890), p. 240.

Die Stimme der Zeit will mehr als Griechisch und Lateinisch. und es ist vorauszusehen, dass diese Forderung sich mit dem Laufe der Zeit verstärken wird. Die Schulmänner sind ihr nachzugeben gezwungen worden: sie haben dem Griechischen und Lateinischen etwas Mathematik zugesellt. $\mathrm{Ob}$ es ihnen Ernst damit war, oder nur geschah, um dem Drange so weit nachzugeben, dass ein Schein erzeugt, die lateinische und griechische Schule aber gerettet wurde, kann man beurtheilen, wenn man die bessere Rolle der Sprachen auf den Schulen mit der wirklich traurigen der Wissenschaften vergleicht. Es lässt sich, in der That, Beides nicht vereinigen; unsere lateinischen Schulen können eben so wenig wissenschaftliche werden, als durch diese etwas Anderes erlangt werden kann als ein Wechsel der Rollen. Einseitigkeit muss immer bleiben, aber sie ist verschiedener Art. So viele gute und schlechte Schriften das Alterthum uns auch hinterlassen haben mag, so vielfältig auch sein mag was man daraus hervorsuchen kann - so sind Natur und Mathematik doch unendlich viel reicher: so dass die Benennung Einseitigkeit für Beide einen verschiedenen Sinn erhält. 
Wenn richtig ist, was ich auszuführen gesucht habe, so habe ich ... die Hoffnung, dass über kurz oder lang die Wissenschaft des Himmels und der Erde in das Leben des Volks treten wird, und dass dereinst Fehler gegen den Euklid, oder falsche Ansichten der Natur, eben so bezeichnende Andeutungen mangelnder Bildung sein werden, als jetzt ein falscher Casus. $^{1}$ )

Besser an den Minister Theodor von Schön.

Königsberg, 25. IX. 1828.

s. "Aus den Papieren des Ministers ... Theodor von Schön“, Bd. 4 (Berlin 1876), p. 470 u. 473.

Il n'y a pas de plus grands barbares, me disait souvent Fourier, que certains mathématiciens; ils n'estiment que les mathématiques, et voudraient qu'on $y$ appliquât d'abord les jeunes gens. C'est l'idée la plus fausse, la plus contraire à l'esprit philosophique, à la société et à l'humanité. Il faut maintenir soigneusement dans les colléges l'étude des langues anciennes, du grec et du latin. Car en apprenant le latin, ce n'est pas seulement une belle langue qu'on étudie, c'est un commerce intime qu'on institue avec des hommes sages et d'un génie excellent, un Cicéron, un Virgile, un Horace, un Tite-Live, un Sénèque. Que de belles et bonnes choses on y apprend!

Les humanités terminées, il faut résumer et développer les études grecques et latines par un cours de philosophie. dans lequel on insistera particulièrement sur la morale. - - -

Quand l'homme est ainsi formé, alors appliquez-le aux mathématiques. Il y marchera d'autant plus vite, et il s'en servira comme il faut s'en servir, dans un esprit philosophique et pour la plus grande utilité des hommes.

\section{VICTOR Cousin.}

„Fragments et souvenirs", 3. éd. (1857), p. 374 et 375.

1) vgl. S. 118 (Schluß des Citats H. V. v. Unrub).

A hrens, Scherz und Ernst i. d. Mathem. 
Ich bin auf mein altes Projekt einer école polytechnique zurïckgekommen, für welches auch Alexander Humboldt hier geworben hat. Ich habe aber bei der Gelegenheit recht kennen lernen, dass unsere deutschen Philologen ebenso intolerant wie die Jesuiten sind, und dass eine wahre Verbrüderung Statt findet, die Mathematik nicht aufkommen zu lassen. Ich hoffe, dass die Gauss'sche Angelegenheit [Gauss' Berufung nach Berlin] nun endlich zu Stande kommt und dass wenn er hier ist ich eine Stütze an ihm finde, damit wir die Mathematik in unserem Staate etwas in die Höhe bringen. Ich habe dem Könige gesagt, dass der Staatsunterricht in der Mathematik bei andern Nationen da anfängt, wo er bei uns schliesst.

Generalstabschef von Mürflixg an v. Lindenau.

[Berlin], 1. IV. 1823.

s. K. Bruhns, „Briefe zw. A. v. Humboldt und Gauss" (Leipzig 1877), p. 10/11.

Wie Gauss, Jacobi und andere hervorragende Mathematiker hatte Weierstrass sich gründliche Kenntnisse in allen Zweigen des Gymnasialpensums angeeignet. Auch wusste er die Wichtigkeit einer solchen Bildung während seines ganzen Lebens wohl zu schätzen. Als in den 70er Jahren der Antrag gestellt wurde, die philosophische Fakultät der Universität Berlin in eine historisch-philosophische und eine mathematischnaturwissenschaftliche zu trennen, arbeitete Weierstrass ein Gutachten aus, worin er die Zusammengehörigkeit aller in der philosophischen Fakultät vertretenen Fächer betonte und es als notwendig hinstellte, dass sie durch die Philosophie als ihr gemeinsames Band auch wirklich vereinigt würden.

\section{W. KiLLING.}

„Karl Weierstrass", Rektoratsrede Münster Akad. 1897. s. Natur und Offenbarung 43 (1897), p. 707.

Wie der Schreiber dieser Zeilen .... gehörte auch Lommel zu jenen Nichtphilologen, die ihren Homer noch im Mannesalter immer und immer wieder lasen. Wie kommt es, 
dass diese Menschengattung nun auszusterben scheint? Beim helläugigen Kinde des Ägisträgers! der Sinn für Schönes und Grosses ist unseren Söhnen nicht abhanden gekommen; diese lauschen doch Shakespeare's Worten, Beethoven's Tönen mit der gleichen Begeisterung wie einst wir. Beginnt etwa der Stern Homer's zu erbleichen in der Morgenröte des 20. Jahrhunderts, oder treffen die wissenschaftlich doch weit besser geschulten Philologen von heute Herz und Gemüt der Jugend nicht mehr so wie unsere Lehrer? Würde wohl gar, wenn sie heute lebten, Achill dem Gymnasium mit seiner ganzen Schnellfüssigkeit entlaufen und Sokrates an die Spitze des Vereines für Mittelschulreform treten?

L. Bolitzanan.

„Eugen von Lommel“, Deutsche Nathem.-Verein. Jahresber. 8, 1899, p. 51.

Kegelschnitte! Kein griechisches Scriptum mehr!

Emil du Bois-Reymond.

„Culturgeschichte und Naturwissenschaft", Vortr. Köln 24.III. 1877.

s. Reden, 1. Folge (1886), p. 295.

Wir werden auch ferner das Ideal menschlicher Gesittung fortfahren auf gut lateinisch Humanitït und denjenigen, welcher den Homer meint mit der Zeit durch die Lehre von den Kegelschnitten ersetzen zu können, auf gut griechisch einen Banausen zu nennen, und wir rechnen für dieses Latein und dieses Griechisch auch ferner auf das Verständniss und das Einverständniss des deutschen Publicums.

\section{Theodor Mommsen.}

(„Die nationale Einigung ein Werk der nationalen Nothwehr im Alterthum wie in der Neuzeit"),Festrede i. d.Berl.Akad.20.III. 1884.

s. Berl. Sitzungsber. 1884, p. 246.

Die Muttersprache und fremde Sprachen, welche man allein durch Übung lernt, nehmen nicht das bewusste logische Denken in Anspruch; wohl aber kann man an ihnen das Gefühl für 
künstlerische Schönheit des Ausdrucks üben. Die beiden classischen Sprachen, Griechisch und Lateinisch, haben neben ihrer ausserordentlich feinen künstlerischen und logischen Ausbildung den Vorzug, den die meisten alten und ursprünglichen Sprachen zu theilen scheinen, dass sie durch sehr volle und deutlich unterschiedene Flexionsformen das grammatikalische Verhältniss der Worte und der Sätze zu einander genau bezeichnen. Durch langen Gebrauch werden die Sprachen abgeschliffen, die grammatikalischen Bezeichnungen im Interesse praktischer Kürze und Schnelligkeit auf das nothwendigste zurückgeführt und dadurch unbestimmter gemacht. Das lässt sich auch an den modernen europäischen Sprachen in Vergleich mit dem Lateinischen deut. lich erkennen; am weitesten ist in dieser Richtung des Ab. schleifens das Englische vorgeschritten. Darin scheint es mir auch wesentlich zu beruhen, dass die modernen Sprachen als Unterrichtsmittel viel weniger geeignet sind, als die älteren.

\section{HeLmholtz.}

„Über das Verhältniss der Naturwissenschaften zur Gesammtheit der Wissenschaft", Prorectoratsrede Heidelberg 22. XI. 1862.

s. Vorträge u. Reden, Bd. 1 (1884), p. 134.

Indem ich so doch einige Zeit mich ernstlich mit der Philologie beschäftigte, gelang es mir einen Blick wenigstens zu thun in die innere Herrlichkeit des alten hellenischen Lebens. so dass ich wenigstens nicht ohne Kampf dessen weitere Erforschung aufgeben konnte. Denn aufgeben muss ich sie für jetzt ganz Der ungeheure Koloss, den die Arbeiten eines Euler, Lagrange, Laplace hervorgerufen haben, erfordert die ungeheuerste Kraft und Anstrengung des Nachdenkens, wenn man in seine innere Natur eindringen will und nicht bloss äusserlich daran herumkramen. Über diesen Meister zu werden. dass man nicht jeden Augenblick fürchten muss von ihm er drückt zu werden treibt ein Drang, der nicht rasten und ruher lässt, bis man oben steht und das ganze Werk übersehen kann Dann ist es auch erst möglich mit Ruhe an der Vervollkomm. nung seiner einzelnen Theile recht zu arbeiten und das ganze 
grosse Werk nach Kräften weiter zu führen, wenn man seinen Geist erfasst hat.

\section{G. J. JACOBI}

als Student an seinen Oheim Lehmann.

s. Jacobi, Werke, Bd. 1, herausg. v. Borehardt, p. 5.

Die Regeln der traditionellen Schulgrammatik, denen gleichsam selbstverständlich eine Fülle von Ausnahmen zu folgen pflegte, müssen für jeden mathematisch geschulten Verstand etwas peinliches haben. Um so wohlthuender musste Grassmann von den Versuchen der Sprachwissenschaft berührt werden: statt praktischer Regeln wissenschaftliche Gesetze zu gewinnen. Namentlich musste ihn das Gebiet anziehen, auf welchem vor allem das Wirken bestimmter Gesetze erkennbar ist, die Lautlehre. Ihr waren denn auch seine ersten sprachlichen Arbeiten gewidmet .... . diese zeichnet vor anderen aus die Beharrlichkeit mit welcher dem Stoff so lange zugesetzt wird bis er sich die einfachste Formulirung des Gesetzes hat abringen lassen; es ist die Unermüdlichkeit der mathematischen Abstraction, die in diesen Aufsätzen vorzugsweise zur Erscheinung kommt.

\section{B. DesbrüCK}

[Prof. d. vergl. Sprachforschung in Jena]. „Hermann Grassmann“, Augsburger Allgem. Zeitg. Beilage 18. X. 1877 , p. 4371.

Herr Woepke verdient im höchsten Grade die Unterstützung des Staates, da er, was so überaus selten ist, Kenntniss der höheren Mathematik (Analysis) mit sehr gründlichen arabischen Studien verbindet.... In den Eisbergen des CultusMinisteriums war nichts zu erhalten, aber unterstützt durch unseren grossen Mathematiker Dirichlet (den ersten, seitdem Jacobi gestorben, Eisenstein verhungert und Rosenhain in Breslau politisch verfolgt worden ist), habe ich mich, noch ehe Sie mich dazu aufforderten, theuerste Excellenz, an die 
Akademie der Wissenschaften gewandt, die ihre Fonds (nach den Liebhabereien einer Commission) rersplittert.

Al. Humboldt an Christian Carl Josias Freiherr v. Bunsen. Berlin, 20. II. 1854.

s. „Briefe Alex. v. Humboldt [s] an Chr. C. J. Frhr. v. Bunsen“ (Leipzig 1869), p. 176/177.

Ein noch lebender Geometer, der mit dem grossen Mathematiker des Alterthums die überraschende Eigenthümlichkeit theilt, dass er die deutsch geschriebenen Abhandlungen des Crelle'schen Journals nicht gelesen hat, ist der Archimedes unseres Jahrhunderts genannt worden.

C. F. Geiser.

„Zur Erinnerung an J. Steiner" (1874), p. 37.

Le mémoire du grand géomètre [Göpel] qui a été publié après sa mort: Lieber Projectivat der Kegelschnitte, als Kummen Gebilde. ${ }^{1}$ )

Charles Hermite.

Discours 5. VIII. 1889.

voir "Inauguration de la Nouvelle Sorbonne"

(Paris, Delalain frères), p. 16.

J'ai toujours été et jusqu'à mon dernier jour je serai encore un disciple de ros grands géomètres, de vos maitres illustres, Gauss, Jacobi, Dirichlet; d'autres commt Kronecker, Borchardt, Mr Lipschitz etc. ont été les compagnons de mes études et mes amis dévoués.

Hermite à E. Jahnke.

Paris, 25. XI. 1900.

voir Arch. Math. Phys. (3) 1 (1901), p. 20

Mathematik, Naturkunde, Theologie, alte und neue Spraches bewältigte Schläfli mit seltener Sicherheit und durch seine»

1) sic! statt: „Über Projectivität der Kegelschnitte als krummer Ge bilde"; s. J. f. Math. 36 (1848), p. 317-356. 
Scharfsinn und seine Belesenheit, durch seine hervorragenden Publikationen, mehr als sechzig an der Zahl, hat er besonders die Mathematik nicht nur bei uns, sondern auch im Ausland zu hoher Anerkennung gebracht. - - - - - - -

Er beherrschte die Sprachen in seltenem Maasse, schrieb er doch mit derselben Leichtigkeit italienische, englische und französische Abhandlungen. Die Vertiefung in die orientalischen Sprachen war für ihn eine Erholung, sein letztes Studium galt dem Persischen, der Keilschrift und dem Hebräischen. In den Naturwissenschaften, ganz besonders in der Botanik, excellirte er durch hervorragende Kenntnisse.

\section{J. H. GraF}

bei der Beerdigung Schläfli's 23. III. 1895.

s. Bern Naturf. Ges. Mitth. 1895, p. 144/145.

Gestern ist auch St[einer] eingerückt, was eben nicht für mich zur Verschönerung der Reise wesentlich beitragen wird und hat noch einen Schweizer Jüngling mitgebracht, der ein mathematisches Genie sein soll, sicherlich aber ein Rindvieh ist. Was kann man aber auch erwarten, wenn Einer Herr Schläfli heisst.

Rebecka Dirichlet an ihre Schwester Fanny Hensel. Florenz, 21. X. 1843.

s. S. Hensel, „Die Familie Mendelssohn“, 1. Aufl, Bd. 3 (1879). p. 57.

Auf Schläflis Reklamation hin ist die Stelle in der 2. Ausgabe weggeblieben.

J. H. GraF.

Bern Naturf. Ges. Mitth. 1897 (Bern 1898), p. 115.

Als Schüler war Lie nahezu in allen Fächern gleich vorzüglich und schwankte daher bei Beginn seines Studiums, ob er die Sprachen oder die Realfächer wählen sollte, entschied sich aber schliesslich für die letzteren. Auch als Student 
zeigte er noch keine ausgesprochene Vorliebe für Mathematik und weder er selbst noch einer seiner Lehrer ahnte das in ihm schlummernde mathematische Genie.

Da endlich, es war im Jahre 1868, kamen ihm ganz zufällig die Schriften von Poncelet und Plücker in die Hände: eine neue Welt ging ihm auf und es wurde ihm plötzlich klar, wo sein Beruf lag. Der Trieb zur mathematischen Produktion erwachte in ihm und mit um so grösserer Macht, da er bisher so lange verborgen geblieben war. Kein Wunder daher, dass er Zeit seines Lebens für Poncelet und Plücker eine besondere Verehrung hatte.

F. ENGeL.

„Zur Erinnerung an Sophus Lie“, Rede Kgl. Sächs. Ges. d. Wissensch. 14. XI. 1899.

s. Leipziger Ber. 51 (1899), Math.-phys. Cl., p. XIII-XV.

Ich schlage vor, die unendlich fernen imaginären Kreispunkte einer Ebene als ihre Poncelet'schen Punkte, und dementsprechend den unendlich fernen imaginären Kugelkreis des Raumes als den Poncelet'schen Kreis zu bezeichnen. Die Einführung dieser Begriffe ist doch Pon c e let's originellste Leistung, wenn auch dieser grösste Geometer unseres Jahrhunderts, der leider so früh von der Geometrie weggezogen wurde, nicht dazu Gelegenheit fand, die Tragweite dieser Begriffe nach allen Richtungen klar zu stellen.

\section{Sophus Lie.}

Leipziger Ber. 48 (1896), Math.-phys. Cl., p. 470.

Noch will ich zufügen, dass meine Begriffserweiterungen für einen Schüler Plücker's sehr naheliegend sind; Plücker würde es gemacht haben, wenn er sich überhaupt mit partiellen Differentialgleichungen 1. $\mathrm{O}$. beschäftigt hätte.

Sophus Lie.

„Allgemeine Theorie der partiellen Differentialgleichungen erster Ordnung“", Math. Ann. 9 (1876), p. 246. 
Trotzdem sich Kronecker gern als Schüler von Dirichlet bezeichnete, will es mir doch scheinen, als ob dieser grosse Forscher auf die Richtung seiner wissenschaftlichen Thätigkeit nicht in solchem Masse bestimmend gewirkt hat, wie man gewöhnlich annimmt. Dirichlet selbst hat dies wohl empfunden, da er sich nur rïhmt, Kronecker in die unteren Regionen einer der Wissenschaften eingeführt zu haben, auf deren Höhe dieser als Meister einherschreite, und sich an seiner algebraischen Grösse völlig unschuldig erklärt. ${ }^{1}$ ) So weit Kronecker Schüler war, war er der Schüler von Kummer - - -

\section{G. Frobenius.}

„Gedächtnissrede auf L. Kronecker“, Berlin Akad. 29. VI. 1893. s. Berliner Abh. 1893, p. 8.

Dass die Ergebnisse [meiner Arbeiten] den Begriff des Idealen anderweit begründeten und beglaubigten, welchen die Wissenschaft meinem Freunde Kummer verdankt, befriedigte vor Allem - um dessen eignen Ausdruck zu gebrauchen mein mathematisches Herz.

\section{Kronecker.}

Antrittsrede in der Berl. Akademie.

s. Berl. Nonatsber. 1861, p. 639.

Für die überaus grosse Freude, welche mir die Mittheilung Ihrer schönen Entdeckungen [über complexe Multiplication der elliptischen Functionen und ideale Zahlen] verursacht hat, finde ich keinen passenderen Ausdruck als Ihnen aus vollster Überzeugung macte virtute zuzurufen. Zugleich kann ich Ihnen nicht verhehlen, dass sich dieser Freude etwas Egoismus beimischt, da ich mir bei aller Bescheidenheit das Zeugniss nicht versagen kann, dass ich Sie zuerst in die unteren Regionen einer der Wissenschaften eingeführt habe, auf deren Höhen Sie jetzt als Meister einherschreiten. Ich rede absichtlich nur

1) s. das übernächste Citat. 
von einer dieser Wissenschaften, denn an Ihrer algebraischen Grösse muss ich mich völlig unschuldig erklären.

Dirichlet an Kronecker. Göttingen, 4. VII. 1857.

s. Dirichlet, Werke, Bd. 2, p. 401

$=$ Göttinger Nachr. 1885, p. 374.

Certains géomètres planent majestueusement dans les hautes régions de l'espace, sans qu'il soit aisé de dire qui leur a frayé le chemin. Ajoutons que ce chemin était ordinairement établi sur un échafaudage dont personne n'a pris soin quand l'oeuvre a été accomplie.

Arago.

Oeuvres, t. 2 (1854), p. 126

= Werke, Bd. 2 (1854), p. 103.

Bekanntlich war Weierstrass selbst kaum Jemandes persönlicher Schüler gewesen. Der Mathematiker aber, dessen Schriften auf seine Entwicklung den grössten Einfluss gehabt haben, ist Abel. Einer der ersten Schüler von Weierstrass in Berlin, der ihn im Anfang seiner wissenschaftlichen Lehrthätigkeit am genauesten gekannt hat, sagte mir: „Ich kann mir Weierstrass nicht anders denken als mit den Werken Abels in der Hand und stets auf Abel hinweisend“1).

G. Mittag-Leffler.

"Weierstrass", Acta mathem. 21 (1897), p. 81.

Die Mathematiker unserer Zeit wurden erst durch das Studium von Abel's durchsichtigen und tiefen Untersuchungen in den Stand gesetzt, Galois' ebenso schwer zugängliche als tiefe Ideen zu verstehen.

Nach meiner Ansicht kann man den Mathematikern noch heute keinen besseren Rath geben, als Abel's Werke im Original zu lesen.

Sophus Lie.

Leipziger Ber. 49 (1897), Math.-phys. Cl., p. 242.

1) vgl. a. S. 137 (Weierstrass). 
Laplace, qui pourtant n'avait pas pris, dans ses écrits, pour modèle le célèbre géomètre de Bâle, ne cessait de répéter aux jeunes mathématiciens ces paroles mémorables que nous avons entendues de sa propre bouche: Lisez Euler, lisez Euler, c'est notre maître à tous.

G. LiBRI.

Journal des Savants 1846 , p. 51 .

[Franz] Neu mann verehrte in wissenschaftlicher Beziehung ganz besonders die Franzosen und bezeichnete geradezu einige derselben (insbesondere z. B. Fourier) als seine Lehrer. Er stellte Fourier fast neben Newton, und erzählte häufig, wie er durch das Studium von Fourier's Wärmetheorie erst in die Mathematik eingeführt worden sei.

\section{Carl Neumann.}

s. P. Volkmann, „Franz Neumann“ (1896), p. 7.

Poisson . . has done much, but he does not seem to me to have nearly so logical a mind as Cauchy, great as his talents and his clearness are: and both are in my judgment very far inferior to Fourier, whom I place at the head of the French School of Mathematical Philosophy, even above Lagrange and Laplace, though I rank their talents above those of Cauchy and Poisson.

W. R. Hanilion to John T. Graves. Observatory [Dublin], 15. X. 1833. ef. Graves, „Life of Sir William Rowan Hamilton“, Vol. 2 (1885), p. 58.

To the Memory of Fourier.

Fourier! with solemn and profound delight, Joy born of awe, but kindling momently To an intense and thrilling ecstasy, I gaze upon thy glory and grow bright: 
As if irradiate with beholden light;

As if the immortal that remains of thee

Attuned my to thy Spirit's harmony,

Breathing serene resolve and tranquil might.

Revealed appear thy silent thoughts of youth,

As if to consciousness, and all that view

Prophetic, of the heritage of truth

To thy majestic years of manhood due:

Darkness and error fleeing far away,

And the pure mind enthroned in perfect day.

W. R. Hamizín.

1. X. 1832.

ef. Graves, „Life of Sir William Rowan Hamilton",

Vol. 1 (1882), p. 596.

Gauss hat sich öfter gegen uns geäussert, dass Archimedes der Mann des Alterthums gewesen sei den er am höchsten schätze, er denke sich ihn als einen durchaus edel aussehenden würdigen Greis, nur könne er ihm nicht verzeihen, dass er bei seiner Sandrechnung das decadische Zahlensystem ${ }^{1}$ ) nicht gefunden habe. „Wie konnte er das übersehen", sagte er bewegt, „und auf welcher Höhe würde sich jetzt die Wissenschaft befinden, wenn Archimedes jene Entdeckung gemacht hätte."

Sartorius v. Waltershausen.

"Gauss zum Gedächtniss" (1856), p. 84.

Gauss äusserte sich gesprächsweise einmal, es habe nur drei epochebildende Mathematiker gegeben: Archimed, Newton, Eisenstein.

M. Cantor.

Artikel „Gotthold Eisenstein" in „Allg. Deutsche Bíogr.“, Bd. 5 (1877), p. 774.

Ihr Brief endigt mit Worten, die mir nicht gefallen können, wenn Sie sie nicht im Scherz gesagt: „Ich hoffe natürlich durch

1) lies statt dessen: „die Positionsarithmetik“. 


\section{$-285$}

die Eigenschaften, die ich an diesen Formeln entdeckt, ein zweiter Newton zu werden!!“ So etwas sagt man ja nie von sich selbst. Glücklicherweise steht es in einem Briefe an mich. Ich schreibe gewiss morgen nach Karlsruhe ${ }^{1}$ ) und werde Sie nicht einen $\mathrm{New}$ to n nennen, was den Eindruck meines ganzen Briefes vernichten würde.

A. von Humbold an Eisenstein 1847.

s. Karl Bruhns, ,Alexander v. Humboldt“, Bd. 2 (1872), p. 345/346.

Soll ich noch eine ganz unerhebliche Kleinigkeit erinnern, so ist es das, dass es mir nicht so ganz gefällt, wenn Sie in der Abhandlung [,Über die Figur des Saturns" ete., Monatl. Corresp. von Zach 15 (1807), p. 239-260] immer von sich in der mehrern Person reden. Dies ist bei anonymen Schriften, Recensionen u. s. w. gebräuchlich und schicklich: aber sonst muss ich gestehen, kömmt es mir nur bei Schriftstellern, die schon ihr Ansehen als ganz ausgemacht selbst vor der Welt betrachten dürfen, z. B. La Place, La Grange etc. recht passend vor. Auch ist man bei französischen Mathematikern mehr daran gewöhnt als bei deutschen.

\section{Olbers an Bessel.}

Bremen, 19. I. 1807.

s. Briefw. Olbers-Bessel, herausg. v. Erman, Bd. 1 (1852), p. 70.

The name of Sir Isaac Newton has by general consent been placed at the head of those great men who have been the ornaments of their species. . . . . The philosopher*, ndeed, to whom posterity will probably assign the place next to Newton, has characterized the Principia as pre-eminent above all the productions of human intellect.

* The Marquis La Place. - See Systême du Monde, p. 336.

David Brewster.

"The Life of Sir Isaac Newton" (London 1831), p. 1/2.

1) um Eisenstein für einen Lehrstuhl in Heidelberg zu empfehlen. 
L'ouvrage de Clairaut sur la forme de la terre est peut-être, de tous les écrits mathématiques composés depuis deux siècles, celui qui, par la forme sévère et la profondeur ingénieuse des démonstrations, pourrait le mieux être comparé, égalé même, aux plus beaux chapitres du Livre des principes.

\section{J. Bertrand.}

„L'Académie des sciences et les académiciens de 1666 à 1793 "

(Paris 1869), p. 263.

M. Lagrange, qui citait souvent Newton comme le plus grand génie qui eût jamais existé, ajoutait aussitôt: et le plus heureux; on ne trouve qu'une fois un systême du monde à etablir.

\section{Delambre.}

"Notice sur la vie et les travaux de M. Lagrange", Mémoires de la classe des sciences mathém. et phys. de l'Institut de France 13, 1812, Histoire de la classe mathém., p. XLVI $=$ Oeurres de Lagrange, t. 1 (1867), p. XX.

Vous venez, mon cher confrère, d'exposer des choses très-élégantes [sur la théorie des lignes de courbure à l'ellipsoide]; je voudrais les avoir faites.

Lagrange à Monge.

voir Arago, Oeurres, t. 2 (1854), p. 445

$=$ Werke, Bd. 2 (1854), p. 362.

Avec son application de l'analyse à la représentation des surfaces, ce diable d'homme [Monge] sera immortel!

\section{Lagrange.}

voir Arago, Oeurres, t. 2 (1854), p. 447/448

$=$ Werke, Bd. 2 (1854), p. 364. 
Sa Majesté [Napoleon I.], qui accueillait Lagrange toujours avec la plus noble distinction, le nommait la haute pyramide des sciences mathématiques.

Virey et Potel.

„Précis historique sur la vie et la mort de Joseph-Louis Lagrange" (Paris 1813), p. 11/12.

Tait dubbed Maxwell $\frac{d p}{d t}$, for according to thermodynamics

$$
\frac{d p}{d t}=J C M
$$

(where C denotes Carnot's function) the initials of [J. C.] Maxwell's name. On the other hand Maxwell denoted Thomson by $T$ and Tait by $T^{\prime \prime}$; so that it became customary to quote Thomson and Tait's Treatise on natural philosophy as $T$ and $T^{\prime \prime}$.

Alexander Macfarlane. "Peter Guthrie Tait, his life and works", Bibl. math. (3) 4 (1903), p. 187.

Die stete Beschäftigung mit tiefen und schweren Aufgaben verlieh Weierstrass bei seinem Genie und Scharfsinn eine Überlegenheit über andere Zeitgenossen, welche einst in einem Kreise von Mathematikern, die um ihn versammelt waren, mit folgenden Worten charakterisirt wurde: „Weierstrass hat doch etwas übermenschliches an sich. Man kann ihm nie etwas neues erzählen. Er weiss alles schon im voraus."

G. Mittag-Leffler.

„Weierstrass", Acta mathem. 21 (1897), p. 80/81.

Ich erwartete, in W. Thomson, der einer der ersten mathematischen Physiker Europas ist, einen Mann, etwas älter als ich selbst, zu finden, und war nicht wenig erstaunt, als 
mir ein sehr jugendlicher hellblondester Jüngling von ganz mädchenhaftem Aussehen entgegentrat. . . . . . . Er iibertriftt übrigens alle wissenschaftlichen Grössen, welche ich persönlich kennen gelernt habe, an Scharfsinn, Klarheit und Beweglichkeit des Geistes, so dass ich selbst mir stellenweise neben ihm etwas stumpfsinnig erscheine.

Hecmuortz an seine Frau.

Kreuzuach, 6. VIII. 1855.

s. L. Königsberger, „Hermann von Helmholtz", Bd.1 (1903), 1. 255.

Mr. Cayley, of whom it may be so truly said, whether the matter he takes in hand be great or small, „nihil tetigit quod non ornavit" - - -

\section{J. J. SYLVESTER.}

Philos. Transactions, vol. 154, part 3 (1864), 1. 605

Le talent mathématique de Cayley avait pour caractères la clarté et l'extrême élégance de la forme analytique; il était secondé par une incomparable puissance de travail qui a fait comparer l'illustre savant à Cauchy.

Hermite.

"Notice sur M. Cayley", C. R. de l'acad. des sc. de l'aris. t. 120 (1895), p. 234.

. . . ein Forscher, der schon in verhältnissmässig frühe! Zeit dem mathematischen Königreich eine neue Provinz hinzu. gefügt hat.

\section{Auwers}

von L. Fuchs in der Antwort auf dessen Antrittsrede i. d. Berl. Akademi! . s. Berl. Sitzungsber. 1884, p. 747.

Je priai un jour M. Lambert de me classer selon so 1 idée les plus célèbres géomètres vivans. „Le premier géomètı 
vivant, me répondit-il, c'est M. Euler et M. d'Alembert, ou M. d'Alembert et M. Euler: je les place au même rang, et n'en fais, pour ainsi dire, qu'un seul de tous les deux, non pas qu'ils se ressemblent en tout, mais parce que chacun d'eux a des qualités éminentes qui compensent celles de l'autre: M. Euler a plus de naïveté et de facilité, peut-être même plus d'abondance: M. d'Alembert a plus de finesse, de sagacité, et d'élégance: tous deux sont féconds et profonds au même degré: il n'est pas possible de préférer l'un à l'autre; et il faut, comme je le fais, dire de chacun d'eux qu'il est sans contredit le premier des géomètres vivans. M. de la Grange est aujourd'hui le second: j'ajoute ici le mot aujourd'hui, parce qu'il y a tout lieu de s'assurer qu'il tardera peu à les atteindre. Le troisième c'est moi: je ne porte pas cette classification plus loin, vu que je n'en vois aucun autre qui mérite d'être cité ensuite."

ThiéBault.

„Mes souvenirs de vingt ans de séjour à Berlin", t. 5 (Paris 1804), p. 31/32.

Gleich weit von Eitelkeit, und Schmeicheley entfernt urtheilte Lambert über sich, wie über andere, ohne Partheylichkeit; allein eben dieser Gewohnheit, sich als ein äusseres Subject zu behandeln, und von seinen eigenen Verdiensten, wie von seinen Fehlern, eben so entscheidend und unbefangen als von fremden zu sprechen, gab ihm oft den Schein einer ohnverzeichlichen Prahlerey bey denen, die ihn nicht kannten. Auch hieng er fest an seinen Urtheilen, und war nicht leicht davon abzubringen. - - - -

Bey seinem Aufenthalt in Amsterdam hatte er das Unglück, durch einen Fall so beschädigt zu werden, dass seine Augen von geronnenem Blute ganz schwarz worden, und er denselben und folgenden Tag über ohne Bewustseyn lag, so, dass er auch, wegen des dadurch in seiner Rechnung verlohrnen Tages, sich lange nicht wollte überreden lassen.

s. „Leben der berühmtesten vier Gelehrten unseres Philosophischen Jahrhunderts, Rousseau's, Lambert's, Haller's und Voltaire's" (Frankfurt \& Leipzig 1779), p. 43/44 u. 45/46.

Ahrens, Scherz und Ernst i. d. Mathem. 
Le professeur Euler est un homme très-peu amusant, mais un très-grand géomètre.

\author{
D'Alember'T à Voltaire. \\ Paris, 3. III. 1766.
}

voir Oeurres compl. de Voltaire, t. 69 (Gotha 1789), p. 389.

Doctissimo atque ingeniosissimo Viro Juveni Leonhardo Eulero [9. I. 1728].

Viro Clarissimo ac Mathematico longe acutissimo Leonhardo Eulero [2. IV. 1737].

Viro Celeberrimo atque longe Eximio Le on hardo Eulero [9. XII. 1739].

Viro Celeberrimo atque Excellentissimo Leonhardo Eulero [1740-1743].

Viro Incomparabili Leonhardo Eulero, Mathematicorum Principi [23. IX. 1745].

Mathematicorum Principi Le onhard o Eulero [24.V.1746]

Aus den Anreden in den Briefen JoH. Bernonlur's an Euler.

s. Correspondance mathém. et phys. de quelques célèbres géomètres du 18 ème siècle, t. 2 (1843).

Lagrange était né pour inventer et pour agrandir toute: les sciences de calcul. Dans quelque condition que la fortun. l'eût placé, ou pâtre ou prince, il aurait été grand géomètre il le serait devenu nécessairement, et sans aucun effort: $c$. qu'on ne peut pas dire de tous ceux qui ont excellé dans cett science, même dans les premiers rangs.

\title{
Fourier.
}

„Éloge historique de M. le Marquis de Laplace." voir Mém. de l'Acad. des Sc. de Paris, 10, 1831, Histoirc, p. LXXXV. 
S'il avait eu de la fortune, a dit Lagrange lui-même, il n'eût probablement pas fait son état des Mathématiques.

\section{Delambre.}

„Notice sur la vie et les ouvrages de M. Lagrange“, Mémoires de la classe des sciences mathém. et phys. de l'Institut de France, 13, 1812, Histoire de la classe mathém., p. XXXV

$=$ Oeuvres de Lagrange, t. 1 (1867), p. X.

Lagrange a été le réformateur de l'analyse. En rendant plus évidentes quelques-unes des bases de cette science, il lui a donné plus de force en même temps que par ses immortelles découvertes il en a étendu le domaine. Un de nos plus grands géomètres s'est plu à célébrer la perfection de son style analytique. Pures et faciles comme les vers de Racine, les formules de Lagrange ont augmenté le nombre des adeptes de la science en même temps qu'elles ont facilité leurs travaux.

Laplace, en appliquant aux lois de l'univers les facultés d'un géomètre de premier ordre, est devenu le législateur des mouvements célestes. Par les vastes conquêtes qu'il a faites sur la nature, il a mérité d'être appelé le Newton de la France.

Legendre, plus profond que populaire, a été notre Euler; comme Euler et à son exemple, il a légué à l'avenir une foule de ces résultats analytiques que le génie seul sait obtenir et qui enrichissent pour toujours le domaine de l'esprit humain.

\section{Élie de Beaumont.}

"Éloge historique de Legendre."

voir Mém. de l'acad. des sc., t. 32, 1864, p. LXXXVI/LXXXVII.

Lagrange, Laplace, Legendre et Monge, ont été connus de nos contemporains, et il m'a été donné plus d'une fois de les entendre juger par ceux dont ils avaient encouragé la jeunesse. M. Poinsot, dans quelques lignes finement travaillées, s'était plu à marquer les traits principaux de leur caractère et 
de leur talent, et, malgré l'injustice très-apparente envers l'un des plus illustres, il avait assez bien réussi pour que dès la première lecture on n'hésitât pas un instant sur le véritable nom des géomètres $A, B, C, D$.

$A$. Va d'un air simple à la vérité qu'il aime: la vérité lui sourit et quitte volontiers sa retraite pour se laisser produire au grand jour par un homme aussi modeste.

$B$. Ne l'a jamais vue que par surprise. Elle se cache à cet homme vain qui n'en parle que d'une manière obscure. Mais vous le voyez qui cherche à tourner cette obscurité en profondeur et son embarras en un air noble de contrainte et de peine comme un homme qui craint d'en trop dire et de divulguer un commerce secret qu'il n'a jamais eu avec elle.

$C$. Il faut bien, se dit-il, qu'elle soit en quelque lieu. Or il va laborieusement dans tous ceux où elle n'est point, et comme il n'en reste plus qu'un seul qu'il n'a pas visité, il dit qu'elle y est, qu'il en est bien sûr, et il s'essuie le front.

$D$. D'un tempérament chaud, la désire avec ardeur, la voit, la poursuit en satyre, l'atteint et la viole.

\section{J. Bertrand.}

"L'Académie des sciences et les académiciens de 1666 à 1793 " (Paris 1869), p. 294/295;

voir aussi du même „Éloges académiques", Nouvelle série (Paris 1902), p. 19/20.

Il Dirichlet era un ammiratore delle. innumerabili ed importanti Memorie pubblicate dal Jacobi in tutte le parti delle Matematiche, ed il Jacobi riconosceva il Dirichlet come una maravigliosa e penetrante intelligenza nella ricerca delle più difficili questioni matematiche, specialmente scelte nella teorica dei numeri. Il Jacobi più volte dicea che la mente del Dirichlet per le matematiche era paragonabile alla mente del Lagrange ${ }^{1}$ ), espressione superiore a qualunque elogio.

\section{B. ToRTolini.}

„G. Lejeune Dirichlet", Annali di matem. 2 (1859), p. 196.

1) s. Note 18 am Ende des Buches. 
Dirichlet gehörte nicht, wie Euler und Jacobi, zu jenen universellen Analysten, denen jeder Gedanke zur Formel sich verkörpert und jede Formel wiederum zu einem Gedanken Anlass giebt, auch nicht zu jenen unerschrockenen Rechnern, die durch ein Labyrinth von Formeln hindurch zu dem gewünschten Ziele vordringen, er war vielmehr einer jener wenigen Mathematiker, von denen gesagt werden kann, dass ihre ganze Thätigkeit ein Wirken des Gedankens sei.

BORCHARDT.

„Gustav Lejeune-Dirichlet", J. f. Math. 57 (1860), p. 91

$=$ Borchardt, Werke, p. 477/478.

Combien de fois ai-je entendu Brioschi se plaindre des mathématiciens modernes, qui n'ont plus l'habitude de faire de longs calculs, et combien de fois suis-je resté surpris en entendant des louanges pour un travail, ou pour certaine partie d'un travail, parce qu'il contenait des calculs bien faits. - - - -

Betti était d'une nature différente, je dirai même opposée à celle de Brioschi. Ce qui manque à l'un, l'autre le possède. $\mathrm{Si}$ on les avait réunis, on aurait eu un esprit complet.

Le désir d'un but à atteindre n'empêchait pas Betti de voir ce qui l'entourait et de s'attarder à mi-chemin pour trouver des rapports et des comparaisons avec bien d'autres choses.

Cette sorte de paresse toscane, qui n'est pas de la paresse intellectuelle, faisait qu'il aimait plutôt penser que travailler d'une manière mécanique.

C'est pourquoi ces longs calculs, que le rude Lombard aimait, lui étaient insupportables. Bien souvent ils l'auraient conduit à des fautes sans le flair mathématique très fin qu'il possédait. Son esprit large et cultivé aimait plutôt les systèmes philosophiques.

\section{Volterra.}

„Betti, Brioschi, Casorati.“

voir Compte rendu du deuxième congrès intern. des mathém.

Paris 1900 (Paris 1902), p. 50/51. 
Euler qu'on aurait pu appeler presque sans métaphore, et certainement sans hyperbole, l'analyse incarnée.

Arago.

Oeuvres, t. 2 (1854), p. 443

$=$ Werke, Bd. 2 (1854), p. 359.

Cette méthode d'embrasser ainsi toutes les branches des mathématiques, d'avoir, pour ainsi dire, toujours présentes à l'esprit toutes les questions et toutes les théories, était pour II. Euler une source de découvertes, fermée pour presque tous les autres, ouverte pour lui seul. ${ }^{1}$ )

\title{
Condoreet.
}

„Éloge de M. Euler."

voir Condorcet, Oeurres, Édition d'Arago, t. 3 (1847), p. 26.

Le 7 septembre 1783 . . . Euler cessa de calculer et de vivre.

\author{
Condorcet. \\ „Éloge de M. Euler." \\ voir Condorcet, Oeuvres, Édition d'Arago, t. 3 (1847), p. 41.
}

Tel jour, Poisson cessa de professer et de vivre.

ARaGo.

Oeuvres, t. 2 (1854), p. 658

$=$ Werke, Bd. 2 (1854), p. 540.

La vie n'est bonne qu'à deux choses: à faire des mathématiques et à les professer.

Poisson.

voir Arago, Oeuvres, t. 2 (1854), p. 662

$=$ Werke, Bd. 2 (1854), p. 544.

1) vgl. S. 126 (Dirichlet). 
Praecipuum docentis officium est docere discendi vias.

Отто Hesse.

These der Habilitationsschr. 1840.

s. Ges. Werke (1897), p. 714.

Praecipuum docentis munus est, docere discendi vias.

C. Neumann.

These der Habilitationsschr. Halle 1858.

M. Euler préférait l'instruction de ses disciples à la petite satisfaction de les étonner, et il croyait n'en pas faire assez pour la science, s'il n'ajoutait, aux vérités nouvelles dont il l'enrichissait, l'exposition nä̈ve des idées qui l'y avaient conduit.

D'ailleurs, peut-être ne faudrait-il pas le louer de cette candeur, quand même elle lui aurait coûté un peu de sa gloire: les hommes d'un grand génie ont rarement ces petites ruses de l'amour-propre, qui ne servent qu'à rapetisser aux yeux des juges éclairés ceux qu'elles agrandissent dans l'opinion de la multitude; soit que l'homme de génie sente qu'il ne sera jamais plus grand qu'en se montrant tel qu'il est, soit que l'opinion n'ait pas sur lui cet empire qu'elle exerce arec tant de tyrannie sur les autres hommes.

Condorcet.

„Éloge de M. Euler."

voir Condorcet, Oeuvres, Édition d'Arago, t. 3 (1847), p. 26/27.

Ich bin unter Anderm mit der Herausgabe einer deutschen Algebra beschäftigt, welche mein Vater [Leonhard Euler] gleich zu Anfang des Verlustes seines Gesichtes einem seiner Bedienten, der ein Schneider von Profession ist, dictiret und also eingerichtet hat, dass derselbe wirklich in den Stand gesetzt 
wurde, die schwersten algebraischen Aufgaben ohne alle fremde Hülfe selbst aufzulösen.

Joh. Albert Euler an Kästner.

[Petersburg], 1/12. IX., 1769.

s. R. Wolf, „Biographien zur Culturgesch. der Schweiz", Bd. 4 (1862), p. 104.

Jacobi's Vorträge zeichneten sich nicht durch diejenige Deutlichkeit aus, welche auch der geistigen Armuth oft zu Theil wird, sondern durch eine Klarheit höherer Art. Er suchte vor Allem die leitenden Gedanken, welche jeder Theorie zu Grunde liegen, darzustellen, und indem er Alles, was den Schein der Künstlichkeit an sich trug, entfernte, entwickelte sich die Lösung der Probleme so naturgemäss vor seinen Zuhörern, dass diese Ähnliches schaffen zu können die Hoffnung fassen konnten ${ }^{1}$ ).

\section{DiRICHLET.}

"Gedächtnissrede auf Carl Gustav Jacob Jacobi."

s. Abhandl. d. Berl. Akad. 1852, p. 20

$=$ Dirichlet, Werke, Bd. 2, p. 245/246

$=$ Jacobi, Werke, Bd. 1, p. 21/22.

Jamais professeur ne fut moins didactique, mais ne fut plus vivant [qu'Hermite].

\section{E. Picard.}

"L'oeurre scientifique de Charles Hermite", Acta mathem. 25 (1902), p. 107.

Monge enseignait ordinairement ce qu'il avait lui-même découvert. ......Monge ne suivait pas strictement, devant ses auditeurs, la marche qu'il s'était tracée dans le silence du cabinet; il s'abandonnait souvent à des inspirations subites; on apprenait alors de lui comment les esprits créateurs font avancer les sciences, comment les idées naissent, percent l'obscurité qui d'abord les entoure, et se développent. Dans les

1) vgl. S. 126 (0. Hesse). 
occasions dont je parle, mon expression ne sera que juste: Monge pensait tout haut.

.... Il y a toujours un grand avantage à faire professer les sciences par ceux qui les créent.

Arago.

Oeuvres, t. 2 (1854), p. $451=$ Werke, Bd. 2 (1854), p. 366/367.

Mit Hintansetzung peinlich strenger Systematik knüpfte Kronecker [in seinen Vorlesungen] völlig eigenartig an Untersuchungen an, die ihn augenblicklich beschäftigten, oder er liess sich umgekehrt durch den vorgetragenen Stoff zu eigenen, tiefsinnigen Forschungen anregen und gab dann neue, oft von gestern auf heut gefundene Resultate und Beweise. ${ }^{1}$ ) Man wäre geneigt, seine Vorlesungen „esoterische“ zu nennen.

E. NetTo.

Vorwort zu L. Kronecker, Vorlesungen I (Integrale).

In 1875 the Johns Hopkins University, at Baltimore, was founded, and the Trustees sought the advice of the president, Daniel C. Gilman, in the selection of the professorial staff. He replied „Enlist a great mathematician and a distinguished Grecian; your problem will be solved. Such men can teach in a dwelling-house as well as in a palace. Part of the apparatus they will bring; part we will furnish. Other teachers will follow them." Joseph Henry ${ }^{2}$ ) also advised that liberal salaries should be paid and the best men in the world secured. He brought Sylvester's name prominently forward, and finally the latter was offered the post of Professor of Mathematics.

P. A. M[aC Mahon].

London Roy. Soc. Proc. 63 (1898), p. XXI.

1) s. a. Frobenius, "Gedächtnissrede auf L. Kronecker", Berl. Abh. 1893, p. 18/19, sowie eine ähnliche Äusserung A. R. F[orsyth]'s über Cayley in London Roy. Soc. Proc. 58 (1895), p. XI.

2) berühmter amerikanischer Elektriker, Sekretär der ,Smithsonian Institution". 
Sylvester methods! he had none. "Three lectures will be delivered on a New Universal Algebra", he would say; then "the course must be extended to twelve". It did last all the rest of that year. The following year the course was to be "Substitution Theory, by Netto". We all got the text. $\mathrm{He}$ lectured about three times, following the text closely, but stopping sharp at the end of the hour. Then he began to think about Matrices again. „I must give one lecture a week on these", he said. He could not confine himself to the hour nor to the one lecture a week. Two weeks passed and Netto was forgotten entirely and never mentioned again.

\section{E. W. Davis}

[pupil of Sylvester at the Johns Hopkins University]. cf. London Roy. Soc. Proc. 63 (1898), p. XXII.

W. Thomson ist so schnell in seinen Gedanken, dass man ihm erst durch eine lange Reihe von Fragen, zu deren Beantwortung er schwer zu bringen ist, die nöthigen Angaben über die Einrichtung der Instrumente u. s. w. herauswinden muss. Wie seine Studenten ihn verstehen, die ihn nicht so festhalten können bei der Sache, wie ich es mir erlauben durfte, begreife ich nicht - -

H. von Helmholtz an seine Frau von Schottland aus, Frühjahr 1864.

s. Königsberger, „Hermann von Helmholtz“, Bd. 2 (1903), p. 51/52

Der mathematische [Schul-] Unterricht, so wollte Clebsch, sollte nicht mit einer Reihe schwerverständlicher abstracter Definitionen beginnen, sondern von der Anschauung ausgehen, und durch die Anschauung Interesse erwecken. Das eben war auch bei seinen späteren Universitäts-Vorträgen das Charakteristische: der Gegenstand des Vortrages erwuchs vor den Zuhörern in organischem Aufbau. Und das in seinem ganzen Wesen und Denken zu Tage tretende, selbst in seinen einzelnen 
Abhandlungen erkennbare Streben nach plastischer Darstellung und künstlerischer Abrundung gab seinen Vorträgen eine seltene Vollendung und Anziehungskraft, und verwandelte den Gegenstand des Vortrags in ein Bild von wahrhaft idealer Schönheit. Es war, im höchsten Sinne des Wortes, ein ästhetischer Genuss, seinem Vortrage zu folgen.

\section{Neumann.}

„Zum Andenken an Rudolf Friedrich Alfred Clebsch", Göttinger Nachr. 1872, p. 551/552.

Der hochberühmte Jacob Steiner ist eine Zeit lang, ich glaube 1828 mein Lehrer gewesen. Aber leider dauerte die Freude nicht lange. Denn als er merkte, dass ich durch seine geometrischen Herrlichkeiten im Grunde mehr unterhalten sein, als dieselben ernstlich studiren wollte, und besonders als ich ihn mit Aufgaben aus der Arithmetik quälte, die ihm zuwider war, gab er seine Stunden auf. - -

Jedenfalls freut es mich, dass ich einmal mit ihm in regem Verkehr gestanden habe.

. . Sein Geist besass in Bezug auf geometrische Lehrsätze eine, man möchte sagen kaleidoscopische Kraft. Das Einfachste gestaltete sich vor seinem inneren Auge zu vielseitiger harmonischer Erscheinung. Ein Sechseck wurde zu funfzehn Sechsecken, deren Ausstrahlungen sich wieder in Knotenpunkten vereinigten, und diese Punkte durch wei tere Linien verbunden bildeten neue Figuren. Die meisten seiner Sätze konnte er nur im Kopfe construiren, weil keine Zeichnung im Stande war seinen Complikationen nachzukommen. .... Es war ein hoher Genuss ihn solche Sätze entwickeln zu hören, und die Stunden, während welcher er mir in seiner unbeholfenen Sprache dergleichen Dinge vortrug, werden mir ewig unvergesslich bleiben. So unverständlich war sein Schweizerdialect damals noch, dass ich anfangs glaubte, er sei ein Franzose, und ihn bat französisch zu sprechen, weil ich ihn dann besser zu verstehen hoffte. Zu meinem Erstaunen erwiderte er, dass er gar nicht französisch 
reden könne. - Vermochte ich zuweilen seine Meinung gar nicht aufzufassen, so rief er mir halb ärgerlich, halb komisch zu: Zinkedöh! wobei er zwei Finger in die Höhe hob. Dies Wort erklärte er auf meine Frage durch Zinke deux, d. h. zwei Zinken oder Hörner, also ein Ochse!

Steiner war durchaus und in jeder Beziehung ein Original, und zwar ein solches, wie kaum alle hundert Jahre Eins geboren werden mag.

Prof. [iur.] Felix Eberty.

„Jugenderinnerungen eines alteu Berliners" (Berlin 1878),

p. 293,296 u. 297.

Überall ist in Möbius' Arbeiten das Bestreben sichtbar, seine Ziele auf kürzesten Wegen, bei geringstem Aufwand von Mitteln, durch die angemessensten Mittel zu erreichen. Nicht nur in den Ergebnissen, welche die mathematischen Wissenschaften bereichern, zeigt sich der Meister, sondern auch in der Auswahl und Handhabung der Mittel, durch welche die Sätze der Wissenschaft begründet und in Verbindung gebracht werden. Grandia parvis ist bei Werken von künstlerischer Tollendung ein besonderer Vorzug, durch welchen die von Möbius verfassten Schriftwerke sich auszeichnen; denselben Vorzug hatten auch die academischen Vorlesungen, in welchen Möbius meist auf engere Gebiete sich beschränkte und durch seine eigenthümlichen Anschauungen anregte.

R. BALtzer.

Vorrede zu A. F. Möbius, Ges. Werke, Bd. 1 (1885), p. VII.

Wenn Helmholtz der genialere und umfassendere Forscher ist, so ist Kirchhoff der geschultere Physiker und der bewährtere Lehrer; während Helmholtz immer productiv, mit immer neuen Problemen innerlich beschäftigt ist, hat Kirch h off mehr Lust und Liebe zum Lehren, seine Vorträge sind durch musterhafte Klarheit und Abrundung ausgezeichnet; er ist auch, 
um Arbeiten von Anfängern zu leiten, nach allem, was wir hören, geeigneter als Helmholtz.... Wenn nun noch die Erwägung hinzukommt, dass Kirchhoff leichter als Helmholtz zu gewinnen sein dürfte, so glaubt die Facultät berechtigt zu sein, wenn sie zunächst den Professor Kirchhoff als denjenigen namhaft machte, welcher Ew. Excellenz als G. Magnus' Nachfolger zu berufen ehrerbietigst gebeten werden.

Die philos. Facultät der Berliner Universität an den preuss. Cultusminister v. Mühler, 1870.

s. Königsberger, „Hermann von Helmholtz“, Bd. 2 (1903), p. $179 / 180$.

Kirchh off's Vortrag fesselte jeden sofort durch die äusserliche Eleganz und Präcision der Darstellung. Kein Wort zu wenig, kein Wort zu viel; nie kam ein Irrthum, eine Unklarheit, ein Schwanken im Kleinsten vor. Bewundernswerth war auch die Eleganz der Rechnung - eine dem Laien schwer zu definirende Eigenschaft. Der ganze Stoff baute sich vor dem Zuhörer in Gestalt eines ungemein kunstvollen, classisch formvollendeten logischen Fachwerkes auf, in welchem jeder Theil sich streng aus dem andern ergab, so dass es einen geradezu ästhetischen Genuss gewährte, den Kir ch h off' schen Deductionen zu folgen. Demgemäss mussten Kirchhoff's Vorlesungen, obgleich sie innerlich zu den schwierigsten gehörten, Jedem, auch dem Unbegabtesten, in sich verständlich sein, vorausgesetzt natürlich, dass er das Werkzeug, die mathematische Sprache kannte. Es konnte vorkommen und ist thatsächlich oft vorgekommen, dass einer das Gebotene nicht einzuordnen wusste, nicht begriff, warum und zu welchem Zwecke Kirchhoff gerade so und nicht anders deducirte; aber dem Gedankengange folgen, ihn nachdenken und richtig wiedergeben, musste Jeder können. So paradox es klingt, wäre es daher nicht unmöglich, ohne Kirchhoff je eigentlich verstanden zu haben, seine Vorlesung nach der Niederschrift als vorzügliches Buch herauszugeben. In dieser Eigenschaft der Kirchhoff'schen Dialektik, 
der absoluten Klarheit und Geschlossenheit in sich, lag wohl ein grosser Theil seines Erfolgs als Lehrer.

Robert von Helmholtz [Sohn Hermann v. H.'s]. „Gustav Robert Kirchhoff", Deutsche Rundschau 54

(1888), p. 236/237.

Tiele von [Franz] Neumann wesentlich durchgearbeitete Probleme, die er Schülern zur Veröffentlichung überliess, gehen unter fremden Namen, und wie seine zarte Art, den Arbeitenden zu leiten, jenen oft im Unklaren liess, was eigentlich sein eigener Antheil an dem schoenen Resultat war, so war er zu selbstlos, um den seinigen ausdrücklich bezeichnen zu lassen. ${ }^{1}$ ) Ich hörte ihn einst, ohne die mindeste Empfindlichkeit, nur gewissermassen verwundert, von einer sehr geschätzten Arbeit sagen: „Seltsam, - der Mann glaubt, das sind seine Formeln, und es sind doch die meinigen."

Es ist hiernach begreiflich, dass eine volle Würdigung der wissenschaftlichen Bedeutung Fr. Neumann's viel schwieriger ist, wie z. B. die seines grossen Schülers Kirchhoff, der, trotz seines vielleicht weiter strahlenden Ruhmes, dennoch seinen Meister bezüglich des Umfanges und der Tiefe seines Einflusses bei weitem nicht erreicht hat.

W. VoIGT.

„Zur Erinnerung an F. E. Neumann“, Göttinger Nachr. 1895. Math.-phys. Kl., p. 249.

Wenn Weierstrass in seinen Vorträgen der frïheren Zeit einmal den Faden verlor, so freuten wir uns über solche Entgleisungen; denn bei den Überlegungen, die er dann mitzutheilen pflegte, entwickelte er aphoristisch eine Menge fruchtbarer Gedanken, von denen manche durch seine Schüler zu

1) vgl. hierzu jedoch P. Volkmann, „Franz Neumann“ (1896), Vorbemerk., p. V/VI. 
Abhandlungen verarbeitet wurden. Dabei fiel es ihm nie ein, solche Gedanken als sein Eigenthum in Anspruch zu nehmen; man wird danach den Ausspruch eines seiner Schüler verstehen: Weierstrass freue sich über jeden Gedanken, der ihm gestohlen werde, wenn er denselben bei dem Entwender wieder finde.

Indem Weierstrass seine eigensten Gedanken auf seine Schüler vererbte, hatte er an ihren Arbeiten, wenn sie in seinem Sinne ausfielen, dieselbe Freude, wie ein Vater an den Erfolgen seiner Kinder.

\section{E. LAMPE.}

„Karl Weierstrass", Gedächtnisrede Berlin Phys. Ges. 5. III. 1897 , p. 21 u. 22

$=$ Deutsche Mathem.-Verein. Jahresber. 6, 1896:1897 (Leipzig 1899), p. 42.

Nichts scheint dem eigenthümlichen Geiste Weierstrass' weniger sympathisch gewesen zu sein als eine . Verschmelzung verschiedener, anscheinend heterogener Gebiete mathematischer Erkenntniss. Einheitlichkeit der Methode und der Darstellung gieng ihm ïber alles ${ }^{1}$ ); in der einheitlichen und strengen Durchführung aller Beweise lag seine Hauptstärke; in dieser Richtung hat er am nachhaltigsten auf seine Schüler und seine Zeitgenossen gewirkt. - - - - - - - - - -

Auf . Zusammenhänge auch nur hinzuweisen, war nicht Weierstrass' Sache. In diesem strengen Festhalten am einheitlichen Aufbau, im Vermeiden jeden seitlichen Ausblickes auf verwandte Gebiete, in dem fast ängstlichen Verweilen auf dem schmalen, einmal vorher abgesteckten Pfade liegt die Grösse des Weierstrass'schen Systems, vor allem bei Untersuchungen prinzipieller Natur, darin liegt aber auch die Schwäche des Systems in seiner Wirkung auf die Schüler, denen es nicht immer vergönnt sein kann, durch eigene Arbeiten den Ausblick auf andere Wege zu gewinnen, die dem gleichen Ziele der Er-

l) vgl. S. 173 (Poincaré). 
kenntniss zustreben, oder vom erreichten Gipfel Umschau ïber ein weites Gebiet zu halten.

F. Lindeniann.

Münchener Ber. 27 (1897), Math.-phys. Kl., p. 404 u. 407/408.

Weierstrass war nicht so vielseitig produktiv wie Euler und Gauss; dafür waren die Zeiten vorüber; aber innerhalb der nicht engen Grenzen, die er sich gesteckt hatte, herrschte er mit unbedingter Sicherheit. Was er veröffentlichte, drang nicht bloss bis in die innersten Kernpunkte der Wissenschaft vor, sondern es war auch absolut fertig bis auf das kleinste formale Hilfsmittel. Da war kein Symbol, kein Buchstabe, der etwa noch durch einen geeigneteren hätte ersetzt werden können. Neidlos wurde er von allen Nationen der Erde, die überhaupt Mathematik zu ehren vermögen, als der grösste Mathematiker anerkannt. . . . . In Stockholm wurde ein eigenes internationales Journal für die Weierstrasssche Schule unter dem Titel Acta mathematica gegründet.

\section{O. StTaude.}

"Die Hauptepochen der Entwicklung der neueren Mathematik", Rektoratsrede Rostock Univ. 28. II. 1902.

s. Deutsche Mathem.-Verein. Jahresber. 11 (1902), p. 290.

Während andere Disciplinen mancherlei Arbeiten erfordern, die den Bearbeitern ,aufgegeben“ werden können, und auch solche, die geradezu von vereinten Kräften geleistet werden müssen (die Astronomie bietet ja hierfür viele Beispiele), während es also fast in allen anderen naturwissenschaftlichen Disciplinen vorkonmt, dass, „wenn die Könige bauen, die Kärrner zu thun haben", muss bei uns jeder Forscher König und Kärrner zugleich sein. Darum geben wir Mathematiker eigentlich das Beispiel einer echten Gelehrtenrepublik, in welcher jeder einzelne seine volle Forscherselbständigkeit bewahrt. Ich mag auch deshalb bei uns nicht den Ausdruck "Schüler" gern; 
wir wollen und brauchen keine Schule, sondern wir gehen nur in den Wegen fort, die uns ein Lehrer oder Vorgänger geebnet und gewiesen hat, wenn wir meinen, auf diesen Wegen weitere Ziele erreichen zu können. „Wir wollen und brauchen keine Schule", weil in unserer absolut klaren Wissenschaft jede neue Entdeckung die bisherige Schulweisheit wertlos machen kann.

L. Kronecker an G. Cantor.

Jugenheim a. d. Bergstr., 18. IX. 1891.

s. Deutsche Mathem.-Verein. Jahresber. 1, 1890/1891, p. 23/24.

Ein naher persönlicher Verkehr der Studierenden nicht nur mit den Professoren, sondern auch unter einander scheint im Bereiche der Mathematik ein ganz besonders wichtiges Moment. Wo immer ich Gelegenheit hatte, die wissenschaftliche Entwickelung eines hervorragenden Mathematikers näher zu beobachten, da hat sich bestätigt, dass daran der tägliche, Jahre hindurch fortgesetzte intensive Verkehr mit Gleichstrebenden einen hervorragenden Anteil hatte.

F. Klein.

„Zur Frage des höheren mathematischen Unterrichts."

s. Verhandl. d. 1. intern. Mathem.-Congr. Zürich 1897

(Leipzig 1898), p. 302.

Das Glück eines persönlichen wissenschaftlichen Verkehrs habe ich in reichlichem Masse genossen und weiss es also aus Erfahrung zu schätzen; die etwa 20 Jahre von 1856 bis nahe 1876, in denen wir drei, Kummer, Weierstrass und ich, des engsten und lebhaftesten wissenschaftlichen Verkehrs uns erfreuten, haben nicht bloss uns selbst, sondern auch vielen andern, die $a b$ und zu an unserem Verkehr teilnahmen, reiche Früchte und den Segen wahrer geistiger Erbauung gebracht.

L. Kronecker an G. Cantor.

Jugenheim a. d. Bergstr., 18. IX. 1891.

s. Deutsche Mathem.-Verein. Jahresber. 1, 1890/1891, p. 24. 
Wer einmal mit einem oder einigen Männern ersten Ranges in Berührung gekommen ist, dessen geistiger Maassstab ist für das Leben verändert; zugleich ist solche Berührung das Interessanteste, was das Leben bieten kann.

\section{H. Henmholtz.}

„Über die akadem. Freiheit der deutschen Universitäten", Rectoratsrede Berlin Univ. 15. X. 1877.

s. Vorträge u. Reden, Bd. 2 (1884), p. 207.

A Leyde Lambert eut une avanture plaisante avec Musschenbroek, et il me semble qu'on peut aisément se représenter cette scene comme une des plus risibles. Le Professeur déjà blanchi dans son métier, en recevant la visite de M. Lambert, crut que c'étoit l'hommage d'un Écolier, ou tout au plus d'un Commençant. Il se mit done à l'endoctriner, et à lui dire des choses communes auxquelles les connoissances de M. Lambert étoient fort supérieures. Celui-ci lui répondit avec ce ton ferme et cette volubilité qu'il avoit à son commandement; et ayant bientôt fait perdre terre au bon homme, les Interlocuteurs changerent de personnage, Lambert fut le Maître et Musschembroek le disciple.

FORMEY.

„Éloge de MI. Lambert.“

voir Histoire de l'acad. roy. de Berlin, 1778, p. 80.

Olbers hat in gelegentlichen Mittheilungen wohl geäussert. er betrachte es als sein Hauptverdienst um die Astronomie dass er Bessel dafür gewonnen.

ENCKE.

"Gedächtnissrede auf Bessel."

s. Berl. Abhandl. 1846, p. XXVII

Was die rein wissenschaftliche Thätigkeit anbetrifft, sı steht ihr die allgemeine Ermüdung des Geistes, daher dir 
Häufigkeit logischer Irrthümer und Schwerfälligkeit des Herauswindens aus wirklichen oder vermeintlichen Widersprüchen entgegen. Ich bin über pflichtmässige Unterrichtsthätigkeit äusserst froh und bemühe mich, meine mathematische Hartknochigkeit und Routine auf andere zu übertragen. Ich trachte immer im Unterricht so frisch und unmittelbar als möglich zu sein und, wenn es mir gelingt in dieser Thätigkeit mich aufzubrauchen, so will ich mich für glücklich halten.

\section{LUDWIG SCIILÄFLI}

an seinen Freund, den Chemiker Hugo Schiff.

s. J. H. Graf, „Ludwig Schläfli“,

Bern. Mittheil. Naturf. Gesellsch. 1895, p. 147.

Ich muss meine Zeit theilen zwischen Collegia lesen (wogegen ich von jeher einen Widerwillen gehabt habe, der, wenn auch nicht entstanden, doch vergrössert ist durch das Gefühl, welches mich immer dabei begleitet, meine Zeit wegzuwerfen) und praktisch astronomische Arbeiten.

Göttingen, 14. III. 1824.

Wenn ich meinen Kopf voll von theoretischen Untersuchungen habe, spannt mich immer das Heranrïcken der Stunden, wo ich Collegia zu lesen habe, auf die Tortur, und das Abspringen in den Ideen macht mir dann zuweilen die an sich erbärmlichsten Dinge unbeschreiblich schwer und angreifend. Ich habe viel schoenes gefunden, aber mitunter Monate lang auf einem Problem vergeblich zugebracht.

Göttingen, 12. III. 1826.

Gauss an Bessel.

8. Briefw. Gauss-Bessel (1880), p. $428 / 429$ u. 458 . 
Schellbach war das Lehren der Mathematik Religion: „Auch die geistig Armen sollten“, wie er sich auszudrücken pflegte, „Teil haben an dem Himmelreich.“

Felix Müller.

„Carl Heinrich Schellbach“, Gedächtnisrede 29. X. 1892

(Berlin 1893), p. 28.

Lud wig Sc heeffer hatte keine Neigung für die Universitätskarriere, da er die Einseitigkeit der Mathematik fürchtete, obgleich er andererseits sich aus vollster Überzeugung dieser Wissenschaft gewidmet hatte. Er machte daher . . . das Examen pro facultate docendi . . . und absolvirte sein pädagogisches Probejahr..... Während der Zeit keimte bei ihm das Bewusstsein, dennoch zum höheren [akademischen] Lehrfache berufen zu sein - - -

\section{G. Cantor.}

„Ludwig Scheeffer (1859-1885). Necrolog", Bibl. mathem. (2) 1885 , p. 197.

D'Alembert dans ses écrits mathématiques manque d'élégance et de clarté. Comment ce savant universel, nourri aux études classiques, habile à disserter spirituellement et avec éloquence sur les sujets les plus divers, cet écrivain célèbre pour la vigueur et la précision de son style, perd-il son habileté et sa grâce en rédigeant ses belles découvertes? Je hasarderai une explication. D'Alembert à aucune époque de sa vie n'a voulu être professeur.

Joseph Bertrand.

„D'Alembert" (Paris 1889), p. 55

Ich habe die Paedagogik immer als meine starke Position angesehen, als die am wenigsten angreifbare Seite meiner eigener Thätigkeit. Gewisse Erfolge, die ich in paedagogischer Hinsich 
errungen habe, liegen äusserlich greifbar zur Hand, und es stört mich wenig, wenn jemand hinterher sagen wollte: die Erfolge sind da, aber sie sind durch verkehrte Methode errungen.

Felix Klein.

„Über Aufgabe und Methode des mathematischen Unterrichts an den Universitäten", Vortr. Naturf.-Vers. Düsseldorf 1898.

s. Deutsche Mathem.-Verein. Jahresber. 7, 1897/1898, p. 129.

Ferien, Ferien! in meiner eignen Stube genossen! Wenn die Stunde schlägt, keine, keine Collegia! Du grosser Himmel! das geht über alles. Bin ich ausser Göttingen, da weiss ich, dass es keine Collegia giebt, aber hier, hier in loco, das zu fühlen, ist wie eine Brautnacht.

\section{LICHTENBERG}

an A. Chr. Fr. Amelung (Fabrikbesitzer im Braunschweigischen). Göttingen, 26. III. 1787.

s. Lichtenbergs Briefe, herausg. v. Leitzmann u. Schüddekopf, Bd. 2 (1902), p. 296.

Eigenschaften des Gemüths wie des Geistes trugen in völlig gleichem Masse dazu bei, „dem alten Kummer" unter den Studenten eine Zuneigung zu gewinnen, die zuweilen bis zur Schwärmerei ging. . . . . Neben mir, auf den Bänken des grossen Hörsaals im Castanienwäldchen, wo der greise Mathematiker sein Colleg über Zahlentheorie las, sass ein Commilitone - jetzt selbst schon angesehener Professor der Mathematik auf einer sïddeutschen Hochschule - der zu sagen pflegte: „Für den alten K u m mer könnt' ich einen Spiegelsextanten nebst Wasserwage verschlucken." - _ - - -

Der alte Kummer verschmähte es auch keineswegs, seinen Vortrag mit guten alten Humoresken zu würzen, und es kann nicht geläugnet werden, dass Hörer, die eines seiner Collegs zweimal hörten, auch diese Scherze, zum Theil wenigstens, zweimal genossen. $\Lambda$ ber so arm war Kummer nicht an guten Einfällen, dass er sein Repertoire nicht erneuern konnte, und 
nicht so eitel, all die Anekdoten, die er meisterhaft vortrug, jedesmal vor seinen Zuhörern auszuschütten. . . . . es gehörte zu den Momenten heiterster Erfrischung, die je in einem mathematischen Colleg vorgekommen sein werden, wenn der etwas steifbeinige alte Herr uns pantomimisch veranschaulichte, wie d'Alembert dem wissbegierigen König [Friedrich d. Gr.] das Princip der virtuellen Geschwindigkeiten vordemonstrirte.

O. N.-H.

„Eduard Kummer †“, Münchener Allg. Zeitg. Nr. 139 (20. V. 1893)

$=$ Zeitschr. math. naturw. Unterr. 24 (1893), p. 310-311.

Wer diesen Factor unbeachtet lässt, ist einem Menschen zu vergleichen, der eine Pflaume isst und den Kern verschluckt, während er das Fleisch ausspuckt.

Vorlesungs-Ausspruch ron E. E. Kunmer.

s. E. Lampe, „Nachruf für Ernst Eduard Kummer“, Deutsche Mathem.-Verein. Jahresber. 3, 1892/1893, p. 18

$=$ Naturw. Rundschau 8 (1893), p. 363.

Tout entier . . aux développements d'une théorie difficile, il arriva à Ampère, dans le feu de la démonstration, de prendre le torchon saupoudré de craie pour son mouchoir. Le récit, grossi, amplifié, de cette méprise, assurément bien innocente, se transmit de promotion en promotion; et quand A mpère paraissait pour la première fois devant une d'elles, ce n'était plus le savant analyste qu'elle cherchait de préférence: elle guettait plutôt le moment où il l'égaierait par la distraction, dès longtemps promise, et dont elle était très-peu disposée à le tenir quitte.

Arago.

Oeuvres, t. 2 (Paris 1854), p. 33

$=$ Werke, Bd. 2 (Leipzig 1854), p. 29. 
It cannot be said that he [Sylvester] was a great success at Woolwich [at the Royal Military Academy] as a teacher, being too far beyond his pupils who, for the most part, regarded mathematics as an irksome duty. He had the reputation of being eccentric and irritable. When not actually engaged in teaching, the mind of Sylvester would occasionally become abstracted from earthly affairs, and it is stated that on one occasion he suddenly looked up from a paper in the hall of study and demanded of the corporal on duty, "What year is it?" An explosion of laughter in the room led to a "scene", and the subsequent infliction of many punishments upon the cadets.

The sight of Sylvester leaving his house pursued by his landlady carrying his collar and necktie is said to have been not an unusual one. He came into collision on more than one occasion with the authorities at the Academy and with the War Office.

$$
\text { P. A. M[ac Mahon]. }
$$

London Roy. Soc. Proc. 63 (1898), p. XVIII/XIX.

Als Weierstrass 1856 in Berlin seine Vorlesungen begann, waren dieselben zwar durch Originalität, durch Tiefe der Gedanken und durch den Reichthum an Gesichtspunkten gleich ausgezeichnet; aber er hatte als Vortragender noch nicht die ruhige Sammlung gewonnen, durch welche die übersprudelnden Ideen für den Hörer in übersichtliche Entwickelungen zu bringen waren. In seinem freien Vortrage versah er sich leicht und war dann genöthigt, in der nächsten Vortragsstunde ganze Partien der vorangehenden Vorlesung zurückzunehmen und neu darzustellen. Dazu kam ein anderer, ihn belästigender Umstand. Wenn er beim Schreiben dicht vor der Tafel stand, so schienen sich ihm die Buchstaben an der Tafel zu bewegen, und damit war der Beginn von Schwindelanfällen gegeben. Gegen diesen unangenehmen Einfluss schützte er sich nach den Erfahrungen der ersten Jahre seit 1862 dadurch, dass er einen Zuhörer zum Schreiben an der Tafel 
benutzte, ${ }^{1}$ ) während er selbst, in einiger Entfernung sitzend, den Vortrag hielt und zugleich den Anschreiber überwachte.

E. LANiPE.

„Karl Weierstrass", Gedächtnisrede Berlin Phys. Ges. 5. III. 1897 , p. $19 / 20$

$=$ Deutsche Mathem.-Verein. Jahresber. 6, 1896/1897 (Leipzig 1899), p. 40/41.

Der mündliche Vortrag verursachte Riemann in den ersten Jahren seiner akademischen Lehrthätigkeit grosse Schwierigkeiten. Seine glänzende Denkkraft und rorahnende Phantasie liess ihn meist, was besonders bei zufälligen mündlichen Unterhaltungen über wissenschaftliche Gegenstände zum Vorschein kam, sehr grosse Schritte nehmen, denen man nicht so leicht folgen konnte, und wenn man ihn zu einer näheren Erörterung einiger Zwischenglieder seiner Schlüsse aufforderte, so konnte er stutzig werden und es verursachte ihm einige Mühe, sich in den langsameren Gedankengang des Anderen zu fügen und dessen Zweifel rasch zu beseitigen. So hat ihn auch bei seinen Vorlesungen die Beobachtung der Mienen seiner Zuhörer ... oft empfindlich gestört, wenn er, bisweilen ganz gegen sein Erwarten, sich genöthigt glaubte, einen für ihn fast selbstverständlichen Punkt noch besonders zu beweisen. Dies hat sich aber nach längerer Übung verloren - - -

H. WeBER.

„Bernhard Riemann's Lebenslauf."

s. Riemann, Werke, herausg. v. H. Weber, 2. Aufl. (1892), p. 550/551.

Arago avait l'habitude, quand il montait en chaire, de chercher dans l'auditoire le visage qui lui semblait le moins intelligent; il ne le quittait plus, semblait ne parler que pour

1) An der zweiten der angegebenen Stellen lautet das Folgende: "während er selbst, in einiger Entfernung bequem sitzend, mit seinem in der Hand gehaltenen Schlüsselbunde oder auch mit seinem Spazierstocke den Vortrag in nachdrücklichen Bewegungen begleitete und zugleich den Anschreiber überwachte." 
lui et continuait sa démonstration, en la variant, jusqu'au moment où ce visage montrait à des signes certains que son propriétaire avait compris; à fortiori, tous les auditeurs devaient être dans le même cas, et le professeur pouvait continuer; quelquefois ce propriétaire naïf, heureux de l'attention dont il avait été l'objet, venait remercier, sans deviner la cause d'un si grand honneur.

\section{JAMIN.}

„François Arago“, Revue scientifique (3) 9 (1885), p. 263.

Briot se fâchait volontiers; il n'avait aucun égard pour la dignité (déjà chatouilleuse) des élèves de l'École normale; les injures tombaient dru sur celui qui répondait mal, ou ne répondait pas; il bondissait de sa chaise, le secouait rudement par le bouton de la redingote et lui tirait les oreilles.

Plus que personne, il a contribué à nettoyer l'enseignement; on n'a plus idée, aujourd'hui, des horreurs qui se débitaient sous le nom de mécanique; il les a balayées. Sa méthode, dans l'enseignement de la mécanique, était nettement géométrique..... cet homme irrespectueux traitait de "faribole" la Mécanique analytique de Lagrange.

... il s'intéressait au travail de ses élèves, d'abord, mais aussi à tous les détails de leur vie. Il envoyait chez le coiffeur l'un de mes meilleurs amis qui avait la déplorable habitude d'oublier, pendant de longs mois, que ses cheveux étaient hors de toute mesure. Un jour, celui-ci, par esprit de pénitence, sans doute, était allé de lui-même chez ce maudit coiffeur; pendant que, revêtu du costume traditionnel, essayant d'échapper par le rêve aux parfums maussades qui l'envahissaient, il livrait aux ciseaux alertes et bruisants sa tête mélancolique, Briot, qui passait dans la rue, entra brusquement dans la boutique pour le féliciter.

\section{Jules Tannery.}

voir „Le centenaire de l'école normale" (Paris 1895), p. 392 et 393 ("L'enseignement des mathématiques à l'École"). 
Ob ich mich Steiners erinnere? Fr steht ${ }^{1}$ ) in aller Deutlichkeit vor mir, als hätt' ich ihn gestern gesehen, auf und abschreitend auf dem schmalen Raum zwischen Katheder und erster Bank (die nicht meine Bank war) und dabei Rechenaufgaben diktierend. . . . . Was sich mir am tiefsten eingeprägt hat, ist das, dass sich in seinem ganzen Wesen eine gewisse Resignation aussprach, eine leichte Schwermut darüber, sich mit einem, an ihm gemessen, so minderwertigen Material abquälen zu müssen. Vielleicht waren Einige von Talent unter uns, aber was wollte das sagen! Aufs Ganze hin angesehen, stand ein Aristoteles vor abc-Schützen. - - - - - -

Ich eroberte mir von einem bestimmten Zeitpunkt ab sein Interesse. Meine grandiose Nicht-Beanlagung für alles, was mit Zahlen zusammenhängt, das gänzliche Fehlen jedes Organs für das, was er vortrug, machte zuletzt einen rührenden und doch auch wieder erheiternden Eindruck auf ihn, und wenn er mich dann, amüsiert über meine blöden Antworten, schelmisch anblinzelte, so lag darin jedesmal ein bestimmtes Mass von Anerkennung, etwa wie wenn er sagen wollte: „das lass ich mir gefallen, den lob ich mir, der ist in seiner Art vollendet.“ Th. Fontane an Prof. J. Lange.

31. I. 1898.

s. J. Lange, „Jacob Steiners Lebensjahre in Berlin" (Berlin 1899), p. 41.

Steiner's Vorträge erheischten ein sehr fleissiges Mitarbeiten der Zuhörer und wurden deshalb nicht von vielen andauernd besucht, abgesehen davon, dass Steiner's Gewohnheit, mitten im Winter die Fenster des Hörsaales aufzureissen, ihm manchen Schüler abspenstig machte.

\section{Cantor.}

Artikel „Jakob Steiner" in „Allg. Deutsche Biogr.", Bd. 35 (1893), p. 703.

1) als Oberlehrer an der Berliner "Gewerbeschule", der späteren "Friedrichs-Werderschen Oberrealschule". 
In der Wochenrechnung von Steiner befand sich ein Posten für Heizung des Zimmers, bemessen nach der vom sparsamen Mieter genau vorgeschriebenen Anzahl der jedesmal zu verwendenden Stücke Holz und Torf. Nun argwöhnte Steiner, dass die Wirtin in seiner Abwesenheit eine geringere Anzahl wirklich verwendete. Um sie auf frischer That zu ertappen, ersann er folgenden Plan. Pohlke, ein anhänglicher Schüler und enthusiastischer Bewunderer des grossen Geometers, sollte sich von diesem in den Kleiderschrank des Zimmers einschliessen lassen und durch ein Astloch in der Thüre des Schrankes die Wirtin beim Heizen beobachten. Dessen weigerte sich Pohlke, weil er es einerseits nicht für anständig hielt, auf diese Weise den ungesehenen Denunzianten zu spielen, und weil er andererseits mit seiner langen Figur nicht die Qual der gebückten Stellung in einem verschlossenen Schranke stundenlang aushalten wollte. Diese Weigerung führte dann zur Entzweiung des Meisters mit seinem Schüler.

E. Lampe.

„Zur Biographie von Jacob Steiner", Bibl. mathem. (3) 1 (1900), p. 137.

Einen jungen Studenten, der in Nachahmung des grossen Geometers sich nur mit synthetischer Geometrie beschäftigen wollte, zankte Steiner deswegen tüchtig aus; es solle nicht jeder meinen, es ihm gleich thun zu können, oder mit einer Lieblingswendung: es werden nicht alle, die zu mir Herr, Herr sagen, ins Himmelreich kommen. Pflicht des Studenten sei es, etwas Ordentliches zu lernen; besonders riet er das Studium der Zahlentheorie an, die zur Schärfung des Geistes vornehmlich geeignet sei.

E. LAMpe. „Zur Biographie von Jacob Steiner", Bibl. mathem. (3) 1 (1900), p. 134. 
Von solchen, die bisher im Ministerium [als Nachfolger Dirichlet's in Berlin] genannt worden sein sollen, wären Kummer (Breslau), Weierstrass (in Braunsberg), der grosse Heine (in Bonn). .... In Crelle finden sich Aufsätze vom besagteu Weierstrass; eliminiren Sie ihn.

\section{Steiner an L. Schläfli.}

12. VI. 1855.

s. J. H. Graf, „Der Briefwechsel zwischen Jakob Steiner und Ludwig Schläfli“, Bern Naturf. Ges. Mitth. 1896, p. 247/248.

Wie mir Weierstrass einmal erzählte, hatte er seinem Freunde und Kollegen Jacob Steiner kurz vor dessen Tode versprochen, er wollte dafür sorgen, dass die neuere synthetische Geometrie an der Universität Berlin nicht verwaise. Da nach Steiners Tode niemand da war, der das Fach übernehmen wollte, so arbeitete Weierstrass sich selbst hinein, und hielt mehrere Jahre hindurch ab und zu ein Kolleg über neuere synthetische Geometrie. Im Seminar regte er oft zu andern als funktionentheoretischen Studien an.

\section{SCHUBERT.}

„Zum Andenken an Karl Weierstrass †“,

Rede Hamburg Mathem: Gesellsch. 1. III. 1897.

s. Zeitschr. math. naturw. Unterr. 28 (1897), p. 230 [dort angegeben als abgedruckt aus dem "Hamburger Correspondent"].

Richelot hatte mit seinen Vorlesungen einen so durchschlagenden Erfolg, dass er bereits im September 1832 Extraordinarius wurde, - noch keine zehn Jahre, nachdem ihm (20. Februar 1821) in einer Decanatsprüfung das eben nicht verheissungsvolle Zeugniss gegeben war, „das seine mathematischen Kenntnisse mittelmässig waren, indem er vieles aus früherer Zeit Erlernte wieder vergessen hat."

H. Prutz.

„Die Königliche Albertus-Universität zu Königsberg i. Pr. im 19. Jahrh." (Königsberg 1894), p. 163. 
[Examinateur et Candidat.]

[Legendre:] Comment vous appelez-vous?

[Arago:] Arago.

[Legendre:] Vous n'êtes donc pas Français?

[Arago:] Si je n'étais pas Français, je ne serais pas devant vous, car je n'ai pas appris qu'on ait été jamais reçu à l'École [polytechnique] sans avoir fait preuve de nationalité.

[Legendre:] Je maintiens, moi, qu'on n'est pas Français quand on s'appelle Arago.

[Arago:] Je soutiens, de mon côté, que je suis Français, et très-bon Français, quelque étrange que mon nom puisse vous paraître.

[Legendre:] C'est bien; ne discutons pas sur ce point davantage, et passez au tableau.

[Legendre:] La méthode que vous suivez ne vous a pas été donnée par le professeur. Où l'avez-vous prise?

[Arago:] Dans un de vos Mémoires.

[Legendre:] Pourquoi l'avez-vous choisie? Était-ce pour me séduire?

[Arago:] Non, rien n'a été plus loin de ma pensée. Je ne l'ai adoptée que parce qu'elle m'a paru préférable.

[Legendre:] Si vous ne parvenez pas à m'expliquer les raisons de votre préférence, je vous déclare que vous serez mal noté, du moins pour le caractère.

voir Arago, Oeuvres, t. 1 (1854), p. 10/11 $=$ Werke, Bd. 1 (1854), p. 8/9.

Im August [1798] wurde ich Doctor der Philosophie. . . . Nachmittags war das mündliche Examen über Physik, Geschichte und Philologie. In der Pause ergötzte es mich, wie 
meine Examinatoren über die Geringfügigkeiten des Lebens im steifen Tone der Zunftgelehrten sich unterhielten, wo unter Anderem Hindenburg [der Mathematiker] an den zeitigen Decan Beck $^{1}$ ), der ein kleines Landgut besass, die Frage richtete: „Sind bei Eurer Spectabilität die Kartoffeln gut gerathen?“

K. F. BURdACH.

"Blicke ins Leben“, Bd. 4, "Selbstbiographie des Verfassers" (Leipzig 1848), p. 60.

Man chicanirte mich anfangs hier mit der Doctorwürde, die ich, wie Sie wissen, nicht besitze. Indess erklärte ich, dass ich als königlicher Professor der Astronomie über diese Wissenschaft lesen könne, ohne zu einer Facultät zu gehören . . . . . . Späterhin hatte einer der neuen Professoren die alten so gestimmt, dass sie mir, wie ich wohl merkte, ihr Diplom mittheilen wollten; allein es schien mir, dass ich es nach der Taxe bezahlen sollte, und da liess ich mir denn merken, dass jeder Preis mir zu hoch sein würde. ..... Wenn man mich ferner mit dieser Sache belästigen sollte, so nehme ich vielleicht meine Zuflucht zu Ihnen, und bitte Sie, mir dort das Diplom zu verschatfen, welches bei Ihrem Einflusse, und da man mich dort kennt, wohl keine Schwierigkeiten haben kann.

Besser an Gauss.

Königsberg, 26. VIII. 1810.

Mit dem allergrössten Vergnügen eile ich, Ihnen lieber Bessel anzuzeigen, dass Ihre Promotion jetzt vollzogen ist.

Gauss an Bessel.

Göttingen, 6. IV. 1811.

s. Briefw. Gauss-Bessel (1880), p. 123 u. 145.

1) Chr. Dan. Beck, Prof. „graccarum et latinarum litterarum" in Leipzig. 
Als der verstorbene Ellendt des Altst. Gymnasiums zum Kolloquium $^{1}$ ) zu dem Mathematiker Jakoby [C. G. J. Jacobi] kam - denn damals wurden die Akte der Prüfungskommission noch alle in der Behausung jedes einzelnen Mitgliedes vorgenommen, sagte er: So! ich soll mit ihnen kolloquiren. Nun - zunächst nehmen Sie einmal eine Cigarre. Hierauf Cigarren und Konversation. Endlich: Doch um nun auf unser Geschäft zu kommen: Können Sie mir die Versicherung geben dass Sie als Direktor für den mathematischen Unterricht am Gymnasium so viel thun werden, als in Ihren Kräften steht? Bejahung. Nun dann sind wir fertig. Solcher Verstand existirt nun freilich gewiss in den Prüfungskommissionen nicht mehr. - -

Lehrs $^{2}$ ) an seinen Freund C. F. W. Müller.

Königsberg, 1. VI. 1872.

s. „Ausgew. Briefe von und an Chr. A. Lobeck und K. Lehrs", herausg. v. Arthur Ludwich, Th. II (Leipzig 1894), p. 881.

A la fin de 1829, Galois se présenta, pour la seconde fois, à l'École polytechnique. Il ne fut pas jugé capable d'y entrer. Après les travaux importans qu'il avait déjà faits, jl lui était permis de croire à une admission certaine. - - -

Auguste Chevalier.

Revue encyclopédique 55 (1832), p. 746.

Wer weiss! wer weiss! die Seele des Pythagoras ist vielleicht in einen armen Kandidaten gefahren, der durch das Examen fällt, weil er den pythagoraeischen Lehrsatz nicht beweisen konnte, während in seinen Herren Examinatoren die Seelen jener Ochsen wohnen, die einst Pythagoras, aus

1) zwecks Erlangung einer Direktor-Stelle am Gymnasium.

2) s. S. 129. 
Freude ïber die Entdeckung seines Satzes, den ewigen Göttern geopfert hatte.

Heinrici Heine.

"Reisebilder I: Norderney".

s. Werke, z. B. 12-bändige Ausg. (Leipzig, Gustav Fock), Bd. 5, p. 68/69.

Da der Examinandus [J. Steiner] die Prüfung in den alten Sprachen und den historischen Disziplinen ganz verbeten hatte, als gänzlich unkundig dieser wissenschaftlichen Zweige, so blieb zunächst nur die Mathematik als einziger Gegenstand des Examens übrig. . . . . Die von dem Examinandus gelieferte Abhandlung [,Über Bildung, Verbindung und Summierung der Elementarzahlenreihen"] zeichnete sich aus durch Gründlichkeit und Genauigkeit und gab von des Verfassers Erfindungskraft und Scharfsinn einen erfreulichen Beweis, indem der Gegenstand auf eine ganz eigentïmliche Weise behandelt worden ist.

... Mit der Elementargeometrie war er zwar wohl bekannt, aber fast gänzlich fehlte ihm die Kenntnis der höheren Analysis, obgleich sein Aufsatz das Gegenteil vermuten liess. Auf Differenzial- und Integralrechnung konnte er sich fast gar nicht einlassen, ja sogar die Kenntnis der Algebra scheint bei ihm nicht über die Auflösung der Gleichungen vom zweiten Grade zu gehen, wenigstens wie ihm dieselbe gegenwärtig war. Ebenso hatte er selbst in der ebenen Trigonometrie keine Fertigkeit, und die sphärische fehlte ihm gänzlich.

Protokoll der Kgl. wissenschaftl. Prüfungs-Commission

\section{zu BerLin}

vom 9. VI. 1821.

s. J. Lange, „Jacob Steiners Lebensjahre in Berlin“

(Berlin 1899), p. 7. 
Bequem, wie der alternde Geometer [Steiner] war, und unbeholfen in der Abfassung von Schriftstücken, entzog er sich gern den kleinen amtlichen Geschäften. Mehrere von uns [Studenten] mussten zum Zwecke der Erlangung oder des weiteren Bezuges von Benefizien Dekanatszeugnisse beschaffen, die auf Grund einer von einem Professor angestellten Prüfung über den erfolgreichen Besuch der Vorlesung des Professors ausgefertigt werden. Da Steiner seine Zuhörer ziemlich genau kannte, so bedurfte es im Grunde keiner Prüfung bei ihm. Um ihm nun aber auch die unangenehme Mühe des Schreibens zu ersparen, war einer von uns auf einen Ausweg verfallen, dessen die anderen, welche in gleicher Lage waren, sich dann mit Vergnügen bedienten. Wir schrieben uns nämlich selbst das Zeugnis, indem wir uns nur das Notwendigste aus den zu erfüllenden Bedingungen bescheinigten. Dieses Schriftstück legten wir ihm in seiner Wohnung zur Unterschrift vor. Nun galt es, den alten Herrn, der unseren Kniff sehr wohl durchschaute, dahin zu bringen, seinen Namen unter die vorgelegten Zeilen zu setzen. Ehe er das that, hielt er uns eine derbe Strafrede über die Unverschämtheit einer solchen Zumutung, über unsere Faulheit während des Semesters, über die Geringfügigkeit unseres Wissens, über die Bedrängnis, in die wir sein Gewissen brächten; zuletzt aber, wenn alles, was ihn bewegte, herausgesprudelt war, wobei er listig mit halb zugekniffenen Augen uns anschaute, drückte man ihm die Feder in die Hand, und er unterzeichnete, um den lästigen Dränger los zu werden.

\section{E. LAMPE.}

"Zur Biographie von Jacob Steiner", Bibl. mathem. (3) 1 (1900), p. 136.

Dirichlet gratulire ich, wenigstens schriftlich, nicht, da er's über's Herz hat bringen können, mir auch bei diesem Anlass [Geburt von Dirichlet's erstem Kind] nicht ein A hrens, Scherz und Ernst i. d. Mathem. 
Wort zu schreiben, er hätte doch wenigstens schreiben können: $2+1=3$.

Abraham Mendelssohn [Schwiegervater Dirichlet's] an seine Tochter Rebecka Dirichlet.

London, 9. VII. 1833.

s. S. Hensel, „Die Familie Mendelssohn", Bd. 1

(Berlin 1884), p. 340/341.

On accusait Poinsot de négligence et de paresse. C'était un faux jugement. - -

Poinsot écrivait peu et ne lisait guère; il aimait la méditation. Quand un visiteur inconnu frappait à sa porte, le valet de chambre lui demandait son nom et, sans prétexter que Monsieur fût sorti, revenait bientôt déclarer qu'il ne pouvait pas recevoir. - - - - - - - - - -

Lui seul peut-être pouvait dire aux savants les plus illustres de son époque: „Je vous ignore“, et marcher auprès d'eux en restant leur égal. Il a vu naître les plus grandes: découvertes $\mathrm{du}$ siècle et les a tenues dans l'indifférence. Ni li théorie des ondes lumineuses, ni celle de la polarisation, ni l'électricité dynamique, ni la théorie mathématique de la chaleur, ni celle de l'élasticité, ni les propriétés des fonctions imagi naires et des fonctions doublement périodiques n'ont pu, mêm pour un jour, captiver son attention.

\section{J. Bertrand}

„Eloges académiques", Nouvelle série (Paris 1902), p. 1 et 2\%.

A mpère lisait beaucoup, toutes sortes de livres, particulièr. ment l'Encyclopédie, d'un bout à l'autre. Rien n'échappait à sa curiosité d'intelligence; et, une fois qu'il avait conçu, rien $\mathrm{n}$ ว sortait plus de sa mémoire. Il savait done, et il sut toujour", entre autres choses, tout ce que l'Encyclopédie contenait, y compris le blason. Ainsi son jeune esprit préludait à cetle universalité de connaissances qu'il embrassa jusqu'à la fin. S'l 
débuta par savoir au complet l'Encyclopédie du XVIII ${ }^{\circledR}$ siècle, il resta encyclopédique toute sa vie..... . ses derniers travaux sont un plan d'encyclopédie nouvelle [„Essai sur la philosophie des sciences, ou Exposition analytique d'une classification naturelle de toutes les connaissances humaines"].

SaInte-Beuve.

„M. Ampère“, Revue des deux mondes (4) 9 (1837), p. 392.

La partie la plus essentielle du vaste plan de notre confrère ${ }^{1}$ ) était un projet de classification de toutes les connaissances humaines. . . . . Ses deux points de vue principaux, ses deux règnes, sont l'étude du monde, la cosmologie, l'étude de la pensée, l'ontologie.

Les sciences cosmologiques se divisent, à leur tour, en deux sous-règnes, savoir les sciences qui traitent des objets inanimés, et les sciences qui envisagent seulement les objets animés. Le premier sous-règne des sciences cosmologiques donne lieu à deux embranchements: les sciences mathématiques, les sciences physiques. En poursuivant cette division toujours par deux, Ampère n'arrive à rien moins qu'à former un tableau où l'ensemble des sciences et des arts se trouve disposé

En deux règnes,

En quatre sous-règnes;

En huit embranchements,

En seize sous-embranchements;

En trente-deux sciences du premier ordre,

En soixante-quatre du second ordre,

En cent vingt-huit du troisième ordre.

Cent vingt-huit sciences! Voilà done ce qu'il faudrait étudier pour être au fait de l'ensemble des connaissances humaines! .... A mpère n'est arrivé à trouver cent vingt-huit sciences distinctes dans les résultats des travaux accumulés de quarante siècles, qu'en dépeçant, qu'en morcelant ce qu'on avait jusqu'ici laissé réuni; qu'en transformant en sciences

1) s. das vorhergehende Citat. 
séparées de simples chapitres des sciences actuelles; qu'en leur appliquant des noms qui ont trouvé plus d'un contradicteur, tels que canolbologie, cybernetique, terpnognosie, technesthétique, etc., etc.

Arago.

Oeuvres, t. 2 (Paris 1854), p. 75 et 78/79

$=$ Werke, Bd. 2 (Leipzig 1854), p. 61 u. 64/65.

Belesenheit und Nachsuchen ist nicht meine Stärke.

Göttingen, 13. I. 1839.

Vollständigkeit in [litterarischer] Erudition ist niemals meine Liebhaberei gewesen oder ich habe dazu nie die erforderliche Ausdauer gehabt.

Göttingen, 16. IX. 1846.

Gauss an Schumacher

s. Briefw. Gauss-Schumacher, Bd. 3 (1861), p. 220

u. Bd. 5 (1863), p. 205.

Unter den ältern Mathematikern giebt es verschiedent Sorten von Menschen, ein trivialer Satz, der aber doch vieles erklärt. Mein lieber Freund Kummer z. B. hat in der Zeit. wo er seine ganze Kraft an die Auffindung der Beweise fü die höheren Reciprocitätsgesetze setzte, sich nicht und nachher, nachdem er sich daran erschöpft, erst recht nicht mehr un das, was auf mathematischem Gebiete geschehen ist, gekümmert; er verhält sich, wenn nicht ablehnend, doch gleichgültig da gegen. Wenn Du ihm sagst, die Euclid'sche Geometrie fuss' auf einem unbewiesenen Grundsatze, so giebt er Dir das zu. von dieser Einsicht ausgehend aber nunmehr die Frage so zu stellen: Wie gestaltet sich denn die Geometrie ohne dieser: Grundsatz? Das ist seiner Natur zuwider, die darauf ge richteten Bemühungen und die daran sich reihenden allgemeinen von dem empirisch Gegebenen oder Angenommenen sich lo: 
machenden Untersuchungen sind ihm müssige Speculationen oder gar ein Gräuel. Kronecker ist anders, er macht sich mit allem Neuen rasch bekannt, sein leichtes Auffassungsvermögen befähigt ihn dazu, aber es geschieht nicht in eindringender Weise; er besitzt nicht die Gabe, sich mit einer guten fremden Arbeit mit dem gleichen wissenschaftlichen Interesse wie mit einer eigenen Untersuchung $\mathrm{zu}$ beschäftigen. ${ }^{1}$ )

Dazu kommt ein Mangel, der sich bei vielen höchst verständigen Menschen, namentlich bei denen semitischen Stammes findet, er besitzt nicht ausreichend Phantasie (Intuition möchte ich lieber sagen $)^{2}$ ) und es ist wahr, ein Mathematiker, der nicht etwas Poet ist, wird nimmer ein vollkommener Mathematiker sein. Vergleiche sind lehrreich: Der allumfassende auf das höchste, das Ideale gerichtete Blick zeichnet Abel vor J acobi, Riemann vor allen seinen Zeitgenossen (Eisenstein, Rosenhain), Helmholtz vor Kirchhoff (obwohl bei dem letztern kein Tröpfchen semitischen Blutes vorhanden) in ganz eclatanter Weise aus.

\section{WeIERstrass an S. Kowalevski.}

27. VIII. 1883.

s. Mittag-Leffler, "Une page de la vie de Weierstrass", Compte rendu du deuxième congrès intern. des mathém. Paris 1900 (Paris 1902), p. 148-150.

Von den vielen Geistesgaben, welche Helmholtz zu Theil geworden waren, war vielleicht die wichtigste der innere Zwang und die Fähigkeit, ein jedes Problem in der einfachsten Weise $\mathrm{zu}$ erfassen und auf das zweckmässigste durchzudenken und von allen Seiten zu betrachten, bis seine Lösung, sei es vollständig, sei es wenigstens für eine Reihe von Fällen zu einem klaren gesicherten Abschluss gekommen war. Diese Begabung verlieh den Forschungen von Helmholtz die eminente Klarheit, mit der er jederzeit zielbewusst seine grossen Arbeiten durchführte. Dazu half ihm eine ganz ausserordentliche mathe-

1) s. Note 19 am Ende des Buches.

2) vgl. S. 148 (Felix Klein). 
matische Befähigung. Gegen Ende seiner Studienzeit reichten seine Kenntnisse kaum weiter, als wie sie etwa dem Werk von Navier über Differentialrechnung entsprechen und dennoch las er bald darauf mit grosser Leichtigkeit schwierige Abhandlungen von Poisson. Durch eigenes Arbeiten erreichte er bei der Behandlung mathematisch-physikalischer Probleme eine Höhe, wie nur wenige Mathematiker und mathematische Physiker, sowohl in der ihm freilich stets nur als Mittel dienenden formalen Behandlung, als auch in der Conception weittragender allgemeiner Fundamentalsätze.

G. WiEdEMANN.

„Hermann von Helmholtz' wissenschaftliche Abhandlungen." s. Helmholtz, „Wissensch. Abhandlungen“, Bd. 3 (1895), p. XVI/XVII.

Nach dem Mittagessen, zu einer Zeit, wo andere Leute gern schlafen, lag Hermann [Helmholtz] auf dem Sofa, und erinnere ich mich beispielsweise, dass er auf diese Art Jacobis Lehre von den elliptischen Funktionen studirt hat.

Отто Helnholtz [Bruder Hermann's].

s. S. S. Epstein, „Hermann von Helmholtz als Mensch und Gelehrter" (Stuttgart etc. 1896), p. 21

$=$ Deutsche Revue, herausg. v. R. Fleischer, $21_{2}$

(April 1896), p. 37.

Chasles lisait fort peu....... Il s'élevait très haut dans la science et aimait qu'on le suivit, mais il ne suivait personne.

\section{J. Bertrand.}

„Éloges académiques", Nouvelle série (Paris 1902), p. 51.

Cayley was singularly learned in the work of other men, and catholic in his range of knowledge. Yet he did not read a memoir completely through: his custom was to read only 
so much as would enable him to grasp the meaning of the symbols and understand its scope. The main result would then become to him a subject of investigation: he would establish it (or test it) by algebraical analysis and, not infrequently, develop it so as to obtain other results. This faculty of grasping and testing rapidly the work of others, together with his great knowledge, made him an unvaluable referee; his services in this capacity were used through a long series of years by a number of societies to which he almost was in the position of standing mathematical adviser.

A. R. F[ORSYTH].

London Roy. Soc. Proc. 58 (1895), p. XI/XII.

Sylvester seemed incapable of reading mathematics in a purely receptive way. Apparently a subject either fired in his brain a train of active and restless thought, or it could not retain his attention at all. To a man of such a temperament, it would have been peculiarly helpful to live in an atmosphere in which his human associations would have supplied the stimulus which he could not find in mere reading. The great modern work in the theory of functions and in allied disciplines, he never became acquainted with. . . . . . . . . What would have been the effect if, in the prime of his powers, he had been surrounded by the influences which prevail in Berlin or in Göttingen? It may be confidently taken for granted that he would have done splendid work in those domains of analysis, which have furnished the laurels of the great mathematicians of Germany and France in the second half of the present century.

\section{Fabian FrankLin}

"Address commemorative of Professor Sylvester." ef. Johns Hopkins Univ. Circulars 16 (1897), p. 54. 
Die kritisch sichtende Thätigkeit hat viel dazu beigetragen, dass den Deutschen, von welchen sie vornehmlich geïbt wird, die Führerschaft in der Mathematik zufiel.

E. Czuber.

„Aphorismen zur Entwickelungsgeschichte der Mathematik im neunzehnten Jahrhundert",

Rectoratsrede Wien Techn. Hochsch. 13. X. 1894.

s. Zeitschr. math. naturw. Unterr. 26 (1895), p. 614.

Quant aux sciences nous ne manquons ni de physiciens ni de mécaniciens; mais le goût de la géométrie ne prend pas encore. J'ai beau dire à mes concitoyens qu'il faut des successeurs à Leibniz, il ne s'en trouve point.

Frédéric Le Grand ${ }^{1}$ ) à d'Alembert 28. I. 1773.

voir Oeurres de Frédéric le Grand, t. 24

(Édition Decker 1854), p. 594.

Die Deutschen haben vermöge ihrer Unpartheiligkeit und Neigung, die Wahrheit anzuerkennen, wo sie auch zu finden seyn mag, so wie vermöge ihrer Beharrlichkeit und ihrer Vorliebe für das Ergründen, gerade für die Mathematik einen vorzüglichen Beruf; wie es auch die Geschichte dieser Wissenschaft beweiset.

\section{A. L. Crelle.}

Vorrede zu Bd. 1 des J. f. Math. (1826), p. 1.

Ich meines Theils .. nehme alle wahren wissenschaftlichen Fortschritte, die jenseit des Rheins und jenseit des Canals gemacht werden, freudig auf, aber wenn sie drüben dumme Streiche machen . . . . ., so ist dabei weiter nichts zu thun, als eben gar keine Notiz davon nehmen - - Gauss an Schumacher.

Göttingen, 17. VIII. 1836.

s. Briefw. Gauss-Schumacher, Bd. 3 (1861), p. 108.

1) vgl. S. 124 (Friedrich d. Grosse). 
Eine der merkwürdigsten Thatsachen auf dem Gebiete der mathematischen Literatur ist die weitgehende Trennung, die zwischen den englischen Publicationen auf der einen Seite und denen der übrigen europäischen Nationen andererseits besteht, und die sich in den letzten Decennien, wenn wir von ganz modernen Ansätzen absehen, eher verschärft als gemildert hat.

\section{F. KLEIN.}

Vorwort zu Routh, „Die Dynamik der Systeme starrer Körper“, Bd. 1 (Leipzig 1898), p. III.

Die Engländer, bei denen bis vor Kurzem aus Pietät gegen ihren grossen Landsmann Newton die Mathematik völlig still stand, haben sich neuerdings eng an Deutschland angeschlossen und Gelehrte, wie Hamilton, Cayley, Sylvester, Salmon u. s. w., sind auf das Lebhafteste in der Wissenschaft thätig.

\section{Hermann Hankel.}

„Die Entwickelung der Mathematik in den letzten Jahrhunderten“, Akadem. Vortrag Tübingen 29. IV. 1869, 2. Aufl.

(Tübingen 1884), p. 22.

It is a curious fact that, although the United Kingdom has possessed some of the very greatest of pure mathematicians in the second half of this century, the subject has there received but a scant share of attention as compared with that which it has found in France, in Germany, in Italy, in Sweden and Norway, or in the United States. I am not oblivious of the magnificent contributions to other parts of our science made alike by British leaders and British followers; their fame is known to the world. But apathy rather than attention has been the characteristic feature of our attitude towards pure mathematics; and it seems to me a misfortune, alike for the intellectual activity of the nation and for the progress of the subject, that 
English thought has had relatively so small an influence upon its vast modern developments.

A. R. Forsyti.

Address Toronto 1897.

ef. Report of the 67 th meeting of the British Assoc. for the advancement of Science, p. 543.

Mr. Tait kennt hier [in dem Badedort St. Andrews] nichts anderes als golfing. Ich musste gleich mit, die ersten Schläge gelangen mir, nachher traf ich entweder nur die Erde oder die Luft. Tait ist eine eigenthümliche Art von Wildem Mann, lebt hier, wie er sagt, nur für seine Muskeln, und erst heute am Sonntag, wo er nicht spielen durfte, aber auch nicht in die Kirche ging, war er zu vernünftigen Gegenständen zu bringen. ... Wir hatten Dinner bei Professor Brown, bei dem noch ein grosser Mathematiker Sylvester einquartiert war, der von Mr. Gladstone sehr schlecht behandelt worden ist, worüber grosse Aufregung unter den Herren herrschte - - - -

W. Thomson hat die Freiheit des Umganges jetzt so weit getrieben, dass er stets sein mathematisches Heft mit sich führt, und sobald ihm etwas einfällt, mitten in der Gesellschaft zu rechnen anfängt, was man allgemein mit einer gewissen Ehrfurcht betrachtet. Wie wäre es, wenn ich die Berliner auch daran gewöhnte? Am nairsten aber fand ich es, dass er sich am Freitag die Gesellschaft auf seine Yacht eingeladen hatte und dann, sobald das Schiff auf seinem Curse war, und sich jeder einen gegen Schwankungen möglichst gesicherten Platz auf dem Deck gesucht hatte, in die Cajüte verschwand, um dort zu rechnen, während sich die Gesellschaft, soweit sie noch Lust dazu hatte, wechselseitig unterhalten mochte, natürlich nicht gerade sehr lebhaft.

\section{H. HeLmholtz an seine Frau \\ von Schottland aus, Herbst 1871.}

в. Königsberger, „Hermann von Helmholtz“, Bd. 2 (1903), p. $197 / 198$ u. 199. 
Die Bibliothek des grossen Physikers Cavendish war mir [1790 bei einem Aufenthalt in England] geöffnet unter der Bedingung, dass, wenn er das Unglück hätte, mir dort zu begegnen, ich ihn nie begrüssen noch weniger anzusprechen wagen sollte. Der sonderbare Mann ahndete nicht, dass 1810 ich im Institut seine Stelle als einer der 8 Associés étrangers einnehmen würde.

Al. Humboldt an Christian Carl Josias Freiherr v. Bunsen. Berlin, 20. II. 1854.

s. „Briefe von Alexander von Humboldt an Christian Carl Josias Freiherr von Bunsen" (Leipzig 1869), p. 178.

Grass-mann (quere, is his Christian name Nebuchadnezzar?) - - - -

A. De Morgan to W. R. Hamilton. Camden Town, 29. X. 1852.

I have recently been reading (and it is curious that sometimes, when otherwise in mental activity, I seem to myself unable to read a page, or almost a sentence of German) more than a hundred pages of Grassmann's Ausdehnungslehre, with great admiration and interest. Previously I had only the most slight and general knowledge of the book, and thought that it would require me to learn to $s m o k e^{1}$ ) in order to read it. If I could hope to be put in rivalship with Des Cartes on the one hand, and with Grassmann on the other, my scientific ambition would be fulfilled! But it is curious to see how narrowly, yet how completely, Grassmann failed to hit off the Quaternions.

W. R. Hamilton to A. De Morgan. Observatory [near Dublin], 31. I. 1853.

I am not quite so enthusiastic to-day about Grassmann as I was when I last wrote. But I have read through nearly all of what $I$ could procure of his writings, including a

1) s. die nächste Seite. 
subsequent commentary (in German) by Moebius. Grassmann is a great and most German genius; his view of space is at least as new and comprehensive as mine of time - - -

W. R. Hamilton to A. De Morgan. Dublin, 2. II. 1853.

If you have any curiosity to know anything of the result of my recent Nebuchadnezzarological reading (my daughter looking over my shoulder is amused at the folly of philosophers), it will be quite consistent with my humour to inform you. To the public I am likely to say but little at present about Grassmann; for I find, that beyond the rule for adding lines, which he seems to have indepedently worked out, whereas I took it from Warren, we have scarcely a result in common, except one thing which is (in my view) important, namely the interpretation of $\mathrm{B}-\mathrm{A}$, where $\mathrm{A}$ and $\mathrm{B}$ denote points, as the directed line $\mathrm{AB}$.

W. R. Hamilton to A. De Morgan. Observatory [near Dublin], 9. II. 1853.

I did not at first see the Nebuetc. logical pun, though I thought of Grass, and feeding with the beasts of the field. ${ }^{1}$ ) .... I should like to hear about Grassmann - whom I am not likely to read. Between ourselves, I am disappointed with Germans - almost always. I have a new theory - take it. German intellect is an excellent thing, but when a German product is presented it must be analysed. Most probably it is a combination of intellect $(I)$ and tobacco-smoke $(T)$. Certainly $I_{3} T_{1}$ and $I_{2} T_{1}$ occur; but $I_{1} T_{3}$ is more common, and $I_{2} T_{15}$ and $I_{1} T_{20}$ occur. In many cases metaphysics occurs $(M)$; and I hold that $I_{a} T_{b} M_{c}$ never occurs without $\mathrm{b}+\mathrm{c}>2 \mathrm{a}$.

N.B. - Be careful, in analysing the compounds of the three, not to confound $T$ and $M$, which are strongly suspected

J) De Morgan forgot that the joke on the name was his own. Supra, p. 425. [Note of the editor Graves]. 
to be isomorphic. Thus, $I_{1} T_{3} M_{3}$ may easily be confounded with $I_{1} T_{6}$. As far as I dare say anything, those who have placed Hegel, Fichte etc., in the rank of extenders of Kant have imagined $T$ and $M$ to be identical.

A. De Morgan to W. R. Hamilton. Camden Town, 12. II. 1853.

cf. Graves, „Life of Sir William Rowan Hamilton", Vol. 3 (1889), p. 425, 441, 442, 444, 446.

Ich habe mich über Hamilton geärgert, dem ich über seine neue Methode in Dynamics ein Compliment machte, die das wichtigste ist, was seit 100 Jahren in England mathematisches gemacht worden, und der vornehm thun wollte, und mir sagte, er hätte sie schon wieder vergessen.

C. G. J. JACOBI an H. C. Schumacher 1849.

s. Briefw. Gauss-Schumacher, Bd. 6 (1865), p. 55.

As regards my old researches in Physical Astronomy, .....Jacobi, very early, did me the honour to take them up as a commentator: and, of course, as an enlarger - nil fere non tetigit; nil tetigit, quod non ornavit.

W. R. Hamilton to Dr. Ingram.

14. VIII. 1857.

cf. Graves, „Life of Sir William Rowan Hamilton“, Vol. 3 (1889), p. 89.

Wer wird der eigentliche Erfinder des lenkbaren Luftschiffes sein? Maxim ist es heute noch nicht. Nur derjenige wird es sein, der in der That in willkürlich gewählter Richtung, so lange ein grösserer Kraftvorrat reicht (etwa eine Stunde lang) mit und gegen den Wind in der Luft zu fliegen vermag. Diese Erfindung ist noch nicht gemacht; noch wäre es Zeit, dass wir den Engländern den Rang ablaufen. Freilich, 
durch Grossartigkeit der Mittel können wir es nicht; Maxims Maschine soll über 300000 Gulden gekostet haben. Aber wie so manches hat der Deutsche schon mit kleinen Mitteln durch die Feinheit seiner Ideen geleistet! Wer möchte dies hier in Wien bezweifeln, wo die Zauberflöte, die neunte Symphonie und die Missa solemnis geschrieben worden? Das sollen sie uns nachmachen in der ganzen übrigen Welt, wenn sie's können!

\section{Boltzmann.}

„Über Luftschifffahrt", Vortrag Naturf.-Vers. Wien 1894. s. Zeitschr. math. naturw. Unterr. 25 (1894), p 615/616

Ich weiss sehr wohl dass das Land [der U. S.] die eigentliche Zukunft der civilisirten Menschheit repräsentirt, und dass es eine grosse Zahl interessanter Menschen einschliesst, während wir in Europa das Chaos oder die russische Weltherrschaft immer näher rücken sehen $-\ldots$

H. von HeLmholtz vor seiner amerikanischen Reise an seinen Freund Knapp in New-York.

20. VI. 1893.

s. Königsberger, „Hermann von Helmholtz", Bd. 3 (1903), p. 72.

Unsere öffentlichen Zustände werden immer düsterer. Ich weiss nicht, welcher Philosoph es war, der die Lehre aufstellte, schlimme Zeiten solle man weder betrauern noch verlachen, sondern verstehen. ${ }^{1}$ ) Ich gestehe, das erste Verbot ist sehr schwer zu erfüllen, aber noch schwerer ist die Ausführung des dritten Gebots. Zuweilen scheinen mir die Recht zu haben,

1) Spinoza, Tractatus politicus, Cap. I, § IV (s. Opera, herausg. v. J. van Vloten u. J. P. N. Land (1882), 1, p. 282): „ut ea, quae ad Politicam spectant, eadem animi libertate, qua res Mathematicas solemus, inquirerem, sedulo curavi, humanas actiones non ridere, non lugere, neque detestari, sed intelligere" - - 
die da glauben, nicht bloss die Paulskirche ${ }^{1}$ ), sondern fast ganz Deutschland sei ein grosses Tollhaus geworden.

Gauss an Schumacher.

Göttingen, 17. V. 1849.

s. Briefw. Gauss-Schumacher, Bd. 6 (1865), p. 24.

La politique occupe une place considérable dans la vie d'un grand nombre des savants modernes. .... Rien n'est plus connu que les infortunes et la fin lamentable de Bailly et de Cond or cet pendant la tourmente révolutionnaire ........ Fourcroy, député à la Convention, membre du Conseil des Cinq-Cents, devient membre du Conseil d'Etat sous l'Empire; Laplace, ministre de l'Intérieur en Brumaire, puis président du premier Sénat, se retrouve Pair de France sous la Restauration; Monge, membre du Comité de Salut public et ministre de la marine en 1793, est plus tard transformé en sénateur impérial, il reçoit le titre de comte et possède un blason avec des armoiries. ..... Arago, après avoir été, comme député, membre influent de l'opposition sous le règne de Louis-Philippe, se laisse acclamer, en 1848, membre du Gouvernement provisoire, et ne considère pas comme trop lourde pour ses épaules la tâche de diriger deux ministères: celui de la Guerre et celui de la Marine.

\section{VALSON.}

„La vie et les travaux d'André-Marie Ampère" (1886), p. 342/343.

Mon ami, prenons une prise de tabac; demain je serai privé de ce plaisir, puisque j'aurai les mains attachées derrière le dos.

\section{BAILLY}

à son neveu la veille de son exécution [12. XI. 1793]. voir Arago, Oeuvres, t. 2 (1854), p. 406

$=$ Werke, Bd. 2 (1854), p. 329.

1) In der Paulskirche zu Frankfurt a. M. tagte 1848/1849 bekanntlich die deutsche Nationalversammlung. 
Captis .. Syracusis Marcellus machinationibus Archimedis multum ac diu victoriam suam inhibitam senserat. Eximia tamen hominis prudentia delectatus, ut capiti illius parceretur edixit, paene tantum gloriae in Archimede servato quantum in oppressis Syracusis reponens. At is dum animo et oculis in terra defixis formas describit, militi, qui praedandi gratia domum inruperat strictoque super caput gladio quisnam esset interrogabat, propter nimiam cupiditatem investigandi quod requirebat nomen suum indicare non potuit, sed protracto manibus pulvere 'Noli', inquit, 'obsecro istum disturbare', ac perinde quasi neglegens imperii victoris obtruncatus sanguine suo artis suae liniamenta confudit.

Valerius Maximus.

Memorabilia, Liber VIII, C. 7, Ext. \& 7.

Euler quitta Pétersbourg pour se rendre à Berlin, où le roi de Prusse l'avait appelé. Il fut présenté à la reine mère: cette princesse se plaisait dans la conversation des hommes éclairés, et elle les accueillait avec cette familiarité noble qui annonce dans les princes les sentiments d'une grandeur personnelle, indépendante de leurs titres, et qui est devenue un des caractères de cette famille auguste. Cependant, la reine de Prusse ne put obtenir de M. Euler que des monosyllabes; elle lui reprocha cette timidité, cet embarras qu'elle croyait ne pas meriter d'inspirer: Pourquoine voulez-vous donc pas me parler, lui dit-elle? Madame, répondit-il, parce que je viens d'un pays où, quand on parle, on est pendu.

\section{CONDORCET.}

„Éloge de M. Euler".

voir Oeuvres de Condorcet, Édition d'Arago, t. 3 (1847), p. 6.

Friedrich [d. Gr.] ernannte Lambert zum Rath bein Generaldirectorium im Fache des Bauwesens ...... und als Lambert sich .. nach dem. Generaldirectorium begab um 
sich hier installiren zu lassen, sprach er ganz unumwunden zu den Ministern: „Neine Herren, ich hoffe nicht dass Sie von mir erwarten, ich werde Ihre kleinlichen Rechnungen über Bauwesen durchsehen: das ist eine Arbeit die jeder Commis machen kann und hiesse meine Zeit verschwenden. Wenn Ihnen jedoch etwas vorkömmt was Sie in Verlegenheit setzt, dann schicken Sie es mir nur zu und ich will es gern zu lösen suchen. Ich glaube Ihnen dies voraussagen zu müssen, damit Sie nicht wähnen, ich könnte eine Stelle annehmen, deren Obliegenheiten mich zu Ihren Schreibern herabsetzen würden.

Thiḱbault.

„Friedrich der Grosse" [Deutsche Ausg. der „Souvenirs de Vingt ans de séjour à Berlin"], Th. 2 (Leipzig 1828), p. 171/172;

s. a. Franz. Ausg., t. 5 (Paris 1804), p. 33.

Je ne connais de M. Lambert qu'un seul ouvrage, qui est bon, mais qui ne me parait comparable à aucun de ceux de M. Euler; et si ce dernier est à genoux devant M. Lambert, comme V. M. me fait l'honneur de me l'écrire ${ }^{1}$ ), il faudra dire de M. Euler ce qu'on a dit de La Fontaine, qu'il fut assez bête pour croire qu'Ésope et Phèdre avaient plus d'esprit que lui. Ce n'est pas que je prétende rien ôter au mérite de M. Lambert, qui doit être très-réel, puisque toute l'Académie en juge ainsi; mais il y a dans les sciences plus d'une place honorable, comme il y a, si on en croit l'Évangile, plusieurs demeures dans la maison du Père céleste; et M. Lambert peut être très-digne d'occuper une de ces places. On assure d'ailleurs qu'il a fait plusieurs excellents ouvrages, qui ne me sont point parvenus. Je le trouverais encore assez bien partagé, quand il serait à M. Euler (pour parler mathématiquement) . . . . . . .

1) s. das Citat S. 350/351, wo es heisst: „jusqu'à M. Euler, toute l'Académie.."; bekanntlich schliesst ,jusqu'à" ein, während ein „bis auf" mit Ausschluss durch, „à M. Euler près" zu geben gewesen wäre. Lambert's Urteil über Euler und sich selbst s. übrigeus S. 288/289.

Ahrens, Scherz und Ernst i. d. Mathem. 
en même proportion que Marc-Aurèle et Gustave-Adolphe sont à un monarque que je n'ose nommer.

D'Alembert à Frédéric le Grand.

Paris, 1. III. 1765.

voir Oeuvres de Frédéric le Grand, t. 24 (Édition Decker 1854), p. 394.

A l'intérieur, le ministre $Q u$ inette fut remplacé par Laplace, ${ }^{1}$ ) géomètre du premier rang; mais qui ne tarda pas à se montrer administrateur plus que médiocre; dès son premier travail, les consuls s'aperçurent qu'ils s'étaient trompés: Laplace ne saisissait aucune question sous son vrai point de vue; il cherchait des subtilités partout, n'avait que des idées problématiques, et portait enfin l'esprit des infiniment petits dans l'administration.

NAPOLÉon I.

"Mémoires pour servir à l'histoire de France, écrits à Sainte-Hélène, sous la dietée de l'empereur",

t. 1, dieté au général Gourgaud (Londres 1823), p. 111/112.

Nous avons enterré M. Laplace: c'est une des grandes gloires de moins. La haine politique qu'on lui portait parce qu'il n'avait aucune élévation de caractère et courait toujours au secours du plus fort, a fait moins sentir sa perte ici. C'est une injustice cependant.
A. Нumboldt à son frère „Bill“ [Wilhelm].
Paris, 12. III. 1827.

voir „Briefe Alexander's von Humboldt an seinen Bruder Wilhelm“ (Stuttgart 1880), p. 150.

Auf unsern grossen Laplace soll die Wiederkunft des furchtbaren Despoten mächtig gewirkt haben. Er hatte seine Sprache etwas stark geändert, wie der „Essai philos. sur les

1) nach Einführung der Konsularregierung, 9. Nov. 1799. 
probabilités" zeigt, und man hat mir erzählt, dass er die Dedication der "Théorie des Probabilités" an Napoleon cassirt und eine andere an Ludwig XVIII. habe drucken lassen ${ }^{1}$ ).

Olbers an Bessel.

Bremen, 26. I. 1816.

s. Briefw. Olbers-Bessel, herausg. v. Erman, Bd. 2 (1852), p. 12.

I. de Laplace a eu l'honneur de présenter ses ouvrages [,L'exposition du système du monde"] à S. A. I. C'est la récompense de ses longs travaux, d'avoir, Madame, le bonheur de les déposer aux pieds d'une Princesse distinguée par les grâces de son esprit.

Comtesse de Laplace ${ }^{2}$ ) à Élisa Napoléon.

\section{VI. 1808.}

voir "Lettres de Madame de Laplace à Élisa Napoléon“, réunies .... par P. Marmottan (Paris 1897), p. 123.

$\mathrm{Au}$ précieux avantage d'avoir toujours la paix au-dedans, M. de la Grange joignit celui d'avoir la paix au-dehors. Jamais il n'a été accessible à aucune sorte d'intrigue ou d'esprit de parti; et si quelquefois il $\mathrm{y}$ a eu de légères divisions dans l'académie, il y a toujours été étranger, et même il a paru les ignorer. Ce n'est pas qu'on pût l'intimider. Je me souviens que M. le ministre de Sch. ........ ayant fait adopter au roi un projet de caisse pour les veuves, et M. d e la Grange nous ayant lu à l'académie, un Mémoire où il démontroit que cette caisse finiroit nécessairement pour une banqueroute assez prompte, le ministre fit dire à l'académicien, qu'au lieu de publier ce Mémoire, il auroit dû le remettre au gouvernement; sur quoi ce dernier répondit, $1^{\circ}$. qu'il n'avoit point rendu son Mémoire

1) Die erste Ausg. der "Théorie analytique des probabilités" (Paris 1812) trägt die Widmung "A Napoléon-le-Grand", die zweite (1814) ist ohne Widmung, ebenso übrigens auch die dritte (1820).

2) Die Gattin des berühmten Mathematikers war „dame d'honneur" von Napoleon's Schwester Élisa. 
public, et qu'il s'étoit contenté de remplir un devoir d'amitié, en avertissant ses confrères du danger qu'il y auroit pour eux à s'intéresser à ce projet; et $2^{\circ}$. que n'ayant pas été engagé pour être aux ordres des ministres, il ne se croyoit point tenu d'aller faire antichambre chez eux, pour leur offrir des lumières qu'ils ne lui demandoient pas; que c'étoit à eux à choisir les personnes auxquelles ils vouloient s'en rapporter pour les calculs dont ils avoient besoin; et qu' enfin il ne pouvoit mériter aucun reproche, tant qu'on n'avoit pas recours à lui. Cette réponse... réduisit M. de $\mathrm{Sch}$........ au silence.

Thiébault.

„Mes Souvenirs de vingt ans de séjour à Berlin", t. 5 (Paris 1804), p. 40/41.

Il me semble qu'il y auroit ici en ce moment une acquisition digne du Roi de France...... Lillustre la Grange, le premier géometre qui ait paru depuis $\mathrm{Newton}$, et qui, sous tous les rapports de l'esprit et du génie, est l'homme de l'Europe qui m'a le plus étonné, la Grange, le plus sage, et peut-être le seul philosophe vraiment pratique qui ait jamais existé, recommandable par son inperturbable sagesse, ses moeurs, sa conduite de tout genre, en un mot l'objet du plus tendre respect $\mathrm{du}$ petit nombre d'hommes dont il se laisse approcher, est depuis vingt ans à Berlin, où il fut appellé dans sa premiere jeunesse par le feu Roi, pour remplacer Euler, qui l'avoit désigné ${ }^{1}$ ) lui-même comme le seul homme capable de marcher sur sa ligne Il est très-mécontent; il l'est en silence,

1) An der Berufung Lagranges nach Berlin hat Euler jedenfalls keinerlei Anteil gehabt, vielmehr teilt er in einem - Berlin, 3. V. 1766 datierten Brief Lagrange mit, dass er, von der russischen Kaiserin mit der Wiederherstellung der russischen Akademie beauftragt, in erster Linie Lagrange als Akademiker für Petersburg in Vorschlag gebracht habe, und bedauert dann später (Petersburg, 20. I. 1767), dass Lagrange den Ruf nach Russland nicht angenommen habe (s. Oeuvres de Lagrange, t. 14 (1892), p. $208-210$ ). 
mais il l'est irrémédiablement, parce que c'est du mépris que sont nés ses dégoûts Les fougues, les brutalités, les folles jactances de M. de Hertzberg ${ }^{1}$ ), l'association de tant d'hommes auprès desquels la Grange ne peut avec pudeur rester assis, la crainte très-sage de se trouver pressé entre le repos philosophique qu'il regarde comme le premier des biens, et le juste sentiment $d u$ respect de lui-même, qu'il ne laissera pas blesser, tout le convie à se retirer d'un pays où rien n'absout du crime d'être étranger, et où il ne supportera pas de n'être pour ainsi dire qu'un objet de tolérance.

„Histoire secrète de la cour de Berlin“, t. 2 (1789), Lettre LI (28. XI. 1786), p. $97 / 98$.

[Le Ministre X.:] Monsieur, êtes-vous véritablement des nôtres?

[Fresnel:] Si j’ai bien compris, Monseigneur, je répondrai qu'il n'existe personne qui soit plus dévoué que moi à l'auguste famille de nos rois et aux sages institutions dont la France lui est redevable.

[Le Ministre:] Tout cela, Monsieur, est trop vague; nous nous entendrons mieux avec des noms propres. A côté de quels membres de la Chambre siégeriez-vous, si vous deveniez député?

[Fresnel:] Monseigneur, à côté de Camille Jordan²), si j’en étais digne.

[Le Ministre:] Grand merci de votre franchise - [et le lendemain un inconnu obtint la place demandée par Fresnel].

voir Arago, Oeuvres, t. 1 (1854), p. 182

$=$ Werke, Bd. 1 (1854), p. 146.

1) Delambre ("Notice sur la vie et les ouvrages de M. Lagrange", Histoire de la classe des sc. math. et phys. de l'institut de France 13, année 1812, p. LXI/LXII) erklärt auf grund zuverlässiger Information diese Motivierung von Lagrange's Fortgang von Berlin für völlig unwahr und die Beschuldigung des Ministers Hertzberg für ganz unbegründet.

2) Camille Jordan, 1771-1821, Grossvater des gleichnamigen Nathematikers, bekannter Politiker, Freund u. Landsmann von A.-M. Ampère. 
Monge conservait les habitudes révolutionnaires à une époque où tout le monde les répudiait; on rappelle, par exemple, qu'à l'École normale, en 1794, dans les séances qui portaient le nom de débats, il était le seul professeur qui tutoyât les élèves.

Monge, toujours enthousiaste de la Marseillaise, la chantait chaque jour à pleine voix avant de se mettre à table.

\section{Arago.}

Oeuvres, t. 2 (1854), p. 479 et 516 $=$ Werke, Bd. 2 (1854), p. 390 u. 421.

On pourrait croire Monge ... un homme terrible: Quand. la guerre fut décidée, il monta à la tribune des· Jacobins, et déclara qu'il donnait d'avance ses deux filles aux deux premiers soldats qui seraient blessés par l'ennemi; ${ }^{1}$ ) ce qu'il pouvait faire à toute rigueur, pour son compte; mais il prétendait qu'on y obligeât tout le monde et voulait qu'on tuât tous les nobles, etc. - Or Monge était le plus doux, le plus faible des hommes, et n'aurait pas laissé tuer un poulet, s'il eût fallu en faire l'exécution lui-même, ou seulement devant lui. Ce forcéné républicain, à ce qu'il croyait, avait pourtant une espèce de culte pour moi, c'était de l'adoration: il m'aimait comme on aime sa maîtresse.

\section{NAPOLÉON I.}

voir Cte de Las Cases, "Mémorial de Sainte-Hélène", t. 2 (Londres 1823), 4 ième partie, p. 177.

Pour témoigner leur reconnaissance envers leur collègue, dont la réputation scientifique s'était déjà étendne sur toute l'Europe, les professeurs de Cambridge nommèrent Newton,

1) Freunde und Angehörige des berühmten Mathematikers haben den Inhalt dieser Erzählung entschieden in Abrede gestellt mit dem Hinweis darauf, dass Monge nachweislich nie bei den Jakobinern erschienen sei (s. das oben citierte Werk von Las Cases, t. 3, 6ième partie, p. 280). 
mais, il faut le dire, à une faible majorité, pour les représenter au parlement, en 1688. - - - -

Sa carrière parlementaire fut sans éclat. Pendant toute sa durée, il ne prit, dit-on, la parole qu'une seule fois, et ce fut pour inviter l'huissier de la chambre des communes à fermer une fenêtre d'où partait un courant d'air qui aurait pu enrhumer un orateur placé non loin de là.

Arago.

Oeuvres, t. 3 (1855), p. $329=$ Werke, Bd. $3(1855)$, p. 264.

Das Jahr 1837 ist für Euere Universität polternd zu Ende gegangen. Ich habe mit grosser Befriedigung gesehen, dass Dein Name sich niemals in den langen Zeitungsartikeln aus Göttingen befand. Nicht dass ich bei Deinen 7 Kollegen die ehrliche Vertretung ihrer Ansichten missbillige; aber wir, die wir praktische Wissenschaften ausüben, müssen niemals mehr als: Noli turbare circulos sagen. - Ich habe oft mit grosser Betrübnis gesehen, wie der im Observatorium grosse Arago in der Deputiertenkammer eine kleine und zuweilen lächerliche Figur ist.

Berzelius an Wöhler. Stockholm, 1. I. 1838.

Es ist doch ein kurioser Zufall, dass [Wilh.] Weber den Spruch, den Du mir in Deinem letzten Briefe in Beziehung auf die hiesigen Vorfallenheiten schreibst, als Motto unter sein lithographirtes Portrait gewählt hat - Noli turbare circulos obgleich ich ihm durchaus nichts davon gesagt hatte. Auch passt es ja bei ihm gar nicht, da er gerades Wegs gegen das Princip gehandelt hat.

WöHLER an Berzelius. Göttingen, 11. II. 1838.

s. „Briefw. zw. J. Berzelius und F. Wöhler“, herausg. v. 0. Wallach,Bd. 2 (Leipzig 1901), p. 1 u. 10/11. 


\section{$-344-$}

Gerade in der jetzigen Bedrängniss unserer armen G[eorgia] A[ugusta], kann ich mich von einer gewissen Pietät nicht los machen, und gerade jetzt würde es mir schwerer sein Göttingen zu verlassen, als zu irgend einer andern Zeit, wenigstens so lange ich noch nicht alle Hoffnung aufgeben muss, alles, was mir persönlich darin lieb ist, $\mathrm{zu}$ verlieren.

Gauss an Schumacher.

Göttingen, 7. I. 1838.

s. Briefw. Gauss-Schumacher, Bd. 3 (1861), p. 193/194.

Gauss war eine aristokratische durch und durch conservative Natur ..... Von der Intelligenz und Moral der grossen Menge hatte er nur einen sehr geringen Begriff und hat dieses namentlich in Bezug auf politische, religiöse und wissenschaftliche Dinge oft ausgesprochen. Mundus vult decipi, pflegte er $\mathrm{zu}$ sagen und darum verfolgte er auch Agitatoren und Wühler mit dem Auge des Misstrauens, mit einem beständigen Falkenblick. Von unsern constitutionellen Regierungssystemen hatte er nur eine sehr geringe Meinung und er war unablässig bemüht unsern parlamentarischen Grössen entweder logische Fehler oder Mangel an Sachkenntniss nachzuweisen, was ihm denn auch öfter gelungen ist.

Sartorius v. Waltershausen. „Gauss zum Gedächtniss“ (1856), p. 94.

Sogenannte Literaten, d. i. Leute, die ohne etwas gelernt zu haben, über Alles schwatzen und schreiben.

Gauss an Schumacher.

1. I. 1845 .

s. Briefw. Gauss-Schumacher, Bd. 4 (1862), p. 385.

Bei Gelegenheit seines siebzigsten Geburtstages erzählte Weierstrass mit grossem Behagen, dass er unter den vielen ihm in seinem Leben zugefallenen Pflichten vor 1848 in Deutsch- 
Krone das Amt eines Censors für das dortige Localblatt ausgeübt hätte. Der königliche Beamte, dem die Überwachung übertragen war, hatte eine Abneigung gegen die schönwissenschaftliche Literatur . . . . und übertrug daher die Beaufsichtigung des belletristischen Theiles dem Mathematiker Weierstrass. Da nun gerade zu jener Zeit die Herwegh'schen Freiheitslieder erschienen und Weierstras s zugesandt wurden, so machte es ihm ein besonderes Vergnügen, unter den Augen des streng conservativen Censors die revolutionären Freiheitslieder abdrucken zu lassen, sicher, dass jener die Gedichte nicht lesen würde. Endlich wurde durch Einschreiten der vorgesetzten Behörden diesem burschikosen Spasse ein Ende gemacht - - -

\author{
E. Lampe. \\ "Karl Weierstrass", \\ Gedächtnisrede Berlin Phys. Ges. 5. III. 1897, p. 16 \\ $=$ Deutsche Mathem.-Verein. Jahresber. 6, 1896/1897, p. 38.
}

Der talentvolle Rosenhain, welchen man eines unbeliebten, Jacobi'schen, ${ }^{1}$ ) etwas erhöhten Chromatismus beschuldigte, hat den Preuss. Dienst verlassen. Ich habe ihn sehr warm, da ihn die ruhige Zeit entfärbt hat, an den ... . Oestr. Cultusminister empfohlen.

Al. Humbold' an Gauss.

Potsdam, 26. X. 1851.

s. K. Bruhns, „Briefe zw. A. v. Humboldt und Gauss“ (Leipzig 1877), p. 61.

Schon in ihrer Jugend verkehrte Sonja Kowalewski in ihrem Heimatlande in nihilistischen Kreisen. ... . Und wenn sie auch in Folge ihrer besonderen Wirksamkeit zu einer aktiven Bethätigung ihrer politischen Anschauungen nicht kam, so liess sie sich durch keine ihr von den Grossen dargebrachte

l) vgl. S. 128 . 
Huldigung blenden, sondern bewahrte treu die Ideale der Jugend. Sie blieb nicht nur bis ans Ende im Herzen Sozialistin, sondern scheute auch keineswegs vor einer gelegentlichen rückhaltslosen Aussprache ihrer Überzeugung zurück. Sie war auf dem internationalen Sozialistenkongress von Paris 1889 zugegen. Insbesondere verfolgte sie die Fortschritte der deutschen Sozialdemokratie mit grösstem Interesse und höchster Sympathie. Als sie während der Zeit des ärgsten Druckes des Ausnahmegesetzes $^{1}$ ) wiederholt in Berlin weilte, führten ihre Wege nicht selten von der sie feiernden offiziellen Gesellschaft unmittelbar zu gehetzten Sozialdemokraten, mit denen sie befreundet war.

G. [ron] Volmmar [Mitglied des deutschen Reichstags]. „Sonja Kowalewski“, Die Neue Zeit, Jahrg. IX, Bd. 1 (1891), p. 844.

Rempublicam summam societatis humanae formam esse nego.

L. Kronecker.

Doctorthese Berlin 1845. s. Werke, Bd. 1, p. 73.

Tout en rétablissant l'obligation du serment [politique], Napoléon III. résolut d'en dispenser quelques hommes à qui il était moralement impossible de le demander. Deux exceptions célèbres furent faites dans le monde savant, l'une pour Arago et l'autre pour Cauchy.

$$
\text { C.-A. Valson. }
$$

„La vie et les travaux du baron Cauchy" (Paris 1868), t. 1, p. 106.

Le premier géomètre de l'Italie [Brioschi] a été SousSecrétaire d'État et Sénateur du royanme. Il a pris, au Sénat, une grande part dans le travail des Commissions du budget; il a été l'organisateur des chemins de fer de la péninsule; il

1) Gemeint ist das unter dem Namen „Sozialistengesetz" bekannte deutsche Reichsgesetz rom 21. X. 1878 ,gegen die gemeingefährlichen Bestrebungen der Socialdemokratie", das am 1. Oktober 1890 ausser Kraft trat. 
a été délégué, par le Gouvernement italien, à la Commission internationale du Mètre, à Paris. Notre illustre Confrère appartenait à la plupart des Académies et Sociétés savantes de l'Europe et de l'Amérique, il était Président de l'Académie des Lincei, les plus hautes distinctions, les honneurs dont il a été comblé, les grandes situations qu'il a occupées l'ont toujours laissé simple et modeste.

\section{Hermite.}

„Notice sur M. F. Brioschi",

Paris C. R. de l'académie des sc. 125 (1897), p. 1141.

Il est d'usage, en Russie, d'accorder des titres militaires à des hommes très-étrangers au service; e'est rendre hommage au préjugé qui faisait regarder cet état comme la seule profession noble, et avouer en même temps qu'on en reconnaît toute la fausseté: quelques savants ont obtenu jusqu'au grade de général-major; M. Euler n'en eut et n'en voulait avoir aucun: mais quel titre pouvait honorer le nom d'Euler?

\section{Condorcet.}

"Éloge de M. Euler".

voir Condorcet, Oeuvres, Édition d'Arago, t. 3 (1847), p. 39.

An S. Durchlaucht, den Herzog von ....... zu schreiben, würde ganz ausserhalb meiner Handlungsweise liegen, da ich niemals mich jemandem aufdringen mag. Selbst die Klugheit verbietet eine solche Zudringlichkeit, da der Erfolg leicht sein könnte, zu hören, dass Sr. Durchlaucht mein Name völlig unbekannt wäre.

Gauss an Schumacher.

Göttingen, 12. II. 1837.

s. Briefw. Gauss-Schumacher, Bd. 3 (1861), p. 152.

Wie viele Gebildete, die sich nicht verzeihen würden, von einem Clavier- oder Geigen-Virtuosen nicht alles Erdenkliche 


\section{$-348$}

zu wissen, haben keine Ahnung von der Grösse eines Gauss, eines Faraday!

\section{Emil du Bois-Reymond.}

"Gedächtnissrede auf Hermann von Helmholtz", p. 4 in Berl. Abhandl. 1896.

Neuton fut plus connu que jamais à la Cour sous le Roi George. La Princesse de Galles, aujourd'hui Reine d'Angleterre, avoit assés de lumieres et de connoissances pour interroger un homme tel que lui, et pour ne pouvoir être satisfaite que par lui. Elle a souvent dit publiquement qu'elle se tenoit heureuse de vivre de son temps, et de le connoître. Dans combien d'autres Siécles et dans combien d'autres Nations auroit-il pû être placé sans y retrouver une Princesse de Galles!

Fontenelle.

„Eloge de M. Neuton".

voir Oeuvres de Fontenelle, t. 6 (1758), p. 356/357.

J'ai vu M. la Grange, qui a bien voulu tempérer la sublimité de son langage en raison inverse des carrés de mon ignorance; il m'a conduit d'abstraction en abstraction dans un labyrinthe d'obscurités où mon pauvre esprit se serait perdu, si notre bon Suisse M. Merian ne m'avait retiré des sublimes régions infinitésimales pour me remettre sur ce globe abject et brut où je végète.

Frédéric le Grand à d'Alembert.

(I. 1780.)

voir Oeuvres de Frédéric le Grand, t. 25 (Édition Decker 1854), p. $139 / 140$.

Über Alles sollte der Alles Wissende [A. v. Humboldt] [seinem königlichen Freunde Friedr. Wilh. IV.] Auskunft geben, bald über ein ernstes Problem, bald über ein müssiges 
Curiosum, so über die Frage, warum die Produkte der Zahl 9 immer die Ziffersumme 9 ergeben. ${ }^{1}$ )

\section{H. v. TreitschKe.}

„Deutsche Geschichte im 19. Jahrh.“, T. 5, 4. Aufl., p. 29.

Bei einer sehr geringen mathematischen Kenntniss, die aber doch das Rührende, vielleicht die Ironie Vermildernde hat, dass ich in meinem sechzigsten Jahre mir noch täglich achtzehn Monate lang Privatunterricht von Duhamel über mathematische Physik geben liess, bei der Unwissenheit, die ich so gern eingestehe, hat mir doch der lange Umgang mit Lagrange, Laplace, Fourier einiges Ahndungsvermögen über den relativen Werth meiner Zeitgenossen eingeflösst. - - -

\section{A. von Humboldt an C. G. J. Jacobi.}

s. Karl Bruhns, „Alexander von Humboldt“, Bd. 2 (1872), p. 7/8.

Lambert ging nach Potsdam, mit Empfehlungsbriefen versehen, worin aber gewarnt wurde, doch alles Mögliche anzuwenden, dass er dem Könige [Frie drich d. Gr.] nicht persönlich vorgestellt würde. „Ihro Majestät,“ sagte man dem Monarchen, „Herrn Lamberts Gepäck ist noch nicht angekommen." „Ihr Herren scherzt", entgegnete er; "seit wann glaubt Ihr dass ich Kleider und nicht Menschen sehen will?" „Nun“, fuhr man fort, "wir wollen Ihro Majestät gestehen, dieser Gelehrte, der so viel Verdienst hat, kündigt durch seine äussere Haltung sich nicht gut an." „Wir wollen die Lichter auslöschen; bringt mir den Mann des Nachts; ich will ihn nicht sehen, sondern hören", erwiederte Friedrich. Lambert kam; die Lichter wurden nicht ausgelöscht; der König sah und hörte ihn. Sie führten folgendes Gespräch: K. Guten Abend, mein Herr! Machen Sie mir das Vergnügen mir zu sagen, welche Wissen-

1) Nach Karl Bruhns, „Alexander von Humboldt", Bd. 2 (1872), p. 301 musste Humboldt sich selbst erst bei Encke hierüber informieren. 
schaften Sie besonders erlernt haben. L. Alle. K. Sind Sie also auch ein geschickter Mathematiker? L. Ja. K. Und welcher Professor hat Sie in der Mathematik unterrichtet? L. Ich selbst. K. Sie sind demnach ein zweiter Pascal? L. Ja, Ihro Majestät. Jezt drehte ihm der König den Rücken, indem er sich des Lachens kaum enthalten konnte und gieng in sein Cabinett. Bei Tische äusserte der Monarch, man habe ihm den grösten Dummkopf für seine Akademie vorgeschlagen, den er je gesehen.

M. GraF.

„Lambert's Leben“, p. 15

in: Daniel Huber, „Joh. Heinr. Lambert" (Basel 1829);

s. a. Formey, „Floge de M. Lambert", Hist. de l'acad. de Berlin 1778, p. 84, sowie Thiébault, „Mes sourenirs de vingt ans de séjour à Berlin“, t. 5 (Paris 1804), p. 26-28.

Lambert, der zu wenig Erfahrung hatte um zu merken, dass er nicht gefallen habe, kam ganz vergnügt wieder.

„Johann George Sulzer's

Lebensbeschreibung von ihm selbst aufgesetzt"

(Berlin \& Stettin 1809), p. 39.

On m'a, pour ainsi dire, presque forcé de prendre la plus maussade créature qui soit dans l'univers pour la mettre dans notre Académie. Il se nomme Lambert, et quoique je puisse attester qu'il n'a pas le sens commun, on prétend que c'est un des plus grands géomètres de l'Europe. Mais comme cet homme ignore les langues des mortels et, qu'il ne parle qu'équations et algèbre, je ne me propose pas de sitôt d'avoir l'honneur de m'entretenir avec lui. En revanche, je suis très-content de M. Toussaint, dont j'ai fait l'acquisition. Sa science est plus humaine que celle de l'autre. Toussaint est un habitant d'Athènes, et Lambert un Caraïbe, ou quelque sauvage de la côte de la Cafrérie. Cependant, jusqu'à M. Euler, toute l'Académie est à genoux devant lui, et cet animal tout crotté du buorbier de la plus crasse pédanterie reçoit ces hommages 
comme Caligula recueillait ceux du peuple romain, chez lequel il voulait passer pour dieu.

FrÉdÉric le Grani à d'Alembert (1764).

voir Oeuvres de Frédéric le Grand, t. 24 (Édition Deeker 1854), p. 391/392.

„Monsieur, disoit le pasteur Hachard à Lambert, il ne faut pas vous impatienter: le roi vous nommera certainement à son académie; mais en ce moment il est fort occupé. - Oh! monsieur, je n'en suis pas inquiet: il y va de sa gloire; et s'il ne me nommoit pas, ce serait une tache dans son histoire."

\section{Thinḱbaulta.}

„Mes souvenirs de vingt ans de séjour à Berlin", t. 5 (Paris 1804), p. 28.

M. La m bert a quelque chose de singulier dans son maintien et dans sa conversation qui déplaît au premier abord, et je ne suis pas surpris que le roi ne l'ait pas gaîté, ayant eu moi-même de la peine à m'accommoder à ses manières. Il était ou du moins il me parut si plein de lui-même, lorsque j'arrivai ici, que je pris le parti de ne pas le fréquenter, mais en même temps de ne laisser échapper aucune occasion de le rabaisser; cela l'a rendu beaucoup plus traitable, et à présent nous sommes assez bons amis.

Lagrange à d'Alembert.

Berlin, 15. VII. 1769.

voir Oeuvres de Lagrange, t. 13 (Paris 1882), p. 142.

Vous ne voulez pas vous engager à la cour, fût-ce même en qualité de casuiste chargé de faire les équations algébriques des péchés du souverain et des peines qu'il encourt.

Frédéric le Grand à d'Alembert.

4. VIII. 1768.

voir Oeuvres de Frédéric le Grand, t. 24

(Édition Decker 1854), p. 439. 
Son amour pour l'indépendance va jusqu'au fanatisme, au point qu'il se refuse souvent à des choses qui lui seroient agréables, lorsqu'il prévoit qu'elles pourroient être pour lui l'origine de quelque contrainte; ce qui a fait dire avec raison à un de ses amis, qu'il étoit esclave de sa liberté.

\section{D'Alembert.}

„Portrait de l'auteur, fait par lui-même", 1760. voir Oeuvres posthumes de d'Alembert, t. 1 (Paris 1799), p. 29 $=$ J. Bertrand, „D'Alembert" (Paris 1889), p. 190.

M. Daniel Bernoulli ne s'est point marié. Dans sa jeunesse, on lui proposa un parti très-avantageux; mais l'extrême économie de la femme qu'on lui destinait l'eut bientôt décidé à rompre avec elle. Depuis ce temps, il n'a plus pensé au mariage que pour se souvenir qu'il avait été sur le point de perdre en un jour sa liberté et son repos, et pour se fortifier dans la résolution de ne plus s'exposer au même péril.

\section{Condorete.}

„Éloge de M. [Daniel] Bernoulli", voir Coudorcet, Oeurres, Édition d'Arago, t. 2 (1847), p. 573.

M. Leibnitz ne s'étoit point marié; il y avoit pensé à l'age de 50 ans: mais la personne qu'il avoit en vue voulut avoir le temps de faire ses réflexions. Cela donna à M. Leibnitz le loisir de faire aussi les siennes, et il ne se maria point.

Fontenelle.

„Eloges des académiciens de l'acad. des sc., morts depuis l'an 1699", t. 1 (1766), p. 501.

Mon cher et illustre ami, on m'écrit de Berlin que vous avez fait ce qu'entre nous autres philosophes nous appelons le saut périlleux et que vous avez épousé une de vos parentes....; recevez-en mon compliment, car je compte qu'un grand mathé- 
maticien doit avant toutes choses savoir calculer son bonheur et qu'après avoir fait ce calcul vous avez trouvé le mariage pour solution.

D'Alembert à Lagrange. Paris, 21. IX. 1767.

Je ne sais si j'ai bien ou mal calculé, ou plutôt je crois n'avoir point calculé du tout, car j'aurais peut-être fait comme Leibnitz, qui, à force de réfléchir, ne put jamais se déterminer. Quoi qu'il en soit, je vous avouerai que je n'ai jamais eu du goût pour le mariage et que je ne m'y serais jamais engagé si les circonstances ne m'y avaient en quelque façon obligé. Étant dans un pays étranger, sans amis et sans liaisons, avec une santé assez délicate, j'ai cru devoir engager une de mes parentes, que je connaissais depuis longtemps et avec qui j'avais déjà vécu quelques années dans la maison de mon père, à venir partager mon sort et avoir soin tant de moi que de tout ce qui me regarde. Voilà l'histoire exacte de mon mariage. Si je ne vous en ai point fait part, c'est qu'il m'a paru que la chose était si indifférente d'elle-même, qu'elle ne valait point la peine de vous en entretenir.

LAGRANGE à d'Alembert. Berlin, 20. XI. 1767.

voir Oeuvres de Lagrange, t. 13 (Paris 1882), p. 100 et 102.

A l'avis de Descartes, il y avait trois choses également difficiles à rencontrer: une belle femme, un bon livre et un bon prédicateur, et il était également difficile à satisfaire sur ces trois points.

\section{C.-A. Valson.}

„Les savants illustres du XVIe et du XVIIe siècle“, t. 2 (1880), p. 94.

Sauveur a été marié deux fois. A la premiere il prit une précaution assés nouvelle; il ne voulut point voir celle qu'il 
devoit épouser, jusqu'à ce qu'il eût été chés un Notaire faire rédiger par écrit les conditions qu'il demandoit; il craignit de n'en être pas assés le maître après avoir vû.

Fontenelle.

„Eloge de M. Sauveur".

voir Oeurres de Fontenelle, t. 5 (1758), p. 480.

Dans ce temps-là [au XVIII e siècle] . . . les savants, les mathématiciens, surtout, étaient regardés dans le monde comme des êtres d'une nature à part. On aurait voulu leur interdire le concert, le bal, le spectacle, comme à des ecclésiastiques. Un géomètre qui se mariait semblait enfreindre un principe de droit. Le célibat paraissait la condition obligée de quiconque s'adonnait aux sublimes théories de l'analyse.

Arago.

Oeurres, t. 2 (1854), p. $181=$ Werke, Bd. 2 (1854), p. 148.

Vous étiez dans l'âge convenable pour vous marier; un homme destiné à passer beaucoup de temps dans les travaux du cabinet, a besoin d'une compagne qui s'occupe de tout le détail du ménage et qui affranchisse son mari de tous ces petits soins minutieux dont un homme n'est guère capable.

LE Gendre à C. G. J. Jacobi.

Sans date, timbré Paris, 30. juin 1832.

voir "Correspondance mathém. entre Legendre et Jacobi", éd. par Borchardt, J. f. Math. 80 (1875), p. 278

$=$ Jacobi, Werke, Bd. 1, p. 460 .

J'étais heureux et fier quand je dînais dans la rue de Tournon chez le grand géomètre [Laplace]. Mon esprit et mon coeur étaient très-disposés à tout admirer, à tout respecter chez celui qui avait découvert la cause de l'équation séculaire de la lune, trouvé dans le mouvement de cet astre les moyens de calculer l'aplatissement de la terre, rattaché à l'attraction 
les grandes inégalités de Jupiter et de Saturne, etc., etc. Mais, quel ne fut pas mon désenchantement, lorsque, un jour, j'entendis madame de Laplace s'approcher de son mari, et lui dire: „Voulez-vous me confier la clef du sucre?"

\section{Arago.}

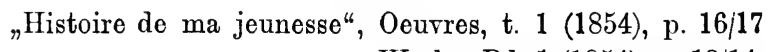

$=$ Werke, Bd. 1 (1854), p. 13/14.

Après avoir demeuré près de quarante ans dans la maison de sa nourrice, sa santé l'obligea de quitter le logement qu'il [D'Alembert] occupait chez elle, et l'âge de cette femme respectable ne lui permit pas de le suivre: tant qu'elle vécut, deux fois chaque semaine il se rendait auprès d'elle. - Condorcet.

„Éloge de M. D’Alembert",
voir Condorcet, Oeurres, Édition d'Arago, t. 3 (1847), p. 106.

Uxor mea ... peperit filiolam, et ita me jam sextae prolis parentem fecit.

Vides qualia nobis uxoratis negotia facessant familiae nostrae jam satis numerosae et subinde adhuc accrescentes.... Mille sunt curae et sollicitudines, quae nos occupant, a quibus coelibes sunt liberi: habet matrimonium sua commoda et habet sua incommoda in variis vitae generibus, sed qui literis se totum dedere cupit, discat is prius vitam suam in coelibatu transigere, quo nomine Te felicem reputo.

\section{Johann Bernoulli an Leibniz.}

Basel, 11. IX. 1706.

s. Leibniz, Mathem. Schriften, herausg. v. C. Gerhardt, Bd. 3, Abth. 2 (1856), p. 799/800.

Gaudeo etiam Dn. Filium Tuum [Niclaus B. 1695-1726] Bernoullizare, et hereditarium familiae decus tueri.

Leibniz an Joh. Bernoulli.

Hannover, 4. XI. 1715.

s. Leibniz, Mathem. Schriften, herausg. v. C. Gerhardt, Bd. 3, Abth. 2 (1856), p. 948. 
Ma situation actuelle est telle qu'elle ne me laisse rien à désirer; il est vrai que je suis marié, mais je n'ai point d'enfants et je ne souhaite pas d'en avoir; ma femme, qui est une de mes cousines et qui a même vécu assez longtemps dans ma famille, est très-bonne ménagère et n'a d'ailleurs aucune espèce de prétention, de sorte qu' à tout prendre mon mariage ne m'est pas une charge.

LAGRANGE à d'Alembert.

Berlin, 15. VII. 1769.

voir Oeuvres de Lagrange, t. 13 (Paris 1882), p. 143.

Wahrlich nur wer Vater ist hat das volle Bürgerrecht der Erde.

$$
\text { Gauss an W. Bolyai. }
$$

Braunschweig, 20. VI. 1803.

8. „Briefw. zw. C. F. Gauss u. W. Bolyai", herausg. v. Schmidt u. Stäckel (1899), p. 54.

Lasse Dich . . etwa durch mein Beispiel nicht gereitzt werden, trau den Mädchen nicht, weun sie Dir auch in lichtloher Flamme ewige Treue schwüren, das helle Feuer brennt $a b$, und die Aschen sind dunkel - trau den betrügerischen Augenhlicken nicht, frage die Zeit um Rath, wie schön steht die Rose da, die nach einigen Stunden wüste bleibt -

W. BOLyai an Gauss.

Domáld, 11. IX. 1802.

Leyder ist es wol gewiss dass wer heirathet in eine Lotterie setzt wo es viele Nieten und wenig Treffer giebt. Der Himmel gebe dass auch ich, wenn ich einst greifen sollte keine Niete ziehe.

Gauss an W. Bolyai. Braunschweig, 3. XII. 1802.

Ich bin mit den Schwiegereltern in einer sehr stechenden Lage .... Diese verdammte Verbältnisz hat meinen Freude- 
kelch so vergiftet, dass ich mit Dryden sagen muss: „Das Weib ist die Hölle, wo der brave Mann hinverdammt ist.].]

W. Bolyai an Gauss. Clausenburg, 27. II. 1803.

s. „Briefw. zw. C. F. Gauss u. W. Bolyai“, herausg. v. Schmidt u. Stäckel (1899), p. 43,46 u. 49.

Sie, lieber Gauss, haben uns gezeigt, dass Interesse für ein Weib und Interesse für eine Wissenschaft sich vereinigen lassen; ich hotfe Thnen hier nachzueifern.

Bessel an Gauss.

Königsberg, 26. III. 1812.

Gewiss werden auch Sie erfahren, dass unter allen Gütern des Lebens das Glück das aus einer wohlgetroffenen ehelichen Verbindung entspringt das grösste und reinste ist, was allen übrigen erst die Krone aufsetzt.

Gauss an Bessel. Göttingen, 5. V. 1812.

s. Briefw. Gauss-Bessel (1880), p. 167 u. 169.

Mit Vergnügen habe ich gesehen, dass Sie schon die Bahn der Vesta berechnet haben; auch der von Ihnen gewählte Name ist herrlich, und gewiss allen Freunden auch deshalb angenehm, weil er ihnen zeigt, welcher Göttin Sie opfern.

Bessen an Gauss.

Lilienthal, 6. V. 1807.

s. Briefw. Gauss-Bessel (1880), p. 53.

Bei der Taufe seiner Tochter spielte Ewald [Gauss' Schwiegersohn] mit Gauss, Weber und der ïbrigen Gesellschaft auf dem Rasen Ball, nachdem es vorher Chokolade gegeben hatte.

J. Wellhausen.

„Heinrich Ewald“, Festschr. zur Feier des 150-jähr. Bestehens d. Kgl. Ges. d. Wiss. zu Göttingen (Berlin 1901), p. 80/81. 
Von allen Trostgründen, die ich versucht habe, ist mir keiner kräftiger gewesen, als der, dass ich, wenn das Schicksal mir die Alternative vorgelegt hätte, mein gegenwärtiges Unglück zu wählen oder selbst zu sterben und die Seelige [G.'s erste Frau] trostlos zurückzulassen, ich doch das hätte gutheissen müssen, was jenes nun entschieden hat.

\section{GaUsS an Schumacher. \\ Göttingen, 23. XI. 1809.}

s. Briefw. Gauss-Schumacher, Bd. 1 (1860), p. 13.

Vor acht Tagen ist die sterbliche Hülle, welche eine Hauptquelle der unbeschreiblichen Leiden der armen Dulderin [G.'s zweiter Frau] war, der Erde zurückgegeben, und noch immer kann ich keinen Augenblick ohne die innerste Erschütterung an diese Leiden denken. Früher konnte ich Ihnen nicht schreiben. Mit der Zeit wird ja endlich über das Gefühl der Zuspruch der Vernunft Platz gewinnen, dass ihr, wie allen, Glück zu wünschen ist, von einem Schauplatz geschieden zu sein, wo die Freuden flüchtig und nichtig, die Leiden, Fehlschlagungen und schmerzlichen Täuschungen die Grundfarbe sind. Wie sehr sehnte auch ich mich, davon abtreten żu können, wenn nicht so vielfache Bande mich fesselten.

GaUsS an Schumacher.

Göttingen, 24. IX. 1831.

s. Briefw. Gauss-Schumacher, Bd. 2 (1860), p. 282.

Als Weihnachtsgabe habe ich von Ihrer Schwester [A. Charl. Leffler] einen Artikel von Strindberg erhalten, worin er ebenso klar, wie zweimal zwei vier sind, beweist, dass eine solche Monstrosität, wie ein weiblicher Professor der Mathematik, schädlich, unnütz und unangenehm ist. Ich finde, dass er im Grunde ganz recht hat; das einzige, wogegen ich protestiere, ist, dass sich in Schweden eine so grosse Anzahl männlicher 
Mathematiker finde, die mir überlegen seien, und dass es nur aus Galanterie geschehen sei, dass man mich berufen habe.

\section{Sonja Kovalevsky an G. Mittag-Leffler.} 27. XII. 1884.

s. A. Charl. Leffler, „Sonja Kovalevsky“, Deutsche Ausg., Reclam-Bibl. No. 3297/3298, p. 71.

Ich bin für Zulassung der Frauen zum akademischen Studium. . . . . Wir zwingen die Frauen, mit einer niedrigeren Bildung sich zu begnügen, als wir sie empfangen; natürlich wissen sie dann weniger, aber das gestattet doch nicht den Schluss, dass sie weniger befähigt sind.

R. Sturm.

Wenn eine Frau, was nicht häufig, aber doch bisweilen vorkommt, für die Aufgaben der theoretischen Physik besondere Begabung besitzt und ausserdem den Trieb in sich fühlt, ihr Talent zur Entfaltung zu bringen ....., werde ich ihr gerne ..... den probeweisen und stets widerruflichen Zutritt zu meinen Vorlesungen und Übungen gestatten, und habe in dieser Beziehung auch bis jetzt nur gute Erfahrungen gemacht.

Andererseits muss ich aber daran festhalten, dass ein solcher Fall immer nur als Ausnahme betrachtet werden kann, und dass es insbesondere höchst verfehlt wäre, durch Gründung besonderer Anstalten die Frauen zum akademischen Studium heranzuziehen, wenigstens sofern es sich um die rein wissenschaftliche Forschung handelt. Amazonen sind auch auf geistigem Gebiet naturwidrig.

Max Planck.

Ich selbst habe in Freiburg wie in Berlin Zuhörerinnen gehabt, welche unter den Studenten sassen; dabei war das Benehmen der letztern, soweit ich sehen konnte, tadellos. Auch 
kommt in der Physik nichts für Franen Anstössiges vor. Anders ist es in der /\%oologie und Chirurgie.

Emil Warburg.

s. Arthur Kirchhoff, „Die akademische Frau“ (1897), p. 242,256 u. 257.

In gewissem Sinne kann man sagen, das Mathematische ist der Gegensatz des Weiblichen. Möchte dieses in grenzenlosen Gefühlen verschwimmen, so gipfelt männliche Klarheit in der Exactheit, d. h. dem Zahlenmässigen. Auch bringt die Tolksmeinung Liebe und Mathematik in Gegensatz. . . . . . . . Auf jeden Fall ist die Mathematik dem Liebeswerke weniger abträglich als die philosophische Neigung, denn die meisten grossen Wathematiker scheinen glückliche Familienväter gewesen zu sein und manche, die nicht verheirathet waren, haben natürliche Kinder hinterlassen, wie Galilei, Descartes, Leibniz.

Sieht man von den wenigen weiblichen Wesen ab, die „aus der Art geschlagen" sind, so giebt es kaum einen schrofferen Gegensatz als den Mathematikerkopf und den Weiberkopf einerseits, den Mathematikergeist und den Weibergeist andererseits.

Der Kopf des Durchschnittsmannes steht in der Mitte zwischen dem Mathematikerkopfe und·dem Weiberkopfe, sodass jener als die Steigerung des specifisch männlichen Typus erscheint.

P. J. Moebius.

"Über die Anlage zur Mathematik“ (Leipzig 1900), p. 8485 u. $148 / 149$.

On a vu deux prodiges; l'un que Newton ait fait cet ourrage [,Principia mathem. philosophiae naturalis"], l'autre qu'une dame l'ait traduit et l'ait éclairci.

VOLTAIRE.

„Éloge historique de Madame la Marquise du Châtelet." voir Oeurres de Voltaire, t. 47 (Gotha 1787), p. 71 = Voltaire, „Mélanges philosophiques, littéraires, historiques"..., t. 1 (Genève 1771), p. 1. 


\section{$-361$}

Si madame du Châtelet ${ }^{1}$ ) est une femme à composition, je lui propose de lui emprunter son Voltaire à gage. Nous avons ici un gros cyclope ${ }^{2}$ ) de géomètre que nous lui engagerons contre le bel esprit.

\section{FrÉdÉRIC Le GRANd à Voltaire.}

Potsdam, 29. XI. 1748.

voir Oeuvres de Frédéric le Grand, t. 11 (Édition Decker 1849), p. 128.

Les recherches arithmétiques ont pour moi un attrait particulier et j'admire toujours avec un nouveau plaisir l'enchaînement des vérités exposées dans votre livre; malheureusement, la faculté de penser avec force est un attribut réservé à un petit nombre d'esprits privilégiés, et je suis bien sûre de ne rencontrer aucun des développements qui, pour vous, semblent une suite inévitable de ce que vous avez fait connaî[t]re.

\section{Sophie Germain à Gauss.}

Paris, 20. II. 1807.

voir Sophie Germain, Oeuvres philosophiques, édition de Stupuy (1896), p. 272.

Le goût pour les sciences abstraites en général et surtoût pour les mysteres des nombres est fort rare: on ne s'en étonne pas; les charmes enchanteurs de cette sublime science ne se decelent dans toute leur beauté qu'à ceux qui ont le courage de l'approfondir. Mais lorsqu'une personne de ce sexe, qui, par nos moeurs et par nos préjugés, doit rencontrer infiniment plus d'obstacles et de difficultés, que les hommes, à se familiariser avec ses recherches epineuses sait neansmoins franchir ces entraves et penétrer ce qu'elles ont de plus caché, il faut sans doute, qu' elle ait le plus noble courage, des talens tout à fait extraordinaires, le génie supérieur. En effet rien ne pourroit

1) s. das vorhergehende Citat.

2) Euler, der damals bereits auf einem Auge erblindet war. 


\section{$-362$}

me prouver d'une manière plus flateuse et moins équivoque, que les attraits de cette science, qui ont embelli ma vie de tant de jouissances, ne sont pas chimeriques, que la predilection, dont vous l'avez honorée.

Bronsvic, ce 30 Avril 1807, jour de ma naissance.

Gauss à Sophie Germain.

voir Sophie Germain, Oeurres philosophiques, édition de Stupuy (1896), p. 275/276.

Während meines ganzen Lebens hat mich die Mathematik .. mehr durch ihre philosophische Seite angezogen und war mir stets diejenige Wissenschaft, die ganz neue Aussichten eröffnet.

\section{Sofia Wassildiluewna Kowalewsky.}

s. Deutsche Rundschau 108 (Juli 1901), p. 120: Übersetzung einer in der russ. Zeitschr. Russkaja Starina 1891 erschienenen Autobiographie, in's Deutsche übertragen durch Sophie von Adelung.

Le monde des abstractions ${ }^{1}$ ) est le seul qui vaille la peine qu'on l'habite.

AMpère à son ami J. Bredin 1805 .

voir "André-Marie Ampère et Jean-Jacques Ampère." Correspondance et souvenirs, recueillis par Madame H. C., 2.éd. (Paris 1875), t. 1 , p. 9 .

La figure de ce monde passe. Si tu te nourris de ses vanités, tu passeras comme elle. - Mais la vérité de Dieu demeure éternellement; si tu t'en nourris, tu seras permanent comme elle. - Mon Dieu! que sont toutes ces sciences, tous ces raisonnements, toutes ces découvertes du génie, toutes ces

1) zum Verständnis vgl. das Citat auf S. 236 oben. 
vastes conceptions que le monde admire et dont la curiosité se repaît si avidement? En vérité, rien, que de pures vanités.

AMpère 1805.

voir "Journal et Correspondance de André-Marie Ampère", recueillis par Mme H. C. (Paris 1872), p. 354.

À l'exception des sciences exactes, il n'y a presque rien qui lui paroisse assez clair pour ne pas laisser beaucoup de liberté aux opinions; et sa maxime favorite est que prèsque sur tout on peut dire tout ce qu'on veut.

D'Alembert.

„Portrait de l'auteur, fait par lui-même“, 1760.

voir Oeuvres posthumes de d'Alembert, t. 1 (Paris 1799), p. 27 $=$ J. Bertrand, „D'Alembert" (Paris 1889), p. 188.

Geometria principiis utitur non indubitatis solum ac discursivis, sed sub obtutum mentis cadentibus, et evidentia in demonstrationibus (quae est claritas certae cognitionis, quatenus assimilatur sensuali) non solum in ipsa est maxima, sed et unica, quae datur in scientiis puris, omnisque evidentiae in aliis exemplar et medium, quia, cum Geometria spatii relationes contempletur, cuius conceptus ipsam omnis intuitus sensualis formam in se continet, nihil potest in perceptis senso externo clarum esse et perspicuum, nisi mediante eodem intuitu, in quo contemplando scientia illa versatur.

\section{IMMANUEL KaNT.}

"De mundi sensibilis atque intelligibilis forma et principiis." s. Werke, herausg. v. Rosenkranz u. Schubert, Th. 1 (Leipzig 1838), p. 322.

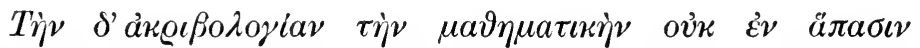

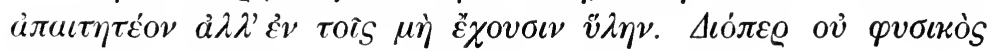

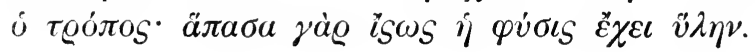


[Nathematische Strenge ist nicht in allen Dingen zu fordern, wohl aber in den unmateriellen. Deshalb ist dies nicht die Weise der Naturwissenschaften; denn die ganze Natur ist doch natürlich materiell.]

\section{ARISTOTELES.}

Metaphys. lib. I, 3, 995.

v. Bibl. Didotiana 10 (= Aristotelis Opera II), p. 488, 23-26.

Ich glaubte mit aller Gewissheit, deren Dinge, die nicht mathematische Theoreme sind, fähig sind, versichern $\mathrm{zu}$ können - - -

Gauss an Schumacher.

Göttingen, 13. II. 1835.

s. Briefw. Gauss-Schumacher, Bd. 2 (1860), p. 391.

Sic incertum, ut, stellarum numerus par an impar sit _ _ _ Cicero.

Academica, lib. II, cap. $10, \S 32$.

v. Bibl. Teubneriana: Ciceronis Scripta, Pars IV, Vol. 1, p. 36.

„Die Mathematik“, äusserte sich Gauss einmal, „sei die Königin der Wissenschaften und die Zahlenlehre die Königin der Mathematik “. ${ }^{1}$ ) Wenn dieses Wort wahr ist, so kann man es dahin fortsetzen, dass man die Disquisitionen die Magna Charta der Zahlenlehre nennt. Das war der Vortheil, den die Wissenschaft aus der . . . allzuzögernden Veröffentlichungsweise von Gauss gezogen hat; was er in Druck gab, ist heute wahr und wichtig wie am ersten Tage; es sind Gesetzbücher, darin den menschlichen Anordnungen überlegen, dass nie und nirgend ein Fehler darin nachgewiesen worden ist. So kann man auch das stolze Urtheil verstehen und billigen, welches Gauss am Abende seines Lebens über die erste grössere Jugend-

1) в. S. 67 . 
arbeit fällte: „Die Disquisitiones arithmeticae gehören der Geschichte $a n$ "1).

M. Cantor.

Artikel "Gauss" in "Allg. Deutsche Biographie", Bd. 8 (1878), p. 435.

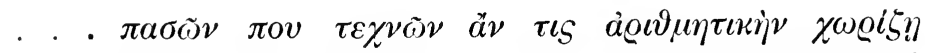

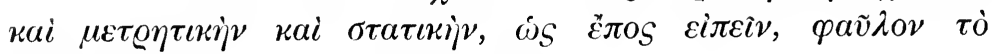

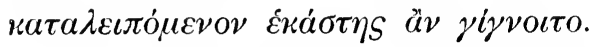

[ . . wenn jemand von allen Künsten wegnehmen würde die Rechenkunst, die Messkunst und die Wägekunst sozusagen, so würde das von jeder Übrigbleibende wenig sein].

$$
\text { Plato. }
$$

Philebus $55 \mathrm{e}$.

v. Opera I, Bibl. Didotiana 45 (Paris 1891), p. 432, 27-30.

Die Weltzustände gleichen der Wasserbouteille, die d'Alembert schüttelte, damit ein Gewebe verschiedeneckiger Blasen entstand. Er sagte dann, um das hydraulische Wissen, in dem er doch selbst so gross war, zu verspotten: „Calculez-moi cela!"

A. v. Humbold' an Varnhagen von Ense.

Potsdam, 2. VII. 1850.

s. „Briefe von Alexander von Humboldt an Varnhagen von Ense aus den Jahren 1827 bis 1858" (Leipzig 1860), p. 253.

Après avoir consumé ses premières années dans la méditation et le travail, il a vu, comme le sage, le néant des connoissances humaines; il a senti qu'elles ne pouvoient occuper son coeur, et s'est écrié avec l'Aminte du Tasse: „J'ai perdu tout le tems que j'ai passé sans aimer"2). d'Alembert.

„Portrait de l'auteur, fait par lui-même“, 1760.

voir Oeuvres posthumes de d'Alembert, t. 1 (Paris 1799), p. 33/34 $=$ J. Bertrand, „D'Alembert" (Paris 1889), p. 193.

1) s. S. 96 .

2) In Tasso's Schäferspiel „Aminta" sagt Dafne (Atto I., Scena I.): "Perduto è tutto il tempo Che in amar non si spende". 
Je commence à sentir que ma force d'inertie augmente peu à peu, et je ne réponds pas que je fasse encore de la Géométrie dans dix ans d'ici. Il me semble aussi que la mine est presque déjà trop profonde, et qu'à moins qu'on ne décourre de nouveaux filons il faudra tôt ou tard l'abandonner.

La Physique et la Chimie offrent maintenant des richesses plus brillantes et d'une exploitation plus facile; aussi le goût du siècle paraît-il entièrement tourné de ce côté-là, et il n'est pas impossible que les places de Géométrie dans les Académies ne deriennent un jour ce que sont actuellement les chaires d'arabe dans les Universités.

LAGRANGE à d'Alembert.

Berlin, 21. IX. 1781.

voir Oeuvres de Lagrange, t. 13 (Paris 1882), p. 368.

Ce que nous connaissons est peu de chose, ce que nous ignorons est immense ${ }^{1}$ ).

LAPLACE.

voir Fourier, „Éloge historique de M. le Marquis de Laplace", Mém. de l'Acad. des Sc. de Paris 10, 1831, Histoire, p. C.

Wir Mathematiker und Physiker dürfen das stolze Bewusstsein hegen, dass wir ein Wissensgebiet unser eigen nennen, welches der Menschheit fortschreitend immer neuen äusseren Erfolg und innere Einsicht bietet, und diese Freude an unserem Besitz, die müssen wir und wollen wir, wenn sie je uns verloren gegangen sein sollte, wiedergewinnen!

F. KLeIN.

"Allgemeines über angewandte Mathematik",

Vortr. Feriencurs für Oberlehrer, Göttingen 1900.

s. Klein u. Riecke, ,Über angewandte Mathem. u. Phys." (1900), p. 25.

1) Es sollen dies Laplace's letzte, allerdings undeutlich gesprochene Worte gewesen sein, die er zur Antwort gab, da man ihm seine wissenschaftlichen Entdeckungen aufzählte; vgl. hierzu das Citat auf S. 2/3. 
Entschuldigen Sie es mit meiner armseligen Lage, dem fast gänzlichen Mangel an geselligem Verkehr und der daraus hervorgehenden gedrückten Stimmung, dass ich fast den ganzen Sommer der morphologischen Botanik widmete. Wenn ich nur wïsste, die reine Mathematik mit objektiver Wirklichkeit $z u$ verbinden! Es war früher mein Wunsch, mathematische Physik zu studiren; aber wenn man nicht die Mittel hat, um eigene Versuche (zu machen), so ist da kaum etwas zu leisten. SCHLÄFLI an Steiner.

Bern, 3. I. 1852.

s. J. H. Graf, „Der Briefwechsel zwischen Jakob Steiner und Ludwig Schläfli“, Bern Naturf. Gesellsch. Mitth. 1896, p. 76.

Ich hörte mich anklagen, als sei ich ein Widersacher, ein Feind der Mathematik iiberhaupt, die doch niemand höher schätzen kann als ich, da sie gerade das leistet, was mir zu bewirken völlig versagt worden.

\section{Goethe.}

"Über Mathematik und deren Missbrauch".

s. Werke, Grosse Weimarische Ausg., Abth. II, Bd. 11 (1893), p. $78 / 79$

$=$ Goedeke'sche 10-bändige Ausg., Bd. 9, p. 706.

„Welch ein erhabner Gedanke! uns lehrt der unsterbliche Meister ${ }^{1}$ )

Künstlich zu spalten den Strahl, den wir nur einfach gekannt."

Das ist ein pfäffischer Einfall! denn lange spaltet die Kirche

Ihren Gott sich in drey, wie ihr in sieben das Licht.

GöTHE an Schiller.

Weimar, 10. X. 1795.

s. Goethe, Werke, Grosse Weimarische Ausg., Abth. IV, Bd. 10 (1892), p. 312.

1) Newton. 
Indem ich diese Woche verschiedne physische Schriften wieder ansahe ist es mir recht aufgefallen, wie die meisten Forscher die Naturphänomene als eine Gelegenheit brauchen die Kräfte ihres Individuums anzuwenden und ihr Handwerk zu üben. Es geht über alle Begriffe wie zur Unzeit Newton den Geometer in seiner Optik macht, es ist nicht besser als wenn man die Erscheinungen in Musik setzen oder in Verse bringen wollte, weil man Kapellmeister oder Dichter ist.

GötHe an Schiller.

Weimar, 13. I. 1798.

s. Goethe, Werke, Grosse Weimarische Ausg., Abth. IV, Bd. 13 (1893), p. 19/20.

Und Jakoby ${ }^{1}$ ) bestelle, sobald er die blaue Grotte differenzirt, werde ich die Marmorfelsen von Carrara in Musik setzen; eher kann er mir es nicht zumuthen.

Felix Mendelssohn-Bartholdy

an seine Schwester Rebecka Dirichlet.

Leipzig, 29. X. 1843.

s. S. Hensel, „Die Familie Mendelssohn“, Bd. 2 (Berlin 1884), p. 250 .

Non mihi si linguae centum sint, oraque centum, enumerare possem, quam multis in rebus usus sit numerorum.

Philipp Melanchthon.

s. Michael Stifel, „Arithmetica integra“

(Nürnberg 1544), Praefatio.

.... Pascal fit une application singulière du calcul des probabilités. Il observa qu'il y avait une différence infinie entre le sort qui attend les impies, s'il y a des peines éter-

1) der Mathematiker C. G. J. Jacobi, der damals mit Dirichlet, Borchardt, Steiner und Schläfli in Italien weilte. 
nelles, et le peu qu'ils ont à gagner, s'ils subissent un anéantissement total; et il en conclut qu'il y a un avantage infini à préférer, dans sa conduite, l'opinion de l'éternité des peines, pour peu que la probabilité ne soit pas infiniment petite: c'est-à-dire, en langage ordinaire, pourvu qu'elle ne soit pas absurde.

Condorcet.

„Éloge de Pascal."

voir Condorcet, Oeuvres, Édition d'Arago, t. 3 (1847), p. 608/609.

Eines Abends sass Frau . . v. Salis mit Hausgenossen und Freunden zu Chur vor ihrer Wohnung. Lambert [damals Hauslehrer in dem S.'schen Hause] war bei ihnen. Sein Blick erhob sich in die höhere Sphäre der Dünste. Plötzlich verkündigte er: „Jezt regnet es in Spanien!“ — - -

Er dachte einen neuen Schnitt zu Hemden aus, wobei der siebente Theil der Leinwand, die man sonst braucht, erspart werden sollte. - - -

Auf einem Spaziergange, wo er rom Regen übereilt wurde, rechnete er im Laufen den kürzesten und trockensten Weg aus. - -

Ein starker Schnupfen überfiel ihn im Winter 1775.... Die Krankheit zog sich in die Länge und machte starke Fortschritte. . . . Er nahm zusehends ab. . . . Er hoffte immer und berechnete, dass er achttausend kleine Abscesse in den Lungen auszuhusten hätte, worauf er sich besser befinden und noch fünfzehn Jahre leben würde. ${ }^{1}$ )

M. GRAF.

„Lambert's Leben“, p. 35, 36 u. 44

in: Daniel Huber, „Joh. Heinr. Lambert" (Basel 1829).

A l'Académie des Sciences de Paris, on discutait, il n'y a pas un grand nombre d'années, une question relative au choc

1) Lambert + 25. IX. 1777.

Ahrens, Scherz und Ernst i. d. Mathem. 
des corps. Cauchy avait pour tous les cas des formules et des lois qu'il exposait longuement, lorsque M. Poinsot, alors octogénaire, l'interrompit par une phrase ${ }^{1}$ ) du docteur Akakia, qui excita un rire universel. Les académiciens admirèrent l'esprit toujours jeune de leur éminent confrère, mais bien peu s'aperçurent qu'il avait fait une citation.

J. Bertrand.

„Éloges académiques", Nouvelle série (Paris 1902), p. 283

Den Hrn. D'Alembert halte ich für einen grossen mathematicum in abstractis; aber wenn er einen incursum macht in mathesin applicatam, so höret alle estime bey mir auf: seine Hydrodynamica ist viel zu kindisch, dass ich einige estime für ihn in dergleichen Sachen haben könnte. Seine pièce sur les vents will nichts sagen und wenn Einer alles gelesen, so weiss er so viel von den ventis, als vorhero. Ich vermeinte, man verlange physische Determinationen und nicht abstracte integrationes. Es fängt sich ein verderblicher goût an einzuschleichen, durch welchen die wahren Wissenschaften viel mehr leiden, als sie avancirt werden, und wäre es oft besser für die realem physicam, wenn keine Mathematik auf der Welt wäre.

Daniel Bernoulli ${ }^{2}$ ) an Euler.

Basel, 26. I. 1750 .

s. "Correspondance mathém. et phys. de quelques célèbres géomètres du 18 ème siècle", t. 2 (1843), p. 649650.

Mr. Dan. Bernoulli a été plus sage que les Euler, les d'Alembert, etc., il ne s'est jamais jeté dans l'Analyse comme

1) jedenfalls folgende Stelle:

„les lois sur le choc des corps parfaitement durs sont puériles et imaginaires, attendu qu'il n'y a aucun corps connu parfaitement dur, mais bien des esprits durs sur lesquels nous avons en vain tâché d'opérer".

Voltaire.

„Diatribe du docteur Akakia."

s. Oeuvres complètes, t. 46 (Gotha 1787), p. 24.

2) vgl. a. S. $463 / 464$. 
eux pour avoir enfin de ces horribles formules dont la vue fait frissonner, comme me le disait Mr. D. Bernoulli, et qui d'ailleurs ne servent à rien [dans l'Hydrodynamique]. . . M. Bernoulli m'a souvent dit de me défier de toutes ces formules compliquées, il croit que la nature est trop simple pour y mener, et que si on en trouve, c'est qu'on a fondé ses calculs sur de fausses hypothèses.

JEANNERET $^{1}$ ) à Jetzler $^{2}$ ).

21. XI. 1778.

voir R. Wolf, „Biographien zur Kulturgesch. d. Schweiz“, Bd. 3 (1860), p. 176.

Il est un temps où j'aurais lu avec intérêt votre traité du calcul des probabilités ${ }^{3}$ ); aujourd'hui je dois me borner à vous témoigner la satisfaction que j'éprouve toutes les fois que je vous vois donner de nouveaux ouvrages qui perfectionnent et étendent cette première des sciences [les mathématiques]. Ils contribuent à l'illustration de la nation. L'avancement et la perfection des mathématiques sont intimement liés à la prospérité de l'État.

NAPOLÉon I. à Laplace.

Vitebsk, 1. VIII. 1812.

voir „Correspondance de Napoléon I. publiée par ordre de l'empereur Napoléon III.", t. 24 (1868), p. 112, No. 19028.

De bonnes mœurs valent mieux pour la société que tous les calculs de Newton.

FrÉdÉric le Grand à d'Alembert.

(Berlin), 4. I. 1770.

voir Oeuvres de Frédéric le Grand, t. 24 (Édition Decker 1854), p. 469.

1) Privatgelehrter in Yverdun und Mitarbeiter an einer Encyklopädie.

2) Oberforstmeister und Prof. d. Math. in Schaffhausen.

3) Gemeint ist die Napoleon gewidmete erste Ausgabe der ,Théorie analytique des probabilités" (Paris 1812), vgl. S. 339, Anm. 1. 
Rien n'est moins applicable à la vie, qu'un raisonnement mathématique. Une proposition en fait de chiffres est décidément fausse ou vraie; sous tous les autres rapports le vrai se mêle arec le faux d'une telle manière, que souvent l'instinct peut seul nous décider entre les motifs divers, quelquefois aussi puissans d'un côté que de l'autre. L'étude des mathématiques habituant à la certitude, irrite contre toutes les opinions opposées à la nôtre; tandis que ce qu'il y a de plus important pour la conduite de ce monde, c'est d'apprendre les autres, c'est-à-dire de concevoir tout ce qui les porte à penser et à sentir autrement que nous. Les mathématiques induisent à ne tenir compte que de ce qui est prouvé; tandis que les vérités primitives, celles que le sentiment et le génie saisissent, ne sont pas susceptibles de démonstration.

$$
M^{\text {me }} \text { DE STÄ̈L. }
$$

„De l'Allemagne", t. 1 (Paris 1823), Chap. XVIII (,Des universités allemandes"), p. 135/136.

Une des plus honorables conquêtes des mathématiques sur les préjugés cupides des administrations de certaines villes a été, de nos jours, la suppression radicale des maisons de jeu.

Arago.

Oeurres, t. 2 (1854), p. 368

=Werke, Bd. 2 (1854), p. 298.

Ich könnte Ihnen erzählen, wie eine der ältesten und angesehensten [Versicherungs-] Gesellschaften an den Rand des Abgrundes gebracht worden ist, weil der Herr Staats-Commissar "Plus" und „Minus" mit einander verwechselt hatte.

\section{KiEPERT.}

„Über die mathem. Ausbildung von Versicherungstechnikern", Vortrag Wien 1894.

s. Deutsche Mathem.-Verein. Jahresber. 4, 1894/1895, p. 118/119 $=$ Zeitschr. math. naturw. Unterr. 26 (1895), p. 4. 
Es wurde die Frage erörtert, ob die Fraunhofer'schen Linien auch die Anwesenheit von Gold in der Sonne ergäben. Kirchhoff's Bankier bemerkte dazu: „Was nützt mich Gold in der Sonne, wenn ich es nicht herunterholen kann?" Kirch hoff erhielt in Folge seiner Entdeckung eine englische Medaille und deren Goldwerth. Als er diesen dem Bankier brachte, meinte er: „Sehen Sie, da habe ich doch Gold von der Sonne geholt."

Robert von HeLmholtz.

„Gustav Robert Kirchhoff“, Deutsche Rundschau 54 (1888), p. 242.

[Mannigfache] Enttäuschungen harrten der ausländischen Gelehrten, die dem Rufe nach Petersburg gefolgt waren. Das Gehalt wurde ihnen während der Zeit der Reaktion so unregelmässig ausgezahlt, dass sie genöthigt waren, durch Ertheilung von Privatunterricht oder andere Arbeiten sich ihren Unterhalt zu erwerben. .... Euler, Daniel Bernoulli und Leutmann ${ }^{1}$ ) erhielten 1732 den Auftrag, die Frage zu studiren, wie die berühmte grosse Glocke in Moskau wieder gehoben werden könnte.

A. Diezmann.

„Die russischen Universitäten“ etc., Preuss. Jahrbücher 110 (1902), p. 63.

Eine knappe Ernährung ist offenbar der Mathematik nicht abträglich, denn viele hervorragende Mathematiker haben hungern müssen.

P. J. Moebius.

"Über die Anlage zur Mathematik" (Leipzig 1900), p. 110/111.

Mittel ein Fuder Holz zu bekommen.

Tobias Mayer in Göttingen besass ein Kunststück, von einem Gemälde mit leichter Mühe eine Menge gleicher und

1) Joh. George Leutmann, 1667-1736, ursprünglich Pastor in Dabrun (Prov. Sachsen), von 1725 an Prof. der Mechanik u. Optik an der neuen Akademie der Wissenschaften zu Petersburg. 
ähnlicher Copien darzustellen. Die Erfindung zu erklären, ist hier nicht der Ort. ${ }^{1}$ ) Ein französischer General, der sich in Göttingen befand - Mayer starb im Anfange 1762 - wünschte eine dergleichen Copie zu haben. Es war zu einer kalten Jahreszeit; Mayer äusserte, die Copie zu machen, erfordere Wärme, und er habe kein Holz. Der General schickte ihm einen Wagen mit Holz.

\section{A. G. KästNer.}

s. Ges. schönwissensch. Werke (Berlin 1841), Th. I, p. 163/164 $=$ Reclam-Bibl. No. 1035, p. 45.

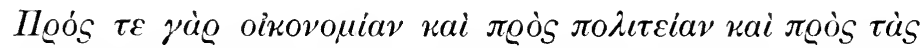

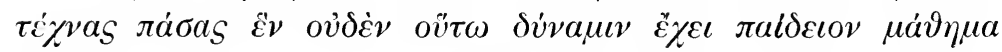

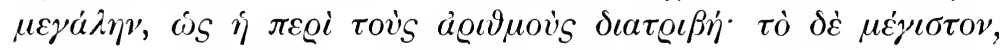

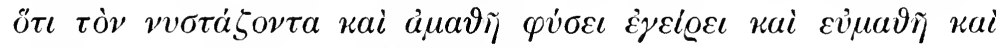

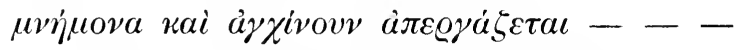

[Denn für die Verwaltung des Hauswesens wie des Staates, sowie für alle Künste besitzt kein Unterrichtsfach so grosse Bedeutung, wie die Behandlung der Zahlen; das wichtigste ist aber, dass sie einen schlafenden und von Natur ungelehrigen aufweckt und ihn gelehrig und scharfsinnig macht und ihm ein gutes Gedächtnis verleiht - - - ]

Plato.

Nó́uo [Gesetze] V, $747 \mathrm{~b}$.

v. Opera II, Bibl. Didotiana 46 (Paris 1883), p. 348, 5-10.

Um bei seinen Spaziergängen sicher zu sein, Nichts vergessen zu haben, was er unterwegs brauchen könne, versäumte Moebius nie von einer mnemonischen Regel Gebrauch zu machen, an die „3 S und Gut" zu denken: Schlüssel, Schirm, Sacktuch, Geld, Uhr, Taschenbuch vorstellend.

M. CANTOR.

Artikel „A. F. Moebius" in „Allg. Deutsche Biographie", Bd. 22 (1885), p. 41.

1) vgl. indessen W. Schur in „Festschr. zur Feier des 150-jähr. Bestehens d. Kgl. Ges. d. Wiss. zu Göttingen" (Berlin 1901), p. 108/109. 
Euler étoit en état de réciter, sans interruption, l'Enéide d'un bout à l'autre, et d'indiquer les premiers et les derniers vers de chaque page de son édition.

\section{N. Fuss.}

„Éloge d’Euler" (Pétersbourg 1783), p. 68.

Mein Gedächtniss hat die Schwäche (und hat sie von jeher gehabt), dass alles Gelesene bald spurlos daraus verschwindet, was im Augenblick des Lesens sich nicht an etwas unmittelbar interessirendes anknüpft.

GuUss an Schumacher.

Göttingen, 12. II. 1841.

s. Briefw. Gauss-Schumacher, Bd. 4 (1862), p. 9.

Ich sprach neulich mit Lübsen ${ }^{1}$ ) über Ihre bewundernswürdige Fertigkeit im numerischen Rechnen. Er gestand, dass diese Fertigkeit ihm ganz unbegreiflich sei, wenn Sie nicht eigene Vortheile dabei hätten. Auf meine Frage, welche Vortheile er meine, nannte er die - biquadratischen Reste. Es war dies kein Scherz, .... sondern sein voller Ernst.

Schumacher an Gauss. Altona, 13. XI. 1841.

Meine jetzt fast 50 jährigen Beschäftigungen mit der höhern Arithmetik haben an der mir zugeschriebenen Fertigkeit im numerischen Rechnen in so fern einen grossen Antheil, als dadurch von selbst vielerlei Zahlenrelationen in meinem Gedächtniss unwillkürlich hängen geblieben sind, die beim Rechnen oft zu Statten kommen. Z. B. solche Producte, wie $13 \times 29=377,19 \times 53=1007$ und dergleichen, schaue ich unmittelbar an, ohne mich zu besinnen, und bei andern, die sich aus solchen sogleich ableiten lassen, ist des Besinnens so wenig, dass ich mir desselben kaum selbst bewusst werde. Übrigens habe ich niemahls Rechnungsfertigkeit absichtlich irgendwie

1) Der durch seine Schulbücher bekannte Mathematiker war ein Schüler von Gauss. 
cultivirt, sonst hätte sie sich ohne Zweifel viel weiter treiben lassen; ich lege darauf gar keinen Wert, ausser in so fern sie Mittel nicht aber Zweck ist.

Gauss an Schumacher. Göttingen, 6. I. 1842.

s. Briefw. Gauss-Schumacher, Bd. 4 (1862), p. 44 u. 49.

Sir Isaac Newton, though so deep in Algebra and Fluxions, could not readily make up a common account: and, when he was Master of the Mint, used to get somebody to make up his accounts for him.

The Rev. J. Spence.

„Anecdotes, observations, and characters, of books and men. Collected from the conversation of Mr. Pope, and other eminent persons of his time", Second edit. by S. W. Singer (London 1858), p. 132.

Dem liebenswürdigen Poisson warf Madame Biot neulich in meiner Gegenwart vor, dass er nicht so gut rechnen könne als ihre Köchin. Er schien seine Schwäche in der gewöhnlichen Arithmetik zuzugeben, und liess sich von ihr exaniniren, z. B. wie viel 13 und 29 machten. Man bewundert es an Ihnen und Gauss, dass Sie beide so grosse Analytiker, zugleich so ungemeine Rechner sind.

Olbers an Bessel.

Paris, 4. VI. 1812.

s. Briefw. Olbers-Bessel, herausg. v. Erman, Bd. 1 (1852), p. 334.

Es war ausgemacht, dass Kummer [in den Vorlesungen] sich immer verrechnete, so wie es sich um die vier Species handelte. Er hantirte mit Differentialquotienten und Integralen, mit Determinanten und elliptischen Functionen, wie ein Jongleur mit seinen Bällen, aber addiren konnte er nicht. Im Laufe der Jahre hatte sich diese kleine Schwäche zur Coketterie ausgewachsen. Nahte eine Addition oder eine Division auf der 
grossen schwarzen Wandtafel, so kündigte sich dieses bedeutsame Ereigniss durch ein verlegenes Räuspern der mathematischen Autoritït an, die da auf dem Katheder stand. Der alte Herr kehrte sich mehrmals um, schaute verlegen, hülfesuchend ins Auditorium und liess sich unter seltsamen Kehllauten von einem steifen Bein aufs andere fallen. Endlich setzte er unter steigender Heiterkeit der Zuhörer an, und verrechnete sich. Er ging sonst in seinen Darlegungen flott vorwärts, aber bei solchen nichtigen Rechnereien konnte er kostbare Minuten verweilen. Das nahmen ihm Manche übel. Wie thöricht! Es geschah so liebenswürdig, ja graziös, dass man sich sagte: das gehört zum alten Kummer!

$$
\text { O. N.-H. }
$$

„Eduard Kummer †“, Münchener Allg. Zeitg. Nr. 139 (20. V. 1893) $=$ Zeitschr. math. naturw. Unterr. 24 (1893), p. 312.

Un savant distingué à qui je témoignais un jour ma surprise de le voir, en pleine séance académique, entreprendre la multiplication de deux énormes lignes de chiffres pris au hasard: „Vous oubliez, me répondit-il sur-le-champ, vous oubliez le plaisir que je trouverai tout à l'heure à faire la preuve de ce calcul par la division."

Alrago.

Oeuvres, t. 2 (1854), p. $4=$ Werke, Bd. 2 (1854), p. 5/6.

Numero deus impare gaudet.

VERGIL.

Ecloga VIII, 75.

v. Corpus poëtarum latin., ed. Postgate, t. 1 (London 1894), p. 111.

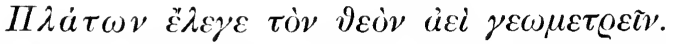

[Plato sagte, dass Gott immer geometrisch verfahre.]

\section{Plutarch.}

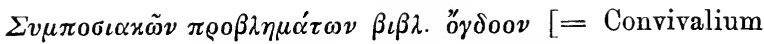
disputationum liber VIII, 2].

v. Bibl. Didotiana 52 (Paris 1890), p. 875. 
Aber du hast alles geordenet mit mas, zal und gewicht. Weisheit Salomonis XI, 22. [Luthers Übers.]

Die letzten Tage seines [Gauss'] Lebens waren .... oft recht schmerzlich - aber er behielt doch immer dabei die Freiheit und Grösse seines Geistes, die zweifelloseste Überzeugung seiner persönlichen Fortdauer, die festeste Hoffnung auf dann noch tiefere Einsicht in die Zahlenverhältnisse, die Gott in die Materie gelegt habe und die er dann auch vielleicht in den intensiven Grössen werde erkennen können, denn

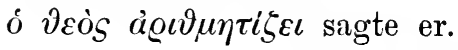

$$
\begin{gathered}
\text { BAUM [Gauss' Arzt] }{ }^{1} \text { ) an A. v. Humboldt. } \\
\text { Göttingen, 28. V. 1855. }
\end{gathered}
$$

s. K. Bruhns, „Briefe zw. A. v. Humboldt und Gauss“ (Leipzig 1877), p. 75.

Die ganzen Zahlen hat der liebe Gott gemacht, alles andere ist Menschenwerk.

\section{KRONECKER ${ }^{2}$ )}

in einem Vortrag auf der Berliner Naturf--Vers. 1886.

s. H. Weber, „Leopold Kronecker", Math. Ann. 43 (1893), p. 15

$=$ Deutsche Mathem.-Verein. Jahresber. 2, 1891/1892, p. 19.

Die Zahlbegriffe entstehen normalerweise in erster Linie durch das Hören und Vergleichen von Tönen und werden dann durch das Sehen und Tasten befestigt.

W. Preyer.

„Über den Ursprung des Zahlbegriffs" etc. in Festschrift zu H. von Helmholtz' 70. Geburtstag (1891), p. 4.

Hermite croyait que les nombres forment un monde ayant son existence propre en dehors de nous, monde dont nous pouvons

1) vgl. S. 185 .

2) vgl. S. 59,60 . 
saisir seulement ici bas quelques-unes des harmonies profondes. Dans l'antiquité il eût été platonicien, et au moyen âge, dans la longue querelle entre le réalisme et le nominalisme, il aurait suivi Guillaume de Champeaux avec les réalistes.

E. Picard.

"L'oeuvre scientifique de Charles Hermite", Acta mathem. 25 (1902), p. 108.

Dieu seul est infini, hors de lui tout est fini. Les êtres spirituels et les êtres corporels sont en nombre fini, et le monde a ses limites dans l'espace comme dans le temps. L'infinité, l'éternité, sont des attributs divins qui n'appartiennent qu'au Créateur, et que Dieu lui-même ne peut communiquer à ses créatures, non que sa puissance soit bornée en aucune manière, mais parce qu'il y aurait contradiction dans les termes, si l'idée de l'infini était appliquée à ce qui est susceptible de variation et de changement. En vertu de sa toute-puissance, Dieu peut augmenter indéfiniment le nombre des êtres qu'il a créés, il peut prolonger indéfiniment la durée de ces mêmes êtres; mais il ne s'en suivra pas que ces êtres soient éternels dans la véritable acception de ce mot.

\section{Augustin Cauchy.}

„Sept leçons de physique générale", Turin 1833, avec appendices par l'abbé Moigno (Paris 1868), p. 27/28.

Wenn die Glaubenssätze zu ihrer Stütze eines so grundfalschen Satzes, wie derjenige von der Unmöglichkeit der actualunendlichen Zahlen, bedürften, wäre es mit ihnen sehr schlecht bestellt und es scheint mir höchst bemerkenswerth, dass der h. Thomas v. Aquino in seiner Summa theologica [theologiae] p. 1, q. 2, a. 3, wo er mit fünf Argumenten die Existenz Gottes beweist, von diesem fehlerhaften Satze nicht Gebrauch macht, obwohl er kein Gegner desselben ist; jedenfalls erschien er ihm für diesen Zweck doch zu unsicher. Es ist zu bedauern, dass dieses Beispiel nicht überall befolgt worden ist. 
So hoch ich Cauchy schätze, so sympathisch mir seine Frömmigkeit ist und so sehr mir im Besondern auch jene „Sept leçons de physique générale“, abgesehen von dem in Rede stehenden Irrthum, gefallen, muss ich doch entschieden gegen seine Autorität protestiren, da, wo er gefehlt hat. - _ -

Alle sogenannten Beweise wider die Möglichkeit actual=unendlicher Zahlen sind dadurch fehlerhaft, und darin liegt ihr $\pi \varrho \tilde{\omega} \tau o \nu \psi \varepsilon \tilde{v} \delta o s$, dass sie von vornherein den in Frage stehenden Zahlen sämmtliche Eigenschaften der endlichen Zahlen zumuthen oder vielmehr aufdringen, während die actual=unendlichen Zahlen doch andererseits, wenn sie überhaupt aufirgend eine Weise denkbar sein sollen, durch ihren Gegensatz zu den endlichen Zahlen ein ganz neues Zahlengeschlecht constituiren müssen, dessen Beschaffenheit von der Natur der Dinge durchaus abhängig und Gegenstand der Forschung, nicht aber unserer Willkür oder unserer Vorurtheile ist.

G. Cantor an G. Eneström.

4. XI. 1885.

s. Natur und Offenbarung 32 (1886), p. 47.

Dieu et la vérité: telle sera ma devise.

CAUChY.

voir C.-A. Valson, „La vie et les travaux du baron Cauchy“ (Paris 1868), t. 1, p. 85.

When I wrote my Treatise about our System, I had an eye upon such principles as might work with considering men, for the belief of a Deity; and nothing can rejoice me more than to find it useful for that purpose. But if I have done the public any service this way, it is due to nothing but industry and patient thought.

I. Newton to Dr. Bentley. Cambridge, 10. XII. 1692.

cf. I. Newton, Opera IV (London 1782), p. 429. 
Euler était pénétré de respect pour la religion: sa piété était sincère et sa dévotion pleine de ferveur. Il a rempli, avec la plus grande attention, tous les devoirs du chrétien.

N. Fuss.

„Éloge d'Euler" (Pétersbourg 1783), p. 69.

Euler était très-religieux; on a de lui une preuve nouvelle de l'existence de Dieu et de la spiritualité de l'âme: cette dernière même a été adoptée dans plusieurs écoles de théologie.

Condorcet.

„Éloge de M. Euler".

voir Condorect, Oeuvres, Édition d'Arago, t. 3 (1847), p. 36.

Gleich den meisten grossen Mathematikern war Euler tief religiös ohne Bigotterie. Er leitete selbst die allabendliche Hausandacht seiner Familie, und eine der wenigen polemischen Schriften, die er verfasste, war seine „Rettung der Offenbarung gegen die Einwürfe der Freygeister", deren Veröffentlichung 1747 in Berlin in nächster Nähe des Hofes Friedrichs d. Gr. einen gewissen sittlichen Muth voraussetzte, welcher über die Angriffe blosser Spötter sich erhaben fühlt.

M. CANTor.

Artikel „Leonhard Euler" in „Allg. Deutsche Biogr.", Bd. 6 (1877), p. 427.

Par le fond même de sa nature, le génie d'A mpère était essentiellement chrétien, et avait besoin d'un milieu chrétien pour arriver à son complet développement. Longtemps comprimé, sous la funeste influence $d u$ naturalisme moderne, il serait certainement demeuré stérile, si, par un effort suprême, le savant n'avait fini par échapper à ses étreintes; ....... Qu'on se représente au contraire Ampère naissant et vivant dans un siècle chrétien, donnant le libre essor à son génie au milieu d'une société chrétienne; A mpère contemporain, par exemple, de Képler, de Descartes, de Newton, de Leibnitz; quel 
changement dans toute cette vie, et probablement que de travaux et de découvertes qui auraient vu le jour, et qui ont été ravis à la science!

\section{C.-A. VAlson.}

„La vie et les travaux d'André-Marie Ampère“ (1886), p. 47/48.

Dunkel sind die Wege, die eine höhere Hand uns hier gehen lässt ..... halten wir fest an dem Glauben, dass eine schönere erhabnere Lösung der Räthsel des Erdenlebens da seyn und uns einst zu Theil werden wird.

Gauss an Schumacher.

Göttingen, 9. II. 1823.

s. Briefw. Gauss-Schumacher, Bd. 1 (1860), p. 298.

Man wird bei solchen anscheinenden Zufälligkeiten [- es handelt sich um die Momente, welche G. veranlassten, den Ruf nach Berlin abzulehnen - ], die zuletzt einen so entscheidenden Einfluss auf das ganze Leben hervorbringen, geneigt, darin Werkzeuge einer höheren Hand zu erkennen. Das grosse Lebensräthsel wird uns hier unten nie klar.

Gauss an Bessel.

Zeven, 25. IV. 1825.

s. Briefw. Gauss-Bessel (1880), p. 453.

Pascal a prétendu que, pourvu que la religion chrétienne ne fût pas impossible, il fallait la croire, et se conduire comme si elle était vraie, parce qu'il y avait peu à gagner, et beaucoup à risquer en ne la croyant pas. ${ }^{1}$ ) Il s'ensuivrait de cet argument que, s'il se trouvait sur la terre cinq ou six religions, qui toutes menaceraient les non-conformistes de peines éternelles, il faudrait les croire et les pratiquer toutes à la fois, ce qui pourrait devenir embarrassant.

\section{CONDORCET.}

„Éloge de Pascal“, Préface.

voir Condorcet, Oeuvres, Édition d'Arago, t. 3 (1847), p. 576/577.

1) s. S. $368 / 369$. 
... C'est le moyen de ne pas manquer le paradis; d'un côté un géomètre, de l'autre un jésuite; avec cette escorte, il faut faire chemin, ou l'on n'en fera jamais.

FrÉdÉRic le Grand à d'Alembert. 25. VII. 1771.

voir Oeuvres de Frédéric le Grand, t. 24 (Édition Decker 1854), p. 543.

Je suis chrétien, e'est-à-dire que je crois à la divinité de Jésus-Christ, avec Tycho-Brahé, Copernic, Descartes, Newton, Fermat, Leibnitz, Pascal, Grimaldi, Euler, Guldin, Boscowich, Gerdil, avec tous les grands astronomes, tous les grands physiciens, tous les grands géomètres des siècles passés. Je suis même catholique avec la plupart d'entre eux; et, si l'on m'en demandait la raison, je la donnerais volontiers. On verrait que mes convictions sont le résultat, non de préjugés de naissance, mais d'un examen approfondi.

\section{CaUChy.}

voir C.-A. Valson, „La vie et les travaux du baron Cauchy“ (Paris 1868), t. 1, p. 173.

Cauchy fut l'un des premiers mathématiciens de l'Europe, et aussi l'un des premiers chrétiens du monde..... au milieu de la sécheresse puissante de ses chiffres et de ses prodigieux calculs, il avait l'âme tendre comme une soeur de charité.

Dupanloup, évêque d'Orléans.

Discours prononcé en faveur des églises d'Orient $\left.{ }^{1}\right)$,

Rome, en juin 1862.

voir C.-A. Valson, „La vie et les travaux du baron Cauchy“ (Paris 1868), t. 1, p. 227/228.

1) Cauchy ist der geistige Urheber der sogenannten „OEuvre des Écoles d'Orient"; näheres s. Valson 1. c. p. $223 \mathrm{ff}$. 
La franchise de Cauchy était sans limites. Le Père de Ravignan, dans la chaire de Notre-Dame, avait risqué une digression sur la science. Le sermon à peine fini, Cauchy, son auditeur assidu, accourt, et du plus loin qu'il l'aperçoit, lui crie, devant de nombreux témoins: „Ah! de grâce, mon Père, vous qui savez tant de choses et qui les dites si bien, ne parlez jamais de ce que vous ne savez pas! “

Le Père de Ravignan eut l'esprit d'en rire, et d'en faire son profit.

\section{J. Bertrand.}

„Éloges académiques", Nouvelle série (Paris 1902), p. 108/109.

Cauchy excellait dans l'art de toucher les coeurs endurcis et de ramener à la foi les âmes égarées. On résistait rarement à ses paroles bienveillantes, à ses manières simples et affectueuses: on cédait volontiers à la bonté sympathique qui débordait de son coeur et rayonnait au dehors dans toute sa personne. ...... Il ne laissait passer aucune occasion de placer une parole d'édification, un conseil de vertu; et lorsque les circonstances le permettaient, il engageait volontiers, avec ses interlocuteurs, des discussions en règle sur ces vérités de la religion dont il était si vivement pénétré.

\section{C.-A. VALSON.}

„La vie et les travaux du baron Cauchy" (Paris 1868), t. 1 , p. $199 / 200$.

On pouvait prévoir, dès les premiers pas de Cauchy dans la vie, l'ardeur et le zèle de piété qui devait plus tard lui attirer, de ses ennemis l'accusation de partialité, de plus d'un ami même celle d'intolérance. Sa bienveillance pour tous était irréprochable . . . . ; mais on la savait plus empressée, plus cordiale et plus dévouée, lors même qu'il parlait au nom de la science, quand il avait la joie de louer ceux dont il savait la foi et approuvait la vie. 
Cauchy, ... dans une des discussions qui précèdent nos élections, avait, avec une autorité prophétique, déclaré l'un des candidats très supérieur à tous les autres. Un de nos confrères, curieux d'autographes, ayant eu, sans songer à mal, l'indiscrétion de jeter les yeux sur les bulletins de vote, lui dit après la séance, sur un ton d'amical enjouement: „M. Cauchy, vous n'avez pas voté pour votre candidat! - J'ai voté suivant ma conscience" répondit Cauchy. _ _ _ -

Jamais Cauchy, il faut s'empresser de le déclarer, n'a refusé ses louanges au vrai mérite.

\section{J. Bertrand.}

„Éloges académiques", Nouvelle série (Paris 1902), p. 107/108.

Lorsqu'en 1839, le Bureau des longitudes perdit le savant et vénérable Prony, aux candidats, nombreux comme toujours, qui briguaient d'honneur de le remplacer, et quelle que fût leur illustration, Arago se bornait à répondre: Cauchy se met sur les rangs! - Si l'on insistait, il répétait: Je vous ai dit, monsieur, que Cauchy se met sur les rangs!

\section{J. Bertrand.}

„Éloges académiques", Nouvelle série (Paris 1902), p. 101.

\section{Venerandae memoriae}

Eximii viri Augustini Cauchy.

Hoc qualecumque munus

Libenter accipias,

Et, ex beata aeternae felicitatis sede Animum sanctae amicitiae pie memorem

Benigne aspicere digneris.

\section{Hermite.}

„Sur la théorie des équations modulaires et la résolution de l'équation du cinquième degréu" (Paris 1859), Dédicace. 
Der fromme Sinn, der im Vaterhaus gepflanzt war, blieb Riemann durch das ganze Leben, und er diente, wenn auch nicht in derselben Form, treu seinem Gott; mit der grössten Pietät vermied er, Andere in ihrem Glauben zu stören; die tägliche Selbstprüfung vor dem Angesichte Gottes war, nach seinem eigenen Ausspruche, für ihn eine Hauptsache in der Religion.

\section{H. WEBER.}

„Bernhard Riemann's Lebenslauf.“

s. Riemanu, Werke, 2. Aufl. (1892), p. 557/558.

So unvollkommen meine mathematische Bildung geblieben ist, so verdanke ich ihr doch die Überzeugung, dass die Existenz solcher Formen und, was die quantitative Bestimmtheit angeht, solche Formeln, wie die analytische Geometrie sie darbietet, unmöglich ein Werk des Zufalls sein können, sondern eine berechnende Intelligenz voraussetzen. Wie sollten zufällige Aggregate von Atomen ein System von Ordinaten und Coordinaten hervorbringen können! Und doch sind auch solche Verhältnisse, wie die lebendige Wechselwirkung der konischen Sectionen sie entwickelt, nur ein Moment in dem ungeheuren Formenspiel der Natur!

Karl Rosenkranz.

„Von Magdeburg bis Königsberg“" (Berlin 1873), p. 159.

Decuit . . eum, qui res ommes creavit, easdem disponere quoque et in ordinem collocare. Quae si vera rerum origo fuit; jam indignum erit philosopho, alias mundi condendi rationes exquirere, vel comminisci quemadmodum e Chao per meras leges naturae mundus universus oriri potuerit; quamvis, formatus cum sit, possit is jam per istas leges in multa quidem secula perdurare. Nam dum cometae moventur in orbibus valde eccentricis, undique et quoquoversum in omnes coeli partes; utique nullo modo fieri potuit, ut caeco fato tribuendum 
sit, quod planetae in orbibus concentricis motu consimili ferantur eodem omnes; exceptis nimirum irregularitatibus quibusdam vix notatu dignis, quae ex mutuis cometarum et planetarum in se invicem actionibus oriri potuerint, quaeque verisimile est fore ut longinquitate temporis majores usque evadant, donec haec naturae compages manum emendatricem tandem sit desideratura.

\section{Newton.}

„Optice", latine redd. S. Clarke (1740), Quaest. XXXI, p. 327.

Mr. Newton et ses Sectateurs ont encore une fort plaisante opinion de l'Ouvrage de Dieu. Selon eux, Dieu a besoin de remonter de tems en tems sa montre, autrement elle cesseroit d'agir. Il n'a pas eu assez de vûe, pour en faire un mouvement perpétuel. .... Selon mon sentiment, la même force et vigueur y subsiste toujours, et passe seulement de matière en matière, suivant les loix de la Nature, et le bel ordre préétabli.

\section{Leibniz à la Princesse de Galles.}

Au mois de Novembre 1715.

voir Leibnitii Opera, ed. Dutens, II, Logica et Metaphys., p. 110,111 .

Wollen die Gegner der Wunder annehmen, dass die ganze Weltentwickelung mechanisch nach den paar Naturgesetzen, die sie aufgefunden haben, fortschreite? Das wäre in der That eine äusserst dürftige, aller wissenschaftlichen Grundlage, ja aller Analogie ermangelnde Anschauung, bei der jene mechanischen Naturgesetze das die Welt Beherrschende wären und die ganze Weltgeschichte wie ein Uhrwerk abrollte.

\section{Hermann Grassmann.}

„Über den Abfall vom Glauben“ (1878), p. 17.

Le nom de Laplace est le plus éclatant de ceux que l'incrédulité revendique, et cependant il convient de ne pas 
même le lui abandonner sans réserve. Le reproche le plus grave qui pèse sur la mémoire de Laplace, e'est d'avoir exprimé, à l'occasion de son célèbre Traité sur la Mécanique céleste, l'opinion que „Dieu etait une hypothèse dont il n'avait pas eu besoin."

$$
\begin{gathered}
\text { C.-A. VALSON. } \\
\text { "La vie et les travaux du baron Cauchy" (Paris 1868), } \\
\text { Introduction, p. XV; } \\
\text { voir aussi du même „Les savants illustres du XVIe et du } \\
\text { XVIIe siècle", t. 2(1880), p. 251. }
\end{gathered}
$$

Wie wunderbar doch die Engländer zu allen Zeiten sind! Der in der Geschichte der inductiven Wissenschaften ganz verständige Prof. Whewell Master of Trinity College Oxford, hielt es für absolut nothwendig, in einer eigenen Schrift „on the Plurality of Worlds" zu beweisen dass kein anderer Weltkörper als die Erde von intelligenten Wesen bewohnt sein kann, da alle intelligente Wesen nach ihrer Natur sündhaft sind und die Erlösung (Kreuzigung) doch nicht auf so viel Million Rossischer Nebelflecken wiederholt werden könne.

Al. Humbold an Gauss. Berlin, 6. III. 1854.

H. Whewell hat mir sein Werk auch geschickt; ich will nicht in Abrede stellen, dass, wer streng an die buchstäbliche Wahrheit der christlichen Dogmen glaubt, kaum umhin kann, auch die Whewell'schen Schlüsse gelten zu lassen..... aber es wäre sehr voreilig, .. den [dem] Mond mir nichts dir nichts alle Einwohner abzusprechen. Die Natur hat mehr Mittel als der arme Mensch ahnen kann.

Gauss an A. von Humboldt. Göttingen, 21. V. 1854.

s. K. Bruhns, „Briefe zw. A.v. Humboldt und Gauss" (Lcipzig 1877), p. 71 u. $72 / 73$. 
Bei solchen feierlichen Gelegenheiten ${ }^{1}$ ) geräth mein Geist n eine höhere Stimmung, in eine andere geistige Welt; die scheidewände des Raumes verschwinden, unsere kothige kleinliche Welt mit allem wass uns hier so gross dünkt, uns so unglücklich und so glücklich macht verschwindet, und ein unsterblicher reiner Geist stehe ich vereinigt mit allen den Guten und Edlen die unsern Planeten zierten und deren Körper Raum oder Zeit von dem meinigen trennten, und geniesse das höhere Leben die bessere Freuden, die ein undurchdringlicher Schleier jetzt bis zu dem entscheidenden Augenblicke unserm Auge verbirgt.

Gauss an W. Bolyai.

Helmstedt, 16. XII. 1799.

s. „Briefw. zw. C. F. Gauss u. W. Bolyai“, herausg. v. Schmidt u. Stäckel (1899), p. 37/38.

Ich unternehme nicht Sie zu trösten [über den Verlust der Mutter], es giebt bei solchen Ereignissen keinen Trost, keinen, als die verstärkte Überzeugung, dass wir hier in Ultima sitzen, und dereinst der Reihe nach zu einer höheren Schule befördert werden. ${ }^{2}$ )

GaUss an Schumacher.

Göttingen, 10. XI. 1822.

s. Briefw. Gauss-Schumacher, Bd. 1 (1860), p. 291.

Sie [Sonja Kovalevsky] hatte keinen bestimmten religiösen Glauben, aber sie glaubte an ein ewiges Leben des Individuums, glaubte daran und zitterte davor.

A. Charl. Leffler.

„Sonja Kovalevsky“, Deutsche Ausg., Reclam-Bibl.

No. $3297 / 3298$, p. 151.

1) Jahrhundertwechsel, als welchen Gauss den Übergang von 1799 zu 1800 ansah (s. a. a. O. die dem obigen Citat unmittelbar vorhergehenden Worte).

2) vgl. a. das Citat S. $390 / 391$. 
A l'exemple d'Archimede qui voulut orner son Tombeau de sa plus belle Découverte Géométrique, et ordonna que l'on y mît un Cylindre circonscrit à une Sphere, M. [Jacques] Bernoulli a ordonné que l'on mît sur le sien une Spirale Logarithmique, avec ces mots, Eadem mutata resurgo; allusion heureuse à l'espérance des Chrétiens, représentée en quelque sorte par les propriétés de cette Courbe.

\section{Fontenelle.}

„Eloge de M. [Jacques] Bernoulli."

voir Oeurres de Fontenelle, t. 5 (1758), p. 112/113.

Die öffentlichen Disputationen [Johann Bernoulli's] über physikalische Gegenstände gaben Veranlassung zu einem heftigen Streite mit den Gröninger Theologen, welcher durch mehrere Jahre sich hindurchgezogen hat. Bernoulli hatte die Meinung geäussert, dass wegen der beständigen Erneuerung der Bestandtheile des menschlichen Körpers nach Verfluss eines Jahres nur wenig mehr von den anfänglichen Stoffen übrig bleibe, der Körper daher bei der Auferstehung nicht mit der gesammten Substanz erscheinen könne, die ihm jemals angehört habe. Er wurde deshalb von den Kanzeln als Socinianer und Ketzer verschrieen, und da er sich über diese Anschuldigungen lustig machte, wurde der Lürm so gross, dass er sich veranlasst fand, eine Dissertation de Nutritione im Mai 1699 öffentlich zu vertheidigen, was, wie er berichtet, im Beisein einer unglaublichen Zuhörermenge geschah, die begierig war, dem Streit zwischen dem Physiker und den Theologen beizuwohnen.

\section{P. Merian.}

„Die Mathematiker Bernoulli“ (Basel 1860), p. 24.

Es ist das traurige Loos des Alters, nach und nach so vieles, was uns nahe stand und theuer war vor uns abscheiden und sich selbst immer mehr vereinsamt zu sehen, und es gibt 
labei keinen Trost, als den Hinblick auf die höhere Weltrrdnung, die einst alles ausgleichen wird.

Gauss an Schumacher.

Göttingen, 1. IX. 1846.

s. Briefw. Gauss-Schumacher, Bd. 5 (1863), p. 196.

Ich lebe [achtzigjährig] noch ganz gern. Aber von der andern Seite bin ich doch ein Conviva satur, der alles Gute was dieses Erdenleben darbieten kann, hinreichend genossen und geschmeckt hat, und nun ohne Widerwillen davon Abschied nehmen kann: einen Abschied, den das Gefühl, jetzt ein ganz unnützes und völlig überflüssiges Mitglied der menschlichen Gesellschaft zu sein, und die Neugierde, einmal selbst zu erfahren, was aus dem Menschen nach dem zeitlichen Tode wird, noch mehr erleichtern werden.

Olbers an Bessel.

Bremen, 5. VII. 1838.

s. Briefw. Olbers-Bessel, herausg. v. Erman (1852), Bd. 2, p. 427.

Gauss sprach sich eines Tages über die persönliche Fortdauer nach dem Tode so aus: „Es gibt in dieser Welt einen Genuss des Verstandes, der in der Wissenschaft sich befriedigt und einen Genuss des Herzens der hauptsächlich darin besteht, dass die Menschen einander die Mühsale, die Beschwerden des Lebens sich gegenseitig erleichtern. Ist das aber die Aufgabe des höchsten Wesens, auf gesonderten Kugeln Geschöpfe zu erschaffen und sie, um ihnen solchen Genuss zu bereiten 80 oder 90 Jahre existiren zu lassen, so wäre das ein erbärmlicher Plan (- das Problem wäre wie er sich ein anderes Mal ausdrückte schofel gelöst.) $\mathrm{Ob}$ die Seele 80 Jahre oder 80 Millionenjahre lebt, wenn sie ein Mal untergehen soll, so ist dieser Zeitraum doch nur eine Galgenfrist. Endlich würde es vorbei sein müssen. Man wird daher zu der Ansicht gedrängt, für die ohne eine streng wissenschaftliche Begründung so Vieles andere 
spricht, dass neben dieser materiellen Welt noch eine andere zweite rein geistige Weltordnung existirt mit ebenso viel Mannigfaltigkeiten als die in der wir leben - ihr sollen wir theilhaftig werden."

Sartorius v. Waltershausen. "Gauss zum Gedächtniss" (1856), p. 103.

Le but de l'homme n'est pas cette vie; ses plus nobles facultés se rapportent à une autre existence; elles seraient de vrais contre-sens dans l'être borné destiné à une durée si bornée, ces facultés qui s'élèvent à l'infini et saisissent l'éternité. Il n'y a pas, je le répète, de contre-sens pareil possible.

André-Marie Ampìre à son fils Jean-Jacques

Paris 1818.

voir „André-Marie Ampère et Jean-Jacques Ampère“. Correspondance et souvenirs recueillis par Madame H. C.,

2. éd. (Paris 1875), t. 1, p. 143.

Ozanam disait en propres termes, qu'il appartient aux Docteurs de Sorbonne de disputer, au Pape de prononcer, et au Mathématicien d'aller en Paradis en ligne perpendiculaire.

Fontenelue.

„Éloges des académiciens de l'académie des sciences, morts depuis l'an 1699", t. 1 (1766), p. 516.

It is not at all improbable that Sir Is a ac Newton, though so great a man, might have had a hankering after the French prophets. $^{1}$ ) There was a time when he was possessed with the old fooleries of astrology; and another when he was so

1) aus Frankreich gekommene epileptische Sektierer, welche den Anbruch eines tausendjährigen Reiches und einer allgemeinen, über die ganze Erde verbreiteten Theokratie predigten. 
far gone in those of chemistry, as to be upon the hunt after the philosopher's stone.

The Rev. J. Spence.

„Anecdotes, observations, and characters, of books and men. Collected from the conversation of Mr. Pope, and other eminent persons of his time," Second edit. by S. W. Singer (London 1858),

p. 54; see also l. c. p. 43 and 81 .

Huyghens ne chercha pas même à voir les autres satellites [de Saturne découverts par Cassini après Huyghens]; on aura peine à croire par quelle raison: cet homme célèbre tenait encore à des préjugés antiques .... Il croyait que le nombre des satellites ne devait pas surpasser celui des planètes principales.

\section{Condoreter.}

„Éloge d'Huyghens.“

voir Oeuvres de Condorcet, Édition d'Arago, t. 2 (1847), p. $62 / 63$.

Ich bin sehr aberglïubisch, allein ich schäme mich dessen gar nicht, so wenig als ich mich schäme zu glauben, dass die Erde stille steht. - - -

Jedes Kriechen eines Insects dient mir zur Antwort auf eine Frage über mein Schicksal. Ist das nicht sonderbar von einem Professor der Physik?

\section{LiCHTENBERG.}

Ausgew. Schriften, Reclam-Bibl. No. 1286-1289, p. 27 und 16.

Die jetzigen Tagsthorheiten habe ich ziemlich mit Gleichmuth betrachten, ja über einige Genrebilder wie die Versuche der Heidelberger Juristenfacultät mit dem Tischdrücken herzlich lachen können. Ich bin seit langer Zeit gewöhnt, von der Gediegenheit der höhern Cultur, welche die s. g. höhern Stände durch Lesen populärer Schriften oder Anwohnen populärer 
Vorlesungen erwerben zu können glauben, wenig zu halten. Ich bin vielmehr der Meinung, dass in wissenschaftlichen Gebieten probehaltige Einsicht nur durch Aufwendung eines gewissen Maasses eigner Anstrengung und eigner Verarbeitung des von andern dargebotenen erlangt werden kann.

C. Gauss an A. v. Humboldt.

Göttingen, 10. V. 1853.

s. K. Bruhns, „Briefe zw. A. v. Humboldt und Gauss" (1877), p. $66 / 67$

$=$ Karl Brubns, „Alexander von Humboldt“, Bd. 2

(1872), p. 432/433.

In diesen Tagen las ich, in Kästner's gesammelten schönwissenschaftlichen Werken (Berliner Ausgabe von 1841 in 4 Bänden) ${ }^{1}$ ), dass Tobias Mayer von den Ärzten ungefähr eben so gedacht hat wie ich, oder vielmehr viel schlimmer noch. Von ihm rührt der Ausspruch her, die Astronomen hätten sehr unklug gehandelt, dass sie selbst den Glauben an die Astrologie bei den Layen zerstört hätten; und als Kästner diesen Ausspruch nur mit Lachen, wie über einen Spass, erwiderte, fuhr Mayer fort, die Ärzte hätten es viel klüger gemacht; ihre Wissenschaft sei nicht mehr werth als die Astrologie, aber sie hätten es besser verstanden, das Volk beim Glauben zu erhalten.

Gauss an Schumacher.

Göttingen, 22. XII. 1845.

s. Briefw. Gauss-Schumacher, Bd. 5 (1863), p. 95.

Früher führte man den Studenten der Medizin ins Collegium logicum, und es muss zugegeben werden, dass eine Vorlesung über Logik die Lust am Denken fördern kann. Aber nötig ist Logik zum Denken ebensowenig wie Kenntnis der Magenphysiologie für die Verdaunng; dazu kommt, dass der zu behandelnde Gegenstand gar weit abliegt von dem sonstigen Ge-

1) s. 1. c., Th. 4 , p. $166 / 167$. 


\title{
$-395-$
}

sichtskreis des Mediziners. Am besten eignete sich vielleicht eine „Einführung in die höhere Mathematik“. Denn die Mathematik wird in naher Zukunft eine Hauptrolle in der Medizin spielen: schon mehren sich die Anzeichen dafür, dass Physiologie und erklärende Anatomie, Pathologie und Therapie der mathematischen Legitimation nicht entraten können.

\section{Max Dessoir}

\author{
[Dr. phil. et med., Prof. der Philosophie]. \\ "Der Beruf des Arztes", Westermann's Monatshefte 77, \\ $1894 / 1895$, p. 380.
}

Das Studium der Mathematik entfaltet seine bildende Kraft vollauf erst mit dem Übergange von den elementaren Lehren zur analytischen Geometrie. Unstreitig gewöhnt schon einfachste Geometrie und Algebra den Geist an scharfes quantitatives Denken, sowie daran, nur Axiome oder schon Bewiesenes für richtig zu nehmen. Die Darstellung von Functionen in Curven oder Flächen aber eröffnet eine neue Welt von Vorstellungen und lehrt den Gebrauch einer der fruchtbringendsten Methoden, durch welche der menschliche Geist seine eigene Leistungsfähigkeit erhöhte. Was die Erfindung dieser Methode durch Viète und Descartes der Menschheit ward, das wird Einführung in sie noch heute jedem für diese Dinge nur einigermaassen Begabten: ein für das Leben epochemachender Lichtblick. Diese Methode wurzelt in den letzten Tiefen menschlicher Erkenntniss und hat dadurch an sich ganz andere Bedeutung, als der sinnreichste, einem besonderen Falle dienende analytische Kunstgriff. - - - - - - - - - - -

Der mathematische Lehrplan des Gymnasiums müsste die Discussion der Gleichung zweiten Grades und einige andere ebene Curven umfassen, wie auch durch die Tangententheorie den Blick in die Differentialrechnung eröffnen. Hierzu müssten freilich der Mathematik mehr Stunden, statt vier sechs bis acht, eingeräumt werden. Bei den Versetzungs- und Reifeprüfungen müsste Mathematik den alten Sprachen und der Geschichte wirklich gleichstehen. Die Gleichberechtigung der 
Mathematik-Lehrer mit den Lehrern jener Fächer würde dann auch eine Wahrheit werden.

Emil، DU BoIs-ReYmond.

"Culturgeschichte und Naturwissenschaft", Vortr. Köln 24.III.1877. s. Reden, 1. Folge (1886), p. 287 u. 292.

Könnte man nicht allgemein im Lehrplan wenigstens der Realanstalten ausreichenden Raum für die Anfangsgründe der Differential- und Integralrechnung, sowie der analytischen Geometrie, - also den sogenannten niederen Teil der höheren Mathematik -, vorbehalten? - _ - _ - _ - -

Es würde sich zunächst darum handeln, gewisse Kategorien Studierender, die nicht gerade weitgehende mathematische Kenntnisse brauchen, an der Hochschule von den mathematischen Studien überhaupt zu entlasten, oder auch ihnen die erforderlichen mathematischen Kenntnisse in einer Weise zugänglich zu machen, wie es an der Hochschule aus allerlei Gründen doch nicht erreicht wird. Ich rechne dahin die Architekten, dann aber auch die Chemiker und überhaupt die Studierenden der sog. beschreibenden Naturwissenschaften. Ich meine aber auch - und das ist eine alte Lieblingsidee von mir —, dass es den Studierenden der Medizin sehr nützlich wäre, mathematische Kenntnisse in dem bezeichneten bescheidenen Umfange zu erwerben; scheint es doch unmöglich, weitergehende physiologische Forschungen zu verstehen, wenn man zurückschreckt, sobald man ein Differential- oder Integralzeichen sieht!

\section{Felix Klein.}

s. „Verhandl. üb. Fragen des höheren Unterrichts, Berlin, 6.-8. Juni 1900", 2. Aufl. (Halle a. S. 1902), p. 154

$=$ Deutsche Mathem.-Verein. Jahresber. 11 (1902), p. 131

$=$ Zeitschr. math. naturw. Unterr. 33 (1902), p. 116/117.

Nach meinen Wahrnehmungen existirt unter der jüngeren Generation eine Kategorie, welche Logik in höherem Masse 
entwickelt. Das sind sonderbarerweise die jungen Juristen. Die fangen früher an in strenger Weise logisch zu denken als die Zöglinge anderer Fächer. Wir bringen es in der Medizin nicht zu Stande, so regelrechte, logische Sätze zu hören, so korrekte Ausführungen zu einem bestimmten Zwecke durchgeführt zu sehen, als das bei den Juristen häufig der Fall ist.

Der Jurist mit seinem Gesetzbuch ist glücklich daran; er bewegt sich in Formen, die sehr genau überlegt, von erprobten, erfahrenen Männern vorgesehen sind, und er lernt sie auch. So etwas haben wir anderen nicht. Die einzige Disciplin, welche dem ungefähr entspricht, ist die Mathematik. Da giebt es nicht die vielen Handbücher, man kann nicht auf verschiedene Weise rechnen, es giebt nur eine Möglichkeit des korrekten Rechnens, und wer überhaupt rechnen will, muss danach verfahren. Daher werden die Mathematiker besser geschult als die anderen, weil diese bei ihrem Umhertappen bald dies, bald jenes ergreifen, ohne rechten Fuss fassen zu können.

\section{Rudolf Virchow}

im preuss. Abgeordnetenhause am 13. März 1899. s. Stenogr. Ber. 1899, Bd. 2, p. 1385.

Ich habe gezeigt, dass die Quadrate aller Primzahlen $>3$ von der Form $6 \mathrm{n}+1$ sind. - - - -

Auf Vereinfachungen, welche die Lehre von der Teilbarkeit grosser Zahlen durch die in dieser Mitteilung begründete Ordnung aller Zahlen in sechsgliedrige Reihen erfährt, will ich nicht eingehen. Andere in der Zahlentheorie besser Bewanderte werden damit viel eher zum Ziele kommen, als einem mehr mit empirischen Fragen beschäftigten Physiologen möglich ist.

\section{W. Preyer.}

„Über den Ursprung des Zahlbegriffs" etc.

in Festschrift zu Helmboltz' 70. Geburtstag (1891), p. 35 u. 36. 
Ich erklärte mir selbst meine guten Erfolge [- in der medicinischen Forschungsperiode - ] wesentlich aus dem Umstande, dass ich durch ein günstiges Geschick als ein mit einigem geometrischen Verstande und mit physikalischen Kenntnissen ausgestatteter Mann unter die Mediciner geworfen war, wo ich in der Physiologie auf jungfräulichen Boden von grosser Fruchtbarkeit stiess, und andrerseits durch die Kenntniss der Lebenserscheinungen auf Fragen und Gesichtspunkte geführt worden war, die gewöhnlich den reinen Mathematikern und Physikern fern liegen.

\section{Hermann von Helmholtz.}

s. „Ansprachen u. Reden gehalten bei der am 2. XI. $1891 \mathrm{zu}$ Ehren von Hermann von Helmholtz veranstalteten Feier"

(Berlin 1892), p. 53.

Als im Jahre 1871 durch den Tod von Magnus die Professur der Physik in Berlin erledigt war, konnte nur Helmholtz berufen werden. Er folgte dem Rufe, vielleicht auch, weil er vorauszusehen glaubte, dass ihn die weitere Beschäftigung mit der Physiologie zu vivisectorischen Arbeiten führen müsste, welche seiner Gemüthsrichtung weniger zusagten.

\section{G. Wiedentann.}

„Hermann von Helmholtz' Wissenschaftliche Abhandlungen“ in Helmholtz, „Wissensch. Abhandl.", Bd. 3 (1895), p. XXIV.

Magnus ... betrachtete die experimentelle und die mathematische Physik als getrennte Gebiete. Im Gegensatz zu der Königsberger Schule warnte er wiederholt vor zu eingehender Beschäftigung mit der Mathematik, ja auch vor den Arbeiten seiner eigenen Schüler, welche etwa ein Gebiet der Physik mit einem anderen scheinbar ferner liegenden vereinigten.

\section{G. Wiedemann.}

"Hermann von Helmholtz' Wissenschaftliche Abhandlungen" in Helmholtz, „Wissensch. Abhandl.“, Bd. 3 (1895), p. XIII/XIV. 
Die Chemie ist der unreinliche Theil der Physik.

Peter Riess.

s. A. W. v. Hofmann, "Zur Erinnerung an vorangegangene Freunde", Bd. 2 (1889), p. 177.

Je ne m'attends pas que mes idées soient adoptées tout d'un coup; l'esprit humain se plie à une manière de voir, et ceux qui ont envisagé la Nature sous un certain point de vue, pendant une partie de leur carrière, ne reviennent qu'avec peine à des idées nouvelles; c'est donc au temps qu'il appartient de confirmer ou de détruire les opinions que j'ai présentées: en attendant, je vois avec une grande satisfaction, que les jeunes gens qui commencent à étudier la Science sans préjugé, que les Géomètres et les Physiciens [Laplace, Monge, Meusnier etc.] qui ont la tête neuve sur les vérités chimiques, ne croient plus au phlogistique dans le sens que $S^{2} \operatorname{lalh}^{1}$ ) l'a présenté, et regardent toute cette doctrine comme un échafaudage plus embarrassant qu'utile pour continuer l'édifice de la Science chimique.

\section{LAVOISIER.}

"Réflexions sur le phlogistique",

Paris Mém. de l'acad. roy. des sciences 1783 (Paris 1786), p. 538.

Il ne leur a fallu qu'un moment, me disait Lagrange, pour faire tomber cette tête [Lavoisier], et cent années peut-être ne suffiront pas pour en reproduire une semblable.

\section{Delambre.}

„Notice sur la vie et les travaux de M. Lagrange." voir Mém. de la classe des sc. mathém. et phys. de l'Institut 13, 1812, Histoire, p. LXVII $=$ Oenrres de Lagrange, t. 1 (1867), p. XL.

1) Lavoisier schreibt überall Stalh statt Stahl. 
Noch neulich hörte ich einen unserer hervorragendsten Chemiker sich dahin äussern, dass Lavoisier und Bunsen keine Chemiker, sondern Physiker waren, und letzterer drückte den inneren Zusammenhang beider Wissenschaften gelegentlich durch den Satz aus: „Ein Chemiker, der kein Physiker ist, ist gar nichts."

\section{J. H. VAN'T HoFF.}

"Über die Entwicklung der exacten Naturwissenschaften im 19. Jahrhundert", Vortr. Naturf.-Vers. Aachen 1900.

s. „Verhandl. d. 72. Vers. d. Ges. deutscher Naturf. u. Ärzte“, Th. 1, p. 31 .

Es ist lustig zu sehen, wie sich in die allgemeine norwegische Gutmütigkeit ein kindliches Selbstvertrauen auf eigene Denkkraft einmischt, worüber sich zu ärgern verkehrt wäre. Ein . Kandidat, der jetzt . . in Berlin sein soll und Abel heisst, schreibt in dem Norwegischen Magazin, „dass die Berliner öffentliche Bibliothek an Reichtum an mathematischen Büchern der norwegischen Universitätsbibliothek weit nachstehe, ${ }^{1}$ ) und im allgemeinen wisse man in Deutschland wenig Mathematik, die Kenntnisse der Mehrzahl erstreckten sich nur auf ein bischen Geometrie und auf die Kombinationslehre". - Unsere lieben Kollegen in Christiania sind recht gute Leute, aber es scheint, als würde es ihnen gut thun, etwas in die Welt hinauszukommen und zu lernen, sich gebiuhrend zu betragen.

Berzelius an Wöhler [damals in Berlin]. 28. ШI. 1826.

s. „Briefw. zw. J. Berzelius und F. Wöhler", herausg. v. O. Wallach, Bd. 1 (Leipzig 1901), p. 115.

1) vgl. hierfür auch einen Brief N. H. Abel's an Hansteen (Berlin, 5. XII. 1825) in "Niels Henrik Abel, Mémorial publié à l'occasion du centenaire de sa naissance": „Correspondance d'Abel" (Traduction française), p. 10. 
Arago. Er war mir einer der interessantesten, obgleich wir ${ }^{1}$ ) nur wenige Mal, nur bei Gay-Lussac und bei Thénard so wie im Institut ihn zu sehen Gelegenheit hatten. Er ist in der That einer der schönsten Männer, die ich gesehen habe, und ist wohl von allen der geistreichste. Aber immer noch ein wüthender Republicaner. - - - - - - -

Ampère. Ein Original wie es wohl wenige mehr gibt. Ein ziemlich grosser alter Mann, vom Alter etwas gebückt, mit dicker hängender Unterlippe, ziemlich zahnlos, mit hervorstehenden, stier blickenden Augen, eine Perrïcke, die hier und da den Kahlkopf durchblicken lässt, gekleidet in schwarzem Frack, der sehr alt und abgeschabt ist, und die Wäsche stets braun von Schnupftabak, den er in zwei Dosen mit sich führt. Dessen ungeachtet war mir dieser Mann einer der merkwürdigsten und respectabelsten. Den Neckereien und Witzen, die er von den anderen alten, namentlich von Arago und Thénard, zu erdulden hat, entgegnet er mit einer grossen Gutmüthigkeit und nicht selten mit komischem Witz. Nichts verdriesst ihn, und er bleibt stets in demselben guten Humor. Er ist ohne Zweifel einer der tiefsten speculativen Köpfe und scheint eine ungeheuere allgemeine Gelehrsamkeit zu besitzen. Er ist selbst in den neuesten ch e mi s chen Entdeckungen ganz im Detail zu Haus.

WöHLER nach seiner Pariser Reise (1833) an Berzelius. Cassel, 13. X. resp. 27. X. 1833.

s. „Briefw. zw. J. Berzelius und F. Wöhler“, herausg. v. O. Wallach, Bd. 1 (Leipzig 1901), p. 529 u. 534.

An und für sich ist die Mathematik niemals schaffend, sie ist nicht productiv, sie verarbeitet stets nur das Gegebene, sie ist die Dienerin des Gegebenen, kein neuer Gedanke kann sich aus ihr entwickeln, sie flösst keine Gedanken ein; ein begeisterter Mathematiker, ein höherer Verstand, welcher neue Gedanken schafft, gehört der Mathematik nicht mehr an, er wird zum

1) G. Magnus und Wöhler.

Ahrens, Scherz und Ernst i. d. Mathem. 
Naturforscher. Wenn sie irgendwo Nutzen schafft, so geschieht dies durch ihre Anwendungen.

Ein jeder Mensch, dem die Natur Vernunft gegeben hat, kann bei einiger Gedächtnissgabe sich mit allen Lehrsätzen der Nathematik auf's vollkommenste bekannt machen; er kann es in ihrer Handhabung durch Übung zu einer grossen Fertigkeit bringen, ohne im Stande zu sein, sich die Lösung auch nur der kleinsten praktischen Aufgabe zu setzen, ohne mit einem Worte in irgend einer Weise nützlich zu sein. - - -

Sobald er aber die Fühigkeit und das Talent besitzt, eine höhere Frage zu lösen und die Wahrheit seiner Resultate selbst zu prüfen; so hört er auf Mathematiker zu sein, er wird zum Naturforscher.

Man bemerkt leicht, wie sich hier die Mathematik von der Naturforschung trennt, und dass ein hoher Grad von Einbildungskraft, Scharfsinn und Beobachtungsgabe sich mit der Mathematik verschwistern müsse, um einen Physiker hervorzubringen; allein im gewöhnlichen Leben geht es wie in allen Dingen, der Effect wird mit der Ursache verwechselt. Man schreibt den Dampfmaschinen zu, was dem Feuer, den Steinkohlen, was dem menschlichen Geiste angehört. Es giebt keine Wissenschaft, in welcher sich mehr Geistesarmuth, mehr Unfähigkeit zum Denken, ein grösserer Mangel an wahrer Einsicht und Verstand, mehr Kurzsichtigkeit und Schwäche unter dem Mantel des Wissens und der Gelehrsamkeit versteckt hält, als in der Mathematik.

Wie oft muss nicht bei Facultätsprüfungen die beste Nummer in der Mathematik Examinanden gegeben werden, welche in allen übrigen Fächern nicht den einfachsten gesunden Menschenverstand bewähren. - $-\ldots$

Wie gering an Zahl sind Mathematiker, die ihre eigene Wissenschaft um einen kleinen Schritt nur voran gebracht haben, die etwas anderes als Lehrbücher hervorzubringen vermögen. Diese seltnen Männer, sie sind höher zu achten als die ausgezeichnetsten Forscher in irgend einem andern Felde, 
eben weil sie alles aus sich selbst und nichts von Aussen schöpfen.

\section{J. VON LIEBIG.}

„Über das Studium der Naturwissenschaften und den Zustand der Chemie in Preussen", 1840.

s. Liebig, "Reden und Abhandlungen", herausg. v. M. Carriere (Leipzig \& Heidelberg 1874), p. 15-18.

. ganz besonders fühle ich mich von Kronecker angezogen. Kronecker ist ein ganz kleines Männchen, aber in dem kleinen Körper wohnt ein grosser Geist, schlägt ein grosses Herz. Ich bin nur Wenigen von gleichem Scharfsinn begegnet. Keinem, der wie er befähigt und geneigt gewesen wäre, gelegentlich seinen Scharfsinn für die Lösung der Aufgaben auch seiner Freunde einzusetzen ${ }^{1}$ ). Man hat mir einmal, halb im Scherz, halb im Ernst den Vorwurf gemacht, ich sei ein Egoist, der seine Freunde in dem Maasse schätze, als er etwas von ihnen lernen könne. Dies trifft nun für Kronecker wirklich zu. Ich komme nie mit ihm zusammen, ohne das Gefühl eines geistigen Gewinnes mit mir hinweg zu nehmen. Dabei ist er keineswegs nur ein Verstandesmensch; den Ansprüchen des Gemüthes wird in gleicher Weise Rechnung getragen.

Aug. Wilhelm v. Hofmann [der Chemiker] an seinen Bruder Fritz.

Aug. 1865 [etwa 3 Monate nach Hofmann's Übersiedelung nach Berlin, wo er L. Kronecker erst kennen lernte].

s. Volhard u. Fischer, „August Wilhelm von Hofmann“, Sonderbeft der Ber. der Deutschen chem. Ges., Jahrg. 35 (Berlin 1902), p. 102.

Rien chez les hommes n'est aussi rare qu'une amitié parfaite et désintéressée. On n'en cite qu'un exemple: celui de la fable.

\section{PoInsot.}

voir Bertrand, „Éloges académiques", Nouvelle série (Paris 1902), p. 19.

1) vgl. hierzu jedoch S. 325 (Weierstrass). 
Am ersten Mittwoch des October lud mich Sturm mit Bertrand und Catalan zum Frühstück. . ein. . Sturm platzte mit dem Urtheil heraus: dass Ihr Aufsatz über elliptische Transcendenten in Grunert's Archiv [Bd. 14 (1850)] das Beste wäre, was er über denselben Gegenstand gelesen habe. Potz Donnerwetter! wie zuckte das mir durch die Glieder.

Als ich von Ihren andern Arbeiten (Wiener Abhandlungen) [Denkschriften Wiener Akad. Bd. 4, 1852] sprach, musste ich - zu Ihrem grossen Leid! - auch Ihre unmenschliche Seite berühren, zur Erklürung, warum Sie noch keine Exemplare geschickt haben und meine Befehle fruchtlos blieben. Da baten mich Sturm und Bertrand, die Deutsch lesen, Sie nochmals zu puffen, was sogleich (folgenden Tags) ich zu thun versprach; aber es gelang mir nicht, - auch entmuthigte mich die wahrscheinliche Erfolglosigkeit. Hätte ich Macht über den Ziegel ${ }^{1}$ ), so würde ich jetzt noch donnernd befehlen sogleich Exemplare zu schicken und zwar an: 1) die Akademie, 2)Poncelet, 3)Lamé, 4)Liouville, 5)Chasles, 6) Bienaymé, 7) Binet, 8) Hermite, 9) Bertrand, 10) Serret, 11) Dr. Wertheim, 12) Abbé Moigno, 13) Dr. Wöpcke (arabisch drauf), 14) Terquem (ein Wort hebräisch drauf), und Andere. Wenn dieser Befehl binnen 8 Tagen nicht ausgeführt wird, so hol Sie - - - -

\section{STEINER an Schläfli.}

Berlin, 20. IV. 1856.

s. J. H. Graf, „Der Briefwechsel zwisehen Jakob Steiner und Ludwig Schläfli", Bern Naturf. Gesellsch. Mittheil. 1896, p. 253.

Personne ne parle de nous en notre présence comme i] en parle en notre absence. L'union qui est entre les hommes n'est fondée que sur cette mutuelle tromperie.... Si tous

1) Übername, den Steiner Schläfli gab, weil der letztere auf äusser Formen nicht viel Gewicht legte [Anm. von J. H. Graf, l. c. p. 103]. 


\section{$-405-$}

les hommes savaient ce qu'ils disent les uns des autres, il n'y aurait pas quatre amis dans le monde.

Pascal.

„Pensées", Edition complète de J.-F. Astié (Paris 1883), p. 385.

Lie's gerades offenes Wesen war jeder Verstellung feind und erweckte unwillkürlich Vertrauen. Man fühlte vom ersten Augenblicke an, dass man ihm gegenüber ganz offen sein durfte, dass er sich so gab, wie er war. Über ihm unsympathische Persönlichkeiten sprach er sich mit rückhaltloser Offenheit aus: besonders war ihm jede Art der Intrige verhasst, er war daher mit der Benennung Intrigant ziemlich freigebig und überhaupt wohl etwas zu sehr geneigt, Intrigen zu wittern.

F. ENGeL.

"Zur Erinnerung an Sophus Lie",

Rede Kgl. Sächs. Ges. d. Wissensch. 14. XI. 1899.

s. Leipziger Ber. 51 (1899), Math.-phys. Cl., p. LII.

Quand Lagrange parlait, c'était toujours sur le ton du doute, et sa première phrase commençait ordinairement par je ne sais pas.

\section{Delambre.}

"Notice sur la vie et les ouvrages de M. Lagrange."

voir Ném. de la classe des sciences mathém. et phys. 13, 1812, Histoire de la classe mathém., p. LXXVI $=$ Oeurres de Lagrange, t. 1 (1867), p. XLVIII.

Lorsqu'une fois Lambert avoit entamé une discussion, quelle qu'elle fût, il n'étoit plus possible de l'arrêter ou de l'interrompre: on étoit sûr que dès le début, il voyoit si bien le plan qu'il avoit à suivre, et y étoit si fidèle, que rien ne pouvoit l'en détourner. L'ordre de ses idées étoit toujours régulier et parfait: si on lui faisoit quelques objections, il ne s'arrêtoit qu'autant qu'il falloit pour laisser dire ce que l'on vouloit: mais jamais il n'y répondoit: il reprenoit la suite de son raisonnement comme si on ne l'eût pas interrompu, parce que l'objection qu'on lui avoit faite, devoit se retrouver dans 
un moment et dans un ordre plus convenable, et que la discussion n'auroit eu qu'à perdre à s'écarter du plan qu'il s'étoit tracé d'abord. Je l'ai mis cent fois à l'épreuve à cet égard, et jamais je n'y ai été trompé. C'étoit vraiment une machine à dissertations, mais une machine parfaite. ${ }^{1}$ )

\section{Thiśbaulit.}

„Mes souvenirs de vingt ans de séjour à Berlin, t. 5 (Paris 1804), p. 30/31.

Étant un soir avec ses amis, Camille Jordan ${ }^{2}$ ) et Degérando ${ }^{3}$, Ampère se mit à leur exposer le système du monde; il parla treize heures avec une lucidité continue; et comme le monde est infini, et que tout s'y enchaîne, et qu'il le savait de cercle en cercle en tous les sens, il ne cessait pas, et si la fatigue ne l'avait arrêté, il parlerait, je crois, encore. O Science! voilà bien à découvert ta pure source sacrée, bouillonnante! - Ceux qui l'ont entendu à ses leçons, dans les dernières années au Collége de France, se promenant le long de sa longue table ............ et discourant durant des heures, comprendront cette perpétuité de la veine savante. Ainsi en tout lieu, en toute rencontre, il était coutumier de faire, avec une attache à l'idée, avec un oubli de lui-même qui devenait merveille. Au sortir d'une charade ou de quelque longue et minutieuse bagatelle, il entrait dans les sphères.

SAInte-Beuve.

„M. Ampère“, Revue des deux mondes (4) 9 (1837), p. 421/422.

La conversation de Sophie Germain avait l'élégance d'une belle formule de Laplace.

Fayolle et Parisot.

"Sophic Germain“ daus „Biographie universelle“ (Michaud), t. 16 (1856), p. 333.

1) s. a. eine ähnliche Äusserung von Joh. III. Bernoulli über Iambert bei R. Wolf, „Biographien zur Kulturgesch. der Schweiz“, Bd. 3 (1860), p. 345 .

2) s. S. 341, Anm. 2.

3) de Gérando. Philosoph, Freund und Verwandter des ersteren. 
J'ai soupé dans une des meilleures maisons de Paris avec M. de Mairan; c'est un petit homme fort doux, d'une grande politesse, qui parle avec beaucoup d'aisance, qui dit de fort bonnes choses, et n'a rien de l'encolure du géomètre. Il y a autant de différence de sa conversation à celle de M. Euler qu'il y en a entre les écrits d'Horace et ceux du savantissime et pédantissime Wolffius ${ }^{1}$ ).

Marquis d'Argens à Frédéric le Grand.

Paris, 15. VIII. 1747.

voir Oeuvres de Frédéric le Grand, t. 19 (Édition Decker 1852), p.18/19.

Assis [un jour] à la table de personnes que tout lui commandait de ménager, Ampère se crut un moment dans sa propre maison et s'écria, avec l'accent d'un mécontentement profond: „Vraiment, ce dîner est détestable! Ma soeur comprendra-t-elle enfin qu'elle a tort d'accepter des cuisinières, sans s'être assurée de leur savoir-faire!“

\section{Arago.}

Oeuvres, t. $2(1854)$, p. $93 / 94$ = Werke, Bd. 2 (1854), p. 76 .

Plücker kam mir zuerst etwas vornehm vor, wie es die Bonner Professoren meistens sein sollen, nachher aber wurde er ganz gemüthlich, als er auf den Einfall kam, meine Anwesenheit durch eine ganz besonders gute Flasche Wein zu verherrlichen. Hesmioltz über seine erste Begegnung mit Plücker, in Bonn 1853.

s. Leo Königsberger, „Hermann von Helmholtz“, Bd. 1 (1903), p. $193 / 194$.

Ich finde nichts abscheulicher, als die Gesellschaft fremder Leute; jedes Vergnügen kann sie mir verbittern und selbst

1) Chr. Wolff (auch Wolf, vgl. S. 224), + 1754 . 
nach unserem Olbers gehe ich, wenn Mehrere dort sind, nur im äussersten Notfalle. ${ }^{1}$ )

Bessel 1805.

s. „Bessel als Bremer Handlungslehrling“, herausg. v. d. Gesellsch. [Kaufm. Verein] „Union“ (1890), p. 74.

Einige Stunden mit Ihnen $^{2}$ ), theurer Freund, sind mir lieber als alle Sectionen der sogenannten Naturforscher, die sich in solchen grossen Massen und so gastronomisch bewegen, dass des wissenschaftlichen Verkehrs für mich nie genug gewesen ist. Ich habe mich am Ende immer gefragt wie der Mathematiker am Schluss der Oper ,en dites-moi franchement ce que cela prouve“.

\section{AL. Humboldt an Gauss.}

Teplitz, 27. VII. 1837.

s. K. Bruhns, "Briefe zw. A.v. Humboldt und Gauss" (Leipzig 1877), p. 30.

Die einzelnen Vorträge [in den Sektionen] beschlugen meist die höchsten Probleme der Mathematik, wo der Vortragende sicher zu Hause ist, aber nicht verlangt werden darf, dass jeder Zuhörer es auch sei. Darum machte einer die Bemerkung, es würde oft genügen, das Trapez zu besteigen, sich vorzustellen und nach Abgabe der Visitenkarte zu verschwinden.

\section{J. H. GraF.}

„Rückblicke und Ausblicke bei Anlass des ersten intern.

Mathematikerkongresses . . in Zürich."

s. Zeitschr. math. naturw. Unterr. 28 (1897), p. 554 [dort angegeben als abgedr. aus dem Berner „Bund“ (16. VIII. 1897)].

Kaum der tausendste Teil der reinen, also von allen Anwendungen abgesonderten Mathematik ist mehr als hohler nichtssetzender Spekulationskram. Sicherlich sind 999 Teile die taiube Frucht alberner Kombinationen und geistleerer Ausspinnungen, die keinen Zuwachs an charakteristischer Wahrheit ergeben. Denkt man sich nun diese Masse von Geröll noch

1) vgl. S. 76 .

2) aus Anlass der Univ.-Saecularfeier in Göttingen 1837, die mit der Naturf.-Vers. in Prag zeitlich zusammenfiel. 
durch die verfehlten, ja oft ungereimten Anwendungen und müssigen Spielereien in Mechanik, Physik und Chemie vermehrt, so begreift man, wie die gegenwärtig uns überall in die Augen fallenden Schutthaufen übel gestalteter und ebenso übel angebrachter Mathematik sich haben auftürmen können. . . . Die Mathematik ist diejenige Wissenschaft, mit der man in den höhern kaleidoskopischen Würfeleien der andressierten Kalkülroutine, wegen der geringen Zahl der an der jedesmal fraglichen Lektüre aktiv Beteiligten, das andre, mehr passiv hinnehmende Publikum am leichtesten düpieren kann.

Es giebt . . Narrheiten der Mathematik sogut wie Narrheiten der Philosophie, nur dass im ersten Gebiet diese Früchte die Ausnahme, im letzteren, wo das Delirieren zu den herkömmlichen Privilegien gehört, die Regel bilden.

\section{Eugen DüHRing.}

„Die Überschätzung der Mathematik und die Erschöpfung des Wesentlichen eines Wissenszweiges in einfachen Kombinationen." s. Zeitschr. math. naturw. Unterr. 14 (1883), p. 563 u. 564 [dort angegeben als abgedruckt aus des Verfassers „Logik und Wissenschaftstheorie"].

Fast noch grösser als in der Geometrie war Steiner im Schimpfen. Ich habe das Vergnügen gehabt, mit Männern zu verkehren, die unter den jetzt Lebenden in dieser Hinsicht eine hervorragende Stellung einnehmen, aber ohne Jemanden beleidigen zu wollen, muss ich gestehen, dass Keiner von ihnen auch nur im Entferntesten an den alten Steiner heranreichte. Seine originellen Ausdrücke, sein plastisches und drastisches Darstellungsvermögen kamen ihm dabei trefflich zu statten: legte Er los, so erbebten die Höhen des Olymps und Sonne, Mond und Sterne verbargen ihr Licht hinter den grollend aufziehenden Donnerwolken. Ich bin fest überzeugt, hätte er vor ein paar Jahrhunderten gelebt, so würden wir in ihm jetzt einen der grössten Theologen seiner Zeit verehren.

C. F. GEISER [Grossneffe Steiner's]. „Zur Erinnerung an Jakob Steiner“, Vortr. schweiz. naturf. Vers. Schaff hausen 22. VIII. 1873 (Schaff hausen 1874), p. 34. 
Ich rerlange auf die entschiedenste Weise und krafft meines Amtes von Ihnen, dass Sie Sich der entwürdigenden und niedrigen Schimpfworte in den Klassen durchaus enthalten, die Anspielungen auf die Religion der jüdischen Zöglinge durchaus unterlassen, sowie jede thätliche Äusserung Ihres Zornes und Ihrer Hitze durch Schlagen, Zupfen u. dergl. vermeiden. ....... Schon ist die Stadt voll von dem Gerede über Ihr gewaltiges Schimpfen - - - - - - - -

KLöDen, Direktor der Gewerbeschule zu Berlin, an J. Steiner, damals Oberlehrer an dieser Anstalt.

22. XII. 1831.

s. J. Lange, „Jacob Steiners Lebensjahre in Berlin“ (1899), p. 34.

Mit Stolz hob Steiner oftmals hervor, wie er auf den Weiden des entfernten, in blauem Schimmer erglänzenden Jura die Kühe sich habe bewegen sehen und nicht ohne ironische Befriedigung fügte er hinzu, dass ihm anch im spätern Alter das Rindvieh selbst auf grössere Distanzen erkennbar gewesen sei.

$$
\text { C. F. GeISER. }
$$

„Zur Erinnerung an Jakob Steiner", Vortr. schweiz. naturf. Vers. Schaff hausen 22. VIII. 1873 (Schaffhausen 1874), p. 4.

\section{[Weierstrass' erster Besuch bei Steiner].}

[Steiner:] „Sie kommen wohl hauptsächlich nach Berlin, um [Martin] Ohm kennen zu lernen?“

[Weierstrass:],Nein, ich wolltezu Ihnen und zu Dirichlet.“ [Steiner:] „Dann haben Sie Grütze im Kopf; wer Grütze hat, kommt zu Dirichlet und mir, die andern gehen zu Ohm." C. F. Geiser und L. Maurer.

„Elwin Bruno Christoffel“, Math. Ann. 54 (1901), p. 329, Anm.

.... le mathématicien $\mathrm{Ohm} . .$. , qui, lors de son arrivée à Berlin, ayant pris logement dans une rue depuis longtemps nommée la rue $0 \mathrm{hm}^{1}$ ), répondait d'un ton modeste, quand on

1) s. Note 20 am Ende des Buches. 
lui demandait son adresse: „On a bien voulu donner le nom de $\mathrm{Ohm}$ à la rue que j'habite".

J. Bertrand.

„Éloges académiques", Nouvelle série (Paris 1902), p. 318.

Dirichlet war in Breslau [1827-1828 als Privatdocent] zwar als fein gebildeter und liebenswürdiger junger Mann in allen geselligen Kreisen gern gesehen und gesucht, aber gerade als Mathematiker wurde er im Vergleich zu seinem Vorgänger, der ein Lehrbuch der analytischen Geometrie geschrieben hatte, nur gering geachtet.

Kummer.

"Gedächtnissrede auf Gustav Peter Lejeune Dirichlet." s. Abhandl. Berl. Akad. 1860, p. 12

$=$ Dirichlet, Werke, Bd. 2, p. 322 .

Lie war unter seinen Landsleuten als Fussgänger berühmt . . Freilich wusste man damals [1884] in Norwegen den Fussgänger Lie weit mehr zu schätzen als den Mathematiker.

F. ENGeL. „Zur Erinnerung an Sophus Lie",

Rede Kgl. Sächs. Ges. d. Wissensch. 14. XI. 1899. s. Leipziger Ber. 51 (1899), Math.-phys. Cl., p. LI.

Prof. Kummer hat zum Glück seine schoene Theorie der complexen Zahlen noch bei Zeiten durch Encke von der Akademie zurücknehmen lassen; denn sie enthielt zuviel Revolutionsstoff, ich wäre z. B. rasend geworden; man kann durch dieselbe beweisen, dass zu jeder Determinante nur ein e quadratische Form gehört und dergl. Unsinn mehr. Kummer hofft die Theorie leicht zu ergänzen [publ. in Berliner Ber. 1846, p. 87 bis 96]; es erhebt sich ein leiser Zweifel in meinem Gemüte. ${ }^{1}$ ) Eisenstein an M. A. Stern. (1844, etwa im Juli.)

s. Abh. zur Gesch. d. Math. 7 (1895), p. 173.

1) Die Zukunft hat bekanntlich Eisenstein nicht Recht gegeben [Anm. der Herausgeber des Briefwechsels, A. Hurwitz und F. Rudio]. 
Die Mannigfaltigkeit mathematischer Auffassungen und Interessen erschwert eine Bezugnahme von Mathematiker zu Mathematiker ausserordentlich. Die Verschiedenheit der Sprache tritt fast zurück hinter der Verschiedenheit der mathematisehen Denkweise.

\section{F. KLEIN.}

„Zur Frage des höheren mathematischen Unterrichts.“" s. Verhandl. d. 1. intern. Nathem.-Congr. Zürich 1897 (Leipzig 1898), p. 300.

In Betreff Berliner Neuigkeiten, so wissen Sie, dass Prof. Jakobi (der Grosse) für immer hier angestellt, und dass Prof. Dirichlet (der Liebenswürdige) . . . a aus Italien zurück ist Aber wir haben auch Prof. Kummer hier als Gast auf einige Zeit... Noch habe ich ihn nicht ordentlich geniessen kömnen, da er gleich nach der Insel Rügen abgereist ist, hoffe dies aber nach seiner Rückkehr hierher nachzuholen. . . . . . . Mein Freund Kronecker, dessen ich mich gegenwärtig, wie im vorigen Jahre des Heine, als meines wöchentlichen und tïglichen Hausfreundes bediene, arbeitet an seiner Promotion und ist jetzt nach Rügen gereist mit Kummer, da ihn diese Reise in seinem Fleisse aufhält, später geht er nach seinem Gute in Schlesien, um dort zu verbauern;... der reiche Dr. Borchardt, dessen unmenschliches Geld ihn ausserhalb des Bereiches aller menschlichen Berechnungen setzt _ _ _ _ -

Eisenstein an M. A. Stern.

Berlin, 20. VIII. 1845.

s. Abh. zur Gesch. d. Nath. 7 (1895), p. 176/177.

Schon längst hatte die französische Akademie das Perpetuum mobile in dieselbe Kategorie wie die Quadratur des Zirkels gestellt, und beschlossen keine angeblichen Lösungen dieses Problems mehr anzunehmen. Das muss doch als der Ausdruck einer unter den Sachverständigen weit verbreiteten Überzeugung angesehen werden. Ich selbst habe diese Überzeugung schon 


\section{$-413$}

während meiner Schulzeit oft genug aussprechen und die Unvollständigkeit der dafür $\mathrm{zu}$ erbringenden Beweise erörtern hören. Die Frage nach dem Ursprung der thierischen Wärme forderte eine sorgfältigere und vollständige Erörterung aller Thatsachen, die darauf Bezug hatten. Als ich an diese Arbeit [„Über die Erhaltung der Kraft" (1847)] ging, habe ich sie immer nur als eine kritische betrachtet, durchaus nicht als eine originale Entdeckung, um deren Priorität es einen Streit geben könnte. Ich war nachher einigermassen erstaunt über den Widerstand, dem ich in den Kreisen der Sachverständigen begegnete; die Aufnahme meiner Arbeit in Poggendorff's Annalen wurde mir verweigert, und unter den Mitgliedern der Berliner Akademie war es nur C. G. J. Jacobi, der Mathematiker, der sich meiner annahm.

\section{HeLmholtz 1881.}

s. Ostwald's Klassiker d. exakt. Wiss. Nr. 1 (Leipzig 1889), p. 58/59.

Einen hiesigen tapferen Mathematiker, der aber etwas confus in Bezug auf nicht mathematische Logik ist und der hierselbst die Mechanik vorträgt ${ }^{1}$ ), habe ich nach schwerem Kampfe endlich zur Erhaltung der Kraft bekehrt, so dass dieselbe an hiesiger Universität wohl officinell werden wird. Dem Neumann ist etwas schwer anzukommen, er ist hypochondrisch, scheu, aber ein Kopf ersten Ranges.

Helmholtz an Emil du Bois-Reymond.

Königsberg, 1850.

s. Leo Königsberger, „Hermann von Helmholtz“, Bd.1 (1903), p.117.

Der hervorragendste Zug in Helmholtz' wissenschaftlicher Gestalt ist. . . . sein transcendentes mathematisches Talent.

Während Brücke und ich, seine nächsten Freunde uns in die dem preussischen Gymnasiasten heute bekanntlich höheren

1) Im W. S. 1849/1850 las in Königsberg nur O. Hesse über Mechanik, im S. S. 1850 und W. S. $1850 / 1851$ niemand. 
Ortes untersagte analytische Geometrie ${ }^{1}$ ) auf eigene Hand hineinarbeiteten, ahnten wir nichts von der ungeheuren Stärke, welche damals noch, wohl ihm selber halb unbewusst, in ihm schlummerte, sondern erblickten in ihm nur einen besonders gescheidten Mediciner

Am 23. Juli 1847 trug er in der Physikalischen Gesellschaft seine Abhandlung ,iiber die Erhaltung der Kraft" vor, in welcher er sich zu unserem Erstaunen mit Einem Schlage als einen jeder Aufgabe gewachsenen Physico-Mathematiker offenbarte.

Es wäre ein Wunder gewesen, wenn eine Aufstellung von so unermesslicher Tragweite, durch welche die materielle Welt zu einem verständlichen Mechanismus wird, ohne Gegenrede geblieben wäre. Die älteren Berliner Physiker, Magnus, Dove, Riess, wollten nichts davon wissen, selbst Mathematiker, wie Lejeune-Dirichlet und Eisenstein schüttelten dazu den Kopf, nur Jacobi erwies sich einsichtiger. . . . Von hoher militärischer Seite wurden ihm [Helmholtz] die wärmsten Lobsprüche gespendet für die wichtige praktische Richtung, die er seinen Studien zu geben gewusst habe. Sein Gönner hatte nämlich geglaubt, dass es sich um die Erhaltung einer ganz anderen und für den Laien allerdings interessanteren Kraft handele, als der von Helmholtz gemeinten.

Emil du Bois-Reymond.

"Gedächtnissrede auf Hermann von Helmholtz", p. 4, 5, 9, 10 in Berl. Abhandl. 1896.

Die älteren Berliner Physiker, auch Poggendorff als Redacteur der Annalen der Physik und Chemie, erkannten den hohen Werth von Helmholtz' Arbeit [über die Erhaltung der Kraft] nicht. Indess ist ihnen daraus nicht, wie häufig geschieht, ein Vorwurf zu machen. Sie waren eben in der

1) vgl. S. 275 u. 395 . 
realistisch-empirischen Methodik aufgewachsen, fürchteten ein neues Eindringen der rein speculativen Richtung und konnten die Bedeutung so weittragender und umfassender Vorstellungen nicht in sich aufnehmen. Auch uns Zeitgenossen, als Schülern dieser Generation, wurde es nicht ganz leicht, uns sofort in dieselben hineinzufinden, dann aber erfüllten sie uns mit um so grösserer Begeisterung.

\section{G. Wiedemann.}

„Hermann von Helmholtz' Wissenschaftliche Abhandlungen“" in Helmholtz, „Wissensch. Abhandl.“, Bd. 3 (1895), p. XXVI.

Die Gelehrten der Pariser Akademie sollen im Jahre 1775, als sie beschlossen, kein angebliches perpetuum mobile mehr zu berïcksichtigen, dabei nicht nur an das mechanische, sondern an das physische perpetuum mobile gedacht haben. ${ }^{1}$ ) Wenn man aber den betreffenden Beschluss liest*, so sieht man sofort, dass die Akademie nur an das mechanische perpetuum mobile dachte. Und wenn man sich ein wenig mit der Geschichte der Physik beschäftigt hat, so weiss man, dass die Akademie damals auch an garnichts weiter denken konnte.

Mit solchen lächerlichen historischen oder vielmehr unhistorischen Ausführungen wollte von Helmholtz offenbar beweisen, dass im Jahre $1842^{2}$ ) an der Erhaltung der Energie eigentlich nicht mehr viel zu entdecken war, woraus man selbstverständlich schliessen soll, das Hauptverdienst bei der Entdeckung derselben habe in ihrer angeblich von ihm geleisteten mathematischen Entwickelung bestanden.

* Histoire de l'Académie royale 1775 . p. 65 . . .

Tн. Gross. „Robert Mayer und Hermann v. Helmholtz" (Berlin 1898), p. $165 / 166$.

l) s. S. 412 (Helmholtz).

2) Jahr des Erscheinens von Robert Mayer's „Bemerkungen üb. d. Kräfte der unbelebten Natur" in Liebig's Annalen d. Chem. u. Pharm. 
Nächst der fichlichen Virtuosität, mit welcher Helmholtz alle noch unerledigten Punkte des Mayerschen Programms und noch andere Aufgaben zu bewältigen weiss, tritt uns hier die volle kritische Klarheit des 26 jährigen Mannes überraschend entgegen. Seiner Darstellung fehlt das Ungestüm, der Impetus der Mayerschen. Ihm ist das Prinzip der Energieerhaltung kein a priori einleuchtender Satz. Was folgt, wenn er besteht? In dieser hypothetischen Frageform bewältigt er seinen Stoff.

Ich muss gestehen, ich habe immer den ästhetischen und ethischen Geschmack mancher unserer Zeitgenossen bewundert, welche aus diesem Verhältnisse gehässige.... personale Fragen zu schmieden wussten, anstatt das Glück zu preisen, das mehrere solche Menschen zugleich wirken liess, und anstatt sich an der so lehrreichen und für uns so fruchtbringenden Verschiedenheit bedeutender intellektueller Individualitüten zu erfreuen.

\section{E. MACH.}

"Über das Prinzip der Vergleichung in der Physik", Vortr. Naturf.-Vers. Wien 1894.

s. E. Mach, „Populär-wissensch. Vorlesungen“, 3. Aufl. (Leipzig 1903), p. 274/275.

F. Ch. Schlosser kam in der ersten Abtheilung des zweiten Theils seiner ,[Universalhistor. Übersicht der] Geschichte der alten Welt" (Frankfurt a. M 1828) auch darauf, die Euklidische Methode rïhmen zu wollen, und schrieb bei dieser Gelegenheit, in hochkomischer Zusammenstellung der Namen, S. 225 [f.] Folgendes: „Alle denkende Mathematiker von Archimedes bis auf $\mathrm{K}$ ästner haben diese Methode als die einzig richtige gepriesen". Archimedes und Kästner, in der That eine wunderliche Brüderschaft der Namen! Das Genie, welches uns beinahe allein das ganze mathematische Können des Alterthums repräsentirt, und dicht daneben in gleichheitlicher Zugesellung der seiner Keit sehr gangbare Göttinger Professor, von dem man aber jetzt nichts mehr weiss und dessen Bücher längst verschollen sind Wirklich sind für die Unerfahrenheit derjenigen, die eben nu 
nach der officiellen Schablone studiren, auch heute noch die Archimedes und Kästner von gleichem Gewicht und vielleicht giebt es auch heute Historiker, die geneigt sind, von einer Mechanik oder Physik von Galilei bis auf Helmholtz zu reden!

\section{Eugen DÜHRING.}

„Kritische Gesch. der allgem. Principien der Mechanik", 2. umgearb. Aufl. (1877), p. 550.

Ich möchte Faraday's Zeitgenossen nicht herabsetzen, weil seine Worte ihnen unbestimmt und dunkel erschienen; ich weiss selbst zu wohl, wie oft ich gesessen habe, hoffnungslos auf eine seiner Beschreibungen von Kraftlinien und von deren Zahl und Spannung starrend, oder den Sinn von Sätzen suchend, wo der galvanische Strom als eine Axe der Kraft bezeichnet wird, und Ähnliches mehr. ..... Es war ein Clerk Maxwell nöthig, ein zweiter Mann von derselben Tiefe und Selbständigkeit der Einsicht, um in den normalen Formen des systematischen Denkens das grosse Gebäude auszuführen, dessen Plan Faraday in seinem Geiste entworfen hatte, welches er klar vor sich sah und welches er sich bemühte seinen Zeitgenossen sichtbar zu machen.

\section{HeLnholtz.}

„Die neuere Entwickelung von Faraday's Ideen über Elektricität", Vortrag London 5. IV. 1871.

s. Vorträge u. Reden, Bd. 2 (1884), p. 277 u. 281.

Wie oft konnte man von Kirchhoff noch lange, nachdem er durch seine Spectralanalyse sich unsterblichen Ruhm erworben, die bescheidenen, aber wahren Worte hören: „Ich bin schon zufrieden, wenn ich nur eine Arbeit von Helmholtz verstehen kann, aber ich kann manche Punkte in seiner grossen akustischen Arbeit noch immer nicht enträthseln“.

Leo Königsberger.

„Hermann von Helmholtz", Bd. 2 (1903), p 13. 
Wenn ich nur wüsste, wie ich die Mathematiker dazu bringen könnte sich für die Transformationsgruppen und darauf begründete Behandlung der Differentialgleichungen zu interessiren. Ich bin so gewiss, absolut gewiss, dass diese Theorien einmal in der Zukunft als fundamental anerkannt werden. Wenn ich wünsche bald eine solche Auffassung zu schaffen, so ist es u. A. weil ich dann zehnmal mehr machen könnte.

Sophus Lie an A. Mayer 1884.

s. F. Engel, „Zur Erinnerung an Sophus Lie" in Leipziger Ber. 1899, Math.-phys. Cl., p. XLIX.

Auch die äusseren Ehren, die Lie von allen Seiten zuströmten, vermochten nicht seine [insbesondere seit einer nervösen Erkrankung (1889) gedrückte] Gemüthsstimmung zu verbessern. Dass alle bedeutenden Akademien ihn nach und nach zum Mitgliede wählten - eine Ausnahme machte bezeichnender Weise nur die Berliner Akademie - dass 1897 bei der ersten Verleihung des Lobatschefskijpreises der Preis ihm zuerkannt wurde, Alles das nahm er als einen schuldigen Tribut hin, ohne dass dadurch sein pessimistisches Urteil über die Menschen im Allgemeinen und über die Mathematiker im Besondern gemildert worden wäre.

\section{F. ENGeL.}

„Zur Erinnerung an Sophus Lie“,

Rede Kgl. Sächs. Ges. d. Wissensch. 14. XI. 1899. s. Leipziger Ber. 1899, Math.-phys. Cl., p. LVII

Wenn zu Lie's Leidwesen die Berliner Akademie wegen der ihr nicht zusagenden Form, in der er seine Arbeiten veröffentlichte, ihm nicht die Anerkennung zutheil werden liess die er bei seinen ausserordentlichen Gaben von ihr erwartete so ist einerseits zu bedenken, dass bei derartigen Ehrunger persönliche Beziehungen zunächst meistens eine Hauptroll spielen, andererseits dass die Veröffentlichung der systematischer Darstellung der Lieschen Entdeckungen erst 1893 beendet war 
also zu einer Zeit, wo die Sterne, welche am mathematischen Himmel der Berliner Universität so lange gestrahlt hatten, der Reihe nach erloschen, während ihr Ersatz sich erst allmälig einzugewöhnen hatte.

\section{E. LAMPE.}

„Sophus Lie †. Nachruf", Naturw. Rundschau 14 (1899), p. 28.

Auch in seinen ausführlichen und umfangreichen Abhandlungen vermochte Lie nicht die klassische Ruhe und Klarheit zu erreichen, die wir in der Darstellung der grossen Meister der Mathematik bewundern. Wenn er nun auch in dieser Beziehung hinter den meisten grossen Mathematikern zurücksteht, so darf man darauf nicht allzuviel Gewicht legen. Die Kunst kann Werke schaffen, die niemals veralten, dagegen kommt auch für die Werke der grössten Mathematiker eine Zeit, wo sie anfangen zu veralten, so dass schliesslich nur noch der in ihnen niedergelegte Ideengehalt fortlebt, während die Form, die der Entdecker seinen Ideen gegeben hat, nur noch historisches Interesse hat. Daher wird man auch mit der Zeit vergessen, dass Lie seine Ideen zum Theile in unvollkommener Form dargestellt hat, und wird ihn nur kennen als den Schöpfer einer Reihe grossartiger Theorien, die man zwar inzwischen noch weiter. entwickelt und vervollkommnet haben wird, die aber stets mit dem Namen Lie untrennbar verknüpft bleiben werden. Dann wird man nicht begreifen können, dass es eine Zeit gegeben hat, in der die Grösse Lies nicht allgemein anerkannt worden ist, dann wird er im Urteile aller Mathematiker als der Grössten einer gelten.

\section{F. ENGeL.}

„Zur Erinnerung an Sophus Lie",

Rede Kgl. Sächs. Ges. d. Wissensch. 14. XI. 1899. s. Leipziger Ber. 1899, Math.-phys. Cl., p. XII/XIII. 
On aurait pu hésiter en lisant les Ouvrages de Lie, on n'hésitait plus après avoir causé avec lui; on voyait tout de suite qu'il pensait en images.

H. Poinchré.

„Du rôle de l'intuition et de la logique en mathématiques." voir Compte rendu du deuxième congrès intern. des mathém. Paris 1900 (Paris 1902), p. 117.

Die Lie'sche Theorie der continuierlichen Gruppen bildet wieder einen der Fälle, in denen der Analyst gegenüber dem Geometer - einem Ausspruche Steiner's zufolge ${ }^{1}$ ) - , seine "Schuldigkeit" gethan hat.

G. Bollumann.

Doctorthese Halle 1892.

Cremona sagte bewundernd von Lie's Wirken: „manchmal habe ich mir selbst gesagt, dass ich mit Freude auf alle meine Arbeiten verzichten würde, wenn ich so glücklich gewesen wäre, das entdeckt zu haben, was Lie entdeckt hat.

\section{Elling Holst.}

\section{"Sophus Lie."}

s. Münchener Sitzungsber., Bd. 30, Jahrg. 1900 (München 1901), Math.-phys. Cl., p. 345.

Dopo Abel, la Norvegia e la scienza hanno da piangere la perdita di un altro sommo geometra, degno di stare accanto al primo: Sophus Lie.

\section{Cremona.}

[,Sophus Lie"], Atti della reale acead. dei lincei (5) 8, 1 (1899), Classe di scienze fisiche, matem. e naturali, p. 282.

In Sophus Lie la scienza matematica contemporanea perde uno dei suoi più eccelsi campioni, anzi quello che, per

I) s. auf S. 141 das letzte Citat. 
la singolare genialità ed originalitì dell' indirizzo e dei procedimenti, occupava nel mondo scientifico una posizione quasi del tutto eccezionale, così da poter essere, e non a torto, chiamato il Wagner della matematica.

Beltrami.

Atti della reale accademia dei lincei (5) 8,1 (1899), Classe di scienze fisiche, matematiche e naturali, p. 281.

Un géomètre justement célèbre, physicien peu curieux des faits, tenait par habitude pour la théorie de l'émission. Un concours académique, dont il était juge, le rendit attentif aux démonstrations de Fresnel; il voulut combattre le novateur et crut le vaincre par ses propres armes: „Votre théorie, dit-il à Fresnel, n'a aucune vraisemblance; l'ombre d'un petit disque circulaire éclairé par un point lumineux devrait recevoir au centre, je l'ai calculé par vos méthodes, autant de lumière que si le disque était percé d'un trou!" Sans être troublé par la scandaleuse conséquence, Fresnel accepta l'épreuve. L'expérience était facile, elle fut immédiate; un point brillant marquant le centre de l'ombre vint tourner en preuve l'objection et confondre le géomètre par le triomphe de son calcul; il fallait se rendre ou fermer les yeux. Poisson, dit-on, refusa de les ouvrir.

J. Bertrand.

„Éloges académiques" (Paris 1890), p. 254.

Dur comme un géomètre en ses opinions - - Frédéric le Grand.

„Vers de l'empereur de la Chine."

voir Oeuvres de Frédéric le Grand, t. 13 (Édition Decker 1849), p. 37.

Als junger Mann machen Sie sich schwerlich eine Vorstellung davon, wie sehr es einem im Mittelalter, in welches ich schon seit längerer \%eit getreten bin, an der nöthigen 
Flexibilität fehlt, um sich schnell in Dinge hineinzufinden, mit denen man nicht schon einigermassen vertraut ist $\left.^{1}\right)$

DiRICHLET an Kronecker.

Berlin, 3. V. 1853.

s. Dirichlet, Werke, Bd. 2, p. 389

$=$ Göttinger Nachr. 1885, p. 362.

Ich weiss, dass wenn es mir auch nicht gelingt, in einer bisher vergeblich von mir ersehnten Stellung einen Kreis von Schülern um mich zu sammeln, welche ich mit meinen Ideen befruchten und zur weiteren Entwickelung und Bereicherung derselben anregen könnte, dennoch einst diese Ideen, wenn auch in veränderter Form, neu erstehen und mit der Zeitentwickelung in lebendige Wechselwirkung treten werden. Denn die Wahrheit ist ewig, ist göttlich; und keine Entwickelungsphase der Wahrheit, wie geringe auch das Gebiet sei, was sie umfasst, kann spurlos vorübergehen.

\section{Hermann Grassmann.}

„Die Ausdehnungslehre“ (1862), p. IX/X.

Riemann's Dissertation, „das Buch mit sieben Siegeln“, wie sie früher wol genannt worden ist - - -

H. BURKhaRdT.

„Bernhard Riemann", Vortr. math. Ver. Göttingen 20. VII. 1891 (Göttingen 1892), p. 7.

Als Fourier .... (21. Dec. 1807) zuerst den Satz aussprach, dass eine ganz willkürlich (graphisch) gegebene Function sich durch eine trigonometrische Reihe ausdrücken lasse, war diese Behauptung dem greisen Lagrange so unerwartet, dass

1) Es handelt sich um die von Dirichlet der Berliner Akademie am 20. VI. 1853 vorgelegten und in den Berichten abgedruckten Untersuchungen Kronecker's „über die algebraisch auflösbaren Gleichungen“. 
er ihr auf das Entschiedenste entgegentrat. Es soll* sich hierïber noch ein Schriftstück im Archiv der Pariser Akademie befinden. Dessenungeachtet verweist Poisson überall, wo er sich der trigonometrischen Reihen zur Darstellung willkürlicher Functionen bedient, auf eine Stelle in Lagrange's Arbeiten über die schwingenden Saiten, wo sich diese Darstellungsweise finden soll. Um diese Behauptung, die sich nur aus der bekannten Rivalität zwischen Fourier und Poisson erklären lässt, zu widerlegen - - -

* Nach einer mündlichen Mittheilung des Herrn Professor Dirichlet.

B. Riemann.

„Über die Darstellbarkeit einer Function durch eine trigonometrische Reihe."

s. Werke, herausg. v. H. Weber, 2te Aufl. (1892), p. 232/233.

Petit poisson deviendra grand

Pourvu que Dieu lui prête vie $\left.{ }^{1}\right)$.

Laplace du jeune S. D. Poisson. voir Arago, Oeuvres, t. 2 (1854), p. 692

$=$ Werke, Bd. 2 (1854), p. 571.

Galois est connu par son ardent républicanisme, par les jugemens qu'il a subis et par de longues détentions: il le sera bien plus un jour par son génie scientifique. Sous ce rapport, il est presque complétement ignoré; et cependant il fit de nombreuses tentatives auprès de l'Académie des sciences pour répandre ses découvertes; tous ses efforts furent inutiles. - -

M. Poisson invita Galois à écrire de nouveau les théories qu'il avait soumises à l'académie dans le manuscrit égaré l'année

1) Sprichwörtlicher Anfang der Lafontaine'schen Fabel „Le petit Poisson et le Pêcheur" (Fables, libre 5, fable III); derselbe Ausspruch in gleicher Beziehung wird übrigens bei Arago, Oeuvres, t. 2, p. 597 $=$ Werke, Bd. 2, p. 488 auch Poisson's Lehrer und Freund Billy zugeschrieben. 
précédente. . . . M. Poisson se chargea de présenter le travail $^{1}$ ) à l'Académie; on le nomma pour en rendre compte, et il vint déclarer, après quatre mois d'attente, qu'il n'avait pu le comprendre.

\section{Auguste Chevalier.}

Revue encyclopédique 55 (1832), p. 744 et 747.

Liouville, en publiant, quinze ans après la mort de Galois, le mémoire que Poisson trouvait obscur, annonçait un commentaire qu'il n'a jamais donné. Je l'ai entendu déclarer la démonstration très facile à comprendre. Et au geste d'étonnement qu'il me vit faire, il ajouta: „Il suffit d'y consacrer un mois ou deux, sans penser à autre chose."

\section{J. Bertrand.}

"Éloges académiques", Nouvelle série (Paris 1902), p. 342.

Il n'est pas rare d'entendre les mathématiciens déplorer la brièveté de la vie de Galois: que n'eût pas donné un tel génie si la mort ne l'avait pris à vingt ans! Mais non, Galois, semble-t-il, a rempli toute sa destinée. Si, comme il l'avait ardemment souhaité, il était entré à l'École Polytechnique, il aurait été tué ......... sur une des barricades de Juillet ${ }^{2}$. Et comme il avait raison, deux ans plus tard, en regrettant de

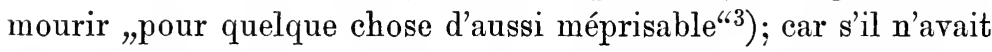
pas péri dans son duel, c'eût été certainement aux journées de juin 1832, et il aurait pu croire alors qu'il mourait pour son

1) "Mémoire sur les conditions de résolubilité des équations par radicaux", s. Oeuvres mathém. de Galois (Paris 1897), p. 33-50.

2) Galois hatte die Aufnahmeprüfung an der École polytechnique bekanntlich zweimal vergeblich versucht (s. S. 319) und war dann in die École normale eingetreten. Während die Schüler der École polytechn. sich an den Strassenkämpfen des Juli 1830 beteiligten, wurden die „Normaliens" von ihrem Studiendirektor Guigniault während jener Tage streng von aller Berührung mit der Aussenwelt ferngehalten (s. hierüber den oben citierten Artikel Dupuy's).

3) s. S. 474 unten. 
pays. Mais la mort avait marqué sa jeunesse, et il le savait bien lui-même, lui qui écrivait au milieu de ses calculs:

L'éternel cyprès m'environne:

Plus pâle que la pâle automne,

Je m'incline vers le tombeau. ${ }^{1}$ )

P. Dupur.

„La vie d'Évariste Galois", Annales de l'école normale (3) 13 (1896), p. 251/252.

Cherchez ailleurs qui vous suive dans vos inventions numériques . . . Pour moi, je vous confesse que cela me passe de bien loin; je ne suis capable que de les admirer.

Pascal à Fermat.

Paris, 27. X. 1654.

voir Fermat, Oeuvres, t. 2 (1894), p. 314

= Blaise Pascal, Oeuvres, t. 4 (1779), p. 443.

[Tischgespräch beim Astronomen Schumacher.]

Gunlögsen [der deutschen Sprache unkundig]: Legi librum tuae theoriae motus corporum coelestium.

Gauss: primum librum?

Gun 1.: nonne primum!

Gauss: ultimum?

Gunl.: nonne ultimum!

Gauss: quem librum legisti?

Gunl.: nedium!

Gauss: quare medium?

Gunl.: Verschuch, sed inutile ${ }^{2}$ ).

Gauss: hm!

s. Briefw. Gauss-Schumacher, Bd. 2 (1860), p. 228 [Anm. des Herausgebers C. A. F. Peters].

1) s. den S. 424 citierten Nachruf von Galois' Freund A. Chevalier l. c. p. 754 .

2) seitdem geflügeltes Wort im Verkehr zwischen Gauss und Schumacher, s. in dem Briefw. ausser der oben citierten Stelle z. B. Bd. 4, p. 358 und Bd. 5, p. $179 / 180$ u. 292. 
Den Sinn, diese Theorie [der elliptischen Functionen] so geschmeidig zu machen, wie sie sich ohne Zweifel machen lässt, hat Jacobi nicht. Hier und in jeder anderen Hinsicht wird Ihre Arbeit wieder klassisches Muster werden.

\section{Bessel an Gauss.}

Königsberg, 30. XI. 1827.

s. Briefw. Gauss-Bessel (1880), p. 476.

Je viens de recevoir le nouveau cahier du Journal de M. Crelle [Bd. 4, Heft II] où il y a trois beaux mémoires de M. Abel et un précis que vous m'aviez annoncé de vos nouvelles recherches. Vous allez si vite Messieurs, dans toutes ces belles spéculations, qu'il est presque impossible de vous suivre; surtout pour un vieillard qui a déjà passé l'âge où est mort Euler, âge où l'on a nombre d'infirmités à combattre, et où l'esprit n'est plus capable de cette contention qui peut vaincre des difficultés et se plier à des idées nouvelles. Je me félicite néanmoins d'avoir vécu assez longtemps pour être témoin de ces luttes généreuses entre deux jeunes athlètes également vigoureux, qui font tourner leurs efforts au profit de la science dont ils reculent de plus en plus les limites. Ce spectacle m'intéresse d'autant plus qu'il m'offre les moyens de perfectionner mon propre ouvrage, en profitant de quelques-uns des matériaux précieux qui sont le résultat de leurs savantes recherches.

\section{Le Gendre à C. G. J. Jacobi.}

\section{Paris, 8. IV. 1829.}

voir "Correspondance mathém. entre Legendre et Jacobi“", éd. par Borchardt, J. f. Math. 80 (1875), p. 258/259.

Abels's Los [als Student] wurde, als ein vielversprechender Anfünger behandelt zu werden, dem man den Rat und die Anweisung erteilte, sich auf seinen künftigen Beruf durch bessere Aneignung der gelehrten Sprachen vorzubereiten, zu derselben Zeit, wo er thatsächlich im Begriffe war, die Grenzen seiner 
Wissenschaft zu erweitern. Uns erscheint das sonderbar; A bel verstand ja überdies Latein genug, um Euler und Gauss zu lesen.

\section{SyLow.}

Festrede zum Abeljubiläum, Christiania 1902.

s. Deutsche Mathem.-Verein. Jahresber. 11 (1902), p. 378.

Je compte parmi les moments les plus heureux de ma vie celui où j'ai vu mes essais meriter l'attention de l'un des plus grands géometres de nôtre siecle [Legendre]. Cela a porté au plus haut degré mon zêle pour mes études.

\section{ABEL à Legendre.}

Christiania, 25. XI. 1828.

voir „Niels Henrik Abel, Mémorial publié à l'occasion du centenaire de sa naissance": „Correspondance d'Abel“, p. 82

$=$ „Mathematische Bruchstücke aus Herrn N. H.Abel's Briefen“, J. f. Math. 6 (1830), p. 73.

En stor Afhandling over en vis Classe transcendante Functioner har jeg udarbeidet for at præsentere den for Institutet. Jeg gjør det paa Mandag. Cauchy viiste jeg den; men han vilde neppe kaste Øjnene paa den. Og jeg tør uden Bram sige at den er god. Jeg er nysgjerrig efter at høre Institutets Dom

(J'ai achevé un grand mémoire sur une certaine classe de fonctions transcendantes pour le présenter à l'Institut. Cela aura lieu lundi. Je l'ai montré à Cauchy; mais c'est à peine s'il a voulu y jeter les yeux. Et j'ose dire sans me vanter qu'il est bon. Je suis curieux d'entendre le jugement de l'Institut ${ }^{1}$ ).

ABEL à Holmboe.

Paris, 24. X. 1826.

voir „Niels Henrik Abel, Mémorial publié à l'occasion du centenaire de sa naissance": „Texte original des lettres", p. 42 ou „Correspondance d'Abel" (Traduction française), p. 46.

1) Es handelt sich hier und in dem folgenden um die berühmte, das „Abel'sche Theorem“ betreffende Abhandlung Abel's „Sur une pro„priété générale d'une classe très étendue de fonctions transcendantes", 
Quelle déconverte de M. Abel que cette généralisation de l'intégrale d'Euler! A-t-on jamais vu pareille chose! Mais comment s'est-il fait que cette découverte, peut-être la plus importante de ce qu'a fait dans les Mathématiques le siècle dans lequel nous virons, étant communiquée à votre Académie il y a deux ans, elle a pu échapper à l'attention de vous et de vos confrères?

C. G. J. ЈАсові à Legendre. Königsberg, 14. III. 1829.

M. Poisson était alors président de l'académie, les commissaires nommés pour examiner le mémoire furent M. Cauchy et moi. Nous nous aperçûmes que le mémoire n'était presque pas lisible, il était écrit en encre très-blanche, les caractires mal formés; il fut convenu entre nous qu'on demanderait à l'auteur une copie plus nette et plus facile à lire. Les choses en sont restées là; M. Cauchy a gardé le manuscrit jusqu'ici sans s'en occuper, l'auteur M. Abel paraît s'en être allé sans s'occuper de ce que devenait son mémoire, il n'a pas fourni de copie, et il n'a pas été fait de rapport. Cependant j'ai demandé à M. Cauchy qu'il me remette le manuscrit qui n'a jamais été entre mes mains, et je verrai ce qu'il y a à faire, pour réparer, s'il est possible, le peu d'attention qu'il a donné, à une production qui méritait sans doute un meilleur sort.

Le Gendre ì Jacobi. Paris, 8. IV. 1829.

voir "Correspondance mathém. entre Legendre et Jacobi“, éd. par Borchardt, J. f. Math. 80 (1875), p. 257/258 et 260.

welche erst nach Cauchy's Tode unter dessen Papieren wieder aufgefunden wurde und dann in den "Mémoires des savants étrangers" 1841 und später in der Sylow-Lie'schen Ausg. von Abel's Werken zum Abdruck gelangte (s. in dem oben citierten Abel-Centenarwerk die historische Finleitung von IIolst, p. 99/100). 
Les travaux d'Abel consistaient alors ${ }^{1}$ ) en une démonstration fort contestable de l'impossibilité de la résolution algébrique des équations du cinquième degré ${ }^{2}$ ) etc. Voilà sur quoi on aurait dû deviner qu'Abel ferait un jour d'admirables découvertes analytiques.

Arago.

Oeurres, t. 3 (1855), p. 531/532

$=$ Werke, Bd. 3 (1855), p. 429.

C'est une grande satisfaction pour moi de voir deux jeunes géomètres, conıme vous et $\mathrm{Abel}$, cultiver avec succès une branche d'analyse qui a fait si longtemps l'objet de mes études favorites et qui n'a point été accueillie dans mon propre pays comme elle le méritait. ${ }^{3}$ ) Vous vous placez par ces travaux au rang des meilleurs analystes de notre époque; nous voyons au contraire ici les talents peu nombreux qui y restent se livrer ¿ des recherches vagues qui ne laisseront que de faibles traces dans l'histoire. Ce n'est pas assez d'avoir du talent, il faut savoir choisir l'objet dont on doit s'occuper.

LE Gendre à C. G. J. Jacobi.

Paris, 9. II. 1828.

voir „Correspondance mathém. entre Legendre et Jacobi“, éd. par Borchardt, J. f. Math. 80 (1875), p. 226.

Crescunt disciplinae lente tardeque: per varios errores sero pervenitur ad veritatem; omnia praeparata esse debent diuturno et assiduo labore ad introitum veritatis novae; jam illa, certo temporis momento, divina quadam necessitate coacta emergit. C. G. J. Jacobi.

Festrede Königsberg (nicht veröffentlicht).

s. W. Dyck, „Über die Beziehungen zwischen künstlerisehem und wissenschaftlichem Erfassen der Natur" (Rede München Techn. Hochsch. 16. I. 1901), Münch. Allg. Zeitg. Beilage No. 35 (12. II. 1901), S. 3.

1) während seines Aufenthalts in Paris 1826/1827.

2) die berühmte Abhandlung aus J. f. Math. 1 (1826), p. 65-84.

3) vgl. hierzu auch Legendre, „Traité des fonctions elliptiques et des intégrales eulériennes", t. 3 (Paris 1828), p. 1. 
À certaines époques, où, après de grands efforts, les sciences mathématiques semblent avoir épuisé toutes les ressources de l'esprit humain, et atteindre le terme marqué à leurs progrès, tout ì coup une nouvelle méthode de calcul vient s'introduire dans ces sciences et leur donner une face nouvelle; bientôt on les roit s'enrichir rapidement par la solution d'un grand nombre de problèmes importants dont les géomètres n'avaient osé s'occuper, rebutés par la difficulté, et pour ainsi dire par l'impossibilité physique de conduire leurs calculs jusqu'à un résultat réel. Peut-être la justice exigerait-elle de réserver, à celui qui a su introduire ces méthodes et les rendre usuelles, une portion dans la gloire de tous ceux qui les emploient avec succès; mais du moins il a sur leur reconnaissance des droits qu'ils ne pourraient contester sans ingratitude.

Condoretet.

Oeuvres, Édition d'Arago, t. 3 (1847), p. 9/10.

La méthode complète du calcul intégral serait une révolution dans la géométrie, semblable à celle de l'application de l'algèbre et à celle de l'invention du calcul différentiel.

Sophie Germain.

"Pensées diverses."

voir Oeurres philosophiques, édition de Stupuy (1896), p. 238.

Nos grands Géomètres, et surtout l'auteur de la Mécanique céleste, paraissent avoir épuisé tout ce qui restait à faire dans l'Astronomie transcendante; ils ont réduit tous les grands problèmes en équations, où l'Astronome, sans être lui-même un analyste bien habile, peut substituer les nombres qu'il tire de ses observations; ce genre de calcul n'est même nécessaire que pour celui qui veut construire de nouvelles tables planétaires, ou perfectionner celles qui sont en usage, c'est-à-dire pour deux ou trois Astronomes par génération. Les autres ont rarement besoin de ces théories relevées; avec des connaissances plus 
communes on peut se rendre très-utile, par le travail des observations et par le soin beaucoup plus pénible de les calculer.

DeLAMBRe.

"Astronomic théorique et pratique" (Paris 1814), t. 1, Préface, p. VI.

Die jetzige Epoche ist wohl eine der glänzendsten für die Astronomie. Vielfache Entdeckungen am Himmel und eben so viele, die die Theorie Ihnen verdankt, werden unsere Zeit verewigen.

Bessel an Gauss.

Königsberg, 17. VII. 1815.

s. Briefw. Gauss-Bessel (1880), p. 218.

Wir leben nicht in einer Zeit, in der auf leichtem und mühelosem Wege unsere Kenntnis zu erweitern wäre, wie das z. B. der Fall war, als mit der Erfindung der Infinitesimalrechnung, und einigermassen auch, als mit der Ausbildung der projektiven Geometrie Hindernisse mit einen Schlage weggeräumt waren, die der Fortentwicklung lange entgegengestanden hatten, und nun der Strom der Bearbeiter sich auf den noch unberührten Boden stürzen konnte. Was am Wege wächst, ist längst abgegrast; in tieferen Urwald darf sich nur wagen, wer mit den schärfsten Werkzeugen ausgerüstet ist.

H. BurkhardT.

"Mathematisches und naturwissenschaftliches Denken", Antrittsvorl. Zürich Univ. 6. XI. 1897.

s. Münchener Allg. Zeitg. Beilage 22. XI. 1897

$=$ Deutsche Nathem.-Verein. Jahresber. 11 (1902), p. 55.

Les époques où l'on a ramené à un principe unique des phénomènes considérés auparavant comme dus à des causes absolument différentes, ont été presque toujours accompagnées 
de la découverte d'un grand nombre de nouveaux faits, parce qu'une nouvelle manière de concevoir les causes suggìre une multitude d'expériences à tenter, d'explications à vérifier; c'est ainsi que la démonstration donnée par Volta de l'identité du galvanisme et de l'électricité a été accompagnée de la construction de la pile, et suivie de toutes les découvertes qu'a enfantées cet admirable instrument.

\section{A.-M. Ampère.}

"Théorie des phénomènes electro-dynamiques"

(Paris 1826), p. 131.

Sicherlich stammen die ersten und ältesten Probleme in jedem mathematischen Wissenszweige aus der Erfahrung und sind durch die Welt der äusseren Erscheinungen angeregt worden.

Bei der Weiterentwickelung einer mathematischen Disciplin wird sich jedoch der menschliche Geist, ermuthigt durch das Gelingen der Lösungen, seiner Selbstständigkeit bewusst; er schafft aus sich selbst heraus oft ohne erkennbare äussere Anregung allein durch logisches Combiniren, durch Verallgemeinern, Specialisiren, durch Trennen und Sammeln der Begriffe in gliicklichster Weise neue und fruchthare Probleme und tritt dann selbst als der eigentliche Frager in den Vordergrund. - - -

Inzwischen, während die Schaffenskraft des reinen Denkens wirkt, kommt auch wieder von neuem die Aussenwelt zur Geltung, zwingt uns durch die wirklichen Frscheinungen neue Fragen auf, erschliesst neue mathematische Wissensgebiete und, indem wir diese neuen Wissensgebiete für das Reich des reinen Denkens zu erwerben suchen, finden wir häufig die Antworten auf alte ungelöste Probleme und fördern so zugleich am besten die alten Theorien. Auf diesem stets sich wiederholenden und wechselnden Spiel zwischen Denken und Erfahrung beruhen, wie mir scheint, die zahlreichen und überraschenden Analogieen und jene scheinbar praestabilirte Harmonie, welche der Mathe- 
matiker so oft in den Fragestellungen, Methoden und Begriffen verschiedener Wissensgebiete wahrnimmt.

\section{Hilbert.}

"Mathematische Probleme“, Vortrag Mathem.-Kongr. Paris 1900.

s. Göttinger Nachr. 1900, Math.-phys. Kl., p. 256/257

$=$ Arch. Math. Phys. (3) 1 (1901), p. 47/48.

Die Weiterbildung der reinen Mathematik erscheint dem Fernerstehenden vielleicht als etwas ganz Willkürliches, weil die Concentration auf einen von Haus aus gegebenen bestimmten Gegenstand wegfällt. Und dennoch giebt es einen Regulator, der in beschränkterem Sinne innerhalb aller anderen Disciplinen wohlbekannt ist - die historische Continuität: Die reine Mathematik wächst, indem man alte Probleme mit neuen Methoden durchdenkt. In dem Maasse, wie wir die früheren Aufgaben besser verstehen, bieten sich neue von selbst.

Felix Kuein.

„Riemann“, Vortrag Naturf.-Vors. Wien 1894.

s. Deutsche Mathem.-Verein. Jahresber. 4, 1894/1895, p. 77.

Die Mathematik ist in ihrer Entwickelung völlig frei und nur an die selbstredende Rücksicht gebunden, dass ihre Begriffe sowohl in sich widerspruchslos sind, als auch in festen durch Definitionen geordneten Beziehungen zu den vorher gebildeten, bereits vorhandenen und bewährten Begriffen stehen.

Wegen dieser ausgezeichneten Stellung, die sie von allen anderen Wissenschaften unterscheidet und die eine Erklärung für die verhältnissmässig leichte und zwanglose Art der Beschäftigung mit ihr liefert, verdient sie ganz besonders den Namen der freien Mathematik, eine Bezeichnung, welcher

A hrens, Scherz und Ernst i. d. Mathem. 
ich, wenn ich die Wahl hätte, den Vorzug vor der üblich gewordenen „reinen“ Mathematik geben würde. ${ }^{1}$ )

G. CANTor.

"Grundlagen einer allgemeinen Mannichfaltigkeitslehre"

(Leipzig 1883), p. 19.

Unsere Wissenschaft ist, im Gegensatze zu anderen, nicht auf eine einzelne Periode der menschlichen Geschichte gegründet, sondern sie hat die Entwickelung der Cultur auf allen ihren Stufen begleitet. Die Mathematik ist mit der griechischen Bildung ebenso verwachsen, wie mit den modernsten Aufgaben des Ingenieurbetriebes. Sie reicht nicht nur den vorwärtsschreitenden Naturwissenschaften die Hand, sondern sie participiert gleichzeitig an den abstracten Untersuchungen der Logiker und Philosophen. Unsere besondere Aufgabe dürfte hiernach sein, in unserer Umgebung die Überzeugung von der Solidarität aller höheren geistigen Interessen zur Geltung zu bringen. Wir nehmen damit innerhalb der heutigen Gegensätze eine wichtige vermittelnde Stellung ein.

\section{Felix KLein.}

„Die Anforderungen der Ingenieure und die Ausbildung der mathematischen Lehramtscandidaten",

Vortrag Hannover mathem. Ver. 20. IV. 1896.

s. Klein u. Riecke, „Über angewandte Mathematik u. Physik“etc. (1900), p. 228.

In den meisten Wissenschaften pflegt eine Generation das niederzureissen, was die andere gebaut, und was jene gesetzt, hebt diese auf. In der Mathematik allein setzt jede Generation ein neues Stockwerk auf den alten Unterbau.

\section{Hermann Hankel.}

„Die Entwickelung der Mathematik in den letztenJahrhunderten“, Akadem. Vortrag Tübingen 29. IV. 1869, 2. Aufl. (Tübingen 1884), p. 25 .

1) Die beiden Abschnitte des Citats sind von mir in ihrer Reihenfolge vertauscht. 
Die Mathematik hat bisher das Glück gehabt, dass bei ihr immer nützliche und verständliche Zeichen geltend geworden sind. Es ist zu hoffen, dass auch künftig weder übel gewählte noch überflüssige Zeichen eingeführt werden mögen, woraus nur Verwirrung entstehen würde.

J. F. Pfaff

von einem jetzt veralteteten Hindenburg'schen "Coefficienten-Zeichen".

s. C. F. Hindenburg, „Der polynomische Lehrsatz, das wichtigste Theorem der ganzen Analysis" (1796), p. 126.

Mein hochgeehrter Lehrer, der vor wenigen Jahren verstorbene Geheime Hofrath Gauss in Göttingen, pflegte in vertraulichem Gespräche häufig zu äussern, die Mathematik sei weit mehr eine Wissenschaft für das Auge ${ }^{1}$ ) als eine für das Ohr. Was das Auge mit einem Blicke sogleich übersieht, und vermöge der glücklichen Zeichensprache die in den mathematischen Schriften mit einer Vollkommenheit sich ausgebildet hat, wie in keiner andern Wissenschaft, besonders auch in Bezug auf die Aufeinanderfolge der auszuführenden Operationen ${ }^{2}$ ) sogleich auffasst; das wird auf dem langen Wege der Übersetzung der Zeichen in Worte, nie so präcise bei dem mündlichen Vortrage dem $\mathrm{Ohr}$ sich darstellen, dass es dem $\mathrm{Zu}-$ hörenden zugänglich genug gemacht werden könnte, um auch für die mit dem Gegenstande weniger Vertrauten, sogléich vergegenwärtigt zu sein. Eben deshalb war Gauss in der Wahl der Bezeichnungen, selbst bei der Auswahl der einzelnen Buchstaben ungemein peinlich.

\section{ENCKe.}

Beantwortung der Antrittsrede von Kronecker i.d.Berl. Akademie. s. Berliner Monatsber. 1861, p. 640.

Ein bescheidenes Hülfsmittel zum Verständnis und zur Kenntnis des neuen Rechts [BGB.] hat sich bisher der Beachtung und Benutzung entzogen, obschon es solcher nicht

1) vgl. S. 251 (Sophie Germain).

2) vgl. ein Citat der nächsten Seite (Gauss an Bessel). 
unwert ist: die Formel, die in ihrer Bedeutung für sinnfällige Darstellung von Grundelementen und Grundsätzen in anderen Wissenschaften, namentlich in der mit der Rechtswissenschaft in mancher Hinsicht zu vergleichenden Mathematik, zum unentbehrlichen Arbeitsgerät gehört.

Prof. Dr. Frommold-Greifswald.

„Die Verwendung der Formel für die Rechtskunde", Deutsche Juristen-Zeitung 5 (1900), p. 158.

Das $\sin ^{2} p$ ist mir jedes Mal fatal, obgleich auch Laplace es gebraucht; fürchtet man, dass $\sin \varphi^{2}$ zweideutig werden könne (was doch vielleicht nie oder höchst selten eintritt, wenn man von $\sin \left(\varphi^{2}\right)$ spräche), ei nun, so schreibe man $(\sin \varphi)^{2}$, aber nicht $\sin ^{2} \varphi$, was der Analogie nach nur $\sin (\sin \varphi)$ bedeuten sollte.

Gauss an Bessel.

Göttingen, 21. XI. 1811.

s. Briefw. Gauss-Bessel (1880), p. 153/154;

vgl. a. Briefe Gauss' an Schumacher vom 23. IX. 1839 u. 3. IX. 1842

(Briefw. Gauss-Schumacher, Bd. 3, p. 292 u. Bd. 4, p. 83).

Kein Mathematiker ist über die Bedeutung der Ausdrücke $d x^{2}$ und $d^{2} x$ in Zweifel. . . . Es ist ...

und

$$
d x^{2}=(d x)^{2}
$$

$$
d^{2} x=d(d x) .
$$

Das Sinuszeichen und das Differentialzeichen spielen hierbei offenbar eine ganz analoge Rolle, und dem Schlusse auf Analogie dürfte hier nichts im Wege stehen. _ _ _ _ _ ...... Der Umstand, dass der Verfasser des bei weitem verbreitetsten mathematischen Schulbuchs, Kambly, die französische Schreibweise $\sin ^{2} x$ adoptiert hat, ist sicherlich für einen grossen Teil der deutschen Mathematiker, vielleicht für den grössten Teil derselben, infolge der ersten Gewöhnung massgebend geworden. Wäre Kambly zufüllig ein Anhänger der 


\section{- $437-$}

Schreibweise $\sin x^{2}$, so würde diese heute die üblichere sein. Man übertreibt nicht, wenn man behauptet: Kambly hat über Gauss den Sieg davongetragen.

F. v. Lühmann.

„ $\sin ^{2} x$ oder $\sin x^{2}$ ?", Zeitschr. math. naturw. Unterr. 16 (1885), p. 417 u. 418.

Eine Definition erklärt Kronecker nur dann für zulässig, wenn sie in jedem Falle durch eine endliche Anzahl von Schlüssen erprobt werden kann. Er bricht damit den Stab nicht nur über alle die neueren Versuche, zu einer logisch verständlichen Auffassung der Irrationalzahlen zu gelangen, sondern auch über Euklid's darauf bezügliche Definitionen.

H. Weber.

„Leopold Kronecker", Math. Ann. 43 (1893), p. 15

$=$ Deutsche Mathem.-Verein. Jahresber. 2, 1891/1892, p. 20.

Führen wir, wenn auch nur für einen Augenblick, die Bezeichnung demoliratisch für diejenigen Gruppen ein, deren sämtlichen infinitesimalen Transformationen unter einander innerhalb der Gruppe gleichberechtigt sind, bezeichnen wir andererseits die nicht demokratischen Gruppen als aristokratisch, - - - -

Sophus Lite.

„Verwerthung des Gruppenbegriffes für Differentialgleichungen“, Leipziger Ber. 47 (1895), Nath.-phys. Cl., p. 271.

Sturm, lorsqu'il parlait du célèbre théorème qu'il a découvert, disait: le théorème dont j'ai l'honneur de porter le nom.

A. REBIÈRe.

„Mathématiques et Mathématiciens", 2. éd. (1893), p. 195. 
Ampère avait l'habitude de désigner chacune de ses conceptions par le lieu où elle était née; c'est ainsi que d'année en année, la théorie d'Avignon, la démonstration de Grenoble, la proposition de Marseille, le théorème de Montpellier, venaient enrichir ses cours publics de l'École Polytechnique et du Collège de France.

\section{Arago.}

Oeurres, t. 2 (1854), p. 110 = Werke, Bd. 2 (1854), p. 89.

Die Zahl der französischen mathematischen Kunstausdrücke ist geringer als die der deutschen. Unsere französischen Fachgenossen scheuen sich nämlich häufiger als wir eine blosse Übersetzung eines fremdsprachlichen Ausdrucks als Kunstwort anzuerkennen und bedienen sich lieber einer Umschreibung desselben.

\section{FeLix MüLLer.}

„Deutsch-französ. Vokabularium“, Vorwort zu Teil II, 1901.

Il me semble que pour perfectionner un peu la langue géométrique, imparfaite à tant d'égards, il faudrait consacrer exclusivement la terminaison en ide aux surfaces, et la terminaison en ique aux lignes courbes. Par là, le nom seul d'une grandeur graphique indiquerait si elle est une ligne ou une surface. Je ferais plus, je consacrerais le genre féminin aux surfaces, tandis que le genre masculin serait réservé aux longueurs et aux volumes.

Essayons d'appliquer cette nomenclature aux surfaces du second degré, et voyons l'avantage qu'elle peut avoir sur l'ancienne, pour la rapidité et la simplicité.

La ligne $d u$ second degré . . . . . . . . . Le Deutérique.

L'aire comprise par une ligne du second degré . . La Deutérique. L'aire de la surface du second degré. . . . . . La Deutéride. Le volume terminépar une surface du second degré. . Le Deutéride. 
Il est à désirer que la nécessité d'un tel changement soit sentie par tous ceux qui cultivent la vraie géométrie, et qu'ils aient le courage d'imiter les chimistes modernes, et de refaire ainsi leur langue. Car il est étonnant que les dénominations d'une science où tout est harmonie et précision soient incohérentes et souvent si peu précises.

\section{Charles Dupin.}

„Développements de Géométrie" (Paris 1813), p. 291/292, Note première: „Idées sur la nomenclature géométrique".

In seinen eigenen Schriften konnte Steiner sich nie genug thun, bis er überall den richtigen Ausdruck seiner Gedanken gefunden hatte, jede seiner Arbeiten wurde zwei bis dreimal sorgfältig umgeschrieben, bis sie ihre endgiiltige Redaction fand. . . . . . Wer erinnert sich nicht daran, wie. sein Bestreben, der Sprache für den scheinbar kalten und todten Stoff Leben, Bewegung und Wärme zu geben, schon in den gewählten neuen Bezeichnungen hervorbricht, die immer in glücklichem Worte etwas dem Wesen der Sache Entnommenes bezeichnen, wie z. B. Strahlbüschel, Kegelschnittschaar, SchaarSchaar, Kerncurve, Flächengebüsch, Voll- und Theil-Curve etc. Wie traurig steht unsre neuere Zeit in dieser Richtung da, die entweder einen übertriebenen Cultus mit Namen treibt, der allerdings bis zu einem gewissen Grade sich rechtfertigen liesse, oder gar in trostloser Langweiligkeit ein paar Curven als „Pippian" und „Quippian“ einführt, blos weil sie in der Abhandlung ihres Entdeckers durch $\mathrm{P}$ und Q charakterisirt sind. C. F. Geiser.

„Zur Erinnerung an Jakob Steiner", Vortr. schweiz. naturf. Vers. Schaffhausen 22. VIII. 1873 (Schaffhausen 1874), p. 31/32.

Leider hat in allerneuester Zeit die Sprachreinigungswut auch unsere Mathematik nicht verschont. Allenfalls kann man diese Verdeutschungen für den Unterricht in der Volksschule rechtfertigen; in den höheren Schulen richten sie nur Ver- 
wirrung an. Für die Wissenschaft ist das Bestreben, fremdsprachliche Kunstausdrïcke verdeutschen zu wollen, überflüssig, wenn nicht gar schädlich. Es giebt zum Glück nur sehr wenige wissenschaftliche Werke, deren Verfasser, von der Puristenseuche befallen, prinzipiell alle Fremdwörter vermeiden. Als merkwürdiges Beispiel führe ich an R. Grassmanns ${ }^{1}$ ) Buch: Die Formenlehre oder Mathematik (1872). Hierin wird für Element - Stift ${ }^{2}$ ), Addition - Fügen, Multiplikation - Weben, Kombinationslehre - Bindelehre etc. gebraucht. Bei der Schwierigkeit, sich mit diesen neuen Terminis zurechtzufinden, darf sich der Verfasser nicht wundern, wenn seinen Schriften weniger Anerkennung zu teil wird, als sie ihrem Inhalte nach verdienen.

FELIX MüLler.

"Über die mathematische Terminologie", Bibl. mathem. (3) 2 (1901), p. 322/323.

In verbis simus faciles; dagegen fordere ich immer, dass der Mathematiker sich stets der Sachen bewusst bleibe, wobei denn natürlich nie etwas unpassendes auf die Phrase gebauet werden kann. $\left.{ }^{3}\right) \ldots \ldots \ldots \ldots$. Unsere neuere Mathematik hat überhaupt, in Rücksicht der Sprache, einen ganz andern Charakter wie die Mathematik der Alten; man erlaubt sich jeden Augenblick solche Redensarten die cum grano salis verstanden werden müssen ${ }^{4}$ ).

Gauss an Schumacher.

Göttingen, 30. IV. 1830.

s. Briefw. Gauss-Schumacher, Bd. 2 (1860), p. 245.

1) Bruder Hermann Grassmann's d. Älteren.

2) Ein „Element" heisst l. c., Erster Zweig, p. 15 u. a. and. Stellen auch ein „Einfaches", die übrigen angegebenen Bezeichnungen s. z. B l. c., Erster Zweig, p. 34 u. 37; Dritter Zweig, p. 29.

3) vgl. hierzu auch die in Note 1 am Ende des Buches ausserdem noch angeführten Stellen.

4) Ausgangspunkt für G. ist die auch in dem Fall gleicher Wurzeln gebrauchte Ausdrucksweise, eine Gleichung nten Grades habe n Wurzeln, eine "Façon de parler", die "man immerhin verstatten möge". 
Die herrschende Meinung, eine Gleichung n-ten Grades habe $\mathrm{n}$ und nur $\mathrm{n}$ Wurzeln, ist irrig. . . . Es folgt . . . keineswegs, dass die Gleichung n-ten Grades nicht mehr als n Wurzeln habe oder dass sich ihre linke Seite nicht in mehrfacher Weise als ein Produkt aus n Faktoren darstellen lasse.

- Der Schluss, dass die Gleichung n-ten Grades nur n Wurzeln habe und sich nur in einziger Weise in n Faktoren zerlegen lasse, ist daher unlogisch, und thatsächlich hat die Gleichung auch mehr als n verschiedene Wurzeln.

\section{H. SChefFler.}

„Das Wesen der Mathematik und der Aufbau der Welterkenntniss auf mathematischer Grundlage", Teil I (Braunschweig 1895), p. 295.

La nomenclature méthodique que j'ai proposée dès 1793 dans mon mémoire sur les transcendantes elliptiques a été adoptée généralement, vous l'avez trouvée établie; quelles sont done vos raisons pour vous écarter de l'usage général? Vous faites schisme avec M. Abel et avec moi, vous faites schisme avec vous-même, puisqu'après avoir appelé fonctions elliptiques les sinus, cosinus et autres fonctions trigonométriques de l'anplitude vous êtes encore obligé d'appeler fonctions de troisième espèce celles que je désigne sous le même nom.

Le Gendre à C. G. J. Jacobi. Paris, 16. VII. 1829.

Comme j'écris ceci en hâte, je ne puis répondre que quelques mots aux reproches que vous m'avez faites dans votre dernière lettre, et pour lesquelles je vous rends grâce mieux encore que pour les éloges que vous m'avez prodigués et que j'ai si peu mérités. Il me fallait absolument une dénomination pour les fonctions sin am, cos am, etc., dont les propriétés répondent parfaitement à celles des fonctions sin, cos, dites circulaires. D'un autre côté, l'application importante qu'on fait de la théorie des Fonctions Elliptiques au Calcul intégral 
rendait nécessaires les distinctions et les dénominations que vous avez introduites dans l'Analyse, et qui ont été accueillies par tous les Géomètres. J'ai donc trouvé convenable d'appeler les intégrales auxquelles vous donnez le nom de Fonctions Elliptiques de la première, seconde, troisième espèce, Intégrales Elliptiques de la première, seconde, troisième espèce et d'étendre ou d'attribuer de préférence la dénomination de Fonctions Elliptiques aux sin am, cos am $\triangle \mathrm{am}$, analogiquement comme on nomme Fonctions Circulaires les sinus, cosinus, etc. Si cela vous déplait, toute autre dénomination me sera agréable.

$$
\text { C.-G.-J. JАCOBI à Legendre. }
$$

Francfort [sur Mein], 19. VIII. 1829.

voir "Correspondance mathém. entre Legendre et Jacobi“, éd. par Borchardt, J. f. Math. 80 (1875), p. 269 et 270 $=$ Jacobi, Werke, Bd. 1, p. 451 et 452.

Mit Kreisfunctionen und Logarithmischen wissen wir jetzt umzugehen, wie mit dem 1 mal 1 , aber die herrliche Goldgrube, die das Innere der höhern Functionen enthält ist noch fast ganz Terra Incognita. Ich habe darüber ehemals sehr viel gearbeitet und werde dereinst ein eignes grosses Werk darüber geben, wovon ich bereits in meinen Disq. arithm. p. 593 einen Wink gegeben habe. Man geräth in Erstaunen über den überschwenglichen Reichthum an neuen höchst interessanten Wahrheiten und Relationen die dergleichen Functionen darbieten (wohin $u$. a. auch diejenigen gehörigen, mit denen die Rectification der Ellipse und Hyperbel zusammen hängt).

Gauss an Schumacher.

Göttingen, 17. IX. 1808.

s. Briefw. Gauss-Schumacher, Bd. 1 (1860), p. 3.

Sie sehen mich eben im Begriff dieses Buch (Legendres exercices) auf die Bibliothek zurückzuschicken, mit welchem ich entschiedenes Unglück habe. Wenn ich soust ein be- 
deutendes Werk studirt habe, hat es mich immer zu eigenen Gedanken angeregt und ist dabei immer etwas für mich abgefallen. Diesmal bin ich ganz leer ausgegangen und nicht zum geringsten Einfalle inspirirt worden.

C. G. J. JACOBI zu einem seiner Freunde.

s. Jacobi, Werke, Bd.1, p. 7 (Dirichlet's Gedächtnissrede auf Jacobi).

Comment se fait-il que M. Gauss ait osé vous faire dire que la plupart de vos théorèmes lui étaient connus et qu'il en avait fait la découverte dès 1808 ? . . Cet excès d'impudence n'est pas croyable de la part d'un homme qui a assez de mérite personnel pour n'avoir pas besoin de s'approprier les découvertes des autres. . . . Mais c'est le même homme qui en 1801 voulut s'attribuer la découverte de la loi de réciprocité publiée en 1785 et qui voulut s'emparer en 1809 de la méthode des moindres carrés publiée en $1805 .{ }^{1}$ )

Le Gendre à C. G. J. Jacobi. Paris, 30. XI. 1827.

Quant à M. Gauss, il n'a rien encore publié sur les Fonctions Elliptiques, mais il est certain qu'il a eu de jolies choses. S'il a été prévenu et peut-être surpassé, c'est une juste peine de ce qu'il a répandu un voile mystique sur ses travaux. Je ne le connais pas personnellement, ayant étudié la philologie à Berlin, où il n'y a pas des géomètres de distinction. C.-G.-J. JACOBI à Legendre Koenigsberg, 12. IV. 1828.

voir "Correspondance mathém. entre Legendre et Jacobi“, éd. par Borchardt, J. f. Math. 80 (1875), p. 217 et 235 $=$ Jacobi, Werke, Bd. 1, p. 398/399 et 416 .

In Wahrheit ist diese Entdeckung weder Legendre noch Gauss zuzuschreiben, sondern Euler, der zuerst - freilich

1) von Legendre in den „Nouvelles Méthodes pour la détermination des orbites des comètes" (Paris 1806), p. 72-80, datiert Paris, 6. III. 1805. 
nur auf dem Wege der Induction - zu jenem Fundamentalsatze der Theorie der quadratischen Reste gelangt ist, welchem Legendre den Namen des Reciprocitätsgesetzes beigelegt hat.

L. Kronecker.

„Bemerkungen zur Geschichte des Reciprocitätsgesetzes", Berliner Monatsber. 1875, p. 267.

Ich habe auf das von mir seit 1794 gebrauchte Verfahren, dem später der Name Methode des moindres quarrés beigelegt ist, niemals grossen Werth gelegt. Verstehen Sie mich recht; nicht in Beziehung auf den grossen Nutzen den sie leistet, der ist klar genug, aber danach taxire ich die Dinge nicht. Sondern deshalb oder in so fern legte ich nicht viel Werth darauf, als vom ersten Anfang an der Gedanke mir so natürlich, so äusserst nahe liegend schien, dass ich nicht im Geringsten zweifelte, viele Personen, die mit Zahlenrechnung zu verkehren gehabt, müssten von selbst auf einen solchen Kunstgriff gekommen seim, und ihn gebraucht haben, ohne deswegen es der Mühe werth zu halten, viel Aufhebens von einer so natürlichen Sache zu machen. Namentlich fiel mir vor allen Tobias Nayer ein, und ich erinnere mich sehr bestimmt, dass ich oft, wo ich mit andern von meiner Methode sprach (wie z. B. während meiner Studirzeit 1795-1798 wirklich vielfach geschehen ist), geäussert habe, ich wolle die allergrösste Wette eingehen, dass Tobias Mayer bei seinen Rechnungen dieselbe Methode schon gebraucht habe. Ich weiss nun jetzt aus.. Papieren [Nayer's], dass ich jene Wette verloren haben würde.

Gauss an Sehumacher.

Göttingen, 6. VII. 1840.

s. Briefw. Gauss-Schumacher, Bd. 3 (1861), p. 387.

Zur Ausarbeitung meiner seit vielen Jahren (1798) angestellten Untersuchungen über die transcendenten Functionen werde ich vorerst wohl noch nicht kommen können, da erst 
noch mit manchen anderen Dingen aufgeräumt werden muss. Herr Abel ist mir, wie ich sehe, jetzt zuvorgekommen und überhebt mich in Beziehung auf etwa ein Drittel dieser Sachen der Mühe, zumal da er alle Entwickelungen mit vieler Eleganz und Concision gemacht hat. Er hat gerade denselben Weg genommen, welchen ich 1798 einschlug, daher die grosse Übereinstimmung der Resultate nicht $\mathrm{zu}$ verwundern ist. $\mathrm{Zu}$ meiner Bewunderung erstreckt sich diess sogar auf die Form und zum Theil auf die Wahl der Zeichen, so dass manche seiner Formeln wie eine reine Abschrift der meinigen erscheinen. Jeder Missdeutung zuvorzukommen bemerke ich jedoch, dass ich mich nicht erinnere, von diesen Sachen irgend jemand etwas mitgetheilt zu haben.

Gauss an Bessel.

Göttingen, 30. III. 1828.

s. Briefw. Gauss-Bessel (1880), p. 477.

. . . je vous engage de ne pas trop tarder à publier les parties essentielles de votre travail. Il y a des gens comme M. Gauss, qui ne se feraient pas scrupule de vous ravir, s'ils le pouvaient, le fruit de vos recherches, et de prétendre qu'elles sont depuis longtemps en leur possession. Prétention bien absurde assurément; car si M. Gauss était tombé sur de pareilles découvertes qui surpassent à mes yeux, tout ce qui a été fait jusqu'ici en Analyse, bien sûrement il se serait empressé de les publier.

LE Gendre à C. G. J. Jacobi. Paris, 14. IV. 1828.')

Je remarque au surplus que votre possession à vous et à M. Abel est maintenant bien assurée. L'envahisseur M. G . . . ne s'avisera point, je pense, d'écrire qu'il avait trouvé tout cela

1) Cette lettre s'est croisée avec celle du 12 avril 1828 adressée par Jacobi à Legendre.

$\mathrm{B}$ [orchardt]. [1. c. p. 235]. 
longtemps avant vous, car s'il disait pareille chose, il se ferait moquer de lui.

Le Gendre à C. G. J. Jacobi.

Paris, 15. X. 1828.

voir "Correspondance mathém. entre Legendro et Jacobi“, éd. par Borchardt, J. f. Math. 80 (1875), p. 236/237 et 246

$=$ Jacobi, Werke, Bd. 1, p. 418 et 428.

Si Gauss, sans s'expliquer davantage, osait dire que les théorèmes de Jacobi [sur les fonctions elliptiques] lui étaient connus, c'est que ses vieux cahiers de notes en contenaient la raison profonde, et l'on pourrait ajouter, lors même que les preuves ne seraient pas devenues évidentes: il fallait le croire, puisque Gauss l'affirmait. Pour l'élévation du caractère, comme pour la puissance du génie, il était le plus grand de tous.

J. Bertikand.

"Éloges académiques", Nouvelle série (Paris 1902), p. 314.

Nach allem stellt sich die bedeutsame Thatsache heraus, dass nicht nur $\mathrm{Abel}$, sondern auch Jacobi wesentlich unter dem Einflusse von Gauss zur Entdeckung der elliptischen Functionen gelangt sind. Während jedoch diese beiden Mathematiker bei der Vertiefung ihrer Auffassung höchst wahrscheinlich im Banne Cauchy's standen, hat Gauss ganz selbständig (man vergleiche den bekannten Brief an Bessel vom 18. Dec. 1811) die fundamentalen Sätze über die Integration auf krummlinigem Wege geschaffen und, wie wohl anzunehmen ist, zur Erklärung der von ihm 1797 gefundenen doppelten Periode - - - - - - - - - -

Es ist sogar mehr als wahrscheinlich, dass auch Cauchy selbst zu seinen Untersuchungen über Integrale auf krumm- 
linigem Wege durch den dritten Beweis von Gauss über den Fundamentalsatz der Algebra angeregt worden ist (Gauss, Ges. W., Bd. III, p. 57-64).

S. Gundelfinger.

„Über die Entdeckung der doppelten Periodicität und Jacobi's Antheil daran", Berl. Sitzungsber. 1898, p. 345.

Dans l'oeuvre si étendue de Cauchy, une part principale doit être donnée à l'idée fondamentale d'étendre la notion première de l'intégrale définie, en faisant passer la variable, d'une limite à l'autre, par une succession de valeurs imaginaires, par un chemin arbitraire. La science n'a point d'exemple d'une conception plus féconde: elle a été la source des plus belles découvertes de son auteur _ _ -

Charles Hermite.

Discours 5. VIII. 1889.

voir "Inauguration de la Nouvelle Sorbonne" (Paris,

Delalain frères), p. 28.

Der Cauchy'sche [Integral-] Satz war schon vorher Gauss bekannt, der ihn in seinem Briefwechsel mit Bessel (18. Dec.1811) ausdrücklich erwähnt; aber es ist doch ein grosser Unterschied, ob Jemand eine mathematische Wahrheit mit vollem Beweise und der Darlegung ihrer ganzen Tragweite veröffentlicht, oder ob ein Anderer sie nur so nebenher einem Freunde unter Discretion mittheilt. Deshalb können wir den Satz mit Recht als das Cauchy'sche Theorem bezeichnen. - - - -

Die neueren Fortschritte der Analysis beruhen wesentlich auf dem Cauchy'schen Satze. Diese sind aber nicht, wie man es gewöhnlich ausdrückt, auf die Benutzung von complexen Variabeln zurückzuführen, sondern einzig und allein auf den Fortschritt von Functionen einer Variabeln zu solchen mit zwei Variabeln. Nicht einer mystischen Verwendung von 
$\sqrt{-1}$ hat die Analysis ihre wirklich bedeutenden Erfolge des letzten Jahrhunderts zu verdanken, sondern dem ganz natürlichen Umstande, dass man unendlich viel freier in der mathematischen Bewegung ist, wenn man die Grössen in einer Ebene statt nur in einer Linie variiren lässt. Die Functionen einer Veränderlichen treten als Grenzfall derer mit zwei Veränderlichen auf; und gerade an diesen Grenzen, diesen Ufern finden sich die Klippen, von denen das hohe Meer frei ist. Gauss hat den Sachverhalt in dem erwähnten Briefwechsel vollkommen klar dargelegt.

L. KRONECKER.

Vorlesungen I (Integrale), herausg. v. Netto (1894), p. 52.

Zuvörderst würde ich jemand, der eine neue Function in die Analyse einführen will, um eine Erklärung bitten, ob er sie schlechterdings bloss auf reelle Grössen (reelle Werthe des Arguments der Function) angewandt wissen will, und die imaginären Werthe des Arguments gleichsam nur als ein Überbein ansieht, oder ob er meinem Grundsatze beitrete, dass man in dem Reiche der Grössen die imaginären $a+b \sqrt{-1}=a+b i$ als gleiche Rechte mit den reellen geniessend ansehen müsse. Es ist hier nicht von praktischem Nutzen die Rede, sondern die Analyse ist mir eine selbständige Wissenschaft, die durch Zurücksetzung jener fingirten Grössen ausserordentlich an Schönheit und Rundung verlieren und alle Augenblicke Wahrheiten die sonst allgemein gelten höchst lästige Beschränkungen beizufügen genöthigt sein würde.

Ich behaupte nun, dass das Integral $\int \varphi_{\mathbf{x}} \cdot \mathrm{d} \mathbf{x}$ nach zwei verschiedenen Übergängen immer einerlei Werth erhalte, wenn innerhalb des zwischen beiden die Übergänge reprësentirenden Linien eingeschlossenen Flächenraumes nirgends $\varphi \mathrm{x}=\infty$ wird. Diess ist ein sehr schöner Lehrsatz, dessen eben nicht schweren Beweis ich bei einer schicklichen Gelegenheit geben werde. Er hängt mit schönen anderen Wahrheiten die Entwicklungen in Reihen betreffend zusammen. Der Übergang nach jedem Puncte 
lässt sich immer ausführen, ohne jemals eine solche Stelle wo $p \mathrm{x}=\infty$ wird zu berühren. Ich verlange daher, dass man solchen Puncten ausweichen soll, wo offenbar der ursprüngliche Grundbegriff von $\int \varphi \mathrm{x} \cdot \mathrm{dx}$ seine Klarheit verliert und leicht auf Widersprüche führt.

\section{Gauss an Bessel.}

Göttingen, 18. XII. 1811.

s. Briefw. Gauss-Bessel (1880), p. 156 u. 157.

Je regarde comme un des pas les plus importants que l'Analyse ait faits dans ces derniers temps, de n'être plus embarrassée des quantités imaginaires et de pouvoir les soumettre au calcul comme les quantités réelles.

\section{LAGRANGe à Lorgna ${ }^{1}$ ).}

Berlin, 20. XII. 1777.

voir Oeurres de Lagrange, t. 14 (1892), p. 261.

Wendet man die durch Grössenoperationen ausgedrückten Abhängigkeitsgesetze zwischen veränderlichen Grössen in einem erweiterten Umfange an, indem man den veränderlichen Grössen, auf welche sie sich beziehen, complexe Werthe giebt, so tritt eine sonst versteckt bleibende Harmonie und Regelmässigkeit hervor. . . . . Beinahe jeder Schritt, der hier gethan ist, hat nicht bloss den ohne Hülfe der complexen Grössen gewonnenen Resultaten eine einfachere, geschlossenere Gestalt gegeben, sondern auch zu neuen Entdeckungen die Bahn gebrochen.

\section{B. Riemann.}

„Grundlagen für eine allgemeine Theorie der Functionen einer veränderlichen complexen Grösse", Inaug.-Diss. Göttingen 1851.

s. Werke, herausg. v. H. Weber, 2. Aufl. (1892), p. 37/38.

1) Antonio Maria Lorgna, 1735-1796, Mathematiker; s. 1. c. p. 252 Anm. 
.. das Imaginäre, dieses Schoosskind complexer Mystik _Eugen Dühring ${ }^{1}$ ).

„Kritische Gesch. der allgem. Principien der Mechanik“, 2. umgearb. Aufl. (1877), p. 517.

Leider scheint noch immer nicht überall das Bestreben erloschen, Prioritäten im Sinne und der Denkungsweise vergangener Zeiten zu reclamiren und zu bestreiten. Für denjenigen, welcher die Geschichte der Wissenschaft ruhig betrachtet, giebt es nicht leicht etwas Unerquicklicheres und Zweckloseres. Können wir doch täglich sehen, wie die Keime wissenschaftlicher Entdeckungen zu gewissen Zeiten überall liegen, und an den verschiedensten Orten gleichzeitig aufgehen.

A. Clebsich.

„Zum Gedächtniss an Julius Plücker", gel. i. d. Kgl. Ges. d. Wiss. zu Göttingen 2. XII. 1871.

s. Göttinger Abhandl. 16, Jahrg. 1871 (Göttingen 1872), Math. Cl., p. 2/3.

Von dem Aufenthalte in Baltimore an [seit 1876] gewann Sylvester kaum noch die Ruhe zur Durchführung von Specialuntersuchungen, die er lieber seinen jüngeren Freunden übertrug. Sein Gedächtniss erwies sich als unsicher und trügerisch, so dass er die Existenz von Sätzen gelegentlich leugnete, die er selber gefunden und veröffentlicht hatte. Obgleich er deshalb die Literatur seines Gegenstandes, den er gerade behandelte, weder gründlich kannte, noch genau citirte, brauste er heftig auf, wenn man ihm eine seiner Entdeckungen durch Erhebung eines Prioritätsstreites rauben wollte.

\section{E. LAMPE.}

„Arthur Cayley † und James Joseph Sylvester †. Nachruf", Naturw. Rundschau 12 (1897), p. 362.

Cauchy a pu laisser paraître quelquefois un peu de susceptibilité; il a pu se montrer trop jaloux de ses droits de

1) vgl. S. 29 . 
priorité; on a pu s'étonner de le voir prendre des précautions, aujourd'hui insolites, pour s'assurer le mérite de ses découvertes; mais comme le disait si bien sur sa tombe un de ses confrères de la section de mécanique, M. Combes, ce n'était ni vanité, ni ambition; c'était seulement une émulation toute juvénile et la passion de la vérité. - - -

\section{L'abbé Moigno.}

voir "Sept lę̧ons de physique générale par Augustin Cauchy", Turin 1833, avec appendices par l'abbé Moigno (Paris 1868), p. XI.

Lagrange racontait que, dans sa jeunesse, il éprouva un si profond chagrin en trouvant, par hasard, dans les oeuvres de Leibnitz, une formule analytique dont il avait parlé à l'Académie de Turin, comme d'une découverte à lui, qu'il s'évanouit complétement.

Arago.

Oeuvres, t. 1 (1854), p. 119

= Werke, Bd. 1 (1854), p. 94/95.

Laplace is so terrible a copyist that I suppose somebody had shown it [,some propositions in the theory of Probabilities"] before him.

W. R. Hamilton to John T. Graves.

Observatory, [near Dublin,] 15. X. 1833.

cf. Graves, „Life of Sir William Rowan Hamilton“, Vol. 2 (1885), p. 57.

Je fais parapher actuellement par le secrétaire de l'Académie l'énoncé de différents problèmes et théorèmes de Calcul intégral, afin de m'en conserver du moins la possession et de constater que je les ai trouvés de mon côté, si ce diable d'homme 
[Euler] me prévient encore sur quelques-uns, ce qui pourrait bien être, car où ne fouille-t-il pas?

D'Alembert à Lagrange.

Paris, 30. VI. 1769.

voir Oeuvres de Lagrange, t. 13 (Paris 1882), p. 137.

The press-work [of the "Historia Coelestis"] goes on but slowly ... Worthy Sir J. Newton has twice or thrice been stopping the press: he does all he can to hinder it, or break off, and to perplex me; but an accident has lately happened, that has discovered his proud and insolent temper, and exposes him sufficiently. He has been told calmly of his faults, and could not contain himself when he heard of them.

Flamsteed to his friend A. Sharp. The Observatory [at Greenwich], 29. V. 1707.

cf. F. Baily, „An Account of the Revd John Flamsteed, the first Astronomer Royal" (London 1835), p. 264.

The Differential Method is one and the same with the Method of Fluxions, excepting the Name and Mode of Notation; Mr. Leibnitz calling those Quantities Differences, which Mr. Newton calls Moments or Fluxions ...... ..... And therefore we take the proper Question to be, not who invented this or that Method, but who was the first Inventor of the Method. . . . . . we reckon Mr. Newton the first Inventor; and are of Opinion that Mr. Keill, in asserting the same, has been no ways injurious to Mr. Leibnitz.

ARBITRI

Societatis Regiae [Londinensis].

v. „Commercium epistolicum" (Londini 1725), p. 241/242.

M. Leibnitz . . . . est le premier qui ait publié ce calcul [des infiniment petits]; et s'il l'avoit pris de M. Neuton, il 
ressembleroit du moins au Promethée de la Fable, qui déroba le feu aux Dieux, pour en faire part aux Hommes.

\section{Fontenelle.}

„Eloge de M. Neuton."

voir Oeurres de Fontenelle, t. 6 (1758), p. 332.

Quod . a ad functiones rationales attinet, ratio ultima, quam earum incrementa evanescentia inter se tenent, multo ante Neutoni ac Leibnizii tempora assignari potuit; ita ut Calculus differentialis, quatenus ad solas functiones rationales applicatur, diu ante haec tempora inventus sit censendus. Tum vero nullum est dubium, quin $\mathrm{N}$ euton o eam Calculi differentialis partem, quae circa functiones irrationales versatur, acceptam referre debeamus; ad quam insigni suo Theoremate de evolutione generali potestatum binomii feliciter est deductus, quo eximio invento limites calculi differentialis iam mirifice erant amplificati. Leibnizio autem non minus sumus obstricti, quod hunc calculum, antehac tantum velut singulare artificium spectatum, in formam disciplinae redegerit, eiusque praecepta tanquam in systema collegerit, ac dilucide explicaverit. Hinc enim maxima subsidia suggerebantur, ad hunc calculum ulterius excolendum, et ea, quae adhuc desiderabantur, ex certis principiis elicienda. Mox igitur studio cum Ipsius Leibnizii, tum Bernouilliorum ad hoc ab eo incitatorum, fines Calculi differentialis etiam ad functiones transcendentes, quae pars adhuc fuerat inculta, sunt promoti, tum vero etiam solidissima fundamenta Calculi integralis constituta; quibus insistentes, qui deinceps in hoc genere elaborarunt, continuo maiora incrementa addiderunt. Neutonus vero etiam amplissima dederat specimina Calculi integralis, cuius prima inventio, cum a prima origine calculi differentialis vix separari queat, non ita absolute constitui potest; et quoniam maxima eius pars adhuc excolenda restat, hic calculus ne nunc quidem pro absolute invento haberi potest; sed potius quantum cuique pro viribus ad eius perfectionem conferre contigerit, id grata mente 
agnoscere debemus. Atque haec de gloria inventionis huius calculi tenenda esse iudico, de qua quidem antehac tantopere est disceptatum.

L. Euler.

„Institutiones calculi differentialis" (1755), Praefatio, p. XV-XVII.

Fermat, le véritable inventeur du calcul différentiel, a considéré ce calcul comme une dérivation de celui des différences finies ..... Newton a depuis rendu ce calcul plus analytique .. Léibnitz a enrichi le calcul différentiel d'une notation très-heureuse.

LAPLACE.

„Théorie analytique des Probabilités" (1812), p. 7.

Selbst abgesehen von der urkundlichen Wahrheit ist es eine Thorheit, Leibniz als Plagiator Newton's hinzustellen. Hätte er auch alle Methoden seines Rivalen gekannt, so würde sein Algorithmus allein ihn unsterblich gemacht haben; mit sicherem Gefühle hat dies bereits die Sprache anerkannt, indem sie Newton's Erfindung „methodus fluxionum et fluentium“, Leibnizens aber den "calculus differentialis et integralis“ genannt hat.

\section{Hermann Hankel.}

„Die Entwickelung der Mathematik in den letzten Jahrhunderten", Akadem. Vortrag Tübingen 29. IV. 1869,

2. Aufl. (Tübingen 1884), p. 10.

Es ist heute anerkannt, dass schon im siebzehnten Jahrhunderte die Infinitesimalrechnung so weit vorbereitet war, dass ihr hauptsächlich eine einheitliche Sprache und eine Schrift fehlte. Beides hat Leibniz ihr selbständig gegeben, so wenig es ihm einfiel in Abrede zu stellen, dass er auf den Schultern 
von Vorgängern stand, dass diese sich ausgiebig und erfolgreich mit Infinitesimalaufgaben beschäftigt hatten. Dass Newton nicht minder selbständig Aehnliches besass, früher besass als Leibniz, wird ebensowenig geleugnet werden, aber sein Wort Fluxion kam erstmalig 1687 in den Principien, seine Bezeichnung, in der er lange schwankte, erstmalig 1693 durch Wallis an die Öffentlichkeit, während Leibnizens Abhandlung von 1684 schon als Markstein in der Geschichte der Mathematik vorhanden war. Wir nennen sie einen Markstein, weil sie . . ..... . den Ausgangspunkt bildete, von wo aus neue Wettbewerber in die Rennbahn traten, vorwärts zu eilen nach entfernten Zielpunkten. Das Ende der Bahn ist in den mehr als zweihundert Jahren, die inzwischen verflossen sind, weiter und immer weiter hinausgeschoben worden, aber Leibnizens Abhandlung von 1684 bildet nach wie vor den Sammelpunkt, an welchem Jeder vorbei muss, der sich am Rennen betheiligen will, Leibnizens Sprache, Leibnizens Schrift sind die unerlässlichen Eintrittskarten, ohne welche Niemand zugelassen werden kann. Und Leibniz ahnte diese grosse Zukunft. Er hat frühzeitig die Bedeutung der mathematischen Form erkannt, die seine Gegner nicht sahen, oder nicht sehen wollten. Auch über den Prioritätsstreit als solchen haben die Ansichten sich geklärt, leider dahin geklärt, dass seine gründliche Durchforschung allen Betheiligten ohne irgend eine Ausnahme zum Nachtheile gereicht.

\section{CAn'tor.}

„Vorlesungen über Geschichte der Mathematik“, Bd. 3, 2. Aufl. (Leipzig 1901), p. 328.

Es dürfte richtig sein, zu sagen, dass die Begriffe Differentialquotient und Integral, deren Ursprung jedenfalls bis auf Archimedes zurückgeht, dem Wesen der Sache nach durch die Untersuchungen von Kepler, Descartes, Cavalieri, Fermat und Wallis in die Wissenschaft eingeführt worden sind. . . 
Die capitale Entdeckung, dass Differentiation und Integration inverse Operationen sind, gehört Newton und Leibniz.

Sophus Lik.

„Zur allgemeinen Theorie der partiellen Differentialgleichungen beliebiger Ordnung", Leipziger Ber. 47 (1895), Math.-phys. Cl., p. 53.

Newton était en possession de toutes ses grandes découvertes mathématiques à un âge que Leibnitz n'avait pas encore atteint lorsqu'il se livra à l'étude de ces sciences, et Leibnitz fut peu d'années après l'un des inventeurs de l'analyse infinitésimale; mais il faut ajouter que Leibnitz . . s'était consacré à d'autres études dès sa première jeunesse: il arait acquis, si l'on peut parler ainsi, les moeurs littéraires, et son esprit était exercé aux longues recherches.

Fourier.

„Éloge de M. Delambre."

voir Histoire de l'acad. des sc. de Paris, t. 4, 1819-1820, p. CCXXII.

Je vous suis très obligé de la Harangue inaugurale qui m'a été remise de votre part, et je suis sensible à la mention obligeante que vous $y$ avez fait de moi; mais il me semble que vous auriez pu rendre plus de justice à mes travaux mathématiques, et surtout ne pas attribuer à d'autres ce qui m'appartient.

Il m'importe assez peu, Monsieur, d'être un écrivain nerveux, il m'importe encore moins d'avoir été appellé à des Cours; mais il m'importe que des hommes tels que vous, faits pour connoître et pour juger, et pour lesquels je suis plein d'estime, me rendent justice sur les découvertes que je crois avoir faites dans les sciences.

\section{D'Alembert à J. F. Hennert. ${ }^{1}$ )}

voir Ch. Henry, „Correspondance inédite de d'Alembert", Bullettino di bibliografia e di storia delle scienze mat. e fis. 18 (1885), p. 538/539.

1) Prof. in Utrecht. 
Poncelet habe ich wahrlich mehr genutzt als irgend jemand, und dass ich ihn nicht so oft citirt, als du es wünschest, ist seine und des Pöbels Schuld, denn dieser ist ohne dies geneigt, alles was er von mir gelernt, ihm zuzuschreiben, und er selbst hat mich bisher gänzlich ignorirt.

Steiner an C. G. J. Jacobi.

. Berlin, 31. XII. 1833.

s. E. Jahnke, „Auszüge aus drei Briefen Steiners an Jacobi," Arch. Math. Phys. (3) 4 (1903), p. 272.

Ihren Aufsatz in No. 27 der Europa hatte ich schon gelesen und gefunden, dass Sie von mir wohl hätten in einem etwas höhern Tone reden können, statt mich einigermaassen mit dem Helmholz zu parallelisiren. Sagen, „er und ich ständen auf demselben Boden" ist wie sagen, der Montblanc und ein Maulwurfshaufen neben ihm ständen auf demselben Boden. Vor einem Jahr hat er einen abgeschmackten Aufsatz über Goethe's Farbenlehre ${ }^{1}$ ) verfasst. Sie hätten ihn dafür, dass er über das $\mathrm{Sehn}^{2}$ ) schreibt, ohne mich zu kennen, oder kennen zu wollen, herunterhunzen sollen, und nach Noten.

Schopenhauer an J. Frauenstädt.

Frankfurt a. M., 15. VII. 1855.

s. J. Frauenstädt, ,Arthur Schopenhauer. Von ihm. Über ihn" (1863), p. 653.

Einen „Montblane neben einem Maulwurfshaufen“ nennt sich Schopenhauer*, wenn er sich mit einem Naturforscher vergleicht.

* Arthur Schopenhauer, von ihm, über ihn von Frauenstädt und Lindner. Berlin 1863 . S. 653.

HeLmholtz.

„Das Denken in der Medicin“, Festrede militärärztl. Bildungsanst. Berlin 2. VW. 1877.

s. Vorträge u. Reden, Bd. 2 (1884), p. 184.

1) „Über Goethe's naturwissenschaftliche Arbeiten“, Vortr. Koenigsberg Frühling 1853, zuerst veröffentlicht in der ${ }_{n}$ Kieler Monatsschrift“, Mai 1853; s. „Vorträge und Reden“, Bd. 1 (1884), p. 1-23.

2) „Über das Sehen des Menschen“" Vortr. Königsberg 27. II. 1855, erschienen als Broschüre 1855; s. „Vorträge und Reden“, Bd. 1 (1884), p. $365-396$. 
[Robert] Grant's History of Physical Astronomy is very valuable, and very creditable to its composer. But your humble servant may be pardoned if he finds himself somewhat amused at the title, „History of Physical Astronomy, from the Earliest Ages to the middle of the Nineteenth Century" [London 1852], when he fails to observe any notice of the discoveries of Sir W. R. Hamilton in the theory of the Dynamics of the Heavens.

I heard Jacobi speak of me, at Manchester, in 1842, when I was sitting, by invitation, beside Dr. Peacock, the then President of the Mathematical Section of the British Association, as ,le Lagrange ${ }^{1}$ ) de votre pays“; but Mr. Grant ignores my existence. I can bear it!

W. R. Hamilton to A. De Morgan.

Observatory, near Dublin, 11. VI. 1856.

ef. Graves, „Life of Sir William Rowan Hamilton“, Vol. 3 (1889), p. 508/509.

Dans le frontispice de son ouvrage Daniel Bernoulli n'avait pris qu'un titre, le seul, qu'il eût alors, celui de fils de Jean Bernoulli ${ }^{2}$ ), et il continua de prendre ce même titre à la tête de tous ses mémoires, dans un temps où il pouvait $\mathrm{y}$ en ajouter de bien honorables, et où son nom n'avait plus besoin de se parer d'aucun éclat étranger.

\section{CONDORCET.}

„Éloge de M. [Daniel] Bernoulli“, voir Condorcet, Oeuvres, Édition d'Arago, t. 2 (1847), p. 550.

1) vgl. S. 292 (B. Tortolini).

2)

Danielis Bernoullii Basileensis

Joh. Fil.

Exercitationes quaedam

mathematicae.

Venetiis. 1724. 
Ich für mein Theil hab im höchsten Grad Ursach mich über meines Vaters Opera, sonderlich den letzten tomum zu beschweren: Die nova problemata mechanica sind meistens von mir, und hat mein Vater meine Solutionen sogar gesehen, ehe er sie suo modo solvirt hat, und wird doch meiner mit keinem Worte gedacht, welches mir um so verdriesslicher fällt, als meine Solution noch nicht publicirt. Meine erstere Solution circa centrum spontaneum rotationis, petitam a natura minimae inertiae, hat er lang contestirt auch meprisirt, und endlich hat er sie als die seinige publicirt. . . . . Meiner ganzen Hydrodynamic, von welcher ich doch in Wahrheit meinem Vater kein jota zu verdanken habe, werde ich auf einmal völlig beraubt und verliere also in einer Stunde die Früchte von einer 10jährigen Arbeit.

Dieses Alles ist noch das Wenigste, worüber ich mich beschweren kann. Anfangs wollte es mir schier unerträglich fallen; endlich aber hab ich Alles mit einer Resignation aufgenommen: hab aber auch einen dégoût und Verachtung für meine bisherige studia geschöpft, dass ich lieber wollte das SchumacherHandwerk als die Mathematic erlernt haben. Ich hab auch seithero mich nicht mehr resolviren können etwas Mathematisches auszuarbeiten.

\section{Daniel Bernoulli an Euler.}

Basel, 4. IX. 1743.

s. "Correspondance mathém. et phys. de quelques célèbres géomètres du 18 ème siècle", t. 2 (1843), p. 530 u. 532;

vgl. a. ibidem p. 504/505.

Mon Frére nous propose ... deux Problémes, s'adressant particulièrement à moi. ------------
Quelques difficiles que ces Problémes paroissent, je n'ai pas manqué de m'y attacher à l'instant même que je les ai lus: mais voyez avec quel succès; au lieu de trois mois qu'on me donne pour sonder le gué, et au lieu de tout le reste de cette année pour trouver la résolution, je n'ai employé en tout que trois minutes de tems pour tenter, commencer, et achever 
d'approfondir tout le mystére; et bien au delà: Car je donnerai les resolutions mille fois plus generales que ne sont les.. Problémes - -

\section{JoHANn BERNoULLI}

dans l'Histoire des Ouvrages des Sçavans, Juin 1697.

Avant que de publier ma Réponse aux solutions de mon Frére, je le prie de repasser tout de nouveau sur sa derniére, d'en examiner attentivement tous les points, et de nous dire ensuite si tout va bien; lui déclarant, qu'après que j'aurai donné la mienne, les prétextes de précipitation ne seront plus écoutés.

\section{Jacob Bernoulli}

au Journal des Savans, 26. V. 1698.

Je n'ai que faire de repasser sur mes solutions des Problémes de mon Frére: Je sai qu'en penser, et mon temps sera assûrément mieux employé à faire de nouvelles découvertes.

\section{Johann Bernoulli}

au Journal des Savans, 23. VI. 1698.

Je n'ai jamais cru que mon Frére possedât la véritable méthode pour le Probléme des isopérimétres; mais maintenant j'en doute plus que jamais, vu la difficulté qu'il fait de repasser sur ses solutions. Car enfin pourquoi nous refuser une chose si-tôt faite, si ce n'est qu'il ne se fie pas lui-même à sa méthode? S'il n'a emploié que trois minutes de temps, comme il le dit, pour tenter, commencer, et achever d'approfondir tout le mystére, il y a apparence que la revuë de ce qu'il a trouvé, ne lui en côtera pas davantage: d'ailleurs quand il y en mettroit le double, est-ce que six minutes, emploiées à cet examen, diminueroient tant le nombre de ses nouvelles découvertes?

\section{JACOB Bernouli.}

au Journal des Savans, 4. VIII. 1698.

voir Johannis Bernoulli Opera (Lausanne \& Genève 1742), t. 1, p. 201-202, 220, 221, 230. 
Lorsque je proposai, dans les Journaux de Leipsic, à mon Frére quelques Problémes de Géométrie, ce fut principalement dans la vûë, et dans l'espérance qu'il nous en donneroit un jour la solution. Car outre que je considerois, que nous pouvons avoir bonne part à la gloire de ceux qui se rendent habiles dans une Science, dont il n'y a long-temps que nous leur avons donné les premiéres ouvertures; j'avois encore des raisons particuliéres pour souhaiter qu'il y pût réüssir, et gagner le petit prix qui y a été joint par un de mes amis.

\section{JaCOB Bernoulli}

au Journal des Savans, 4. VIII. 1698.

On ferait bien mieux de se taire, que de prétendre nous avoir donné quelque ouverture dans cette Science: Je crois que ces ouvertures se sont données mutuellement; et si nous voulions entrer en compte, je ne sais à qui on seroit en reste. Je prie seulement mon Frére de se ressouvenir à qui il est redevable de la premiére Théorie des chainettes, de laquelle il se sert présentement en Maitre dans toute sa Lettre: les gens qui le savent, sauront qu'en penser; ces sortes de reproches sentent trop la vanité.

\section{Johann Bernoulli}

au Journal des Savans, 8. XII. 1698.

voir Johannis Bernoulli Opera (Lausanne \& Genève 1742), t. 1, p. $222 / 223,232 / 233$.

M. Euler se crut obligé par reconnaissance de défendre le principe de la moindre action de Maupertuis. Le moyen que choisit M. Euler ne pouvait guère être employé que par lui; c'était de résoudre par ce principe quelques-uns des problèmes les plus difficiles de la mécanique: ainsi, dans les temps fabuleux, les dieux daignaient fabriquer, pour les guerriers qu'ils favorisaient, des armes impénétrables aux coups de leurs adversaires.

\section{CONDORCET.}

„Éloge de M. Euler."

voir Condorcet, Oeuvres, Édition d'Arago, t. 3 (1847), p. 30. 
Des querelles littéraires s'élèvent rarement entre les géomètres; ils ont peu de juges $^{1}$ ); ces juges ne peuvent être ni éblouis ni séduits, et ce qui est plus précieux encore, ils ne peuvent être injustes; on leur démontrerait bientôt qu'ils se sont trompés dans leur jugement.

\section{Condorcet.}

Oeuvres, Édition d'Arago, t. 2 (1847), p. 576.

Je n'ai rien à reprendre en ceux qui enseignent la Géométrie. Cette science est la seule, qui n'ait point produit de Sectes; elle est fondée sur l'analyse, sur la Synthèse et sur le calcul; elle ne s'occupe que de vérités palpables; aussi a-t-elle la même méthode en tout pays. Je me renferme également dans un respectueux silence à l'égard de la Théologie. On dit que c'est une science divine, et qu'il n'est par permis aux profanes de toucher à l'encensoir.

\section{Frédéric le Grand.}

„De la littérature allemande; des défauts qu'on peut lui reprocher" etc. (Berlin 1780, Decker), p. 26/27 $=$ Oeuvres, t. 7 (Édition Decker 1847), p. 100.

Suivant une habitude que le silence de nos savants confrères a peut-être trop encouragée, M. Cauchy ne veut pas laisser aux autres le dernier mot, et, à cet effet, il change le terrain de la discussion [née à l'occasion d'une Note de Cauchy sur le choc des corps élastiques].

.... déjà plusieurs fois, j’avais rencontré M. Cauch y comme juge et rapporteur assez peu sympathique aux idées et aux doctrines appartenant à cette géométrie générale et pure que, depuis, on a nommé géométrie moderne, géométrie supérieure, jusquelà à peu près étrangère, je puis le dire, aux études de M. Cauchy. $\Lambda$ une revendication aussi circonspecte que respectueuse, mais au fond très-positive, de ce que je considérais alors comme un droit au point de vue des théories mécaniques, notre confrère n'a fait qu'une réponse évasive à la p. 375 du t. XII du

1) vgl. S. 184 (Condorcet). 
Bulletin des Sciences Mathématiques [1829], sous la forme d'un laconique et dédaigneux Post-scriptum. Cette inexplicable réticence ... me convainquit, j'en fais ici la remarque avec regret, que nous n'étions plus au temps où Huygens, Euler, Bernoulli, Lagrange, etc., jetèrent les premiers fondements de la mécanique, de la dynamique rationnelle; temps auquel, bien loin de craindre les discussions scientifiques, on les recherchait; on les provoquait même, pour en faire jaillir la lumière.

..... je me permets de faire observer que l'immense réputation de notre confrère, et, je le dis en toute franchise, l'éblouissement, la fascination en quelque sorte, que me fait subir ainsi qu'à d'autres, son merveilleux talent de manier et retourner les formules ou équations de l'analyse algébrique, m'ont fait accorder, dans mes précédentes observations sur le point de doctrine en discussion, quelques concessions plus apparentes dans leur forme explicite que réelles au fond.

\section{Poncelet}

dans l'académie des sciences de Paris, séance du 26 janvier 1857.

voir C. R., t. 44 , p. $105-107$.

Herr Maupertuis hat mir . . ein miraculum miraculorum gemacht aus einem jungen D'Alembert, welcher eine Mechanicam und Hydrodynamicam hätte drucken lassen, bis ich endlich sagte, es sey nicht möglich, in diesen Wissenschaften in dem $20^{\text {ten }} \mathrm{Jahr}$ seines Alters alle principia einzusehen und sogar wunderbare Progressen zu machen. Unterdessen hat mich dieses bewogen, mir obbemeldte Werke anzuschaffen, und hab mit Verwunderung gesehen, dass ausser einigen wenigen Sachen in seiner Hydrodynamica nichts als eine impertinente suffisance hervorleuchte. Seine criteria sind bisweilen recht puerilisch, und zeigen nicht nur, dass er kein sonderbarer Mann ist, sondern sogar, dass er es niemals werden wird, indem seine Praesumtion viel zu gross, um von andern Leuten, 
und seine eignen Einsichten viel zu gering, um von sich selbsten etwas sonderliches zu lernen. .......... an vielen . Orten refutirt er mich, aber zugleich, welches mich freuet, die berïhmtesten Männer macht er sich kein Bedenken als des petits garçons zu critisiren. - - -

\section{Daniel Bernoulli an L. Euler. Basel, 7. VII. 1745.}

s. "Correspondance mathém. et phys. de quelques célèbres géomètres du 18 ème siècle", t. 2 (1843), p. 577; vgl. a. ibidem p. 584 f., 594, 621 .

Aus des Hrn. D'Alembert Hydrodynamic hab ich gesehen, dass er in mathesi applicata über die Maassen schwach ist. Dass er praetendirt für alle Jahreszeiten die Direction und vim ventorum pro omni climate per formulas difficillimas integrales hergeleitet zu haben, darauf kann ich nichts anders sagen, als verba sunt, welche der Mathematic mehr Schand als Ehre machen. . . . . . wenn es bei Hrn. Maupertuis stünde, so würden Ew. und Herr Clairaut nur Dii minorum gentium seyn, und Herr D'Alembert als ein Apollo, von welchem alle Wissenschaften, als der wahren source, herfliessen, erhoben werden; da es doch bei mir stünde, ihn wegen seiner Hydrodynamic allen Leuten zum Gespött zu machen. Ich werde .... aber vielmehr seine wahren mérites ... admiriren, als seine lächerliche suffisance, welche ich seiner Jugend zuschreibe, releviren, sonderlich da ich vorsehe, dass er auch in dem, was ihm dato mangelt, ein grosser Mann werden wird.

Daniel Bernouldi ${ }^{1}$ ) an L. Euler.

\section{Basel, 29. VI. 1746.}

s. „Correspondance mathém. et phys. de quelques célèbres géomètres du 18 ème siècle", t. 2 (1843), p. 603/604; vgl. a. ibidem p. 646 , sowie auch p. 608 .

1) vgl. zu dieser und der vorhergehenden Briefstelle auch die auf S. 370, sowie ferner S. 365 (A. v. Humboldt an Varnhagen). 
C'est assez, ce me semble, le défaut des jeunes gens de s'enfler de leurs premiers succès; mais la présomption diminue ensuite à mesure que la science augmente.

\section{Lagrange à Condorcet.}

Berlin, 18. VII. 1774.

voir Oeurres de Lagrange, t. 14 (1892), p. 27.

Lubens cognovi tibi meam solutionem chordae vibrantis probari, quam Alembertus variis cavillationibus infirmare est conatus, idque ob eam solam rationem quod non ab ipso esset profecta. Minatus est se gravem refutationem esse publicaturum; quod an fecerit, nescio. Putat se per eloquentiam semidoctis fucum esse facturum. Dubito an serio rem gerat, nisi forte amore proprio sit penitus occoecatus. Voluit nostris Commentariis, non demonstrationem, sed nudam declarationem inseri: meam solutionem maxime esse vitiosam; ego vero opposui novam demonstrationem omni rigore adornatam. Sed praeses noster, beatae memoriae [Maupertuisius], noluit ipsi nostram Academiam tanquam palaestram concedere; unde etiam meam confirmationem lubens suppressi; ex quo judicabis quantas turbas, si praesidio decoratus ${ }^{1}$ ), sit acturus. Equidem omnia tranquillus expecto, nihil negotii cum illo mixturus.

L. Euler an Lagrange.

Berlin, 2. X. 1759.

s. Oeurres de Lagrange, t. 14 (1892), p. 162/163.

Ce grand génie [d'Alembert] me semble un peu trop enclin à détruire tout ce qui n'est pas construit par lui-même.

L. Euler à Lagrange. Berlin, 26. II. 1765.

voir Oeuvres de Lagrange, t. 14 (1892), p. 205; voir aussi la page 206 de la même lettre.

1) als Nachfolger des am 27. VII. 1759 verstorbenen Maupertuis, wofür Friedrich d. Gr. d'Alembert bekanntlich ausersehen hatte. 
Je regarde les disputes comme très inutiles à l'avancement des sciences et comme ne servant qu'à faire perdre le temps et le repos.

LAGRANGe à Laplace. Berlin, 5. VII. 1779.

voir Oeuvres de Lagrange, t. 14 (1892), p. 85.

Mit Encke lebe ich in alter Zärtlichkeit trauernd über den nordischen Krieg [zwischen Bessel in Königsherg und Encke]. Jacobi ist geistreich, lebendig und fett. Dirichlet ist mehr in sich gezogen, fein und liebenswürdiger.

Al. Hunbold an Gauss.

Paretz, 18. VI. 1839.

s. K. Bruhns, „Briefe zw. A. v. Humboldt und Gauss" (Leipzig 1877), p. 46.

Von Zeit zu Zeit erscheinen in dem Gebiete der Astronomie und anderer Theile der Mathematik Irrlichter . . . . Sie, theuerster Freund, haben ein eigenes Talent, solche Afterastronomen zu behandeln, wenigstens glaube ich Ihre Feder öfters in Recensionen der Jenaischen Literaturzeitung erkannt zu haben, die mir immer das grösste Vergnügen machen, und wo man glauben sollte es könne nun nicht fehlen, dass solche Leute sich selbst in ihrer Blösse sehen müssten. Es scheint aber doch auch Ihnen nicht zu gelingen. - - -

Gauss an Bessel. Göttingen, 5. IX. 1817.

s. Briefw. Gauss-Bessel (1880), p. 255.

Ich habe einen grossen Widerwillen dagegen, in irgend eine Polemik gezogen zu werden, ein Widerwille, der mit jedem Jahre vergrössert wird aus ähnlichen Gründen, wie die- 
jenigen, die Göthe in einem Briefe an die Frau von Wolzogen vorträgt (S. der letztern literarischen Nachlass 1. Bd. [Leipzig 1848] S. 445). ${ }^{1}$ )

Gauss an Schumacher.

Göttingen, 4. X. 1849.

s. Briefw. Gauss-Schumacher, Bd. 6 (1865), p. 47.

Es gibt in der Mathematik keine wahren Controversen.

Gauss an Bessel.

Göttingen, 18. XII. 1811.

Es kann in der That nichts klarer sein, als dass Widersprüche immer die Schuld der Mathematiker selbst sind; und dass sie immer nur aus einem Mangel an Consequenz in der Festhaltung der Begriffe entstehen. Freilich findet die Consequenz oft nicht Statt und ist doch so scheinbar, dass ein Auge wie das Ihrige dazu gehört, ihren Mangel zu entdecken.

Bessel an Gauss.

Königsberg, 12. I. 1812.

s. Briefw. Gauss-Bessel (1880), p. 156 u. 161.

It is not so long since, during one of the meetings of the Association, one of the leading English newspapers briefly described a sitting of this Section in the words, „Saturday morning was devoted to pure mathematics, and so there was nothing of any general interest": still, such toleration is better than undisguised and ill-informed hostility. But the attitude of respect, I might almost say of reverence, is even more trying: we mathematicians are supposed to be of a different mould, to live far up the heights above the driving gales of controversy, breathing a rarer intellectual atmosphere, serene in

1) Goethe sagt hier u. a., dass ,in hohen Jahren, wo man mit der Zeit so haushältig umgehen muss, man über sich .... wegen vergeudeter Tage höchst ärgerlich wird." 
impenetrable calm. It is difficult for us to maintain the gravity of demeanour proper to such superior persons; and perhaps it is best to confess at once that we are of the earth, earthy, that we hare our differences of opinion and of judgment, and that we can even commit the Machiavelian crime of making blunders.

\section{A. R. FonsytiI.}

Address Toronto 1897.

ef. Report of the 67 th meeting of the British Association for the advancement of Science, p. 542.

Are you sure that it is impossible to trisect the angle by Euclid? I have not to lament a single hour thrown away on the attempt, but fancy that it is rather a tact, a feeling, than a proof, which makes us think that the thing cannot be done. No doubt we are influenced by the cubic form of the algebraic equation. But would Gauss's inscription of the regular polygon of seventeen sides have seemed, a century ago, much less an impossible thing, by line and circle?

\section{W. R. Hamilton to A. De Morgan. Observatory, [near Dublin,] 18. XII. 1852.}

As to the trisection of the angle, Gauss's discovery increases my disbelief in its possibility. When $x^{17}-1$ is separated into quadratic factors, we see how a construction by circles may tell. But, it being granted $a x^{3}+b x^{2}+c x+d$ is not separable into a real quadratic and a linear factor, I cannot imagine how a set of intersections of circles can possibly give no more nor less than three distinct points.

I never convinced a squarer or trisector who proceeded on mathematical principles - permanently. I once persuaded a R. C. priest, who came from South America with a square circle in his pocket to get the reward, that he had better learn geometry first. The argument which seemed to weigh most with him was that the only known instance of a cure was 
one effected on a Jesuit ${ }^{1}$ ) mentioned by Montucla. But he relapsed, for his book was advertised (I mean my priest's book) a few days afterwards.
A. De Morgan to W. R. Hamilton.
Camden Town, 24. XII. 1852.

cf. Graves, „Life of Sir William Rowan Hamilton", Vol. 3 (1889), p. 433 and 434/435.

Un géomètre français m'écrit avec emphase qu'il a découvert la quadrature du cercle, et que toute l'Europe est jalouse de lui. Autant que je m'entends à ces matières, cette quadrature est impossible, à cause que les sections sont impaires, et même que si par son calcul il en approchait de plus près que ses devanciers, cette découverte n'en serait pas moins inutile. Ces hautes sciences ne deviennent utiles à la société qu'autant qu'on les applique à l'astronomie, à la mécanique et à l'hydrostatique; d'ailleurs, elles ne sont qu'un luxe de l'esprit.

Frédéric le Grand à d'Alembert.

(1. V. 1780.)

voir Oeuvres de Frédéric le Grand, t. 25 (Édition Decker 1854), p. 149.

. . en 1873, deux géomètres annoncèrent presque simultanément qu'ils avaient démontré le théorème de Chasles [concernant le nombre des coniques satisfaisant à cinq conditions données quelconques]. L'un était Clebsch, l'un des savants les plus justement célèbres de l'Allemagne, l'autre était Halphen . . . Le théorème était inexact pourtant. N'y a-t-il pas là de quoi scandaliser un peu ceux qui croyaient les mathématiciens infaillibles?

Comment peut-on se tromper dans ces sciences dont la méthode semble exclure l'erreur? On admettrait à la rigueur des fautes de calcul, mais non des fautes de raisonnement. Cependant la plupart des grands géomètres en ont commis; il

1) „Richard Albius" (White); s. Montucla, Histoire des mathémamatiques, t. 4 (Paris, an $\mathrm{X}=1802$ ), p. 628 . 
est vrai que, le plus souvent, ils ont été les premiers à s'en apercevoir. Je ne citerai qu'Abel qui avait résolu les équations du cinquième degré avant de démontrer qu'elles sont insolubles.

. Encore ai-je oublié de citer deux autres savants qui, quelques années après, ont cru de leur côté démontrer le théorème inexact de Chasles.

\section{H. Poincaré.}

„Notice sur Halphen", Journal de l'école polytechnique, cah. 60 (1890), p. 145 et 152.

Les plus grands géomètres ont écrit sur le calcul des probabilités; presque tous ont commis des erreurs: la cause en est, le plus souvent, an désir d'appliquer des principes à des problèmes qui par leur nature échappent à la science.

D'Alembert commet la faute opposée: il nie les principes. Imposer aux hasards des lois mathématiques est pour lui un contresens; il rejette le problème et détourne les yeux. Les géomètres, sur ce point, n'avaient qu'un parti à prendre, celui de ne pas le lire. Il n'a jamais connu la question. Daniel Bernoulli l'a invité à se mettre au fait des matières dont il parle. D'Alembert l'a traité d'impertinent: ils avaient tous les deux raison.

Joseph Bertrand.

„D'Alembert" (Paris 1889), p. 52/53.

Depuis longtemps, presque tous les Membres de l'Académie sont convaincus que M. Chasles est victime d'une fraude, et, pour mon compte, je n'en ai jamais douté. Mais notre honoré confrère, connaissant seul et voulant seul connaître la source d'où émanaient les. Pièces qu'il produisait, l'Académie, par déférence pour son caractère, a laissé se prolonger devant elle une discussion qu'elle ne supportait qu'avec impatience et douleur. - - - - - - - - 
On peut se tromper, on peut être trompé; mais du moment où notre éminent confrère reconnaissait que les accusations qu'il avait portées devant l'Académie et devant le monde savant, contre Newton et contre $\mathrm{Huyghens}$, reposaient sur des Pièces fabriquées, ne devait-il pas, avant tout, réparation à deux mémoires dignes de respect et cruellement compromises? -

Dans la dernière séance, notre savant confrère s'est surtout attaché à démontrer sa bonne foi. Mais avait-elle jamais été contestée?

Il a oublié, qu'il me permette de le dire, ce qui était dû à Huyghens, l'honneur de sa patrie; à Newton, l'honneur de l'humanité.

L'Académie voudra protester contre cet oubli. Elle fermera cette discussion regrettable, mais elle ne peut pas demeurer solidaire de la conclusion de notre savant confrère, qui ne consent pas encore à absoudre ceux qu'il accusait. S'il croit qu'il reste un mystère à pénétrer, et que jusque-là il n'y a rien à conclure, qu'il reste du moins seul à le croire.

Jean-Baptiste Dumas

[Secrétaire perpétuel de l'académie des sciences]. voir Comptes rendus de l'académie des sciences, t. 69 (1869), p. 677-678 (Séance du 20 septembre 1869).

Aus meiner eigenen Erinnerung weiss ich, dass Petzval die Reden, welche er bei der 32. Versammlung deutscher Naturforscher und Ärzte, die im Jahre 1856 in Wien stattfand, gehalten hat - in denen er u. a. den oft citierten Ausspruch getan hat, „er habe drei Wissenschaften beinahe von der Grundfeste bis an den Gipfel ausgebildet" (Theorie der Differentialgleichungen ${ }^{1}$, Dioptrik und Akustik) - sowie die offenbar absichtlich unrichtigen Mitteilungen über die Ergebnisse seiner nicht publizierten Unternehmungen, die er damals in Vorträgen und gesprächsweise seinen Berliner Fachkollegen Kummer und Weierstrass gegenüber machte, von dem ersteren niemals

1) s. Note 21 am Ende des Buches. 
vergeben wurden, obwohl sich mein unvergesslicher Lehrer Weierstrass in seiner bekannten milden, selbst die geringste Leistung anerkennenden und jedermann aufmunternden Weise wiederholt bemühte, ihm von Petzval eine günstigere Meinung beizubringen. Kummer brach derartige Erörterungen stets mit der Bemerkung ab „Was er uns in Wien vorredete, zeigt, dass er ein ganz dummer Kerl ist", worauf Weierstrass zu erwidern pflegte: „Dumm ist der nicht, er hat nur uns für dumm gehalten."

\section{Gegenbauer.}

„Ein vergessener Österreicher", Deutsche Mathem.-Verein. Jahresber. 12 (1903), p. 332.

J'ai toujours remarqué que les prétentions dans tous les genres sont exactement en raison inverse du mérite; c'est un de mes axiomes de morale.

LAGRANGE à d'Alembert. Berlin, 12. VII. 1771.

Je suis enchanté du contraste de votre modestie avec la bonne opinion que d'autres géomètres ont d'eux-mêmes, quoiqu'assurément ils n'y aient pas le même droit. Vous prouvez bien ce que vous me disiez il y a quelque temps, que les prétentions sont en raison inverse du mérite.

D'Alembert à Lagrange. Paris, 15. XI. 1774.

voir Oeuvres de Lagrange, t. 13 (Paris 1882), p. 206 et 293.

Von niemand ist das Dopplersche Prinzip über den Einfluss der Bewegung der Ton- oder Lichtquelle oder des Beobachters auf die Höhe des von demselben gehörten Tones, bezw. die Farbe des von ihm empfundenen Lichteindrucks, dessen fundamentale Bedeutung heute allseitig anerkannt ist, 
sowie sein Entdecker so grimmig angefeindet worden als gerade von Petzval, der das Wesen dieses Prinzips völlig verkannte. Der kurze Ausdruck und die ungemein einfache Begründung desselben durch den Entdecker widersprach nämlich gänzlich der Meinung Petzvals, dass "gegenwärtig neue Wahrheiten nur hinter gewaltigen Entwicklungen des Kalkuls mehr hervorgezogen werden können." "Was kann man“, meinte er, "mit einer Theorie von acht Zeilen machen, und was soll ein Theorem für einen Wert haben, das durch lineare Gleichungen begründet werden kann?" ...... Am meisten scheint Doppler der Anwurf beleidigt zu haben, „er treibe kleine Wissenschaft", während Petzval sich nur mit ,grosser" beschäftige.

L. Gegenbauer.

„Ein vergessener Österreicher“, Deutsche Mathem.-Verein. Jahresber. 12 (1903), p. 333/334.

An einem Abende gerieten die zuerst so befreundeten Jacobi und Steiner an einander und trennten sich im Zorn. Kaum nach Hause gekommen, sandte Steiner seinem Widerpart eine Herausforderung auf Pistolen. Darauf schrieb Jacobi seinem Dutzfreunde in seiner schlagfertigen, spöttischen Art: Wenn Du des Lebens überdrüssig bist, so kaufe Dir Pistolen und schiesse Dir selbst eine Kugel in den Kopf; mich hast Du dazu nicht nötig. Damit war die Sache erledigt.

E. LAMPe.

„Zur Biographie von Jacob Steiner“, Bibl. mathem. (3) 1 (1900), p. 138.

In dem merkwürdigen Beleidigungsprozess des Regierungsassessors gegen einen Berliner Universitätsprofessor, der jenen Herrn als Kartellträger zur Thür hinauskomplimentirt hatte, ist der Termin zur mündlichen Verhandlung für heute angesetzt. Mit Rücksicht hierauf braucht auch der Name des 


\section{$-474-$}

Professors, der seinen Standpunkt zur Duellfrage so nachdrücklich gewahrt hat, nicht weiter verschwiegen zu werden. Es handelt sich um den Ordinarius der Mathematik, Professor Hermann Amandus Schwarz, Mitglied der Akademie der Wissenschaften. Professor Schwarz, der Schwiegersohn des berühmten Mathematikers Kummer, hat beilüufig als aktiver Soldat den Krieg von 1866 mitgemacht. Wo es also darauf ankam, hat auch er mit der Waffe in der Hand seinen Mann gestanden!

.... Professor Schwarz führte seine Sache mit wirkungsvoller Beredtsamkeit. Was er als Christ, Staatsbürger, Beamter und Familienvater über das Duell sagte, kann dem besten, was in dieser Materie geäussert worden ist, an die Seite gestellt werden.

\section{Berliner Tagebiatt}

No. 615 (3. XII. 1896), 1. Beibl. und No. 618 (4. XII. 1896).

In einer Garnison forderten 13 Cavallerie-Officiere Johann Bolyai; er nahm die Forderungen an, mit der Bedingung, dass ihm gestattet werde, nach je zwei Duellen ein Stück auf der Violin zu spielen. - Er blieb Sieger über alle.

\section{Franz Schnimt.}

„Lebensgeschichte des ungarischen Mathematikers Johann Bolyai", Abh. zur Gesch. d. Math. 8 (1898), p. 141.

Je prie les patriotes, mes amis, de ne pas me reprocher de mourir autrement que pour le pays.

Je meurs victime d'une infâme coquette, et de deux dupes de cette coquette. C'est dans un misérable cancan que s'éteint ma vie.

Oh! pourquoi mourir pour si peu de chose, mourir pour quelque chose d'aussi méprisable! 
Je prends le ciel à témoin que c'est contraint et forcé que j'ai cédé à une provocation que j'ai conjurée par tous les moyens.

Je me repens d'avoir dit une vérité funeste à des hommes si peu en état de l'entendre de sang-froid. Mais enfin j'ai dit la vérité. J'emporte au tombeau une conscience nette de mensonge, nette de sang patriote.

Adieu! j'avais bien de la vie pour le bien public.

Pardon pour ceux qui m'ont tué, ils sont de bonne foi.

E. Galois

à tous les républicains, ses amis.

Paris, 29. V. 1832.

voir Revue encyclopédique 55 (1832), p. 753.

Die nächtliche Zeit, welche sonst von jungen Leuten sehr oft zur Beobachtung der irdischen Venus angewendet wird, widmete Tycho-Brahe [als Student in Leipzig] der himmlischen, oder, wenn man auch das Äusserste von einem Manne in seinen Jahren vermuthen wollte, so theilte er die Zeit doch so ein, dass seine Nachtwachen mehr gute als böse Folgen hatten.

Von Leipzig reiste er.... nach Wittenberg und Rostock. ...... Ein Zufall, der aber wohl nicht von seinen astronomischen Geschäften herrührte, verursachte, im eigentlichen Verstande, eine silberne Nase; und man versichert, dass er in diesen Universitätsjahren beym Duell um eine Nasenlänge zu kurz gekommen sey.

„Nachrichten von dem Leben und den Erfindungen der berühmtesten Nathematiker" (Münster 1788), p. 268.

Zwei Schatten seh' ich schweben

In später, später Nacht;

Wisst Ihr, wohin sie streben? -

- Beide auf Numero acht! 
Der Hausknecht, als es läutet,

Mit einem Fluch erwacht;

Er weiss schon, was es bedeutet:

Beide auf Numero acht!

„Alt Holland steht in Nöthen,

Weh' uns, die wilde Jagd!

Weh' uns, die alten Schweden

Beide auf Numero acht!

„Heisst das als fleissiger Schreiber

Ein neues Buch gemacht,

Ihr grausamen Überkneiper

Beide auf Numero acht?

„Heisst das als frommer Pastor

An die Gemeinde gedacht?

Ihr sündenharte Laster

Beide auf Numero acht?!"

Der Hausknecht, ungewaschen

Murrt ers und ungeschlacht,

Da lärmts: „He! noch zwei Flaschen,

Beide auf Numero acht!"

Und weiter singt es und klingt es

Und jubilirt und lacht,

Und bis zum Hausherrn dringt es:

„Beide auf Numero acht!“"

Der spitzt betrübt die Füsse,

Die Bettstatt seufzt und kracht;

Stumm nimmt er eine Prise;

„Beide auf Numero acht!!“

J. V. v. Scherfer.

„Gaudeamus“, „No 8 im Holländer Hof zu Heidelberg“" [bezieht sich auf Otto Ludwig Hesse und dessen Freund, den Pfarrer Sehmetzer von Ziegelhausen, s. Hesse, Ges. Werke (1897), p. 719 Anm.]. 
Als eifriges Mitglied des Korps Saxonia fehlte Weierstrass [in Bonn als stud. jur.], wie er später gern erzählte, keinen Abend auf der Verbindungskneipe.

E. Lampe.

"Karl Weierstrass",

Gedächtnisrede Berlin Phys. Ges. 5. III. 1897, p. 4/5

$=$ Deutsche Mathem.-Verein. Jahresber. 6, 1896/1897

(Leipzig 1899), p. 28/29.

Mathematicus non est collega.

\section{Geflügelites Wort}

an den humanistischen Anstalten des 18. Jahrhunderts.

Nach der Cirkular-Verfügung vom 24. Oktober v. J. will das Ministerium das Prädikat Oberlehrer ausschliesslich den Klassen-Ordinarien beilegen, welche sich als solche während längerer Zeit in jeder Beziehung bewährt und ausgezeichnet haben. Die Lehrer der Nathematik und der Naturwissenschaften sind, vorausgesetzt, dass sie die zu einem gelehrten Schulamte nöthige allgemeine wissenschaftliche Bildung besitzen, und neben den für ihr spezielles Fach bestimmten Lektionen auch in anderen Lehrgegenständen unterrichten können, von der Wirksamkeit der Klassen-Ordinarien nicht ausgeschlossen und dem Ministerio sind nicht wenige Gymnasien bekannt, wo die Lehrer der Mathematik und Naturwissenschaften mit dem besten Erfolge auch als Klassen-Ordinarien thätig sind. Überdies hält das Ministerium für den Lehrer der Mathematik, wenn es für ihn eines besondern Praedikats bedarf, das des Mathematikus, welches bei vielen Gymnasien herkömmlich ist, für das passendste. Auch behält sich das Ministerium vor, ausgezeichneten Lehrern der Mathematik und der Naturwissenschaften, die sich durch namhafte wissenschaftliche Leistungen vortheilhaft bekannt gemacht und während längerer Zeit in ihrem Fache mit einem besonders glïcklichen Erfolge gelehrt 
haben, zu ihrer Aufmunterung das Praedikat Professor beizulegen.

Reskript des kgl. Preuss. Unterr.-Minist. (v. Altensteis) an das Provinz.-Schul-Kolleg. zu Münster. 27. II. 1838.

s. von Kamptz's Ann. d.Preuss. Staats-Verwaltung, Bd. 22, p. 103/104.

Ein Rector in Sachsen hiess in der Studienstunde einen Schiiler die mathem. Arbeit weglegen mit den Worten: „Was für harbarica treiben sie da?" Ein anderer beehrte die Mathematik mit dem Titel „dummes Zeug“, ein dritter sehr glimpflich mit „Nebenfach“. Diese (übrigens verbürgten) Übergriffe konnten bei dem trefflichen alten Gymnasialschulregulativ [vor 1848] passiren!

J. C. V. Hofrinans.

Zeitschr. math. naturw. Unterr. 1 (1870), p. 2/3 Anm. (,Vorwort des Herausgebers").

Nicht eher wird die Mathematik in weiteren Kreisen ihre richtige Würdigung finden, als bis man in den Schulen mehr als das Abe derselben lehrt, und die unglückselige Meinung beseitigt ist, dass sie im Unterrichte weiter keinen Zweck habe, als den Geist formal zu bilden. Die Mathematik hat ihren Zweck im Inhalt; ihre Form ist nebensächlich und muss nicht notwendig diejenige sein, die sie historisch geworden ist, weil sie unter dem Einflusse griechischer Logik zuerst feste Gestalt gewann.

Mathematik mag man ihres formalen Nutzens wegen mit demselben Rechte treiben, als Geschichte - zur Stärkung des Gedächtnisses.

\section{Hermann Hankel.}

„Die Entwickelung der Mathematik in den letzten Jahrhunderten“, Akadem. Vortr. Tübingen 29. IV. 1869,

2. Aufl. (Tübingen 1884), p. 6. 
Vorschläge zur Abänderung des Gymnasial=Lehrplans ... zu Gunsten einer weiteren Ausdehnung des mathematischen, physikalischen und naturgeschichtlichen Unterrichts im Ministerium zu besprechen fand sich 1860 eine merkwürdige Veranlassung. Beinahe gleichzeitig waren mir vom Min. v. Bethmann=Hollweg und von Seiten der Generalinspection des Militair=Bildungswesens Schreiben des Kronprinzen übersandt; worin dieser über ein beigefügtes Promemoria ein Gutachten erforderte. Jenes war von einem Gymnasialprofessor in Berlin [Schellbach] verfasst, dessen mathematischer und physikalischer Unterricht sich durch anregende und zur Selbstthätigkeit nöthigende Methode auszeichnete, wodurch er auch die Aufmerksamkeit des Kronprinzen auf sich gezogen hatte. ${ }^{1}$ ) Er suchte in seiner Denkschrift nachzuweisen, dass weder in den Civil= noch in den Militair=Bildungsanstalten Mathematik und Physik die ihnen gebührende Stellung einnehmen, dass darin auf beiderlei Anstalten in Deutschland weniger geleistet werde als in Frankreich, und dass es an der Zeit sei, im Gymnasium ... die genannten Wissenschaften ins Centrum zu versetzen.

Die Entwickelung der menschlichen Gesellschaft habe eine andre Richtung eingeschlagen, wofür die Staatsregierung offene Augen haben müsse. - - - - - - - - - -

Als der Professor den Vorwurf der Vernachlässigung so generalisirte, als ob auf den preuss. Gymnasien überhaupt wenig oder keine Mathematik gelernt werde, konnte ich nicht nur den Zweifel aussprechen, ob er eine so umfassende Kenntnis des Thatsächlichen besitze, sondern mich auch auf die Urteile von Männern wie Richelot und Neumann in Königsberg, Heine in Halle und anderer Mitglieder der wissenschaftl. Prüfungscommissionen für Mathematik und Physik über die Leistungen der Gymnasien berufen . ... Dabei schlug ich vor, dem Professor durch ein besonderes Commissorium Gelegenheit zu geben, sich mittels einer Revision des mathematischen und

1) Schellbach war bekanntlich der Lehrer des Kronprinzen, späteren Kaisers Friedrich gewesen. 
physikalischen Unterrichts an beliebigen Gymnasien von dessen Einrichtung und Ergebnissen selbst zu überzeugen. Der Minister erklärte sich zu einem solchen Auftrage bereit...... Nicht lange darauf besuchte der Professor zu dem erwähnten Zweck das Gymnasium und die Realschule einer benachbarten Stadt, und später noch ein anderes Gymnasium. Sein Bericht sprach eine unerwartete Befriedigung aus, und er setzte die Revisionen nicht fort. So ging dieser Angriff vorüber.

Der, welcher ihn unternommen hatte, war ein liebenswürdiger Enthusiast für seine Wissenschaft; Mathematiker waren ihm die idealst gerichteten und glücklichsten Menschen. Darum wünschte er nichts mehr, als dass die Schulen endlich dahin kommen möchten einzusehen, welches Glücks sie sich berauben, wenn sie sich noch immer von den alten Sprachen dominiren lassen: „wie viel reicher und tiefer sei doch ein Euklidischer Gedanke als ein Ovidischer!" Er war indess keineswegs unduldsam gegen die Ansprüche seiner philologischen Collegen, wie ich es ein paar mal an Gymnasien erlebte, wo vom Director der Schutz des Ministers gegen die Übergriffe des Mathematikus angerufen war. Nicht viel öfter als dies unfriedliche Verhältnis ist mir das entgegengesetzte wünschenswertheste vorgekommen, dass ein bedeutender Nathematiker nicht nur gute Kenntnis der alten Sprachen besass, sondern auch gern darin unterrichtete und die Verbindung beider Gebiete fruchtbar zu machen wusste. Dabei denke ich an Männer wie ... Hrm. Grassmann in Stettin, und an Directoren wie August in Berlin .... Das Gewöhnliche ist nach meiner Wahrnehmung an Gymnasien ein ziemlich indifferentes Nebeneinander.

\section{WIESE}

[Wirkl. Geh. Ob.-Regierungsrath im preuss. Cultus-Ministerium]. „Lebenserinnerungen und Amtserfahrungen", Bd. 1 (1886), p. $217-220$.

Wenn ich kinder hette und vermöchts, Sie müsten mir nicht alleyne die sprachen und historien hören, sondern auch 
singen und die musica mit der gantzen mathematica lernen. Denn was ist dis alles denn eyttel kinder spiel? darynnen die Kriechen yhre kinder vor żeytten zogen, da durch doch wunder geschickte leut aus worden zu allerley hernach tüchtig.

\section{LUTHER.}

„An die Ratherren aller Städte deutsches Lands, dass sie christliche Schulen aufrichten und halten sollen" (1524).

s. Werke, Kritische Gesammtausgabe, Bd. 15

(Weimar 1899), p. 46

$=$ Werke (Ausg. v. Irmischer), Bd. 22 (Erlangen 1833), p. 191.

Bei einem Kinde . . muss man im Unterrichte allmälig das Wissen und Können zu verbinden suchen. Unter allen Wissenschaften scheint die Mathematik die einzige der Art zu seyn, die diesen Endzweck am besten befriedigt.

\section{InMANUEL KaN'T.}

„Über Pädagogik."

s. Werke, herausg. v. Rosenkranz u. Schubert, Th. 9 (Leipzig 1838), p. 409.

. . o

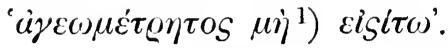

[. . Plato, vor dessen Lehrplatz angeschrieben war: "Ohne Kenntnis der Geometrie trete man nicht ein."]

\section{Johnnnes Philoponus [6. Jahrh.]}

"In Aristotelis de anima libros commentaria", I $3 \mathrm{~d} 3 \mathrm{v}$.

s. Commentaria in Aristotelem graeca (Ausg. der Berl. Akademie) 15 (1897), p. 117.

Die Mathematik ist ... ihrem Weseu und Aufbau nach eiu Gymnasiallehrfach in ganz besonderem Sinne. Insbesondere

1) $\mu \eta \delta \varepsilon i_{S}$ nach Eliae (olim Davidis) in Aristotelis Categorias Commentarium $226 \mathrm{r}$ et $226 \mathrm{v}$; s. Commentaria in Aristotelem Graeca (Ausg. der Berl. Akad.) 18, Pars I (1900), p. 118, 18 u. 119, 4.

A hrens, Scherz und Ernst i. d. Mathem. 
die höhere Mathematik, auch wenn sie nur in ihren ersten Grundzügen dargestellt wird, vereinigt alle diejenigen Eigenschaften in sich, welche ein Lehrfach des Gymnasiums haben muss. Sie packt, sie regelt, sie befruchtet, sie schärft, macht aufmerksam, ebenso bedächtig als findig, flösst Mut und Selbstvertrauen, ebenso wie Bescheidenheit und Unterordnung des eigenen Sinns unter die gegebene Wahrheit ein. Sie giebt von allem das Wesen und den Kern, ist knapp in der Form und übersprudelnd im Reichtum des Inhalts. Sie deckt die Tiefe und Weite des Gesetzes und den geistigen Inhalt hinter der Oberfläche der Erscheinungen auf, sie reisst ron einem Punkt zum andern fort und trägt in sich selbst den Antrieb zum Fortschritt, sie wirkt ebenso anregend auf das künstlerische Terständnis, auf den Geschmack im Urteil und im Schaffen wie auf die wissenschaftliche Auffassung der Dinge. Mehr als irgend ein anderes Fach macht sie daher die Jünglinge begierig, man möchte sagen lüstern, immer tiefer in den Grund der Dinge einzudringen und die eigene Kraft mit Selbständigkeit zu verwenden, sie sammelt den Geist und lenkt ihn auf einen einzigen Punkt, sie weckt dadurch das eigene Forschen, das Selbstvertrauen, die Lust an der Arbeit, reizt durch die Ausblicke, die sie eröffnet und gewährt durch die allseitige Gültigkeit ihrer Betrachtungsweise Sicherheit und Zuversicht. . . . . Ein so vorbereiteter Jüngling dürstet nach Weisheit und ist reif fïr die Hochschule und ihre Wissenschaften.

Darum wäre es sachlich begründet und sprachlich gerechtfertigt, wenn das Wort Realgymnasium, dessen Zusammensetzung und fremdartiger Klang schon etwas Anstössiges hat, in mathematisches Gymnasium verwandelt würde.

\section{Dillmanan.}

„Die Nathematik die Fackelträgerin einer neuen Zeit" (Stuttgart 1889), p. 40/41.

Der gewissenhafte mathematische Unterricht bildet das Anschauungsvermögen und den Intellect so aus, dass jede andere 
Geistesthätigkeit Nutzen daraus ziehen kann. Die übermittelten Kenntnisse sind so vieler Anwendungen fähig, ihr weiterer Ausbau ist so reizvoll, dass sie für das spätere Leben zu einem sicheren Schutz gegen geistige Vereinsamung werden. Deshalb sollte den gescheidtesten und wohlwollendsten Lehrern die Einführung der jugendlichen Geister in die Mathematik anvertraut werden. Die einzelnen Klassen, eigens für den mathematischen Unterricht gebildet, sollten die Schülerzahl von zwölf nicht übersteigen; nur dann ist der Lehrer im Stande, zu beurtheilen, ob ein Jeder ihn verstanden hat; Frage und Antwort, Beispiele und Anschauung liefern ihm dazu die Mittel.

\section{Paul Güssfeldt.}

„Die Erziehung der deutschen Jugend“, Deutsche Rundschau 62 (1890), p. 245.

Auf dem Gymnasium in Neisse war mir die Mathematik ein Greuel, in Königsberg wurde innerhalb dreier Monate die Mathematik mein Lieblingsfach. In Neisse wurde . . . diese Wissenschaft rein zur Gedächtnissache gemacht. In Königsberg bestand der mathematische Unterricht hauptsächlich aus Aufgaben. Selbst auf Findung der Lehrsätze wurden wir hingeleitet; jedenfalls bestand nach dem Vortrage eines Lehrsatzes die erste Aufgabe in dem Suchen des Beweises. Die Schüler mussten geistig thätig sein, sie lernten mathematisch denken. Nur unter dieser Bedingung ist die Mathematik ein wirkliches und sehr erfolgreiches Bildungsmittel. Wenn auf den meisten Gymnasien auch jetzt nach sechzig Jahren in der Mathematik so wenig geleistet wird und dies Wenige nach ein paar Jahren wieder vergessen ist, so liegt dies unzweifelhaft an der Methode und an den Lehrern. Zu deren Entschuldigung muss indessen angeführt werden, dass die angedeutete Art des mathematischen Unterrichts eine sehr bedeutende Kraft und Anstrengung des Lehrers voraussetzt, die älteren Männern nur selten zugemutet werden kann. Die fortwährende Anregung und Heranziehung 
der Schüler zur Selbstthätigkeit, die stete Erfindung neuer Aufgaben, welche den Kreis des bis dahin Gelernten nicht überschreiten, aber vollständig ausfüllen und nicht in jedem Schüler zugänglichen Hilfsbüchern, wie Meier Hirsch ete, stehen dürfen; die genaue Durcbsicht der Hefte und die Kritik derselben in der Klasse, wobei sich jedesmal evident ergibt, ob sich der Schüler hat helfen lassen, ob er gar abgeschrieben hat; die steten Repetitionen durch Aufgaben und Lösung derselben an der Tafel in der Klasse, das alles nimmt die eigene geistige Thätigkeit des Lehrers unendlich mehr in Anspruch als der Vortrag nach einem Kompendium und seltene Aufgaben aus ganz bekannten Schmökern.

Es gibt ein ganz sicheres Merkmal dafür, ob der mathematische Unterricht nach richtiger Methode gut erteilt wird oder nicht. Im ersten Fall macht die ganze Klasse mit sehr wenigen Ausnahmen augenscheinliche Fortschritte und das Gelernte sitzt fest; im andern Fall folgen nur einige begabte und fleissige Schüler dem Vortrage, die Mehrzahl bleibt teilnahmlos und kommt nicht vorwärts. Man lege doch diesen Prüfstein bei unseren Gymnasien an, und man wird über das Resultat bei den meisten erschrecken.

\section{Hans Victor v. UnRuh}

[der bekannte Politiker und Ingenieur].

„Erinnerungen aus dem Leben“, herausg. von H. v. Poschinger (1895), p. 20.

Meyer ${ }^{1}$ ) [„Procurator" am Paedagogium Kloster Lieben Frauen zu Magdeburg] beschränkte sich, wozu fast alle Lehrer der Mathematik und Physik neigen, auf eine Elite und liess uns Übrige treiben, was wir wollten. Gewöhnlich lasen wir Romane. Nur zuweilen richtete er an uns Pöbel einige Fragen, mit offenbarem Humor, uns etwas einzuschüchtern, damit wir nicht zu weit in den Freiheiten gingen, die uns unser Unverstündniss gestattete. Ich höre noch seine Stimme: „Wenn das Licht aus einem dünneren Medio in ein dickeres übertritt, was geschieht?"

1) Christoph Jakob Eusebius Meier, † 20. XI. 1835. 
Hierauf oder auf die umgekehrte Frage antworteten wir stereotyp: „Es bricht sich“. Damit waren wir abgefunden.

Karl Rosenkranz.

„Von Magdeburg bis Königsberg“ (Berlin 1873), p. 112/113.

Soll ich mich in allgemeinem Sinne über Paedagogik äussern, so will ich folgende Betrachtung vorausschicken: Man kann das paedagogische Problem mathematisch formulieren, indem man die individuellen Qualitäten des Lehrers und seiner n Schüler als ebensoviele Unbekannte einführt, und nun verlangt, eine Function von $(1+n)$ Variablen:

$$
\mathrm{F}\left(\mathrm{x}_{0}, \mathrm{x}_{1}, \cdots \mathrm{x}_{\mathrm{n}}\right)
$$

unter gegebenen Nebenbedingungen zu einem Maximum zu machen. Liesse sich dieses Problem eines Tages entsprechend den bis dahin realisirten Fortschritten der psychologischen Wissenschaft direct mathematisch behandeln, so wäre die (praktische) Paedagogik von da ab eine Wissenschaft, so lange das aber nicht der Fall ist, muss sie als Kunst gelten.

\section{Felix Klein.}

„Über Aufgabe und Methode des mathematischen Unterrichts an den Universitäten", Vortr. Naturf.-Vers. Düsseldorf 1898.

s. Deutsche Mathem.-Verein. Jahresber. 7, 1897/1898, p. 133.

Die Vorbereitung auf die einzelnen Lektionen muss man sich selbst machen, und zwar im Anfange so, dass das Bild der Stunde vorher genau so vor den Augen des Lehrers steht, wie sie sich nachher abrollt. Der Lehrer muss, ehe er die Klasse betritt, genau wissen, welche Frage er welchem Schüler fünf Minuten vor Schluss vorlegt, nur dann hat er jene „drüberstehende Sicherheit", die dem Lehrer und Leiter der Klasse geziemt. Gerade die Vorbereitung für den elementarsten Unterricht, den Rechenunterricht in der Sexta und Quinta, und die Planimetrie in der Quarta ist die mühsamste.

\section{Max Srmon.}

"Rechnen und Mathematik" in Baumeister's "Handbuch der Erziehungs- und Unterrichtslehre für höhere Schulen", Bd. IV, 2 , r, p. 36 . 


\section{$-486$}

Es ist mir . . . nicht zweifelhaft, dass für einen im höchsten Sinne erfolgreichen mathematischen Unterricht eine gewisse spezifische Begabung erforderlich ist, womit nicht bestritten werden soll, dass es sehr wohl möglich und für die logische Zucht des Denkens notwendig ist, einem jeden normal begabten Schüler ein mathematisches Wissen und Verstehen in gewissem Umfang zu gewähren, die ihm bei jedem künftigen Studium von Nutzen sind. - _ - - - -

... dass ein Schüler ohne jedes Verständnis durch die mathematischen Lehrstunden geht und schliesslich doch als reif entlassen wird, kommt jetzt wohl kaum noch vor.

Es ist dies ein unverkennbarer Fortschritt; nur darf er nicht auf Kosten des innern Gehaltes gemacht werden, damit auch der mathematisch tiefer Angelegte bei dem neuen Systeme noch zu seinem Rechte kommt. Dies geschieht aber nicht dadurch, dass man die bessern Schüler möglichst weit über die Grenzen der Elementarmathematik hinaus in das Gebiet der höheren Analysis führt. Dadurch werden künftige gründliche mathematische Studien eher gehemmt als gefördert. Viel fruchtbarer, bildender und belebender ist die Vertiefung des Inhalts des elementaren Unterrichtes, der innerhalb der alten Grenzen einen unerschöpflichen Reichtum an Stoff bietet.

Es soll hier dem Lehrer vollkommene Freiheit gelassen werden, unter dieser Fülle je nach seiner wissenschaftlichen Neigung die Auswahl zu treffen. Denn nur da kann eine fruchtbare Einwirkung auf den Schüler erwartet werden, wo auf der Seite des Lehrers selbst noch Interesse an dem Gegenstand des Unterrichts lebendig ist.

\section{H. WEBER.}

Vorrede zu Bd. 1 der „Encyklopädie der Elementar-Mathematik ${ }^{4}$ von H. Weber und J. Wellstein (Leipzig 1903), p. VI u. VII.

Als vor etwa zehn Jahren ein Manuskript für das von Kronecker redigirte Journal für Mathematik einlief ${ }^{1}$ ), zog

1) anscheinend: J. Worpitzky, „Studien über die Bernoullischen und Eulerschen Zahlen", J. f. Math. 94 (1883), p. 203-232; hierzu Bemerkungen von Kronecker l. c. p. $268 \mathrm{f}$. 


\section{$-487$}

dieser aus dem Kasten ein Primanerheft seiner Gymnasialzeit, in welchem die Grundgedanken der eingelieferten Abhandlung über die Bernoulli'schen Zahlen nach einer von Kummer [Kronecker's Lehrer auf dem Gymn.] gestellten Aufgabe behandelt worden waren.

E. LAMPe.

„Leopold Kronecker †. Nachruf“, Naturw. Rundschau 7 (1892), p. 129.

Die technische Hochschule beansprucht von dem eintretenden Studirenden nur die Beherrschung der Elemente der Mathematik. Einige Realgymnasien haben bis in die neueste Zeit Differential-Rechnung gelehrt! Die analytische Geometrie steht noch heute auf dem Lehrplane. Wozu das, wenn Universität und technische Hochschule doch von vorn beginnen? Weder die Hochschule noch das Publikum beansprucht solche Fachkenntnisse. Nur die Lehrer der Realgymnasien halten an ihnen fest, überfüllen damit den Lehrplan und halten einen angeblich wesentlichen Unterschied zwischen Gymnasium und Realgymnasium auf diese Weise aufrecht.

\section{G. Holzmülluer.}

„Zur Frage der Berechtigung der Realgymnasien“, Zeitschr. d. Ver. deutscher Ingenieure, Bd. 29, No. 41 (1885), p. 791 $=$ Zeitschr. math. naturw. Unterr. 16 (1885), p. 546.

Es giebt . . e einige Lehren in der niedern Mathematik, welche derjenige, der später auch die höhere kennen lernt... erst wieder los werden muss, und gerade das Losreissen von eingewurzelten Vorstellungen erschwert das Studium bisweilen recht sehr. Zudem widerspricht es dem Charakter der Mathematik ganz und gar, denn dies ist ja eben ihr grosser Ruhm vor den andern. Wissenschaften, dass beim Aufbau ihres erhabenen Gebäudes kein Umbau je nothwendig sei; das muss dann aber auch ganz und gar wahr sein. Daher fort mit dem scharfen Gegensatze, den die niedere Geometrie zwischen 
parallelen und sich schneidenden Geraden bildet und den die höhere wieder aufhebt; parallele und sich schneidende Gerade haben viel mehr Gemeinsames als Unterscheidendes.

\section{R. STURM.}

„Über einige Incorrectheiten, die sich in der Sprache, besonders der elementaren Mathematik eingeschlichen haben",

Zeitschr. math. naturw. Unterr. 1 (1870), p. 277.

Die Schwäche liegt in der Methode der höheren Geometrie, nicht in der niederen.

\section{J. KOBER.}

„Über die Definition des Parallelismus. Bemerkung zu dem Aufsatze Sturms" . ., ibidem, Bd. 1 (1870), p. 493.

Meine früheren Bemerkungen tragen gewiss, weil sie noch einer Zeit entstammen, wo ich mir noch nicht so klar über die Sache war, wie jetzt, manches Unvollkommene an sich und was der Unklarheit, um mich mathematisch auszudrücken, stets proportional ist, sind vielleicht auch etwas zu schroff. -

Machen wir uns alle an die saure Arbeit der Aufstellung einer auch schon für Tertianer verständlichen und der neuen Anschauung sich anbequemenden (ihr wenigstens nicht widersprechenden) Definition. - - - - - - - - -

Der grosse Historiker der inductiven Wissenschaften Whewell thut einmal die Äusserung: „Das Wesen der Triumphe der Wissenschaft und ihres Fortschritts besteht darin, dass wir veranlasst werden, Ansichten, welche unsere Vorfahren für unbegreiflich hielten und unfähig waren zu begreifen, für evident und nothwendig zu halten." Die Geschichte der Mathematik beweist hinreichend, dass dieselbe auch für diese Wissenschaft gilt. Wo wären wir, wenn wir heute Euclid's Satz (Elemente X, 7): „Incommensurable Grössen verhalten sich nicht wie Zahlen zu einander", für richtig hielten, wenn wir noch nicht gelernt hätten, die incommensurablen Grössen zu begreifen, und die alte beschränkte Anschauung von den Zahlen nicht längst abgeworfen hätten? Wo, wenn wir noch die negativen 
Zahlen (die quantitates falsae) als unbegreifliche Dinge abfertigten, so wie wir es heute noch in der Schule - wohl grösstentheils aus Mangel an Zeit - mit den imaginären Zahlen thun? Das Paradoxe muss man begreifen lernen, wenn es sich aller Orten in der Forschung darbietet und wenn es zudem sich als etwas für die Forschung höchst werthvolles gezeigt hat, wie das mit den negativen, den irrationalen, den imaginären Grössen und so auch mit der neuen Anschauung von den Parallelen der Fall ist.

\section{R. Sturu.}

„Über die unendlich entfernten Gebilde", ibidem, Bd. 2 (1871), p. 392, 393 u. 398.

Schade, dass Herr Sturm seine Schulzeit nicht in Dresden verlebt und unsre Anstalt als Schüler besucht hat. Dann würde er wahrscheinlich den grössten Theil seines Aufsatzes, sicherlich aber die „Bitte“ an „erfahrenere Collegen“, „Versuche“ mit der "neuen Anschauung“" zu machen [1. c. Bd. 2, p. 407], weggelassen haben, da er sich überzeugt hätte, dass dieselben wenigstens bei uns längst aufgehört haben, „Versuche“ zu sein.

Es scheint übrigens, dass ich mich an der angefochtenen Stelle ${ }^{1}$ ) nicht ausführlich genug ausgesprochen habe; sonst würde man wohl nicht aus derselben herausgelesen haben, dass ich die Sicherheit der Methode und der Resultate der höheren Mathematik anzweifle. Ich hätte vielleicht in dem Schlusssatze .... statt „Schwäche" ein milderes Wort schreiben sollen.

\section{J. KoBer.}

„Bemerkung zu Dr. Sturm's vorstehendem Aufsatze“, ibidem Bd. 2 (1871), p. 408.

I think that the study of natural and experimental science and mathematical culture should go on hand in hand together .... I should rejoice to see mathematics taught with that life and animation which the presence and example of her young and buoyant sister could not fail to impart, short roads preferred to long ones, Euclid honourably shelved or buried „deeper

1) s. das Citat der vorigen Seite. 
than did ever plummet sound" out of the schoolboy's reach, morphology introduced into the elements of Algebra - projection, correlation, and motion accepted as aids to geometry - the mind of the student quickened and elevated and his faith awakened by early initiation into the ruling ideas of polarity, continuity, infinity, and familiarization with the doctrine of the imaginary and inconceivable.

\section{SYLVESTER.}

Address Meeting of the British Association for the advancement of Science, Exeter 1869.

cf. Report, Notices and Abstracts, p. 6 .

During the last few years some determined efforts have been made to displace Euclid's Elements in our schools, but the majority of experienced teachers still regard it as the best foundation for geometrical teaching that has yet been published.

\section{W. W. Rouse BaLL.}

„A short history of Mathematics" (1888), p. 51.

Heute ist die Geometrie des Euclides aus unseren hervorragendsten Fachzeitschriften beinahe völlig verdrängt, und auch aus dem akademischen Unterricht ist sie nahezu verschwunden. Die noch vorhandene, an Umfang keineswegs geringe Production trägt vielfach ein antiquirtes Gepräge.

E. Study.

"Sphärisehe Trigonometrie, orthogonale Substitutionen und elliptische Functionen", Leipziger Abhandl. 33 (1893), (20. Bd. der Math.-phys. Cl.), p. 88.

Es ist sehr zu bedauern, dass die Richtung der mathematischen Wissenschaften auf den Universitäten den Anschluss an dasjenige, was Aufgabe der Schule ist, immer mehr aufgiebt.

\section{WIESE}

[Wirkl. Geheim. Ober-Regierungsrath im preuss. Cultus-Ministerium]. „Lebenserinnerungen und Amtserfahrungen“, Bd. 2 (1886), p. 198. 
Der Hochschulunterricht . . . wird erst dann von nachhaltigem Erfolge sein, wenn er von einem systematischen, wenn auch kurzen . . . . Repetitorium der Elementarmathematik . . ... begleitet wird. Man wird selten einen unmittelbar von der Mittelschule gekommenen Zuhörer finden, der beispielsweise eine klare Anschauung davon hätte, dass dem Satze ", $\mathrm{a}^{0}=1^{\text {“ }}$ zwei ganz verschiedene Bedeutungen zukommen, einmal lediglich als Einführung einer Bezeichnung, das andere Mal aber als ein zur Begründung der Potenzlehre notwendiger Grenzsatz. W. Franz Meyer.

"Zur Ökonomie des Denkens in der Elementarmathematik", Vortr. Naturf.-Vers. Düsseldorf 1898.

s. Deutsche Mathem.-Verein. Jahresber. 7, 1897/1898, p. 147/148.

Unsere Studirenden wachsen zur Zeit heran, indem sie gleich anfangs alle die intricaten Verhältnisse kennen lernen, welche die moderne Analysis als möglich aufgedeckt hat. Das ist gewiss gut, aber es hat eine bedenkliche Folgeerscheinung, dass nämlich die jungen Mathematiker sich vielfach scheuen, überhaupt bestimmte Sätze zu formuliren, dass ihnen die Frische fehlt, ohne welche auch in der Wissenschaft kein Erfolg errungen werden kann. Auf der anderen Seite glaubt die Mehrzahl der Praktiker sich den angedeuteten schwierigen Untersuchungen einfach entziehen zu dürfen. Sie lösen sich dadurch von der strengen Wissenschaft $a b$ und entwickeln für ihren Hausgebrauch eine besondere Mathematik, die wie ein Wurzelschössling neben der veredelten Pflanze emporschiesst. Wir werden alles einsetzen wollen, dass die hier vorliegende gefährliche Spaltung überwunden wird. Sei es dem entsprechend gestattet, mit zwei Sätzen meine eigene Stellung in dieser Sache zu präcisiren:

Erstlich glaube ich, dass die von mathematischer Seite gerügten Mängel der räumlichen Anschauung nur temporäre sind, dass man die Anschauung üben kann, so dass man mit ihrer Hülfe die abstracten Entwickelungen der Analytiker jedenfalls in ihrer Tendenz versteht.

Ich glaube ferner, dass bei der so geforderten Ausbildung 
der Anschauung die Anwendungen der Mathematik auf Gegenstände der Aussenwelt in der Hauptsache ungeändert bestehen bleiben, sofern man sich nur entschliesst, dieselben durchweg als eine Art von Interpolation gelten zu lassen, welche die Verhältnisse mit einer den praktischen Anforderungen genügenden, aber doch nur begrenzten Genauigkeit darstellt.

\section{F. KLEIN.}

„Riemann“, Vortrag Naturf.-Vers. Wien 1894.

s. Deutsche Mathem.-Verein. Jahresber. 4, 1894/1895, p. 86/87.

Sollen wir in den einführenden Vorlesungen über höhere Analysis schon versuchen, unentbehrliche Fundamentalbegriffe mit angemessener Gründlichkeit zu erörtern, oder uns statt dessen mit gewissen alt hergebrachten Surrogaten begnügen? Die Beantwortung dieser Frage wird immer bis zu einem gewissen Grade vom individuellen Geschmack, von allerhand Erfahrungen und von Nebenumständen verschiedener Art abhängen. Mir persönlich will es scheinen, dass jener angeblich leichtere Weg sich gar bald als ein recht dornenvoller Umweg erweist. Und das bekannte Sprichwort: „Wer billig kauft, kauft teuer", halte ich, wie zumeist im gewöhnlichen Leben, so auch hier für zutreffend. ....... Ich bin zu der Überzeugung gekommen, dass die Anstrengung, ohne welchenun einmal Mathematik nicht gelernt werden kann, durch logische Verschïrfung der Lehrmethode keine wesentliche Steigerung erfährt.

A. Pringshem.

„Zur Frage der Universitäts-Vorlesungen über InfinitesimalRechnung“, Vortrag Naturf.-Vers. Düsseldorf 1898.

s. Deutsche Mathem.-Verein. Jahresber. 7, 1897/1898, p. 142 u. 143.

Die Studierenden der ersten Semester haben noch das Recht, an irrationale Zahlen zu glauben, das komplexe Gebiet als ein unentbehrliches Hilfsmittel dankbar und kritiklos hinzunehmen, jeden Grenzprozeß unbefangen mitzumachen und zum Unendlichen Stellung zu nehmen wie zum kleinen Einmaleins.

Eughen NetTo.

"Elementare Algebra“ (Leipzig 1904), Vorwort, p. III. 
Bacon de Verulam a dit que l'admiration est le principe du savoir ${ }^{1}$ ); sa pensée qui est juste en général, l'est surtout à l'égard de notre science, et je m'en autoriserai pour exprimer le désir qu'on fasse, pour les étudiants, la part plus large aux choses simples et belles qu'à l'extrême rigueur, aujourd'hui si en honneur, mais bien peu attrayante, souvent même fatigante, sans grand profit pour le commençant qui n'en peut comprendre l'intérêt.

Cimarles Hermite à E. Jahnke. Paris, 25. XI. 1900.

voir Arch. Math. Phys. (3) 1 (1901), p. 20.

Es ist ein Irrtum zu glauben, dass die Strenge in der Beweisführung die Feindin der Einfachheit wäre.

Das schlagendste Beispiel . für meine Behauptung ist die Variationsrechnung. Die Behandlung der ersten und zweiten Variation bestimmter Integrale brachte zum Teil äusserst complicirte Rechnungen mit sich, und die betreffenden Entwickelungen der alten Mathematiker entbehrten der erforderlichen Strenge. Weierstrass zeigte uns den Weg zu einer neuen und sicheren Begründung der Variationsrechnung ... [und] die Verfolgung dieses Weges bringt zugleich eine überraschende Vereinfachung der Variationsrechnung mit sich.

D. HiLiberT.

„Mathematische Probleme“, Vortrag Mathem.-Kongr. Paris 1900.

s. Göttinger Nachr. 1900, Math.-phys. Kl., p. 257/258

$=$ Arch. Math. Phys. (3) 1 (1901), p. 48/49.

En devenant rigoureuse, la Science mathématique prend un caractère artificiel qui frappera tout le monde; elle oublie ses origines historiques; on voit comment les questions peuvent se résoudre, on ne voit plus comment et pourquoi elles se posent.

Cela nous montre que la logique ne suffit pas; que la Science de la démonstration n'est pas la Science tout entière et que l'intuition doit conserver son rôle comme complément,

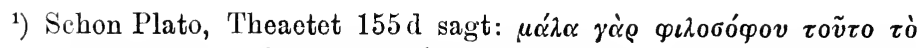

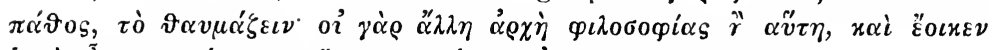

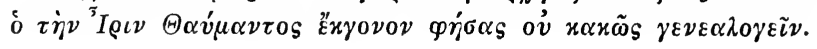


jallais dire comme contrepoids ou comme contrepoison de la logique.

\section{H. Poincaré.}

„Du rôle de l'intuition et de la logique en mathématiques." voir Compte rendu du deuxième congrès intern. des mathém. Paris 1900 (Paris 1902), p. 123/124.

Ich glaube, dass gerade der leichtfertige und inconsequente Gebrauch des Wortes "Grösse" in den Köpfen der Mathematiker (nicht bloss der Anfänger) viel Unheil angerichtet hat, und sollte daher meinen, dass es allgemein als ein wahrer Segen empfunden werden müsste, wenn dieser in seiner quallenhaften Unbestimmtheit geradezu gemeingefährliche terminus technicus aus dem Unterrichte möglichst verschwindet.

A. Pringsilem.

"Über den Zahl- und Grenzbegriff im Unterricht",

Vortrag Naturf.-Vers. Braunschweig 1897.

s. Deutsche Mathem.-Verein. Jahresber. 6, 1896/1897, p. 80.

Wer .., wie Herr Pringsheim, das propädeutischmethodische Verfahren mit einem "molluskenhaften“ identificieren will, dem bleibt dies unbenommen, nur ist er dann nicht als Lehrer der höheren Schule zu gebrauchen, sein Feld ist vielmehr die Universität, wo er die absolute Strenge zum äussersten treiben mag, weit über Pringsheim und Peano hinaus, was voraussichtlich auch einmal nötig werden mag.

G. HolzmülLer.

„Zur Methodik des mathematischen Unterrichts auf Grund der preussischen Lehrpläne von 1891. Ein neves Begleitwort zu meinem methodischen Lehrbuche der Elementar-Mathematik", p. 8. Hagen i/W., 5. I. 1901.

Ich habe ein unbegrenztes Vertrauen zu der Kraft der akademischen Jugend. Man mache höhere Ansprüche und sie werden erfüllt werden! - _ _ _ - _ - -

Der Grundsatz meiner akademischen Thätigkeit ist: Will man gewiss sein, ein mittleres Ziel zu erreichen, so stelle man 
sich das höchste; wer nur nach Mittelmässigkeit strebt, wird auch diese nicht erreichen.

\section{Hermann Hankel.}

„Die Entwickelung der Mathematik in den letzten Jahrhunderten“, Akadem. Vortrag Tübingen 29. IV. 1869,

2. Aufl. (Tübingen 1884), p. 26.

Die „praktische“ Erfahrung an unseren [technischen] Hochschulen zeigt uns, dass bei den jungen Leuten, welche sich der Technik zuwenden, eine ausgesprochene Begabung für die Mathematik nicht sehr häufig vertreten ist.

\section{R. Fricke.}

"Über den mathematischen Hochschulunterricht",

Vortr. Ver. f. Naturw. Braunschweig 19. III. 1902.

s. Deutsche Mathem.-Verein. Jahresber. 11 (1902), p. 245.

Mit der Verkümmerung des Unterrichts geht der Mangel brauchbarer Lebrbücher Hand in Hand. Es giebt dafür kein schlagenderes Beispiel als das folgende. Die Analysis des Unendlichen hat in den letzten 25 Jahren eine wesentliche Umgestaltung erfahren. Wir haben zunächst die Verschärfung der Grundbegriffe des Differentiierens und Integrierens. Wir haben sodann die Theorie komplexer Variabler mit ihrer ganz neuen Einsicht in das Wesen des Funktionsbegriffs. Wir haben endlich eine wesentlich vollständigere Kenntnis der algebraischen Differentiale und Differentialgleichungen. Aber wo ist das für den allgemeinen Gebrauch bestimmte Lehrbuch der Differential- und Integralrechnung, das von alle dem Rechenschaft gäbe? Unsere besseren Bücher sind immer noch diejenigen, welche auf Cauchy's Cours d'analyse zurückgehen, und der ist jetzt nahe 60 Jahre erschienen.

\section{F. KLEIN.}

„Über die Beziehungen der neueren Mathematik zu den Anwendungen", Antrittsrede Leipzig Univ. 25. X. 1880. s. Zeitschr. math. naturw. Unterr. 26 (1895), p. 537/538. 


\section{Noten.}

1. Man vergleiche hier zu einander: S. $43 / 44$ (Gauss an Schumacher), S. 152/153 (Gauss an Olbers) und S. 252 (Gauss an Schumacher), sowie auch S. 440 (Gauss an Schumacher).

2. Zu S. 59: vgl. a. S. 378 .

3. Zu S. 80: vgl. hierzu S. $246 / 247$.

4. Zu S. 81: vgl. hierzu a. S. 238 und 239.

5. Zu S. 93: s. S. 214 (Shakespeare).

6. Zu S. 99: Als 1848 die Herausgabe des Briefwechsels Gauss-Bessel zusammen mit der des Briefwechsels Bessel-Olbers geplant wurde, äusserte Gauss, „er wisse recht gut, wie viel von den vielen Complimenten, die sich in fast allen Briefen Bessel's fänden, abzuziehen sei, auf den Grund vou Bessel's Liebhaberei, den Leuten gern etwas Angenehmes zu sagen, oder etwas wovon er voraussetzte, dass sie es gern hörten"; für diese Stelleu bat er daher - wenigstens bei einer Veröffentlichung zu seinen Gauss') Lebzeiten - um ein "(nöthigenfalls unbarmherziges) Wegs(chneiden" (s. den Brief Gauss' an Schumacher rom 23. XII. 1848 in Briefw. Gauss-Schumacher, Bd. 6, p. 11).

7. Zu S.S. 105, 112, 133 u. 136: vgl. hierzu S. 442 (Gauss an Schumacher), S. 444/445 (Gauss an Bessel), sowie S. 446/447 (S. Gundelfinger); man vergleiche zu diesen Stellen übrigens auch das von F. Klein herausgegebene Tagebuch Gauss' in der „Festschr. zur Feier des 150-jähr. Bcstehens d. Kgl. Ges. d. Wiss. zu Göttingen" (Berlin 1901) und zwar besonders die Fussnote Klein's zu No. 111 des Tagebuchs.

8. Zu S. 124: vgl. hierzu S. 328 (Friedrich d. Gr. an d'Alembert).

9. Zu S. 126: vgl. hierzu S. 296 (Dirichlet).

10. Zu S. 127: vgl. a. S. 413 und 414 .

11. Zu S. 136: s. S. 426 (Legendre an Jacobi).

12. Zu S. 159: s. S. 294 oben (Arago).

13. Zu S. 173: vgl. hierzu S. 254 (C.-A. Valson) u. S. 255 (Arago).

14. Zu S. 219 : s. S. 481.

15. Zu S. 228: Sylvester hatte in der handschriftlichen Aufzeichnung dies Gedicht, in dem eine überaus grosse Zahl ungewöhnlicher Worte, vor allem Eigennamen, vorkommen, mit erläuternden Anmerkungen versehen, die jedoch, als nach dem Tode des Verfassers die hier reproduzierte Probe publiziert wurde, nicht mit abgedruckt wurden. Da indessen auch schon diese Probe derartig ist, dass man es begreiflich findet, wenn es in der "Academy“, l. c. p. 358 heisst: "An examination paper in Spring's 
Delut would floor most candidates", so mögen hier noch folgende, jedenfalls ausreichende Erläuterungen Platz finden:

Cymbeline (= Cunobelinus resp. Kymbelinus), sagenhafter englischer König, berühmt als das Original von Shakespeare's "Cymbeline".

Imogen, bei Shakespeare Tochter von Cymbeline.

Nell Gwynne (= Eleanor Gwyn, 1650-1687), berühmte Schauspielerin und Maitresse König Karls II.

Evelyn, John (1620-1706), Verfasser verschiedener Schriften, unter denen eine der bekannteren ist: „Sylva; or a discourse of Forest Trees and the propagation of timber"...

Maturin, Charles Robert (1782-1824), Novellist und Dramatiker.

Evangeline, Heldin eines bekannten Epos von Longfellow.

16. Zu S. 230 : s. S. 325 .

17. Zu S. 251 : s. S. 435 (Encke).

18. Zu S. 292: vgl. hierzu a. S. 458 (Hamilton an I)e Morgan).

19. Zu S. 325: s. indessen S. 403 (A. W. v. Hofmann).

20. Zu S. 410: Hermann Vogt, „Die Strassen-Namen Berlins", Heft XXII der „Schriften des Vereins für die Geschichte Berlins" (Berlin 1885) giebt p. 69 hierüber folgendes an:

Ohm-Gasse. Laut Allerhöchster Kabinets-Ordre vom 19. September 1827 wurde „die bisher unbenannte, von der Köpnicker Strasse bis zur Wasser-Gasse bezw. nach dem Wusterhausenschen Holzmarkte führende Nebengasse" Ohm-Gasse benannt. Diese Benennung erfolgte auf Vorschlag des Königl. Polizei-Präsidiums nach dem Professor der Mathematik an der Königlichen Universität M. Ohm, welcher das Grundstück Nr. 2 besass.*

* Gärtner Olım, nach dem, anderen Erklärern nach, die Gasse ihren Namen erhalten haben soll, besass 1812 das Grundstück Köpnicker strasse Nr. 70 an der Holzmarkt-Gasse.

21. Zu S. 471: In der Vorrede zum 1. Band seines grossen Werkes über „Integration der linearen Differentialgleichungen mit constanten und veränderlichen Coefficienten" (Wien 1853), p. III bezeichnet Petzval es als den Zweck jenes Werkes, "diese Wissenschaft .... zu erschöpfen" und bemerkt weiter, dass man darin "mitunter auch die Resultate der Forschungen Anderer findet". In der Vorrede zum 2. Bande (Wien 1859), p. VIl sagt er sodann über die Aufnahme, die der erste Band beim mathematischen Publikum gefunden hatte: „Man hat seine [des Verfassers] Rechnungen zerlegt in kleine Parzellen und hat finden wollen, dass diese nicht neu seien. Siehe da, hat man gesagt, diess ist ein Stein, der sich bereits in einem anderen Lehrgebäude Seite $x$ Zeile $y$ vorfindet, mithin ist es nicht neu. Möge sich der Leser durch solche Recensentenkünste nicht irre führen lassen. Die Untersuchungsmittel, Kunstgriffe

Ahrens, Scherz und Ernst i. d. Mathem. 
der Rechnung, kurz die einzelnen kleineren Bestandtheile sind allerdings alt, aber das daraus aufgeführte Gebäude ist doch neu, und wenn der Leser auch nur bei irgend einem Paragraphe sich zur Annahme verleiten lässt, er habe lauter alte, völlig bekannte Dinge vor sich, so wird er den Standpunkt des Verfassers verlieren und sich selbst das Verständniss erschweren." Allerdings hatte auch Petzval selbst nicht auf eine sofortige Anerkennung seiner Leistungen gerechnet, denn (2. Band, p. V) „brauchte das einbändige Werk [Newton's, Principia nova'] vierzig Jahre zu seiner Aufnahme, so ist diess um so mehr von dem zweibändigen zu erwarten, das ich gegenwärtig rorlege".

\section{Weitere Zusätze und Berichtigungen.}

S. 9, Z. 1 der Anm. lies "Das" statt "Dies".

S. 16: Der Titel der Abhandlung von B. Riemann ist zu verändern in: „Versuch einer Lehre von den Grundbegriffen der Mathematik und Physik als Grundlage für die Naturerklärung" (Fragment).

Zu S. 61 , Z. 3 v. u.: s. S. 67 (Sartorius v. Waltershausen).

S. 101, Z. 12 v. u. lies "und" statt „und und".

Zu S. 126 (Dirichlet über Jacobi): vgl. S. 294 (Condorcet über Euler)

S. 143, Z. 16 v. o. lies "dass" statt "das".

S. 153 , Z. 4 v. o. lies "1814" statt „1831“.

Zu S. 156: Die Originalstelle des ersten Citats (Moritz Cantor) ist: Deutsche Revue, Jahrg. 28 (1903), 1, p. 364.

$\mathrm{Zu}$ S. $165 \mathrm{f}$. (Lagrange-d'Alembert): vgl. S. 253 (Lagrange an d'Alembert).

Zu S. 166, Anm. 1: vgl. a. S. 380 (I. Newton).

S. 189 , Z. 7 v. u. lies "Gardez-vous-en" statt "Gardez-vous en".

S. 230 , Z. 4 v. o. lies "preuves" statt "preuve".

Zu S. 236,379 u. 397 (Citate aus W. Preyer, „Üb. d. Urspr. des Zahlbegriff's"): Um einer Verwechselung mit einer aus gleichem Anlass erschienenen ophthalmologischen Festschrift vorzubeugen, sei hier der genaue Titel des citierten Sammelbandes, wie folgt, angegeben:

„Beiträge zur Psychologie und Physiologie der Sinnesorgane. Hermann v. Helmholtz als Festgruss zu seinem siebzigsten Geburtstag dargebracht". . Gesamm. u. herausg. v. Arthur König (Hamburg \& Leipzig 1891).

Zu S. 242 , Z. 5-8 v. 0.: s. d. M. Annaeus Lucanus Gedicht „Pharsalia", wo im 10. Gesang Cäsar bei einem ihm von Cleopatra gegebenen Gastmahl den Priester Acoreus zu einem Vortrage über die unbekannten Quellen des Nils auffordert; in der darauffolgenden längeren Rede des 
Priesters (Liber X, 194-331 in der Ausg. von C. M. Francken) heisst es in Zeile 295-296:

Arcanum Natura caput non prodidit ulli,

Nec licuit populis parvom te, Nile, videre,

Zu S. 253/254 u. 295 (Citate aus Condorcet): Bei der Teilung eines Citats in zwei ist ein Satz zweimal abgedruckt.

S. 278 , Z. 5 v. o. verbessere den Titel des citierten Werkes in: „Briefe von Alexander von Humboldt an Christian Carl Josias Freiherr von Bunsen".

Zu S. 282, Anm.: vgl. a. S. 325.

S. 284, Z. 3 v. o. lies "me" statt "my".

Zu S. 285 (Citat Brewster): An der angegebenen Stelle bei Laplace, "Exposition du Systême du Monde", seconde édition (Paris, an VII $=1799$ ), p. 336 heisst es: . . "Malgré ces défauts inévitables; l'importance et la généralité des découvertes, un grand nombre de vues originales et profondes qui ont été le germe des plus brillantes théories des géomètres de ce siècle, tout cela présenté avec beaucoup d'élégance, assure à l'ouvrage des principes mathématiques de la philosophie naturelle, la prééminence sur les autres productions de l'esprit humain."

S. 285, Z. 4 des letzten Citats lies "indeed " statt „ndeed“.

Zu S. 291, Z. 4-6 des 2. Citats: vgl. S. 22 (Fourier).

S. 306, Z. 2 des 2. Citats lies „Musschembroek“ statt „Musschenbroek“.

Zu S. 337, Anm.: Nach einem Brief Johannes' III. Bernoulli an Mallet war Lambert eine der Ursachen dafür, dass Euler aus Berlin fortging (s. R. Wolf, „Biographien zur Kulturgesch. der Schweiz“, Bd. 3 (1860), p. 344/345).

Zu S. 390 , Z. 8-6 v. u.: "sub incredibili confluxu auditorum, qui me philosophum theologice disputantem nunquam antea audiverant" schreibt Joh. Bernoulli hierüber an Leibniz, 3. VI. 1699 (s. Leibnizens gesammelte Werke, Ausg. von Pertz, 3. Folge, 3. Bd. (Halle 1856), p. 590).

$\mathrm{Zu}$ S. 398, 2. Citat: vgl. S. 300/301.

S. 419 , Z. 6 v. o. lies "p. $218^{\prime \prime}$ statt „p. $28 . "$

S. 426, Z. 5 v. u. lies "Abels" statt "Abels's".

Zu S. 496, Note 4: vgl. a. S. 246. 


\section{Register.}

Die Zahlen beziehen sich auf die Seiten des Buches: Sie sind fett gedruckt, wenn die betreffende Person, Körperschaft etc., zu deren Registertitel die Zahl gehört, Autor des betreffenden Citats ist. Bezieht sich eine Zahl auf eine Fußnote oder auf die Noten resp. Zusätze am Ende des Buches oder auf die Stellenangabe eines Citats, so ist dies durch Kursivsehrift (Italiques) angedeutet, während die gewöhnlichen Typen (Antiqua) auf den Text der Citate selbst hinweisen. Kommt ein Name in den Stellenangaben etc. nur beilïufig vor, z. B. als der des Übersetzers oder Herausgebers eines Werks etc., so ist dies im liegister nicht berücksichtigt, um letzteres nicht unnötig zu überladen; ebenso sind im allgemeinen die Hinweise in Kursivschrift fortgelassen, wenn schon ein anderer Hinweis auf dieselbe Seite erfolgen musste. Bei den fett gedruckten Zahlen ist ein mehrmaliges Vorkommen durch eine danebengesetzte kleinere Ziffer in Klammern (2), angedeutet.

Die Rücksicht auf den Raum machte den Gebrauch vou Abbreviaturen notwendig, von denen die wichtigsten die folgenden sind:

Math. $=$ "Mathematik"; Mather = "Mathematiker"; math. = "mathe-

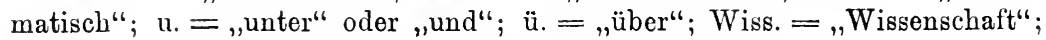
Wissen = "Wissenschaften"; wiss. = ,wissenschaftlich".

Abel, N. H. 21, 31, 133-138, 178, $237,282,420,441,455 f$., 470 .

-, Verkennung s. Genie's u. s. Leistungen $426-429$.

- ü. divergente Reihen $45 \mathrm{f}$.

- ü. Cauchy $181 \mathrm{f}, 42 \hat{\text { o }}$.

- ü. Dirichlet $181 \mathrm{f}$.

- ü. Gauss 102f.

- an Hansteen 47, 103, 400.

- an Holmboe 46, 103(2), 169, 182, 248, 428.

- ü. Jacobi 134.

- ü. Laplace 18\%.

- an Legendre 134, 423.

- ü. Legendre $181 \mathrm{f}$.

- ü. Poisson 182.

- s. a. u. "Arago", „Berzelius", "Bjerknes", "Fourier", „Gauss", "Holst", „Jacobi", "Legendre", "Lie", "Sylow", "Weierstrass". „abelsch" ("abélien“) 138; vgl. a. 136.
Abel'schesTheorem 42\%, 428, vgl. a. 134. Aberglaube 29, 392f.

Acoreus, Priester 498.

Acta mathematica 304.

"Adam", mathematischer 262.

Adelung, Sophie v. -, Cousine S. Kovalevsliy's 23\%, 362 .

Aesop 337.

aesthetisch s. u. künstlerisch.

akademische $u$. andere Wahlen 183 ,

241, 255 f., 385, vgl. a. 351 .

Akakia, Docteur - 195, 370.

Albius, Richard 469.

Alembert, d'-23, 240, 245, 308,370, $\mathbf{4 5 6}$.

- ü. Math. 75, 75, 244(2), 252.

- $\quad$ i. sich selbst $88 \mathrm{f}$., 165,160 , 244(2), 252, 352, 363, 365.

- u. d. schoene Liter. $244(2)$, 245 ; vgl. a. $80,308,363$.

- ü. Euler sð, 250, 290, 33ิ $451 \mathrm{f}$. 
Alembert, d' - an Friedrich d. Gr. 75, 8\%, s9, 338, vgl. a. 310 .

- an Lagrange 16:, 1\%0, $244(2), 250,353,452,472$.

- ü. Lagrange $8 \%$.

- an Voltaire 290.

- s. a. u. „Bernoulli, Daniel", "Euler", "Friedrich d. Gr.", „Lagrange", "Lambcrt", "Laplace".

- 's Nährmutter 21S, 355.

Alerz, Arzt in Rom 130.

Alexander v. Macedonien 88.

Algebra, moderne $53 \mathrm{f}$., 62, 142, 145, 174, 178, 262.

Altenstein, Minister $v$ - $47 \mathrm{~S}$.

Alter und Abnahme der geistigen

Fähigkeiten 136, 143, 158, 170 ,

265,426 ; vgl. a. 171, 366, 370, 422 .

Amerika 11, 334, vgl. a. 329 .

Ampère, A.-M. 18, 50, 183, 236, 267, $341,362,432$.

- , encyklopädisches Wissen 322 -324, 401.

- , Lehrtätigkeit 310, 406, 438.

- , religiöse Ansichten 363, 381, 392.

- , Zerstreutheit u. Nachlässigkeit $267,310,407$.

s.a. u. "Sainte-Beure", "Wöhler.".

Analogien 15, 19, 162 f., 432.

Analysis -- eine Naturwiss. 60.

Analysis u. Synthesis $140 \mathrm{ff}$, vgl. a. 219.

Analytiker u. Synthetiker 140-145, $146 \mathrm{ff}, 420,462 \mathrm{f}$.

antike Geometrie $33 \mathrm{ff}, 58$; s.a. $324,490$.

Anwendungen d. Math., ungewöhnliche $368 \mathrm{ff}$.

Apfel Neuton's 165, vgl. a. 166.

Appell, P. E. 66.

Approximationen, Linear- - 201.

Approximationsmath. $37 \mathrm{f}$., 492.

Araber 38.

Arago 20, 162, 181, 185, 156, 192, 223, 249, 26.), 267, 28:, 294, $297,310,317,324,341,342$,
$343,354,355,3 \% 2,37 \%, 40 \%$, $423,438,451,496$.

Arago ü. Abel 429 .

- ü. Cauchy 255, 385, 496.

- ü. Euler 159, 294.

- ü. Math. $72,74$.

- , Lehrtätigkeit $312 \mathrm{f}$.

- als Politiker 335, 343, 346, 401.

- s. a. u. "Monge", "Wöhler".

Archimedes 84, 175, 223, 245, 278, $416 \mathrm{f}$., 455.

- $\quad$ Tod 336 .

- , Grabmal 390.

— : $\quad$ :ós $\mu \circ \iota \pi \circ \tilde{v} \sigma \tau \tilde{\omega} \ldots 155$.

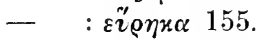

- s. a. u. "Gauss".

Argens, Marquis d'- an Friedrich d.

Gr. 40\%; s. a. u. ,Friedrich d. Gr.". "aristokratische" Gruppen 437.

Aristoteles 80, 218, 245, 314, 364, 481. Arithmetik u. Geometrie 25, 41, $57 \mathrm{ff}$., $78 \mathrm{f}$.

- , Königin der Math. 61, 67, 364 ; s. a. u. ,\%ahlentheorie".

Arithmetisierung der /ahlentheorie, sowie überh. der Math. 57, $59 \mathrm{ff}$,, vgl. a. 378 .

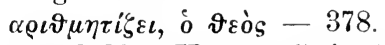

Aronhold an Hesse (ü. Steiner) 143, 145. u. Steiner 143.

Astrologie 392, 394.

Astronomie 3, 67, 76, 93, 116, 121, 193, 216, 223f., 252f., 304, 306, 430 f., $466,469$.

Atomistik 6, 8, 9, 16, 32, $176 \mathrm{f}$.

Auge, Math. eine Wiss. f. das 251, 435, vgl. a. 143.

Augenspiegel 187.

August, E. F., Realgymn.-Dir. 480. Auwers 193.

- $\quad$ ü. L. Fuchs 288 .

Bach, C. v. $-\mathbf{2 0 4}$.

Baco v. Verulam 493.

Bacon-Shakespearefrage $244 \mathrm{f}$.

Baer, K. E. v. - 27.

Bailly 335, 335. 
Ball, W. W. Rouse - 490.

Baltzer, R. 300 .

Barclay 71.

Bartels, J. M. Chr. 95.

barycentrischer Calcul 42 .

Baum, Gauss' Arzt, an A.v. Humboldt ü. Gauss 185, 378.

Beaumont, Élie de - 291.

Beck, Chr. Dan. 318.

Beer, A. 146.

Beethoren 73, $234 \mathrm{f}$., 275.

Beharrlichkeit beim Arbeiten 165f., 253, 380, 429, 439; vgl. a. u. „Cauchy“.

Beiname, Benennung etc. für d'Alembert 252; Aronhold 143; Cayley 263; Dirichlet 140, 412, vgl. a. 292; Eisenstein 285; Euler 159, 294, vgl. a. 290; Gauss 101 f., vgl. a. 28 u. 61 ; Grassmann $331 \mathrm{f}$; Hamilton 458; Jacobi 140, 412; Lagrange 287; Laplace 291; Lie 421; Maxwell 287; Newton 286; Schläfli 404; H. C. Schumacher 100; W. Thomson u. Tait 287; ? 278.

Belesenheit 279, $322 \mathrm{ff} ., 326 \mathrm{f}$., 450, vgl. a. 259.

Beltrami ü. S. Lie $419 \mathrm{f}$.

Berliner philos. Fakultät216, 301, 443.

- math. Schule, Fak. u. dgl. 64, 203, 327, 418f.

- Tageblatt 474.

Bernoulli's, die 453, 463.

Bernoulli, Daniel 352, 370 f., 373.

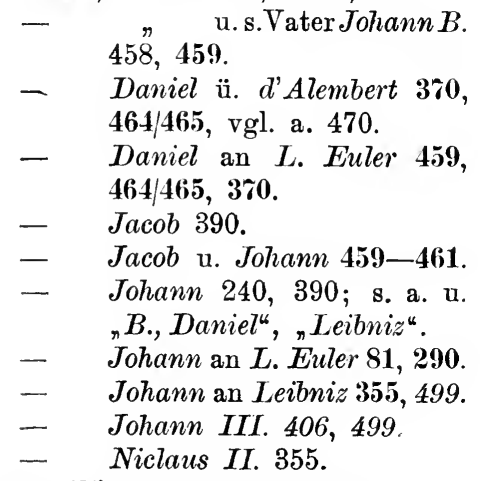

Bernoulli'sche Zahlen 486, 487.
"Bernoullizare" 355.

Bertrand, J. 75, 148, 175, 180, 183, $221,241,252,272,286,292$, $308,322,326,370,384$, 385(2), 404, 411, 421, 424, $446,470$.

- ü. C. G. J. Jacobi $13 \%$.

Berzelius, $J$. ü. Abel 400.

- an J. Liebig 13.

- an F. Wöhler 343, 400.

„beschreiben" 14 f., vgl. a. $18 \mathrm{f}$.

Bessel 2, 76, 92, 113, 408, 466.

- , Lehrtätigkeit 117, 118.

- an Gauss $28,57,97(2), 98,99(2)$, $105,112,114(2), 117,129,269$, $318,357(2), 426,431,467,496$.

- ü. Jacobi 129, 426.

- an Olbers 117, 23s, 496.

- an d. Minister Th. v. Schön 273.

- s. a. u. "Gauss", "Olbers".

Bestimmung der Math. 69.

Betäubungsmittel, Math. ein - $76 \mathrm{ff}$., $230 \mathrm{f}$.

Bethmann-Hollweg, v., Minister 479. Betriebsmathematik 201.

Betti 293.

Bewohnbarkeit der Himmelskörper $10 \mathrm{f}, 388,391$.

Bibel 12, 378.

Bienaymé 404.

Bjerknes, C. A. ü. Abel u. Jacobi $135 \mathrm{f}$. bildender Wert der Math. 69, 70, 79 f., $200,202 \mathrm{f} ., 249,371,374,395$, $397,482 \mathrm{ff}$; s. a. u. "ethisch", "formal ", ,kritisch", , künstlerisch". Bilder der Erscheinungen 16f.

Billy 423.

Binet 404.

Biot 157, 181, 184, 249.

- , Madame 376.

biquadratische Reste 157, 375.

Blanc, Le - s. S. Germain.

Boas, E. 224.

Boeckh 129.

Boguslawski, v. -97.

Bohlmann, G. 420.

Boileau 246. 
Bois-Reymond, Emil du - 4, 5, 9, 49, 71, 200, 239, 269, 275, 348, 396.

- : "Ignorabimus" 7, 20.

- $\quad$ ü. Helmholtz $413 \mathrm{f}$.

- s. a. u. "Helmholtz".

Bois-Reymond, Paul du - 7, 12, 15(2), $51,60$.

- $\quad$ an Kronecker 18.5.

Boisiobert, de - 227.

Boltzmann, L. 17, 19, 7\%, 188, 275, 334.

- $\quad$ ü. s. Lebensaufgabe $176 \mathrm{f}$.

- ü. H. Hertz 1t, 18.

- ü. G. Kivchihoff $13 \mathrm{f}$., $234 \mathrm{f}$.

- $\quad$ u. Maxwell $23+\mathrm{f}$.

Bolyai, Joh. 28 f., 474.

Bolyai, W. 106.

- an Gauss 356, 357.

- ü. Gauss 28, 93 f.(2).

- an Sartorius v. Waltershausen 94.

- s. a. u. "Gauss", "Sartorius v. $W . "$

Bonaparte 184, s. a. Napoleon.

Boole, George 261 .

Böoter 28.

Borchardt 278, 293, 368, 412, 445.

Boscovich, R. G. 383.

Bosscha, J. $22 \%$.

Bradley 226.

Brandes, H. W. 221.

Brewster, D. 221, 255, 499.

Brioschi 293, 346.

Briot 313.

ü. Cayley 14\%, $1 \%$.

Brown 330.

Brücke, Ernst v. - 413.

Bruhns, K. resp. C. 119, 122, 185, 250, 349.

Brunellesco 234.

Buch, das erste - 129.

Buff, Prof. d. Physik 74.

Bunsen, Robert v. - 400 .

Burdach, K. F., Prof. der Medizin 318.

Burkhardt, H. 38, 40, 21\%, 422, 431.
Cuesar 88 f., 498.

Calculs, besondere $42 \mathrm{ff}$., 430, 454 .

Caligula 351.

Cambridge, Herzog v. -100.

Cantor, G. 65, 231 245, 308, 434.

- an G. Eneström 380.

- ü. Gauss $47 \mathrm{f}$.

- s. a. u. "Kronecker".

Cantor, M. 156, 168, 261, 314, 374, 381, 498.

- $\quad$ ü. Gauss 91f., 215, 284, 365.

- ü. Leibniz u. Newton 455.

Carlyle 48 .

Carnot, Sadi, der Wärmetheoretiker 287.

Cassini 393.

Castillon, de, d. Ältere 189.

Catalan, E. Ch. 404.

Catharina II. v. Tussland 86, 340, s. a. 228.

Cauchy 39, 50, 8*, $130 \mathrm{f} ., 136, \mathbf{1 6 3}$, $171,178,183,234,283,346$, $385,427 \mathrm{f}$, $450 \mathrm{f}$.

- , Fruchtbarkeit 255, 370.

- , religiöse Ansichten u. Bestrebungen 11, 131, 182, 379, $380,380,383,383 \mathrm{f} .$, vgl. a. 346 .

- , Ungeduld u. Unbeständigkeit bei d. Arbeit 173, 254 f., vgl. a. $450 \mathrm{f}$.

- Cours d'analyse 495.

- s. a. u. "Abel ${ }^{4},{ }_{\text {"Arago", }}$ "Cayley", "Dühring", "Hermite", "Poncelet".

- scher Integralsatz 446-449.

Causalität $1 \mathrm{ff}$.

Cavalieri 455.

Cavendish 331.

Cayley 194, 264, 297, 326, 329.

- , d. Naturforscher unter den Mathern 263.

- , Vergl. mit Cauchy 288.

- - mit Euler 175.

- s. a. u. „Brioschi ${ }^{\star}$, „Hermite Chamisso 72. "Noether", „Sylvester".

Champeaux, Guillaume de - 379 . 
Charlotte, Sophie - v. Preussen 48. Charpentier, Jacques 272.

Chasles 35, $147 \mathrm{f}$, 172, 326, 404, $469 \mathrm{f}$. - s. a. u. ,Dumas, .J.-B.“

Châtelet, Marquise du- 222, $360 \mathrm{f}$. an Friedrich d. Gr. 74.

Chemie, Chemiker $32 \mathrm{f} ., 171,197,366$, 393, 396, 399-403, 409, 439.

Chemiker ü. Math. u. Mather 74, 399-403, vgl. a. 226.

(hevalier, Auguste 319, 424, 425.

Chladni 20.

Chopin 228.

Christoffel 62.

Cicero 273, $\mathbf{3 6 4}$.

citieren $179 \mathrm{f} ., 423,456 \mathrm{ff}$.

Claivaut 97, 241, 286, 464.

Clebsch. 149, 183, 450, 469.

- , Lehrtätigkeit 298 f.

- s. a. u. "Jacobi".

- Freunde 14\%, 149.

\section{Cleopatra 498.}

Clifford, W. K. 199.

Combes, P.-Ch.-M., Ingenieur u. Nitgl.

d. Pariser Akad. 451.

Condorcet 10, 153, 184, 190, 245, 254, 294 (2), 295, 335, 336, 347, $352,355,369,381,382,393$, 430, 458, 461, 462, 498.

- s. a. u. "Lagrange".

Conduitt, geb. Katherine Barton, Nichte Newton's 165.

Conversation 73, $349 \mathrm{ff}$., $405 \mathrm{ff}$.

Cousin, Victor 273 .

Crelle, A. I. $103 \mathrm{f}$., 316, 328, 426.

Cremona 203.

- ü. S. Lie 420, $\mathbf{4 2 0}$.

Culmann, C. 203.

('urtius, E. 69.

Cuvier 200, 249, 261.

Cymbeline 228, $49 \%$.

Czuber, E. 328.

\section{Daguerre 223.}

Darboux, G. 35, 172, 175. darstellende Geometrie $210 \mathrm{f}$.

Darwin'sche 'Theorie 49, 72; s. a. Descendenzhypothese.
Davis, E. W. 298.

Dedekind, R. 59, 65; s. a. u. „Grauss". Definitionen $262 \mathrm{f}$, 437.

Degérando s. Gérando.

Delambre 181, 237, 241, 286, 291, $341,399,405,431$.

Delbrïck, B. 27\%.

„demokratische" Gruppen 437.

Denderah 11.

Descartes 71, 175, 225, 242, 249, 331, $353,360,381,383,395,455$. an Fermat $18 \%$.

Descendenzhypothese 11 ; s.a. Darwin. Despréaux 246.

Dessoir, M. 39).

Determinanten 39, 53, 139, 376, 411. deus, numero - impare gaudet 377 . deutsche resp. germanische Mather 107, 124, 148, 327 f., 332 f., 436, 438. Deutschland, math. Wiss. in - $129 \mathrm{f}$., $329,400$.

- , math. Unterr. in - resp. Preussen 124 ff., 274, 479.

Diaetetik, geistige 54, 165-171.

Dichter, Mather als - 82, $223 \mathrm{f}, 226$ bis $228,232,283 \mathrm{f}$, $496 f$.

s. a. Literatur, schoene.

Dichter u. Mather 245-247, 248f., vgl. a. $223 / 224$.

Dichtkunstu. Math. 226, 235, 247f., 325.

Diderot 2:6, 252.

Diezmann, A. 373.

Differentialgleichungen 5, 48, 67, 150 , $166,171,238,280,418,471,495,49 \%$.

Differentialquotient, Existenz des - en $49 \mathrm{ff}$.

Differentialrechnung auf der Schule? $395 \mathrm{f}$, $486 \mathrm{f}$. aufderUnivors.? $124 \mathrm{f}$.

Dillmann, C. $\mathbf{7 1 , 4 8 2 .}$

Diophant 61.

Dirichlet, P. G. Lejeune- - 35, 50, $62,73,75,96,115,125$, $130 \mathrm{f} ., 140,156,161,178$, $185,188,218,242,277 \mathrm{f}$., $281,294,316,321,368$, $410 \mathrm{f}, 414,423,466,496$. 
Dirichlet, Art zu arbeiten 161, 293; vgl. a. $96,421 \mathrm{f}$.

- u. Jacobi $292 \mathrm{f}$.

- ü. Jacobi 126, 138, 139, 296 ; vgl. a. $292,498$.

- $\quad$ an Kronecker 282, 422.

- $\quad$, Vergl. mit Lagrange 292. s. a. u. "Abel", „Eisenstein", "Jacobi", "Kronecker".

Dirichlet, Rebecka an Fanny Hensel 127,130 f., 279.

- $\quad$ ü. Jacobi $127 \mathrm{f}$., 130.

- s. u. a. "Hensel, Fanny", "Mendelssohn, Felix".

Diskontinuitätsfaktor 156.

Disquisitiones arithmeticae 55, 56, $94 \mathrm{ff}$., 112, 115, 154, 361, $364 \mathrm{f}$.

Dissen, G. L. 100.

"Dissertationsmaschine" 406.

divergente Reihen 44 ff.; s. a. u. "Abel". Doppler, Chr. $472 \mathrm{f}$.

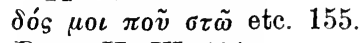

Dove, H. W. 414.

Drobisch, M. W. 124.

Dronke, A. 146.

Dryden, John 357.

Duelle 473-475.

Duhamel 349.

Dïhring, E. 58, 131, 132, 409, 450.

- $\quad$ ü. Cauchy, Jacobi, Lagrange $131 \mathrm{f}$.

- $\quad$ ü. Gauss $29,131 \mathrm{f}$,, vgl. a. 450.

- ü. Helmholtz $4 \mathbf{1 6} \mathrm{f}$.

Dunnas, J.-B. ü. die Betrugsaffäre Chasles $470 \mathrm{f}$.

Dupanloup, F. A. Ph. 383.

Dupin, Ch. 21, 36, 145, 439.

Dupuy, P. ü. Galois $424 \mathrm{f}$.

Dürer, A. 234.

Dyck, W. v. - 19, 205, 429.

Wadem mutata resurgo 390 .

Eberty, Felix 300.

École Polytechnique 184, 206, 266, $317,319,424,438$, vgl. a. 274.

Ehe $83,352-358,360$.
Einseitigkeit d. Mather resp. d. Math. $80 \mathrm{ff}$., $89 \mathrm{f} ., 272,308,367,372,402$. Einsilbigkeit $336,343$.

Eisenstein 229, 240, 285, 325, 414.

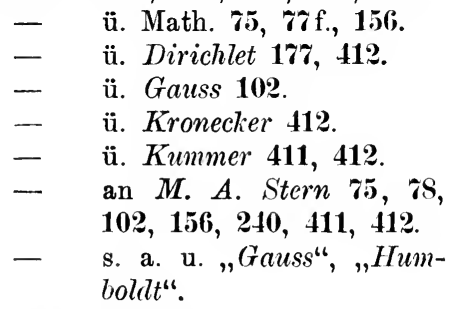

Eleganz i. d. math. Darstellung 22, 3 ร̃., 56, 191, 251, 288, 289, 301, 308. elementare u. höhere Nath. $200 \mathrm{f}$., 487-491.

Math. auf der Univers. 491.

Elektricität, Wesen der $-6,19$.

Elie de Beaumont s. Beaumont.

Elias, Neuplatoniker 481.

Ellendt, E. 319.

elliptische Funktionen $62,112,133 \mathrm{ff}$., $281,322,326,376,404,426,429$, $441-446$.

Encke 125, 119, 122, 306, 349, 411,

435, 466, 497; s. a. u. "Gauss", "Jacobi".

Eneström, G. 267; s. a. u. „Cantor, Gr." Engel, F. 95, 254.

- ü. S. Lie 279 f., 40.), 411, 41s, 419 .

England, Engländer 25, 84f., 234, $329 \mathrm{f} ., 333,388$.

Epikur 247.

Epstein, S. S. 238.

„erklären“ 1, 5, 14f., 49.

erste, Wer ist resp. sind die ersten

Mather? 73, $94 \mathrm{f} .$, 131, $136 \mathrm{f} ., 283$

bis 286, 288f., 304, 340, 446, 464.

Esneh 11.

ethischer Wert der Math. u. der math.

Bildung 68/69, 76, 480, 482f., vgl.

a. 72 .

Euklides 26, 27, 57, 115, 153, 172, $239,241,260,272 \mathrm{f} ., 324$, $416,437,468,480,488,490$.

- , Urteile ü. ihn - 33f., 57. 
Euklid's Elemente als Schulbuch $498 \mathrm{f}$.

Euler, J. Alb. an Kästner 296.

Euler, I. 54, 61, 81, 83, 163, 193, 201, $241,245,336,340,347,370$, $373,375,407,426$ f., 443, 454, 461, 463, 498 .

- , Art des Arbeitens 39, 153, 159, 190.

- , analytische Gewandtheit 126, 159, 293 f.; vgl. a. 83, 190, 289.

- ü. die selbständige Bedeutung des Calculs $189 \mathrm{f}$.

- , Art seiner schriftst. 'Tätigkeit 159,189 f., 253 f., 295 f.; vgl. a. $83,249 \mathrm{f}$.

- , Schöpfer d. modern. math. Stils 251, vgl. a. 283.

- , Fruchtbarkeit 126, 257, 276, $289,304,451 / 452$.

- , umfassendes Wissen u. Gedächtnis 126, 294, 375 .

- Theoria Musicae 81.

- , Lettres à une Princesse d'Allemagne $249 \mathrm{f}$.

- Fortgang aus Berlin 85-88, 499 ; s. a. $256,340$.

- , religiöse Ansichten u. Schriften $381,383$.

- ü. d'Alembert 465 (2).

- an Lagrange 88, 340, 465 (2).

- u. Lambert 337, 350 f., 499.

s. a. u. "Alembert", „Arago",

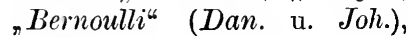
"Cayley", „Friedrich d. Gr.", "Gauss", ,Jacobi", „Lambert", "Laplace", „Legendre", „Sutzer".

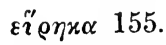

Evelyn, John 228, 497.

Exald, Heinr. 357.

Examina 146, 158, 316-321, 424, $496 f$.

Fälschungen $470 \mathrm{f}$.

Faraday 348 ; s. a. u. "Helnholtz". Faulheit 168f., 293, 322.

Faust 49, 53, 82 .

Fayolle, Fr.-J.-M. 406.
Federmesser, Math. ein - 74 .

Fehler in math. resp. verwandten $\mathrm{Ab}$ handl. 152, 158, 164, 189, $252 \mathrm{f}$., $465-470$.

Ferien 309.

Fermat $56 \mathrm{f} ., 61,73,215,383,454 \mathrm{f}$.

- an Mersenne 254 .

- an Pascal 187.

- s. a. u. „Descartes“, „Pascal“.

- 'scher Satz 56 f., 215.

Fernkraft 6 f., 12 ; s. a. $84,247$.

Fichte 333.

Figuren, Mechanik ohne - (Lagrange) $21 \mathrm{f}$.

Flamsteed, John 452.

Flexibilität des Geistes 422, 426.

„Fliegende Blätter“ 45.

Flügel der Seele, Arithm. u. Geom. $-78 \mathrm{f}$

Fontane, Th. ü. s. Lehrer Steiner 314. Fontenclle 200, 242, 348, 35\%, 354, $390,39 \%, 453$.

formaler u. logischer Nutzen der math. Bildung 397, 478, 482f.; s. a. u. "bildend".

Formeln 21, 23, 33, 37, 59, 62, 82, 125,144 f., $153,163,168,188,199$, $209,232,235,238,251,285,291$, $293,302,370 \mathrm{f}$., $386,436,445,451$, 463,464 ; s. a. u. "Laplace".

Formey 306, 350.

Forschungsmethoden, verschiedene der Mather $147 \mathrm{f}$, $149 \mathrm{ff} ., 172 \mathrm{ff}$., 293 ; s. a. u. „Logiker", „Logik“, "Analytiker u. Synthetiker", sowie u. „Cayley“" (263), „Dirichlet" (293), "Euler" (39, 159, 293), „Gauss" (55, $154 \mathrm{f} ., 156,157)$, „Helmholtz" (163 ff.), „Sylvester" (259f.).

Forsyth, A. R. 175, 196, 264, 297, $327,330,468$.

Fortschritt, Art des - s der Wissen, insb. der Math. 23, 27, 36, $196 \mathrm{f}$., 200, 368, 429-434, 450, $487 \mathrm{f}$.

Fourcroy, d. Chemiker 335.

Fourier 171, 182, 273, 349, 456.

- ü. Abel u. Jacobi 191.

ü. Lagrange 22, 290, 499. 
Fourier ü. Laplace $175 \mathrm{f}$.

- u. Poisson 423.

- s. a. u. "Hamilton", „Neumann, F. E."

'sche Reihen 50, $422 \mathrm{f}$.

Franklin, Fabian 327.

Frankreich, Franzosen 79, 129 f., 222, $234,255,283,285,299,317,327$, $329,436,438,469,479$, vgl. a. 148. Frauen in den math. Wissen $76 \mathrm{f}$, $130 \mathrm{f} ., 358$ - 362 .

Frauenstudium $359 \mathrm{f}$.

Fraunhofer'sche Linien 373.

Frege, G. 263.

"freie" Math. 433.

Fremdwörter 268, 440.

Frénicle de Bessy, Bernard 254.

Fresnel 341, 421.

Freundschaft 76, $93 \mathrm{f} ., 101,106,187$, 281, 316, 403, 404/405, 412, 473. Fricke, R. 209, 495.

Friedrich I. v. Preussen 48.

Friedrich II. der Grosse 83, 86, 130, $310,465$.

ü. Math. 195, 222, 328, $331,462,469$.

ü. Mather $80 \mathrm{f}$., $83-85,89$, 124,246 f., $383,421,462$. an d'Alembert 88, 89, 257, $328,348,351(2), 371,383$, 469,496 .

- $\quad$ ü. d'Alembert $\mathbf{8 0}$ f.; vgl. a. 465.

- an Marquis d'Argens 81.

- an Euler 85-87.

- $\quad$ ü. Euler $85,239,246,256$ f., 361, vgl. a. 381 .

- $\quad$ ü. Lagrange 348.

- $\quad$ ü. $I$. Newton 80, 84f., 247, 371, vgl. a. 222 .

- $\quad$ u. Lambert 336, 349 f., 350 f., 351.

an Voltaire $83,84,85,195$, 222, 361.

- s. a. u. "Alembert", "Ar. gens", "Châtelet", ,Voltaire".

Friedrich Wilhelm III. v. Preussen 98.
Friedrich Wilhelm IV. v. Preussen 130, 131, 139, 348.

Friedrich Wilhelm, Kronprinz v.

Preussen (späterer Kaiser u. König Friedrich III.) 479.

Frischauf, Joh. 30, 31.

Frobenius, G. 52, 54, 62, 168, 297.

- $\quad$ ü. Kronecker 62, 178, 281.

Frommhold, Prof. iuris 436.

Fruchtbarkeit 126, 175, 255, 257, 260, 289 , vgl. a. 288 ; s. a. u. „Cauchy“, "Euler".

Fuchs, L. s. u. "Auwers".

Funktionentheorie 62, 65f., 178.

Fürsten, Umgang mit - 336, 338f., $342,347-350,351$; vgl. a. $88 \mathrm{f}$.

Fuss, N. 257, 375, 381.

- , P. H. 83.

Fußgänger 71, 411.

Gährungsmittel, Math. ein - 69

Galilei 175, 184, 360, 417.

Gall 225.

Galois 52, $136 \mathrm{ff} ., 178,282,319$, $423-425,475$.

- , Verkennung s. Genie's u. s. Leistungen $423 \mathrm{f}$.

- s. a. u. "Dupuy, P.“

Galton, Francis 148.

Galvani 196.

Gariel, C. M. 198.

Gauss, C. F. 1, 11, 23, 29, 50, 52, $61 \mathrm{f} ., 73,90-107,125,136$, $201,211,212,234,242,248$, $274,278,304,348,427$.

ü. sich selbst 92, 106, 185, $240,324,375,466 \mathrm{f}$.

- , ü. - Art zu arbeiten, dio Darstellung in s. Schriften u. s. Forschungen $26,55,56$, 94, 98, 108-114, 115, 119 f., $154 \mathrm{f} ., 156,157,166 \mathrm{f} ., 183$, $258,262,268,268$ f., 270 f., $307,364,444$.

- , ü. - ' spätes Publizieren 28, 97, 109-114, 258, 264f., 443, $444 \mathrm{f}$; vgl. a. $270 \mathrm{f}$.

— , ü. _' Lehrtätigkeit 109, 110, 
11\%, 119-124, 119, 121, 12:, $30 \%$.

Gauss, C.F.ü. Lehrtätigkeit im allgemeinen 92, 115-117, 119, 123. ü. formale Fragen der math. Kunstsprache 435, 436, 437, 440.

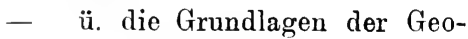
metrie 25-28.

- ü. seine Disquisitiones arithmeticae resp. ü. höhere Arithmetik 55, 56, 61, 67, $\mathbf{6} \mathbf{6}, \mathbf{9 6}$, 157, 361 f., $364 \mathrm{f}$; s. a. 442.

- , reguläres 17-Eck 94, 10\%, 468 .

- , Unters. ü. elliptische Funktionen 112, 133, 426, 442, $443,444 \mathrm{f}, 445 \mathrm{f}$.

- , Unters. ü. Integration auf krummlinigem Wege 446 bis $448,448 \mathrm{f}$.

- Theoria motus corp. coelest. 123, 269, 425.

- , Urteile anderer ï. - ' praktische Arbeiten 193f., 214f., vgl. a. 92.

_- Zahlenregister $91 \mathrm{f}$., 116, $214 \mathrm{f}$.

-_ , Nachlass 264f., 265; s. a. 28, 112.

- , Wahlspruch u. Motto auf dem Siegel 93, 97, 107, 114, 115, 214.

- , politische $u$. religiöse Ansichten 25, 29, 90, 106f., $131 \mathrm{f}$., 334f., 344(2), 344, 378, 382(2), 385-391, $391 \mathrm{f}$.

— , Ehe- u. Familienleben $\mathbf{7 6 , 1 0 5 ,}$ $357-358$; vgl. a. $94,100 \mathrm{f}$.

— , Berufung nach Berlin $98 \mathrm{f} ., 274$.

- , Schüler Abel's? 135/136, vgl. hierzu 105.

- $\quad$ ü. Abel 104, 104, 445, vgl. a. 135.

- ii. Archimedes 284(2).

- an Bessel 28, 45, 113, 11 (2), 119(2), 128, 15\%, 186, 194, 265, 269, 307, 318, 357, 382, $435,436,445,446 \mathrm{f} ., 449,466$, $46 \%, 496$.
Gauss, C.F. ü. Bessel (an Schumacher) 186, 496 .

- an W. Bolyai $27,55,10 \%$, 183, $35 \mathbf{5}(2), 389$.

- zu R. Dedekind 122.

- $\quad$ u. Fisenstein 284.

- an Encke 112.

- ü. Euler 153.

- an Gerling $\mathbf{2 8 .}$

- an S. Germain $\mathbf{7 6 , 3 6 2 .}$

- u. Gunlögsen 425.

- an Humboldt 215, 395, 394.

- $\quad$ u. Jacobi 128, vgl. a. 135.

- ü. Kästner 228, 229.

- ü. Lagrange 42, 153, 25:.

- $\quad$ ü. Laplace 152, 186, 436.

- ü. Newton 153, 284.

- an Olbers $\mathbf{2 5}, \mathbf{5 6}, \mathbf{1 0 6}, \mathbf{1 1 6}$, 153, 15.5, 496 .

- an Schumacher 10, 43, 44, $47,56,90,92,104,108,109$, 110, 111, 113, 114, 119, 121, 123, 153, 185f., 213(2), 219, 229, 252, 258, 262, 268f., 271(2), 324, 328, 335, 344(2), $347,358(2), 364,375,376$, $392,389,391,394,440,442$, 444, 467, 496 .

- an W. Weber 156.

- an Frhr.v. Zach. 269.

- s. a. u. "Abel", ${ }_{n} B a u m "$, "Bessel", „Bolyai, W.", „Cantor, G. ", ${ }_{n}$ Cantor, M. ${ }^{*},{ }_{n}$ Dïhring", "Eisenstein", ${ }_{\text {Ger- }}$ main", "Hamilton", "Humboldt", „Jacobi", „Kronecker", "Lagrange", „Laplace", „Legendre", „Olbers", „Schumacher", "Waldeck".

Gay-Lussac 401.

Gedächtnis 154, 161, 253, 322, 374f., $402,450$.

Gegenbauer, I. 73, 472, 473.

Geiger, Ludwig $\mathbf{2 5 6 .}$

Geiser, C. F. 139, 149, 2శ8, 409, $410(2), 439$.

generalisieren $5,36 \mathrm{f}$., $162 \mathrm{f}$., 172, $263 \mathrm{f}$; vgl. a. $432,448,460$; s. a. Induktion. 
„Generalstabsoffiziere" d. Technik 208. Geodäsie $193 \mathrm{f}$.

$\gamma \varepsilon \omega \mu \varepsilon \tau \rho \varepsilon \tilde{\iota}$, ò $\vartheta \varepsilon \dot{o} s-377$.

Geometrie, analytische $142 \mathrm{ff}$., 174, $386,395,396,411,414,487$.

- , darstellende $210 \mathrm{f}$.

- $\quad$, euklidische $33 \mathrm{ff}$., $324,490$.

- , Grundlagen der - 24, $25 \mathrm{ff} ., 217,324$.

- , mehrdimensionale 31f., 41.

- , nichteuklidische $29 \mathrm{ff}$., 66 .

- projektive 150,431 ; s. a. 142, 172, 462.

synthetische $140 \mathrm{ff}$., 145 , $147,315 \mathrm{f}$.

- s. a. u. "Arithmetik", "Mechanik".

Georg I. v. England 348.

Gérando, de - 406.

Gerdil, H.-S., Cardinal 383.

Gergonne, J. D. 266.

Gerling, Chr. L. 119, 122 ; s. a. u. „Gauss".

Germain, S. 58, 251, 406, 430, 435.

\section{- an Gauss 95, 361.}

- s. a. u. „Gauss".

Geschichtsforschung in der Math. 261; s. a. $416 \mathrm{f}$.

Gesellschaft, menschl. - u. Math. 371 f., 374,469 ; s. a. 79 f., 82,212 , $222,273,338,339$.

Gesetze, Natur- $1 \mathrm{f} ., 386 \mathrm{f}$. "Gewaltige", der - (Gauss) $101 \mathrm{f}$.

Gilman, Daniel C. 297.

Gladstone 330.

Glaisher, J. W. L. 175.

Gleichungen 5. Grades 6, 20, 104, 429, 470.

Gluck 239.

Goethe $7,8,34,49,53,82,248,457$, 467.

- ü. Math. 220, 367.

- ü. Mather 79.

- ü. $I$. Newton 220(2),367, 368.

-- s. a. u. "Schopenhauer".

Göpel 55, 278.

Gott u. Nath. 155, $377 \mathrm{ff} ., 386 \mathrm{ff}$; vgl. a. $221 \mathrm{f}$.
Gott - eine Hypothese (Laplace) 388. Göttingen 100,103, 208, 211, 242, 309, 327, 343 f., $373 \mathrm{f} ., 408,416$.

Göttinger philos. Fakultät 132. - "Sieben" $343 \mathrm{f}$.

Graf, J. H. 258, 259 (2), 404, 408.

$-, M .350,369$.

Grant, Robert 458.

Grassmann, Herm. 387, 440.

- , Urteile ü. s. Methoden $40 \mathrm{f}$., $68,331 \mathrm{f}$., 422.

- als Philolog 277, 480.

Grassmann, $R$. 68, 68, 440.

"Gräuel" $103 \mathrm{f}$.

Graves, John Thomas 332.

Gravitation $6 \mathrm{ff} ., 49$; s. a. Fernkraft. Gregor XVI., Papst $130 \mathrm{f}$.

"Grenze", Begriff der - 51f., 60; vgl. a. 26, 492.

Grenzen des Naturerkennens 7, 20.

Gretschel, Heinr. 124.

Griechen $33 \mathrm{ff} ., 481$; vgl. a. 58.

Grimaldi 383.

Grimm, Jacob 206.

Gross, Th. 415.

Grunert, J. A. 404.

Gruppenbegriff 52, 150.

Guericke, Otto v. - 12.

Guigniault 424.

Guldin 383.

Gundelfinger, S. 447, 496.

Gunlögsen u. Gauss 425.

Günther, S. 261.

Güssfeldt, P. 483.

Gustav Adolf 338.

Gutenberg 267.

Gutzmer, A. 65.

Gwynne, Nell - 228, 497.

Gymnasien s. Mittelschulen.

Gymnasium, ,math.“ 482.

Hachard 351.

Hachette $181 \mathrm{f}$.

Haller, Albrecht v. -260 .

Halphen 174, 259, 469.

Hamilton 40 f., 284, 329.

- $\quad$ an A. DeMorgan 42, 331f., $458,468,497$. 
Hamilton ü. Fourier $283 \mathrm{f}$.

- ü. Gauss $41 \mathrm{f}$.

- u. Jacobi 333, 333, 458.

- ii. Laplace 451.

- an verschiedene 283,333 , 451.

s. a. u. "Morgan, $A$. De - ".

Handwerk, Math. ein - 73, 368.

Hankel, Hermann 33, 36, 50, 52, 159,

162, 172, 329, 434, 454, 478, 495.

Hannibal 250 .

Hänselmann, L. $22 S$.

Hansteen an H. C. Schumacher 133.

Hauck, G. 194.

s. a. u. ${ }_{n} A b e{ }^{4}$.

Hausdorff, $F$. s. Mongré.

Hegel 127, 129, 218, 333.

Heine, Ed. 146, 185, 316, 412, 479.

Heine, Heinr. 320.

Heliotrop 11.

Helmholtz, Hermann 1, 2, 10, 15, 17, 69, 73, 197, 216, 231, 234, $236,238,276,306,334$, $40 \%$.

- $\quad$ ü. s. Forschungen, s. Forschertätigk. u. Art zu arbeiten 12\%, $163 \mathrm{f}$., $166 \mathrm{f}$., 187, 258, 395, 413.

- $\quad$ als Mediziner 398, 398.

- , Verkennung u. Verunglimpfung 12\%, 413(2), 414 bis 417 .

- an E. du Bois-Reymond 413.

- $\quad$ ü. Faraday $41 \%$.

- ü. H. Hertz 17.

- $\quad$ an seine erste Frau 288. - - zweite-298,330.

- $\quad$ ü. C. G.J. Jacobi 12\%, 413.

- u. G. Kirchhoff 300 f., 325 , 417.

- $\quad$ ü. $F$. Neumann u. O. Hesse 413.

- u. Schopenhauer 457, 457.

- $\quad$ ü. $W$. Thomson 287 f., 298 , 330.

- s. a. u. "Bois-Reymond,
E. du-", "Dühring", "Helmholtz, Otto", „Koenigsberger" ", "Kronecker", "Wiedemann".

IIelmholtz, Otto ü. s. Bruder IIermann 326.

Helmholtz, Robert v. - 302, 383.

Hennert, J. F. 456.

Henry, Joseph 297.

Hensel, Fanny ü. Jacobi $12 \% \mathrm{f}$.

- an Rebecka Dirichlet 12\%.

- s. a. u. "Dirichlet, Rebecka".

Herbart 8.

Hermite, Ch. 278(2), 347, 378, 404.

- , Lehrtätigkeit 296, 493.

- ü. Cauchy 255, 385, 447.

- ü. Cayley 288.

Herrmann, E. 37.

Herschel, John 42.

Hertz, Heinr. 6, 16.

-, Urteile ü. _- Mechanik 14, $17,18,19$.

- s. a. u. „Boltzmann“, „Helmholtz".

Hertzberg, Minister $v$. -341 .

Herwegh 345.

"Herz, math. _-" 281.

Hesse, O. 126, 141, 145, 295, 296.

- s. a. u. "Aronhold", "Helmholtz", "Jacobi", "Scheffel".

Heun, K. 18, 202.

Hilbert, D. 20, 24, 59, 61, 66, 15`, 433, 493.

Hindenburg, C. F. 318, 435.

Hippokrates 260.

Hirn, G. A. 198.

Hirsch, Meyer 484.

„Histoire secrète de la cour de Berlin" 341.

historischer Sinn unter den Mathern $260 \mathrm{f}$.

van't Hoff, J. H. 400.

Hoffimann, J. C. V. 478.

Hofmann, Aug.Wilh. v. - ü. L.Kronecker 403, 497.

Hölder, O. 14, 25.

Holst, Elling 133, 237, 420, 428.

- ü. Abel u. Jacobi 136. 
Holzmüller, G. 201, 487, 494.

Homer 177, 245, $274 \mathrm{f}$.

Hôpital, $L^{\prime}-221,241$.

Horaz 226, 253, 273, 407.

Humboldt, Al.v. - 98 f., 185, 261, 274, $348 \mathrm{f}$.

- $\quad$ an Chr.C.J.Fr.hr.v.Bunsen 278, 331, 499.

- $\quad$ an Eisenstein 285.

- ü. Eisenstein 140, $27 \%$.

- an Gauss 98, 100, 130, 140, 180, 345, 388, 408, 466.

- $\quad$ ü. Gauss 98, 99.

- an Jacobi 349.

- ü. Jacobi 128, 130, 131, $139 \mathrm{f}$., 466.

- an Schumacher 128, 131.

- $\quad$ an s. Bruder Wilhelm 338.

- an Varnhagen von Ense $365,464$.

- s. a. u. "Baum", „Gauss", ${ }_{n} J a c o b i$.

Hurwitz, A. 411.

Huxley 70, 71.

Huygens, Christian 227, 227, 247, $393,463,471$.

Huygens, Constantin $22 \%$.

Hypothesen $8 \mathrm{ff}$., 18 f., 190, 235, 371, 388 .

- , Widersprüche der unter einander 12.

Hypotheses non fingo 8, 13.

Jacobi, C. G. J. 50, 5.5, 62, 105, $125-140,130,154,178,188$, 206, 211, 234, 274, 277, 277f., $319,326,368,429,443,446$, 412.

- , Lehrtätigkeit 50, $125 \mathrm{f}$., 135 , 296.

— , Schüler von ihm: Clebsch 149; Hesse 145.

- , politische Rolle 128, 128, 345.

- $\quad$ ü. Abel 133, 134, 428.

- ü. Dirichlet 292.

- u. Encke 256.

- , Vergl. mit Euler 126, vgl. hierzu 294 (Condorcet).

- $\quad$ ü. Gauss 131, 443.
Jacobi, C. G. J. u. Hamilton 333, $333,458$.

u. Helmholtz 414, sowie u. „Helmholtz".

an Humboldt 224, 244.

- $\quad$ ü. Laplace 129.

- an Legendre 133, 134, 191, $428,442$.

an H. C. Schumacher 128, 333.

u. Steiner 473.

s. a. u. "Abel", "Bertrand", "Bessel" ${ }^{4}{ }_{n}$ Bjerknes", ,Divichlet" (P. G. Lejeune- u. Re-

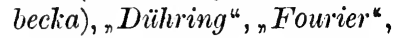
"Gauss", "Hensel, Fanny", "Holst", "Humboldt", "Jacobi, M. H." , "Legendre", "Lie“, "Schumacher", „Steiner", "Weierstrass".

Jacoli, M.H., Bruder des Vorigen 130. an Sylvester ü. s. Bruder 164. Jacobi, Friedr. Heinr. 200.

Jahrhundertwechsel 389.

Jamin 313.

Jeanneret 371.

Jean Paul 83, 248.

Jesuiten 131, 274, 383, 469.

Jesus Christus 383.

Jetzler, Chr. 371.

"Ignorabimus" 7.

in der Math. 20.

Ignoramus 7, 363, 366.

imaginäre Zahlen 29, 42, 59, 147, $322,447-450,489,492,495$.

Inder 35.

Induktion in der Math. $70 \mathrm{f}$., $162 \mathrm{ff}$., 444 ; s. a. ngeneralisieren".

Infinitesimalrechnung, Erfindung 452 bis 456 ; vgl. a. $430 \mathrm{f}$.

Ingenieur u. Mather 195, 201 ff., 209, $266,495$.

"Ingenieur", Etymologisches $205 \mathrm{f}$.

Ingenieure, math. Unterr. für 199-204, 206, 209.

Intrigen $145 \mathrm{f}$., $339,405,452$.

Intuition $u$. Logik $33 \mathrm{f} ., 35,51,147 \mathrm{f}$, 151 f., 491,493 f.; s. a. $23,325,420$; vgl. a. „Synthesis", „Synthetiker". 
Invarianten $52 \mathrm{f} ., 150$.

Johannes Philoponus $\mathbf{4 \$ 1 .}$

Jordan, Camille, der Mather 341.

— , derPolitiker 341, 406.

irrationale Zahlen $59 \mathrm{ff}$., 437, 489, 492.

"Judencoeffizient" 139.

Jüdische Nather 132, 148, 325.

Junghans, F. 41.

Jurisprudenz u. Math. 397, $435 \mathrm{f}$.

\section{Kambly $436 \mathrm{f}$.}

Kant 219, 220, 233, 333, 363, 481.

Karagiannides, A. $\mathbf{3 1 .}$

Karl II. v. England 497 .

Kästner, A. G. 3̧4, 416 f., 394.

- s. a. u. "Euler, J. All.", "Gauss", "Lichtenberg".

Katharina II. v. Rusbland 86, 330, s. a. 228 .

Katholizismus 182, 383.

Keill, Joln 452.

Kelvin, Lord ( $\boldsymbol{H}^{r}$. Thomson) $\mathbf{2 0 9}, 287$;

s. a. u. "Helmholtz".

Kepler 163, 242, 381, 455.

Kiepert, $T$. 37..

Killing, W. 'ist.

Kirchhoff, G. 4, 302, 373.

- , ü. die Aufg. der Mechan"k nach - 14, $14 \mathrm{f} ., 24$, vgl. a. $3 \mathrm{f}$.

— , ü._'s. Mechanik 13, 14, 15, 19 ; s. a. 235.

- , Lehrtätigkeit 300-302.

- , Strenge u. Vollkommenheit s. Arbeiten 176, 235; s. a. $301 \mathrm{f}$.

- u. Helmholtz 300 f., $325,417$. "Kladderadatsch“ "215.

Klein, Felix 25, 48, 145, 14i, 148, 152, 197, 206 f., 208, 210, 305, $325,329,366,433,434,396$, 41\%, 45.5, 492, 49.5, 496.

- ü. sich selbst $30 \mathrm{f}$.

Klöden, Karl Friedrich v. - 410.

Klügel, G. S. 45.

Knapp, G. F. 74.

kneipen, „Überkneiper" 476, 477.
Knör, Obristforstmeister $v,-83$.

Koler, J. 48s, 489.

Koenigsberger, L. 216.

ü. Helmholtz $\mathbf{2 9 ,} 2 \mathbf{2 1}$, $41 \%$.

kombinatorische Schule 400, 435.

Kongresse, wiss. 408, 471.

Königin der Wissen, Math. - 67, s. a. u. "Arithmetik".

Königsberg i. Pr., Universität u. Verwandtes $129,271 \mathrm{f} ., 318,398,413$; s. a. 483 .

Königsweg zur Geometrie resp. Math. $153 \mathrm{f} ., 172$; vgl. a. 164.

Kontroversen, sachliche - unter

Mathern 29 ff., 240 f., 422 f., 461 bis 470.

Kopernikus 84, 124, 383.

Kovalevsky, S. 76, 76, 169, 229, 231, $237,243,345,362,389$.

- $\quad$ an G. Nittag-Leffler 359

- s. a. u. "Weierstrass".

Kraft, Wesen der - $6 \mathrm{f} ., 13 \mathrm{f}$., 16.

Kräfte, Natur- 1, 3 ff.

Krause, MI. 209 .

kritische Bedeutung der Math. 69, $72,200$.

Kronecker, L., 49, 56, 50 (2), 59, 60, 62, 65, 73, 125, 216, 2206, $230,278,281,29 \%, 346$, $338,422,437,486 \mathrm{f}$.

- ü. s. Art zu arbeiten 1əaf., 178, 258.

- , Lehrtätigkeit 297.

- an G. Cantor $305(2)$.

- an Dirichlet 167, 178 (2), 258.

- $\quad$ ü. Gauss 105, 115, $443 \mathrm{f}$., $44 \mathrm{f}$.

an H. v. Helmholtz $21 \hat{\text {. }}$.

- u. Weierstrass $64 \mathrm{f}$., $\mathbf{3 0 5}$

- s. a. u. "Bois-Reymond, P. du -", "Dirichlet", "Eisenstein", "Frobenius", "Hofmann, $A . W$. v.-", "Poincare", , Weierstrass".

Krücke, Math. eine - 33 . 
Kummer, E. E. 38, 96, 120, 126, 161, $178,193,218,226,233$, $265,281,305,316,411$, $471 \mathrm{f} ., 474,487$.

- , Lehrtätigkeit $309 \mathrm{f}$., 310, $376 \mathrm{f}$.

- s.a.u. „Eisenstein", „Weierstrass".

Kunst u. Math. 73, 231, 234, 419; s. a. u. "Dichtkunst".

Kunst u. Mather 148f.; s. a. u. „Musik“". Kunstgriffe 23, 254, 395, 453; vgl. a. $126,253,296,497$.

Künstler u. math. Wissen 234.

künstlerischer u. aesthetischer Charakter u. Wert d. Math. 73, 226, 229-236, 243, 245, 251, $299 \mathrm{ff}$., $325,448,449$, 482; vgl. a. 37,162 , 196.

Kürschner, J. 69.

Lacepède 239.

Lafontaine 337, 423.

Lagrange 25, 39, 61, 79, 98, 99, $179 \mathrm{ff} ., 183,193,201,210$, $236,240,256,261,271,276$, $283,285,349,405,422 \mathrm{f}$., 449, 458, 463 .

- $\quad$ ü. sich selbst 178f., 291,451.

— , Wahlspruch 256.

- , Art zu arbeiten 153, $165 \mathrm{f}$., 170 f., 236 f., $252,253$.

- , analytischer Stil 22, 35, 252 , 291; vgl. a. 251 .

- , Stellung in Berlin u. Fortgang von dort $87 \mathrm{f}$., 256 , $339 \mathrm{ff}$.

- , Abwendung v. d. Math. 171, 366, vgl. a. 291.

- , Einleitungen u. Vorreden 22,108 ; vgl. a. 261.

- , Mécanique analytique $21 \mathrm{f}$., 22 f., 180,313 ; vgl. a. 118 .

- $\quad$ an d'Alembert 165f., 218, 250 , 253, 351, 353, 356, 366, 472, 498.

- an Condorcet 465.

- an Gauss 95.

Ahrens, Scherz und Ernst i. d. Mathem.
Lagrange ü. Lambert 351.

- an Laplace 179, 251, 253, 466.

- $\quad$ ü. Laplace 97 f.; vgl. a. 130 , 180.

- $\quad$ u. Lavoisier 399.

- zu resp. ü. Monge 286 (2).

- u. Newton 286.

- , Vergl. mit Newton 340.

- s. a. u. "Alembert", ,Dirichlet", "Dühring", „Euler", ,Fourier", „Friedrich d. Gr.", "Gauss", "Lambert", "Laplace", "Napoleon", "Poinsot".

Lalande $\mathbf{2 5 3 .}$

$$
\text { leon", "Poinsot". }
$$

Lalanne, L. 250.

Lambert 160, 289, 306, 351, 369, 405, 406.

- $\quad$ u. d'Alembert, Euler, Lagrange $\mathrm{u}$. sich selbst $288 \mathrm{f}$.

- u. Euler 337, 350f, 499.

- u. Friedrich d. Gr. 336, 349 bis 351 .

Lamé 404.

s.a.u.,Lagrange", „Sulzer".

Lampe, E. 151, 160, 262, 265, 303, $312,315(2), 321,345,419,450,473$, $47 \%, 45 \%$.

Lamprecht, Karl 69.

Laplace $10,25,157,184,242,249,276$, 283, 285, 349, 354f., 366, 387 f., 399, 406, 454, 499 .

- , dominierende Stellung $179 \mathrm{ff}$, $182 \mathrm{f}$, vgl. a. $354 \mathrm{f}$.

- , politische Tätigkeit 335, $338 \mathrm{f}$.

- , Mécanique céleste 97, 118, 129, 157, 180, 388, 430.

- , Weltformel 3, 4, vgl, a. 366.

- an d'Alembert 179.

- $\quad$ u. Euler 283.

- $\quad$ ü. Gauss 95 .

- an Lagrange 57, 183, 190.

- u. Napoleon $338 \mathrm{f}$.

- , Vergl. mit Newton 291; vgl. a. 285 , sowie $\mathbf{4 9 9}$.

- von Poisson 423. 
Laplace s. a. u. "Abel", ,Fourier", „Gauss", "Hamilton", ,Jacobi", "Lagrange", "Napoleon", „Olbers", "Poinsot". Laplace, Comtesse de - 339, 355.

Las Cases, Comte - de 342.

Lasswitz, Curd $\mathbf{5 3}$.

Latein für math. Abhandl. 110, 268 bis 271 ; vgl. a. 427.

Laugier, P. A. E., Astronom 255.

Latoisier 399, 400.

- s. a. u. "Lagrange".

„Leben der berühmtesten 4 Gelehrten" etc. 289.

„lebendige Analysis" (Euler) 159, 294. Leffler, A. Ch. 77, 169, 231, 243, $358,389$.

Leffler, Mittag-, G. s. u. Mittag.

Legendre 55, 61, 136, 317, $442 \mathrm{ff}$.

- an Abel 134.

- $\quad$ ü. Abel 135, 428 .

- $\quad$ ü. Abel u. Jacobi 134, 135, $426,429$.

- , Vergl. mit Euler 291.

- $\quad$ ü. Gauss 443, $445 / 446$.

- an Jacobi 354, 426, 428, $\mathbf{4 2 9}, \mathbf{4 4 1}, \mathbf{4 4 3}, \mathbf{4 4 5} \mathrm{f}$. 496.

- s. a. u. "Abel", "Jacobi", "Poinsot".

Lehrbücher, geringe Zahl der math. - 397, 495; vgl. a. 257 f., 402.

Lehrer u. Schüler 123, 139, 240, 306, $313,315,342,461$; s. a. Lehrtätigkeit.

Lehrfreiheit 31, 486.

Lehrs, $K$. an verschiedene Freunde $129,319$.

Lehrtätigkeit $124 \mathrm{ff}$, 294-304, 307 bis $316,483 \mathrm{ff}$; s. a. u. "Lehrer", sowie u. "Ampère", "Arago", „Bessel", ,Clebsch", ,Gauss", ,Hermite", ,Jacobi", ,Kirchhoff", ,Kronecker", "Kummer", "Moebius, A. F.", „Monge", „Poisson", „Riemann", „Schläfli", „Steiner", ,Sylvester", "Weierstrass".

Leibniz 48, 71, 124, 175, 225, 328, $352 \mathrm{f} ., 360,381,383,451 \mathrm{f}$.
Leibniz an Joh. Bernoulli 3.55.

- u. Newton 387, 452-456.

- s. a. u. „Bernoulli, Joh.“, "Cantor, $M$."

Lektionskataloge $271 \mathrm{f}$.

Lemonnier (=le Monnier) 181, 226.

Lenclos, Ninon de - 227.

Lesage, A.-R. 182.

Lesage, G.-I. 6.

Letterkästen des Buchdruckers 9.

Leutmann, J. G. 373.

Leverrier 223.

Libri, G. 283.

Lichtenberg, G. Chr. $\mathbf{2 6}, \mathbf{8 9}, \mathbf{1 6 6}$, $309,393$.

- $\quad$ an Kästner 209.

Lie, Sophus 52(2), 67, 267, 279, 280, $437,456$.

- (an A. Mayer) ü. s. Theorien 418.

-, Mangel an Anerkennung 118, $418 \mathrm{f}$.

- ü. Abel 136, 282 .

- ü. Jacobi 136 f., 137.

- ü. Poncelet 280.

- ü. Riemann 137.

- s. a. u. "Beltrami", „Bertrand", ${ }_{\text {"Cremona }}$, , Engel ${ }^{*}$, Noether", ${ }_{\text {"Poincaré }}$.

Liebig, J. v. - 74, 403; s. a. u. ${ }_{n}$ Berzelius".

Liebmann, O. 223.

Lindemann, $F$. 304.

Lionardo da Vinci 234.

Liouville, J. 266, 404, 424.

Lipschitz, R. 21, 278.

Literaten 344.

Literatur, schoene - u. Mather 76, 226, $229 \mathrm{ff}$., 243 f., 246 f., 308, 345, s. a. u. "Alembert, $d$ '-".

Livius 250, 273.

Lobatschefskij 95, 263.

Lobatschefskijpreis 418.

Lobeck, Chr. A. $271 \mathrm{f}$.

Logik u. Intuition $33 \mathrm{f}$., 35, 51, $147 \mathrm{f}$,, 151 f., 491,493 f., s. a. 23 ; vgl. a. "Analysis u. Synthesis“, „Analytiker".

- s. a. u. "Philosophio". 
Logiker, Forschungsmethode der $151 \mathrm{f} ., 162$.

Lommel, E. v. - 274.

Lorgna, A. M. 449.

Lossow, P. v. - 199, 20\%.

Lotophagen, Mather - 244.

Louis-Philippe 335.

Lübsen 375 .

Lucanus, M. Annaeus 242, 498.

Ludwig XIV. 115.

Ludwig XVIII. 339.

Lühmann, F. v. - 437.

Luther, Martin 481.

Macfarlane, Al. 41, 287.

Mach, E. 4, 6, 14, 19, 33, 37, 40, $43,416$.

Machiavelli 468.

Mac Mahon, P. A. 151, 259, 297, 311.

Magendie 242.

Magnus, G. 301, 398, 401, 414.

Maharbal 250.

Mairan, de - 407.

Maitresse, Math. eine - 75 .

Marc-Aurel 88, 338.

Marcellus 336.

Marie, M., 107.

"Marquis" 140.

Mars, Mlle 248.

Marseillaise 342.

Martius, v. -, Prof. d. Botanik u.

Sekr. d. Münch. Akad. 123.

MaBstab f. d. Wert wiss. Leistungen 187 f., 195, 214, 444.

Math. - Königin d. Wissen 67, 364.

Mathematicus non est collega 477.

"Mathematikus" 477.

Maturin 228, 497.

Maupertuis 195, 461, $463 \mathrm{f}$., 465.

Maurer, L. 410.

Mausefallenbeweis 34 .

Maxim $333 \mathrm{f}$.

Maxwell, J. C. 15, 19, 287, 417; s. a. u. „Boltzmann“.

Mayer, J. R. 14, 415, 416.

Mayer, Tobias (der Ältere) 116, $373 \mathrm{f}$., 394, 444.
Mazarin 228.

Mécanique analytique в. Lagrange.

- céleste s. Laplace.

Mechanik, Aufgabe der - 14 f., 24.

- u. Geometrie 24, 25, $59 \mathrm{f}$.

- , Grundlagen u. Axiome der $-24 \mathrm{f} ., 217$.

- , Strenge der $-4,24$.

- , Systeme der - 17, 21.

- , technische 18.

- s. a. u. „Figuren“.

mechanische Weltanschauung $3 \mathrm{ff}$., 18 f., 387.

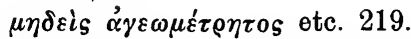

Medizin, Wiss. u. Studium 195f., 216, 268, 394-398, 413f.; vgl. a. 206 .

- , höhere Math. für Studenten der $-395,396$.

Mediziner ü. Math. 395-397; s. a. 275. Meier, Chr. J. E. 484.

Melanchthon 78, 368.

Mendelssohn, Abraham 322.

- , Felix an s. Schwester Reb. Dirichlet 368.

Mephistopheles 49, 53, 82.

Merian, Hans Bernh. 348.

Merian, P. 390.

metamathematische Probleme 51.

Meusnier 399.

Meyer, W. Franz 491.

Mezzofanti s. 158.

Michelangelo 234.

Minding, $F .54$.

Ministerium, Preussisches Cultus- $216,277,316,478,479$.

Mittag-Leffler, G. 77, 229, 282, 287.

B. a. u. "Kovalevsky".

ittelschulen, math. Unterr. an -

$272 \mathrm{f}$., $319,439 \mathrm{f}$., $478-490$.

Möbius, A. F. 42, 124, 146, 235, 332, 374.

- , Schriften u. Lehrtätigkeit300. Möbius, P. J., Enkel des Vorigen $225,235,242,360,373$.

Moigno, l'abbé - 173, 254, 404, 451.

Molière 248.

Mollweide, K. B. 124. 
Mommsen, Th. 25.

Monge 184, 210, 399.

— , Lehrtätigkeit $296 \mathrm{f}$.

— , politische Tätigkeit 335, 342.

- zu Arago 166.

- s. a. u. "Lagrange", "Napoleon", "Poinsot".

Mongré, Paul 69, 170, 245, 248.

Montucla 469.

Moor, Karl 13.

Morgan, A. De - an W. R. Hamilton

$331,333,469$; s. a. u. "Hamilton".

Mozart 73, 234, 239.

Müffling, v. - an B. v. Lindenau 274.

Mïller, Felix 308, 438, 440.

Münchow, K. D. v. -145.

Musik u. Math. 73, 231, 234-236, $368,474,481$; vgl. a. $347 / 348$, 378.

- u. Mather 81, 128, 161, 168, $236-240,474$.

Musschembroek: 306.

0. N. 310, 377.

„Nachrichten v. d. Leben d. berühmt. Mather" 224, 475.

Napoleon I. 13, 129, 184, 339; s. a. Bonaparte.

- $\quad$ ü. Lagrange 287.

- an Laplace $3 \mathbf{3 1 .}$

- $\quad$ ü. Laplace 338.

Napoleon III. 346.

Napoléon, Élisa 339.

Naturerkennen, Grenzen des -'s 7, 20.

Navier 326.

"Nebuchadnezzar" $331 \mathrm{f}$.

Nees von Esenbeck 218.

Nernst, W. 211.

Nerrlich, P. 248.

Netto, E. 297, 298, 492.

Neumann, C. 270, 283, 295, 299.

Neumann, F'. E. 124 f., 191, 271, 479.

- u. s. Schüler 302.

- ü. Fourier 283.

- s. a. u. "Helmholtz".

Newton, I. 1, 2, 6, 24, 98, 166, 175,
$184,215,221 \mathrm{f} ., 226,240 \mathrm{f}$., $250,283,285,329,348,376$, $471,498,499$.

Newton, I. ü. sein Lebenswerk 221; vgl. a. $165,166,380$.

- : Hypotheses non fingo 8 ; vgl. a. 9, 13.

- , religiöse Ansichten 380, 381, 383 , 386 f., 387, vgl. a. 392 .

- , parlamentarische Tätigkeit $342 \mathrm{f}$.

- 's Apfel 165; vgl. a. 166, 280.

- u. Leibniz 387, 452-456.

- s. u. a. "Cantor, M.", ,Friedrich d. Gr.", „Gauss", ,Goethe", "Lagrange", ,Laplace", "Schopenhauer", "Voltaire". nichteuklidische Geometrie $29 \mathrm{ff} ., 66$. Nicolai, Astronom 122.

Noether ü. Cayley $263 \mathrm{f}$.

- $\ddot{\text { u. }}$ Lie $149 \mathrm{f}$.

- ü. Sylvester 259 f., 262.

"noli turbare" etc. 336 , vgl. a. 343. "Novellen" (Euler's Schriften) 159;

vgl. hierzu 266, 267.

Numa 88.

"numero deus impare gaudet" 377 .

iffentliche Meinung, Math., Mather u. die - - 354, $467 \mathrm{f}$.

Ohm, M. 410 f., 497.

Ohm, Gärtner 497 .

ökonomische Aufgabe der Naturwiss. $5,37$.

Ökonomie, geistige - in der Math. $37 \mathrm{ff}$., $42 \mathrm{f}$., 234,300 .

Olbers 408 .

- an Bessel 102, 111, 181, 265, 285, 339, 376, 391, 496.

- ü. Bessel 306.

- ü. Gauss 102, 111, 376.

- ü. Laplace 181.

- s. a. u. "Bessel", „Gauss".

Omar 257.

Organ, math. 242 f.; vgl. a. 225.

Orlow 257.

Ovid 480.

Ozanam, Jacques 392. 
Paedagogik u. Math. 485.

Papperitz, E. 204.

Pappus 155.

parallele Linien, Parallelenaxiom 20, $26 \mathrm{ff}$., $488 \mathrm{f}$.

Parisot 406.

Pascal 80, 80, 230, 239, 249, 350, 405.

— , religiöse Ansichten 368, 382.

- , an Fermat 74, 186, 425.

- s. a. u. "Fermat".

„Pauca, sed matura" s. Gauss, Motto. Paul, Jean - 83, 248.

Peacock, George 458.

Peano 494.

Perpetuum mobile 6, 412, 415.

Perthes, Buchhändler 269.

Peters, C. A. F. 425.

Petzval, J. 73, 471-473, $497 f$.

Pfaff, Joh. Friedr. 95, 116, 124, 167, 435.

Phaedrus 337,

Phaenomenologie 18.

Philidor 239.

Philologen u. Mather 271f., 274, 319, $395 / 396,480$; s. a. $129,297,478$.

Philologen, Mather als - 276f., 443, 480.

Philoponus, Johannes - 481.

Philosophen ü. Math. u. Mather $33 \mathrm{f}$.,

67, 78 ff., 189, 219 f., 222 f., 233, 363

bis $365,374,377,386,481$.

Philosophie, Mather ü. - u. Philosophen 129, $218 \mathrm{f}$., 225f., $249 \mathrm{f}$., $274,333$.

Philosophie resp. Logik u. Math. $30,67 \mathrm{ff} ., 224 \mathrm{ff} ., 409$; vgl. a. 360 . philosophische(erkenntnistheoretische resp. logische) Bedeutung der Math. $5,67,68$ f., 74, 78f., 189, 217, 219 f., $362,366,395,434$, vgl. a. 64 ; s. a. u. „kritisch".

philosophische Fakultät Berlin 216, 274, 301.

philosophischeFakultätGöttingen 132. Phrenologie 225, $242 f$.

Physik, Aufgabe der - 18f, 398.

- , math. u. experimentelle 398.

- u. Chemie 399, 400.
Picard, E. 174, 296, 379.

Piccini 239.

Pietzker 30.

Plagiate 144, 247, 443, 445f., 451, 454,459 ; vgl. a. 471 .

Planck, Max 359.

Plato 67, 78, 80, 94, 189, 218, 365, 374, 377, 493 .

- Inschrift vor seiner Akademie $219,481$.

Plinius 260.

Plücker 146, 280, 407.

- , Mather oder Physiker? $145 \mathrm{f}$. - u. Steiner 142f., 145.

\section{Phatarch 37\%.}

Poggendorff, J. C. $413 \mathrm{f}$.

Pohlke u. Steiner 315.

Poincaré, $H$. 51, 139, 148, 152, 162, 174, 232, 243, 259, 303, $470,494$.

- $\quad$ ü. Lie 420.

- $\quad$ ü. Kronecker u. Weierstrass 64 f.

- $\quad$ u. Riemann u. Weierstrass 151.

- $\quad$ ü. Weierstrass 51, 64f., 151, 173.

Poinsot 144, 179 f., 183, 322, 370, 403.

- $\quad$ ü. Lagrange $22 \mathrm{f}$., $180,291 \mathrm{f}$.

- ü.Lagrange,Laplace,Legendre u. Monge $291 \mathrm{f}$.

Poisson 130, 171, 181, 191, 255, 283, $326,376,421,423 \mathrm{f} ., 428$.

— , Lehrtätigkeit 294, 294.

- u. Fourier 423.

- s. a. u. "Abel".

Politik u. Mather 128, $334 \mathrm{f}$., 338f., $341-346$, 423f., vgl. a. 474/475; s. a. u. "Arago", ,Gauss", ,Jacobi“, "Laplace", "Monge", „Newton".

Polyhistoren 278f., $322 \mathrm{f}$., $348 \mathrm{f}$., 350 ;

s. a. u. „Ampère", „Schläfli".

Poncelet 201, 404; s. a. u. "Lie".

- ü. Cauchy $462 \mathrm{f}$.

- u. Steiner 457.

Pope, Alexander 221.

Porphyrius 244.

Positionsarithmetik 284. 
Potel 181, 240, 287.

Praezisionsmathematik 150 ; vgl. a. 35 f., 491.

Preuss 239.

Preyer, W. 236, 375, 397, 498.

Pringsheim, A. 492, 494, 494.

Prioritätsfragen $9,41 \mathrm{f}$., 112, 133, $143 \mathrm{f}$., $179 \mathrm{f}, 302 \mathrm{f}, 413,415 \mathrm{f} ., 423,430$, $443-447,450-457,459-461,471$, $49 \%$ f., vgl. a. $331 / 332$.

Probleme, Lösbarkeit der math. $20 \mathrm{f}$.

Proclus Diadochus $\mathbf{1 5 4 .}$

Prony, G. C. F. M. Riche de - 181, 385.

Propheten, "französische _-" 392.

Prüfungs - Commission, wiss. - zu Berlin $\mathbf{3 2 0}$.

Prutz, H. 316.

Ptolemäus 153, 172.

Purismus $439 \mathrm{f}$.

„Purus mathematicus, purus asinus" 79.

Pythagoras 72, 88, 319; s. a. 234.

Pythagoräischer Lehrsatz 34, 72, 319.

Quadrate, Meth. der kleinsten - 443f.; s. a. 91,122 .

quadratische Reste, Reciprocitätsgesetz $324,443 \mathrm{f}$.

Formen 188, 411.

Quadratur des Kreises 6, 20, 412, $468 \mathrm{f}$.

Quaternionen $40 \mathrm{ff}$., 331.

Quinette, Minister 338.

Race, math. Talent in Abhängigkeit v. d. - 132, 148; vgl. a. 124, 293. Racine 291.

Radicke, G. 146.

Raphael 234.

Raumer, Karl v. - 11 s.

Raumvorstellungsvermögen $148 \mathrm{f}$., 152 , 203, 210, 299.

Ravignan, de - 384 .

Rebière, A. 437.

Recensionen 466, $49 \%$.
Rechenfertigkeit (Zahlenrechnen) 91, $92,240,375-377$; vgl. a. 85, 337 . Rechnen in der Math. 23, 126, 145, $152 \mathrm{f} ., 222 \mathrm{f}$., $252,293 \mathrm{f}$; в. a. 473. Rechtswissenschaft $\mathrm{u}$. Math.397, $435 \mathrm{f}$. ,reine“ Math. 434; vgl. a. 69, 193, 216. Religion u. Wiss. $11 \mathrm{f}$., 308, $362 \mathrm{f}$., 368 f., 379 ff., $386-388,390$ bis 392 .

- u. Mather 379-392, 410; s. a. u. „Ampère" ", „Cauchy", „Euler", „Gauss", „New-

„Rex" 140. ton", "Pascal".

Ricci, G. 106.

Richelot 316, 479.

Riedler, A. 32, 197, 206-20S(3), 210, 211.

Riemann, Bernh. 8, 16, 29, 31, 105, 178, 201, 386, 423, 449, 498.

- , Lehrtätigkeit 312 .

- 's Dissertation 422.

- s. a. u. "Lie", „Poincaré", "Weierstrass".

Riess, Peter 399, 414.

Rosenhain 168, 277, 325, 345.

Rosenkranz, $K$. 386, 485.

Rosse, W. Parsons, Earl of - 388.

Rossel 181.

Routh 17.

Rudio, F. 188, 234, 249, 251, 257, 411.

Runge, C. 188.

Russen, Russland etc. 86 f., 257, 334, $336,340,345,347,373$.

Sainte-Beuve ü. A. M. Ampère $322 \mathrm{f}$., 406.

Salis, v. -369 .

Salmon, G. 329.

Salomonis, Weisheit - 378.

Sartorius $v$. Waltershausen 42, 67, 93, 96, 114, 122, 153, 167, 215, 240, 249, 284, 344, 392, 498.

- an W. Bolyai 96 .

- s. a. u. "Bolyai, W."

Sauveur, Joseph 353.

Scheeffer, Ludurig 32, 308. 
Scheffel, J. V. v. - ü. O. L. Hesse $476 \mathrm{f}$.

Scheffler, H. $4 \mathbf{4 1}$.

Schellbach, C. H. 308, $479 \mathrm{f}$.

Schelling 131, 218.

Schering, Ernst 95.

Schiedsrichter der Royal Society in London 452.

Schiller 13, 223, 224, 249.

Schilling, C. 154.

Schimpfen 313, 409f.; vgl. a. 127 (Grobheit).

Schläfli, L. 368.

- , Lehrtätigkeit 307.

- , vielseitiges Wissen $278 \mathrm{f}$.

- an Steiner 158, 367.

- s. a. u. "Steiner".

Schleiermacher 58.

Schlosser, F. Ch. 416.

Schmetzer, Pfarrer 476 .

Schmidt, Franz 474.

Schönlein 127.

Schopenhauer, A. 34(2), 223(2).

ü. Newton u. Goethe 220.

u. H. Helmholtz 457, 457.

Schreibfaulheit $321 \mathrm{f}$.

Schröder, E. 68, 68.

Schröter, Heinr. 31.

Schubert, Franz 234.

Schubert, Hermann $\mathbf{3 1 6}$.

"Schulen" in der Math. 304f.

Schulen, Math. auf den - 272f., 319, $439 f$., $477-490$.

Schüler, wessen - war Joh. Bernoulli? 461.

- wessen - war Clebsch? 149.

- wessen - war Gauss? 135/ 136.

- wessen - war Hermite? 278.

- wessen - war Jacobi? 135/ 136.

- wessen - war Kronecker? $281 \mathrm{f}$.

- wessen - war Lie? 280.

- wessen - war Franz Neumann? 283; s. a. 124 f., 191.
Schüler, wessen - war Weicrstrass? 282.

Schumacher, H. C. 47, 101, 108, 266, 425.

- $\quad$ an Gauss $91,97,99,100(2)$, $101,102,108,109,110$, $115(2), 121,129,133,135$, $213,268,375$.

- $\quad$ ü. C. G. J. Jacobi 129.

- s. a. u. „Gauss", „Hansteen", "Humboldt", ${ } J a-$ cobi".

Schur, F. 30.

Schur, W. 228, 374.

Schwarz, H. A. 474.

s. a. u. "Weierstrass".

Schwerkraft s. Gravitation.

Schwierigkeit d. Math. 68f., 109, 154 $-157,163 \mathrm{f} ., 166,218$, $276,306 \mathrm{f}$; ; vgl. a. 56 . , math. Abhandl. u. Vorträge zu verstehen 36 , $136,251,408,412,422$, 424 ff.; vgl. a. 107, 118, $264,266,417$.

des math. Schul-Unterrichts $483 \mathrm{ff}$.

Seebeck 167.

selbständige Bedeutung der Math.

54, 188-196, 231 f.; s. a. 448.

Seneka 273.

Separatabzüge, Versendung 404.

Serret, J. A. 404.

Setzervorwitz 266.

Shakespeare 214, 275, 496, 497.

$$
\text { - -Baconfrage } 245 .
$$

Simon, Max 485.

$\sin ^{2} \varphi$ oder $\sin \varphi^{2}$ ? $436 \mathrm{f}$.

Smith, Adam 14.

Society, Royal - in London 452.

Sokrates 194, 275.

Somerville, Mary, Astronomin $130 \mathrm{f}$.

Sontag, Henriette $237 \mathrm{f}$.

Sozialdemokratie 346 .

Spence, J. 221, 376, 393.

Spielhäuser 372.

Spielzeug, Math. ein -75 ; vgl. a. 469. 
Spinozu 219, $33 \pm$.

Spirale, logarithmische 390 .

Sprache, Nath. die - der - n 71; vgl. a. 79.

Sprachen, alte, ü. ihren bildenden Wert 272-276.

sprachliche Kenntnisse bei Mathern $168,274,276-279,320$, $404,480$.

, Notwendigkeit alt- $-\mathrm{r}$ Keuntnisse für Mather $272,426 \mathrm{f}$; s. a. 480.

Sprachstil u. Math. 249; vgl. hierzu 308.

Staat s. Gesellschaft.

Staël, Nme de - 3\%2.

Stahl, G. E., Begründer der 'Theorie vom Phlogiston 399.

Staude, O. 304.

Staudt, v. - 123.

Steiner, Jacob 3\%, 40, 148, 279, 320 f., $368,409 \mathrm{f}, 420,439$.

- , Lehrtätigkeit 299f., $314 \mathrm{ff}$., 410.

- u. die Analysis 140, 141, 141(2), $142 \mathrm{f}$., $143 \mathrm{f} ., 145,147$, 15), 315, 320; s. a. 299.

- u. Aronhold 143.

- an Jacobi 140, 455.

- ü. Jacobi 138f́., vgl. a. 473.

- u. Plücker 142f., 145.

- u. Pohllie 315.

- u. Poncelet 4.5\%.

- an Schläfli 140, 143, 158, 316, 404.

- u. Weierstrass 316, 316, 410.

- s. a. u. "Fontane, Th.", "Schläfli".

Stern, M. A. 92, 214; s. a. u. "Eisenstein".

Stil mather Darstellungen 22, $35 \mathrm{f}$., $250 \mathrm{ff}$., $263 \mathrm{f} ., 266,268,291,304$, 308, 419, 439, 454 f.; s. a. u. "Euler", "Gauss", "Kirchhoff", "Lagrange", "Moebius, A. $\boldsymbol{E}$.", "Sylvester", "Weierstrass", sowie u. „Eleganz", "Kunstgriffe", „Rechnen", „Strenge ${ }^{*}$.
Stodola, A. 5, 200.

Strenge u. Klarheit der Math. 5, 61, 305,363 f., 462, 487; s. a. 439.

Strenge, Mangel an - u. Praezision in math. Discipl. resp. Abhandl. $35,44 \mathrm{ff}$., $49 \mathrm{f}$, 68 , 137, 157, 158, 259 f., 262 f., 419, 493; vgl. a. $63,150$.

- , absolute - in den Anfangsvorlesungen? $491 \mathrm{ff}$.

Strindberg, Aug. 358.

studentisches Leben 475, 477.

Study, E. 35, 208, 490.

Sturm, Ch. 404, 437.

Sturm, R. 31, 359, 488, 489, 489.

Sulzer, J. G. ü. L. Euler 85, 86 .

- ü. Lambert $\mathbf{3 5 0}$.

Sylow, L. ü. Abel $133 \mathrm{f}$, $426 \mathrm{f}$.

ü. Gauss u. Abel 104.

Sylvester, J. J. 53, 70, 73, 150, 26:, 264, 297, 329, 330, 490.

- , Gedichte 227f., 232, $496 f$. Lehrtätigkeit 298, 311.

- , ü. _'s wiss. u. schriftst. Tätigkeit259, 260, 327, 450.

- $\quad$ u. Cayley 288 .

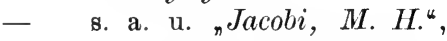
"Noether", "Weierstrass".

Symbole, Symbolik 40, 53, 107, 149,

157, 304; s. a. "Zeichen".

Synthesis u. Analysis $140 \mathrm{ff}$.

Syuthetiker u. Analytiker 140-145, $146 \mathrm{ff}$., 420, $462 \mathrm{f}$.

Tacitus 226.

Tageblatt, Berliner $4 \mathbf{7 4}$.

Tait, $P$. G. 41, 287, 330 .

Takt, math. 162, 229, 468; vgl. a. $90 / 91$.

Talent, math. - angeboren $147 \mathrm{f}$, $290 \mathrm{f}$.

Tannery, J. 313.

Tasso 365.

Taylor 240.

Technik u. Wiss. 84 f, 197-200, 205, 211, 214, 434. 
„Techniker ${ }^{4}$, Etymologisches 205.

Technische Hochsch. u. Universität $206 \mathrm{ff}$.

technische Mechanik 18.

Telegraphie 11, 211-214.

Terminologisches aus der Math. 138f., $262,437-442$; vgl. a. 494.

Terquem, Olry 404.

Theater 239, 248, 408.

Thénard, Chemiker 401.

Theologie, Theologen, Geistliche etc. $89,278,354,367,381,390,409$, 462, 468f., 476, 499; s. a. 351, $383 \mathrm{f}$.

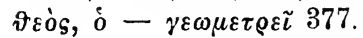

-, - - $\alpha \varrho \iota \vartheta \mu \eta \tau i \zeta \varepsilon \iota 378$.

Thetafunktionen 31, 138, 168.

Thiébanlt, Dieudonné 81, 86, 161, 171, 190, 289, 337, 340, 350, 351, 406.

Thomas v. Aquino 379.

Thomson, W. (Lord Kelvin) 209, 287;

s. a. u. ${ }_{n}$ Helmholtz".

Thorwaldsen 93.

Titel 100, 131, 335, 347, 458, $477 \mathrm{f}$.

Titel, Buch ohne - 267.

Tod, Leben nach dem -e 25, 90, 368f., 378, 379, 382f., 389-392; vgl. a. 267.

Tortolini, B. 292, 458 .

Toussaint, F.-V. 350.

Tralles 125.

Transformationsgruppen 52, 418, 420, 437.

Treitschle, Heinr. v. - $\mathbf{3 4 9}$.

Trisektion des Winkels 468.

trocken, Math. e. -e Wiss. 230, 236, $245,383$.

Tycho.Brahe 383, 475.

Tzetzes 219.

Unendliche, das - 29f., 45, 47f., 379 f., 492.

Unendlich-Kleine, das - 48f., 149, $338,452$.

unnütz, Math. - 73f., 195, 199, 226, 408 f., 469 ; vgl. a. $75,372,401 \mathrm{f}$. Unruh, H. V. v. - 118, 273, 484. Urteilsfähige, geringe Zahl der - $-\mathrm{v}$ in d. math. Wissen 27, $184 \mathrm{f}$., 462; vgl. a. 409 .

\section{J. V. 45.}

Vahlen, K. Th. 270.

Valentin, G. 270.

Valerius Maximus 336.

Valson, C.-A. 249, 254, 335, 346, 353, 382, 383, 384, 388, 496.

Vandermonde 238.

Variationsrechnung 42, 493.

Vaterschaft 321 f., $342,355 \mathrm{ff}$., 360 ; vgl. a. $458 / 459$.

Vergil 273, 37\%; s. a. 375.

Verkehr, wiss. $305 \mathrm{f}$. - , geselliger 76, 90,330, 354, $406 \mathrm{ff}$; s. a. 322 .

Verkennung grosser Forscher u. hervorragender Leistungen 411-429, vgl. a. 41 ; s. a. u. ,Abel", „Galois", „Helmholtz", „Lie“.

Verleger 257, 266, 269; s. a. 259.

vermittelnde Stellung der Math. 434.

"Verschuch, sed inutile" 425 .

Versicherungsgesellschaften 372 ; vgl. a. 339.

Verwunderung - halbe Entdeckung $161 \mathrm{f} ., 493$.

Vieta 395.

Villers, Ch. F. D. de - 248 .

Virchow, R. 397.

Virey 181, 240, 28\%.

Vitruv 155.

Vivisection 398.

Vogt, Hermann 497.

Voigt, W. 13, 125, 176, 191, 211, 302.

Volkmann, P. 302.

Vollmar, G. v. - 346.

Volta 432.

Voltaire 227, 270, 3\%0.

- $\quad$ an Friedrich d. Gr. 84, 195.

- $\quad$ ï. Nevton 84, 165, $221 \mathrm{f} ., 360$.

- $\quad$ s.a.u. ,Alembert", ,Friedrich d. Gr."

Volterra, V. 293.

Vorurteile 43, 64, 85, 383, 393, 399.

Voss, A. 105. 
Wagner, Richaid 421; s. a. 238.

Wagner, Rudolf 225, 243.

Wahrscheinlichkeitsrechnung 24, 92, $368,470$.

Waldeck, Mimna, Gauss' zweite Frau 100, 358.

Wales, Prinzessin $v .-348$.

Wallis 455.

Waltershausen s. Sartorius.

Warburg, E. 360.

Warren 332.

Wassiljef 9.

Weber, Heinrich, Prof. d. Math. 312, $386,43 \%, 486$.

Prof.d.Physik $16 \%$.

Weber, Wilhelm 15,90,167, 211f., 357.

- s. a. u. "Gauss", "Wöhler".

Weber, Lina, Schwester d. Vorigen 90.

Weierstrass, 50, 65, 178, 192, 230,274, $282,471 \mathrm{f} ., 477,493$.

ü. s. Lebenswerk $63 \mathrm{f}$. als Gymnasiallehrer 159f., 344f.

als Universitätslehrer $302-304,311$ f.; s. a. 63.

Einheitlichkeit seiner Methoden 173, 303f.

Überlegenheit über seine Zeitgenossen 287, 304.

- $\quad$ 'sche Schule 304; vgl. a. 63, $302 \mathrm{f}$.

ü. Abel 13\%, 325 .

- ü. Abel u. Jacobi 325

- an S. Koralevsky 64, 255, 325.

- u. Kronecker 64 f., 305.

- $\quad$ u. Kronecker 62-64, $325,403$.

- $\quad$ u. Kummer 324f.

- $\quad$ u. Riemann 66, 151, 325.

- $\quad$ an H. A. Schwarz 66.

- u. Steiner 316, 410.

- $\quad$ u. J. J. Sylvester 262.

Wellhausen, J. 357.

s. a. u. "Poincare".

Weltformel Laplace's $3 \mathrm{ff}$.

Werkzeug, Math. ein - 74 .

Wertheim, W. 404.
Whewell 388, 488.

White, s. Albius.

wichtigste Begriffe der Math. $51 \mathrm{f}$.

- math. Disciplin $66 \mathrm{f}$; s. a. $146,188$.

- resp. fruchtbarste Entdeckung des 19. Jahrh. 188, $428,445,447,448$; vgl.a. 333 .

Wiedemann, G. 398, 415.

ü. Helmholtz 325 f., 398, s. a. 414.

Wiener, Chr. 225, 242.

Wiese, L. 480, 490.

Wild, philosophisches 51 .

Wimn, Mary 227.

Winnecke, F. A. T. 11, 91, 123.

Wiss., Ziel der - 1, $3 \mathrm{f}$.

- Z Zweck der - 1 ff., $191 \mathrm{f}$.

Wittstein, Th. 121.

Wöhler, $F$. an J. Berzelius ü. Arago u. Ampère 401.

- an J.Berzeliusü.W.Weber $\mathbf{3 . 1 3}$

- s. a. u. "Berzelius".

Wolff, Chr. 224, 407.

Wolzogen, Frau $v .-467$.

Wöpcke, Franz 277, 404.

Worpitzky, J. 48\%.

Wunder 283, 387.

Zach, Frhr. v. -116; s. a. u. "Gauss". Zahlensystem, dekadisches 38, 284.

Zahlentheorie, Vorliebe für - $54 \mathrm{f}$., 67,361 f., 378 ; vgl. a. 315,375 .

- , Zurücksetzung u. Isolierung $54,61 \mathrm{f}$.

s. a. Arithmetik.

Zeichen u. Zeichensprache der Math. $39,43,59,60,252,262,435-437$, $445,452,454$; s. a. „Formeln", "Symbole".

Zeichuen $210 \mathrm{f}$.

Zeller, Ed. 216 .

Zerstreutheit $310 \mathrm{f}$., 407.

Zöllner, Friedr. 6.

Zufall 2, 11, 386, 470.

Zwistigkeiten 143, 256, 452, 459-461, $462-466,472 \mathrm{f}$. 


\title{
Mathematische Unterhaltungen und Spiele.
}

\author{
[XII und 428 S.] gr. 8. 1901.
}

In Originalband m. Zeichnung von P. BürkK-Darmstadt n. $\mathscr{M} 10$.Geheftet in 2 Teilen. I. n. $\mathscr{M}$ 5.- II. n. $\mathscr{M}$ 5.-

Urteile der Presse:

Die äußerst schwierige Aufgabe, diese Dinge so zu behandeln, $\mathrm{da} B$ nicht $\mathrm{blo} B$ der Laie mit Verständnis folgen kann, sondern $\mathrm{da} B$ auch das Interesse des Mathematikers von Fach gefesselt wird, hat der Verf. in einer Weise gelöst, die der höchsten Anerkennung wert ist. . . . . Nehmen wir hinzu, daß der Verf. überall, soweit es möglich ist, dem Ursprung und der historischen Entwicklung der behandelten Aufgaben nachgeht und darin eine geradezu erstaunliche Belesenheit zeigt; $\mathrm{daB}$ or trotz der Allgemeinverständlichkeit seiner Darstellung doch nur solche Dinge behandelt hat, denen auch ein wissenschaftliches Interesse alggewonnen werden kann; $d a B$ er die behandelten Aufgaben auch durch eigene Untersuchungen wesentlich gefördert hat, so müssen wir unser Urteil dahin zusammenfassen, $\mathrm{da} B$ sein Buch nicht blob dem Laien und zugleich dem Mathematiker von Fach eine angenehme Unterhaltung bietet, sondern auch für den letzteren eine Quelle der Belehrung und eine Veranlassung zum Nachdenken. Alles in allem liegt hier ein Buch vor, an dem man nur Freude haben kann.

$$
\text { E-l., Univ.-Prof. Literar. Centralbl., Juli } 1901 .
$$

Das Buch bietet, was Reichhaltigkeit des Inhalts sowie Korrektheit und Klarheit der Darstellung betrifft, auch dem anspruchvollsten Rezensenten keine Handhabe zu ernsthaften Ausstellungen.

Prof. G. Wertheim. Zeitschr. f. math. u. naturw. Unterr., 1901.

Ganz hervorragenden Wert besitzen die historischen und literarischen Angaben, die - soviel uns bekannt - noch niemals in dieser Vollständigkeit vereinigt und gesichtet worden sind. Daß der Verfasser an zahlreichen Stellen Eigenes gibt, soll nicht unerwähnt bleiben. Im Übrigen möge noch besonders betont werden, daB sich das vorliegende Buch nicht etwa nur an die Mathematiker wendet: trotz seines wissenschaftlichen Strebens sucht der Verfasser auch dem gebildeten Laien gerecht zu werden, und wir glauben, daß diese der Schrift nicht geringeres Interesse und Vergnügen abgewinnen werden als die Mathematiker.

G., Univ.-Prof. Naturw. Wochenschr., 1901. 
Die Behandlung der Probleme zeugt von pädagogischem Takt.

Prof. Dr. Holzmüller. Zeitschr. f. lateinl. höh. Schulen, 1901.

Les Unterhaltungen ne ressemblent pas aux Récréations de Lucas au point de n'être qu'une espèce de traduction libre, mais on y reconnaît le même esprit et la même méthode. Le tout est traité avec plus d'unité, plus d'ordre, plus de méthode que dans Lucas.

H. Bosmans, S. J. Revue des Questions scientifiques, juillet 1901.

Der Teubnersche Verlag hat mit dem vorliegenden Buch ein sehr bedeutendes Werk auf den Büchermarkt gebracht, das einesteils wegen der umfassenden Behandlung des Gegenstandes, andernteils durch die Gründlichkeit, mit der die meisten Partien abgehandelt sind und die beigefügten Literaturnachweise den Namen eines Standardwerkes verdient.

Joh. Kleiber-München. Bayer. Zeitschr. f. Realschulw.

Wer sich mit diesem Grenzgebiet von Spiel und Wissenschaft, über das ein höheren Anforderungen genügendes Werk bisher im Deutschen noch gar nicht vorhanden war, näher beschäftigen und so gewissermassen sich einen leichteren Zugang zum Verständnis mathematischer Probleme bahnen will, findet in dem vorliegenden Buche außerordentlich reichhaltigen, auf gründlichen mathematischen und geschichtlichen Studien beruhenden Stoff zur Belehrung. Dabei ist die Gruppierung übersichtlich und die textliche Behandlung der einzelnen Probleme sehr anschaulich. was das Studium des Buches wesentlich erleichtert. Das Buch wird zunächst im Kreise der Fachgelehrten, dann aber auch unter mathematisch interessierten Laien zweifellos die verdiente Verbreitung finden; es dürfte sich in hohem Mabe auch als Geschenk fuir eifrige Mathematiker unter den Schüleru höherer Lehranstalten eignen.

C. S. Schles. Zeitg., Sept. 1901.

Zahlreiche ähnliche Besprechungen in wissenschaftlichen Blättern und Tageszeitungen, wie Monatshefte für Mathem. u. Phys. 1901; Archiv der Mathem. u. Phys. 1902; Jahrbuch über die Fortschritte der Mathem. Band 31; Kölnische Volkszeitung 17. Dezember 1900; Magdeburgische Zeitung 15. Dezember 1900; Frankfurter Zeitung 25. Mai 1902; Illustrierte Sonntagszeitung 1900 etc. etc. 



\section{SEVEN DAY RESERVE BOOK}

Return to desk from which borrowed. This book is due on the LAST DATE MPTH $\$ 747$ stamped below. 
$-3,27$

QA

4

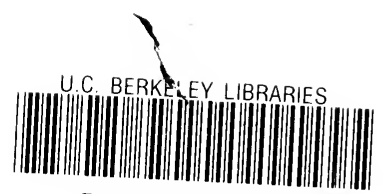

C037442968 
